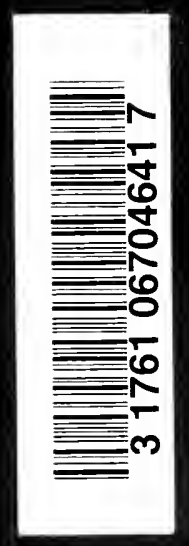





CLINICAL OPHTHALMOLOGY

FOR THE

GENERAL PRACTITIONER 
PUBLISHED BY THE JOINT COMMITTEE OF HENRY FROWDE AND HOLDER \& STOUGHTON, 17 WARWICK SQUARE, NEWGATE STREET; LONDON, E.C. 4 
$R$

\section{Clinical Ophthalmology}

\section{for the}

\section{General Practitioner}

BY

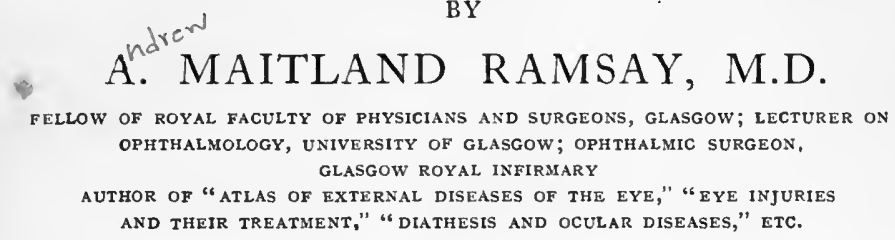

WITH FOREWORD BY

SIR JAMES MACKENZIE, M.D., F.R.S.

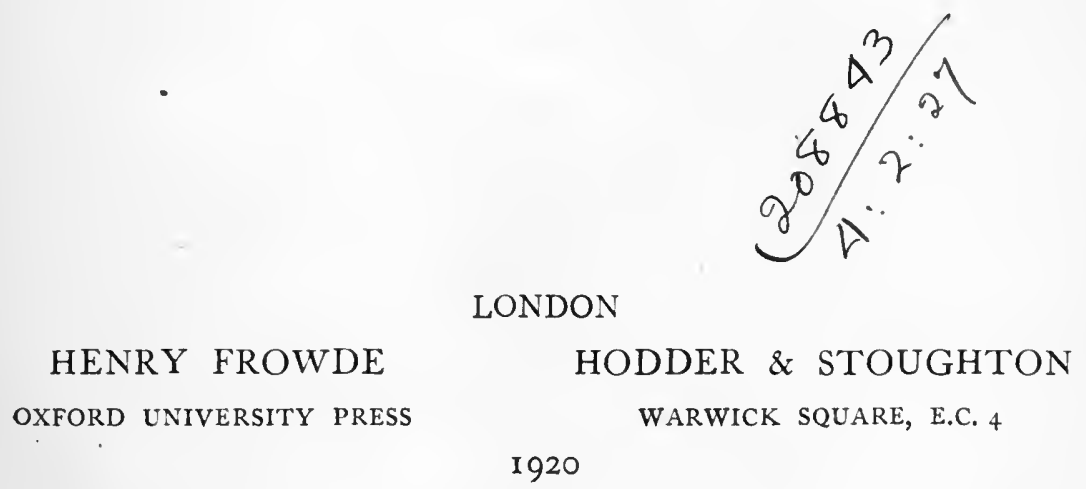

AND THEIR TREATMENT," "DIATHEStS ANd ocular diseases," etc.

\section{LONDON}

HENRY FROWDE OXFORD UNIVERSITY PRESS
WARWICK SQUARE, E.C. 4

\section{HODDER \& STOUGHTON}

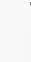


PRINTED IN GREAT BRITAN BI RICHARD CLAY AND SONS, LTD., BRUNGWICK STREET, STAMFORD STREET, S.E. 1, AND BUNGAY, SUFFOLK. 
TO

MY WIFE 


\section{Digitized by the Internet Archive in 2007 with funding from Microsoft Corporation}




\section{PREFACE}

THE author spent ten years in general practice before he specialised in Eye Diseases, and that experience has greatly influenced his outlook on Ophthalmology.

He has written this book, which is the outcome of his personal clinical experience, for the busy general practitioner, and more especially for the country doctor. His aim has been to indicate, as far as possible, the cases which the general practitioner can treat safely on his own responsibility, and those in which it is desirable to obtain a consultation with an expert. $\mathrm{He}$ has tried to bring the treatment of diseases of the eye into line with every-day practice, and to establish the intimate connection between the eye and other parts of the body. A careful study of the eye, in many instances, gives warning of incipient general disease, at a time when treatment offers a reasonable prospect of success, and in cases presenting symptoms of advanced cardiac or renal disease, an ophthalmoscopic examination may often afford valuable assistance in determining which of the two is the primary disorder. The practitioner can also see in the eye the working of a morbid process, and can observe its results. In pneumococcal ulcer, for example, he can see how a microbe acts when it invades the tissues from the outside, and in interstitial keratitis and also in iritis he can see the action on the tissues of a micro-organism conveyed by the blood. In phlyctenular conjunctivitis, on the other hand, he can observe the important influence of soil on all forms of infection; while in chronic glaucoma he has, in all probability, an example of the degenerative changes in structure which occur as a result of the action of chemical toxins due to disordered metabolism.

The book is not meant to take the place of a systematic treatise on Ophthalmology. It is purely clinical, deals with the symptomatology of Eye Diseases, and is an attempt to present the subject as the general practitioner meets it in his daily practice. Each chapter is so far complete in itself; and while that necessarily involves some repetition, it is hoped that the doctor will not find the arrangement a disadvantage, but rather a help in so far as it may enable him the more quickly to obtain the information he seeks in any particular case.

The author desires to thank Dr. George Hunter for reading the manuscript and for many valuable suggestions, and Dr. Walter H. Kiep for assistance in the preparation of the Glossary and in the description of the plates.

A. Maitland Ramsay.

\section{Glasgow,} July 1920. 



\title{
Foreword
}

\section{ON THE WRITING OF TEXT-BOOKS}

\author{
By SIR JAMES MACKENZIE, M.D., F.R.S.
}

WHEN I was asked to write a preface, introduction, or foreword, to Dr. Maitland Ramsay's book on Diseases of the Eye, I at first refused, because I endeavour to avoid saying anything about a subject of which I am ignorant. But when it was pointed out that it was at my instigation Dr. Ramsay had undertaken the onerous work of writing this book, I saw the reasonableness of the request, and I now comply with it, not to comment on affections of the eye, but to give the reasons which influenced me in asking Dr. Ramsay to write.

When I entered general practice, I had a strong belief that medical knowledge had reached such a pitch that everything essential to the diagnosis and treatment of patients was known. Conscious of my own ignorance, as time went on, I got together a small library containing the most recent text-books on a variety of subjects. I remember how I saved to buy that characteristic Teutonic production, Ziemssen's Encyclopadia. After I got that I felt I could tackle any medical problem. But the more I turned for help to my text-books the more bewildered I became. Time after time I failed to get the information I sought, and so implicit was my confidence that I laid the fault down to my own incapacity in not being able to glean from the books the desired knowledge. In a text-book everything is made so simple, and every disease is recorded from every point of view, with full information about ætiology, symptoms, differential diagnoses, prognosis, and treatment, and often with an excursion into the history of the complaint and its discovery. A patient would consult me, and I might detect a heart murmur. It might be a pregnant woman, it might be a man doing heavy work, it might be a man whom I was examining for life insurance. I wanted to know what risk the individual ran. Recognising my inexperience, I turned to my text-book and failed to elicit the desired information. This happened so often and in connection with so many other signs and symptoms that I surmised I had bought the wrong text-books, and so hied me to a library. This I ransacked but failed to find the desired information. Then it gradually dawned upon me that text-books were 
not what they seemed. Further experience elicited the fact that textbooks are rarely written from the standpoint of the practising doctor, but usually from the standpoint of the post-mortem findings, or from the signs of disease observed just before the individual reaches the post-mortem room.

A further experience of men and matters revealed the methods of the writers of text-books. Doctors who have had many years hard work seldom find time to write a text-book after they have gained an experience wide enough to justify them in doing so. The young man who desires to be known as an authority on a subject has the time, so he does not hesitate to write the text-book. His experience is too limited to qualify him for the undertaking; so when he sets to work he sits down at a table and surrounds himself with all the books bearing on the subject that he can lay hands on, and culls from them the pearls of wisdom which he subsequently publishes as his contribution to the subject. The indulgent reviewer is impressed with the wide learning of the author, and praises the book for its professed erudition. A front page advertisement of the book in a medical journal, with a laudatory sentence tagged on from some review, lays the foundation of the author's success as an authority. In later.years the author becomes astonished at his youthful erudition, and may be found quoting, "What I said twenty-five years ago," as if the limits of medical knowledge on that subject had been reached when he gave to the world his text-book.

When one reflects that probably 95 per cent. of the cases a general practitioner meets in his daily work are undiagnosable from the textbook point of view, the conclusion is forced upon one that it is time a change was made in the authorship of these books. The reason that text-books assume their peculiar features is that disease has been classified on the basis of the pathology of individual organs. The pathological state is not recognised until the disease has advanced so far as to produce a physical sign or until the patient has reached the post-mortem table. As, however, people with impaired health rarely show these characteristic signs, and then only late in their history, it is manifest that the text-book description can only refer to advanced stages of disease. As the vast majority of people whom a general practitioner sees are people without those physical signs of advanced disease, it .can be seen why text-books are so useless.

Placing this view of the matter before Dr. Ramsay he recognised its truth, and I then pointed out that, as he had reached mature years and had a very wide experience, he should write a text-book for the general practitioner, drawing his information from his own experience and not copying the beliefs and superstitions of his predecessors. This he has 
done, and written a book to which the general practitioner can turn with confidence to find the knowledge he wants.

My experience has led me to see that in writing text-books, as in teaching, the author or teacher should verify every statement he makes if it does not arise from his own experience. Medical writing has been so slipshod that statements due to hasty and imperfect observation have been accepted as "facts" because some one who had a reputation had given utterance to them. If these "facts" were verified, many a belief cherished and propagated from text-book to text-book would be found to be valueless.

Another matter of importance is that when an author does make a statement from his own experience, he should give the reasons he had for making it, and the proofs on which it is based. I have so often sought for the reasons for accepted beliefs and found them based on such flimsy evidence, that I have no doubt that a vast bulk of what to-day passes as tested knowledge will be scrapped in course of time. If authors would but recognise this, genuine work would be sooner recognised, while the seeker after truth would be spared wading through the vast amount of verbiage which to-day darkens medicine.

July 1920. 


\section{CONTENTS}

\section{CHAPTER I}

THE METHODICAL EXAMINATION OF THE EYE

General principles-Special rules-Method of examining a child's eyesScheme for the examination of the anterior part of the eye-The examination of vision and the refraction of the eye-The examination with the ophthalmoscope.

\section{CHAPTER II}

IMPORTANT SYMPTOMS IN DISEASES OF THE EYE

Importance of the correct interpretation of symptoms-Disorders of general sensibility-Inflammatory pain-Non-inflammatory pain-Retinal and muscular asthenopia-Neuralgia-Disorders of the visual function-Sudden loss of sight-Gradual loss of sight.

\section{CHAPTER III}

DISEASES OF THE TEAR PASSAGES

Significance of a "watery eye "-Causes of over-secretion of tears-Causes of obstruction to the outflow of tears-Conjunctivitis accompanying lachrymal obstruction-Lachrymal catarrh-Blennorrhcea of the tear-sac -Mucocele-Caries of lachrymal bone-Acute dacryocystitis-Lachrymal fistula-Treatment of epiphora-Treatment of lachrymal catarrh-Treatment of catarrh of the lachrymal sac and stricture of the nasal duct-Method of passing a probe-Treatment of acute dacryocystitis-Treatment of permanent enlargement of the lachrymal sac with or without a fistulaOperation for extirpation of lachrymal sac-Summary of methods of treatment.

\section{CHAPTER IV}

THE CLINICAL SIGNIFICANCE OF EEDEMA OF THE EYELIDS • • • CEdema of the lids not a disease per se-Non-inflammatory œdema-Inflammatory œdema: (a) when unaccompanied by disease of eyeball; (b) when accompanied by disease of eyeball without impairment of movements or position; $(c)$ when accompanied by disease and displacement of eyeball with impairment of its movements.

\section{CHAPTER V}

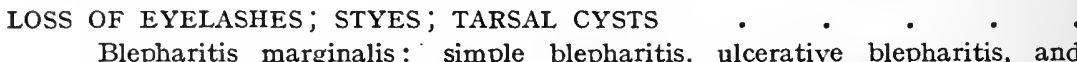
Blepharitis marginalis: simple blepharitis, ulcerative blepharitis, and
hypertrophic blepharitis: symptoms, prognosis, and treatment-Hordeolum or stye : symptoms: differential diagnosis : treatment-Chalazion or tarsal cyst: differential diagnosis, symptoms, course, and treatment.

\section{CHAPTER VI}

BLEPHAROSPASM； LAGOPHTHALMOS ; PTOSIS Blepharospasm : intermittent or permanent-Tic douloureux-Lagophthalmos:-cicatricial, paralytic-Facial palsy and its causes-Neuro-paralytic keratitis-Treatment of lagophthalmos-Ptosis:-hypertrophic, traumatic, congenital, senile, and paralytic: treatment. 


\section{CHAPTER VII}

ENTROPION AND ECTROPION

Entropion: spasmodic and cicatricial : trichiasis : treatment-Ectropion : spasmodic, cicatricial, senile, and paralytic-Epiphora-Blepharitis hypertrophica : treatment.

\section{CHAPTER VIII}

SYPHILIS OF THE EYELID; TUBERCULOSIS OF THE EYELID; EPITHELIOMA; RODENT ULCER .

Syphilis: chancre of eyelid: modes of infection : clinical course and treatment-Tuberculosis: clinical types: treatment-Epithelioma-Rodent ulcer.

\section{CHAPTER IX}

THE CLINICAL SIGNIFICANCE OF A "BLOOD-SHOT" EYE • • . A sign common to all inflammations implicating the anterior segment of the globe-The blood-supply of the front of the eyeball-Conjunctival and ciliary hyperæmia: their signs and symptoms-Chemosis-Presence of discharge diagnostic of conjunctivitis-Keratitis, iritis, and glaucoma as causes of hyperæmia, and the methods of distinguishing between themFascicular injection-Injection in scleritis-Varicose injection-Sub-conjunctival hæmorrhage.

\section{CHAPTER $\mathrm{X}$}

\section{INFLAMMATION OF THE CONJUNCTIVA}

Frequency of conjunctivitis-Presence of discharge-Acute conjunctivitis : catarrhal, purulent, membranous-The importance of discovering the infecting agent-Method of taking a smear-Bacteria found in conjunctivitis-The clinical signs of conjunctivitis-Treatment: local, general, and prophylactic.

\section{CHAPTER XI}

\section{ACUTE INFLAMMATION OF THE CONJUNCTIVA}

Catarrhal conjunctivitis: mode of spread of contagion, clinical course, causal micro-organisms: treatment-Argyrosis-Purulent conjunctivitis: of gonococcal origin: liability to ulceration of the cornea : clinical stages - Metastatic gonococcal conjunctivitis-Treatment of purulent conjunctivitis-Membranous conjunctivitis-Diphtheria of the conjunctiva: treatment.

\section{CHAPTER XII}

OPHTHALMIA NEONATORUM AND ITS CONSEQUENCES • • • • Its frequency as a cause of blindness-Two-thirds of all cases due to gono-
coccus-Other causal micro-organisms-Time of onset and clinical courseAccompanying constitutional disturbance-Occurrence of corneal ulceration -Its sequelæ: leucoma with pyramidal cataract, leucoma adherens, anterior staphyloma, general distension of the globe-Prognosis-Prophylaxis - Treatment-Nitrate of silver and its substitutes-Vaccine treatment.

\section{CHAPTER XIII}

CHRONIC INFLAMMATION OF THE CONJUNCTIVA ..$\quad$..$\quad$. Characteristics of chronic conjunctivitis-Angular conjunctivitis: due to diplobacillus of Morax and Axenfeld, sometimes complicated by corneal ulcer : treatment-Follicular conjunctivitis: treatment-Spring catarrh: treatment-Parinaud's conjunctivitis-Trachoma : complications, pannus, trichiasis, entropion, xerophthalmia, trachomatous ptosis-Treatment of trachoma. 


\section{CHAPTER XIV}

PHLYCTENULAR CONJUNCTIVITIS OR STRUMOUS OPHTHALMIA .

Essentially a disease of children-Intensity of photophobia in this disease -The phlycten-Occurrence of ulceration-Absence of discharge-Connection with tuberculosis-Occurrence of eczema and gastro-intestinal disturbance-Method of examining the child-Treatment: constitutional and dietetic-Tartar emetic-Local treatment-Treatment of complications.

\section{CHAPTER XV}

AFFECTIONS OF THE BULBAR CONJUNCTIVA • • . . . Pinguecula - Pterygium - Lymphectasia - Lipoma - Dermoid cyst-Tuberculosis-Sarcoma-Epithelioma.

\section{CHAPTER XVI}

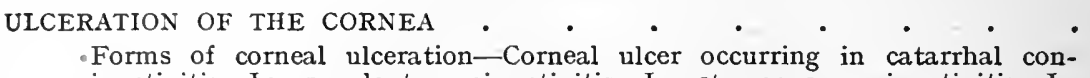
junctivitis-In purulent conjunctivitis-In strumous conjunctivitis-In trachoma-In trichiasis-In cases of abnormal exposure of the eyeballIn diseases characterised by interference with the corneal nerve supplyMarginal keratitis-Herpes cornex-Neuroparalytic keratitis-Keratomalacia-Ulceration due to breaking down of a cicatrix-Methods of examination-Clinical course-Complications--Perforation-Hypopyon-Prognosis -Treatment.

\section{CHAPTER XVII}

SERPIGINOUS ULCER OF THE CORNEA, AND THE DUTY OF THE GENERAL PRACTITIONER IN ITS PREVENTION AND TREATMENT

Occurrence of infection of corneal abrasions-Pneumococcal ulcer-Clinical course and sequel $x$-Prognosis-Pathology-Preventive treatment-attention to tear-passages: first aid-Curative treatment-The use of antiseptics -Ethylhydrocuprein-Vaccines-Cauterisation-Saemisch's operation.

\section{CHAPTER XVIII}

OPACITIES OF THE CORNEA

Causes-Varieties of resulting cicatrices-Prognosis-Treatment-Keratoconus-Keratoglobus--Arcus Senilis.

\section{CHAPTER XIX}

INTERSTITIAL KERATITIS-NON-SUPPURATIVE KERATITIS Varieties-Signs and symptoms-Complications-Stigmata-Treatment.

\section{CHAPTER XX}

THE DIAGNOSIS AND TREATMENT OF IRITIS

Idiopathic iritis-Clinical description "Quiet Iritis"-Acute Iritis-Symptoms-Sequelæ-Treatment.

\section{CHAPTER XXI}

CLINICAL TYPES OF IRITIS AND THEIR TREATMENT $\bullet$ -
Syphilitic iritis: treatment-Arsenical compounds-Mercury-IodidesGeneral-Toxic iritis: types of-Clinical features-" Recurrent iritis"Treatment-Tuberculous iritis-Differential diagnosis from syphilitic iritis -Prognosis-Treatment. 


\section{CHAPTER XXII}

THE COMPLICATIONS AND THE SEQUELE OF IRITIS, AND THEIR TREATMENT

Anatomical consideration-Cyclitis-The clinical importance of the exudate -Occlusion of the pupil-Iris bombé or iridoncosis-Secondary glaucomaShrinkage of the globe-Irido-choroiditis - Treatment: medicinal and dietetic-Renal inadequacy an important cause of chronic uveitis-The use of anti-diphtheritic serum-Operative treatment-Treatment during convalescence.

\section{CHAPTER XXIII}

INFLAMMATION OF THE SCLEROTIC • • • Episcleritis : symptoms--Episcleritis periodica fugax-Sclerotitis : symptoms -Sclerotinising keratitis-Treatment: local: general.

\section{CHAPTER XXIV}

THE CLINICAL SIGNIFICANCE OF A HARD EYE

Increased tension of the eyeball-Secondary glaucoma-Primary glaucoma - Causation-Symptoms, early and late-Alterations in the cornea and sclerotic-Alterations in the uveal tract-Changes in the transparent media - Changes in the retina and optic nerve-Pathology-Non-congestive or simple chronic glaucoma-Symptoms-Glaucoma and cataract-Glaucoma and atrophy of the optic nerve-Congestive or acute glaucoma-Symptoms -Glaucoma and iritis-Hæmorrhagic glaucoma.

\section{CHAPTER XXV}

THE CLINICAL SIGNIFICANCE OF A HARD EYE (continued) . . . The treatment of glaucoma: medical: general, local: surgical-Iridectomy, sclerotomy, sclerectomy-Post-operative .complications.

\section{CHAPTER XXVI}

THE DUTY OF THE GENERAL PRACTITIONER TOWARDS A PATIENT SUFFERING FROM CATARACT-BEFORE, DURING, AND AFTER THE OPERATION Classification of cataract-Symptoms and signs-Procedure in examination - Prognosis-Treatment in early stage-Conditions necessary before undertaking operation - Preparation of the patient - Choice of operationInstruments - Post-operative treatment - Accidents during and after operation.

\section{CHAPTER XXVII}

THE CLINICAL SIGNIFICANCE OF EXOPHTHALMOS .

Exophthalmos a cardinal sign of disease of orbit-Simulated proptosisEtiology of proptosis-Exophthalmos accompanied by signs of inflammation -Tenonitis-Orbital abscess-Cavernous sinus thrombosis-Exoph thalmos unaccompanied by signs of inflammation-Graves' disease-Causes of unilateral proptosis-Pulsating exophthalmos-Proptosis due to tumour.

\section{CHAPTER XXVIII}

SARCOMA OF THE CHOROID, AND GLIOMA OF THE RETINA • . .

Sarcoma: origin, course, termination, treatment-Glioma: site, course, differential diagnosis, prognosis, treatment-Illustrative case. 


\section{CHAPTER XXIX}

GENERAL PRINCIPLES UNDERLYING THE TREATMENT OF INJURIES TO THE EYEBALI.

General considerations-The protective mechanism of the eyes-Methods of preventing infection of the wound-Prophylaxis of ocular injuries.

\section{CHAPTER XXX}

THE TREATMENT OF SUPERFICIAL INJURIES TO THE EYES

Importance from an industrial standpoint-Foreign body beneath the upper lid-Foreign body in the cornea-Dangers of infection of cornea in cases in which tear passages are diseased-Illustrative cases-Recurrent traumatic keratalgia.

\section{CHAPTER XXXI}

BURNS OF THE EYE .

Necessity of a guarded prognosis-Clinical course-Symblepharon-Anchyloblepharon-Treatment-Operations for symblepharon-Electric ophthalmia - X-ray and Radium burns-Burns from warfare gases.

\section{CHAPTER XXXII}

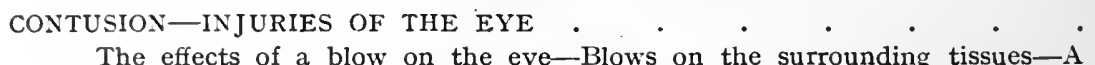
The effects of a blow on the eye-Blows on the surrounding tissues-A
black eye-Emphysema of lids-Injuries to optic nerve-Ophthalmoplegia -Blows on the eyeball itself-Commotio retinæ-Hæmorrhage, sub-conjunctival and intraocular-Lesions of iris and ciliary body-Lesions of choroid-Lesions of retina-Detachment of retina-Dislocation of lensTraumatic cataract-Rupture of eyeball-Treatment.

\section{CHAPTER XXXIII}

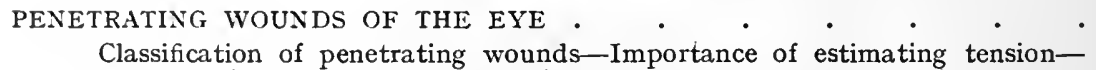
Wounds of the cornea-Wounds of the sclerotic-Involvement of the lensSepsis - Treatment by the general practitioner-Specialist treatmentRecent prolapse-Excision and formation of conjunctival flap-Prolapse complicated by adhesions-Administration of anti-diphtheritic serumTreatment of panophthalmitis-Prothesis.

\section{CHAPTER XXXIV}

PENETRATING WOUNDS OF THE EYE WITH RETENTION OF A FOREIGN BODY

Importance of determining the presence of a foreign body in the eyeSiderosis bulbi-Localisation of the foreign body-Application of the magnet -Fate of the injured eye-After-treatment-Injuries from dynamite explosions and their treatment.

\section{CHAPTER XXXV}

SYMPATHETIC OPHTHALMIA

Conditions in which sympathetic ophthalmia is likely to occur-Time of onset-Sympathetic irritation-Sympathetic inflammation-Theories as to paths by which infecting agent travels from one eye to the other-Intractability of the disease in the sympathising eye-Treatment: prophylactic and curative. 


\section{CHAPTER XXXVI}

EYE INJURIES, WITH SPECIAL REFERENCE TO THE WORKMEN'S COMPENSATION ACT, I906.

The Workmen's Compensation Act of 1906-Necessity of careful examination and note-taking-Pre-existing or acquired disease as a complicationEstimation of the damage to sight from an economic point of view-The visual acuity-The field of vision-Binocular vision-Muscular defectsSympathetic ophthalmia as a complication-Questions involving operation -Malingering.

\section{CHAPTER XXXVII}

LONG SIGHT, SHORT SIGHT, AND ASTIGMATISM, AND THE PRESCRIBING OF SPECTACLES

Apparatus required-The pinhole test-The optical structure of the eye -Accommodation-Presbyopia-Failure of accommodation due to disease - Hypermetropia as a cause of disability for near work-Its diagnosis and treatment-The use of cycloplegics-Myopia : its diagnosis and treatment-Spasm of the ciliary muscle causing low myopia-Astigmatism : varieties, diagnosis, and treatment-High hypermetropia-Insufficiency of convergence.

\section{CHAPTER XXXVIII}

MYOPIA AND ITS TREATMENT • • • • • • • • Myopia a product of civilisation-Symptoms-Dangers of myopia-Causation-Aim of treatment-Importance of attention to general healthOperative treatment-Prophylaxis.

\section{CHAPTER XXXIX}

CONCOMITANT CONVERGENT SQUINT OCCURRING IN CHILDREN

Strabismus-Binocular vision-Paralytic squint-Concomitant squintDifferential diagnosis-Classification of squints-Convergent strabismusEtiology-Latent strabismus-Apparent strabismus-Examination of patient suffering from squint-Treatment of squint: non-operative and operative.

\section{CHAPTER XL}

\section{EYE-STRAIN AND ITS CONSEQUENCES}

The importance of eye-strain as a cause of constitutional troubles-Asthenopia-The mechanism of eye-strain-The use of cycloplegics-A small error of refraction more apt to produce eye-strain in a neurasthenic patient -The importance of exercise and general treatment in dealing with cases of eye-strain-Co-existence of functional disorders of the liver-Medicinal treatment-Fye-strain due to psychical causes.

\section{CHAPTER XLI}

THE PUPIL IN HEALTH AND DISEASE .

Abnormalities in the shape and colour of the pupil-Factors influencing the size of the pupil-Myosis : as an accompaniment of an ocular affection; occurring in the course of acute and of chronic general disease-Mydriasis: as an accompaniment of an ocular affection; occurring in cases of injury to the skull or orbit; occurring in the course of general disease-Inequality of the pupils-Method of testing the pupillary reflexes-Hippus-The paths of the pupillary reflex arcs-Abnormalities of pupillary reactionThe Argyll Robertson phenomenon-Wernicke's hemiopic pupillary reflexIrido-dilatation reflex. 
PARALYSIS OF AN OCULAR MUSCLE-THE CLINICAL SIGNIFICANCE OF DOUBLE VISION .

Anatomy and physiology of the ocular muscles-Homonymous diplopiaCrossed diplopia-Signs and symptoms of paralysis of an ocular muscleHow to examine a patient who is suffering from diplopia-Causation of diplopia-Treatment of diplopia.

\section{CHAPTER XLIII}

THE CLINICAL SIGNIFICANCE OF NYSTAGMUS • • • • • Congenital nystagmus-Hereditary form of the disease-Acquired nystagmus-Associated with disease of the ear and with disease of the nervous system-Occupational nystagmus-Description of miners' nystagmusPrognosis.

\section{CHAPTER XLIV}

THE CLINICAL SIGNIFICANCE OF FAILING SIGHT General considerations on the use of the ophthalmoscope-Points to be
Ge General considerations on the use of the ophthalmoscope-Points to be
borne in mind in testing vision-Determination of the visual acuity, the pupillary reflexes, and the extent of the visual field.

Loss of central vision with preservation of peripheral vision-ScotomataHæmorrhage into the macula-Retrobulbar neuritis-Choroido-retinitisMetamorphopsia, micropsia and macropsia-Toxic amblyopia-Hereditary optic neuritis.

Loss of peripheral vision with complete or partial retention of central vision -Optic atrophy-Retinitis pigmentosa-Chronic glaucoma-Hysterical amblyopia-Retinal detachment-Intraocular tumour-Hemianopsia.

Loss of vision throughout the visual field-Profuse intraocular hæmorrhage - Traumatic asphyxia-Embolism of the central artery of the retinaThrombosis of the central vein-Acute retrobulbar neuritis-Primary optic atrophy-Uræmia.

\section{CHAPिTER XLV}

THE OPHTHALMOSCOPE IN GENERAL MEDICINE • • • • .

Anatomical structure of the fundus and its individual components-Papillitis or choked disc-Post-neuritic atrophy-Primary optic atrophy-Causes and treatment of optic neuritis-Uræmic amaurosis-Retinitis albuminurica-Fundus changes in arterio-sclerosis-Prognosis in albuminuric retinitis, in relation to sight and to life-Treatment-Diabetic retinitisChoroiditis disseminata.

\section{CHAPTER XLVI}

AMBLYOPIA ANI) AMAUROSIS

Definitions-Amblyopia, congenital, hysterical, traumatic, and toxic-

Colour-scotomata occurring in toxic amblyopia-Treatment of toxic amblyopia.

\section{THERAPEUTIC NOTES AND FORMULAE}

GLOSSARY

INDEX 


\section{LIST OF PLATES}

Those marked with an asterisk are coloured.

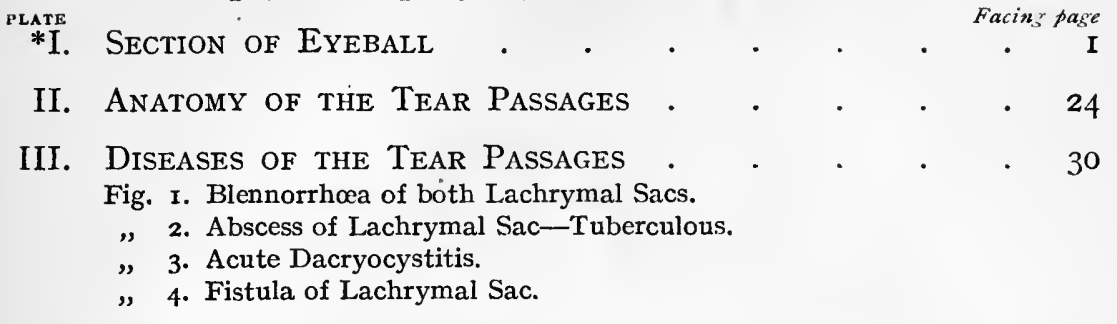

*IV. Syphilis and Tuberculosis of the Eyelid . . . 62

Fig. I. Chancre of Lower Eyelịd.

1) 2. Chancre of Lids with enlargement of Preauricular and Submaxillary Glands.

"3. Tuberculosis of Lower Eyelid.

"4. Tuberculosis of Upper Eyelid.

" 5. Lupus of Eyelids with Implication of Eyeball.

*V. Injection of the Eyeball . . . . . . . . 68

Fig. I. Conjunctival or Superficial Injection.

, 2. Ciliary or Deep Injection.

" 3. Fascicular Injection.

" 4. Varicose Injection.

"5. Chemosis of the Conjunctiva.

" 6. Ecchymosis of the Conjunctiva.

VI. Phlyctenular Conjunctivitis, etc. $\quad . \quad$. $\quad$ IO4

Fig. I. Strumous Ophthalmia.

" 2. Interstitial Keratitis.

" 3. Opacity of Cornea following Strumous Ophthalmia.

" 4. Neuro-Paralytic Keratitis.

*ViI. Affections of the Bulbar Conjunctiva . . . II4

Fig. I. Lipoma.

"2. Dermoid Cyst.

" 3. Tuberculosis of the Bulbar Conjunctiva.

", 4. Syphilis of the Bulbar Conjunctiva.

,5. Sarcoma of the Conjunctiva.

, 6. Epithelioma of the Conjunctiva.

*ViII. Affections of the Cornea and Sclerotic . . . I 48

Fig. I. Opacity of the Cornea.

" 2. Interstitial Keratitis.

" 3. Scleritis with Staphylomata.

*IX. Clinical Types of Iritis . . . . . . . I62

Fig. I. Syphilitic Iritis.

"2. Toxic Iritis.

"3. Miliary Tuberculosis of Iris.

" 4. Conglomerate Tubercle of Iris.

" 5. Tubercle of Iris.

"6. Tuberculous Kerato-Iritis.

xix 
pLATE Facing page

X. Complications ANd Sequele of Iritis . • . . $\quad$ I74

Fig. 1. Iridoncosis.

2. Occlusion of Pupil-Sympathetic Iritis.

3. Section of Eyeball after Occlusion of Pupil.

4. Ciliary Staphylomata.

5. Distended Globe with Staphylomata.

6. Section of Eyeball with Staphylomata.

XI. GlauCOMA - Fig. I. Normal Corneo-Iridic Angle.
Fin

2. Blocked Corneo-lridic Angle in Glaucoma.

", 3. Normal Optic.Nerve Entrance.

, 4. Cupping of Optic Disc in Glaucoma.

XII. Exophthalmos

Fig. I. Abscess of Orbit.

2. Proptosis and Tumour.

3. Tumour of Basi Sphenoid.'

. 4. Bilateral Pulsating Exophthalmos.

*XIII. Sarcoma of Choroid and Glioma of Retina

Fig. I. Sarcoma of Choroid-Intraocular.

, 2. Glioma of Retina-Intraocular.

"3. Sarcoma of Choroid-Extraocular.

"4. Glioma of Retina-Globe Distended.

"5. Section of Eyeball showing both Intraocular and Extraocular Sarcoma.

6. Section of Eyeball showing Globe filled with a Glioma.

XiV. Recurrent Glioma of the Retina • . . . 234

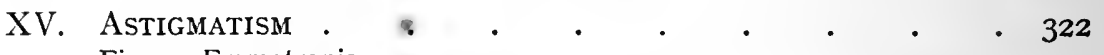

Fig. I. Emmetropia.

2. Simple Hypermetropic Astigmatism.

3. Compound Hypermetropic Astigmatism.

4. Simple Myopic Astigmatism.

, 5. Compound Myopic Astigmatism.

, 6. Miked Astigmatism.

XVI. Mechanism of Accommodation . . . . ' ' 346

*XVit. InNervation of the Iris . . . . . . . 352

*XViII. Paralysis of Ocular Muscles . . . . . . . 362

Fig. I. Action of Ocular Muscles.

„2. Crossed Diplopia.

" 3. Homonymous Diplopia.

" 4. Diplopia upwards.

" 5. Diplopia downwards.

*XiX. The Optic Nerves, the Commissure, and the Optic Traćts 376

*XX. The Ophthalmoscope in Medicine. • . . * 384

Fig. I. Optic Neuritis.

") 2. Post-Neuritic Atrophy.

" 3. Albuminuric Retinitis.

, 4. Primary Atrophy of Optic Nerve.

„5. Choroiditis Disseminata.

, 6 Glaucomatous Atrophy-Deep Cupping. 



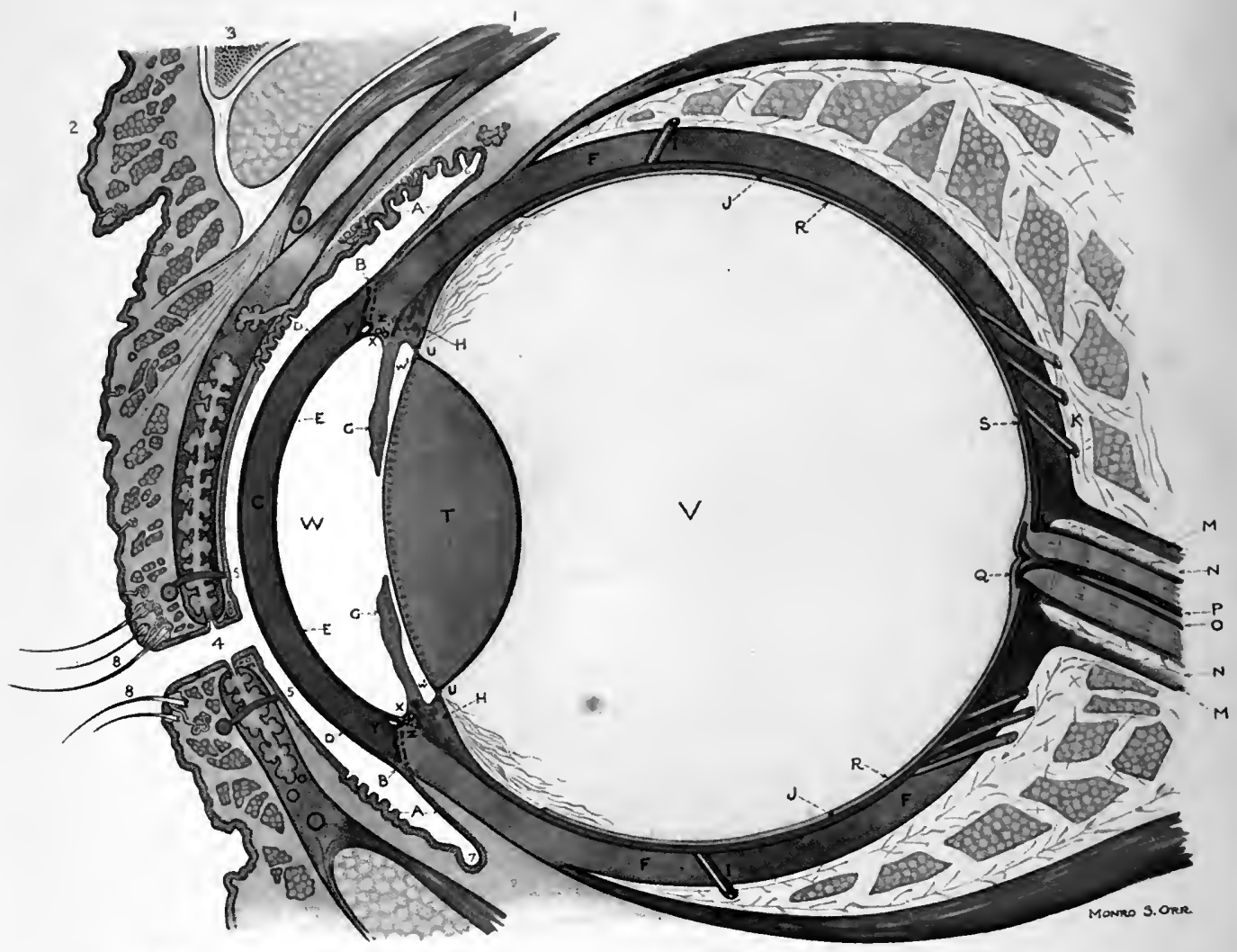


Vertical Section of Lids combined with Horizontal Section of Eyeball (Diagrammatic) Modified from Magnus.
A. Conjunctiva.
B. Limbus corneæ.
S. Macula.
C. Cornea.
T. Crystalline lens.
D. Bowman's membrane.
E. Descemet's membrane.
F. Sclerotic.
G. Iris.
H. Ciliary body.
I. Vena vorticosa.
J. Choroid.
K. Ciliary arteries and nerve.
L. Optic nerve éntrancé.
M. Dural sheath.
N.neArachnoid lc with sub-arachnoidal thespace?
U. Suspensory ligament.
V. Vitreous humour.
W. Aqueous humour.
$W^{1}$. Posterior chamber.
$\mathrm{X}$. Corneo-iridic angle.
Y. Canal of Schlemm.
Z. Spaces of Fontana.
O. Retinal artery.
P. Retinal vein.
Q. Dptic papilla.
R. Retina.
I. Levator palpebræ superioris.
2. Orbicularis pal pebrarum.
3. Orbital margin (frontal bone).
4. Meibomian glands.
5. Tarsal arteries (superior and in- ferior).
6. Conjunctival fornix (superior).
7. Conjunctival fornix (inferior),
8. Cike with glands.

This plate is introduced for the purpose of recalling the main features of the anatomy of the eyeball." In everything pertaining to operations on, and injuries to, the eye, it is absolutely necessary that such a picture be kept constantlyand clearly. before the mind sa The globe is protected by the upper and lower lids, whose edges are fringed with eyelashes. They are closed by the action of the orbicularis muscle, supplied by the facial nerve, and the upper lid is opened tby the levator palpebr: supplied by a branch of the oculp motor. The tissues of which the lids are composed, although continuous with those of contiguous parts, are considerably modified to secure the necessary tightness and mobility. The skin is very thin and loose, containsl few glands or hairs, with little of no fat in the subcutaneous tissues. The shape of each lid is maintained by the tarsal plate, embedded in which are the $M e t$ bomian glands, twenty to thirty in number and opening by minute orifices at the margin of the lid behind the eyelashes: The pal pebral conjunctiva is firmly adherent to the tarsus, is very vascular, and studded by minute papille. It is reflected loosely upon the globe to form the upper and lower retrotarsal folds. At the limbus cornea. it becomes closely adherent to the subjacent structures, and passing over the cornea forms its anterior epithelial layer. Behind this come Bowman's membrane, the substantia propria of the correa, and Descemet's membrane with its lining of endothelial cells. ra Directly continuous i with the cornea, but possessing a different curvature, is the sclerotic, and the two structures together complete the outer or protective tunic of the eyeball. A second tinic is formed by the uveal tract; composed for the mose part of blood-vessels and pigment, and divided from before backwards, for purposes of description; into iris, ciliary body, and choroid. This is the nutritive coat of the eyeball, and is perforated in front to form the pupil, and behind to permit the optic nerve to gain entrance to the interior of the globe. The optic nerve is seen in section surrounded by its subdural and subarachnoidal sheaths; and containing the retinal arteryland vein. At the level of the sclerotic it becomes constricted, and its individual fibres pass through the cribriform plate devoid of their medullary sheaths, so that only naked axis cylinders enter the eyeball as the optic papilla. From this, these expand in all directions to form the retina or sentient tunic. To the outside of the optic papilla and directly in the line of the visual axis is the shallow depression that marks the macula or yellow spot. The cavity of the eyeball is divided by the trystalline lens and its ligament into a large chamber filled by the vitreous, and a small chamber containing the aqueous humonr. The iris is suspended in the aqueous chamber like a movable curtain in front of the lens, with the anterior eapsule of which its pupillary border lies in close contact. Bounded by the iris in front of the lens and its ligament behind is a narrow slit-the posterior chamber-the small size and anatomical position of which serve to explain the ease with which complications and sequelæ occur in inflammation of the iris. At the level of the corneo-iridic angle the canat of Schlemm and the spaces of Fontana are clearly visible. $t$. Note the direo continuation of ivis, ciliary body, and choroid to form the uveal tunic, and the relation of the iris to the capsule of the lens, the posterior chamber, and the corneo-ividic angle. 


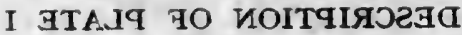

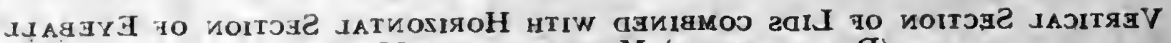

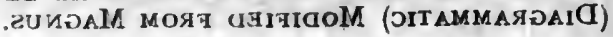

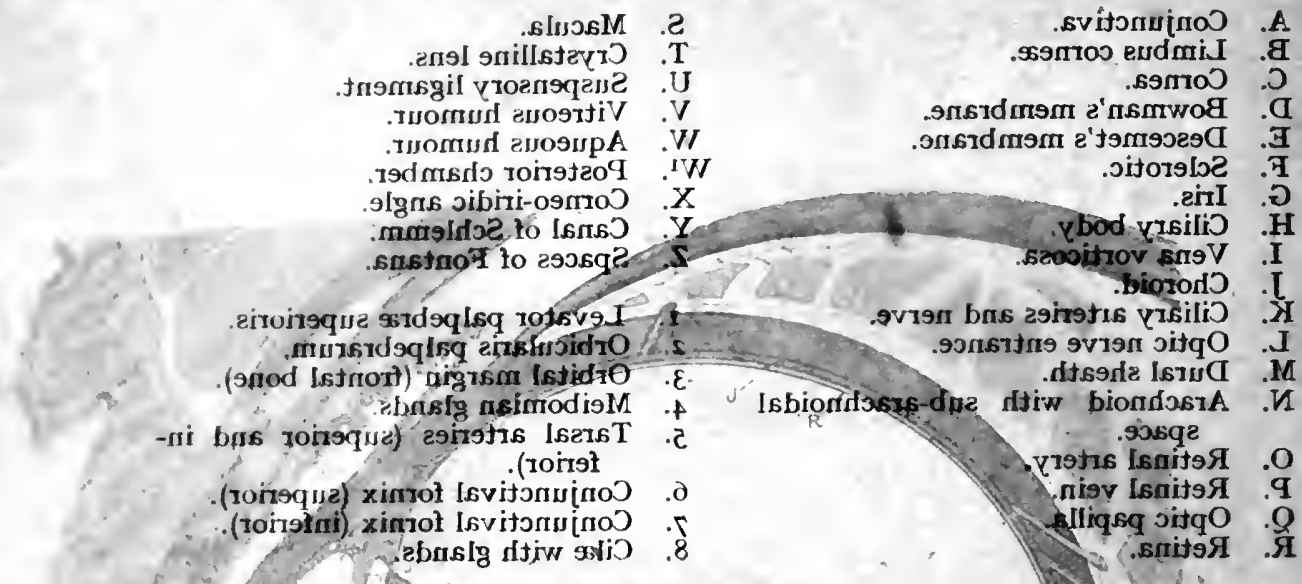

adt to zonutsat aisk ont grills

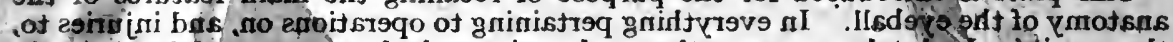

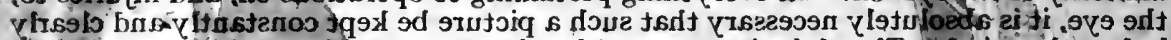

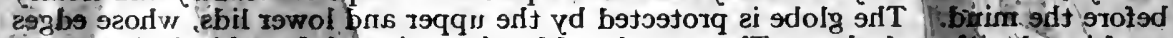

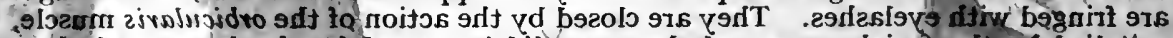

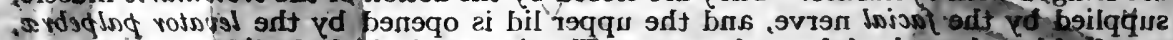

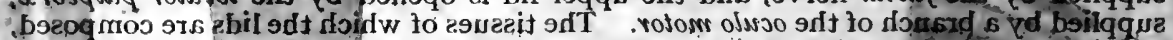

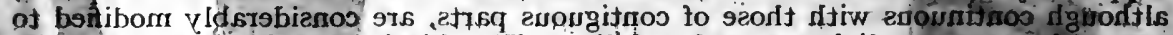

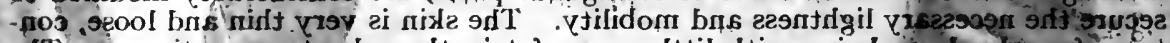

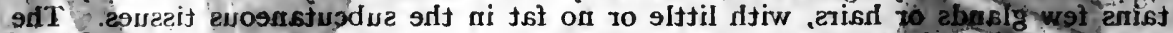

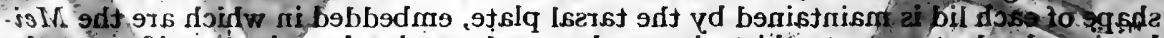

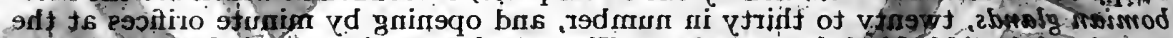

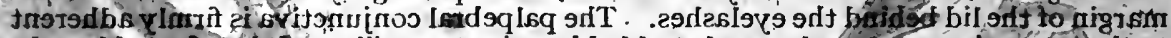

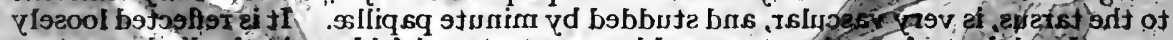

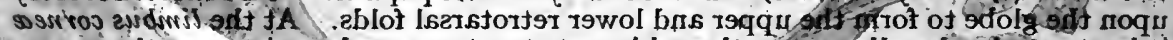

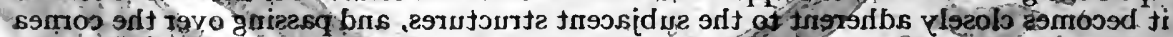

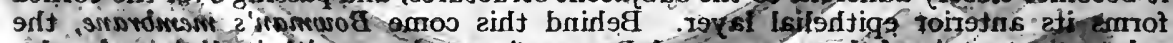

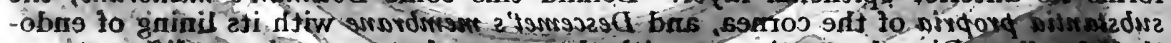

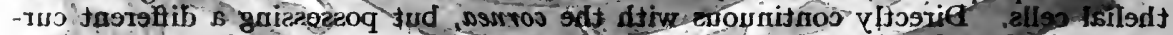

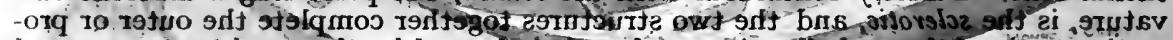

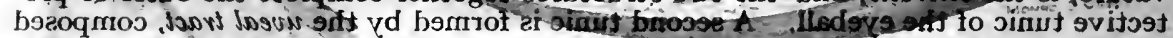
.2bisw'

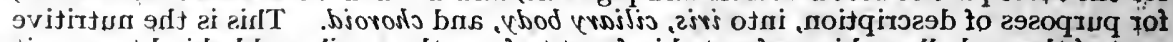

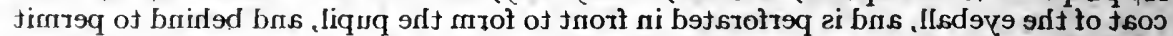

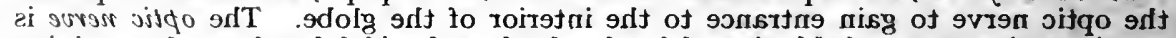

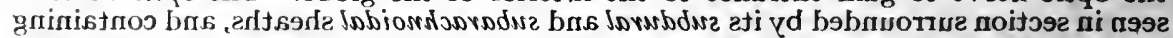

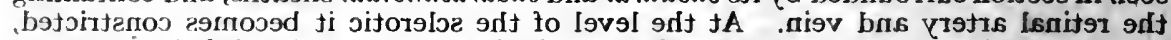

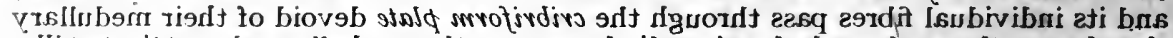

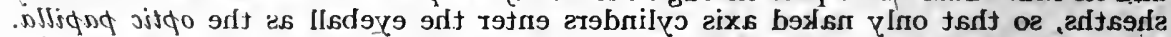

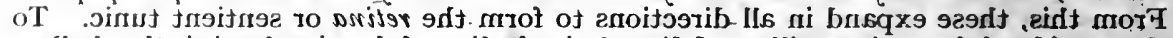

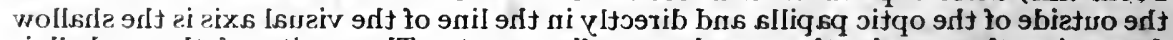

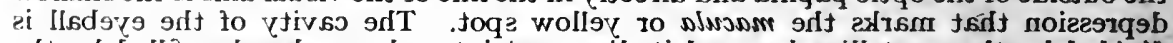

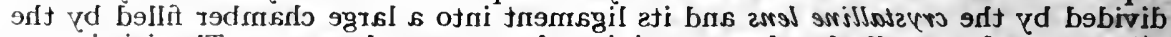

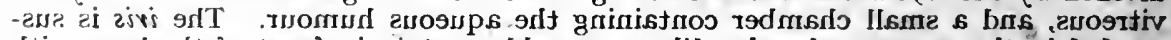

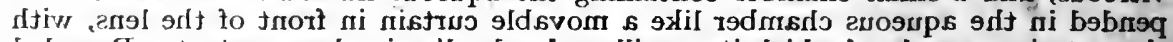

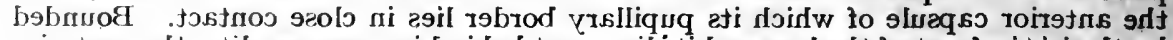

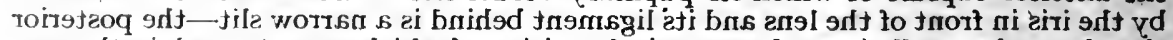

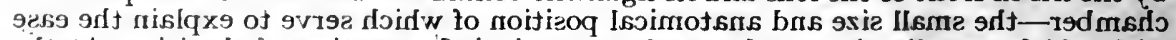

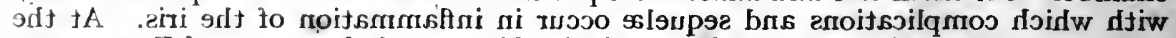

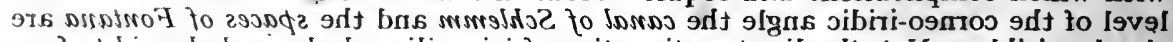

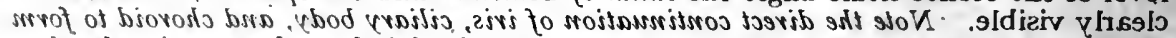

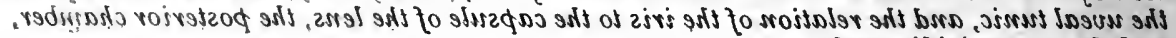




\section{CHAPTER I}

\section{THE METHODICAL EXAMINATION OF THE EYE}

General principles-Special rules-Method of examining a child's eyes-Scheme for the examination of the anterior part of the eye-The examination of vision and the refraction of the eye-The examination with the ophthalmoscope.

WHEN a practitioner is consulted by a patient he is usually guided to a correct diagnosis by observing a sign or a symptom, which his experience has taught him to assess at its proper value. He has acquired that power by careful training. In the early years of his practice he found it necessary to make a thorough examination of every patient as a matter of routine. He thereby taught himself to observe accurately; and as a result of that hard apprenticeship he enabled himself to discover much that would almost certainly be overlooked by a careless and superficial observer. Much time must be given to such detailed examination; but its regular practice inculcates a habit of thoroughness, and supplies the training which is quite essential to enable a doctor to grasp the points that are of real importance in any case presented to him for diagnosis. What is true in regard to medicine in general is equally true of diseases of the eye. When an exhaustive examination is made of every case, constant practice will enable the practitioner to pass quickly in review all the different parts of the eye and its appendages, and enable him rapidly to arrive at a diagnosis.

The following special rules should always be borne in mind-

I. Inspection must always be supplemented by palpation.

2. Artificial light focussed on the eye by a convex lens should be employed, because much is apt to be missed if the eye be examined only by diffuse light.

3. In all cases of ocular inflammation of sudden onset the upper eyelid ought always to be everted, and its conjunctival surface, as well as the superior retrotarsal fold, carefully searched for a foreign body.

4. Suitable spectacles should be worn if the doctor be presbyopic, or suffers from an error of refraction.

Those rules may be said to be self-evident, but they are often disregarded; and to neglect them affords an illustration of the aphorism that 
more mistakes in medical practice occur from want of care than from want of knowledge.

An observant practitioner will also learn much from the manner in which a patient enters the consulting-room. He will see at once whether the patient can come by himself or requires a guide; and if sight be obviously very defective, he will notice much that is of diagnostic value in differences of gait and behaviour. For example, the contrast is very marked between the patient blind from cataract, and one who has lost his sight from atrophy of the optic nerves. The former advances slowly with eyes shaded, and hand outstretched for guidance and protection; whereas the latter walks boldly forwards with eyes wide open and staring straight in front of him. Photophobia is recognised at a glance, and if the patient be a child, the downcast head, the tightly closed eyes, and the eczematous eruption on the face at once tell a tale of long-continued strumous ophthalmia. The character of the discharge from an inflamed eye is quickly seen, and its recognition is of great value in diagnosis : a blood-shot eye that weeps freely, but is unaccompanied by any sticky discharge, must always be regarded seriously, because in these circumstances deep-seated inflammation is indicated and not simple conjunctivitis.

The patient, moreover, must always be examined as well as his eye. Close attention should be paid to the history he gives of his illness. Much valuable information will be got if he be allowed to tell his story in his own words, and a few judicious questions after he has finished will quickly elicit points of importance regarding general health, habits and environment. When the patient is a child, naturally timid and very frightened in case it is to be hurt, the difficulties of properly examining the eyes are very great. They are almost insuperable if the examination be attempted before the child is placed under proper control. The parent or nurse should hold it securely in a good light, and either support the head firmly on her own lap, or else, sitting opposite the doctor, place it between his knees. If those precautions be taken, the subsequent procedure is much simplified. In every case it is absolutely essential that the cornea be properly inspected, and therein lies the chief difficulty. Instillations of a solution of cocain are not of much help when dealing with a frightened child, and in extreme cases it may be necessary to administer a general anæsthetic. The lids are usually separated by the fingers placed as near the margin as possible. This is always resisted by the child, with the result that the lids become everted, and the conjunctiva overlaps the cornea and prevents a satisfactory view of its surface. Not only is much patience necessary, but the attempt to separate the lids must be carried out with great gentleness, because if the cornea be ulcerated any rough manipulation may cause rupture, and so 
damage the eye irretrievably. It is prudent, therefore, to use an elevator to separate the lids; and a satisfactory instrument for the purpose can always be extemporised by bending the curved end of a hairpin into the proper shape, and sterilising it before it is used.

The methodical examination of the eye can be divided into three stages-

I. The examination of the position of the head, the face, the eyebrows, the ocular appendages, and the anterior segment of the globe.

2. The examination of vision and the refraction of the eye.

3. The examination of the fundus oculi with the ophthalmoscope.

In the case of an injury where the diagnosis is evident, the acuteness of vision, the mobility of the pupil, the position and state of the iris, the tension of the globe, and the transparency of the media ought always to be ascertained and carefully recorded.

1. The External Examination of the Eye.-It is convenient to follow an anatomical arrangement, and the following scheme may serve as a guide.

I. Position of the Head-characteristic in paralysis of the extraocular muscles, and may be of great value in the diagnosis of the particular muscle affected.

2. Face-

(a) Asymmetry-paralysis of facial nerve.

(b) Eruption - eczema, herpes, xanthelasma, etc.

3. Eyebrows-One is higher than the other from compensatory over-action of the frontalis muscle in ptosis.

4. Orbits-

(a) Position of eyeball-prominent (exophthalmos), sunken (enophthalmos), squint (strabismus).

(b) Margin-palpation may detect a swelling, painful, fluctuant, solid, hard, osseous.

5. Eyelids-

(a) Palpebral fissure-

(I) Wide-exophthalmic goitre, etc.

(2) Narrow-

(a) cicatricial-trachoma, etc.

(b) congenital-epicanthus.

(b) Skin-swollen, red, eruption, redundancy, ulcer :-it is very important to observe if the pre-auricular gland is implicated, as, e.g. in chancre of the eyelid.

(c) Margin-blepharitis, ectropion, entropion, etc. 
(d) Eyelashes-trichiasis-an ingrowing eyelash is frequently an unsuspected cause of ulceration of the cornea; distichiasis - a double row of eyelashes.

(e) Glands-stye-Meibomian cyst.

(f) Movements-

(I) Cannot be closed-paralysis of 7 th cranial nerve.

(2) Cannot be opened-paralysis of 3rd cranial nerve.

(3) Blepharospasm-spasm of orbicularis.

(4) Retraction of upper lid which does not follow the downward glance-Graves' disease.

(5) Adhesions between eyelid and eyeball due to burns or other injuries-symblepharon.

(g) Lachrymal Sac-

(I) Swelling at inner canthus-note position of tendo oculi, which is the guide in the differential diagnosis between empyema of the frontal and ethmoidal sinuses and inflammation of the lachrymal sac.

(2) Pressure over tear sac-regurgitation of tears, mucus or pus-blennorrhoea of lachrymal sac.

(h) Lachrymal Passages.

(I) Eversion of punctum-always everted if it can be seen before drawing down the lower lid.

(2) Occlusion of punctum-an eyelash may be carried into a punctum and its protruding end cause much irritation to the eye: a wound or a burn at the inner canthus may destroy the canaliculi and lead to persistent watery eye.

(3) Adequacy may be tested by instilling a few drops of 5 per cent. argyrol solution into the conjunctival sac, when, after waiting a minute or two, a brown stain should be seen on the pocket-handkerchief after the patient has blown his nose.

6. Conjunctiva-

(a) Bulbar-transparent-plica semilunaris - caruncle-analogous to skin.

(b) Palpebral-analogous to mucous membrane.

(c) Retrotarsal fold-granulations, follicles.

(d) Injection-

(I) Superficial-characterised by brick-red colour, vessels. arranged in an irregular mesh-work; congested bloodvessels are situated in the conjunctiva and can be moved along with it; congestion is most intense in 
the lids and retrotarsal folds; pressure with the finger through the lid drives the blood out of the vessel, and blanches the part pressed upon, but immediately the pressure is removed, the blood returns into the emptied vessels. Symptomatic of a purely superficial or conjunctival inflammation.

(2) Deep-characterised by pink colour, arranged as a zone of minute hair-like vessels radiating from the cornea; blood-vessels are situated in the episcleral tissue, and are unaffected by pressure-always symptomatic of infammation of cornea, iris or ciliary body.

(3) Dusky congestion formed of tortuous deeply situated veins. Symptomatic of passive congestion, and is found in glaucoma or in any ocular condition in which there has been prolonged increase in the intraocular tension. A localised venous congestion. sometimes marks the site of a tumour of the choroid.

(4) Congestion localised to a leash of blood-vessels of bright red colour is symptomatic of phlyctenular conjunctivitis.

(e) Chemosis-inflammatory, non-inflammatory.

(f) Lymphatics-outline beaded, contents clear.

(g) Secretion-catarrhal, purulent, membranous, or discrete, e. g. phlyctenular, pustular, etc.

7. Sclerotic-

(a) Curvature-staphyloma or tumour-differential diagnosis can readily be made by transillumination, and an ordinary pencil-shaped electric torch is quite sufficient for the purpose.

(b) Colour-congestion, jaundice, blue sclerotic, etc.

(c) Episcleritis and scleritis.

(d) The approximate length of the globe can be readily estimated by asking the patient to look as far inwards as possible and observing the relation of the highest point of the scleral curve to the cornea-hypermetropia-myopia.

8. Cornea-

Sudden onset of pain, intolerance of light and lachrymation frequently arise from the lodgment of a particle of grit in the eye; and whenever a patient complains of those symptoms it is necessary to inspect the cornea in a good light, using both a focussing and a magnifying lens, to make sure there is nothing on its 
surface. If nothing be found, the upper lid should at once be everted, when in all probability the foreign body will be found on its conjunctival surface, usually a short distance from its margin; the search must not be given up until the upper retrotarsal fold has been thoroughly explored.

(a) Size. Average transverse diameter of cornea is $11.6 \mathrm{~mm}$. Priestley Smith's keratometer is the most convenient instrument for measuring the cornea.

(b) Curvature. When the patient looks at a window opposite which he is standing, a perfect image of the window reflected on the cornea shows that at that point of its surface its curvature is to all intents and purposes spherical. If, however, the surface be aspherical or irregular, the reflection of the window will be altered in size and distorted in shape.

(c) Surface-highly polished like a mirror. A superficial abrasion may be difficult to see until it is stained bright green by a solution of fluorescein.

(d) Transparency. Loss of transparency is always the first sign of inflammation. Opacity-scar of past ulceration, or of interstitial inflammation, or spots on posterior surface of cornea, obliterated blood-vessels, etc.

(e) Sensibility. Corneal anæsthesia can be ascertained by touching the cornea with a piece of thread or soft blottingpaper; a very convenient instrument is the head of an ordinary pin which has' been thoroughly sterilised. Sensitiveness is lessened or abolished in glaucoma, herpes, neuroparalytic keratitis, etc.

9. Anterior chamber-

(a) Depth.

(I) Empty. Look for a penetrating wound or a perforated ulcer of the cornea.

(2) Shallow. Think of glaucoma and examine the intraocular tension and the size and shape of the pupil.

(3) Deep. Symptomatic of serous irido-cyclitis, shrinking of the vitreous, etc.

(4) Unequal depth. Usually due to iris bombé, dislocation of the lens, etc.

(b) Contents.

(I) Turbid-inflammatory affections of ciliary body, in the majority of cases accompanied by deposits on the posterior surface of the cornea-keratitis punctata. 
(2) Purulent-hypopyon in serpiginous ulcer of cornea in which the hypopyon is sterile, and in septic wounds of the eye in which it is infected, etc.

(3) Hamorrhage-hyphama-after injuries and acute inflammation of the iris, etc.

(4) Dislocation of the lens-appears like a drop of oil in the anterior chamber.

(5) Foreign bodies.-Metallic particles may or may not. be attracted by the magnet, and of course the prognosis is much more unfavourable if the metal is not magnetisable.

Io. Iris-

(a) Colour.-Always compare the two eyes-discoloration the earliest sign of inflammation.

(b) Surface-shows the delicate pattern with perfect distinctness, only when the iris is healthy. The surface level is altered in disease: iris bombé appears as a round yellowish-brown elevation; anterior synechia when the iris adheres to the cornea after a penetrating wound or a perforating ulcer, etc.

(c) Tremulous.-The iris trembles, with every movement of the globe: think of dislocation of the lens and of fluidity of the vitreous.

II. Pupil--

Affords valuable information not only regarding the eye itself, but also regarding the nervous system generally. During the examination of the pupils each eye must be tested separately, and the patient told to look away in the distance and not to try to open the lids more widely than is natural.

(a) Position. Normally placed in the centre of the iris, but may be excentric from disease or from congenital malformation.

(b) Size. Can be measured by a pupillometer-observe if the pupils are large, medium, or small, and if they are equal or unequal. If inequality of the pupils can be established as a new fact in the history of a case, it adds much to the gravity of the prognosis, because in the majority of instances it is a danger-signal warning of the approach of serious disease of the nervous system. Hippus, when pupils oscillate between contraction and dilatation.

(c) Shape. In the normal condition the pupil is quite circular, but the outline may be broken by a coloboma, due either to a congenital defect or to a surgical operation. An oval pupil ought to make the practitioner think of glaucoma, and 
irregular outline due to synechice is the pathognomonic sign of iritis.

(d) Colour-jet-black in a young, healthy person; becomes less black with age, and in elderly people shows the grey senile reflex which must never be mistaken for opacity of the lens : white and opaque from cataract, or from inflammatory exudation; greenish in glaucoma.

(e) Reaction.

(I) Direct Light Reflex.

(2) Consensual Light Reflex.

(3) Convergence Reflex.

(4) Skin Reflex.

(5) Argyll Robertson Pupil.

(f) Synechia-adhesion of the iris to the anterior capsule of the lens is the pathognomonic sign of iritis : synechiæ may not be visible until a mydriatic is instilled into the conjunctival sac; when the pupil dilates the irregularity of outline indicates the adhesions: when no dilatation occurs after instillation of a mydriatic the pupil is said to be "excluded," -very apt to occur in the " quiet iritis " of elderly patients, a condition which unfortunately is sometimes mistaken for cataract.

I2. Ciliary Body.

Tenderness on pressure over the ciliary region is diagnostic of cyclitis.

I3. Intraocular Tension-is usually estimated by palpation, and the surgeon has always a standard of comparison in his own eye. A hard eye is always dangerous, and usually indicates the presence of glaucoma. Diminished intraocular tension is present after a penetrating wound, in the late stages of irido-cyclitis, detachment of the retina, etc. A difference in tension may be the distinguishing feature between a simple detachment of the retina, and a separation due to sarcoma of the choroid-in the former case it is usually reduced, while in the latter it is frequently increased.

I4. The Extraocular Muscles. The movements of each eye ought, in the first place, to be tested separately, but afterwards the associated movements of both eyes together should be examined. Nystagmus, involuntary movements, may be congenital or arise as a result of occupation-Miners' Nystagmus.

2. The Examination of Vision and the Refraction of the Eye.Acuteness of vision is determined by the size of the object which the eye 
can see clearly at a given distance. Printed letters are usually selected as test objects; and a normal eye, in good daylight, can distinguish letters of such a size, and seen from such a distance, that their individual parts subtend a visual angle of one minute, and each letter as a whole a visual angle of five minutes. All test types-Snellen's, Landolt's, etc.are constructed on that basis, and for those who are unable to read, types are arranged in Snellen's series with the letter E turned so that the divisions of the letter will point in different directions in succeeding figures. All that is required of the patient is to indicate the direction in which the figure is open, and towards which the branches point. The patient is seated twenty feet from the distance test type; but if the consulting-room be too small, special test types constructed for use at a distance less than twenty feet are easily obtainable. Each eye must be examined separately, and it is usually most convenient to use a trial frame, in which an opaque disc is placed before one eye, which must be kept open during the whole time of the examination. The patient is now asked to read the lowest line of letters which he can see distinctly in the board of test types. The measure of the vision is represented by the last line that he is able to read correctly, and it is recorded by a fraction whose numerator is the distance at which the patient is placed from the test types, i.e. twenty feet or six metres, and whose denominator is the figure printed above the line, and which indicates the distance at which a normal eye can read letters of that size. For example, if the patient can only read at twenty feet what a normal eye can read at eighty feet, his visual acuity is said to be $\frac{20}{80}$, or $\frac{6}{24}$ if the fraction be recorded in metres.

I. The patient is guite able to Read the smallest letters oN THE TEST CARD.

$\mathrm{He}$ is either emmetropic or hypermetropic. A + I D. spherical lens should now be placed in the trial frame opposite the right eye.

(a) The vision is blurred - the patient may be regarded as emmetropic.

(b) The vision is unimpaired-the patient is hypermetropic, and stronger and stronger convex glasses should be successively placed in front of the eye until the letters become indistinct, and the number of the strongest convex glass which does not interfere with clear vision represents the degree of the manifest hypermetropia.

In young subjects, whose power of accommodation is very active, results obtained by purely subjective testing are not reliable, because much hypermetropia may remain latent, and only a small part become manifest. In these circumstances no conclusion should be arrived at until the ciliary muscle has been completely paralysed by homatropin. 
2. The Patient is UNABLE TO READ THE SMALLEST LETTERS ON THE TEST CARD.

He is suffering from a high degree of hypermetropia, from myopia, from astigmatism, or from some structural damage to the eye itself, to the visual tract or the visual centres. Defective sight due to an error of refraction can be at once distinguished from failing vision, the result of disease in the eye or its nerve tracts, by asking the patient to look at the test types through a pinhole. If sight is improved, there is an error of refraction, but if vision remains stationary or is made worse, the amblyopia is due to disease.

A differential diagnosis of the different conditions due to errors of refraction can be arrived at by means of lenses.

(a) Convex glasses improve vision-the patient is hypermetropic, and the strongest convex glass which gives the best vision represents the degree of manifest hypermetropia, although no accurate measurement can be made in a young person without the help of a cycloplegic.

(b) Convex glasses make the sight worse, but concave glasses at once improve vision-the patient is myopic and the weakest concave glass which gives the best vision indicates the degree of the myopia.

(c) Neither convex nor concave spherical lenses materially improve sight-the patient is astigmatic. That can be demonstrated subjectively by means of radiating lines, certain of which appear to be much more distinct than others; but if no test chart be available, the face of a watch can be shown to the patient, who, if he be astigmatic, will see certain hours much less clearly than others. If cylindrical lenses either convex or concave be placed before the eye and turned in the trial frame until the proper direction of the axis is found, vision of an astigmatic patient will be improved. In many cases the cylindrical lens must be combined with a spherical in order to obtain standard vision.

After the right eye has been examined in the way just described, the opaque disc must be placed in the right side of the trial frame, and the left eye tested in like manner.

Sometimes the patient, placed at twenty feet from the test types, is unable to see even the largest letter. He should, in these circumstances, be asked to go nearer, and say when the largest letter becomes visiblethat distance will then be the numerator, and 200 the denominator of the fraction which represents the amount of vision. If the patient cannot decipher the letters on the test card at any distance, he is asked, whilst standing with his back to the light, if he can count fingers held against the examiner's coat, and when that is possible, a record is made-counts fingers at-whatever is found to be the maximum distance. At other times the patient can only distinguish hand movements or shadows, or 
tell the position of a light, or of the window of the room in which he is being examined. If he cannot tell light from dark, blindness is absolute.

Whenever it is possible, the subjective tests of vision should be corroborated by objective observation with the ophthalmoscope, the ophthalmometer, and by retinoscopy; but unless the general practitioner has considerable experience in the diagnosis and treatment of diseases of the eye, he should refer patients to an expert whenever he is not satisfied that an error of refraction is properly corrected, and when he fails to obtain standard vision by the aid of lenses. The judicious use of a cycloplegic will be of great assistance in the examination of the majority of patients under forty years of age, and will enable the doctor to obtain much better results than he would have got had he examined the eyes without having previously paralysed the accommodation.

All that has been said refers to the examination of the central part of the retina, i.e. the macula. This is a very small, but a very highly sensitive area, and is wholly concerned with direct vision, in contrast to the much more extensive, but much less sensitive area, which is concerned with peripheral vision. The latter includes nearly the whole of the retina and must be examined separately. It will be described in the chapter on the Field of Vision.

3. The Examination of the Eye with the Ophthalmoscope.The general practitioner should avail himself of every opportunity to use the ophthalmoscope, and ought to regard it as being just as essential as the stethoscope or the clinical thermometer in the daily practice of his profession. In the complete investigation of a case, the examination of the eyes with the ophthalmoscope is as necessary as the examination of the urine by chemical tests.

An ophthalmoscopic examination is conducted in a dark room, and comprises four methods. (I) Oblique or focal illumination; (2) the direct method at a distance from the patient; (3) the indirect method, and (4) the direct method close to the patient. For all those different methods a lamp, a mirror, and a series of lenses are required, but the electric ophthalmoscope greatly simplifies the technique and practically removes all difficulties from the examination. The use of the ophthalmoscope is necessary to assure oneself of the transparency of the crystalline lens, and if such an investigation was carried out as a matter of routine, chronic glaucoma would never be mistaken for incipient cataract. Posterior synechiæ can also be detected at once and will lead to a diagnosis of iritis in an elderly person whose only complaint is of failing sight. In such circumstances the superficial observer is apt to attach undue consideration to the age of the patient, and to rush to the conclusion that he is suffering from opacity of the lens, and, consequently, to counsel him to wait till 
the cataract is ripe before seeking further advice. Such a mistake, so disastrous in its results, is very unlikely to occur if the general practitioner is in the habit of making ophthalmoscopic examination a routine practice.

Great experience is necessary to be able to interpret correctly the various pathological changes visible in the fundus oculi, and such detailed knowledge is not expected from one who is not an expert; but the family doctor who is competent to see the background of the living eye will often find changes there which will enable him to say confidently that the patient is suffering from syphilis, from tubercle, from an intracranial tumour, from disease of the kidney, from general arterio-sclerosis, from glycosuria, or from incipient tabes dorsalis at a time when other signs of the disease are doubtful or have been passed unnoticed by the patient. 


\section{CHAPTER II}

\section{IMPORTANT SYMPTOMS IN DISEASES OF THE EYE}

Importance of the correct interpretation of symptoms-Disorders of general sensibility - Inflammatory pain - Non-inflammatory pain-Retinal and muscular asthenopia - Neuralgia -Disorders of the visual function-Sudden loss of sight-Gradual loss of sight.

A PATIEnT consults a doctor because he feels there is something wrong, and he wishes to know the meaning of his abnormal sensations. Such symptoms become known to the physician wholly by information supplied by the patient himself. They are purely subjective, and if they are to be accurately interpreted, careful attention must be given to the patient's own account of the history of his illness. A doctor should be a good listener. The importance of this cannot be over-estimated, because it is "only after a disease has become developed that its objective signs can be observed, whereas the real beginning of the morbid process is marked by sensations which cause the patient discomfort, but which are apt to be passed lightly over by a physician whose knowledge is insufficient to enable him to assess their real value. It is true that a patient may exaggerate the importance of certain sensations, and disregard others which are much more serious. He may thereby mislead a physician who simply accepts what he is told, and does not trouble to test, by careful physical examination, the truth of the statements that have been made to him. As far as possible, therefore, all the resources of modern clinical medicine should be utilised to explain the patient's symptoms.

In the diagnosis of diseases of the eye, instruments of precision are of enormous value. By their means the practitioner is enabled to make accurate observations quite apart from the patient's sensations, and may thereby discover important facts of which the patient himself is altogether ignorant. The fact that the acuity of vision is normal is no proof that an eye is free from disease. In every case, therefore, if it be possible, the fundus should be carefully examined with the ophthalmoscope, the reaction of the pupils to light accurately tested, and the field of vision measured by the hand. These all form important factors in diagnosis, and to neglect any one of them may lead to serious error. For example, the ophthalmoscope may reveal intense optic neuritis in a case where there has never 
been any complaint of defective sight, and the knowledge thus obtained will prevent a doctor from making the mistake of regarding a complaint of headache as due to gastric disturbance, when in reality it is symptomatic of a tumour of the brain. Nevertheless the true science of medicine consists in being able to give a proper interpretation of the patient's symptoms, and in realising the importance of the subjective as well as the objective phenomena of disease. They are mutually dependent, and are complementary, not merely supplementary. In order, therefore, to arrive at a complete diagnosis, both must be carefully and fully considered, and each looked at in correct perspective and in proper proportion to the whole.

Taking the term "symptom" in its limited sense, as applying to uneasy or unnatural sensations, or to disorders of function, diseases of the eye, in their purely subjective aspect, may be considered under the two heads of, first, disorders of general sensibility, and, secondly, disorders of the function of vision.

\section{DISORDERS OF GENERAL SENSIBILITY}

Of all uneasy sensations pain is the most common and the most important. It ought always to be regarded as a danger signal. It is a purely subjective symptom, for we have no means of measuring it, except by the patient's own statements; and, as some feel pain much more keenly than others, the full value of its significance can be arrived at only after a careful estimate of the patient's constitution and temperament has been supplemented by a thorough examination of the eye itself. Though pain always accompanies inflammation, it may arise from other causes, and so we may arrange all abnormal sensations under two great groups, according as they are inflammatory or noninflammatory.

A. Inflammatory Pain.-When pain is the result of inflammation, much may be learned from a study of its severity, its character, its distribution, and its accompaniments-photophobia, secretion, congestion, œdema, enlargement of glands, suppuration, etc.

The character of the pain varies as the ocular inflammation is superficial or deep. In the former case, in simple conjunctivitis for example, patients complain that they feel as if a foreign body were present between the lids and the ball, and that the irritation is aggravated by every movement of the lids. In deep-seated inflammations, on the contrary, they describe the pain as dull and gnawing, like toothache. Here it radiates along the branches of the fifth nerve, and is always most severe in the early hours of the morning. It may also be associated with tenderness on pressure, and this heightened sensibility of the eye to touch is of the 
greatest value in the diagnosis of inflammation of the ciliary body, and in distinguishing between conjunctivitis and sclerotitis. The pain may be complicated by increase in the intraocular tension, and when that is so, subacute glaucoma should always be thought of, and without a moment's delay the visual acuity and the visual field ought to be carefully tested. The fundus should also, if possible, be examined with the ophthalmoscope, and the size and mobility of the pupil, as well as the depth of the anterior chamber, recorded. Pain occurring from twenty-four to forty-eight hours after an injury to the eye, or after a surgical operation, ought always to be regarded as a danger-signal pointing to probable infection of the wound. In short, the character of the pain may by itself afford valuable indication of the nature of the ocular inflammation. If it be sharp and cutting, as if a foreign body were in the eye, the disease is in all probability superficial. If, on the other hand, it be throbbing and gnawing, radiating outward along all the branches of the fifth cranial nerve, and usually worse late at night and in the early hours of the morning, there is in all likelihood serious implication of the deeper structures.

In reaching a conclusion by the method of differential diagnosis, the accompaniments of pain-photophobia, condition of the pupil, high tension, tenderness on pressure-are most helpful. As to the first of these, which is always most marked in affections of the cornea, and especially in the so-called strumous inflammations, it may be said that the more superficial the lesion the more distressing is the photophobia. In deep and extensive ulceration of the cornea, on the contrary, though the pain may be agonising, there may yet be comparatively little intolerance of light. The state of the pupil enables the surgeon at once to distinguish between superficial and deep inflammation. If it be of normal size and react readily to the stimulus of light, there is no involvement of the iris or of the ciliary body; whereas if it be contracted and sluggish, there is a superficial lesion which is causing great irritation. If, in addition to being contracted, it be irregular, as the result of the formation of adhesions between the iris and the lens capsule-posterior synechice-the anterior chamber being normal or deeper than usual, then iritis is present, and, should the inflammation be acute, it must at once be determined whether or not the ciliary body is involved. This is easily done by gently pressing on the eyeball through the closed lids: if there be much tenderness cyclitis undoubtedly exists. If, on the other hand, the pupil be dilated, subacute glaucoma ought at once to be suspected, and in this case the eyeball must be carefully palpated. Should increase of tension be found associated with a shallow anterior chamber and with impairment of sight, the diagnosis of primary glaucoma will be amply confirmed.

Itching is an uneasy sensation nearly allied to pain. It is often 
associated with inflammation of the edge of the eyelids, but is at other times the result of defective metabolism. Its recognition and right interpretation therefore, not only point the way to a correct diagnosis, but also indicate the proper line of treatment.

B. Non-Inflammatory Pain.-(a) Retinal Asthenopia.-In retinal asthenopia, the symptoms are so distressing that the patient is quite unable to read or write, and in many cases ceases to try to take any active part in life, and thinks and speaks of little besides his or her ocular troubles. One frequently finds that health has originally been impaired by illness, by prolonged sick nursing, or by some serious mental or moral shock, and that the photophobia has come on during the period of convalescence. It is difficult to account for this hypersensitiveness to light. Doubtless an irritation of the fifth nerve is conveyed to, and stimulates, the optic nerve, but there is no exactly similar form of reflex action in any other part of the body.

(b) Muscular Asthenopia.-This. condition-usually the result of an error of refraction or disorder of motility - presents an easily recognised clinical picture. It is impossible to see any minute object distinctly, and every attempt to read is attended by a sense of confusion. The print first becomes unsteady and blurred, and then gradually fades from view; and, though it becomes visible again after the eyes have been rested and gently rubbed for a few minutes, persistence in attempts to read quickly brings about exhaustion. Complaint is usually made of a dull aching pain (sometimes accompanied by twitching of the lids and facial muscles) behind the eyeballs, which feel tight and hot, and may be unduly sensitive to light. There may or may not be signs of congestion in the structures of the eye, but whenever the patient reads or writes there is always headache. The pain may be dull and throbbing, or acute and neuralgic; it may come on while the eyes are being used, or it may not be felt until the following morning on first waking; it may be constant or periodic; it is frequently frontal, just above the eyebrows, but is also often occipital, radiating down the back or along the arm; and at still other times it is felt most severely in one or both temples. In this last form, especially when unilateral, it is almost always very severe, is often accompanied by nausea and vomiting, and may be preceded either by sleeplessness or by excessive drowsiness. Premonition of the onset is often given by the appearance of coloured spectra, or by a transient attack of hemianopia.

(c) Neuralgia.-Pain is, however, often present apart from the use of the eyes. It is then mostly unilateral, confined to one or several branches of the fifth cranial nerve, and accompanied by spots which are painful on pressure. Neuralgia is the usual diagnosis, but a determined effort should always be made to discover a cause. Though the suffering 
may be merely the expression of a lowered condition of general health"the prayer of a nerve for better blood"-it is, in many instances, symptomatic of disease in some distant part—of, for example, a carious tooth, an ulcer of the nasal septum, a morbid growth implicating the Gasserian ganglion, etc. Intense supraorbital pain always precedes an eruption of herpes.

\section{DISORDERS OF THE VISUAL FUNGTION}

In all cases where there is complaint of diminished visual acuity it is necessary to make a careful estimate of the refraction, and to note to what extent suitable glasses improve sight, and what is the amount of defect that remains after the ametropia has been corrected. A simple test is to hold a stenopæic slit, or a visiting card perforated with a pin in front of each eye separately. If sight be improved there is an error of refraction, but if there be no improvement the amblyopia is the result of disease. Sudden loss of sight-complete or partial, and attacking either one eye or both simultaneously-is a different matter, and, in the search for the probable cause, much help is obtained from the patient's account of the onset of the blindness.

A. Sudden Loss of Sight.-When the loss of sight has been sudden and is bilateral, a toxic origin (uræmia is probably the most common) ought always to be suspected. Embolism of the central artery of the retina destroys sight without a moment's warning; but in nearly every case one eye only is affected, and ophthalmoscopic examination leaves no room for doubt regarding the diagnosis. Again, sight may be completely lost immediately after a blow on the forehead, but in that case ophthalmoscopic examination reveals at first nothing abnormal, the reason being that the optic nerve has been injured through fracture of the bones at the optic foramen, or more probably by hæmorrhage into the nerve sheath, and not till some weeks after the accident may atrophy of the optic disc be recognisable. From the outset, however, the pupil on the side affected responds very sluggishly to light, and in many cases the direct light reflex is abolished in the injured eye, and the consensual reflex is also absent in the sound eye.

When the loss of sight is sudden, but incomplete, much information is obtained by testing the field of vision. A blank in a portion of the periphery is due in all likelihood to a detachment of the retina, or to an extensive hamorrhage into the vitreous; should the patient complain that all lights seem to be blood-red in colour; the latter lesion is almost certain to exist.

It ought, however, to be noted that the reason for a patient's assertion that he has suddenly lost the sight of one eye may be merely that he has 
by accident discovered a defect which has existed all through life, or which has been, perhaps, gradually increasing for a long time without having been noticed. In such cases the ophthalmoscope may reveal atrophy of the optic nerve; and this, which might in itself be due to some purely local cause, may, when the clinical associations of the monocular amblyopia are carefully scrutinised, assume an entirely different aspect. There may be no complaint of defective sight in the other eye, nor, on ophthalmoscopic examination, may any disease of the other optic nerve be detected; but the perimeter will reveal contraction of the visual field, both pupils will be found contracted and irresponsive to light, though responding normally in the act of convergence, and the knee-jerks will be absent. All this corroborative evidence makes it certain that the case is one of disease in the posterior columns of the spinal cord, and not simple atrophy of the optic nerve. Here, then, the onset of failing sight in one eye puts the physician on the alert, and suggests the careful examination which enables him to make a correct diagnosis of a disease in which atrophy of the optic nerve is, from the outset, the predominant feature. Another familiar example is when sight of one eye has been lost from chronic simple glaucoma and the patient is either quite unaware of the fact or has paid no attention to it, until the other eye has become affected.

B. Gradual Loss of Sight.-A patient whose age is usually from forty to fifty, may say that for a considerable period his sight has been misty-so much so, indeed, that he has often experienced difficulty in reading ordinary type, and that he cannot get spectacles to improve his sight, which has suddenly become so much worse that he is quite unable to read or write, and cannot even recognise friends when he meets them in the street. He will also add that he sees. best in a'dull light, and may say incidentally that when he is playing billiards he has difficulty in seeing the red ball, or that when he is golfing he cannot recognise the red flags on the greens so easily as the white ones. In such circumstances the trouble is, in all probability, due to excessive indulgence in tobacco, or alcohol, or both; and careful examination will in most instances confirm that opinion.

Then again, on the other hand, a patient may say that he sees fairly well during the day, but after sunset he becomes to all intents and purposes blind. This symptom, commonly called night-blindness, is almost pathognomonic of that progressive form of degeneration of the retina known as retinitis pigmentosa, which sooner or later leads to complete loss of sight.

Sight may also fail for close work so as to cause a patient to change his reading glasses at short intervals time after time, and in speaking of the matter he may say that now and again he suffers from transient 
loss of vision, and that, more or less constantly, he sees coloured halos round gas, lamp, or candle flames. Such an account is most suggestive; for, almost to a certainty, these symptoms are premonitory of glaucoma, if indeed that dread disease be not already fully established. So little may be thought of them, however, that their occurrence is mentioned to the doctor in the most casual way; but their importance cannot be overestimated, for if they be appraised at their proper value, means will at once be taken to check the disease before high tension has become permanently established. If, on the other hand, their significance be not properly appreciated, the patient is allowed to drift till sight is so seriously impaired that it cannot be improved by any form of treatment.

It is very significant when a patient who has required to wear glasses for reading finds that he sees better without them. He is generally much pleased at the discovery, and says he has got his "second sight." If, however, his vision be tested, it will be found that he is now myopic, and requires concave glasses, and very probably he may say that when he looks at the moon it appears doubled or quadrupled-monocular polyopia. Such a combination of symptoms is certainly due to changes taking place in the lens, and is in most instances symptomatic of cataract. It is very important that the disease be recognised at such an early stage, because this allows a careful ophthalmoscopic examination of the fundus to be made, and any pathological changes in the choroid or retina to be detected, at a time when the lens is still sufficiently transparent to permit these structures to be seen.

It occasionally happens that a patient complains that every object looked at is altered in size or is thrown out of proportion and distorted. This symptom is known as metamorphopsia, and is often of very serious import. The distortion may, of course, be due to errors in the optical construction of the eye; but it may also be induced by pathological changes in the retina itself, and is then significant of grave disorder of vision, and usually symptomatic of inflammation of the choroid. In this case it is caused by some change in the relative position of the cones in the bacillary layer of the retina, and is most serious when the macular region is implicated. In some instances, where the percipient elements of the retina are separated by exudation, objects appear too small (micropsia); in others, where the percipient elements are crowded together owing to cicatricial alterations, they seem too large (macropsia); and in still others they assume very irregular shapes. It is sometimes difficult to detect with the ophthalmoscope the changes that give rise to the metamorphopsia, and hence the value of the symptom in leading to a correct diagnosis.

Patients frequently complain of seeing spots or specks (musca voli- 
tantes), but though these often give rise to much annoyance, they do not, as a rule, unless floating bodies be seen on ophthalmoscopic examination, indicate the existence of organic disease. They point, at the most, to a condition of retinal hyperæsthesia, determined, in all likelihood, by congestion of the choroid, the result of prolonged application of the eyes to close work. Under these circumstances the refraction ought to be carefully tested, the urine examined for oxalates or uric acid crystals, and the patient discouraged from looking for the muscæ, as these are only too prone to reappear whenever the attention is directed to them. It is, however, sometimes not easy to draw the line between what may be regarded as physiological and what is pathological. Whenever they are dark in colour so that the patient sees them in variable conditions of illumination, or when they are of large size and change their position with every movement of the eye, a careful ophthalmoscopic examination is necessary, more especially if the patient be short-sighted, for then opacities in the lens, or floating bodies, or hæmorrhages, may be detected in the vitreous chamber, or, most dangerous of all, the muscæ may be premonitory of detachment of the retina; but in that case they are usually accompanied by flashing of light occurring either spontaneously or along with any movement of the eyes.

Up to this point the symptoms referred to have been due to changes in the eyeball itself, but there are others of extraocular origin, having their rise in the ocular nerves, in the optic tracts, or in the cortical visual centres. Each of these groups has, when it is out of order, its own peculiar symptom associated with it-diplopia with the ocular nerves, hemianopia with the optic tracts, and mind blindness with the cerebral cortex. These symptoms may often be of supreme value in the localisation of cerebrospinal disease; but as they are frequently subtle and transient, and mostly subjective, they are in many instances difficult to detect and to appraise at their proper value. For their right appreciation it is necessary to possess an accurate knowledge of the anatomy and physiology of the parts concerned.

Paresis of any of the ocular muscles is usually followed by strabismus, and there is then no difficulty about the diagnosis, but in other cases the patient complains of double vision without there being any obvious squint. In these last there must be careful study of the diplopia in order to find out which muscle, or group of muscles, is at fault, and the knowledge thus obtained may be of great importance in localising the exact site of a cerebral lesion. The principles underlying such an examination are readily enough understood, but it takes considerable experience to carry the examination itself out in detail, and to discover the muscle or muscles at fault. For our present purpose, however, we simply accept the fact 
of the diplopia quite irrespective of the cause, and the first point the physician must determine is whether or not the double vision disappears when either eye is covered. If it persist when one eye alone is used, it is called monocular diplopia, but if it disappear with the closing of one eye, it is spoken of as binocular diplopia. The former condition is a variety of monocular polyopia, one of the earliest symptoms of incipient cataract, and for the present need not engage our attention further; but the latter requires more consideration, for it occasionally affords clinical information of the utmost diagnostic value. Although paresis of any of the ocular muscles may be purely local, and due to disorder of refraction or of motility, or, in a gouty or rheumatic subject, to such a simple cause as exposure to cold, yet its occurrence must in every case excite suspicion regarding the state of the nervous system as a whole. More especially is this so when the diplopia is transient, but recurrent. It is true that the symptoms mentioned may seem to be purely functional in character, but it must be borne in mind that these so-called hysterical manifestations may herald the approach of organic disease. In the circumstances described, the diplopia is probably an early symptom of tabes dorsalis, of general paralysis, or of disseminated sclerosis of the brain and spinal cord, and diligent search requires to be made for other signs confirmatory of such degeneration-Argyll Robertson pupil, incipient atrophy of the optic nerve, absence of knee-jerks, contraction of visual field, impairment of sensation, etc. Even if confirmatory signs be wanting, at the first appearance of the diplopia, the lapse of time alone enables us safely to alter the prognosis.

The chief cause of an ocular paralysis is syphilis, which is also one of the most frequent ætiological factors in degeneration of the nervous system as a whole; consequently if a transient diplopia occur in a person of middle age with a clear history of syphilis, it ought to be regarded as a dangersignal indicative of the approach of widespread organic disease of the nervous system. The fact that in these circumstances diplopia is usually one of the very earliest indications greatly enhances its clinical value; for its correct interpretation leads to an accurate diagnosis at a time when there is some chance of doing good-a very remote probability after the disease has fully declared itself. A Wassermann test of the blood should be made in every case.

A patient may complain that when he looks at an object he sees only part of it; that he has difficulty in reading because he sees only one or two words at a time; or that he cannot see when he looks to the right, although he sees well enough when he turns his eye to the left, or vice versa. A very simple examination will usually reveal the existence of a symmetrical defect in the fields of vision, the explanation of which 
lies in the partial crossing of the optic tracts at the chiasma, each tract supplying the temporal half of the retina of its own side, and the nasal half of the retina on the opposite side. This defect is called hemianopia, and is usually homonymous, but, if the lesion or lesions be situated at the chiasma, it is bitemporal or binasal. A transient attack of hemianopia may be a premonition of the onset of sick headache, in which case the partial blindness is accompanied by balls of light, zigzag lines or the so-called fortification spectra, whose gorgeous colouring always attracts the patient's attention. These visual symptoms usually last from ten to twenty minutes, and are followed by severe head pain —ophthalmic migraine —or in later years by a feeling of heat in the head. When, however, the hemianopia is permanent, it always indicates organic disease, and is a symptom of great localising value. It may be caused by a lesion of the cerebral cortex, or by one situated anywhere in the course of the visual pathway between the occipital lobes and the optic chiasma. It always indicates an intracranial lesion on the same side as the blind half of the retina, and therefore of course on the opposite side to the dark visual fields. Hemianopia is frequently associated with hemiplegia, with monoplegia, with pupillary disturbances, with optic neuritis, with optic atrophy, with contraction of the field of vision, and, what is more important still, with aphasia and mind-blindness.

The term "mind-blindness" is used to imply the loss of the visual memory for words, letters, numerals, forms, and objects generally; and the centre affected is supposed to be situated in the angular gyrus of the left side. Obviously, this area must increase as the individual becomes older and more experienced, and on the other hand a child in whom it is defective or inefficient is unable even to learn the alphabet-the congenital word-blindness of Hinshelwood, Nettleship, and Sydney Stephenson. The furnishing of these dark chambers of imagery, then, with sense impressions of all kinds-letters, words, or what not-conveyed to the brain through the eye, may be regarded as the measure of intellectual capacity, and anything which interferes with the acquisition of new pictures, or which destroys those already acquired, gives rise to nind-blindness. As different classes of pictures are, if I may so speak, stored in different rooms placed side by side, and perhaps communicating with one another, the contents of one chamber may be destroyed, while those of the others remain intact; and hence the particular character which the mind-blindness assumes may, as our knowledge increases, form an important factor in localising cerebral disease. The storage in ordinary circurnstances, with right-handed individuals, takes place only on the left side of the brain; but undoubtedly a sinilar series of cells exists in the right side, and, should the others be destroyed, these may be educated to 
perform the functions of those that have perished. For this, however, it is necessary that the patient should possess quite unusual force of - character, as an immense amount of perseverance and patience is required before the new cells can accumulate fresh memory-pictures capable of being reproduced at will. The following case, one of the most interesting in my own experience, illustrates particularly well the more important clinical facts relating to mind-blindness-

The patient was a gentleman over sixty years of age, who suddenly discovered that he was unable to recognise the objects around him in his own bedroom. His mental powers were quite unaffected, and he told me that when he looked at a timepiece his only idea of what he saw was derived from the sound of the ticking. When he looked at the dial he perceived the hands and the numerals, but they conveyed no meaning whatever to his mind. His wife and daughters were recognised merely by the sound of their voices; and although visitors might not detect anything very far wrong, that was because he had been informed beforehand who they were. Right-sided hemianopia coexisted with the mind-blindness, and throughout the whole course of the case remained unchanged. He very soon reacquired the power of distinguishing persons and the familiar objects around him; but a printed page was just as great a puzzle as it is to a child who has never learned to read-greater, indeed, because he was troubled by a sense of his own incapacity. When, however, it was explained to him that the difficulty was due to a localised cerebral defect, and that a similar area on the opposite side of the brain was ready to be utilised, he at once, with characteristic determination, set himself to learn the alphabet over again. His method of procedure was to provide himself with a number of letter games, which he played constantly with his daughters till he knew all the letters by sight, and was able to combine them to form short words. This was the most difficult part, for after he had got on so far he made much more rapid progress, and was, in less than a year, able to read with comparative ease. He then tried to write, and made the additional discovery that, although he could form the letters and words and compose sentences quite accurately, yet he could not read what he had written. He accordingly set himself to relearn the written characters, and was making excellent progress, but died from an attack of pneumonia before he had time thoroughly to master them.

The family doctor should try to acquaint himself with those subjective disturbances of sight. They are for the most part transient, and on that account easily overlooked or forgotten, but when they are assessed at their proper value, they convey much information at a time when to possess it redounds not only to the benefit of the patient, but also to the credit of the physician. 


\section{CHAPTER III}

\section{DISEASES OF THE TEAR PASSAGES}

Significance of a "watery eye "-Causes of over-secretion of tears-Causes of obstruction to the outflow of tears-Conjunctivitis accompanying lachrymal obstruction-Lachrymal catarrh-Blennorrhœa of the tear-sac-MucoceleCaries of lachrymal bone-Acute dacryocystitis-Lachrymal fistula-Treatment of epiphora-Treatment of lachrymal catarrh-Treatment of catarrh of the lachrymal sac and stricture of the nasal duct-Method of passing a probe-Treatment of acute dacryocystitis-Treatment of permanent enlargement of the lachrymal sac with or without a fistula-Operation for extirpation of lachrymal sac-Summary of methods of treatment.

"WATERY EXE" is the pathognomonic sign of every disorder of the lachrymal apparatus. Under normal conditions there is a balance between the secretion of tears and their outflow by the natural channels; but when people suffer from "watery eye" there is either excessive secretion, or some obstruction in the tear passages. Over-secretion usually affects both eyes, whereas blocking of the lachrymal ducts is, as a rule, unilateral. The chief causes of over-secretion are emotion, some reflex disturbance (for example neuralgia), a foreign body on the conjunctiva or cornea, or some affection of the nose or teeth. In such cases any exposure to wind or light stimulates secretion so greatly that tears are continually gathering on the edge of the lower eyelid, and this, of course, takes place all the more readily when there is any congenital narrowness of the lachrymal passages-as when the bridge of the nose is flat and depressed, or when the features are very sharp. In other cases persistent "watery eye" seems to be due to some optical defect, and then lachrymation is always greatest after reading or writing.

By far the most common cause, however, is either some displacement of the puncta, or some obstruction in the canaliculi. In all injuries involving the inner canthus-e.g. wounds, burns, etc.-the puncta, the canaliculi, and even the lachrymal sac are very liable to be implicated, and after cicatrisation their function may be found to have been very seriously interfered with. In dealing with any such case it is, therefore, necessary, from the very outset, to pay special attention to the condition of the tear-passages, and to take every available means to ensure that their permeability is maintained. The inferior canaliculus is the larger, and plays the more important part in excretion, so that when its punctum 


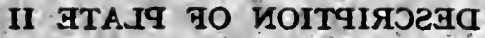

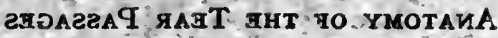

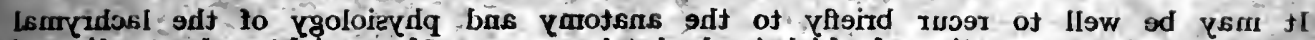

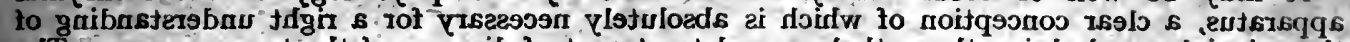

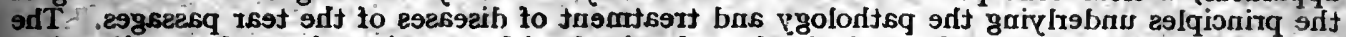

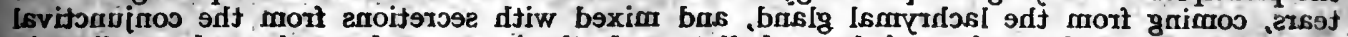

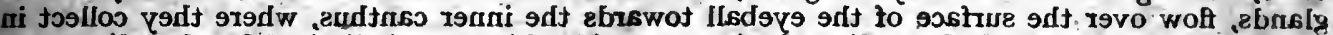

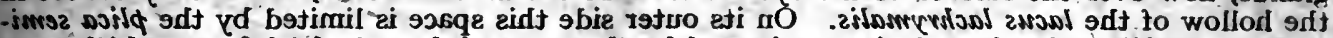

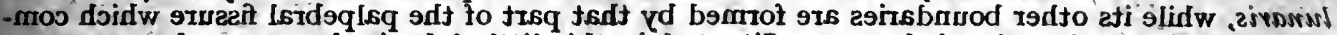

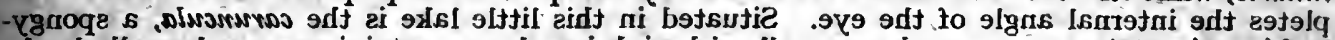

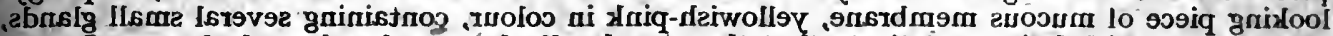
ts tav [-

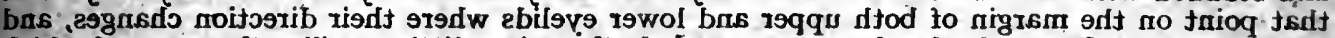

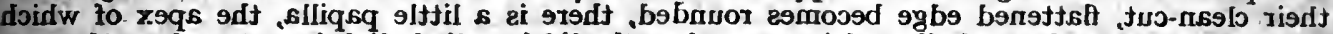

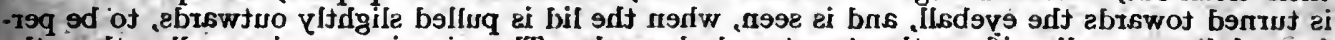

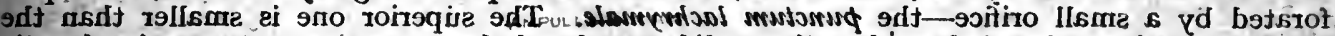

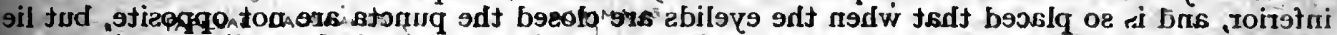

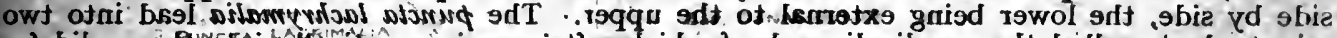

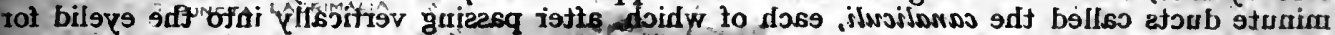
9dt bns abrswni үllstnosinod gininrot

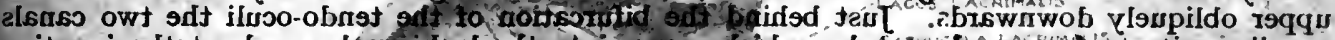

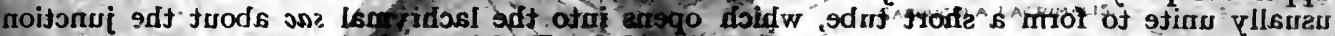

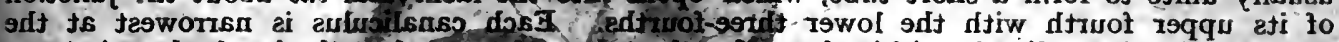
emrut fi

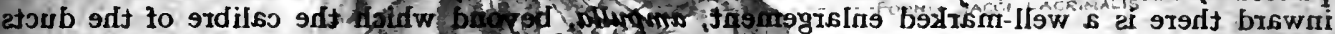

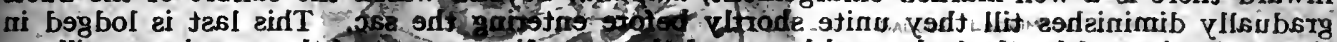

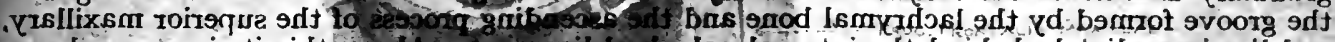

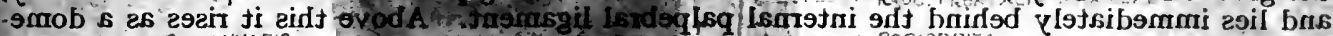

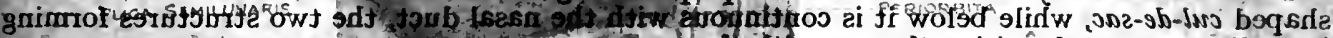

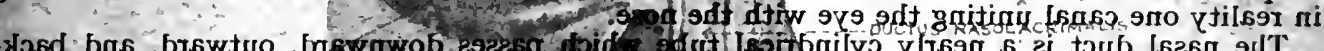

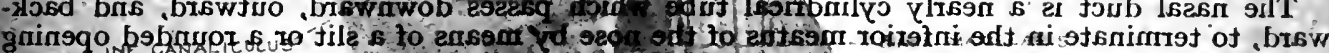
bofsnidiut tongtal ont to yjimsts

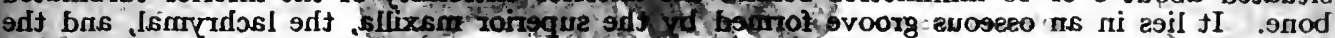

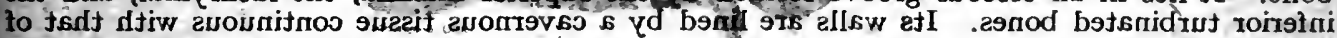

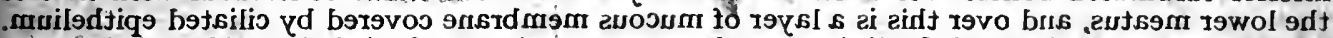

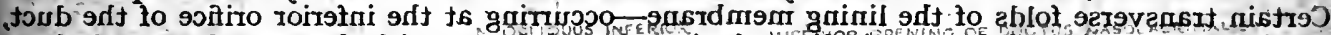

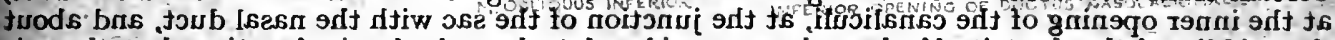

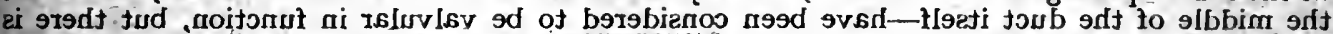
9sir 9d'T . b9noifn

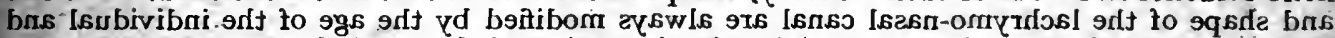

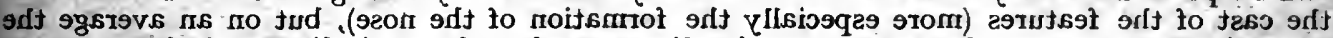

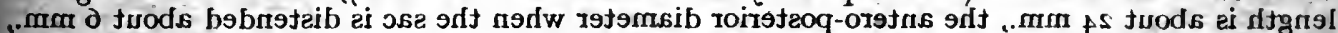

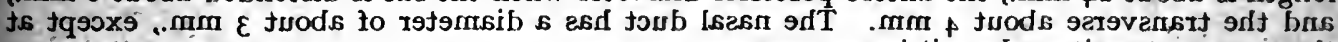

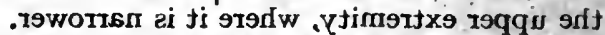

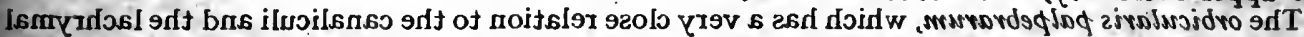

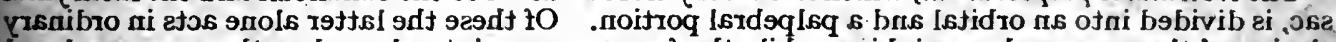

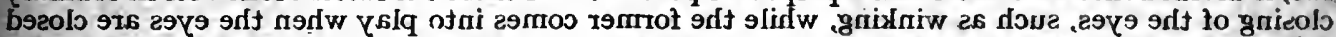

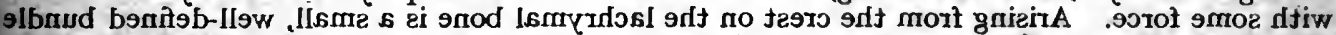

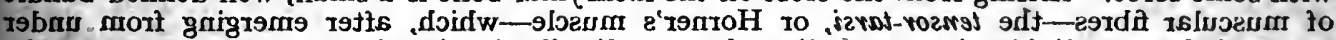

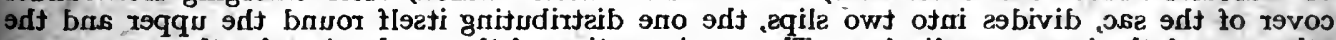

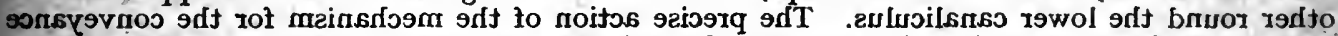

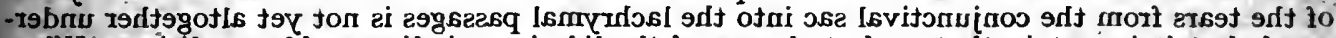

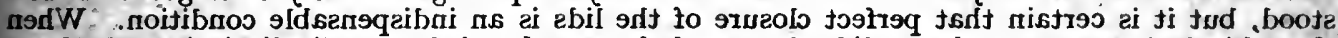

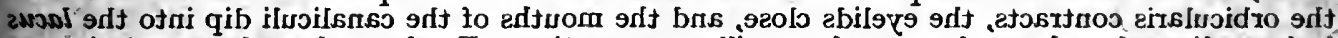

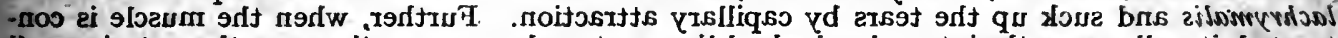

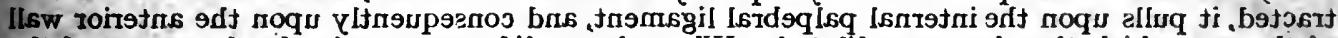

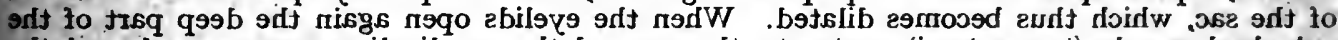

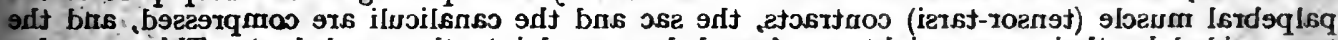

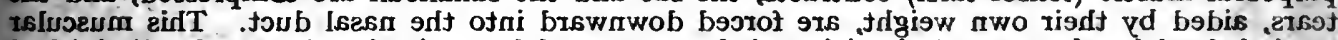

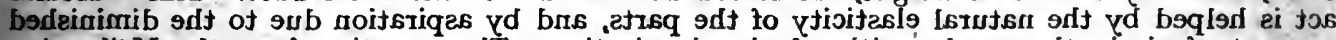

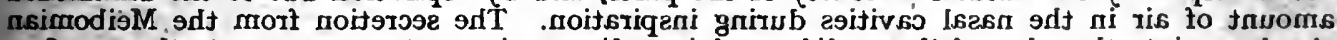

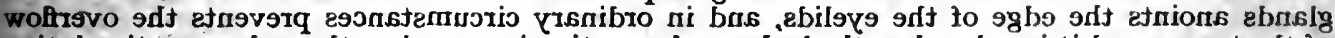

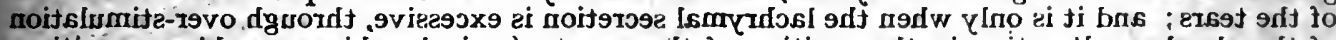

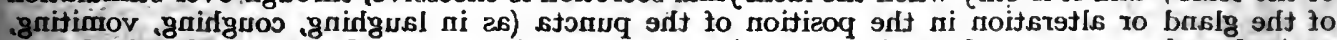
.exlogro 


\section{DESCRIPTION OF PLATE II \\ Anatomy of the Tear Passages}

It may be well to recur briefly to the anatomy and physiology of the lachrymal apparatus, a clear conception of which is absolutely necessary for a right understanding of the principles underlying the pathology and treatment of diseases of the tear passages. The tears, coming from the lachrymal gland, and mixed with secretions from the conjunctival glands, flow over the surface of the eyeball towards the inner canthus, where they collect in the hollow of the lacus lachrymalis. On its outer side this space is limited by the plica semilunaris, while its other boundaries are formed by that part of the palpebral fissure which completes the internal angle of the eye. Situated in this little lake is the caruncula, a spongylooking piece of mucous membrane, yellowish-pink in colour, containing several small glands, and studded with hairs so delicate that they can hardly be seen by the naked eye. Just at that point on the margin of both upper and lower eyelids where their direction changes, and their clean-cut, flattened edge becomes rounded, there is a little papilla, the apex of which is turned towards the eyeball, and is seen, when the lid is pulled slightly outwards, to be perforated by a small orifice-the punctum lachrymale. The superior one is smaller than the inferior, and is so placed that when the eyelids are closed the puncta are not opposite, but lie side by side, the lower being external to the upper. The puncta lachrymalia lead into two minute ducts called the canaliculi, each of which, after passing vertically into the eyelid for a very short distance; changes its direction, the lower turning horizontally inwards and the upper obliquely downwards. Just behind the bifurcation of the tendo-oculi the two canals usually unite to form a short tube, which opens into the lachrymal sac about the junction of its upper fourth with the lower three-fourths. Each canaliculus is narrowest at the punctum, but immediately within the orifice the tube widens, and at the bend where it turns inward there is a well-marked enlargement, ampulla, beyond which the calibre of the ducts gradually diminishes till they unite shortly before entering the sac. This last is lodged in the groove formed by the lachrymal bone and the ascending process of the superior maxillary, and lies immediately behind the internal palpebral ligament. Above this it rises as a domeshaped $c u l-d e-s a c$, while below it is continuous with the nasal duct, the two structures forming in reality one canal uniting the eye with the nose.

The nasal duct is a nearly cylindrical tube which passes downward, outward, and backward, to terminate in the inferior meatus of the nose by means of a slit or a rounded opening situated about 8 or 10 millimetres behind the anterior extremity of the inferior turbinated bone. It lies in an osseous groove formed by the superior maxilla, the lachrymal, and the inferior turbinated bones. Its walls are lined by a cavernous tissue continuous with that of the lower meatus, and over this is a layer of mucous membrane covered by ciliated epithelium. Certain transverse folds of the lining membrane-occurring at the inferior orifice of the duct, at the inner opening of the canaliculi, at the junction of the sac with the nasal duct, and about the middle of the duct itself-have been considered to be valvular in function but there is little evidence in favour of such a theory, except in the case of that first mentioned. The size and shape of the lachrymo-nasal canal are always modified by the age of the individual and the cast of the features (more especially the formation of the nose), but on an average the length is about $24 \mathrm{~mm}$., the antero-posterior diameter when the sac is distended about $6 \mathrm{~mm}$., and the transverse about $4 \mathrm{~mm}$. The nasal duct has a diameter of about $3 \mathrm{~mm}$, except at the upper extremity, where it is narrower.

The orbicularis palpebrarum, which has a very close relation to the canaliculi and the lachrymal sac, is divided into an orbital and a palpebral portion. Of these the latter alone acts in ordinary closing of the eyes, such as winking, while the former comes into play when the eyes are closed with some force. Arising from the crest on the lachrymal bone is a small, well-defined bundle of muscular fibres--the tensor-tarsi, or Horner's 'muscle-which, after emerging from under cover of the sac, divides into two slips, the one distributing itself round the upper and the other round the lower canaliculus. The precise action of the mechanism for the conveyance of the tears from the conjunctival sac into the lachrymal passages is not yet altogether understood, but it is certain that perfect closure of the lids is an indispensable condition. When the orbicularis contracts, the eyelids close, and the mouths of the canaliculi dip into the lacus lachrymalis and suck up the tears by capillary attraction. Further, when the muscle is contracted, it pulls upon the internal palpebral ligament, and consequently upon the anterior wall of the sac, which thus becomes dilated. When the eyelids open again the deep part of the palpebral muscle (tensor-tarsi) contracts, the sac and the canaliculi are compressed, and the tears, aided by their own weight, are forced downward into the nasal duct. This muscular act is helped by the natural elasticity of the parts, and by aspiration due to the diminished amount of air in the nasal cavities during inspiration. The secretion from the Meibomian glands anoints the edge of the eyelids, and in ordinary circumstances prevents the overflow of the tears; and it is only when the lachrymal secretion is excessive, through over-stimulation of the gland or alteration in the position of the puncta (as in laughing, coughing, vomiting, etc.), that the excretory channels become inad equate, and tears overflow on to the cheeks. 


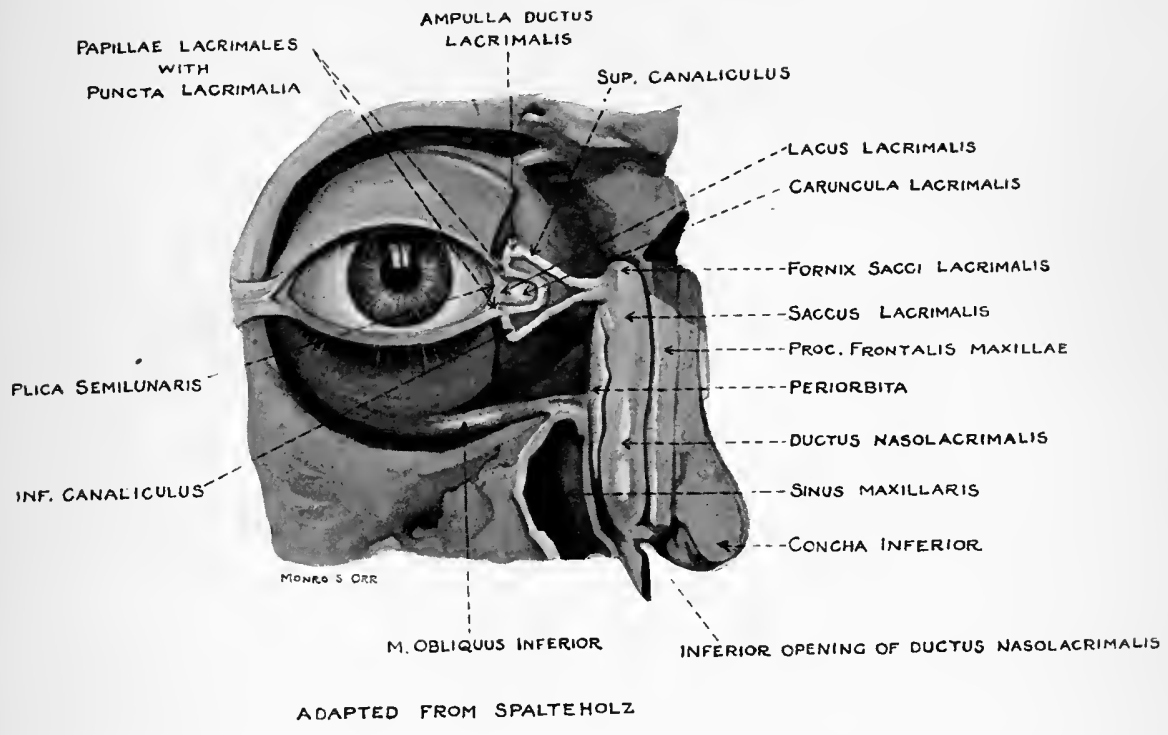



is displaced much lachrymation follows, even though the orifice of the superior duct is in accurate apposition to the eyeball. A lower punctum that can be seen without drawing down the lid is always everted. Displacement of the puncta may arise from want of tone in the fibres of the orbicularis palpebrarum, a condition frequently seen in the aged, the result of the shrinking of the tissues from senile atrophy; or it may occur in paralysis of the facial nerve, or after excision of the upper jaw. Eversion of the eyelids may take place from many different causes, and the ectropion thus produced turns the puncta outwards, and withdraws the mouth of the canaliculus from the lacus lachrymalis, with the result that the conjunctival surface may become fleshy and cuticular. Should the lids be inverted in consequence of burns, wounds, granular conjunctivitis, etc., the puncta are again in a faulty position, and the tears collect at the caruncle and trickle over the cheek. A similar result may be bronght about by the separation of the lid from the eyeball through chronic thickening of the conjunctiva, enlarged caruncle, or polypoid or other tumour of the conjunctiva situated near the inner canthus. The puncta are occasionally congenitally absent, or they may be obliterated from disease of, or injury to, the eyelids. It rarely, however, happens that both upper and lower are destroyed, the latter being more generally closed. Inflammatory swelling of the lining membrane of the canaliculi may become so pronounced as to obstruct the excretion of the tears, or a foreign body-e.g. an eyelash-may find its way into a canaliculus, most frequently the upper, and not only form a barrier to any outflow, but also set up considerable inflammation. The canaliculi may also be obstructed by the formation of calculous deposits in their interior.

The excess of tears renders vision indistinct, the constant wiping of the eyes produces considerable irritation, and a certain amount of conjunctivitis develops. The watering and inflammation always increase with exposure to cold winds, but, as a rule, pass off when the weather becomes warm. An examination of the nose should always be made in such cases, for in nearly all the nasal mucous membrane will be found to be involved. The nostril on the affected side may be dry and slightly inflamed, or there may be a swollen and œdematous condition of the spongy tissue covering the inferior turbinate bone; and as the disease travels towards the eye, the lining membrane of the lachrymal duct becomes swollen, and the calibre of the passage contracted. When the upper eyelid is everted, the retrotarsal fold of conjunctiva may be found hypertrophied, and there is hypersecretion of mucus from Krause's glands.

Certain cases, which present a well-defined clinical picture, have been grouped under the name of "lachrymal catarrh." There is a history of 
" cold in the head," which had been present for a longer or shorter time before the eye-symptoms showed themselves. Then, after some exposure to cold, more especially when the patient has been heated, there is a sensation as if a foreign body had entered the conjunctival sac, and an itchy feeling in the eyelids is followed by a burning sensation. The lids become slightly swollen and are of a dusky red colour, and their edges may be ulcerated. There is excessive lachrymation, and after a time a slight mucous discharge, which is seen adhering to the roots of the eyelashes, and may be so abundant as to glue the lids together during sleep. The conjunctiva is injected, and the caruncle and semilunar fold are swollen. When the eyelid is pulled outward the lachrymal papilla is seen to be enlarged, and the punctum is dilated and patulous-very characteristic symptoms. Pressure over the tear sac usually causes a slight regurgitation of clear watery fluid through the lower canaliculus. There may be slight spasmodic closure of the eyelids and inability to look at the light. If the patient be exposed to cold or damp, such symptoms may persist for a long time, the discomfort being some days greater, other days less, but never entirely gone, and always aggravated by the necessarily constant use of the handkerchief, or by the rubbing of the éyelids to try to obtain relief from the intolerable itching. Opthalmia tarsi frequently co-exists, and the caruncle and semilunar fold are red and swollen. Whenever one eye alone is thus affected suspicion ought at once to fall upon the lachrymal passages as the cause of the trouble. The swelling of the conjunctiva also tends to separate the punctum from the eyeball, and, if the disease be not controlled by treatment, a vicious circle is developed whereby the disease is perpetuated.

After a "watery eye". has persisted for a time-it may have been for months or even years-a slight swelling is observed at the inner canthus, over the region of the lachrymal sac. This, at first insignificant, becomes more and more conspicuous as the disease progresses. - It is painless, and the skin (which is at the outset of a natural colour, but may subsequently become inflamed) glides freely over it. When the tumour is pressed upon, its contents pass downwards into the nasal duct, and the swelling disappears. In a few hours, however, there is a return to the former condition; a fresh accumulation of tears has formed, and by-and-by this is mixed with catarrhal discharge due to inflammation of the wall of the sac. As the tumour grows larger, a point is reached when, the nasal duct having become blocked, the contents can no longer be pressed downwards into the nose, but regurgitate through the puncta and overflow the eye. The discharge usually consists at first of glairy mucus, like white of egg, streaked with pus; but this after a time becomes turbid and more distinctly purulent. The name blennorrhoea, however, 
which is often applied to this form of disease, implies, not that there is a specific inflammation analogous to blennorrhœa of the conjunctiva, but only that the secretion contains pus. Under the microscope the discharge is seen to swarm with micro-organisms of many kinds. The pneumococcus is the most important, and it is so virulently infective that when it comes into contact with an abrasion of the cornea, purulent infiltration speedily occurs and causes ulcer with hypopyon. When it comes into contact with an operation wound most disastrous results follow. In many cases operated on for cataract, the eye has been lost through suppuration arising from a blennorrhœa; and to operate when the tear passages are not permeable is simply to court defeat.

As the tumour becomes still larger, pressing fails to empty it, as the canaliculi are now blocked as well as the nasal duct. The walls of the sac become relaxed and thinned from the pressure of the accumulating discharge; and a'swelling of a bluish-grey colour of a size varying from that of a horse-bean to that of a walnut, fluctuant, and grooved on its anterior surface by the internal palpebral ligament, forms at the inner canthus. This is called a mucocele, and is usually painless, but may produce much disfigurement. It causes constant lachrymation, and patients. often complain of dryness of the nostrils on the same side. When catarrh of the tear sac has existed for a long time, periostitis, followed by caries of the lachrymal bone, always occurs; so that when a probe is passed along the canaliculi it strikes against bare bone.

Blennorrhœa of the lachrymal sac is more frequent among women than men, and more especially in those who, like washerwomen, are exposed to considerable alternations of heat and cold. It is also more likely to occur in those whose strength is below normal, and that is probably why diseases of the tear passages are so common among women who are nursing children. In some cases it is clearly connected with syphilis or tubercle; and in such, caries of the bones surrounding the nasal duct always occurs at an early stage of the disease. When it is met with in infants, it is due to a congenital narrowness of the nasal duct or to closure of its lower end where it should open into the nose, and it passes off, as a rule, spontaneously as soon as the tear passages become fully developed.

In most instances acute inflammation of the tear sac appears as a complication in the course of a chronic catarrh, and comes on as a result of a streptococcal infection after exposure to cold. It may, however, also occur after any injury in the neighbourhood of the sac, in the course of affections of the nasal mucous membrane-especially in subjects whose health has broken down, more particularly in those who suffer from syphilitic disease of the nose-or after measles and scarlatina. 
Occasionally infants are born suffering from dacryocystitis, or it comes on within a few weeks of their birth.

The onset of the disease is usually marked by shivering and rise of temperature, and there is intense pain in the region of the sac, where the skin becomes red and œdematous. Between the root of the nose and the inner canthus a swelling appears, at first well defined, and so painful that the patient shrinks the moment it is touched. Very soon, however, as the surrounding tissues become involved, it gets more diffuse and extends to the lids and cheeks. The symptoms of local inflammation are sometimes so severe that the disease has been mistaken for erysipelas, and this error may all the more readily occur when the initial feverishness is great, or when the disease is bilateral. As the size of the swelling increases, the skin assumes a more angry red and becomes glistening from the tension to which it is subjected. The tumour, at first firmly elastic and resistant, now becomes fluctuant, and at a point below the internal palpebral ligament a yellowish spot appears, over which, if no operation is performed, the tissues give way, and the purulent contents of the sac escape. Immediately after the abscess has opened great relief is felt by the patient. When the opening in the skin is large enough to permit of the free escape of pus, the swelling and redness soon subside; and if the nasal duct be permeable the opening will close and the cure be complete. It often happens, however, that the opening into the abscess cavity remains, and tears and muco-pus continue for a long time to escape through the fistula thus formed. In cases which have been long neglected, especially in scrofulous and syphilitic subjects, the surrounding tissues become inflamed, the adjacent bones become carious, and inflammatory swelling and sprouting granulations appear round the fistula. In a few rare cases the abscess does not burst externally, but perforates the lachrymal bone, and its contents, escape into the nose.

As has been said, acute dacryocystitis may be mistaken for erysipelas, but, as a rule; the diagnosis is not difficult, as in the former the general febrile disturbance is not so high, the redness and swelling of the skin rarely pass over the nose to the opposite side of the face, and quite pathognomonic is the presence at the inner canthus of a well-defined tumour, excessively tender to the touch. The disease may also be confounded with abscess at the root of the canine tooth, but here the internal canthus will be free from tenderness and circumscribed swelling, while palpation of the canine fossa by the mouth will detect a swelling there. In hordeolum, or ordinary "stye," the swelling of the eyelids and the parts around may be very great, but palpation will show that the tender spot is not over the tear sac, but at a point on the margin of the eyelids, where, in a day or two, suppuration will be seen. In the 
diagnosis of all tumours, abscesses of the skin, boils, etc., in the neighbourhod of the sac, the absence of a history of previous "watery eye" is of the first importance.

Treatment.-The treatment of these conditions is always troublesome and protracted, and varies according to the stage of the disease when the patient comes under observation. If the tear-passages are acting satisfactorily, a drop of argyrol instilled into the conjunctival sac will pass. in a few minutes into the nostril, and stain the nasal discharge brown; that is readily detected in the pocket-handkerchief when the patient blows his nose.

1. Simple Lachrymation-Epiphora or Stillicidium.-In every case in which a watery eye comes on suddenly, careful examination must be made of the front of the eye so as not to overlook a misplaced eyelash, an unsuspected foreign body, an abrasion of the cornea, inflammation of the conjunctiva, or a commencing iritis. The cause having been determined, the principles of treatment suggest themselves naturally. Careful examination ought to be made of the teeth, eyes, and nose, and any defects remedied. It is most important to correct any error of refraction with suitable spectacles, and any hypertrophy or inflammation of the nasal mucous membrane must be treated. If there be simply congestion of the erectile tissue over the inferior turbinate bone, much relief will be given by painting the nostrils with a solution of cocain and menthol, or by causing the patient to inhale carbolic and menthol smelling-salts. If the lower punctum be contracted, it ought to be carefully enlarged by means of a conical dilator; and if the lachrymation persist, a 2 per cent. solution of cocain in adrenalin ( $\mathrm{I}$ in IOoo) should be slowly injected along the canaliculus into the lachrymal sac. If there be no obstruction, the fluid will at once pass into the nose ; if, however, the lining membrane of the nasal duct be swollen from congestion the passage of the fluid will be delayed until the adrenalin has had time to constrict the vessels and render the canal pervious. It must be remembered that the use of adrenalin in this way is occasionally followed by acute coryza, which, however, is usually very transient. Force must never be used when injecting the tear passage for fear the wall of the sac ruptures and the fluid escapes and infiltrates the tissue. If such an accident occurs while the lachrymal sac is being syringed with coloured fluids like argyrol or a solution of methylene blue, a permanent discoloration will take place, much to the annoyance of the patient and the mortification of the doctor.

2. Lachrymal Gatarrh. Astringent lotions, or astringents combined with alkalies, diminish secretion and subdue conjunctival inflammation; and dusting the eyelids with calomel, well dried and finely powdered, often gives marked relief to the sensation of itching and burning heat. 
Brushing the palpebral conjunctiva with a solution of nitrate of silver (from I to 2 per cent. according to the amount of the discharge), or pencilling the lids with a crayon of alum, of sulphate of copper, or of mitigated caustic, often aids very materially in restoring the puncta to their natural position, and permitting them to resume their proper excretory function. The application of the actual cautery to the conjunctiva of the lower lid at the inner canthus is often more efficacious than the use of astringents or caustics. When, however, the eversion of the lids is too great to be overcome by such simple means, it is necessary to slit up the canaliculus as far as the lachrymal sac, and so convert the duct into a little gutter along which the tears may escape. This operation is best performed with the small probe-pointed knife devised by Weber. The eyelid having been pulled outwards, so as to keep it on the stretch, the blade is introduced into the canaliculus and pushed horizontally inwards until its point is felt to strike against the lachrymal bone. The cutting edge ought to be inclined backwards and upwards, and the incision of the canaliculus is completed by simply raising the handle of the knife, and the result is more perfect if a very small piece is excised with curved scissors from the posterior lip of the incised canaliculus. For two or three days the lips of the wound must be kept from adhering by passing a probe along their edges.

Sometimes the punctum is partially occluded, or there may be an obstruction in the canaliculi, most frequently just where they unite, or at the point where they enter the sac. Such obstacles must be overcome by the use of small probes, but the employment of these has to be managed with considerable nicety of touch, as, if a false passage be made in the mucous membrane lining the duct, the subsequent cicatrisation will still further obliterate the channel, and matters will be worse than they were at the beginning. The natural drainage of the tears having been established, the progress of the case ought to be quite satisfactory, and in most instances nothing more requires to be done. Any defects in the eyes or nose must be remedied, and the local treatment is greatly assisted by the use of alkaline salines given internally.

3. Catarrh of the Lachrymal Sac and Stricture of the Nasal Duct.-The mucous membrane lining the lachrymal sac is now inflamed and infected by septic micro-organisms, and that fact is of supreme importance in treatment. It never undergoes spontaneous cure, and steadily goes from bad to worse unless it is properly treated. In the milder cases all that is necessary is to keep the sac empty by pressing on it with the tip of the little finger. In addition, the sac and nasal duct ought to be washed out by alkaline antiseptic solutions; and for ordinary purposes nothing answers better than the nasal tabloids with 

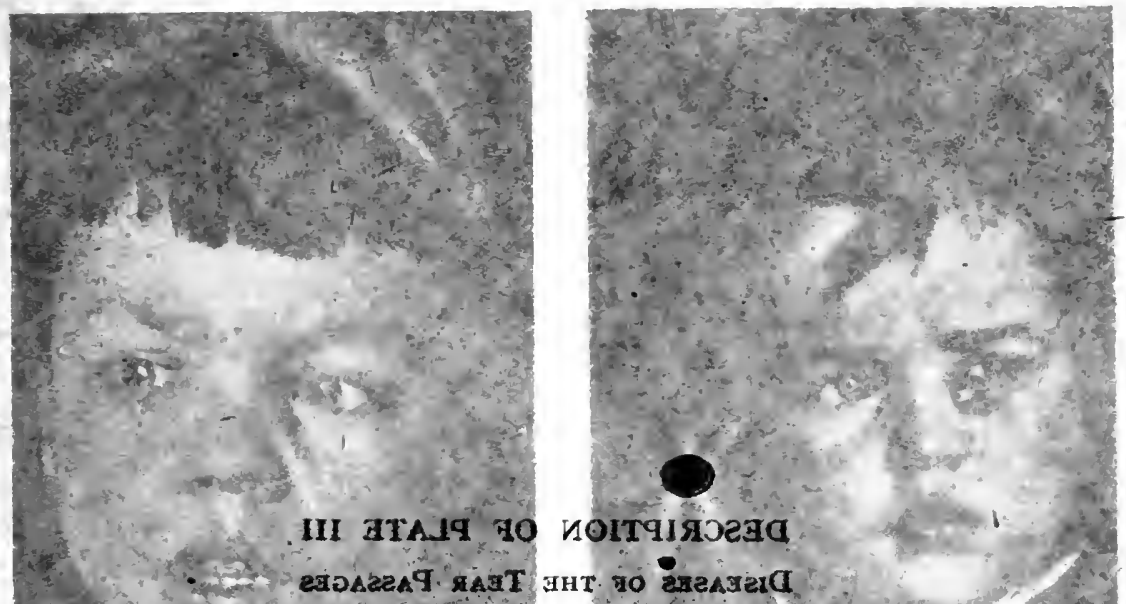

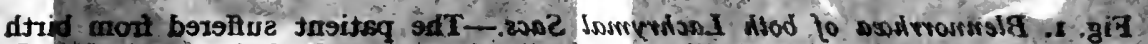

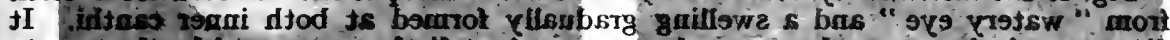

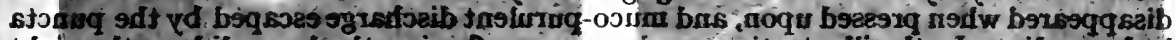

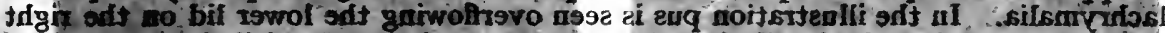

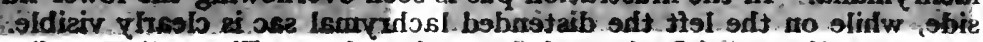

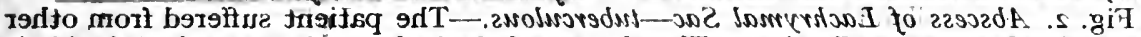

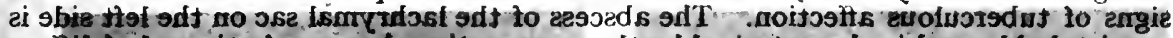

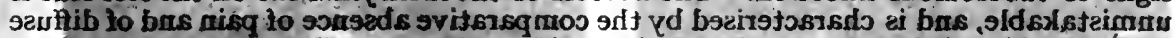

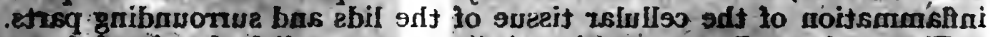

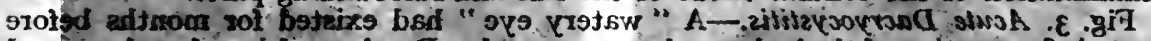

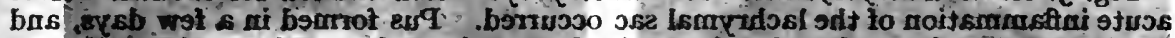

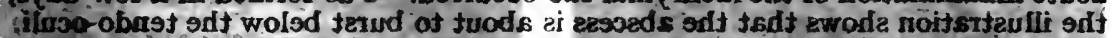

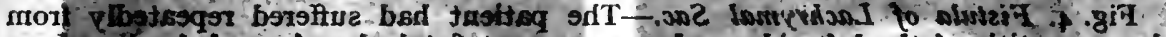

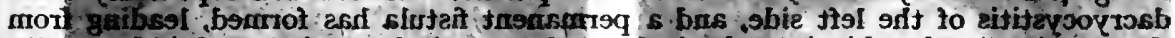

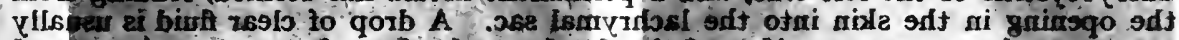

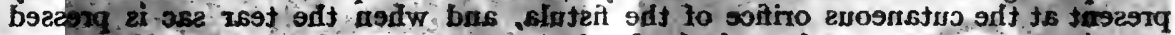

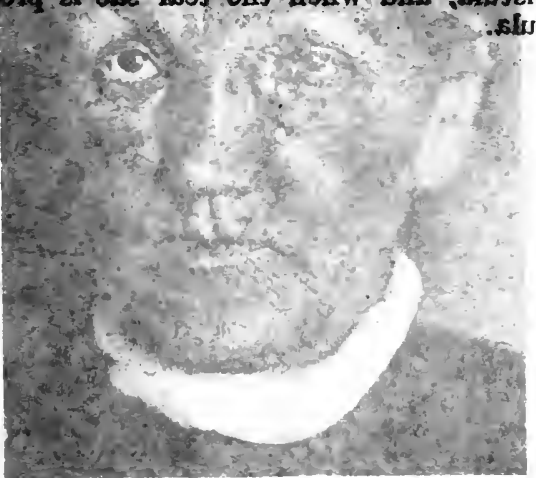

Figill

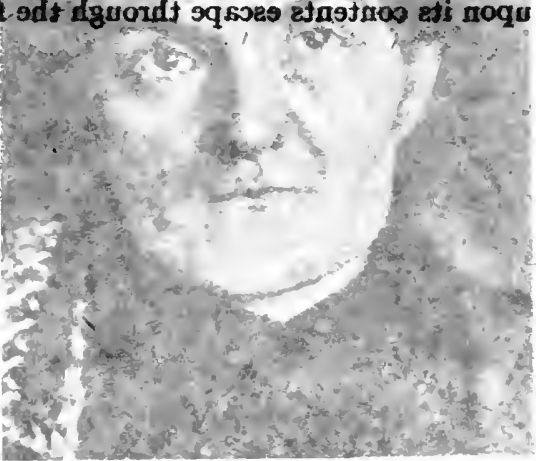

Fig $\mathbb{E}$ 
Whung the palpebral conjunetiva with is solution of nitrate of silves (f1, m I to 2 per cont. arcording to the amount of the discharge), or pencillug the jids mith a cruron of alum, of sulphate of copper, or of miti-

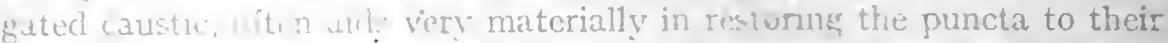
nat ital in

futic

Fig. I. Blennoryhoea of both Lachrymal Sacs, - The patient suffered from birth from " watery eye" and a swelling gradually formed at both inner canthi It disappeared when pressed upon, and muco-purulent discharge escaped by the puncta lachrymalia. In the illustration pus is seen overflowing the lower lid on the right side, while on the left the distended lachrymal sac is clearly visible.

Fig. 2. Abscess of Lachrymal'Sac-tuberculous. The patient suffered from other signs of tuberculous affection. The abscess of the lachrymal sac on the left side is unmistakable, and is characterised by the comparative absence of pain and of diffuse inflammation of the cellular tissue of the lids and surrounding parts.

Fig. 3. Acute Dacryocystitis.-A "watery eye" had existed for months before acute inflammation of the lachrymal sac occurred. Pus formed in a few days, and the illustration shows that the abscess is about to burst below the tendo-oculi.

Fig. 4: Fistula of Lachrymal Sac. The patient had suffered trepeatedly from dacryocystitis of the left side, and a permanent fistula has formed, leading from the opening in the skin into the lachrymal sac. A drop of clear fluid is usually present at the cutaneous orifice of the fistula, and when the tear sac is pressed upon its contents escape through the fistula.

will be wore than they were - bu inning. Tle matumal drandge of the tear having been estaband in most nore rupuires to be done. Anv defoces in the eyes or merlici, and the local treatmint is gratly assisted. by of the Jachrymal Sac and Stricture of the Nasal Duct. ! an mombre lining the lachrymal sac is now inflamed ptic micio-organisms, and that fact is of supreme. 


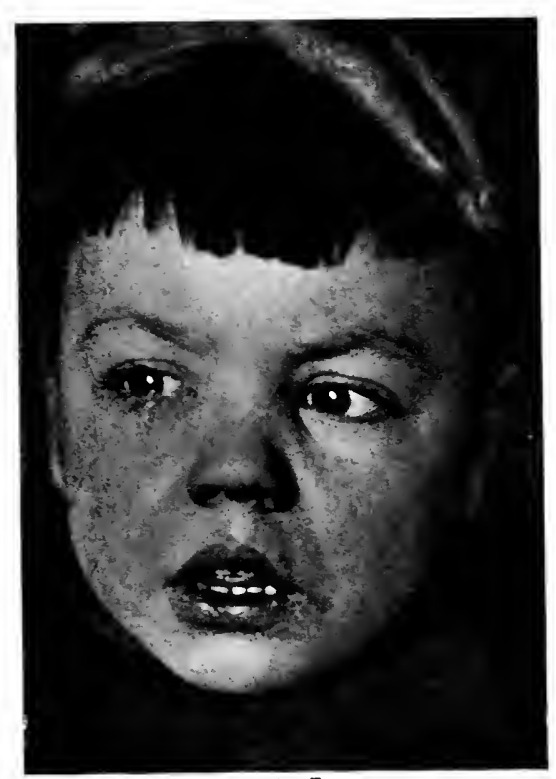

Fig I

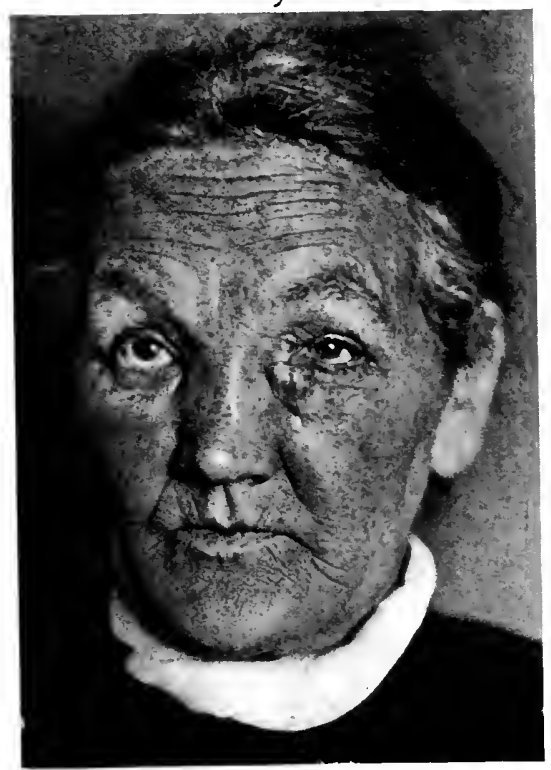

FigIII

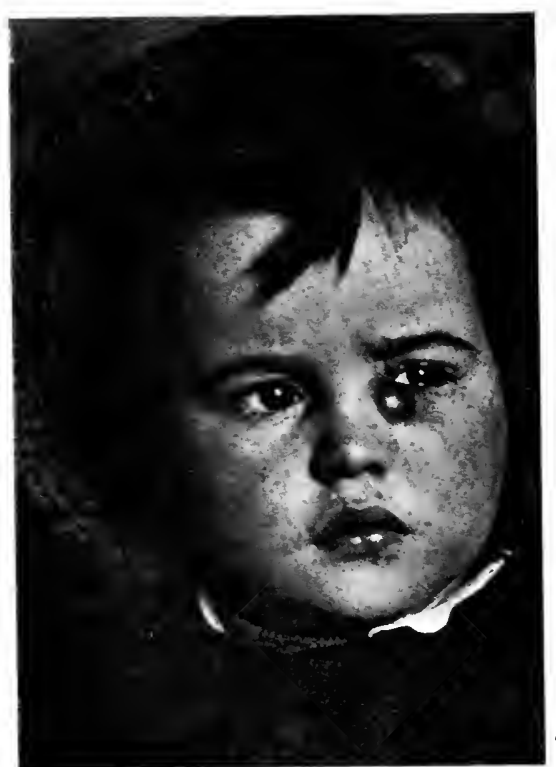

Fig II

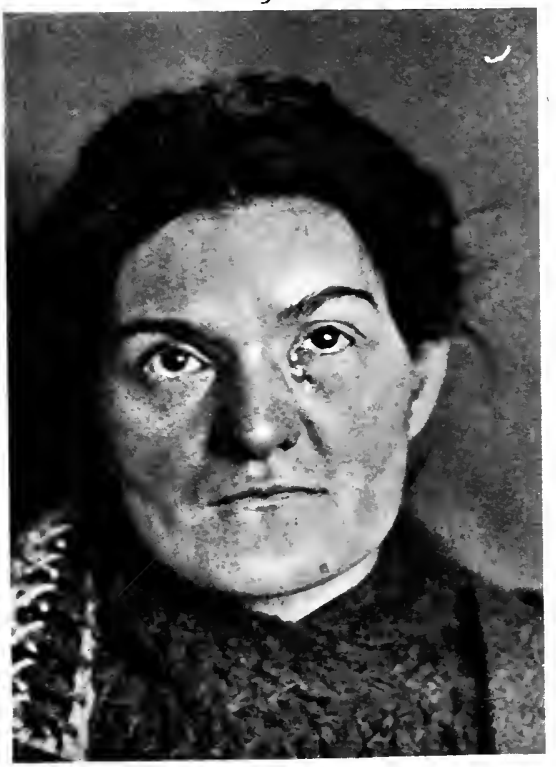

Fig $\square$ 

carbolic acid prepared by Burroughs, Wellcome \& Co. One or two tabloids, each containing five grains of boracic acid, five grains of borax, and half a grain of carbolic acid, are dissolved in a wineglassful of tepid water, and the solution injected into the sac by means of a small glass syringe, provided with a lachrymal nozzle. If the duct be free from obstruction the fluid passes into the inferior meatus, and, if the patient inclines his head forwards, flows out of the nostril. These injections ought to be repeated every day, or every second day. The nasal mucous membrane will also require attention, and a similar solution may be sniffed up the nostrils, or sent through by means of a syphon douche. After the membrane has been thoroughly cleansed, an ointment ought to be applied to its surface. Menthol, cocain, and camphor always afford much relief, from their power of contracting the blood-vessels, and so reducing the congestion of the erectile tissue which covers the inferior turbinate bones; and when such drugs are combined with iodoform; aristol, carbolic acid, etc., the surface soon becomes much healthier. A solution of menthol and iodoform in paroleine or saxol may also be used with a spray atomiser, and this has a certain advantage over ointments, in so far that the medicated spray reaches every part of the nasal mucous membrane.

When, however, the contents of the sac cannot be pressed downwards into the nose, and when fluids injected by the syringe will not pass onwards, but regurgitate through the upper canaliculus, means must be taken to re-establish the permeability of the lachrymal passages. When the obstruction is due to simple inflammatory thickening of the mucous membrane, this may be effected by the passage of small probes through the puncta and canaliculi, and down the sac and duct, into the nostril. Some surgeons make a practice of introducing a probe by the superior canaliculus; but, on the whole, it is more convenient and easier to use the inferior. The operation is performed in the following manner : The lower eyelid is pulled outwards by the forefinger of the left hand, while with the right the probe, previously bent so as to present a concavity forward (this makes its subsequent passage into the duct easier), is pushed into the punctum, at first perpendicularly, and then, the handle being depressed, horizontally along the canaliculus until it enters the sac and is stopped by the bone at the inner wall. The direction of the probe must now be completely changed, in order to reach the nasal duct. While the point is kept firmly against the inner wall, the handle is raised until it assumes a vertical position. The finger of the left hand ought then to be removed from the lower lid, and with the thumb or forefinger the skin of the forehead should be raised and made tense, the passage of the probe into the entrance of the duct being thus facilitated. The probe ought now to be pushed gently but steadily downwards, 
backwards, and a little outwards, following the direction of the duct, until it reaches the nose. Great care must be taken not to tear the mucous membrane and form a false passage; and while firm pressure is often needed to overcome a stricture, anything approaching to violence must be avoided. If the right course has been taken, the handle will rest against the upper and inner margin of the orbit, and will point in a line which, passing through the centre of the internal palpebral ligament, is directed downwards towards the canine tooth of the same side. Any marked deviation from this position implies that a false passage has been made, and the probe ought to be at once withdrawn and no further attempts at probing made until the laceration has had time to heal. If the probe passes into the cheek or into the orbit instead of following the course of the nasal duct, the result may be disastrous: abscess of the orbit has been caused in that way and the eye has been lost. When the probe strikes against dead bone, and a firm stricture is also present in the duct, it is necessary to slit up the lower canaliculus, and open into the sac, according to the method first proposed by Bowman. In cases where the lower canaliculus is obstructed the operation may be performed through the superior. A strong Stilling's knife is then employed, and when its probe point rests against the inner bony wall, its handle is raised and the canaliculus slit right into the sac. The cutting edge is then turned forwards, and the anterior wall, together with the internal palpebral ligament, incised as freely as possible. The knife is now pushed downwards into the duct, and the stricture divided by turning the edge in every direction. It often happens that whenever the anterior wall is divided pus wells out by the side of the knife, and when much dead bone is present the discharge is usually very fœetid. The sac ought, thereafter, to be freely irrigated by antiseptic and astringent solutions; but injections ought not to be made immediately after passing a probe. It is wiser to wait until the following day, when there will be less danger of washing germs from the infected sac into the surrounding tissues. When the disease has existed for a long time, care must be taken not to use too much force with the syringe as the walls are thin and might give way under the pressure, and the injection fluid pass into the subcutaneous tissues. A large probe ought now to be passed along the nasal duct. Couper and Theobald recommend very large probes and claim surprisingly good results from their use, but, as Risley has shown, the less probing lachrymal cases get, the better. What is wanted is not a duct dilated to its utmost capacity, but a channel kept permeable. When I use a large probe, I prefer the one devised by Ziegler, and as the use of all probes of this size is very painful, it is advisable to have the patient under a general anæsthetic, nitrous oxide being, as a rule, 
sufficient. 'After the probe has been passed a style may be introduced into the nasal duct. Nothing is more serviceable for that purpose than a piece of lead wire, because that metal readily moulds itself to any irregularity in the walls of the canal. Formerly the use of styles was unsightly, as they were introduced from the outside immediately below the internal palpebral ligament; but when they are passed from the conjunctival surface, the lead can be so tapered and curved that it lies in the groove formed by the slit canaliculus, and is almost invisible. The style is usually removed in from forty-eight hours to a fortnight after the operation, but it can be worn for months without discomfort. It is better, however, for the patient to have it taken out at regular intervals, and the sac washed with an astringent lotion. Suppuration is diminished by the injection of 25 per cent. argyrol solution, but the prolonged use of either argyrol or protargol may be followed by indelible staining of the conjunctiva. When the duct is closed by a bony stricture it will be necessary to perforate the lachrymal bone and so establish a passage directly into the nose.

In addition to local treatment, careful attention must be paid to the general health. The strength of the patients is often much run down, and good food and protection from exposure to cold will do a great deal to expedite the progress of a chronic case. Tubercular cases are sometimes bilateral, and in them, as well as in those complicated by syphilis, constitutional treatment is most essential. Anything like very forcible dilatation of the lachrymal duct must here be carefully avoided, as being likely to still further damage the bony walls of the canal, which are, in all likelihood, already extensively diseased.

4. Acute Dacryocystitis.-Acute dacryocystitis is rarely seen early enough to permit of anything being done to check its course; but when this is so, the progress may be arrested by pressing upon the sac to evacuate its contents, prescribing an evaporant lotion to diminish pain and subdue inflammation, administering a purgative, and applying leeches. As soon, however, as it is quite clear that pus has formed within the tear sac, use should be made of fomentations, and of opiates or hypodermic injections of morphia to procure relief from pain, and enable the patient to obtain sleep. Whenever fluctuation can be detected, a free incision ought to be made right down into the sac, beginning about the middle of the lower border of the internal palpebral ligament, and enlarged downwards for about half an inch in order that the pus may have free exit. The passage ought then to be cleansed by gentle syringing with warm antiseptic solution, and a probe introduced through the incision into the nasal duct, when, if this channel be successfully opened, the contents of the sac will escape into the nostril and the skin wound 
will close. In the majority of cases, however, it is more satisfactory not to interfere with the canal until all signs of acute inflammation have disappeared, and then to slit the lower canaliculus, pass a large probe into the nasal duct, and introduce a style which can be worn as long as may be deemed necessary.

5. Permanent Enlargement of the Lachrymal Sac, with or without a Fistula.-In cases of very old standing, where the nasal duct has become blocked, and there is extensive necrosis of bone, a permanent fistula, situated in the midst of a fungating mass of granulation tissue, remains. In other instances the walls of the sac become thickened and completely lose their elasticity; and in these, even after a free passage has been re-established, the lachrymation is very little (if at all) relieved, and an unsightly swelling remains at the inner canthus. In the treatment of the cases previously described, the aim was to conserve and restore the function of the natural passages. In this group, on the other hand, in order to effect a cure, the natural passages must be completely obliterated.

Till recently this was accomplished by incising the sac from the outside, burning its walls with chemical caustics or by the actual cautery, packing the cavity, and permitting slow granulation. This method, which goes back to the time of Celsus, is still practised; but it has of late been replaced by complete extirpation of the sac. The operation is performed with local anæsthesia. The tablets of novocain and adrenalin prepared by the Saccharin Co. are very convenient. One or two are dissolved in sterile normal saline, and the solution injected beneath the skin covering the sac as well as deeply into the tissues surrounding it. After waiting twenty minutes anæsthesia is complete, and the bloodvessels are so contracted that hæmorrhage is greatly reduced.

An incision about half an inch in length, curved slightly outwards, is made through skin and subcutaneous tissue down to the bone, beginning above the internal palpebral ligament, which is made more distinct by pulling the lower lid outwards. The ligament must always be divided in order thoroughly to expose the sac, and the incision ought to be as near the inner canthus as possible without actually cutting into it. The lips of the wound are conveniently held apart by retractors, and hæmorrhage is controlled by pressure against the upper and lower extremities of the incision.

After the field of operation is cleared of blood, the fibrous covering of the sac is cut through along the crest of the lachrymal bone, and the sac itself, by means of a "rugine," carefully dissected from the lachrymal fossa, caught by forceps, and kept on the stretch, while (still by means of the rugine) it is gradually freed from its adhesions. Great care 
must be taken to get thoroughly over the dome. After it has been completely cleared, the sac, still held firmly by the forceps, is cut through with curved scissors at its junction with the nasal duct, and the upper orifice of the duct is thoroughly scraped with a small Volckmann's spoon.

The skin is sutured by one or two stitches of fine silkworm gut about the middle of the incision, so that free drainage may take place above and below. The wound and the skin surrounding it are swabbed freely with 3 per cent. tincture of iodine, a graduated compress carefully adjusted, and a bandage applied. The dressing is not removed as a rule for three or four days, by the end of which time union has generally taken place. If necessary, the scar may be protected for a few days after that with collodion. The cicatrix is small, and, if the incision be made in one of the natural furrows of the skin, it is, in a few weeks, hardly noticeable. The diseased sac may also be removed by the intranasal method, but that operation should be performed by a highly trained rhinologist.

Sometimes, where the suppuration has been prolonged, it may be impossible to dissect out the sac owing to the extent of the ulceration in its walls, and it has then to be removed piecemeal with a Volckmann's spoon. This is not nearly so satisfactory as complete extirpation, because, should a small piece of mucous membrane be left behind (as is very apt to happen) it will continue to suppurate, and pus will accumulate at the bottom of the wound. In these cases, the skin will, as a rule, heal perfectly, but the pus finds its way, sooner or later, to the surface, usually accompanied by all the signs of acute inflammation. In order to obviate this, it is my practice to apply trichloracetic acid carefully to the cavity, which is afterwards very lightly packed with gauze; to syringe it out with peroxide of hydrogen lotion a day or two later, and thereafter to apply iodine vasogen, or naphthol and camphor, to every part of the granulating surface. The naphthol and camphor preparation (two parts of naphthol and three parts of camphor) is very effective, but it must be used with great caution on account of the irritating effect on the eyeball. This can, however, in great part be prevented if, during its application, oil be freely applied to the conjunctival sac.

The immediate results of complete extirpation of the lachrymal sac are perfectly satisfactory, for the patient is freed from the disfigurement of the swollen sac, as well as from a continual source of suppurative discharge, with all its attendant dangers. The remote results are, as experience has shown, equally good, because abnormal lachrymation occurs only if the eye be exposed to cold winds or other sources of irritation. If, however, watering of the eye persists, extirpation of the accessory lachrymal gland will usually put an end to the discomfort. 
In my own experience, however, that operation has been required on very few occasions.

It may at first seem strange that a constant epiphora should be cured by obliteration of the tear passages; but an explanation is readily to be found in the fact that the lachrymal gland may be regarded as a reserve apparatus, and that, therefore, under normal circumstances, it secretes very little. The natural moisture on the surface of the eyeball is carried away by evaporation, and tears flow only in response to an irritant. An inflamed lachrymal sac is a constant source of irritation, and whenever it is removed the lachrymal gland ceases to secrete abnormally.

In summing up the different lines of treatment, it may briefly be said that, without doubt, a simple epiphora, with or without a blennorrhœa of the sac, may be quite successfully treated by means of injections and occasional probing. This procedure involves, however, much time, and when there is a considerable suppuration of the sac, there must always remain doubt as to ultimate cure by such conservative means. The simpler class of cases, therefore, may need extirpation, if the patient cannot afford the time necessary for treatment, if he have a cataract requiring operation, or if he suffer from ulcer of the cornea, or be liable in his work to receive any injury to the cornea which might become infected from the lachrymal sac.

In acute inflammation, incision and drainage are, in the first instance, necessary; but, if there be much infiltration of the surrounding tissues, extirpation of the sac may be necessary after the acute symptoms have passed off.

Lastly, in those cases where there is persistent discharge associated with the presence of a permanently distended lachrymal sac, whether the contents be mucous, muco-purulent, or purulent, extirpation is undoubtedly the only rational treatment. 


\section{CHAPTER IV}

\section{THE CLINICAL SIGNIFICANCE OF EDEMA OF THE EYELIDS}

Edema of the lids not a disease per se-Non-inflammatory œdema-Inflammatory œdema : (a) when unaccompanied by disease of eyeball; $(b)$ when accompanied by disease of eyeball without impairment of movements or position; (c) when accompanied by disease and displacement of eyeball with impairment of its movements.

EDEMA of the eyelids is not a disease, but only a sign of a pre-existing local or constitutional state. It is, however, one of those conspicuous symptoms of disease which at once arrests attention, and always causes the patient much anxiety. The family doctor is, therefore, usually consulted.without delay, but as the œedema may be due to many different morbid conditions, he, on his part, may have difficulty in knowing the amount of importance he ought to attach to its occurrence. The ætiological factor may originate in the lids themselves, in the eyeball, in the orbit, or in connection with a constitutional disease. It is very important, therefore, that the examination of the patient be conducted carefully and systematically, in order that an accurate diagnosis be made and a trustworthy prognosis given to the patient or his relatives.

The peculiar structure of the skin of the eyelids, and the absence of fat from its connective tissue render it very liable to œedema. All four lids may be implicated, and the swelling may rapidly become so great that the palpebral fissure is reduced to a mere slit. In every instance the physician's first duty is to discover the true significance of the swelling. Until that is known no prognosis can be given, and no rational treatment can be prescribed. In all cases the first and fundamental distinction is to find out whether the œdema has existed for some time, or is of recent occurrence, and whether or not it is accompanied by signs of inflammation.

1. Non-inflammatory CEdema.-This variety is often associated with œdema of the ankles and other parts of the body. Puffiness of the eyelids is frequently the first sign to give warning of the existence of general disease. It occurs for the most part in the lower lids, is usually bilateral, and is quite free from pain. The natural folds of the skin are obliterated, and its surface presents a characteristic waxy pallor. The swelling is soft to the touch and readily pits on pressure. The absence of crepitation on palpation distinguishes it from emphysema, while the fact that it pits on 
pressure differentiates it from the thickening of the skin in myxcedema. Edema of the eyelids is seen most frequently in the course of disease of the kidneys, heart, or liver, and whenever it is detected the urine ought to be tested, and the heart and circulation carefully examined. Puffiness of the lids on first rising in the morning is often present, especially during the menstrual period, in young women who are weakly or anæmic. This variety of œdema appears to be allied to urticaria, and is due to disturbance in innervation-angio-neurotic cedema. It is characterised by being sudden in onset, of short duration, and usually accompanied by swelling of the skin of other parts of the body. Similar appearances occasionally arise from the long-continued use of arsenic internally-arsenical cedema -and that fact should always be remembered when there is persistent œdema of the eyelids, although repeated examination has failed to discover any evidence of either cardiac or of renal disease. Chronic œdema may also be due to local causes. In children suffering from strumous ophthalmia, the persistent blepharospasm compresses the palpebral veins, and causes œdema of the upper lids. It is occasionally symptomatic of prolonged inflammation of the nasal mucous membrane, and it occurs also as a sign of chronic disease of the orbit or of its accessory sinuses. In such cases the œdema may vary from slight puffiness to a swelling so great that the lids cannot be opened. After repeated attacks of erysipelas the skin of the eyelids sometimes becomes greatly hypertrophied and causes solid cedema, which steadily increases until a condition resembling elephantiasis of the eyelids develops. It is due to a permanent alteration of the lymph channels, consequently the hypertrophy is apt to progress after portions of the thickened skin have been removed. The deformity is, therefore, always liable to recur after operation, and the redundant folds of skin must be excised as often as is necessary to produce the desired cosmetic effect.

All these conditions must be distinguished from " bagginess of the lower eyelids" - a peculiarity which seems natural in some people, and tends to increase with age.

2. Inflammatory Edema.-In this variety the skin is usually discoloured, and the swelling occurs for the most part in the upper lid, is frequently confined to one side, and is usually painful. It may be due to injury or to local disease of the lids themselves or to inflammation of the tear passages, but it may also be symptomatic of morbid changes in the eye or orbit. In every case, therefore, the first thing to be done is to separate the lids, either by the fingers or with retractors, in order to examine the condition of the conjunctiva and the structures of the anterior segment of the eye, and to see the position, and to test the movements of the globe. 
(a) The eyeball is unaffected. The lids are red, shining, swollen, hot, and painful. If the odema is increasing, the skin is smooth and tense, whereas if it be passing off, its natural wrinkles quickly reappear. The cause of the inflammation may be quite apparent, for example, a suppurating wound or a boil. In the former case a bacteriological examination should be made, because wounds in the neighbourhood of the eye are, like injuries in any other part of the body, liable to be infected by spores of tetanus bacilli, streptococci, etc. Acute œdema of sudden onset may be due to the bite or the sting of an insect, and in summer that is a frequent cause of the swelling of an eyelid in an infant or a young child. The mark of the sting is usually visible, but when œdema is pronounced, it may be difficult to find. A vaccine pustule or a primary chancre is always accompanied by intense œdema of the eyelid, and in these circumstances the preauricular and submaxillary glands are usually affected very early in the course of the disease. If no cause for the œdema can be seen, the lid must be carefully palpated with the index finger. In that way the margin of the lids, the outer and inner canthus, and the bony margin of the orbit must be thoroughly examined, and a differential diagnosis is arrived at by the discovery of pain on pressure over a particular spot. If the pain be caused while the finger is being drawn along the margin of the lid, the cause is usually a stye, or a suppurating Meibomian cyst, whereas if the sensitive spot be found at the inner canthus, the first thought should be of acute dacryocystitis. A boil or a periostitis may occur at the inner angle of the eye, but either of them is of rare occurrence when compared with an inflamed lachrymal sac. Moreover, the diagnosis can always be confirmed by the history that a watery eye has been present for a considerable time before the onset of the acute inflammation. If a painful infiltration be found in the substance of the lid it will usually terminate in abscess, whereas when the swelling and redness are uniform, and the lid, though infiltrated, is not tender to touch, the condition is probably erysipelas. Herpes of the ophthalmic division of the fifth cranial nerve is frequently mistaken for erysipelas, but if the occurrence of an eruption and the fact that the disease is confined to one side be observed, there ought to be no difficulty in the differential diagnosis. If tenderness be found along the margin of the bony orbit where a localised thickening can also be felt, a diagnosis of periostitis can be made. The periostitis is usually of tuberculous origin when it occurs at the lower and outer margin of the orbit, whereas it is probably syphilitic if the upper border be affected.

(b) The eyeball is inflamed, but its position and movements are unimpaired.

A foreign body beneath the upper lid may cause considerable œdema, 
and if its presence be unsuspected, the symptoms will steadily increase in severity. Whenever, therefore, the history of the case suggests the possible intrusion of a foreign body, the lids must be everted and their conjunctival surface and the upper and lower retrotarsal folds thoroughly examined. In injuries of greater severity the white of the eye is generally hidden by blood, and the condition may appear much worse than it is in reality, but before giving a prognosis it is necessary to examine the intraocular tension, to observe the size and reactions of the pupil, and to test the acuity of vision. In affections of the conjunctiva it may be said that, as a rule, the greater the œdema the more severe the attack; and acute blennorrhœa or diphtheria ought always to be suspected when there are great pain and swelling of the lids. The secretion ought always to be examined bacteriologically if there be any doubt regarding the diagnosis. Edema of the eyelids is not a predominating symptom either in iritis or glaucoma, but if the patient be an adult, those diseases should 'be looked for, especially if on separating the lids there is a gush of tears instead of an escape of purulent or muco-purulent secretion from the conjunctiva.

(c) The eyeball is not only inflamed, but it is also displaced and its movements are impaired.

Displacement of the globe and limitation of its movements are always accompanied by great chemosis of the conjunctiva and an alarming degree of inflammatory œedema of the eyelids. The principal diseases which give rise to those symptoms are panophthalmitis, frontal or ethmoidal sinusitis, tenonitis, abscess of the orbit, or thrombosis of the cavernous sinus.

In panophthalmitis, pain is very severe, and is often accompanied by vomiting and rise of temperature. Sight is lost very early in the course of the disease, and pus forms in the interior of the globe, which projects from its socket and becomes almost immobile. It is at once distinguished from purulent conjunctivitis. by the fact that the discharge is not abundant and the usual micro-organisms are not found in the secretion. Moreover, in no form of conjunctivitis is the eyeball displaced or are its movements impaired. In simusitis of the cavities accessory to the orbit, the signs are in a measure localised, and the eye is always pushed away from the sinus that is affected. As the disease develops, the cellulitis of the orbit extends, and pus forms, leading to the formation of an orbital abscess. The protrusion of the eyeball is now extreme, sight is reduced, or may be altogether lost, and the ophthalmoscope usually reveals optic neuritis with hæmorrhages into the retina. The patient often suffers from severe pain, high temperature, headache, and vomiting, but, unless in exceptional circumstances, pus never forms either in the aqueous or vitreous chambers. Tenonitis-inflammation of the capsule of Tenon-is frequently due to gout or to rheumatism, and vision is not affected. 
Although at a first glance it resembles orbital cellulitis and abscess, yet the differential diagnosis ought not to be difficult, because in tenonitis the chemosis of the bulbar conjunctiva is always much greater than the degree of the exophthalmos. Accurate diagnosis is of great importance, because tenonitis, although liable to relapse and recurrence, always terminates in recovery, whereas in the other conditions mentioned the prognosis is not nearly so favourable. In thromborsis of the cavernous sinus the obstruction to the circulation leads to protrusion of the eyeball and overdistension of the retinal veins. The œdema of the lids spreads to the mastoid region, and one of the most characteristic features of cavernous thrombosis is that it frequently passes from the one side to the other. Not only is sight endangered, but life is threatened from the outset by the gravity of the constitutional symptoms. 


\section{CHAPTER V}

\section{LOSS OF EYELASHES: STYES AND TARSAL CYSTS}

Blepharitis marginalis: simple blepharitis, ulcerative blepharitis, and hypertrophic blepharitis: symptoms, prognosis, and treatment-Hordeolum or stye: symptoms: differential diagnosis: treatment-Chalazion or tarsal cyst: differential diagnosis, symptoms, course, and treatment.

1. Blepharitis Marginalis or Ophthalmia Tarsi.-Among the poor, and especially among the children of the poor, no form of eye disease is more frequently seen than inflammation of the edges of the eyelids. Though usually described as a blepharitis or ophthalmia tarsi, it is essentially an eczema, its signs and symptoms-redness, swelling, exudation leading to the formation of crusts, itching, etc.-being similar to those presented by eczematous inflammation of the skin of other parts of the body. The margins of the lids are the parts chiefly involved, and hence the name blepharitis marginalis. It frequently originates as a complication and sequela of measles, scarlet fever, or whooping cough, and often occurs in those whose occupations expose the eyes to sudden alternations of heat or cold, to dust or to irritating vapours. It is aggravated by improper forms of food and by want of personal cleanliness. Girls are affected rather more frequently than boys, and as a rule the most severe forms of the disease are met with in blondes of delicate constitution and naturally pale complexion. When weakly or scrofulous children are attacked, the blepharitis is usually associated with glandular swelling, and complicated by the occurrence of phlyctens on the bulbar conjunctiva, particularly when the disease appears during the course of any of the exanthemata. In such cases the conjunctivitis may be very pronounced, and both eyes are usually implicated. When the disease is unilateral, its origin can often be traced to inflammation of the mucous membrane of the nose and of the tear passages. Many children, otherwise to all appearance strong and healthy, suffer from inflammation of the eyelids whenever they read or write. If the use of the eyes for near work be given up, the inflammatory symptoms pass away, only to reappear, however, whenever the strain begins again. Here obviously the blepharitis. is due to an error of refraction, and will not be relieved by any form of treatment, until suitable spectacles have been provided. 
At the commencement of the attack the borders of the lids feel hot and uncomfortable, and are red and somewhat swollen, as if the patient had been weeping. This is the stage of simple blepharitis, or " blear eye." During the day, fine bran-like scales form at the roots of the eyelashes, and during the night the eyelids get stuck together by the increased secretions from the sebaceous and other glands. The formation of little pustules at the roots of the eyelashes marks a further stage of progress, and the hypersecretion is then so abundant that the cilia are matted into pencils, and after sleep the lids are so firmly glued together that they cannot be separated until the crust, which has formed by the drying of the discharge, has been softened by bathing with hot water. When these crusts are removed, a raw bleeding surface, due to little ulcers at the roots of the eyelashes, is exposed along the border of the lid, which is considerably swollen and of a dusky red colour. There are now much heat and itching of the eyelids, and, as a consequence, increased lachrymation, the tears overflowing on to the cheek, and irritating the skin until an eczematous eruption is produced, and the disease is described as blepharitis ulcerans.

If the disease be not arrested, the ulcers eat into the hair follicles, and the eyelashes drop out. Many of them never grow again, owing to the complete destruction of their root sheaths, and in this way the lids may be denuded of cilia, such bald condition of the eyelids being known as madarosis. When the hair follicles are only partially destroyed, cilia are reproduced, but they are sickly and stunted, irregular in their distribution, and so altered in direction that they tend to grow towards the eyeball, and the trichiasis thus produced adds greatly to the patient's suffering.

The more protracted the inflammation, the greater is the infiltration of the margin of the eyelid, more especially of the upper lid; and at length the intermarginal space becomes obliterated, and the lid no longer presents a clean-cut edge, but is rounded, swollen, and bordered by a raised ring of fleshy-looking conjunctiva. After the disease has reached this advanced stage it is termed tylosis or blepharitis hypertrophica. Such changes in the shape of the eyelid always produce a certain amount of eversion, with consequent misplaccment of the puncta lachrymalia; and when there has been considerable eczematous inflammation, the ectropion is aggravated by cicatricial contraction of the skin of the lid. As a result, the tears are not sucked up by the canaliculi, but keep dropping constantly upon the cheek, the eyes being always suffused with water. The constant wiping which follows increases the irritation, and the supervention of conjunctival inflammation adds to the patient's distress by inducing a certain amount of photophobia, with the consequent formation of a 
habit of partial closing of the eyelids, and wrinkling of the brows, in order the better to protect the eyes from the full glare of light. The subjective symptoms to which blepharitis gives rise are, however, remarkably slight, and the patients seek advice chiefly on account of the disfigurement; inquiry, however, leads them to admit that the eyes become easily tired, especially in artificial light, and that they water and feel tender after exposure to wind or to dust. Two sets of causes therefore contribute to produce a blepharitis, the one local, the other constitutional; and the resultant anatomical changes are essentially similar to those seen in eczema of the skin. In some families blepharitis seems to be a hereditary disease, but in most instances it is acquired, and is due to constitutional as well as to local causes. The former may be summed up by the word scrofulous, while the latter include all these conditions likely to induce hyperæmia of the conjunctiva. Among the local causes, however, must be mentioned the parasites pediculus pubis and demodex folliculorum, both of which occasionally find a habitat in the eyelashes, and by their presence cause irritation sufficient to give rise to inflammation. This very rare form of the disease-phtheiriasis-is easily distinguished, as, by the aid of a magnifying glass, the parasites are readily seen usually partially buried in the root sheaths of the eyelashes.

Treatment.-In every case the tendency is for this disease to pursue a very chronic course; but it is always favourably influenced by suitable treatment, although peculiarly liable to relapse whenever the treatment is discontinued. Many cases, which in spite of care and attention have persisted all through childhood, disappear spontaneously as adolescence is reached, and then the patient is said to have outgrown the disease. In the treatment of blepharitis it is important not only to estimate fully the local and constitutional causes of the disease, but also to recognise the stage of its progress. All local causes, such as errors of refraction, inadequacy of the tear-passages, inflammation of the nasal mucous membrane, etc., must as far as possible be removed, and any diathesis present must be carefully considered. In particular, every effort must be made to correct errors of digestion and assimilation, by proper feeding and good hygienic surroundings. Cod-liver oil and the hypophosphates ought not to be prescribed until the child's appetite is healthy and its tongue clean; but when they are given at the proper time, these drugs are of the greatest service, not only in hastening the disappearance of the disease, but also in preventing a relapse. When the lids are hot and red, and the roots of the eyelashes covered by bran-like scales, lead lotions are very useful, particularly when applied to the eyes by means of a douche. If there be much hyperæmia of the conjunctiva, their action is rendered all the more beneficial by the addition of sulphate of zinc. Ointments containing 
mercury, ichthyol, hazeline, oil of cade, or oil of cajuput are always indicated, but care must be taken not to employ them too strong, lest the irritation they induce may prolong rather than cure the hyperæmia of the lid margin. When the disease has lasted long, and has reached the stage of ulcerative blepharitis, the first essential of the treatment is that all the crusts which mat the eyelashes together are thoroughly removed. This is best accomplished by a hot alkaline wash, or by a weak solution of perchloride of mercury, but the process always demands much time and care, and is usually attended by considerable pain. After the scabs are removed the raw bleeding surface on the edge of the lid should be brushed with a 2 per cent. solution of nitrate of silver, and wherever pus is seen in a hair follicle the cilia must be epilated. When the edge of the lid is . thoroughly cleaned, ointments of red or yellow precipitate of mercury, protargol, etc., should be thoroughly applied, and this treatment requires to be repeated at'least twice a day. In order to prevent relapse, it is necessary to continue the use of an ointment at bedtime for several weeks after all sign of inflammation has disappeared from the lids. When the edge of the lid has become hard, thickened, and everted, all the cilia which remain should be epilated, and the lower canaliculus slit up to the lachrymal sac, so as to form a gutter to direct the tears into their natural excretory channels. The lid margin should be touched daily with a crystal of sulphate of copper, or massaged with a precipitate of mercury ointment ; and at bedtime the eyes should be covered by discs of gauze spread thickly with a paste composed of diachylon ointment, linseed oil, and balsam of Peru. When the palpebral conjunctiva is greatly hypertrophied, it may be cauterised by solid nitrate of silver, or a piece may be excised; and in cases where the eversion is very pronounced, it may be necessary to shorten the lid by removing a portion at the outer canthus and stitching the cut edges together, or to puncture the conjunctival surface of the lid with a small galvano-cautery, as Ziegler recommends.

2. Hordeolum or Stye.-A hordeolum may be defined as a localised inflammation at the edge of the eyelid, usually originating in connection with a hair follicle, and as a rule terminating in suppuration.

Though a stye is regarded as a very trifling ailment, it is often accompanied by an amount of suffering out of all proportion to the size of the little boil that is causing it. The severity of the pain may even disturb sleep, and give rise to symptoms of general feverishness. Fortunately, however, the distress is of brief duration, for the suppuration soon reaches its height, and from the moment that the skin breaks, and a drop or two of pus escapes, the pain begins to subside. Styes are, however, prone to occur in crops, and this tendency is specially seen in those whose digestive organs are at fault, and who suffer from habitual constipation or from 
irregularities of menstruation. In a predisposed person, after exposure to a cold wind with dust flying, or to a bright light; or after over-use of the eyes, especially if the patient be hypermetropic, there comes a feeling of heat and itching of the lids. If the eyes be rubbed, pain is felt at a particular spot, which soon afterwards swells. This swollen part is of an angry red colour, and may attain considerable size; whi'e the pain, which was at first sharp and cutting, soon becomes dull and throbbing. After a time, varying from twenty-four hours to three or four days, a yellowish coloration appears round the roots of the eyelashes surmounting the most prominent part of the stye, the skin gives way, and a few drops of purulent matter escape; immediately thereafter the pain subsides, and the swelling lessens, till in the course of a few days the spot, partially denuded of eyelashes, is marked only by a cicatrix.

The general swelling of the lids is occasionally so intense as to mask completely the localised swelling. The latter is, however, always to be detected on careful palpation. Sometimes the inflammatory œdema of the lids and surrounding parts is so pronounced that, at first sight, it might be mistaken for acute inflammation of the lachrymal sac; but here again palpation helps to make the diagnosis clear, for in hordeolum the tenderness is limited to a point or points along the margin of the eyelid, while in lachrymal inflammation it is confined to the region of the tear-sac. As a rule the bulbar conjunctiva remains unaffected, except in the case of styes which form at the outer canthus. These, from the pressure they cause on the blood-vessels and lymphatics, are always complicated by a considerable amount of œdema of the ocular conjunctiva.

Treatment.-A hordeolum can very rarely be aborted, and as a rule the wisest treatment is to encourage suppuration from the very outset by means of the continuous application of hot compresses. Whenever pus forms the abscess should be opened by a free incision parallel to the edge of the lid. The parts ought afterwards to be bathed freely with hot antiseptic lotions, as the tendency to recurrence may be at least partly explained from the infection of neighbouring glands by the micro-organisms in the discharge. For a similar reason, if blepharitis complicates a stye it ought always to be thoroughly treated. Any error in the general health must be corrected, more particularly constipation and anæmia, consequently salines combined with iron are indicated in almost every case. Exceptionally, recurrent styes are due to an error of refraction, and only disappear in these circumstances after suitable glasses are worn.

3. Ghalazion or Tarsal Cyst.-A chalazion is a tumour of the tarsal cartilage, which originates as a result of obstruction and subsequent inflammation of a Meibomian gland.

It was formerly regarded as a hordeolum which had not gone on to 
suppurątion; but, both as regards position on the eyelid, origin, and subsequent progress, each disease presents signs and symptoms quite distinctive and easy of recognition. A hordeolum always occurs at the margin of the eyelids, in line with the eyelashes; whereas a chalazion is placed within the edge of the lids, along the line of the Meibomian glands. When one of these glands becomes obstructed, inflammation of the wall is set up by the retained secretion, and this irritation, spreading to the parts adjacent, produces proliferation of the surrounding tissues, which brings about a gradual swelling of the tarsus. A chalazion occurs much more frequently in adults than in children, and may develop in both upper and lower eyelids, although its most frequent position is in the centre of the upper lid. It is in many instances solitary, but in those who, from digestive and other derangements, are specially liable to the disease, several may be present at the same time, and occasionally there may be one, or more than one, placed symmetrically on the right and left upper or lower lids. There is a close analogy between such cases of chalazion and acne of the skin. As a rule a chalazion develops slowly. It is hard and circumscribed, rolls under the finger like a lead pellet or a hailstone, and seldom exceeds the bulk of a split pea. It is non-inflammatory and quite painless, and patients complain more of the disfigurement which it produces than of any actual discomfort.

When it attains a certain size, however, it interferes to some extent with the movements of the eyelid, the conformation of which is altered, more especially if several are present at the same time. The skin covering a chalazion is freely movable, and at first is unchanged in appearance, but as the tumour grows it gradually becomes of a more or less livid red colour. When the eyelid is everted, the conjunctiva corresponding to the site of the chalazion is seen to be red and swollen, and, in the more advanced cases, a bluish spot occupies the centre of this area, and indicates that the tarsus is becoming thinned by the pressure of the tumour. When perforation occurs, a gelatinous substance escapes, but this is only the central softened portion of the chalazion; and if the harder and more resistant peripheral parts be not removed, fleshy granulations will sprout from the interior, and form a fungoid excrescence upon the inner surface of the eyelid. A small chalazion of the lower lid, more especially when situated near the inner canthus, is nearly always wholly cartilaginous, whereas those which attain a larger size have their contents often wholly liquid. The swelling is then either a cyst or an abscess. In the former case it is filled with a yellowish-brown fluid; in the latter, with pus. When the contents of a chalazion become purulent, some describe it as a hordeolum internum.

An ordinary stye always evacuates its contents through the skin, 
while a suppurating chalazion almost always points and bursts on the conjunctival surface. A chalazion has been considered to be a manifestation of tubercle, but there is no evidence in support of such a view. The earliest stages of tubercle or epithelioma of the lid may, however, be mistaken for a chalazion which has burst externally.

Treatment.-A chalazion may last for months or even years, and in many cases, in the long run, spontaneously disappears. If the tumours are small and multiple, the best treatment is not to excise them, but to prescribe for any digestive or other constitutional disturbance that may be present. In such cases the inunction of the Jids at bedtime with yellow oxide of mercury ointment, and the administration of salines combined with alkalies and carminatives, are often attended by the happiest results. Larger chalazia are best dealt with by operation. The lid should be everted, and the parts rendered as anæsthetic as possible by means of cocain and adrenalin. In the case of the lower lid the tumour may be fixed by ring forceps, which also, by their pressure, prevent hæmorrhage. An incision should be made into the chalazion, perpendicular to the margin of the lid with a sharp Beer's knife. When the contents are evacuated the wall of the cyst must be thoroughly scraped with a small sharp spoon. Immediately after the operation an accumulation of blood within the cyst causes the tumour to appear on the surface as large as it was before the operation, but this blood clot soon absorbs, and in the course of a week or ten days all trace of swelling has disappeared. If the wall of the cyst is not thoroughly scraped granulation tissue springs from the interior, and forms a fungating mass which protrudes from the conjunctival surface. As this causes considerable irritation, and a constant mucopurulent discharge, it ought to be snipped off with scissors, and if necessary the base cauterised. It rarely happens that a chalazion requires to be removed through the skin, but when it is cartilaginous, and situated at the outer or inner canthus, it is easier to remove it by an incision through the skin, rather than through the conjunctiva. It can then be excised completely, and if the edges of the cutaneous wound be neatly stitched together there is very little scarring. 


\section{CHAPTER VI \\ BLEPHAROSPASM; LAGOPHTHAIMOS; AND PTOSIS}

Blepharospasm : intermittent or permanent-Tic douloureux-Lagophthalmos : cicatricial, paralytic-Facial palsy and its causes-Neuro-paralytic keratitisTreatment of lagophthalmos-Ptosis: hypertrophic, traumatic, congenital, senile, and paralytic : treatment.

Blepharospasm is due to spasm of the orbicularis muscle, and may be intermittent or permanent. In the former case it is an exaggeration of the normal winking of the lids, and is frequently seen in children who are anæmic and nervous. The exciting cause may be a follicular conjunctivitis, or an error of refraction. If it be allowed to persist it becomes a habit, and other muscles of the face are implicated. In some cases the movements resemble chorea. The muscular twitchings are always worse under the influence of emotion, and pass off completely if the attention is fully occupied, but they recur at once whenever any reference is made to the eyes.

Treatment.-The treatment consists in removing any local condition-conjunctivitis, error of refraction, etc.-and in paying attention to the general health.

In adults, and especially in the elderly, blepharospasm is much more serious, because it is likely to be permanent. It varies greatly in severity, from a muscular tremor which is felt distinctly by the patient, but which is rarely seen, to a tonic spasm which closes the lids firmly for a longer or shorter time. Tonic spasm may come on suddenly while the patient is walking along the street and render him quite helpless. He is quite blind for the time being, because he is unable to open his eyes until the spasm passes off. This form of blepharospasm is very persistent, is, indeed, often incurable, and the older the patient the worse the prognosis. It may attack one or both eyes. When unilateral, careful examination must be made to discover any local cause such as an inverted eyelash, a foreign body, an abrasion of the cornea, etc. ; but in the majority of cases, and more especially in those that are bilateral, the most careful ophthalmological and neurological examinations fail to find a cause for the spasm, which is usually vaguely ascribed to some degeneration of the facial nerve. The nose and the teeth ought always to be examined. Few 
diseases are more difficult to cure, and senile cases may be absolutely incurable. If it be possible to find points pressure on which causes the lids to open, good may be got by applying the galvanic current to those spots after having instilled cocain into the conjunctival sac. The injection of absolute alcohol along the trunk of the facial nerve where it emerges from the stylo-mastoid foramen is occasionally successful in curing the spasm, but the patient must be warned that paralysis of the muscles supplied by the nerve is sure to follow the injection, that the palsy may persist for a considerable time, and that when it passes off the spasm may recur. In addition to those local measures, bromides with arsenic and iron should be prescribed, along with any other remedies suitable to combat neurosis in general.

Tic douloureux is usually confined to one side, and the spasm is accompanied by very severe pain. In many cases no relief is obtained until after extirpation of the Gasserian ganglion. That operation, however, is by no means free from danger, because it is followed occasionally by destruction of the cornea from neuroparalytic keratitis.

Lagophthalmos.-Inability to close the eyelids may arise from several causes. For example, the cicatrisationi after a burn may so contract the lids that they cannot be closed, or the cicatrix remaining after disease of bone may drag the upper or lower eyelid, or both, away from the globe, and fix them so firmly to the orbital margin as to render it impossible for them to cover the eye. In both of these examples the lagophthalmos will be complicated by ectropion. In other cases, such as. severe exophthalmic goitre, or orbital tumour, the lids will not close, even during sleep-not through any failure of their own position and mobilitybut owing to the great prominence of the eyeballs. Another inducing cause is paralysis of the seventh cranial nerve, in which the paralysis of the orbicularis palpebrarum is accompanied by palsy of the other muscles supplied by the facial nerve.

The lesion producing the paralysis may be central or peripheral, and it is always important to try to localise it as accurately as possible. A central lesion may be cortical, intracerebral, or nuclear; while a peripheral lesion may affect the nerve in its course through the temporal bone-intracranial—or after its exit from the skull by the stylo-mastoid foramen-extracranial. As a rule, it is not difficult to distinguish a peripheral lesion from one of central origin, for in the former the paralysis is complete-involving all the branches of the nerve-and is, in most instances, confined to one side of the face; whereas in the latter the loss of power is not absolute-the branch supplying the orbicularis palpebrarum usually remaining intact-and both sides of the face may be affected. If such an incomplete palsy of the muscles of one side of the 
face exists by itself-monoplegia facialis-it is most probably due to a lesion of the cortex; but as a rule it is accompanied by paralysis of the limbs of the same side of the body, and presents a clinical picture which can with difficulty be distinguished from a similar group of symptoms produced by disease of the internal capsule or of the crus cerebri. Under such circumstances, however, associated symptoms are usually present to aid the diagnosis.

When the site of the lesion is in the pons, the facial paralysis is usually more profound than when it is due to implication of the seventh nerve in other parts of its intracerebral course. In pontine lesions, moreover, the paralysis of the face may be on the side opposite to that of the limbs, and when this "crossed paralysis" occurs, the lesion must obviously be situated in the lower part of the pons, that is, below the point where the fibres of the facial nerve have crossed from one side of the brain to the other. When, however, facial paralysis is connected with spinal disease, or associated with paralysis of any of the ocular or other cranial nerves, there is usually a lesion of the nucleus. Whether a peripheral lesion implicates the nerve in its intracranial or in its extracranial course, the paralysis of the nuscles supplied by it is complete. Injury during passage through the aqueduct of Fallopius is generally caused by suppuration of the middle ear, and disease of the adjoining portion of the temporal bone; and the exact site may be deternined by giving heed to the presence or absence of certain concomitant symptoms, such as paralysis of the palate, abnormal acuteness or impairment of hearing, diminished salivary secretion, or alteration in the sense of taste.

It is in its extracranial course, however, that the nerve is most liable to injury, as, after it emerges from the stylo-mastoid foramen, it lies nearer the surface. It is therefore readily pressed upon by any tumour at the angle of the jaw, or injured by an accident, a gunshot wound, or during a surgical operation; and its function becomes frequently suspended after exposure to cold. This last is the form usually described as due to rheumatism. The chill, however, can only be regarded as the immediate exciting cause of the disease; the true predisposing cause, whether rheumatic, syphilitic, or other, being found in the blood condition existing at the time.

The usual history of a case is somewhat as follows: The patient, while heated, sits down at an open window, or exposes himself to a draught while travelling. He may or may not be conscious of a little stiffness of neck afterwards, but, even if he be, thinks, as a rule, nothing of it. In a few hours, however, he is considerably alarmed to find that he has lost power over the muscles of one side of his face. Try as he will he cannot shut his eye or wrinkle his brow on the affected side. $\mathrm{He}$ 
cannot close his lips firmly, as in the act of whistling, nor can he blow out his cheeks, and when he laughs his face is drawn to the sound side so that the mouth is twisted, the affected side remaining quite flat and expressionless. He may experience difficulty in swallowing, as food tends to lodge between the gum and the cheek, or sometimes, owing to paralysis of the palate, to regurgitate through the nose. The eyelids are never closed, even during sleep, and consequently the eyeball is unprotected from dust, and exposed constantly to the air, with the result that conjunctivitis and corneal ulceration supervene. These complications are aggravated by the constant epiphora due to ectropion; and in severe cases the cornea may lose its sensitiveness, and be completely destroyed.

On account of the slight eversion of the lids, the palpebral fissure is wider than normal, and consequently the eyeball appears more prominent on the affected than it does on the sound side. Patients usually complain that the eye feels cold; but apart from this there is no disturbance of sensation except where paralysis of the seventh nerve is complicated by lesion of the fifth. In these circumstances the cornea is liable to suffer severely-neuro-paralytic keratitis. In this connection, however, it is of interest to mention that herpes zoster now and again occurs as a complication in peripheral paralysis of the facial nerve. In every case of facial paralysis, the sensibility of the cornea should be tested.

As a rule rheumatic facial paralysis passes off in the course of a few weeks, but its duration is extremely variable. The electrical condition of the paralysed muscles is, however, the best guide in prognosis, for if there be no change in their reaction either to the faradic or the galvanic current, the case will probably terminate favourably in from one to three weeks; whereas, if the "reaction of degeneration " be found, recovery is likely to be delayed for many months. Should secondary contractures and spasmodic twitchings of the muscles supervene, the time will probably be still more extended, and, indeed, complete recovery may never take place.

Treatment of lagophthalmos. - In an ordinary case all that is necessary is to tell the patient to wear a shade or a pair of protective glasses. Morning and evening the eye should be bathed with a boracic lotion and anointed with iodoform ointment. If ulceration of the cornea occurs, the lids should be held together by three strips of sticking-plaster; but when the ulcer is spreading, it is safest to stitch the lids together at one or two points, along the palpebral margin.

Medical treatment by mercury, iodides, alkalis, etc., must be prescribed according to the ætiology of the lagophthalmos-syphilis, rheumatism, etc., and the constant current, carefully applied, is often of great service in all cases of old standing. 


\section{PTOSIS}

Ptosis, or drooping of the upper eyelid, may be complete or partial, confined to one side, or bilateral. While it is sometimes the result of purely mechanical causes, it is at others due to paralysis of the oculomotor nerve; and cases may thus be divided into spurious and genuine. For clinical purposes it is, however, more convenient to distinguish five separate groups, namely, the hypertrophic, the traumatic, the congenital, the senile, and the paralytic.

1. Hypertrophic, or Ptosis Adiposa.-Here the lid, through bulk and increased weight, becomes so relaxed and pendulous that it falls over the eye. The levator palpebræ superioris is quite unable to raise it, and the deformity is most prominent on the temporal side. If the relaxed skin be pinched up between the finger and thumb, the patient can by a strong effort raise the eyelid, but if the support be withdrawn the lid falls at once into its former position. In this group may also be included those cases of drooping of the upper eyelid which are the result of chalazion, abscess, œdema, hæmorrhage, or inflammation, more especially erysipelas and trachoma.

2. Traumatic Ptosis occasionally occurs after penetrating wounds of the upper evelid, when the levator palpebræ or its nerve has been injured. Unilateral ptosis that has existed from the time of birth is often attributed to the pressure of the forceps during a prolonged and difficult labour.

3. Congenital Ptosis is usually partial, affects both eyes, and is often hereditary. It is frequently associated with paralysis of the superior recti muscles, and occasionally co-exists with flattening of the bridge of the nose, and the presence of a semilunar fold of skin covering the inner canthus-epicanthus. It is characterised by an atrophic condition of the affected eyelids, and is due either to a defect in the development of the levator palpebræ superioris, or to a congenital abnormality of the nerve centres. In a well-marked case the upper lids droop so much that they almost cover the pupil, and in order to see better the patient gets into the habit of throwing the chin forward, and forcibly contracting the frontalis muscles. This attitude is very characteristic, and not less so is the anxious expression produced by the wrinkling of the skin of the forebead, and the elevation of the eyebrows, due to the over-action of the frontalis from the constant effort of the patient to raise the upper lids. In this connection may be mentioned those cases of unilateral ptosis, where, as a result of some disorder in the central nervous arrangements, the lid cannot be raised by the strongest effort of the will, but the eye opens involuntarily with the contraction of the facial muscles-: $e g$. the affected 
lid moves synchronously with the opening of the mouth. A slight drcoping of the upper lids may appear about puberty. This peculiarity often runs in families, and for the most part affects females rather than males.

4. Senile Ptosis.-It sometimes happens that the power of the levator muscle gradually diminishes with advancing years, so that the upper lids droop, and are raised only with great difficulty. As a result the palpebral fissure is narrowed, and the closing eyelids interfere with sight. In the aged there is also a superabundance of flaccid skin, the presence of which still further impedes the action of the levator muscles. As presbyopia approaches, patients often complain of difficulty in opening the eyes on first wakening from sleep in the morning. This transient ptosis, which may be so complete that the lids require to be raised by the fingers, is simply an evidence of eye-strain, and passes off speedily after the patient begins to use suitable spectacles.

5. Paralytic Ptosis is due to a lesion of the oculo-motor nerve. The paralysis may affect the whole nerve or only some one or more of its branches. It occasionally happens that both third nerves are affected, but rarely are both paralysed completely. If the ptosis is complete, and the paralysed eyelid be raised by the finger, the patient at once complains of double vision and of giddiness. The eyeballs are markedly divergent, and cannot be moved inwards, upwards, or downwards. The pupil is moderately dilated, and does not contract when exposed to the light; and as the ciliary muscle is also paralysed, the power of accommodation is lost, and the patient is unable to read. In some cases the pupil is contracted, the eyeball drawn slightly backwards into its socket, and its intraocular tension reduced. In these circunstances the ptosis, which is usually slight, is due to injury of the sympathetic nerve in the neck.

Paresis, or paralysis of the ocular muscles, may be due to injury, or to pressure from tumours, etc., within the orbit, or it may be consequent upon the existence of such blood states as diphtheria, rheumatism, or syphilis. The lesion may be peripheral or central, the former being characterised by the absence of all cerebral symptoms, the latter almost invariably by their presence. When due to syphilis, it indicates in many instances the commencement of degenerative changes in the cerebrospinal system. The paresis may be so slight that no squinting is noticeable, but if the patient sees with both eyes, he at once complains of double vision. It is well to remember that this diplopia may be of brief duration, and that it may pass off without the aid of medicine, but nevertheless it is of serious import, and the more transitory it is the worse the prognosis, for then it is to be regarded as an early symptom of disease of the spinal cord, more particularly tabes dorsalis. This transient diplopia is 
occasionally accompanied by contracted and unequal pupils, and in such circumstances its significance becomes all the graver. In other cases the paralysis is much more profound, and one muscle after another becomes affected until all the extraocular muscles are involved, and the ophthalmoplegia externa is complete. Similarly the muscles inside the globe may be paralysed, giving rise to complete ophthalmoplegia interna. In syphilitic cases the prognosis is the less favourable the longer the interval of time between the primary infection and the appearance of the diplopia; and naturally more serious results are to be feared in those who from carelessness on their own part, or from other causes, have not been subjected to a thorough course of antisyphilitic treatment at the outset.

Now and again reflex ptosis results from irritation of some branch of the fifth nerve, e.g. a decayed and painful tooth.

Ptosis due to hysteria is also well recognised, but a much more important variety is that known as recurring ptosis. The nature of this disease is mysterious. At regular intervals there is complete paralysis of the oculo-motor nerve. Each attack lasts from a few days to several weeks, and then passes off completely, only, however, to return again after a longer or shorter lapse of time. The disease attacks females oftener than males. It may appear in infancy or early childhood, and although it usually passes off as middle age is reached, it may persist throughout the whole of life. The attacks vary in frequency: when they occur but seldom they are usually of long duration. They are generally unilateral; but either side may be affected alternately, and each paroxysm may be ushered in by severe headache and vomiting. The disease seems closely allied to migraine.

Treatment.-In congenital ptosis, and in old-standing cases of acquired ptosis, the deformity can only be remedied by operative treatment. Operations for ptosis are numerous, but may be grouped under three heads-(a) those which aim at shortening the lid by removal or displacement of tissue; (b) those which depend upon transplantation of the frontalis muscle; and $(c)$ those in which a portion of the superior rectus is made to take the place of the levator. In acquired ptosis every effort must be made to find out the cause of the paralysis, and treatment must be directed to its removal. Many cases are the result of syphilis, and require energetic mercurial medication; while in others rheumatism is the pre-disposing cause, and the appropriate treatment is salicylate of soda, with alkalis and iodide of potassium. In paralysis of the sympathetic it may be necessary to operate on swollen lymphatic glands in the neck, or to treat a gunshot wound or other injury which has implicated the cervical sympathetic.

Double vision may be prevented by the closure of the eyelid, but it 
at once becomes apparent whenever the eyelid is raised. It is necessary, therefore, to make sure that diplopia does not exist before any attempt is made to operate on the ptosis. Mechanical devices to suspend the lid are numerous, but none is really satisfactory, and they should only be employed if the patient is so nervous that he will not submit to operative treatment. 


\section{CHAPTER VII}

\section{ENTROPION AND ECTROPION}

Entropion: spasmodic and cicatricial: trichiasis: treatment-Ectropion: spasmodic, cicatricial, senile, and paralytic-Epiphora-Blepharitis hypertrophica: treatment.

1. Entropion.--Inversion of the eyelid may be due to spasm of the orbicularis palpebrarum muscle, or it may be produced by cicatricial contraction of the eyelid.

(a) Spasmodic entropion usually affects the lower lids only, and the inversion may be so complete that the eyelashes become hidden from view. In an advanced case the margin of the lid is not visible, when the open eye is seen from the front. It has been recorded that some individuals can by an effort of will induce contraction of the ciliary portion of the orbicularis, and cause both lower lids to become completely inverted. The disease is usually seen in old people whose tissues are lax as a result of senile changes. In such cases it may be determined by the application of a bandage, and often proves a troublesome complication after operation for the extraction of cataract. Spasmodic entropion is seen also in persistent strumous ophthalmia, and in bad cases of this disease the blepharospasm is so pronounced that the edges of both upper and lower lids are turned against the globe. In none of these cases, however, is there any organic change in the structure of the lid itself, and the entropion at once disappears for the time being whenever the lid drawn away from the eyeball.

(b) Gicatricial entropion is produced by wounds which involve the edge of the lids, and more particularly by burns, which, when severe, produce so much cicatricial contraction that the lid is greatly destroyed and becomes fixed to the eyeball-symblepharon. It is seen also after those diseases of the conjunctiva which are followed by cicatricial changes, more particularly after granular ophthalmia, in the late stages of which the normal conjunctiva is replaced by a development of connective tissue, which by its contraction produces incurvature of the tarsal cartilage, and consequent inversion of the free margin of the eyelid.

In every case of entropion the cilia are misdirected-trichiasis-and rub against the eyeball, and as a result of this irritation the cornea becomes 
inflamed. Severe corneal complications-opacity, vascularity, ulceration -are more common in entropion of the upper lid; and consequently in. these cases the danger of impaired eyesight is greater than in those where the inversion is confined to the lower lid. It must be remembered, however, that trichiasis may exist without any displacement of the palpebral margin, but the symptoms and the injurious results to the cornea are the same. There is no sharp dividing line between trichiasis and entropion.

Treatment of Spasmodic Entropion.-In a mild case instillation of a 2 per cent. solution of cocain into the conjunctival sac, and the removal of the cause, may be all that is necessary. For example, when the lower lid becomes inverted in the course of the treatment of an operation wound, an ulcer of the cornea, etc., the first thing to do is to discontinue the use of a bandage; or when the lid for want of the support of the globe rolls in after enucleation, the entropion is usually rectified at once by fitting an artificial eye into the empty socket. At other times it is necessary, after the lid has been restored to its proper position by the finger, to keep it in place by a strip of sticking-plaster, or by the application of strong collodion. The latter, if properly employed, is a very satisfactory means of treatment. A small surgeon's probe should be dipped in the collodion and applied immediately to the skin of the lower lid parallel to and about a quarter of an inch below its margin. The probe must be held so firmly that it dents the skin, and should be carefully withdrawn the moment the collodion begins to dry. The walls of the little gutter thus formed are firmly approximated, and the lid is held securely in proper position. The application should be renewed at least once a day. When the skin is lax and has lost its tone, the insertion of the sutures devised by Gaillard or by Snellen is of great service. This method of treatment is specially recommended in the treatment of senile entropion, and its success depends upon the thread causing a certain amount of inflammation so that when it is withdrawn, its track is occupied by a strand of connective tissue. In more severe, cases, however, it is more satisfactory to excise an elliptical fold of skin and the underlying portion of the orbicularis muscle. The amount of skin removed must be sufficient to cause slight eversion after the cut edges have been accurately sutured. The ectropion thus induced, however, quickly disappears.

In cicatricial entropion there is always trichiasis, and the misplaced eyelashes irritate the surface of the cornea. Whenever there is persistent ulceration of the cornea, the margin of the lids should be carefully examined for an inverted eyelash. The simplest method of treatment is to epilate the ingrowing cilia, but as they grow again quickly the operation requires to be repeated every fortnight. It is better, therefore, to try to 
destroy the offending eyelashes by electrolysis, or to remove completely the little strip of skin from which they grow. In cicatricial entropion due to trachonia, plastic operations are required, but these are, as a rule, best left in the hands of the specialist.

2. Ectropion.-Eversion of the eyelids may be produced by many different causes, and varies much in degree. It may be temporary or permanent, and affects the lower lid much more frequently than the upper. It may, on the one hand, be so complete as to draw the eyelid away from the eyeball in its whole extent, or it may, on the other, be so slight as to simply bring about a displacement outwards of the lower punctum lachrymale. The patient complains more of the constant overflow of tears than of anything else. In severe cases of granular ophthalmia both eyelids may for the time being become completely everted, as a result of the extreme inflammation of the conjunctiva, which protrudes as a fleshy mass through the palpebral fissure; and in ophthalmia neonatorum and in phlyctenular keratitis there is often spasmodic ectropion.

The more permanent forms of eversion, however, result from cicatricial contraction of the skin of the eyelids and of the parts adjoining; from morbid changes in the lids themselves; from senile atrophy of the skin and the palpebral portion of the orbicularis muscle; or from paralysis of the facial nerve.

(a) Ectropion due to Gicatricial Gontraction.-Cicatricial ectropion is usually the result of burns by fire, by mineral acids-e.g. in cases of vitriol throwing - or by the fixed alkalies, more especially quicklime; and the amount of eversion depends upon the severity of the original injury. In unhealthy children the adherent cicatrix consequent on the healing of the diseased bone after caries at the lower and outer (hall-mark of tubercle) or upper and inner (generally syphilitic), orbital margin, is a very frequent cause of eversion. Syphilitic or tubercular ulcerations of the lids are also invariably provocative of ectropion. When the ectropion is due to any of these causes both upper and lower eyelids may suffer alike; but when the upper lid is implicated there is much more risk of corneal complications than when the lower only is affected, because the eyeball instinctively tends to seek for protection by rolling upwards. It is, however, often very remarkable how far the lower lid can be raised to cover the cornea when, from eversion, the upper has become inefficient.

When eversion is complete the edge of the lid is drawn away from the globe, and in the case of the lower lid is fixed to the skin of the cheek, and of the upper along the line of the eyebrow. The tarsus is intact, but is usually considerably elongated, and a curved elevation, somewhat pale in colour and fringed with eyelashes, marks the new position of the margin of the lid. The palpebral conjunctiva is wholly everted, and the 
mucous membrane, as a result of constant exposure, becomes so red and hypertrophied as to present the appearance of raw flesh. Whenever an attempt is made to close the lids a gap necessarily remains open, and almost invariably the eyeball becomes inflamed for want of its natural protection. Owing to displacement of the puncta lachrymalia there is constant epiphora, and tears mixed with secretion from the inflamed conjunctiva overflow continually on to the cheek and irritate the skin. After a severe burn the integument round about the orbit may be so completely destroyed that nothing but cicatricial tissue remains; and consequently, when a grafting operation is necessary, the skin must be obtained from another part of the body.

(b) Ectropion from Morbid Changes in the Eyelids themselves is usually seen as a result of long-standing inflammation of the edge of the eyelids. This disease is often neglected in its early stages, and gradually becomes worse and worse until the whole of the structures of the margin of the eyelids become inflamed and hypertrophied-blepharitis hypertrophica. The border of the lids are red and swollen, and instead of the edges being clean-cut, each co-apting perfectly with its fellow, they become rounded, and the well-marked space which is normally present between the line of the cilia and the openings of the Meibomian glands, is obliterated. Many of the eyelashes fall out, the follicles being permanently destroyed; and the cilia which remain are diseased at their roots and consequently stunted in growth, and so misdirected that their free extremities rub against the eyeball. The conjunctiva, as a result of exposure, is hypertrophied and granular, and loses much of its normal sensibility. Owing to this alteration in the form and position of the eyelids, they do not close perfectly during sleep; and partly from exposure due to this cause, partly from the constant lachrymation, the ocular conjunctiva often becomes so inflamed and irritable as to induce an amount of intolerance of light sufficient to cause the patient to keep the lids partially closed, and to produce a reflex contraction of the pupil.

(c) Ectropion from Senile Atrophy occurs as a result of senile changes. The skin of the lids becomes relaxed, and the palpebral portion of the orbicularis muscle loses its normal tonicity. The lower eyelids, in consequence, fall away from the globes, and the everted conjunctiva becomes almost cuticular, and presents a fleshy appearance. The eyes are constantly bathed in tears, which collect like a film over the surface of the cornea, and interfere with vision. The frequent use of the handkerchief in connection with this excessive watering aggravates the conjunctivitis, and induces considerable œdema of the caruncles and semilunar folds, which tend still further to increase the eversion of the nasal 
extremity of the lid, and to separate the punctum from the surface of the eyeball.

(d) Ectropion from Paralysis of the Seventh Granial Nerve.The patient is unable to close the eye on the side affected, and in consequence of the want of tone in the orbicularis muscle the edge of the lower lid tends to fall by its own weight away from the globe, while the tears, unable to drain away by the natural channels, collect in the lower part of the conjunctival sac and overflow at intervals. After a time the margin of the lid becomes everted, and the exposed conjunctiva, becoming inflamed, forms a red ridge behind the eyelashes. The chief danger in paralytic ectropion is that the cornea readily ulcerates, and may be completely destroyed. The sensitiveness of the cornea, therefore, ought always to be tested in a case of paralysis of the facial nerve; because if it be lost the trigeminus is also implicated, and in these circumstances the danger of destructive ulceration of the cornea is greatly increased.

Treatment of Ectropion.-From the point of view of treatment, all cases of ectropion may be divided into cicatricial and non-cicatricial. Of the former the most typical examples are seen after vitriol throwing, or when a child or an epileptic has fallen into the fire. The skin of the lids is usually so thoroughly destroyed that complete eversion takes place. In these circumstances the practitioner should warn the patient's relatives of what is likely to occur, because it is very unlikely that any form of treatment will arrest the process of cicatrisation. All that can be done is to protect the cornea by an ointment containing iodoform or other antiseptic, to keep the parts as clean as possible by bathing with a weak astringent lotion, and to apply a bandage. Later on, after cicatrisation is complete, operation to restore the eyelids will be necessary. The edge of the everted lid must be separated from its attachment to the skin and restored to its normal position. The upper and lower lids should then be stitched together, and the raw surface filled up, either by a flap with a pedicle, or by a graft of skin brought from another part of the body. These operations, however, need not be described further, because as a rule the general practitioner will not operate himself, but will place the patient under the care of a specialist.

In non-cicatricial ectropion it is usually the lower lids that are affected, and as the condition is always aggravated by wiping the eye to get rid of the overflow of tears, the patient should be advised to use his handkerchief from below upwards, and not as he naturally would do from above downwards. A bandage to keep the lid in position may be applied at night, and dark glasses worn during the day to protect the eye from bright light, cold, or dust. An astringent lotion is useful to cleanse the conjunctiva two or three times a day, and Io per cent. argyrol drops, or a 
simple antiseptic ointment, applied to lubricate the cornea. It is often necessary to slit the canaliculus and to pass a probe regularly until the cut edges have healed. A little gutter is thus formed into which the tears fall and drain into the nasal duct. In all cases it is necessary to treat any existing disease of the tear passages or any blepharo-conjunctivitis, and in many instances the cure of these conditions brings about the disappearance of the ectropion. In more advanced cases the edge of the lid must be fixed in proper position by sutures, passed into the most prominent part of the conjunctiva and brought out through the skin about an inch from the border of the lid. The threads are pulled tight until the lid is drawn into its proper position and then tied firnly over.a piece of drainage tube; or the conjunctiva may be punctured or seared deeply with the galvano-cautery. In both these methods of treatment the object is to obtain strands of cicatricial tissue strong enough to hold the edge of the lid in its proper place. In cases which have continued for a long time, the conjunctiva becomes greatly hypertrophied, and throws the edge of the lid more and more away from the globe. In these circumstances the whole of the diseased tissue should be excised and the wound closed by sutures. The good effect of this operation is much increased by excision of a triangular flap of skin at the outer canthus, and accurate approximation of the lips of the wound by sutures. This operation is only applicable to cases where the conjunctiva is diseased, and must never be employed when the mucous membrane is healthy. Numerous plastic operations have been devised for the treatment of non-cicatricial ectropion, but as a rule they ought to be left in the hands of a specialist. 


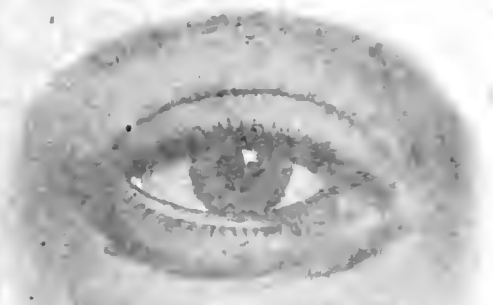

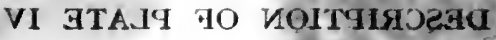

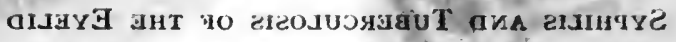

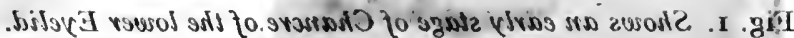

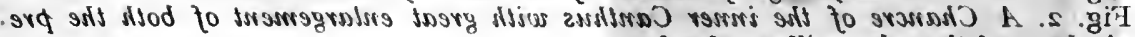

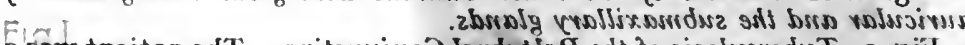

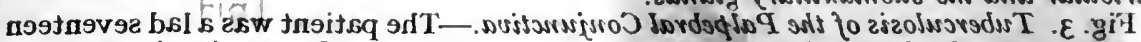

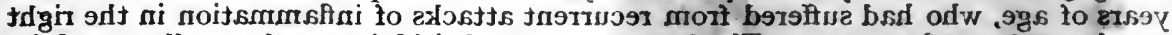

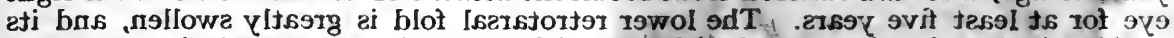

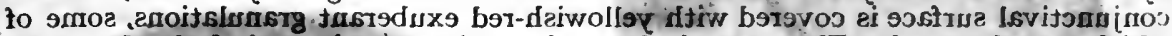

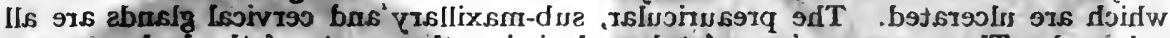

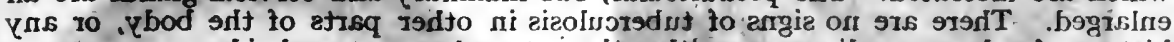

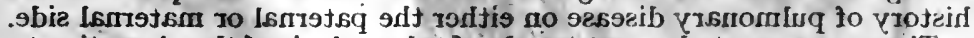

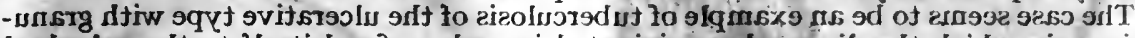

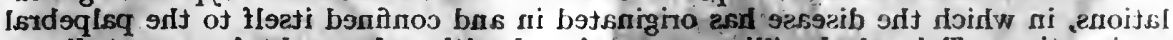

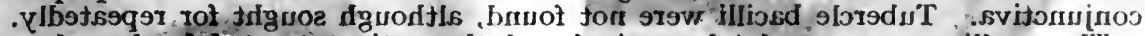

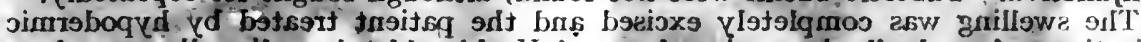
.997t 9eompdis niluorgduf blo e'd

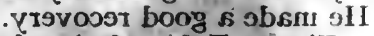

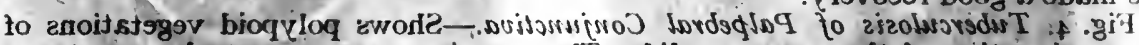

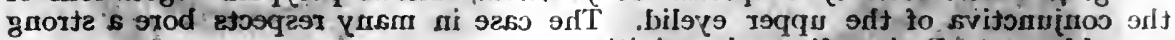

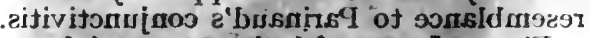

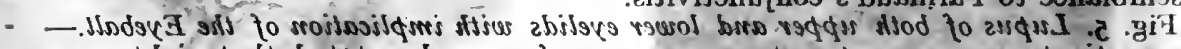

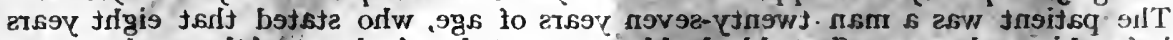

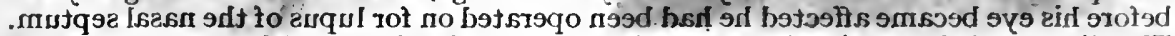

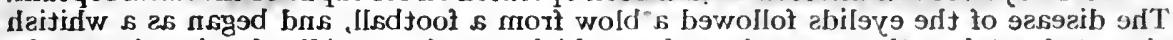

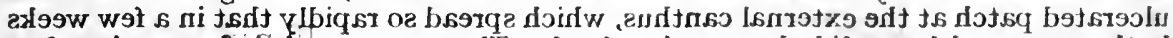

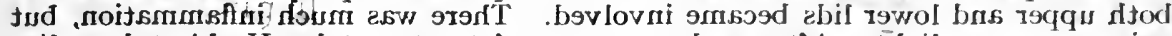

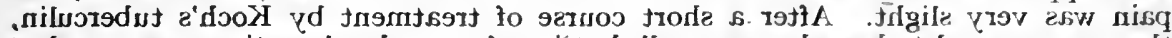

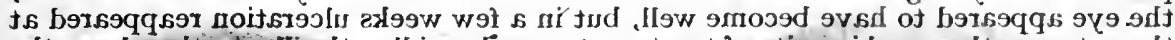

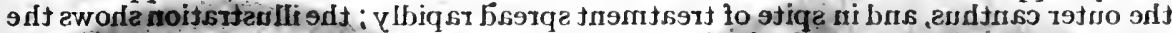

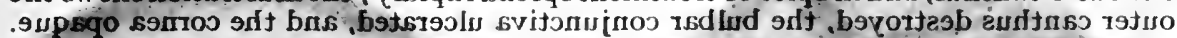

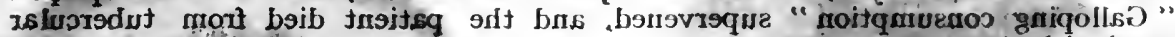
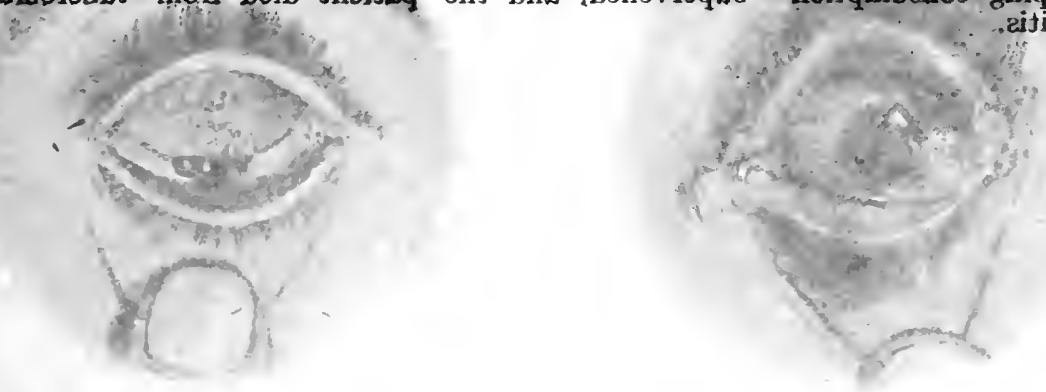


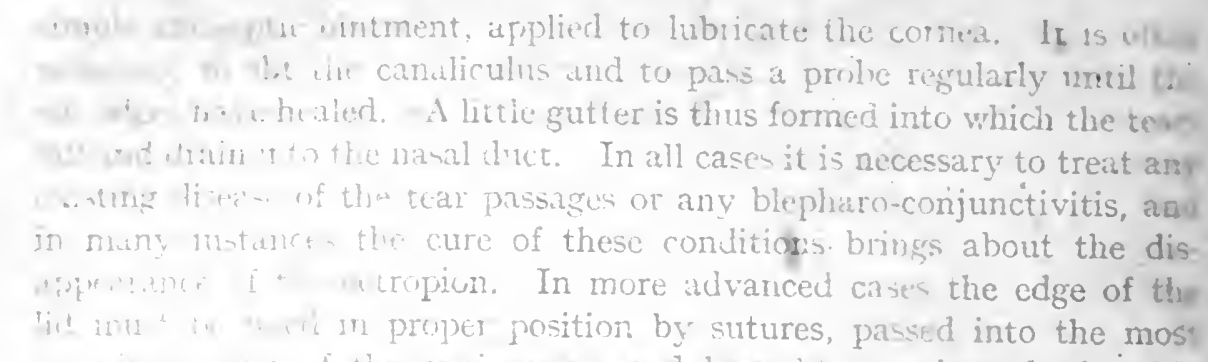

DESCRIPTION OF PLATE IV out through the skin SYPHILIS AND TUBERCULOSIS of THE EYELID an prlled tight

Fig. I. Shows an early stage of Chancre of the lower Eyelid.

Fig. 2. A Chancre of the inner Canthus with great enlargement of both the preed auricular and the submaxillary glands.

Fig. 3. Tuberculosis of the Palpebral Conjunctiva. - The patient was a lad seventen years of age, who had suffered from recurrent attacks of inflammation in the right eye for at least five years. The lower retrotarsal fold is greatly swollen, and its conjunctival surface is covered with yellowish-red exuberant granulations, some of which are ulcerated. The preauricular, sub-maxillary and cervical glands are all enlarged. There are no signs of tuberculosis in other parts of the body; or anynhistory of pulmonary disease on either the paternal or maternal side.

The case seems to be an example of tuberculosis of the ulcerative type with granulations, in which the disease has originated in and confined itself to the palpebrald conjunctiva. - Tubercle bacilli were not found, although sought for repeatedly.

The swelling was completely excised and the patient treated by hypodermic. injections of gradually increasing doses of Koch's old tuberculin, albumose free. is He made a good recovery.

Fig. 4. Tuberculosis of Palpebral Conjunctiva.-Shows polypoid vegetations of the conjunctiva of the upper eyelid. The case in many respects bore alstrong resemblance to Parinaud's conjunctivitis.

Fig. 5. Lupus of both upper and lower eyelids with implication of the Eyeball. The patient was a man twenty-seven years of age, who stated that eight yearst before his eye became affected he had been operated on for lupus of the nasal septum. The disease of the eyelids followed a blow from a football, and began as a whitish ulcerated patch at the external canthus, which spread so rapidly that in a few weeks both upper and lower lids became involved. There was much inflammation, but pain was very slight. After a short course of treatment by Koch's tuberculin, the eye appeared to have become well, but in a few weeks ulceration reappeared at the outer canthus, and in spite of treatment spread rapidly; the illustration shows the outer canthus destroyed, the bulbar conjunctiva ulcerated, and the cornea opaque. "Galloping consumption" supervened, and the patient died from tubercular meningitis. 

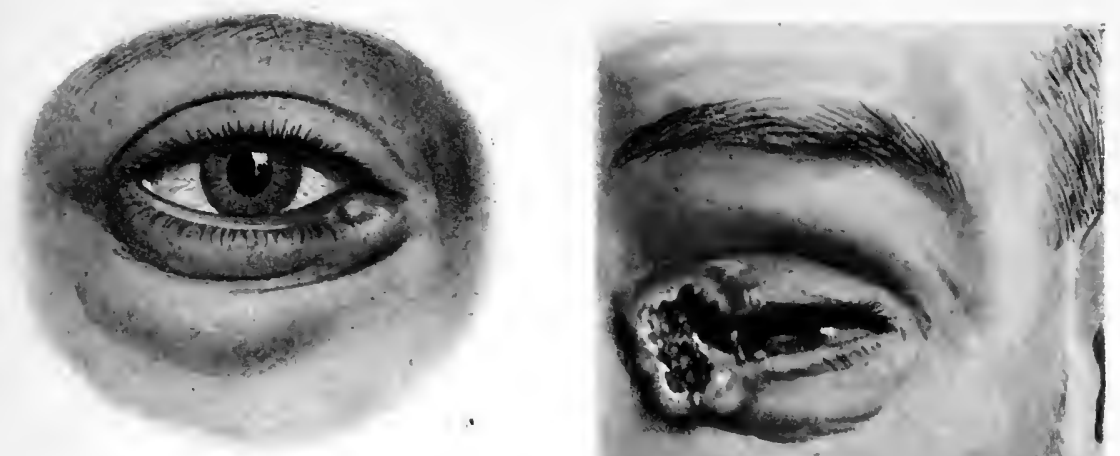

FigI

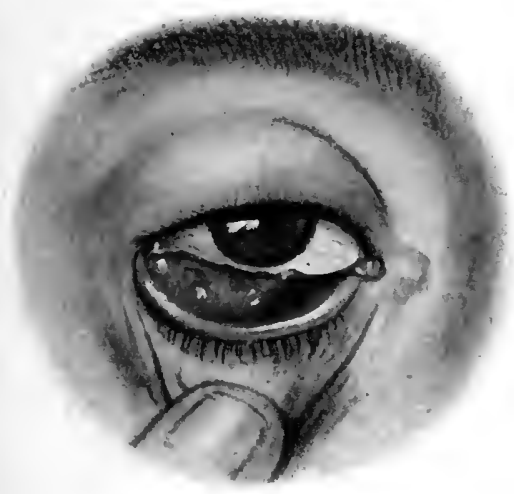

Fig III

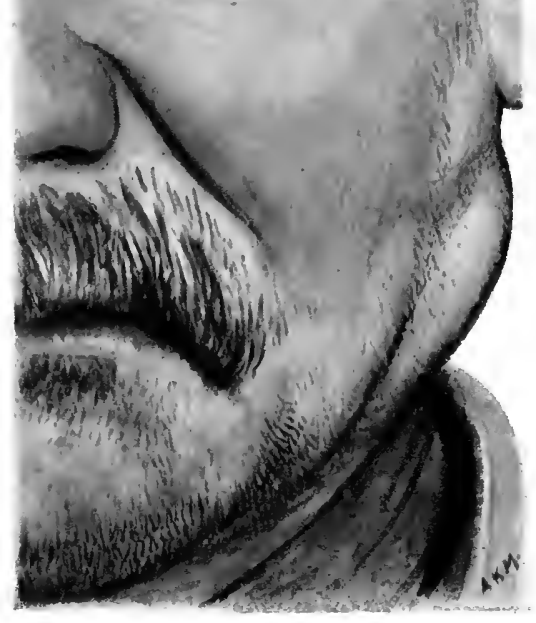

Fig II

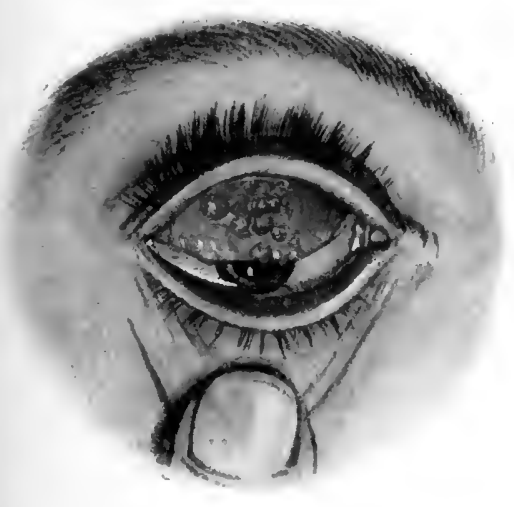

Fig L

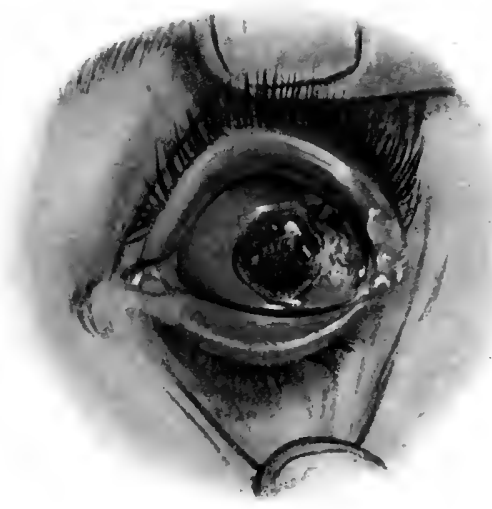

Fig $\nabla$ 



\section{CHAPTER VIII}

SYPHILIS OF THE EYELID; TUBERCULOSIS OF THE EYELID; EPITHELIOMA; RODENT ULCER

Syphilis : chancre of eyelid : modes of infection : clinical course and treatmentTuberculosis : clinical types : treatment-Epithelioma-Rodent ulcer.

1. Syphilis of the Eyelid.-Should syphilitic poison gain entrance to the system through the eye, the primary sore forms upon the eyelids or conjunctiva, the favourite seat of the chancre being at the inner canthus. The disease is met with in persons of all ages, is seen in men oftener than in women, and the lower lid is more frequently affected than the upper. In many instances the infection is purely accidental and non-venereal, and the patients have no suspicion of the nature of the sore. Often, therefore, the source of the contagion can be traced only with difficulty; sometimes it cannot be determined at all.

The most frequent modes of communication, however, are undoubtedly the finger, the niouth, the tongue, or previously contaninated sponges, towels, etc. From this it will be readily understood how medical men suffer so frequently from this particular form of chancre. While a doctor is brushing a syphilitic throat, he may receive infection from the accidental fall of saliva on his face through the patient's coughing; or after touching a syphilitic sore he may accidentally carry his finger to his eye, and thereby inoculate himself. A dentist told me that, while extracting a tooth, his face was bespattered with blood and saliva. Some weeks afterwards, a typical chancre formed on the right lower lid at the inner canthus, and this was in due course followed by all the usual symptoms of secondary syphilis. Infants and young children are often infected through being kissed by a nurse suffering from mucous patches in her mouth. The disease may be spread through the practice, common among the lower classes, of removing foreign bodies from the eye by means of the tongue. Should there be mucous patches about the lips of the operator, the contagion is immediate. Cases of palpebral chancre also arise from the common custom of incising the skin and sucking out the effused blood from a bruise about the eye. "Fasting spittle," or urine, both of which are frequently applied by the poor to inflamed eyes, may also be a source 
of contamination; and as these remedies are employed when the edges of the eyelid are inflamed and partially denuded of epithelium, the syphilitic poison will gain all the more ready access to the system. It has been affirmed that extra-genital chancres, and more particularly those occurring upon the eyelids, are apt to be followed by specially severe secondary symptoms; but it is very doubtful if the site of the primary sore has any influence upon the course of the disease.

In its earliest stages a chancre of the eyelid may easily be mistaken for a stye or a tarsal cyst; but the diagnosis becomes less difficult as the evolution of the lesion progresses. The sore gradually increases in size by eating into the edge of the lid and implicating the conjunctiva; but soon becomes characteristically circumscribed by a hard, raised, clean-cut border. It is usually covered by a glazed greyish membrane, the removal of which exposes a raw bleeding surface. Even in cases where the lid and the bulbar conjunctiva are greatly swollen, and the preauricular, submaxillary, and cervical glands much enlarged, the subjective symptoms are slight-out of all proportion to the severity of the objective signs. The three main points to attend to, therefore, in the clinical diagnosis are-(I) the comparative absence of pain and other subjective symptoms; (2) the glazed appearance and the hard, raised, clean-cut edge of the ulcer; and (3) early and pronounced enlargement of the glands. The diagnosis is confirmed by the demonstration of the spirochæe, but a negative laboratory report does not exclude syphilis, and ought not to be accepted to the prejudice of clinical experience. In many instances, however, the primary manifestations are slight, and little or no attention is paid to them, with the result that the patient does not consult a doctor until well-recognised signs of secondary syphilis have made their appearance. In those circumstances there is no difficulty in the diagnosis, but much valuable time has been lost, because it is early treatment which gives the patient the best chance of a complete cure.

The early recognition and the immediate treatment of 'a chancre of the eyelid are all-important. The copious use of a weak solution ( $\mathrm{I}$ in 5000) of bicyanide or oxycyanide of mercury, and of an ointment containing calomel is all that is necessary locally; but the chief treatment must always be constitutional, and should include the use of arsenical compounds in addition to a thorough course of mercury. The patient must be kept under observation for at least two years, and it is a great advantage if the clinical progress can be controlled by laboratory tests.

It occasionally happens that gummata form in the lids and the retrotarsal folds, and tertiary syphilis may give rise to an enormous thickening of the tarsus. All such lesions, however, disappear rapidly under the influence of antisyphilitic remedies. In these circumstances, secondary 
anæmia and general malnutrition are important complications, and require special modifications to be made in the general treatment.

2. Tuberculosis of the Eyelids. - Of all the various forms of ulceration of the eyelids, the tubercular is that most apt to be confounded with the syphilitic. They closely resemble one another, and both are accompanied by a hard and painless enlargement of the lymphatic glandsusually the preauricular or the submaxillary; but in tubercle the glands are much more prone to suppurate. In lupus, the margins of the ulcer are not so hard as in chancre, and there is nearly always associated with it similar ulcerations of the skin of the nose, the lips, or the cheek. This comparatively rare disease often begins in the palpebral conjunctiva. It progresses slowly, and as it creeps along the surface it also eats into the substance of the lid. It is usually accompanied by a considerable amount of inflammation, so that the whole lid becomes red and swollen. The ulcer is usually saucer-shaped, and is surrounded by an irregular raised margin. When free from discharge, the surface is pale red in colour, bleeds easily, and is somewhat tender to touch.

Palpebral tuberculosis occurs in several types. It forms small ulcers, or trachoma-like granules, or polypoid vegetations on the palpebral conjunctiva, or gelatinous cockscomb excrescences on the retrotarsal folds. It may be primary or secondary. In the former case the contagion is direct, and the bacilli are inoculated into minute abrasions of the conjunctiva, whereas in the latter infection usually reaches the eye by the tear passages from pre-existing disease of the nasal mucous membrane. Primary tuberculosis of the eyelid is frequently benign and strictly localised, although in some instances it forms the starting-point of a general infection; whereas secondary tuberculosis of the lid is much more prone to progress. It destroys the lids, implicates the globe, and interferes with sight as a result of ulceration of the cornea.

The indolent character of the ulceration, the pronounced glandular enlargement, and the fact that the patients are usually children or young adults, are, as a rule, sufficient evidence to enable a diagnosis to be made; but all doubt can at once be removed by inoculation experiments in a laboratory, or by a test injection of Koch's old tuberculin.

The treatment must be both local and constitutional. If the lesion is limited, it ought to be excised as completely as possible, or attempts to destroy it made by the cautery. Darier has recommended guaiacol cacodylate in 2 per cent. solution, either as drops or as a subconjunctival injection; and the results following the use of that remedy are at times so favourable, that in certain circumstances it may be looked upon as a specific. General treatment is, however, most important, and consists in fresh air, proper food, and the judicious administration of tuberculin 
subcutaneously. Needless to say, the treatment must be prolonged, and will, if it is to result in cure, require great patience and perseverance on the part both of the patient and the doctor.

3. Epithelioma of the Eyelids. - Epithelioma attacks elderly patients; and develops most commonly at the junction of skin and mucous membrane. It is oftenest seen in the lips, but not unfrequently attacks the edges of the eyelids. Primarily it consists of an overgrowth of epithelium, which, alike in skin and mucous membrane, has the peculiar cell formation known as "nests." The most trustworthy means of diagnosis, in a doubtful case, is to excise a small piece of tissue at the most actively growing border of the ulcer, and examine it under the microscope. The typical histological characters of epithelioma will at once differentiate it from syphilitic, tuberculous, or other forms of ulceration of the eyelids.

In its earliest stages, the disease may appear either as a wart, a fissure, or a nodule; but in every case ulceration occurs sooner or later, and gives rise to an open sore, with hard and " rampart-like" edges. These three clinical forms of epithelioma are identical in their microscopic structure, and all of them tend to infect the lymphatic glands in the neighbourhood, and to recur after they have been excised. They vary, however, in the rapidity of their growth and the extent of the ulceration, as well as in the degree of malignancy, according as the disease has originated in the papillæ, or the glands of the skin or mucous membranes, or has involved from the beginning the whole thickness of the integument. When the glandular structures are primarily involved, an ulcerating, warty growth projects from the surface. This progresses slowly, and consequently admits of a much more favourable prognosis than is possible when the skin is involved in its whole thickness, for then the disease burrows both deeply and widely. No structure except cartilage is able to resist the steady spread of its attack. From the surface of the ulcer there comes a constant fotid discharge; and every now and then, as the disease eats through the walls of the blood-vessels which lie in its way, free hæmorrhage occurs. Every case of epithelioma will, if left to itself, terminate fatally, as the steadily progressive ulceration and frequent bleeding exhaust the patient's strength. A fatal issue is sometimes also hastened by the occurrence of complications incidental to the infection of the neighbouring lymphatic glands, and the dissemination of the disease through other parts of the body.

The following is a typical example of the superficial or papillary form of epithelioma of the eyelid-

The patient was a farm labourer, sixty-five years of agè, who accidentally noticed a little growth about the size of a split pea projecting 
from the edge of his right upper eyelid. He showed it to his medical attendant, who told him it was a wart, and then and there snipped it off with a pair of scissors. Very soon, however, there appeared in the same place a swelling which grew rapidly, though its full extent was concealed until the upper lid was everted. Then a tumour, about the size of a hazel-nut, could be seen growing from the ciliary border to which its base was firmly attached, though its main portion was non-adherent to, and could be easily separated from, the palpebral conjunctiva. The base was hard and cartilaginous; but the surface of the unattached portion was spongy and friable, roughly lobulated, and so vascular that it bled on the slightest touch. The cutaneous surface of the lid was unaffected, and the skin was freely movable over the tumour; but the ocular conjunctiva was much injected, and its inter-palpebral portion at the inner side of the cornea was degenerated, and of a reddish-grey colour. There was no enlargement of the neighbouring lymphatic glands.

The tumour was removed as thoroughly as possible, and the gap in the lid repaired by a plastic operation. The immediate result was quite satisfactory; but unfortunately the subsequent history of the case cannot be given, because the patient failed to report himself at the hospital, and was lost sight of.

4. Rodent Ulcer of the Eyelids. - The microscopic appearance of the tissues invaded by rodent ulcer hardly differs from that of those affected by epithelioma; and hence some authorities regard the one disease as simply a variety of the other. Since, however, its clinical features-slow ulceration, extending over many years, and absence of infection of the lymphatic glands in its neighbourhood-are so peculiar, it is better to describe rodent ulcer as noncancerous, and call it by a perfectly distinctive name. Moreover, although it is locally malignant, and destroys every tissue which it attacks, yet if in its earliest stages it be freely excised, it does not recur. It is usually seen in elderly patients; and the glands of the skin of the lower eyelid, near the inner canthus, are probably the most frequent point of origin.

Commencing as a soft tubercle covered by smooth skin, the growth at first somewhat resembles a wart, and in this condition it may remain for years, and attract but little attention. As the patient grows older, however, an ulcer develops, with edges sharply cut and hard, but not markedly elevated. Superficial at the outset, it penetrates deeply in the end; and its dry and somewhat glossy surface is frequently covered by a reddish-brown scab. In epithelioma there is usually an overgrowth of tissue accompanying the process of ulceration; but in rodent ulcer there is no such overgrowth, as the disease, when once fairly started, does nothing but destroy. There may at first be cicatrisation and healing, 
but only for the time being, as the disease breaks out afresh, and sooner or later makes steady progress. It may come to a standstill, and after remaining stationary for years, without any apparent cause again become progressive. It strikes deeply and steadily, destroys the lids, and causes, usually by the side of the nose, a deeply excavated ulcer.

The following is a description of a case at a very advanced stage. The patient was a man of about seventy years of age. He had suffered from rodent ulcer for many years; and not only were both the upper and lower eyelids on the right side destroyed, but the eyeball itself had been eaten into and had burst; and the bones bounding the inner wall of the orbit and the side of the nose had been denuded of their periosteum, and were becoming carious. Such was the deplorable condition of this patient when I first saw him; and for nearly four years I was able to watch the further progress of the disease, which, in spite of all that could be done to prevent it, steadily extended its boundaries. It crept down the cheek, and, after destroying the soft parts, laid bare the bones, in part so completely that, through a hideous opening into the right nasal fossa, the turbinals were entirely exposed to view. Nor did the disease confine its ravages to the right side of the face, for, crossing the bridge of the nose, it reached the left eyelids, and after partially destroying them, invaded the orbit. The left globe was attacked; and the ulceration, creeping along the conjunctiva, seriously involved the cornea. The patient's state was then most pitiable. He was almost blind, and his suffering was so acute that he could get rest neither by day nor by night, except by the use of large quantities of laudanum. His pallor was like that of death, his emaciation extreme, and after at least twelve years of continuous suffering, he died from sheer exhaustion.

The treatment, both of epithelioma and of rodent ulcer of the eyelids, is free excision at the earliest possible moment; and after the disease has been removed a plastic operation must be performed to replace the tissues which have been excised. Remarkably good results are, however, frequently obtained by the use of radium, X-rays, or carbon dioxide snow; but the cure is not always permanent, consequently the patient must be told to report himself for examination at regular intervals, in order that relapse may be detected at once, and treatment repeated without any delay. 


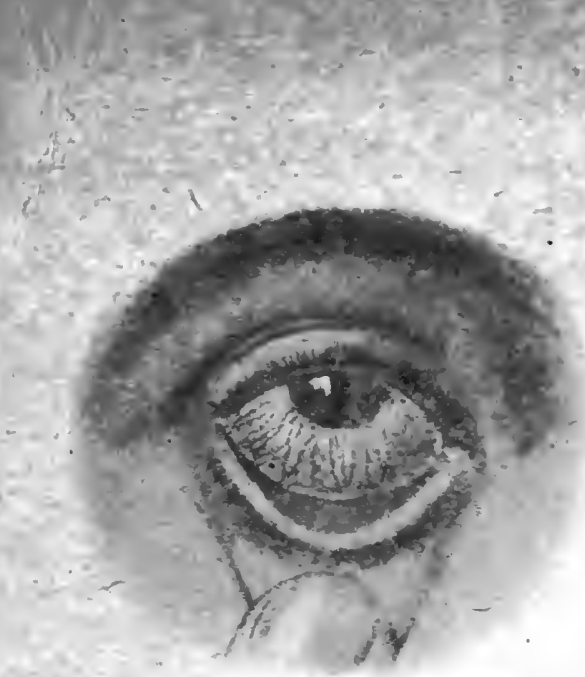

Fig I

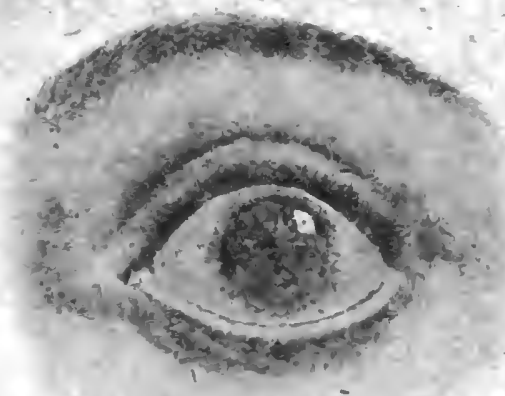

Fig II

\section{V ЗTA.IT НО VOITЯIЯगЕЯด}

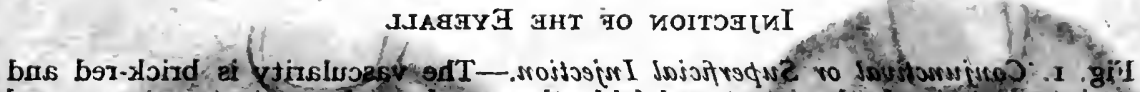

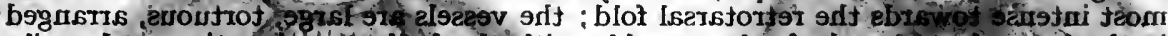

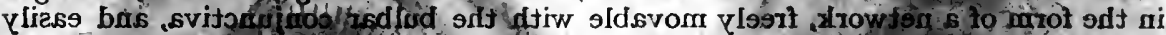

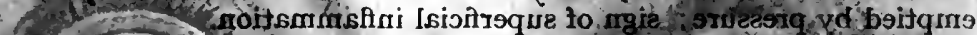

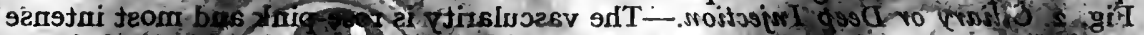

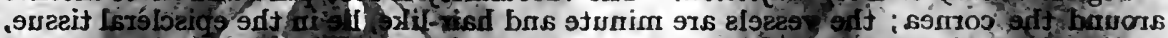

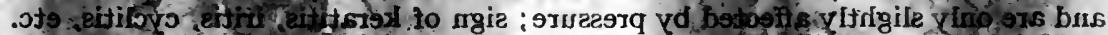

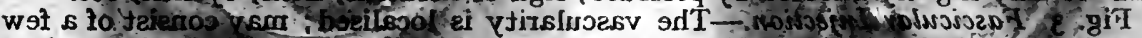

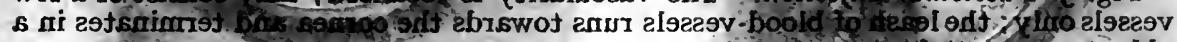

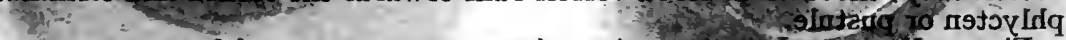

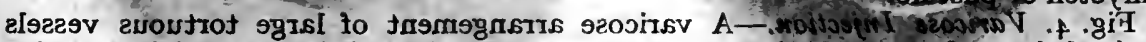

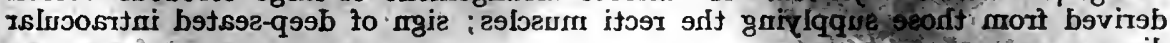
- - -

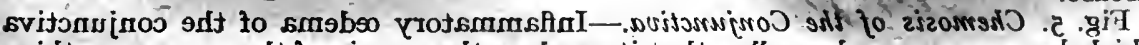

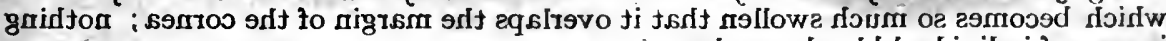
.elszesv-boold Isubivibri to noge $2 i$

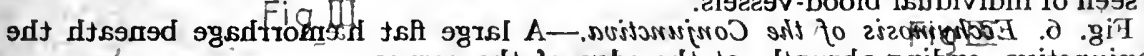

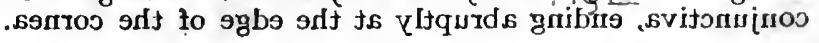

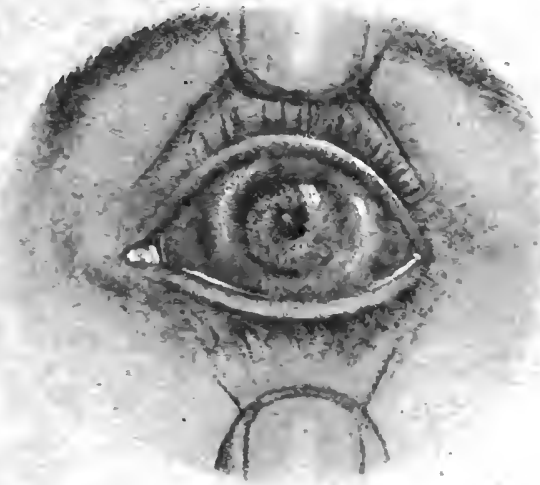

Fig $\bar{T}$

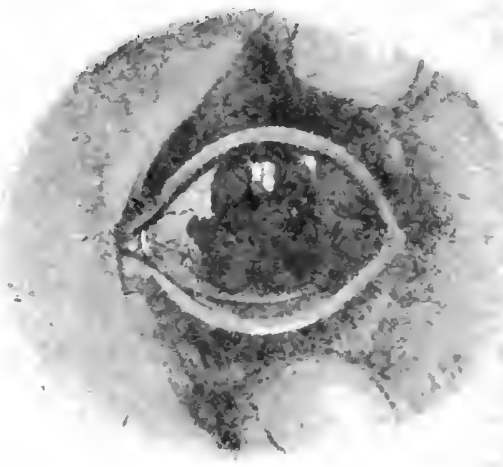

Fig 


\section{(1. 2IT U1, UITHAIMOLOGI}

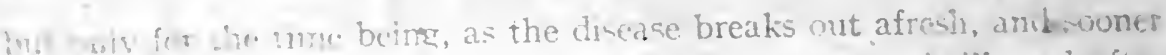
er later unates ctedily progress. It may come to a-standstill, and aftet

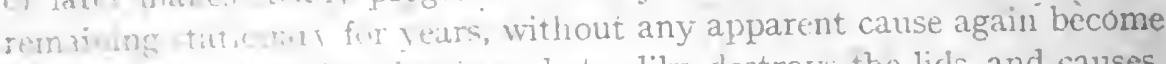
mares. a clecriy and steadily, destrnys the licis, and causes, the nuse, a deeply excavated ulcer.

description of a. case at a very advanced stage. of about seventy years of age. He had suffered and not only were both the upper and ight side destruyed, but the eyeball itself had been and the bones bounding the inner wall of the wul liad burst; and the bone bounding the inner wall of the mine carious, Such was the deplorable condition of this ac1 I first saiv Jim and for nearly four years I was able to

DESCRIPIION OF, PLATE iv spite of all that could InJection of THE EYEBall daries. It crept down

Fig. I. Conjunctival or Superficial Injection. - The vascularity is brick-red and most intense towards the retrotarsal fold; the vessels are large, tortuous, arranged in the form of a network, freely movable with the bulbar conjunctiva, and easily emptied by pressure; sign of superficial inflammation.

Fig. 2. Ciliary or Deep Injection. - The vascularity is rose-pink and most intense around the cornea; the vessels are minute and hair-like, lie in the episcleral tissue, and are only slightly affected by pressure; sign of keratitis, iritis, cyclitis, etc.

Fig. 3. Fascicular Injection. - The vascularity is localised; may consist of a few vessels only; the leash of blood-vessels runs towards the cornea and terminates in' $\mathbf{a}$ phlycten or pustule.

Fig. 4. Varicose Injection.-A varicose arrangement of large tortuous vessels derived from those supplying the recti muscles; sign of deep-seated iptrabcular disease.

Fig. 5. Chemosis of the Conjunctiva. Inflammatory odema of the conjunctiva which becomes so much swollen that it overlaps the margin of the cornea; nothing is seen of individual blood-vessels.

Fig. 6. Echymosis of the Conjunctiva. $\mathrm{A}^{2}$ large flat hxmorrhage beneath the conjunctiva, ending abruptly at the edge of the corneapnt ulcur of the eyelias,

beten 1 movel a plastic operation must be performed to replace the tissues which liwe beun excised. Remarkibly good results are, however, freibtained by the use of radium, X-rays, or carbon dioxide snow is not alvays permanent, consequently the patient must be wrot. himsels for examination at regular intemvals, in order that detected at once, and treatment repated without any 


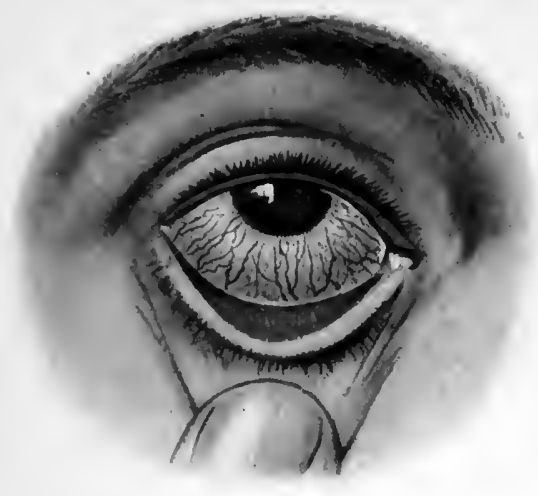

Fig I

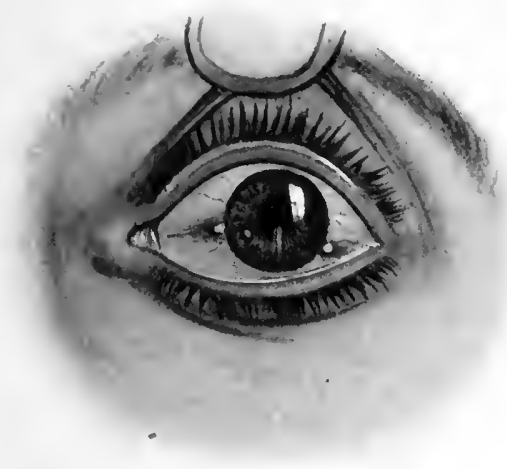

Fig II

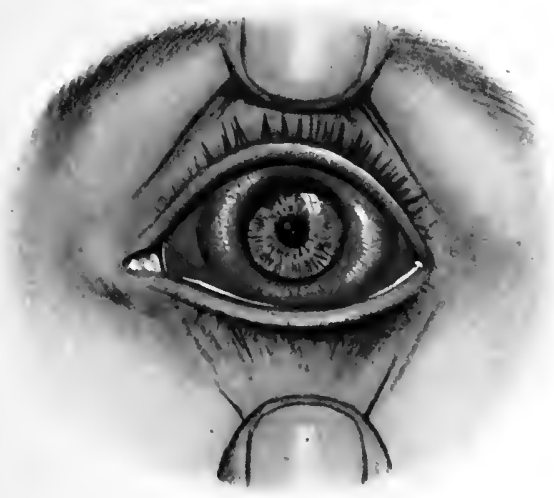

Fig $I$

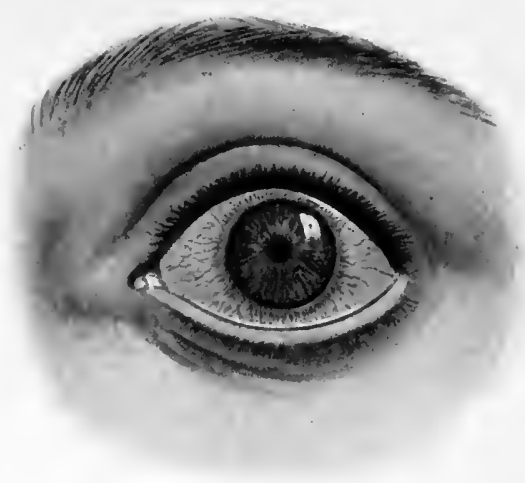

Fig II

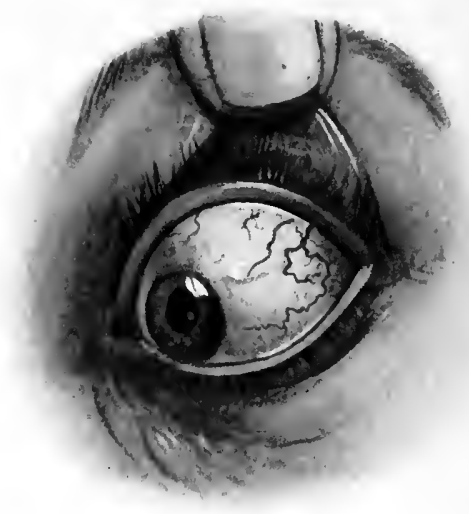

Fig $\mathbb{Z}$

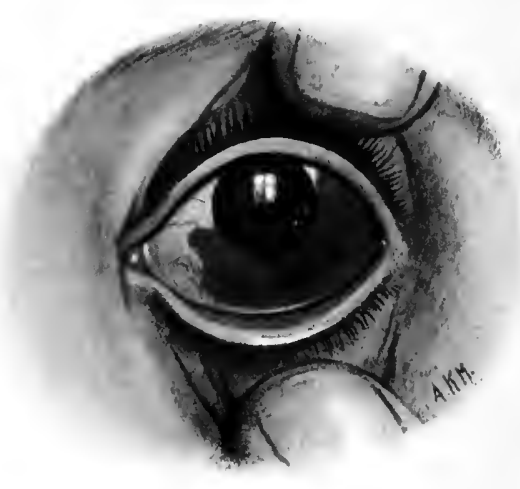

Fig VI 



\section{CHAPTER IX}

THE CLINICAL SIGNIFICANCE OF A "BLOOD-SHOT" EYE

A sign common to all inflammations implicating the anterior segment of the globe -The blood-supply of the front of the eyeball-Conjunctival and ciliary hyperæmia: their signs and symptoms-Chemosis-Presence of discharge diagnostic of conjunctivitis-Keratitis, iritis, and glaucoma as causes of hyperæmia, and the methods of distinguishing between them-Fascicular injectionInjection in scleritis-Varicose injection-Subconjunctival hæmorrhage.

THE conjunctiva is, when healthy, perfectly transparent; for, although it is abundantly supplied with both blood-vessels and lymphatics, these are so small that, with one or two exceptions, they are invisible, and in no way modify the whiteness of the underlying sclerotic. If, however, a particle of dust or any other irritant enters the conjunctival sac, the capillaries become at once congested, and, in proportion to the amount of the hyperæmia, the "white of the eye" is concealed by an intricate meshwork of injected blood-vessels. In popular language, the eye becomes "blood-shot." Redness of the "white of the eye" is, therefore, one of the earliest, as well as one of the most characteristic, signs of conjunctival inflammation; though every eye that is red is not to be regarded as suffering from conjunctivitis. On the contrary, a " bloodshot " eye is a sign common to all inflammations implicating the anterior segment of the globe. It occurs, therefore, not only in conjunctivitis, but also in keratitis, scleritis, iritis and in acute or subacute glancoma. These different diseases vary greatly in their severity and in their danger to sight; consequently to mistake one for the other may lead to deplorable results. There must, therefore, be no error in the diagnosis. The cases which present most difficulty are those in which a "blood-shot eye" is accompanied by signs of active inflammation. The blood supply to the front of the eyeball is derived from three sets of vessels : the conjunctival vessels, which come from the palpebral branches of the ophthalmic artery; the anterior ciliary arteries, which supply iris, ciliary body, and adjacent structures; and the posterior ciliary arteries, which, after supplying the choroid, pass forward to anastomose directly and indirectly with the conjunctival and anterior ciliary vessels in the neighbourhood of the limbus corneæ. Injection of the front of the eye, therefore, is due, 
either to injection of the superficial conjunctival vessels, or to injection of the more deeply situated ciliary blood-vessels.

Careful examination will readily distinguish conjunctival from ciliary injection. In the former condition the redness is of a brick-red tint, and is most intense in the region of the retrotarsal fold. The congested vessels are comparatively large and tortuous, and are arranged in the form of a network freely movable with the ocular conjunctiva. Pressure will, for the moment, drive the blood away and expose the sclerotic. In the latter condition, on the other hand, where the deeper structurescornea, sclera, iris, etc.--are attacked, the redness is rose-pink, and forms a zone of intense injection around the cornea. The distended vessels are minute and hair-like, and lie in the episcleral tissue under cover of the conjunctiva.

The symptoms associated with superficial injection of the conjunctiva are different in character from those that accompany deep inflammation. In conjunctival injection the patient complains that a particle of grit has entered his eye; which he says, feels as if it was full of rough sand or broken glass. It may indeed be very difficult to convince him that the discomfort is not due to the presence of a foreign body, and that the symptom indicates the commencement of an attack of conjunctivitis. The dilated blood-vessels irritate the sensitive nerves of the conjunctiva. This pain is always worse during the day, or when the eyes are exposed to artificial light, or after any attempt to read or write, and passes off when the lids are closed. It is quickly relieved by local remedies. In ciliary injection, on the other hand, the pain is deep-seated and throbbing. It radiates from the eye along the distribution of the fifth cranial nerve, being felt most severely in the supra-orbital, infra-orbital, and the nasal branches. It is always worse at night, and although it disappears for some hours during the day, recurs with unfailing regularity and renewed severity at night, and continues unabated until the early hours of the morning. It is not readily amenable to local remedies, but requires opiates or other internal sedatives, together with blood-letting for its relief. It is always accompanied by more or less intolerance of light and profuse lachrymation.

The first and most important step in diagnosis, therefore, is to distinguish between superficial and deep injection of the conjunctiva. In many instances, hyperæmia of both conjunctival and ciliary vessels co-exist, but it is easy to determine, by careful inspection, which of the two predominates. In some cases, the nature of the injection is obscured by inflammatory œdema of the conjunctiva, which becomes swollen, overlaps the edge of the cornea, and may, in severe cases, protrude between the eyelids. That condition is termed chemosis, and when it 


\section{CLINICAL SIGNIFICANCE OF A “BLOOD-SHOT" EYE 7 I}

occurs, all trace of individual blood-vessels is lost. It always implies that the conjunctiva is acutely irritated. It occurs in inflammation both of the superficial and the deeper structures of the eyeball, and its degree serves as a guide in estimating prognosis. In the presence of pronounced chemosis, differential diagnosis is more difficult, but if close attention be paid to the patient's account of his symptoms, as well as to the different signs invariably associated with inflammation of the individual structures of the anterior segment of the globe, the difficulties will be found to be more apparent than real.

The conjunctiva is a mucous membrane, consequently, when it is inflamed, it behaves like any other mucous membrane in the body, and forms an abnormal amount of secretion which is got rid of as a discharge. The discharge varies in character, according to the severity of the inflammation, and may be catarrhal, purulent, or membranous. In every case of conjunctivitis, therefore, there must be abnormal secretion. It may be so profuse as at once to attract attention, but, on the other hand, it may be so scanty that all that is to be seen at the time of examination of the eyes is a small amount at the inner canthus. The patient will, however, almost invariably volunteer the statement that his lids are sealed after sleep, and that he has difficulty in opening them until after the secretion, dried and hardened from exposure to the air, has been softened and removed by bathing with warm water. In simple conjunctivitis, moreover, the cornea, the iris, and the pupil all appear healthy, and the vision is unaffected. Before a diagnosis of conjunctivitis can be confidently made, therefore, the practitioner must satisfy himself that three cardinal signs are present-redness due to superficial injection, a certain amount of œdema, and an abnormal amount of unhealthy secretion. Before an opinion is given, however, the examination of the patient must be carried a stage further, because conjunctivitis may be a complication of an inflammation of the more deeply seated structures. All the structures of the anterior segment of the eyeball must, therefore, be examined, and if the patient be suffering from uncomplicated conjunctivitis, the cornea will be quite transparent, the iris will appear healthy, the pupil will be circular and active, and the intraocular tension normal. Moreover, after the eye has been bathed to remove any discharge which may be on the surface of the cornea, the patient should be asked to read, in order to make sure that vision has not been affected as a result of the inflammation.

The presence of deep injection ought always to be suspected if photophobia is so distressing that the patient is unable to open his eyes in the light. In these circumstances, a zone of rose-pink injection will be seen immediately surrounding the cornea. Deep or ciliary injection is always 
a sign of danger, for it is a sure indication of keratitis, iritis, or of acute or subacute glancoma. It is not difficult to distinguish these three important diseases; on no account must they be mistaken for conjunctivitis. In keratitis intolerance of light and profuse lachrymation are very pronounced, and the cornea is seen to have lost in whole or in part its transparency and mirror-like appearance. In iritis there is always a certain amount of photophobia and watering of the eyes, but suspicion should be at once aroused if the patient complains of severe circumorbital nocturnal pain, and of his own accord says that his sight is misty. Careful examination will at once show that while the cornea is clear, the iris is discoloured, and the pupil contraicted and irregular in its outline. In glaucoma, on the other hand, the patient may not speak of his eye, but complain loudly of agonising pain over one side of his head, and may be prostrated by acute bilious vomiting. When, however, the eye is examined it is seen to be of a livid red colour from deep diffuse injection, the cornea is steamy, the pupil moderately dilated, the anterior chamber shallow, and the intraocular tension increased. When sight is tested, it is found to be much impaired, or it may be altogether lost.

In the cases dealt with up to this point the injection of the front of the eye is diffuse ; in those now to be considered it is localised. Localised injection may be superficial or deep. The former is sometimes spoken of as fascicular injection, is usually limited to one side of the eyeball, and may only consist of a few enlarged blood-vessels which run towards the cornea. It is characteristic of the eruptive forms of conjunctivitis. The vascularity may surround a pustule in the bulbar conjunctiva, or the fascicle of newly-formed blood-vessels may terminate in a phlycten on the cornea. Intolerance of light and blepharospasm are the outstanding symptoms in those cases, and make the examination of the eye extremely difficult. It may be almost impossible to see the cornea; but whenever a leash of blood-vessels is seen making its way from the conjunctiva over the corneal margin it may safely be assumed that the patient is suffering from phlyctenular keratitis.

Deep localised injection is associated with inflammation of the sclerotic-scleritis. It is characterised by the appearance on the sclera, close to the margin of the cornea, of a circumscribed elevation of dark red or bluish-red colour. This might, at first sight, be mistaken for a pustule, but careful examination will show that the infiltration is not in, but beneath, the conjunctiva. Moreover, the inflamed patch is always tender to touch, and there is usually more or less aching pain in the brow.

Deep-seated localised injection also occurs in the form of large tortuous vessels, especially the veins, supplying one or other of the recti muscles. 
These vessels become varicose, and are always seen in cases of advanced glaucoma and in old-standing inflammation of the uveal tract. A leash of enlarged varicose vessels often appears opposite the site of sarcoma of the choroid, and is a valuable aid in diagnosis.

In all forms of injection of the front of the eye, whether it be diffuse or localised, the brighter the colour the more acute the inflammation, and the more livid the appearance, that is, the more the veins predominate in the congestion, the more chronic the course of the disease.

When a "blood-shot" eye occurs without any sign of inflammation, it is due to subconjunctival hamorrhage, which may occur spontaneously, although it is frequently due to direct injury. The hæmorrhage may be limited to one spot, or, as sometimes happens, may completely cover the sclerotic, and then the conjunctiva projects, and appears like a red collar encircling the cornea. The effused blood forms a clot, visible through the transparent conjunctiva, and bounded in front by the corneal limbus. Now and then, however, these limits are overstepped, and blood finds its way for a short distance into the substance of the cornea. It always more or less completely hides the sclerotic, and this changed appearance of the eyeball is at once noticed by the patient, and usually causes him much anxiety. In order to determine the significance of subconjunctival ecchymosis, it is important to examine the intraocular tension, the state of the pupil, and the acuity of vision. If there be little or no change from normal, it is safe to conclude that the eye has not been seriously injured. The time at which the ecchymosis makes its appearance after an injury is also of great importance. When it occurs immediately after an accident, it is, as a rule, of very little significance, but should it, on the other hand, make its appearance after a lapse of several days, the prognosis is very serious, because it is then one of the most trustworthy indications of fracture through the anterior fossa of the skull. In children suffering from whooping-cough, subconjunctival ecchymosis may appear suddenly after a fit of coughing, and in elderly persons after vomiting, straining at stool, hurrying to catch a train, or any kind of active exertion. As a rule, it is a trifling occurrence in itself, but it should not be regarded lightly until a careful examination has been made of the heart, the blood pressure, and the urine, because, in some cases of arterio-sclerosis, a subconjunctival ecchymosis has been the precursor of cerebral hæmorrhage. 


\section{CHAPTER $\mathrm{X}$}

INFLAMMATION OF THE CONJUNCTIVA

Frequency of conjunctivitis-Presence of discharge-Acute conjunctivitis : catarrhal, purulent, membranous-The importance of discovering the infecting agentMethod of taking a smear-Bacteria found in conjunctivitis-The clinical signs of conjunctivitis-Treatment : local, general, and prophylactic.

INFLAMMATION of the conjunctiva forms from 30 to 40 per cent. of all diseases of the eye. The frequency of its occurrence, therefore, may mislead a general practitioner who is inexperienced in ocular troubles, and encourage him, when he is consulted by a patient suffering from an inflamed eye, to think that the case is probably conjunctivitis, and tempt him to act as if that guess at random was a well-considered diagnosis. That attitude of mind is greatly to be deprecated; for it cannot be repeated too often that a diagnosis of conjunctivitis can only be made with certainty if the injection of the white of the eye be superficial and accompanied by sticky discharge, if the cornea and iris be healthy, the pupil active, and the intraocular tension normal, and if there be no impairment of sight. After he has satisfied himself that the patient is really suffering from uncomplicated conjunctivitis, the practitioner must next determine whether the attack be acute or chronic. That will not be difficult, if attention be paid to the patient's account of the history of his illness. This primary classification of all cases into acute and chronic, although quite arbitrary, is clinically of great value, because acute conjunctivitis is always the result of direct contagion; whereas chronic inflammation of the conjunctiva is due to the prolonged action of the exciting cause, which is not necessarily microbic, but frequently physical or chemical; or may arise in connection with some constitutional state, of which "gout" and "rheumatism" are common examples. Obstinate cases are often associated with disease of the tear passages, or with errors of refraction, and among other ætiological factors may be mentioned chemical irritants, misdirected eyelashes, and foreign bodies hidden in the conjunctival sac.

Acute conjunctivitis may be severe or mild. . The severity of a particular case depends upon two factors, the virulence of the infecting organism, and the resisting power of the patient to infection. 
An estimate may be formed of the gravity of a severe case by observing the character of the discharge, which may be catarrhal, purulent, or membranous. Obviously the prognosis will be most favourable in the catarrhal cases and least favourable in the membranous. The discharge is always contagious, and may be communicated from one person to another by soiled fingers, handkerchiefs, towels, wash-hand basins, etc., etc. It contains infecting organisms, none of which, however, gives rise in every case to the same clinical picture. On the contrary, the same infecting organism may cause in one person a mild catarrhal ophthalmia, while in another it gives rise to severe purulent inflammation. A clinical diagnosis should always be supplemented, therefore, by a bacteriological examination in every case in which the symptoms are very severe, or the course of the disease unduly prolonged. The discovery of the infecting organism is of great assistance in prognosis. At the outbreak of an epidemic, it is very important to have identified the specific microorganism which has caused it, and, in individual cases, to know the particular microbe that has given rise to the infection enables proper precautions to be taken to prevent the spread of the disease to others. A mild case of purulent or membranous conjunctivitis, the true cause being unsuspected, and little care, therefore, being taken to prevent the spread of contagion, may cause deplorable results in other members of the family circle. Take, for example, a mild case of membranous conjunctivitis in which the signs and symptoms are so trivial that little heed is given to the patient's eye, while all the time the Klebs-Loeffler bacillus is present, and constitutes a grave source of danger to others. Diphtheria of the larynx has been contracted in that way, and has resulted in death. That is a case in which the clinical manifestations of the diseases are so slight that its dangerous character is overlooked; but the opposite situation may also arise. The Koch-Weeks bacillus, the pneumococcus, and the gonococcus give rise to types of inflammation of the conjunctiva, which in ordinary circumstances can be distinguished by clinical signs sufficiently characteristic to render a diagnosis possible. In exceptional cases, however, each of those three micro-organisms may produce such acute inflammation, accompanied by so much purulent discharge, that clinically it presents all the features of severe gonorrhceal ophthalmia. The prognosis of such a case depends on the recognition of the causal germ, because, while in gonococcal inflammation there is grave danger that the cornea will be destroyed, in the conjunctivitis due to Koch-Weeks bacillus or to the pneumococcus, corneal complications in the great majority of cases are neither frequent nor dangerous. In all inflammations of the conjunctiva, therefore, where the signs are severe or the course protracted, bacteriological examination must form 
a part of clinical diagnosis. The routine work of a busy general practitioner affords neither time nor opportunity for elaborate laboratory research, but, fortunately, that is not necessary, for, in the great majority of cases, a direct smear preparation is all that is required. And.when the examination has to be made more complete by the preparation of culture tubes, the assistance of an expert can usually be obtained.

The method of examining secretions from the eye can be carried out by any one possessed of an elementary knowledge of bacteriology. Browning gives a clear and simple description of the technique. "The lower lid is everted and a platinum loop sterilised by heating to redness in a flame and allowed to cool, is drawn across the fornix, gathering up any beads of pus or mucus present. The material thus obtained is spread on glass slides in a fairly thin film. Lumps of pus are broken up with the loop and spread evenly. Whenever possible, two slides are made, and one stained by carbol methylene blue and the other by Gram's method counterstained with neutral red." The specimens mounted in the ordinary way are examined with $\frac{1}{1}$ inch oil immersion lens.

The staphylococcus albus and the xerosis bacillus are usually found in the normal conjunctival sac. The other micro-organisms found in pathological conditions can be classified into three groups-

I. Where the same micro-organisms are always found present. Acute catarrhal conjunctivitis always associated with the Koch-Weeks bacillus; chronic catarrhal conjunctivitis dependent upon the diplo-bacillus of Morax-Axenfeld, and purulent conjunctivitis, the exciting cause of which is the gonococcus of Neisser.

2. Where the organisms. may be present in an apparently healthy conjunctiva and only excite inflammation in special conditions. Pneumococcus, bacillus coli, staphylococcus, streptococcus, etc. The conjunctival inflammation frequently arises as a complication in measles, scarlet fever, influenza, etc. ; both the course and duration of the forms of conjunctivitis associated with those germs are profoundly influenced by the constitution of the patient.

3. Where the special micro-organisms only act after the conjunctiva has become inflamed through the agency of the toxins manufactured by other microbes, e.g. membranous conjunctivitis associated with the KlebsLoeffler bacillus, etc.

While bacteriological examination, therefore, is always helpful in diagnosis, it also affords considerable assistance, both in prognosis and treatment, although in the latter its value is much greater in prophylaxis than it is in actual curative treatment.

The clinical signs, however, must always take the first place with the general practitioner, who should be able, by inspection alone, to 
distinguish a mild attack of conjunctivitis from one that is severe. In the former the eyelids are altered very little, either in colour or appearance, and the discharge is so scanty that its presence is only detected by the lids being sealed together after sleep. Those trivial signs are in marked contrast to the striking alterations present in severe conjunctivitis, in which the lids are red and œedematous, difficult to open, and so rigid that it is almost impossible to evert them. The bulbar conjunctiva is swollen and may protrude as a livid red fleshy mass between the lids, and overlap and partially conceal the cornea. The discharge is purulent, is frequently stained with blood, and readily becomes plastic. The acute infectious character of the attack is shown by the fact that the preauricular gland is distinctly swollen and tender; the patient feels ill and suffers much, not only from the eye, but also from general malaise. Such a state of matters is very serious, for ulceration of the cornea may occur very quickly, and then the disease will become much more than a conjunctivitis, and sight be gravely endangered.

Treatment.-The same general principles underlie the treatment of every form of acute inflammation of the conjunctiva. The first and foremost indication is to keep the eyes thoroughly cleansed from all discharge. The secretion must be wiped away from the edge of the lids as soon as it forms. Moist sterile cotton wool should be employed for that purpose, and the soiled swabs must be destroyed immediately after they have been used. An inflamed eye must never be wiped with a handkerchief or a towel. A lotion for bathing the eye is usually prescribed, but its exact composition matters little in comparison with the need for absolute cleanliness. The study of bacteriology encourages the hope that antiseptics will destroy the causal germs, and arrest the inflammation, but the results of treatment inspired by bacteriological investigations are very disappointing. The reason is that no drug, no matter how strong its bactericidal powers, can sterilise an infected conjunctival sac. Its antiseptic power is quenched the moment it comes in contact with blood serum, pus, or other products of inflammation. Moreover, a drug powerful enough to act upon the protoplasm of a microbe, will act just as powerfully on the protoplasm of the tissues, and in the case of a structure so delicate as the conjunctiva strong antiseptics, therefore, cannot be employed. Nevertheless the drugs which have always enjoyed the greatest popularity in the treatment of conjunctivitis are nitrate of silver, sulphate of zinc, and perchloride of mercury, and their usefulness is established beyond all question. Their value, however, depends more on their stimulating than upon their bactericidal properties. When applied to the tissues, they cause hyperæmia and promote recovery, not by their direct action on the micro-organisms, but by attracting to the 
conjunctiva a superabundance of blood rich in antitoxins, and thereby enabling it in virtue of its own energies to overcome the inflammation. The aim, therefore, when local applications, whether caustics, astringents, or antiseptics, are being applied to the inflamed conjunctiva, is to so regulate the strength, as well as the frequency of the applications, that the right degree of stimulation is brought about, and the tissues not overirritated. As far as possible, therefore, the practitioner should apply those remedies himself, in order to ensure that the treatment is efficiently carried out, and to enable him to judge from day to day how far the desired results are being obtained. As a rule, those applications do not require to be repeated more than once, or at most, twice in every twentyfour hours, and in the interval the patient or the nurse must keep the eyes clean with some bland lotion used at blood heat. Solutions can be applied to the eye from an undine, or a dropper, or by means of a pledget of absorbent cotton wool. Syringes or sponges ought never to be used, and care must be taken not to exercise force or any undue pressure when handling the lids. The undine and droppers must be thoroughly boiled after being used, and kept in an antiseptic solution carefully protected from dust until they are required.

The patient must be kept as comfortable as possible. When the inflammation is severe, and the discharge profuse, he ought to be kept in bed in the care of a nurse, who, if possible, has had some training in ophthalmic diseases. The sick-room must be light and well aired; darkness and bad ventilation are inimical to good progress. If photophobia be distressing, the eyes can be shaded from light, but on no account must they be tied up with bandages. Before the patient goes to sleep, the edge of the lids should be smeared with a bland lubricant, because when they are glued together by dried secretion, their separation on waking gives rise to much discomfort. The aim of all treatment is to restore the conjunctiva to its normal condition, and prevent an acute from passing into a chronic attack. It is a wise precaution, therefore, to insist that treatment is not to be discontinued for at least a fortnight after the patient appears to be cured. It must not be forgotten that the causal germs may, after a time, cease from troubling, and lie dormant in the conjunctival sac. They are there, however, ready to resume their activities on the slightest provocation. That is the explanation of the occurrence of relapse after all signs and symptoms of the disease had disappeared. It is prudent, therefore, in all severe cases, to examine a smear preparation and not to discharge the patient unless that examination gives negative results.

Lastly, every precaution must be taken to prevent the disease from spreading. If only one eye be affected, the other should be at once 
covered securely by a Buller's shield, and the patient told to lie on the side affected in order to prevent discharge from flowing over the nose, and thus reaching the sound eye during sleep. Relatives, and any one who is brought into contact with the patient, must be warned of the contagious nature of the discharge if by any means it should come in contact with their own eyes. Children suffering from acute conjunctivitis ought not to attend school, where they would be sure to spread the disease among their companions, through contagion chiefly by means of towels, or wash-hand basins. 


\section{CHAPTER XI \\ ACUTE INFLAMMATION OF THE CONJUNCTIVA}

Catarrhal conjunctivitis: mode of spread of contagion, clinical course, causal micro-organisms : treatment-Argyrosis-Purulent conjunctivitis: of gonococcal origin : liability to ulceration of the cornea : clinical stages-Metastatic gonococcal conjunctivitis-Treatment of purulent conjunctivitis-Membranous conjunctivitis: diphtheria of the conjunctiva : treatment.

OuR knowledge regarding the organisms which cause acute inflammation of the conjunctiva is still so incomplete that the general practitioner is likely in the first instance to fall back on the old clinical classification, and be guided both in prognosis and treatment by the character of the discharge, which may be catarrhal, purulent, or membranous.

1. Gatarrhal Gonjunctivitis is the commonest of all inflammations of the conjunctiva. It is often spoken of as a "cold in the eye" or "pink eye," and certainly atmospheric conditions seem to exercise considerable influence on its "occurrence. The onset takes place most frequently when east winds prevail, and when the weather is changing from warm to cold, or vice versa. The inflammation is very contagious, spreading widely as a result of direct inoculation from common washhand basins, sponges, towels, etc. In that way children at school are very liable to attack, and may, in turn, carry the disease to their homes. Occasionally it is epidemic, and many outbreaks are ushered in by measles or scarlet fever. Both eyes usually are affected, though not simultaneously, the second being attacked just as the first begins to recover. As a rule, it runs a mild course in from three days to as many weeks; but adults suffer almost invariably more severely than children. The disease presents striking clinical features; and nowhere in the body are the classical signs of inflammation-redness, heat, swelling, and pain-better seen. There is first a sensation of itching in the lids, and a feeling as if a gritty substance had entered the conjunctival sac. The discomfort is always greatest in the evening, and especially after exposure to strong artificial light. The lids are red and swollen along their edges, and occasionally the œedema is so great that they remain closed, and the upper overlaps the lower. The palpebral conjunctiva is congested, and, owing to loss of its transparency, the Meibomian glands are not dis- 
tinguishable. In severe cases, the retrotarsal fold is much swollen, and the bulbar conjunctiva so full of turgid blood-vessels that the "white of the eye " is hidden by a brick-red or bright scarlet vascular network, freely movable upon the sclera, the meshes becoming larger as the fornix is reached. Thrombotic spots and minute hæmorrhages are frequently present, and in very acute cases there is considerable chemosis. The muco-purulent secretion, which is.one of the most characteristic features, and appears very early, may be so slight as merely to seal the lids together during sleep, or in the day-time to form little strings of mucus hidden in the retrotarsal fold, or a small yellow bead at the inner canthus. In severe cases, however, the discharge is much more profuse, light yellow in colour, creamy in consistency, hanging about the eyelashes, and forming, when dried by exposure to the air, a thick crist at their roots. The discharge is always very contagious, and in the great majority of cases contains the Koch-Weeks bacillus, although in some epidemics the pneumococcus predominates. There is not, as a rule, much photophobia unless corneal ulceration is present. Ulcers are prone to form in all severe cases. They are superficial, clean cut, of crescentic shape, and situated at the margin of the cornea. Sight is interfered with only in so far as can readily be accounted for by the presence of a flake of mucopus on the surface of the cornea. The coloured lights that it causes by diffraction are not likely to be mistaken for the rainbow halos in glaucoma, because they disappear at once after the patient winks his lids or bathes the eye, thereby removing the floating muco-pus from the surface of the cornea.

Treatment.-Catarrhal conjunctivitis readily yields to simple treatment. The conjunctival sac should be thoroughly cleansed with a solution of borax or of boracic acid three or four times a day, according to the severity of the inflammation and the quantity of the discharge. After the eyes have been bathed, one drop of 20 per cent. argyrol solution should be instilled. At bedtime the edge of the lids should be anointed with an ointment containing 3 per cent. boracic acid. Mackenzie pointed out that the feeling of sand in the eye is uniformly relieved, and the inflammation abated, by the use of a solution of nitrate of silver. It is employed in from I per cent. to 2 per cent. solution, and carefully applied to the everted conjunctiva with a piece of absorbent wool twisted round the end of a probe. A minute or two after the caustic solution has been applied, sharp pricking pain is felt, lasting from ten to fifteen minutes, and then passing off, leaving the eye free from the sensation of grit and the tendency to water. The eye continues easy for five or six hours, when the symptoms of irritation recur, and ought immediately to be checked by the application of the nitrate of silver solution. The use of lunar caustic in the 
manner just described is of great benefit in all cases of catarrhal inflammation of the conjunctiva. The pain it causes is a drawback, but that can be greatly mitigated by instilling a few drops of 2 per cent. solution of cocain five minutes before the nitrate of silver is applied.

The long-continued use of that remedy, however, produces a blueblack staining of the conjunctiva-argyrosis-consequently it ought only to be applied by the practitioner himself, and never prescribed as "drops" for the patient to use at home. Astringents, like sulphate of zinc, and irritants like the yellow oxide of mercury, are to be avoided, and the eyes must not be tied up with bandages. When photophobia is troublesome, a large brown-paper shade may be worn. The treatment ought to be continued until the lids no longer stick together in the morning.

2. Purulent Conjunctivitis may occur at any time of life; but the course of the disease is so much modified by the age of the patient that it is convenient to draw a clinical distinction between the purulent conjunctivitis occurring in the adult, and that seen in the newly-born child. The latter condition is known as ophthalmia neonatorum, and is described in Chapter XII. What follows is a brief description of gonorrhoeal ophthalmia in the adult. When gonococcal infection occurs in the eyes of an adult, the results are much more serious than when it takes place in the infant, because ulceration of the cornea is of very frequent occurrence, indeed, in many instances it cannot be prevented. As a rule the disease is caused by the direct transference of pus from the genitals to the eye, and the more acute the urethritis and the fresher the pus with which the eye is inoculated, the more virulent will be the blennorrhœa of the conjunctiva. The disease is more frequent in men than it is among women; but, considering the large numbers of those who suffer from gonorrhœa, ophthalmia must be regarded as a comparatively rare complication. Moreover, a considerable number of the cases occur in nurses, and in medical practitioners who have been inoculated accidentally in the course of their professional duties. Usually one eye only is affected at the beginning, for obvious reasons most frequently the right. Sudden onset is one of its most striking features. The patient's eye appears quite well at night, but by the morning the lids are acutely inflamed and swollen; the conjunctiva is red and œdematous, protrudes beyond the edge of the palpebral opening, and secretes blood-stained serum in large quantity. The severity of the pain, which is far greater than in other forms of acute conjunctivitis, is another symptom that ought always to arouse suspicion that the case is gonorrhœal. The course of the disease is rapid and destructive.

Ulceration of the cornea is very liable to occur, because the bulbar conjunctiva participates acutely in the inflammation. In the severest 
cases, the conjunctiva bulbi becomes so infiltrated that its blood-vessels are compressed by the exudation, and in consequence its colour changes from red to greyish yellow, and its surface is speckled with hæmorrhages, and at times coated with a fibrinous exudate. It surrounds the cornea like a collar, and, strangulating it at its source of nutritive supply, threatens its vitality from the very onset of the disease. Fortunately, all cases are not so virulent, the severity of an attack seeming to depend upon the activity of the infecting micro-organism, as well as upon the constitution and the previous habits of the patient. In every case, however, the occurrence of purulent inflammation of the cornea is to be feared; and obviously the more pronounced and the more solid the chemosis of the ocular conjunctiva, the more certainly may corneal ulceration be predicted. The course of the disease may be divided into four stages-

(I) Stage of Infection and Incubation.-As yet there are neither signs nor symptoms, but a history of direct inoculation is given. One of the most frequent examples, and one of the most tragic, is when a nurse receives a spurt of pus into her own eye from a baby suffering from ophthalmia neonatorum. In the majority of cases this stage of the disease lasts only a few hours.

(2) Stage of Swelling and Infiltration.-Pain is now very severe; the lids are red, swollen, and tense; they are everted with great difficulty, and the upper frequently overhangs the lower. The bulbar conjunctiva is swollen and infiltrated, and the whole conjunctival surface secretes a large quantity of blood-stained serum.

(3) Stage of Suppuration and Ulceration.-As the signs characteristic of the second stage begin to subside, profuse purulent discharge flows from the conjunctiva. Corneal complications are now prone to occur, and in very acute cases ulceration of the cornea may take place as early as the second or third day from the onset of the disease. It is brought about partly by the pus macerating the epithelium, whereby a way is opened for the gonococci to invade the substance of the cornea, which in the most virulent cases seems to melt away. Descemet's membrane resists, and appears so clear that the inexperienced is apt to be misled into thinking that improvement has set in, and he may be confirmed in that opinion by the patient volunteering the statement that he sees better than he did. The true state of matters, however, becomes quickly apparent, because, in a day or two, Descemet's membrane also gives way, and the eye is hopelessly destroyed. When its destruction is not so rapid, either an abscess forms in the centre of the cornea, or ulceration begins at the margin and spreads rapidly under cover of the swollen conjunctiva.

When this critical stage has been reached, any sudden movement of the head, a cough, or any unequal pressure of the bandage, will 
precipitate perforation. Up to this point, the patient's suffering has been agonising; but with the bursting of the cornea comes an immediate sense of relief, usually followed by sound refreshing sleep. This frequently is the turning-point in the course of the inflammation, and the amount of vision retained by the patient depends upon the extent of the ulceration, and the size and behaviour of the prolapse of iris.

(4) Stage of Chronic Infiammation and Hypertrophy.-The inflammation and suppuration usually subside in from five to six weeks, but in many cases a condition of chronic inflammation of the conjunctiva remains. The lids are no longer swollen, but the palpebral conjunctiva is red, thickened, and granular. The retrotarsal fold is swollen, and the bulbar conjunctiva is hyperæmic - the white of the eye being streaked by large congested blood-vessels. The gonococcus often persists in the conjunctival sac for weeks in spite of treatment, and is responsible for relapse occurring in patients who were supposed to be cured. In these cases, therefore, a bacteriological examination of a smear ought to be made at regular intervals, and it should be found negative on three consecutive occasions before the patient is discharged.

Metastatic Gonococcal Conjunctivitis.-An acute ophthalmia may occur suddenly in a person suffering from gonorrhœe, quite apart from any direct contagion. Such cases are due to metastasis, and occur most frequently in those suffering from arthritis, iritis, scleritis, or some other metastatic gonococcal infection. The discharge contains no microorganisms, and the inflammation usually runs a benign course, varying in its intensity according to the severity of the urethritis.

The prognosis in gonorrhœal ophthalmia is always serious, and is much worse in adults than it is in children. The general practitioner will be well advised, therefore, to consult an ophthalmic surgeon as early as possible. Everything depends on the state of the cornea, and if it appears cloudy in the early stages, the outlook is very unfavourable, because ulceration is almost certain to occur, and even although the eye may not be wholly destroyed, sight is sure to be seriously impaired.

Treatment.-The first care of the surgeon, when called upon to attend a case of purulent conjunctivitis, in the adult, is to protect the sound eye from infection. That is best done by tying it up securely under a Buller's shield, and the patient must be warned on no account to allow his fingers or any soiled dressing to come in contact with the sound eye; and when he is asleep, care must be taken that he lies upon the affected side, for fear pus may trickle over the nose from the inflamed to the sound eye.

From the very outset of the disease, the patient ought to be confined to bed in a well-ventilated room, and every care be taken that he is liberally supplied with nourishment. He ought to have a nurse beside 
him, both by day and by night, as the whole secret of successful treatment depends upon the fidelity and thoroughness with which the cleansing of the eye is carried out. The nurses ought always to be well warned of the virulent character of the disease with which they have to deal, and of the risk to their own eyes if, by means of soiled fingers or the spurting of discharge from the patient's eye, they become infected.

The curative treatment consists in allaying the inflammation, in checking suppuration, in preserving the cornea, and in hastening the return of the conjunctiva to its normal condition.

Whenever it is suspected that the conjunctiva has become infected, the eye must be thoroughly bathed, and a few drops of a 2 per cent. solution of nitrate of silver instilled. The bathing dilutes the virus and so renders it less active, and the nitrate of silver not only exercises its bactericidal influence upon the gonococci, but by irritating the conjunctiva it stimulates it to repel the invasion of the tissues by the microorganisms. When the disease is definitely established, antiphlogistic treatment is clearly indicated at the outset. For the relief of pain, leeches to the temple or free bleeding with the artificial leech, and opium or aspirin internally, are of great value, and iced compresses should be applied to the eye, from which all discharge must be removed by frequent irrigation with ice-cold boracic solution. When the lids are so tense and swollen that their pressure is exercising an injurious influence upon the cornea, the external canthus should be freely divided, and in desperate cases the upper lid may be split vertically. When the stage of suppuration and ulceration has been reached, the application of ice should be discontinued, as the patient derives much more comfort from heat. The eye must now be fomented and kept free from pus by frequent irrigation with a hot solution of boracic acid. The sovereign remedy, however, is silver in the form of a 2 per cent. solution of the nitrate. It is of far more curative value than any of its substitutes or all the other disinfectant solutions in common use. The pain following its use may be greatly mitigated by adding I5 per cent: pure glycerine to the solution. Silver preparations must never be employed until suppuration is well established; but whenever the secretion is distinctly purulent, they should be thoroughly applied to the whole conjunctival surface, the frequency of the application being determined solely by the quantity of the discharge. If ulceration of the cornea occur, the treatment with silver nitrate must be continued and carried out with energy and faithfulness, and atropin or eserin in $\frac{1}{2}$ per cent. to I per cent. solution instilled, according to circumstances. The former soothes locally, and is the best prophylactic,against iritis: while the latter, by reducing intraocular tension, does much to avert perforation 
of the ulcer. After the acute inflammation and suppuration have subsided, the silver salt should be stopped, and astringent collyria substituted: and unless the eye be too irritable, the everted lids should be touched lightly once a day with a crayon of sulphate of copper. The patient must now be encouraged to go out of doors as much as possible, his eyes being shielded from strong light and wind by means of protective glasses, and quinine, iron, or other restorative tonics should be prescribed.

A vaccine, stable and nontoxic and specific against all forms of gonococcal infection, is known as "Dmegon," and is prepared by.M. Ch. Nicolle and $Z$. Blaizot of the Pasteur Institute, Tunis. The appropriate dose is diluted with normal saline immediately before use, and injected deeply into the muscles. Striking results have followed its use, but, in my experience, it can only be relied upon in so far that it is an admirable adjuvant to the classical treatment by nitrate of silver.

3. Membranous Conjunctivitis.-In any form of acute conjunctivitis the discharge is apt to become plastic, and speaking generally, the appearance of a false membrane always indicates that the prognosis has become more unfavourable. It must be remembered, however, that discharge may become plastic and adhere to the conjunctiva, through the over-use of local applications-more especially alum and nitrate of silver-and in these circumstances the membrane disappears at once whenever the use of the offending drugs is discontinued.

For clinical purposes, cases of membranous conjunctivitis are divided into croupous and diphtheritic, but that distinction breaks down at.once the moment the bacteriological test is applied to it. It is simpler and more accurate to speak of the case as superficial or deep. In the former case only the epithelial layers of the conjunctiva are implicated, and is in marked contrast to those cases in which the disease penetrates more or less deeply into the sub-epithelial layers, and presents all the clinical signs of true diphtheria.

Diphtheria of the conjunctiva is the most serious form of membranous conjunctivitis. It is an acute source of danger both to the patient and to those who come into contact with him. It most frequently attacks children between the second and the eighth year. It is seldom primary, usually reaching the eye by spreading along the tear passages from the naso-pharynx. The eyelids are hot, swollen, infiltrated, and brawny. The upper lid often attains a great size, feels hard as a board, and cannot be everted. In severe cases, it is impossible to open the palpebral fissure wide enough to see the cornea. A false membrane, of ashy-grey colour, covers more or less completely the congested livid-red conjunctiva, and is so incorporated with the tissues of the lids that it is separated from them with the greatest difficulty, leaving the surface raw and bleeding. 
No sooner is the membrane removed than it forms again with extraordinary rapidity. It spreads from the lids to the globe, and in malignant cases the destruction of the cornea is inevitable; while in addition, as a result of cicatricial contraction, the lids become adherent to the eyeball. Such disastrous clinical results are due to the fact that, in diphtheria of the conjunctiva, coagulation takes place within the tissues and causes necrosis, which may completely destroy the eyeball. The disease is due to the Klebs-Loeffler bacillus, and in the worst cases streptococci and staphylococci are associated with it. It must be regarded, therefore, as a general infection whose principal local manifestation is in the eye. In all cases, bacteriological examination is imperative, for without it the diagnosis is incomplete, and a reliable prognosis cannot be given. Infection by the Klebs-Loeffler bacillus means, not only that the eye which is attacked may be destroyed, but also that the life of the patient is endangered, whereas membranous inflammations due to other organismsKoch-Weeks bacillus, gonococcus, streptococcus, pneumococcus, etc.may permanently injure sight, but never cause loss of life.

Treatment.-No matter what the causal germ may be, the first thing to do in the treatment of membranous conjunctivitis is to administer antidiphtheritic serum, and to protect the sound eye with a Buller's shield if only one be affected. From two to four thousand units of antitoxin should be injected, and the dose repeated in twentyfour hours. The patient must be isolated, and every precaution taken to prevent the spread of the disease. The eyes should be kept clean by the free use of non-irritating antiseptic lotions, one of the best of which is a solution ( $I$ in 500o) of permanganate of potash, or of calcium. Tweedy recommends a lotion containing I per cent. sulphate of quinine, and excellent results in many cases follow the use of that remedy. Argyrol in 5 per cent. to Io per cent. solution may be instilled into the conjunctival sac five or six times a day, but nitrate of silver and all powerful irritants must be avoided. An ointment containing 3 per cent. iodoform should be smeared along the edge of the eyelids at bedtime. Heat is more grateful than cold, and iced compresses do more harm than good. The eyes require protection from light, but all bandages must be avoided. The lids should be carefully separated as widely as possible, and the palpebral surface gently wiped with an absorbent cotton swab dipped in normal saline solution, but no attempt must be made to remove the false membrane. 


\section{CHAPTER XII}

\section{OPHTHALMIA NEONATORUM AND ITS CONSEQUENCES}

Its frequency as a cause of blindness-Two-thirds of all cases due to gonococcusOther causal micro-organisms-Time of onset and clinical course-Accompanying constitutional disturbance-Occurrence of corneal ulceration-Its sequelæ: leucoma with pyramidal cataract, leucoma adherens, anterior staphyloma, general distension of the globe-Prognosis-Prophylaxis-Treatment-Nitrate of silver and its substitutes-Vaccine treatment.

Ophthalmia Neonatorum is by far the most common cause of loss of sight in children, and accounts for upwards of ro per cent. of all cases of blindness. Bishop Harman states that of every hundred children born in London, one suffers from the disease, and of every 2000 born, one is blinded by it, or has sight seriously impaired. Purulent ophthalmia in the infant is, therefore, one of the most important diseases of the eye which the general practitioner has to treat. It has its origin in specific contagion, and in about twothirds of all the cases the gonococcus of Neisser is found, not only in the pus cells, but also in the superficial layers of the conjunctival epithelium. A valuable clinical generalisation is that the earlier the disease declares itself, the more likely is it to be gonorrhœal and to run a dangerous course; and the opinion is generally held that the most virulent cases are always due to gonococcus, while those associated with other micro-organisms-pneumococcus, staphylococcus, streptococcus, or bacillus coli-are much less serious and run a milder course. In its commonest form the disease is met with in infants whose eyes have been inoculated from the mother at the time of birth ; and though, in these circumstances, it usually makes its appearance during the first two or three days of life, yet it may set in earlier or later, and exceptional cases are on record in which, after a tedious labour with early rupture of the membranes, infection has taken place in utero, and purulent discharge has been well established when the child was born. If the first signs of the disease appear later than the fifth day, the probability is that infection has occurred, not at the time of delivery, but subsequently, by means of fingers, cloths, or sponges, which have been contaminated by coming in contact with infected lochial discharge. Contagion may also pass from one child to another, and the disease be 
spread broadcast, by the neglect of an ignorant or careless nurse to keep her hands scrupulously clean while she is in charge of a case.

Usually both eyes suffer, but in most instances one sooner and more severely than the other. The first signs are redness of the lids and lachrymation, and almost immediately thereafter the eyelids become swollen and glued together by sticky discharge. After twenty-four, or, at most, forty-eight hours, the redness is increased and the swelling so great that the eyes cannot be opened. The upper lid is distended, hot, red, and glazed, and overhangs the lower, and the discharge from exposure to the air dries into a crust which conceals the eyelashes. When the crust is removed and the lids are separated, the purulent secretion, which has been pent up in the conjunctival sac, gushes out so profusely that it wells over on to the cheek, and may even spurt with considerable force from the eye. After the pus has been washed away there remains hidden in the upper retrotarsal fold, stringy muco-pus which is dislodged with difficulty. A few minutes after the eyes have been thoroughly cleansed, a thin yellowish fluid mixed with blood collects rapidly in the conjunctival sac, and in less than an hour the purulent discharge is as profuse as ever. It is amazing with what rapidity the discharge reaccumulates in a severe cass. In certain circumstances the secretion becomes plastic, and adheres so firmly to the palpebral conjunctiva that its removal leaves a bleeding surface.

The conjunctiva lining the eyelids is acutely inflamed, bright red in colour, and so much swollen that its normal transparency is lost. The hypertrophied papillæ are easily seen by the naked eye, and bleed very readily. The bulbar conjunctiva is also red and odematous, but in contrast to what invariably occurs in the adult it seldom overlaps the cornea.

The eyes will, unless cleansed with unfailing regularity, be kept continually bathed in pus, which will macerate and directly infect the cornè. The main object of treatment is to keep the cornea intact; and, with skilled care from the beginning, this is readily accomplished, and the inflammation runs its course in from three to six weeks. The first sign of improvement is a diminution of the purulent discharge, and as this shows itself, the swelling of the lids subsides, and the child begins to open its eyes of its own accord. The palpebral conjunctiva, which is now thrown into folds and ridges studded with papillary granulations, will not, however, be restored to its normal condition for a considerable time, and spasmodic ectropion may temporarily remain even after the discharge has ceased.

A disease attended by inflammatory symptoms so acute, and by purulent discharge so profuse, is, as may be imagined, accompanied by 
constitutional disturbances, and, though these vary according to the healthiness of the child in other respects, there are always present fretfulness, restlessness, disturbed sleep, and (if the symptoms be neglected and the illness prolonged) progressive emaciation, indicated by depressed fontanelles and drawn and shrunken features. In a few cases joint affections arise from metastasis. The larger joints-knee, wrist, ankle, or hip-are most frequently affected, and more than one may be implicated.

If the disease be allowed to run its course untreated, corneal ulceration is inevitable; and if the swollen eyelids be livid, and the purulent discharge profuse and of greenish colour, the case is of an exceptionally grave character. It is at this period very difficult to separate the eyelids so as to obtain a good view of the cornea; and obviously such cases require very careful handling, as any awkward manipulation may precipitate perforation of the cornea, with all its serious consequences. At an early stage there is infiltration of the cornea, at first white, afterwards yellow; and as the epithelial covering gets destroyed, pus begins to collect between its layers, and an ulcer forms, which rapidly extends over the surface, and if not checked, penetrates also deeply into the substance, until the whole of the epithelium and the substantia propria are destroyed, and nothing but the more resistant Descemet's membrane remains. By and by this also gives way, and the iris becomes exposed. In the worst cases, ulceration, commencing at the margin, advances steadily until it forms a complete ring; and as in these virulent cases the ulcer may be in great part concealed from view by the overlapping œdematous bulbar conjunctiva, the critical condition of the eye is not appreciated until the cornea, its nutritive supply being completely cut off, separates en bloc. Such a complication involves complete prolapse of the iris, and as the lens and vitreous humour usually escape at the same time, the eyeball ultimately shrivels.

As long as perforation has not occurred, energetic treatment may arrest the ulceration, and, the recuperative power of the cornea being at this early stage very remarkable, the eye may recover its usefulness far beyond what might have been anticipated.

As soon as the ulcer begins to heal, its base becomes smooth; the dirty yellow colour disappears, being replaced by a pearly white; the breach of surface gradually fills up and becomes coated over by a new formation of epithelium; and only a smooth white cicatrix-leucoma-remains to mark the site. In an infant it is wonderful how completely even a dense leucoma may clear up, and the transparency of the cornea be more or less restored; but when perforation has occurred, the eye is always permanently damaged, the amount of the destruction depending upon the site of the ulcer and the behaviour of the prolapsed iris. 
The following are the most common sequelæ of ophthalmia neonatorum with perforating ulcer of the cornea.

1. Leucoma with Pyramidal Gataract.-The scar of the ulcer of the cornea is represented by a dense leucoma, and behind it is a cataract shaped like a cone, with its base fixed upon the anterior capsule of the lens and its apex drawn out into a fine white thread attached to the posterior surface of the cornea at the site of the ulceration. These appearances are not difficult to interpret.

During the progress of the ophthalmia neonatorum an ulcer formed in the central area of the cornea, and increased in depth until it caused perforation. After the escape of the aqueous humour the cornea became flattened, and lay in contact with the anterior capsule of the lens, and the two got glued together by an outpouring of inflammatory exudation. The opening being thus sealed over, the reaccumulating aqueous restored the anterior chamber, and as the cornea became pushed forward into its natural position, the plastic inflammatory exudation was drawn out, and so a pyramidal mass was left stretching between the anterior capsule of the lens and the posterior surface of the cornea. Usually the cornea breaks away from the cataract, the apex of which thus becomes free.

2. Adherent Leucoma-from the iris coming in contact with the breach of surface accompanying marginal corneal ulceration. It often happens that perforation checks the virulence of the disease, and immediately thereafter recovery begins. During the process of healing, however, the iris gets down towards the ulcer, and becomes firmly fixed to the cicatrix, so that the pupil is hidden behind the leucoma. Sight may be restored by iridectomy, though, from the injurious action of the inflammation on the nutrition of the eyeball, more especially of the optic nerve and retina, improvement in vision may come far short of what was anticipated.

3. Staphyloma Anterior.-Of such cases the following history of a child nine months old affords a characteristic example. For some months before confinement the mother had suffered from a leucorrhœal discharge, and by this the child's eyes were accidentally inoculated during birth. Nothing abnormal was observed until three days after, when it was noticed that the right eye was inflamed, and the lids glued together by a sticky discharge. Within a few hours the left eye became similarly affected. A permanganate of potash lotion was prescribed, but the treatment was carried out so inefficiently that the disease practically ran its course unchecked for nearly three weeks, when, during an awkward attempt to bathe the eyes, the mother noticed a clear substance (evidently the crystalline lens) escape from the right eye on to the cheek She became so alarmed by the occurrence, that without further delay 
she brought her baby to the hospital. It was at once evident, from the profuse discharge and the brawny livid swelling of the eyelids, that the case was one of very exceptional gravity. Every attempt to separate the lids caused the child to cry vigorously, with the result that spasmodic ectropion occurred. The inner surface of the lids was then seen to be fleshy and granular, and so great was the protrusion of folds of chemosed conjunctiva, that the eyeball was almost hidden from view. By means of retractors, however, the lids were opened, and the globes fully exposed, when it was seen that the corneæ were lost, and that the irides lay exposed and prolapsed. It was many weeks before the discharge ceased and the parts healed. In the right eye, six months later, there was a dense flat white cicatrix, and in the left, from which the lens had not escaped, an anterior staphyloma so large and prominent that it interfered with the proper closing of the lids. As a result of the irritation thus produced, as well as from an increase in the intraocular tension, the child suffered much pain.

4. General Distension of the Eyeball.-This may be illustrated by an account of the case of a girl, ten years of age, who had suffered from ophthalmia neonatorum. She had now marked nystagmus; the sight of the right eye was rendered very defective by a central opacity of the cornea and an anterior polar cataract, and the vision of the left was completely destroyed through general distension of the globe. The latter eye was unnaturally prominent; the cornea had become expanded, its normal transparency was gone, and the sclerotic surrounding it had become so stretched and thin that the choroid shining through gave it a slate-blue appearance. There was no anterior chamber, and the iris, so far as could be distinguished, lay closely against the posterior surface of the cornea, to which in the pupillary area it was firmly adherent. This condition is the result of inflammation of the uveal tract after purulent conjunctivitis, and was considered by the older ophthalmic surgeons to be a dropsy of the eyeball.

In addition to the above, nystagmus, strabismus, and impaired sight due to changes in the optic nerve and retina, may be mentioned as occasional sequelæ of ophthalmia neonatorum.

Prognosis.-The prognosis in ophthalmia neonatorum is eminently favourable if the patient be skilfully treated before the onset of ulceration of the cornea. Everything depends upon the power of the cornea to resist the action of the gonococci and their toxins; but, given a strong and healthy child, and provided proper precautions be taken from the outset, corneal complications ought never to occur. Every case, however, no matter how mild the attack may seem, must be treated with all the care that would be bestowed upon one in which the signs and 
symptoms were so acute as to call forth the greatest anxiety from the beginning. Whenever the cornea becomes affected, there is great danger that sight will be lost; and the prognosis largely depends on the time in the course of the disease when ulceration appeared, and on the position and the extent of the ulceration. The danger is proportionately greater if the ulcer occurred early, and if the child be weakly or premature, or the subject of congenital syphilis. Dr. Florence Mann, in a report on ophthalmia neonatorum, stated that blindness occurred in $\mathrm{I}^{\prime} 2$ per cent. of non-syphilitic infants as contrasted with 8 per cent: of the syphilitic. Among other factors which have a farreaching influence on prognosis are the size of the palpebral fissure and the stage of the disease. A narrow fissure prevents proper separation of the lids, and hinders the thorough cleansing of the eyes, and the longer the disease has continued without proper treatment, the greater is the danger that the cornea will be destroyed. It is also vital to prognosis to know whether or not the gonococcus is present, because, as has already been said, the most virulent cases are almost invariably due to that micro-organism. During the progress of the disease, diminution in the quantity of the discharge, and lessening of the swelling of the lids are reliable signs of improvement, which can be regarded as well established whenever the child opens its eyes of its own accord. On the other hand, if the infant loses weight, and keeps the eyes constantly closed, and if there be no diminution of the profuse purulent secretion, it is certain the disease is progressing and hastening on to the destruction of the cornea.

Prophylactic Treatment.-Ophthalmia neonatorum is not only curable, it is also preventable. The older practitioners, as a result of accurate clinical observation, knew that the frequency of the occurrence of the disease was greatly reduced if all discharge adhering to the infant's eyelids and eyelashes were carefully wiped away before the eyes opened, and if at the first bath the face and body were not washed in the same water. It was Crede, however, who, shortly after Neisser discovered the gonococcus, first described the system of prophylaxis which is now generally known by his name. The following is a description of his procedure: "The eyelids are gently separated by an assistant, and by means of a glass. rod a single drop of a 2 per cent. solution of nitrate of silver is instilled into the eyes. For twenty-four hours after the application, the eyes are cooled by means of a linen fold soaked in 2 per cent. salicylic acid solution laid over them." As a result of that method of treatment, ophthalmia neonatorum all but disappeared, and there can be no doubt that whenever the method is rigorously practised the disease is prevented. To prevent infection of the eyes during parturition, therefore, is the basis of all prophylaxis, and if means to that end were adopted as a matter of 
routine, ophthalmia neonatorum might be made to disappear altogether. The whole point is that the nitrate of silver solution should be applied at the earliest possible moment.

The compulsory notification of the disease is indirectly a measure of prophylaxis, because the knowledge that the disease must be notified has a salutary influence on those in charge of the confinement, and provides a strong inducement to practise preventive measures. Moreover, it attracts public attention to the prevalence of the disease, and helps to impress both parents and nurses with its disastrous consequences. The Public Health Regulations define ophthalmia neonatorum as "a purulent discharge from the eyes of an infant commencing within twentyone days from the date of its birth." Consequently there is no excuse for any one making light of the symptoms at the outset and thereby preventing prompt treatment.

Curative Treatment.-The treatment of the disease itself consists principally in keeping the eyes clean and free from discharge. The one all-important indication is to remove every trace of pus as soon as it is formed. It is essential, therefore, to have a good nurse, who ought to have special knowledge of the feeding and general management of an infant. She must be carefully warned of the highly contagious nature of the disease, in order that by scrupulous personal cleanliness she may avoid infecting her own eyes or conveying contagion to others. She ought also to be told that, before separating the eyelids preparatory to bathing the eyes, she should put on large protective spectacles, and should lay a moist swab over the palpebral fissure to prevent spurting pus from reaching her own eye. In all her manipulations she must be instructed to be careful not to injure the cornea by the finger-nails or by putting too great pressure on the eyes; and the fact must be duly impressed upon her that the ultimate issue of the case depends in large measure on her faithfulness and skill. It is not enough, however, for the practitioner to prescribe lotions to be applied by the nurse. It is absolutely essential that he see the cornea at least once, and in bad cases several times, a day, and that he bathes the eyes himself, to make sure that every particle of discharge is washed away, more especially the stringy muco-pus which lodges in the upper and lower conjunctival folds. The particular eyewash employed is not nearly so important as the personal attention on the part of the doctor to see that all secretion is thoroughly removed. Some lotions enjoy greater popularity than others, but any bland solution will serve the purpose of keeping the eyes clean. The following are some of the agents most frequently employed : normal saline solution, saturated solution of boric acid, solution of permanganate of potassium or of calcium ( $\mathrm{I}$ in 5000), solution of corrosive sublimate ( $\mathrm{I}$ in Io,000), hydrogen 
peroxide, etc. While its eyes are being bathed, the child's head should be placed between the knees of the surgeon, and its arms held against the body by the nurse. In this position the infant is under perfect control, and its eyes are easily cleansed by pledgets of absorbent cottonwool dipped in the collyrium. All swabs used for wiping the child's eye must be burned immediately after having been used. A syringe ought never to be employed, as its use is fraught with danger, both to the child and to the nurse. It may abrade the cornea and cause an ulcer; and if there is progressive ulceration, the nozzle of the syringe may burst the eye; while there is always also serious risk of secretion from the child's eye spurting from the syringe and inoculating the eye of the nurse.

Of all drugs the one which most readily destroys the virulence of the disease is silver, which is most commonly used as a 2 per cent. solution of the nitrate. It should be employed by the doctor himself, and ought not to be instilled as drops, but carefully applied with a swab to every part of the everted upper and lower lids, making sure that the liquid reaches the retrotarsal folds. After the silver solution has remained in contact with the tissue for a few minutes, the eye should be bathed with warm saline solution to neutralise any excess of the caustic. This application must be repeated twice a day as long as the discharge is profuse; but whenever the purulent secretion begins to lessen, a longer interval may be allowed to intervene between the applications. The amount and character of the discharge are the sole guides to the frequency with which nitrate of silver should be used. It ought not to be employed before purulent secretion is thoroughly established; while its too energetic use is followed by the formation of a false membrane or the palpebral conjunctiva, owing to the coagulation of the discharge. Nitrate of silver possesses, however, the great disadvantage that its applications always cause much pain; owing to its caustic effect on the superficial layers of the conjunctiva; nevertheless its curative powers are infinitely greater than those of any of its substitutes. The use of cocain in 2 per cent. solution ten minutes before the silver nitrate is applied renders it much less painful at the time, but for the sake of the cornea the less cocain employed in the treatment of ophthalmia neonatorum the better.

Numerous organic salts of silver have been introduced into therapeutics during the last few years as substitutes for the nitrate. They are all less irritating, and even in full strength are not likely to do any harm, consequently the nurse may be trusted to use them in the absence of the doctor. The most important are argyrol, protargol, collargol, collosol argentum, etc. The first is the most popular, and is applied in 25 to 50 per cent. solution every two or three hours. It is a heavy 
fluid, and when instilled into the eye sinks deeply into the retrotarsal folds, cleansing them from secretion. This scavenger action of argyrol is very valuable, and is best utilised by bathing the eye five or ten minutes after it has been instilled, to remove all particles of discharge which have been brought to the surface. After the conjunctival sac has been cleansed, a second instillation of argyrol should be made, in order to obtain its full antiseptic and soothing influence. The basic aluminium acetate in Io per cent. ointment, known as lenicet, is a favourite application with some, but it does not seem to possess any special advantages. When the gonococcus is present and the case is one of exceptional severity, Dmegon-the fluorised curative anti-gonococcic vaccine of Nicolle and Blaizot-ought to be injected into the muscles of the buttock, or beneath the skin of the abdominal wall, every second day. The use of the vaccine has always a favourable action, and, as far as my personal experience of it goes, it never does any harm.

If ulceration of the cornea develops, consultation with a specialist is advisable. The treatment just described must be continued more energetically, if possible, than before, and in addition atropin or eserin in $\frac{1}{2}$ to I per cent. solution should be instilled according as the ulcer is situated towards the centre or the periphery of the cornea. If the ulcer is spreading or threatens to perforate, the eye may be saved by the careful and thorough application of the galvano-cautery.

After the purulent discharge becomes scanty, and the swelling of the lids and the redness of the conjunctiva lessen, the treatment by nitrate of silver may be discontinued gradually; and it is wise at this stage to examine a smear preparation occasionally to make sure that the conjunctival sac is free from gonococci, because these micro-organisms sometimes persist, although no signs or symptoms betray their presence, yet if treatment be stopped prematurely, they are capable of causing a serious relapse. When, however, convalescence has become fairly established, astringent collyria (zinc sulphate or chloride), and the light application of a crayon of sulphate of copper, speedily diminish the hypertrophy of the papillæ and restore the conjunctiva to its normal appearance.

Lastly, infants suffering from ophthalmia neonatorum must be carefully nursed and well fed. If possible, they ought to be nursed by the mother rather than fed artificially. In severe cases especially, when the cornea threatens to slough, every effort must be made to prevent the child from losing weight, and in the majority of cases it is advisable to give a few drops of brandy in milk, or wine whey, at regular intervals. 


\section{CHAPTER XIII}

\section{CHRONIC INFLAMMATION OF THE CONJUNCTIVA}

Characteristics of chronic conjunctivitis-Angular conjunctivitis: due to diplobacillus of Morax and Axenfeld, sometimes complicated by corneal ulcer: treatment-Follicular conjunctivitis: treatment-Spring catarrh: treatment-Parinaud's conjunctivitis-Trachoma: complications, pannus, trichiasis, entropion, xerophthalmia, trachomatous ptosis-Treatment of trachoma.

THE clinical distinction between the acute and the chronic forms of conjunctivitis is that in the latter the symptoms are much less intense and the progress much more protracted. The patient is annoyed by a constant feeling of irritation in the eyes, but the symptoms are never so urgent as to cause alarm. Any form of acute conjunctivitis, if left to itself, or if unskilfully treated, may become chronic. Chronic conjunctivitis is characterised by thickening of the epithelial layer, and hypertrophy of the subconjunctival tissue. When the lids are everted, the surface presents fine elevations due to swollen papillæ. The cause is often physical or chemical, and when one eye only is affected, an ingrowing eyelash, or foreign body hidden in the upper retrotarsal fold, or some inadequacy of the tear passages ought always to be suspected. Those whose occupation exposes them to vicissitudes of weather, to bright light, or to a vitiated atmosphere, are specially liable to attack; and those who strain the eyes at fine work frequently suffer until an error of refraction is corrected by suitable spectacles. Elderly persons are particularly liable to the disease, and in them there is often slight ectropion with displacement outwards of the lower punctum, and inflammation of the edge of the lid. The lids are sealed together after sleep, and a sticky, watery discharge hangs about the eyes during the day. The patients complain of a burning, irritable sensation, which is always worse in artificial light or after any attempt to use the eyes. The palpebral conjunctiva presents a velvety appearance and conceals the Meibomian glands, and the white of the eye is injected by large tortuous bloodvessels.

Treatment.-In the treatment of chronic conjunctivitis, the first care ought to be to correct any error of refraction; and if spectacles have only been worn for near vision to make sure that glasses for distance are not also - required. The nasal mucous membrane and the tear passages should be 
examined, and careful inquiry made into all the circumstances relating to the patient's occupation. Much relief is usually afforded by astringent applications like alum, acetate of lead, or tannin, but as the conjunctiva soon becomes accustomed to the use of any particular drug, it is well, in all cases where the inflammation is resisting treatment, to vary the prescription from time to time. In obstinate cases improvement is often quickly brought about by brushing the everted lids with a 2 per cent. solution of nitrate of silver. A simple ointment of ammoniated mercury applied to the lids at bedtime is useful in preventing them from sticking together after sleep. Fresh air and light are essential, and overheated, badly ventilated rooms are to be avoided. If photophobia be distressing, smoked glasses can be worn, but bandages, shades, or eye-caps ought never to be prescribed. General treatment to promote proper digestion and to help elimination is frequently required, especially in patients of gouty constitution.

Angular Conjunctivitis.-One of the chief forms of chronic catarrhal conjunctivitis is caused by infection with the Morax-Axenfeld diplobacillus, and is known as Angular Conjunctivitis. It is a contagious inflammation, frequently bilateral, and usually chronic. The symptoms are never very pronounced, and the onset of the disease is usually very insidious. There is little or no discomfort during the day, but if the patient tries to read or write in gas-light the eyes begin to burn and to water, and the feeling of irritation becomes so intense that he is forced to desist. Secretion is always scanty, but a little concretion is frequently found at the inner canthus, and the lids are generally sealed together after sleep. The objective signs of inflammation are slight, and are confined to the inner and outer canthi. The caruncle and semilunar fold are red and swollen. In cases that have continued untreated for a long time, superficial ulcers form at the margin of the cornea, but these, as a rule, run a benign course. If, however, the cornea be injured in a patient who suffers from diplobacillary conjunctivitis, the ulcer may assume a grave character and be complicated by hypopyon.

Treatment.-This form of conjunctivitis runs a very chronic course, is very liable to relapse, and is never cured spontaneously. It is, however, rapidly eradicated by the use of sulphate of zinc drops, two grains to the ounce, instilled three or four times a day, and the application to the lids at bedtime of an ointment containing oxide of zinc and ichthyol. The eyes should be bathed with a warm solution of borax or other bland eyewash, and if photophobia is distressing in artificial light, tinted glasses ought to be worn. Treatment should be continued for at least a fortnight after the patient seems to be cured, because of the danger of relapse; and while the attack lasts, he should be warned not to expose 
the eyes to smoke, dust, or impure air, and to avoid straining the eyes by artificial light.

Follicular Gonjunctivitis.-The characteristic objective feature of all forms of chronic conjunctivitis is hypertrophy of the layer of adenoid tissue, which is always present more or less abundantly in the normal conjunctiva. When the inflammation confines itself chiefly to the retrotarsal folds, this adenoid tissue becomes collected into heaps called follicles. These, which are round, pinkish, translucent bodies, about the size of a pin-head, projecting from the surface of the conjunctiva, and arranged in rows parallel to the border of the lid, occur mostly in the lower retrotarsal fold where they are rarely visible till the lower lid is everted. If, as sometimes happens, the inflammation extends to the palpebral conjunctiva, it will be accompanied by catarrhal discharge. In the most typical form of follicular conjunctivitis, however, there is but little redness of the eye, and the chief symptoms are sensations of itching and heat, and difficulty in seeing to read or sew by artificial light. The disease occurs for the most part in children at school, and usually both eyes are affected. It arises most frequently, and may even become epidemic, through overcrowding and bad ventilation; but an acute attack may be excited by the long-continued use of either atropin or eserin. It always runs a prolonged course, is very liable to relapse, and undoubtedly predisposes to attacks of other forms of conjunctivitis. Follicular conjunctivitis never implicates the cornea, and 'after lasting for an indefinite time, passes off, leaving no trace.

Treatment.-The local treatment consists in bathing the eyes several times a day with a lotion containing borax and salicylic acid, and in touching the everted retrotarsal fold once daily with a crystal of sulphate of alum. Attention should also be directed to the refraction, and suitable spectacles prescribed if necessary. When the inflammation is due to the use of atropin or eserin, the drug must be discontinued, and the everted lid brushed with a 2 per cent. solution of silver nitrate. Children who suffer from follicular conjunctivitis also suffer frequently from adenoids, and their general health and environment require attention.

Spring Gatarrh.-Another form of follicular inflammation is Spring Catarrh, so called owing to the peculiar recurrence of the symptoms in the spring-time. It is a rare disease in this country. It only occurs during hot weather, is not contagious, and never epidemic. It assumes two distinct forms, the bulbar and the tarsal, and is rarely mixed.

In the former variety there is usually a reddish or yellowish tumour at the limbus on either the nasal or temporal side of the cornea. This growth is hard, movable, painless, and of uneven surface; and it may become confluent and surround the cornea with a cartilaginous collar. 
The tarsal variety affects only the upper lid, which droops, and the conjunctival surface of which is closely studded with irregular prominences resembling cobble-stones, bluish white in colour as if the epithelium had been brushed over with milk. There is little discharge, and as a rule the cornea remains transparent. The disease affects both eyes, runs a chronic course, and, though it subsides or even wholly disappears in autumn, almost invariably recurs on the return of hot weather.

Treatment.-The disease may last for many years and then spontaneously recover, but treatment is of little or no avail. Cocain and adrenalin drops, or very weak acetic acid and I per cent. ichthyol ointment, relieve the burning and itching, which are often troublesome; and catarrhal symptoms are diminished by douching the conjunctival sac with a solution of borax and boracic acid; but all strong caustics and irritants must be avoided. During the height of an attack well-fitting goggles with Crookes' or chlorophyll glass should be worn.

Parinaud's Conjunctivitis is characterised by the occurrence of granulations on the tarsal conjunctiva and the retrotarsal folds, associated with swelling and suppuration of the preauricular and the submaxillary glands. As a rule one eye only is affected. The disease is accompanied by considerable constitutional disturbance, and may last for months, but usually ends in recovery. -

Treatment is not of much use, and irritant remedies, especially strong caustics, always do harm.

Trachoma or Granular Gonjunctivitis.--In other forms of conjunctivitis, even although sight may be lost, the conjunctiva itself recovers; but in trachoma the inflammatory infiltration is not absorbed, but becomes transformed into connective tissue, which sooner or later undergoes cicatricial contraction. The disease is most insidious in its onset, runs a prolonged course, lasting in many instances for years, often leads to serious and permanent damage to sight, and frequently ends in blindness. It is undoubtedly contagious, but its transmission may be prevented by adequate cleanliness. Poverty, over-crowding, bad sanitation, and dirty living are the most potent factors in its spread. Early diagnosis is of great importance. In its initial stages granular ophthalmia may be confused with follicular conjunctivitis; but in the latter the disease is for the most part confined to the lower retrotarsal fold, and the cornea is never affected. Difficulties in diagnosis occur most frequently among school children, and.in such cases a clue may be found in the knowledge of the child's home surroundings. Trachoma in a mild form is, however, so frequent that in every case of chronic conjunctivitis the upper lid should be everted, and the conjunctival surface and the fornix examined carefully for granulations. The disease 
may present different clinical types according to the stage it has reached at the time of observation.

I. Simple hypertrophy of the papillæ of the conjunctiva and the retrotarsal fold.

2. Inflammatory thickening of the tarsal cartilage.

3. Implication of the cornea-Pannus.

4: Cicatricial contraction of the palpebral conjunctiva.

5. Inversion of eyelashes-Trichiasis.

6. Entropion and shrinking of the whole conjunctival sac, with cicatricial adhesion of the globe to the lids.

7. Xerosis of the conjunctiva.

Although known from time immemorial, trachoma began to attract the serious attention of surgeons only during the Napoleonic wars, when it appeared in epidemic form among the troops of the contending nations. It disabled thousands of soldiers, who, on being disbanded, returned to their homes, and spread the disease among the civil population. In those days the form it assumed was very acute, and as the contagion was conveyed by means of the secretion, its spread was most rapid where, as in public institutions, large numbers of people occupied the same sleeping apartments, and used wash-hand basins, towels, etc., in common.

Nowadays the disease is rarely seen in this virulent form, though as a chronic affection it is of frequent occurrence. Its natural home is in the countries of the East, and thence it has distributed itself over the whole civilised world. Damp and impure air exercise a great influence over its production; and while it is very prevalent in low-lying districts, it seldom or never occurs in regions like Switzerland. It is endemic among certain races, for example, the Irish and the Jews, who, owing to poverty, live huddled together in small badly-ventilated houses, and pay but little attention to personal cleanliness:

The cases may be divided into those accompanied by discharge, and those in which secretion is very scanty. The former group is most dangerous to others, but the specific infecting micro-organism has, up till now, not been discovered. The trachoma may be secondary to another infection; and some affirm that all cases arise from inoculation with modified gonorrhœal pus, and liken the cicatricial changes in the lids to those which lead to stricture of the urethra. That theory, however, will not stand the test of criticism, and for the present it must be admitted that bacteriology has failed to give a satisfactory explanation of the pathology of the disease.

It may be difficult to determine the exact date of onset of trachoma, for the patient usually gives a somewhat indefinite history of " cold in the eye " over a period of years. The following is a brief description of 
the clinical evolution of the disease. Both eyes are usually affected, but occasionally one of them escapes. The lids are slightly swollen, and present a characteristic droop-trachomatous ptosis - which gives the patient a sleepy appearance. When they are everted, the palpebral conjunctiva is seen to be red and rough, from the injection of its bloodvessels and the hypertrophy of its papillæ. The bulbar portion appears normal, except for congestion of its larger blood-vessels, but as a. rule the cornea is hazy, and its upper part is covered by a new formation of minute blood-vessels, which give it the appearance of red velvet-pannus. The palpebral conjunctiva is thick and fleshy, and studded with greyishwhite granulations, which vary in size and are largest and most closely packed together in the outer third of the upper retrotarsal folds. These " sago-grain " bodies are partially concealed by hypertrophied and highly vascular papillæ. When the disease has existed for a long time smooth, whitish-yellow lines and spots, situated near the edge of the lid, mark the beginning of cicatricial changes which will, in the absence of appropriate treatment, terminate in shrinking of the conjunctiva, and destroy its function as a mucous membrane. Those morbid changes, if left to themselves, progress steadily until the whole palpebral conjunctiva is completely converted into cicatricial tissue. As a result of those changes the tarsal cartilage becomes thickened and incurved, and the lids are inverted. This alteration in the shape of the tarsal cartilage is accompanied by change in position and direction of the cilia, so that every movement of the lids causes the misplaced lashes to rub against the eyeball. The ocular conjunctiva becomes inflamed, and a puriform secretion hangs about the margin of the lids. In consequence of prolonged irritation the cornea becomes vascular, as a result of a new formation of blood-vessels from the limbus, and its surface ulcerated, rough, and opaque, so that the iris cannot be distinctly seen; vision thereby being very seriously impaired. Later on the conjunctiva loses its natural moistness and becomes cuticular.

Xerosis.-The absence of its normal secretion produces a sense of dryness and grittiness, almost amounting to torture. The eyes are now opened with difficulty as the retrotarsal fold gets more and more obliterated, and the lid becomes bound to the globe. There is pronounced trichiasis, and the opaque vascular cornea loses its natural curvature and becomes more or less flattened: at this stage vision is lost.

Treatment.--It is very rare nowadays for trachoma to run an acute course with profuse discharge, and to appear in the epidemic form described by the writers of the beginning of the last century. On the contrary, the onset of the disease is in many cases so insidious that it passes unnoticed, and the patient seeks advice to obtain relief, not from inflam- 
mation and swelling of the lids, but from opacity of the cornea. During the stage of inflammation with suppuration, the treatment of trachoma is similar to that described for purulent ophthalmia; but in the chronic stage the whole aim is to remove the infiltration of the conjunctiva as early as possible. Nitrate of silver is the best remedy as long as there is much secretion; but as soon as the discharge lessens, the silver solution should be replaced by sulphate of copper. Bluestone is a more powerful irritant than a 2 per cent. caustic solution, and it should not be employed when the cornea is infiltrated or ulcerated. It is of most use, therefore, in indolent cases with much hypertrophy of the conjunctiva. When the granulations are numerous and succulent, the everted lids should be massaged with boracic acid or iodine ointment, I per cent., or protargol in 5 per cent. solution. The treatment must be faithfully carried out for months, or even years, until the disease is cured, for relapses are very prone to occur if the patient neglects to use the remedies as soon as he is free from feelings of discomfort. Exuberant granulations may be destroyed by the cautery; but it is much better practice to express the trachoma granules from the retrotarsal folds by means of Knapp's roller forceps; or the retrotarsal folds may be excised and the diseased tarsal plate removed. Such operative measures help very materially to shorten the course of the disease. In all cases the treatment is necessarily very prolonged, and as it consists so much in the use of caustics, it is necessary for the surgeon to apply them himself. Carbon dioxide snow, $\mathrm{X}$-rays, and radium have all been tried; but the results are no better than those obtained by the judicious use of sulphate of copper. The patient ought always to be supplied with mild antiseptic collyria, so that the eyes may be kept thoroughly clean; and as the infiltration disappears from the conjunctiva, the more powerful caustics should be applied less frequently, and in the intervals the use of ointments containing oxide of mercury, protargol, or glyceroles of copper or of tannin, should be prescribed. As the conjunctiva improves, the pannus as a rule disappears; but if it be rich in blood-vessels, these must be destroyed by the cautery or by a peritomy operation. In dense pannus there is always a risk of iritis, so atropin must be instilled from time to time. When the cornea is vascular throughout its whole extent, and covered by a considerable development of new-formed tissue, resorption is sometimes obtained by exciting a violent inflammation, either by painting the everted lids. with a 3 to 5 per cent. infusion of jequirity seeds, or by inoculating the conjunctiva with pus from a case of ophthalmia neonatorum. Those methods are seldom employed now, but when a very strong application is required, probably the best is a 4 per cent. solution of perchloride of mercury in glycerine. When trachoma has reached the 
stage of xerosis, it is quite incurable: all that can be obtained by treatment is the amelioration of the patient's sufferings, by removing ingrowing eyelashes, and keeping the eye moist by the frequent use of lubricating ointments.

Every patient suffering from trachoma ought to be supplied with his own washing utensils, towels, etc.; and in schools and reformatories, those suffering from the disease must be rigidly kept apart from their fellows.

In I874 Mr. Nettleship wrote: "There can be no doubt that the best of all ways for getting quit of the present dead weight of actual ophthalmia and of cases of bad granular lids, would be to separate them from all the other children, and to keep them separate for an indefinite time until it is quite certain that all tendency to relapse has ceased."

The Metropolitan Asylums Board has acted on that suggestion, and now provides accommodation in the country for children in the area under its control who suffer from trachoma. The children are boarded and educated under medical observation and treatment, and it has been found that their average residence in those special schools is about two years. Trachoma is now notifiable, so if similar schools were to be provided in the vicinity of all large and densely populated cities, the disease would be prevented from spreading, and in course of time might altogether disappear. 


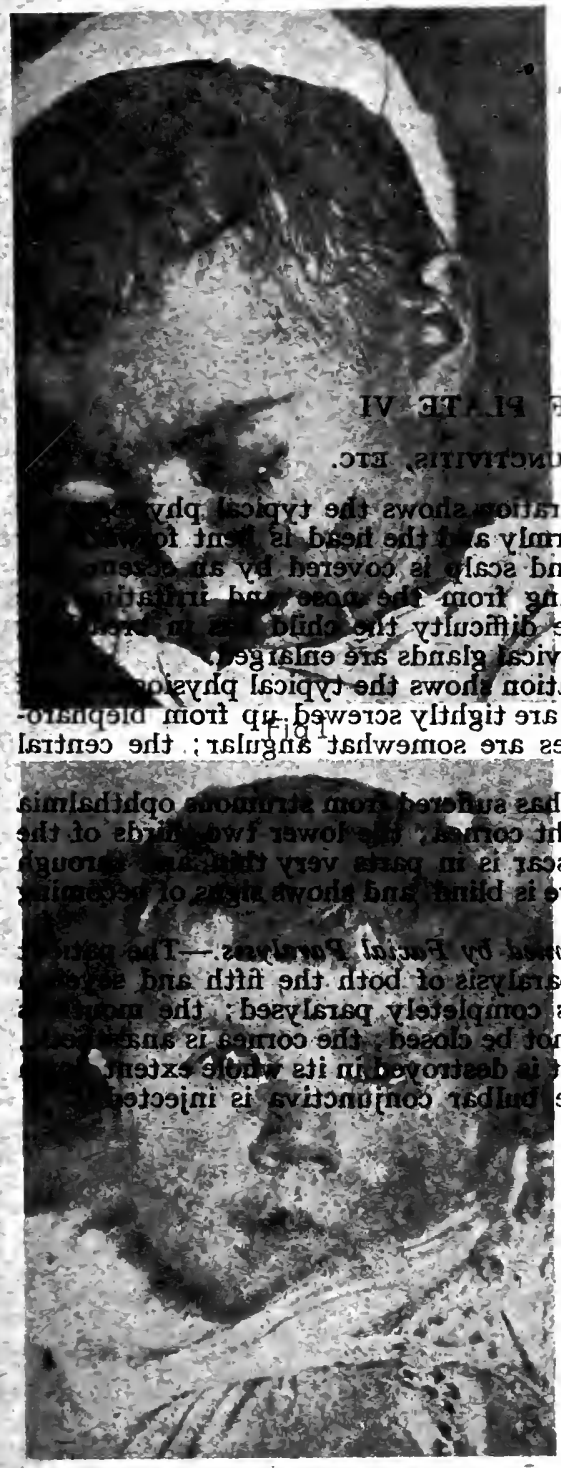

Figlli

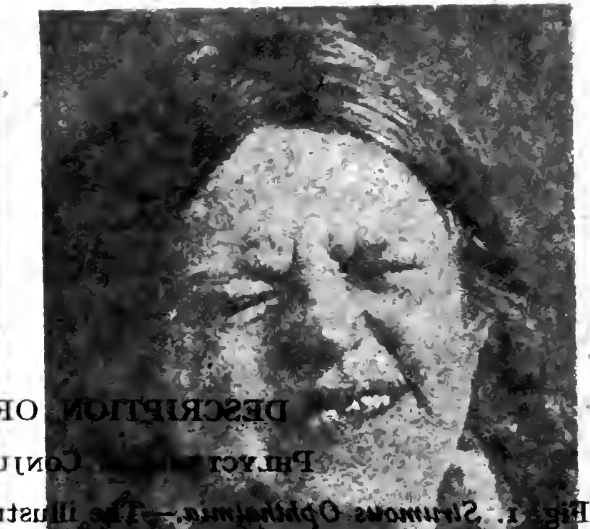

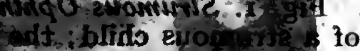
pit iffigil tols

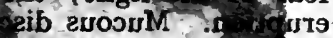

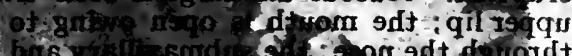
bits

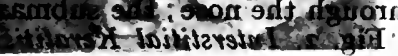

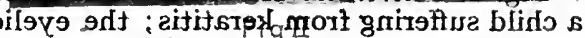
itsot sdt itdgil to son stslotai bas mesq 2ib. 97s choatoni bsinga-

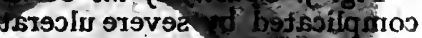

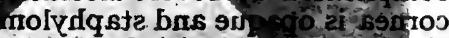
qsiord esd airi orlt e withog 920 rlt

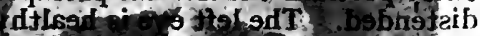

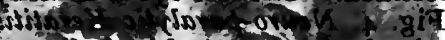

oditios

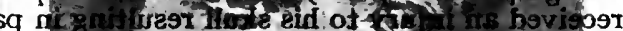

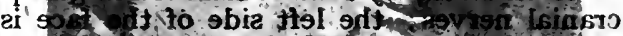

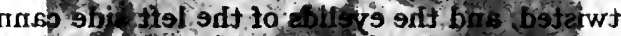

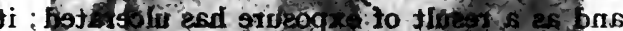
orlt

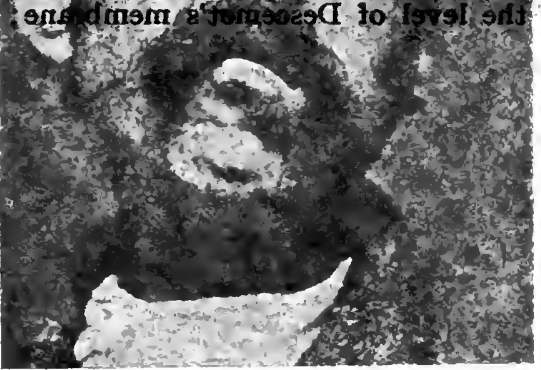

of

Fig $\mathbb{K}$ 

etting quit of the present dead weight of actual ophthalmia of bat granular lids, would be to separate them from all the $n$, and to keep them separate for an indefinite time until it min that all tendency to relapse has ceased."

IT mpolita DESCRIPTION OF PIATEI VI that suggestion, and

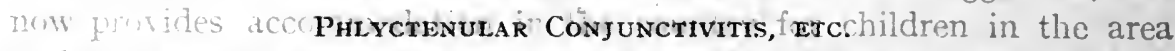

Fig. I. Strumous ophthalmia.-The illustration shows the typical physibghomy of a strumous child; the eyes are closed firmly and the head is bent forward for fear of the light; the skin of the face and scalp is covered by an eczematous eription. Mucous discharge is seen trickling from the nose and irritating the upper/lip; the month is open owing to the difficulty the child has in breathing through the nose; the submaxillary and cervical glands are enlat ged.

Fig. 2. Interstitial' Keratitis. - The illustration shows the typical physiognomy of a child suffering from keratitis; the eyelids are tightly screwed up from blephares spasm and intolerance of light; the features are somewhat angular; the central incisors are distinctly notched.

Fig. 3. Opacity of the Cornea.-The child has suffered from strumous ophthalmia complicated by severe ulceration of the right cornea; the lower two-thirds of the cornea is opaque and staphylomatous; the scar is in parts very thin, and through these portions the iris has prolapsed; the eye is blind, and shows signs of becoming distended. The left eye is healthy

Fig. 4. Neuro-paralytic Keratitis accompanied by Facial Paralysis.-The patient received an injury to his skull resulting in paralysis of both the fifth and seventh cranial nerves; the left side of the face is completely paralysed; the mouth is twisted, and the eyelids of the left side cannot be closed; the cornea is anresthetic, and as a result of exposure has ulcerated; it is destroyed in its whole extent down to the level of Descemet's membrane; the bulbar conjunctiva is injected. 


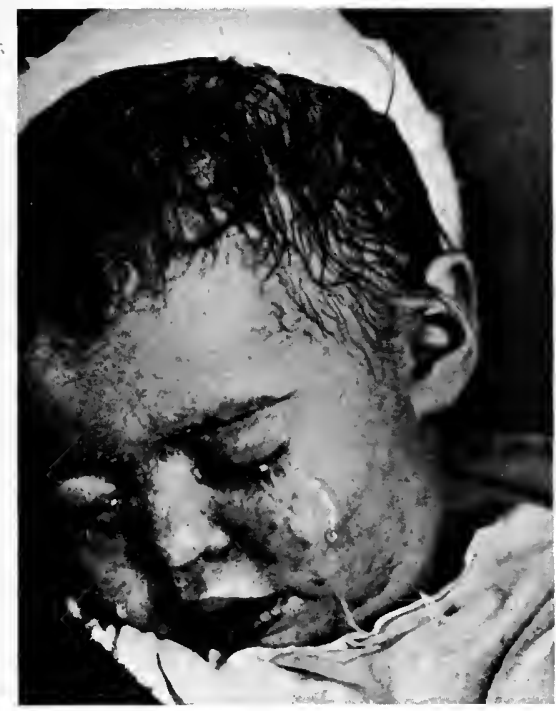

Fig I

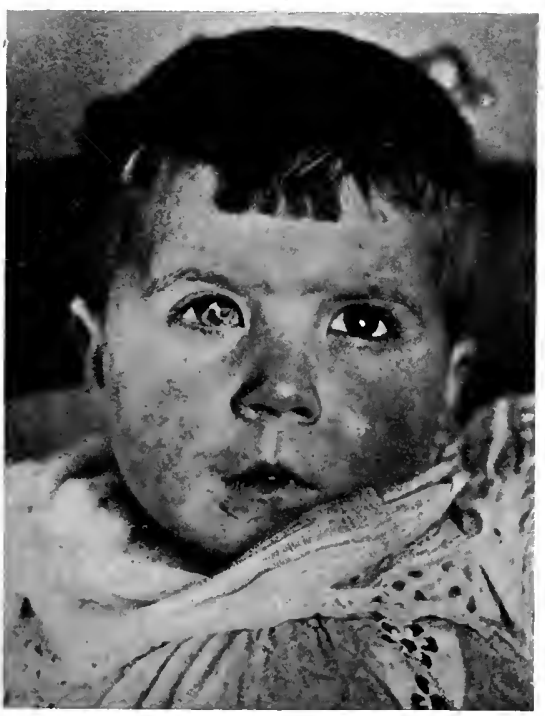

FigIII

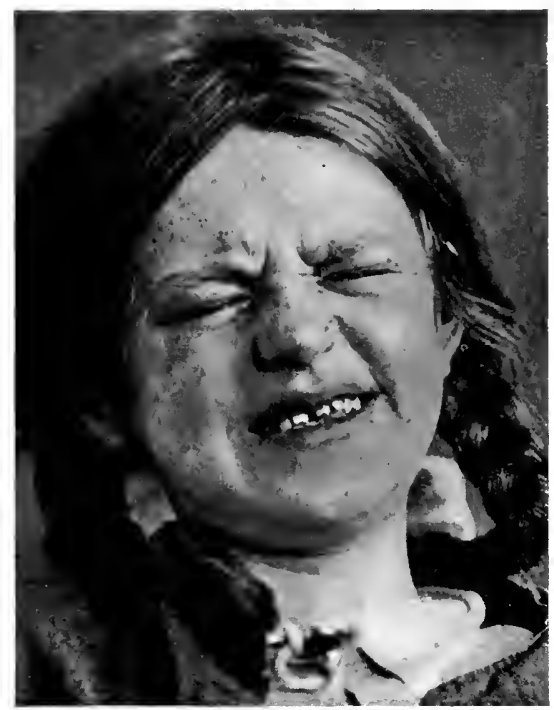

Fig I

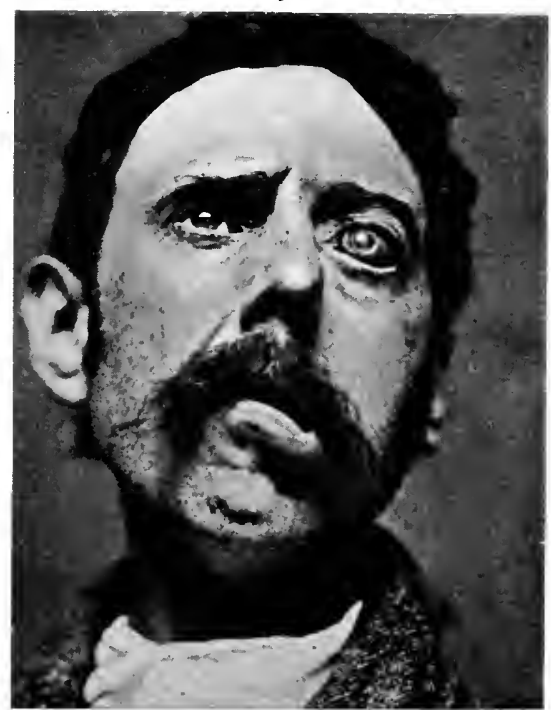

Fig I 



\section{CHAPTER XIV}

\section{PHLYCTENULAR CONJUNCTIVITIS OR STRUMOUS OPHTHALMIA}

Essentially a disease of children-Intensity of photophobia in this disease-The phlycten-Occurrence of ulceration-Absence of discharge-Connection with tuberculosis-Occurrence of eczema and gastro-intestinal disturbance-Method of examining the child-Treatment: constitutional and dietetic-Tartar emetic-Local treatment-Treatment of complications.

StRumous Ophthalmia is used as a convenient name under which to group a large number of cases of inflammation of the conjunctiva characterised by the presence of one or more white-topped papules. The disease is known also as phlyctenular or pustular conjunctivitis, eczema of the conjunctiva, conjunctivitis lymphatica, or, from the frequency with which the cornea is involved, phlyctenular keratitis. The bulbar conjunctiva is primarily affected, and as the corneal epithelium is simply the extension of the conjunctiva over Bowman's membrane, there is little wonder that the cornea is implicated so frequently in the inflammation, which differs from other forms of conjunctivitis in so far that it is discrete in character, and never implicates more than a portion of the surface of the eyeball.

It is essentially a children's disease, for in adults it is almost never seen except in those who have suffered from it in their early years. It sometimes appears during the period of dentition, and is most frequent during the first decade of life. It attacks girls oftener than boys, and in them occasionally comes on for the first time just before menstruation is established. Both eyes are usually affected, one in that case much more severely than the other; and, as relapse is very apt to occur, a case may last for many months. Though strumous ophthalmia occurs in children of all classes, it is naturally much more common among the poor, and especially among those who from their earliest infancy have been improperly fed and scantily clothed, at one time unduly exposed to the vicissitudes of the weather, and at another confined in small and badlyventilated rooms. It frequently follows one or other of the exanthemata, more particularly measles.

Photophobia is one of the most characteristic and most distressing symptoms. The child generally sits on its mother's lap with its face 
pressed closely against her body, and every attempt at examination is met by struggles and screams, entreaties to have the light kept off, and efforts to shield the eyes with hands and arms. After the hands have been drawn away, the eyelids are kept tightly pressed together, and their separation is most strenuously resisted. When at length they are partly opened, there is a gush of tears, and often a violent fit of sneezing expels muco-purulent discharge from the nostrils.

If the disease has existed for a lengthened period, the eyelids, nose, and upper lip are markedly swollen, and in all probability the face is covered by an eczematous eruption. The excoriation of the skin leads to the formation at the outer canthus of a fissure, which, on any rough separation of the lids, bleeds and causes a great deal of pain. When, after much trouble, the balls are at last exposed, one is often surprised to find how slight the local lesions really are. Even when photophobia is very distressing, and when the upper eyelids are swollen and their surface marbled by large congested veins, there may be hardly a trace of redness over the sclerotic. In most cases, however, there are localised congestions, and the ocular conjunctiva is traversed by a leash of bloodvessels running towards a minute phlycten, situated on the surface of the cornea, often right over the centre of the pupil, which, as a result of the irritation of the corneal nerves, is always greatly contracted.

The phlycten is the most characteristic objective feature of this disease, and develops either on the scleral or the corneal conjunctiva. It may be of large size and solitary, or there may be a group of small pustules, but in either case the situation is on the surface of the globe, for the most part on the limbus corneæ. These pustular elevations vary greatly in size, are of a yellowish-grey colour, and in most instances disappear after a few days, leaving no trace. At other times, however, their appearance marks the beginning of ulceration of the cornea. Up to this stage there has been no real pain, except when the eyes have been exposed to light. There is no complaint if the patient be only left alone in a dark corner of a room, or with the face buried in a pillow; but whenever ulceration begins there is more or less active suffering, and pain is very apt to come on violently during the night, and cause the child to start up screaming from its sleep. Such ulcers are always slow in healing, and at times, even after the surrounding infiltration has all cleared up, there remains a transparent dimple which may not disappear for weeks or even months. Sometimes a leash of newly-developed blood-vessels spreads from the corneo-scleral margin to the ulcer, which then, becoming vascular, appears as a red speck, which may persist for a long time. Occasionally the greater part of the cornea becomes vascular-pannus scrofulosus. In unhealthy children these phlyctenular ulcers are prone to suppurate, and 
then pus collects between the layers of the cornea, and accumulates in the anterior chamber. In these severe cases perforation is apt to occur, and to be followed by a prolapse of iris so large that the eyeball is irretrievably damaged.

Although there is, in a typical case of strumous ophthalmia, always profuse lachrymation, there is no sticky muco-purulent discharge, unless the inflammation has spread from the bulbar to the palpebral conjunctiva. Whenever that occurs catarrhal symptoms are present, and the whole conjunctiva becomes injected. In most cases there is also injection of the lymphatics.

Ophthalmia may be the earliest indication of a strumous constitution, and in cases where the conjunctival lesions are slight, severe subjective phenomena may arise from a source of irritation elsewhere than in the eye, e.g. in the stomach or bowels. Although some of the patients may present the outward appearance of good health, most of them are, owing to defective assimilation and excretion, either abnormally pale or unnaturally livid. They have but too obviously inherited the miserable legacy of constitutional weakness; and the inherent vulnerability of their tissues has been intensified by improper dieting and by overcrowding in insanitary houses. They are called scrofulous, but as yet there are no symptoms to show that the tubercle bacillus has effected a lodgment; and careful examination fails, as a rule, to discover it lurking in the conjunctival sac, although it is in children such as these that one would expect to find this micro-organism developing and increasing most rapidly. The Germans speak of them as "candidates for tuberculosis," but bacteriological examination of a pustule excised with antiseptic precautions from the conjunctiva fails to find the tubercle bacillus or any other micro-organism, and if the excised pustule be implanted in the peritoneal cavity of a guinea-pig the result is negative. Nevertheless, those children present a general condition, which if it be not actually tuberculous, is closely associated with a lowered resistance against the tubercle bacillus. The discrete character of the inflammation, and its nodular manifestation, at once indicate the constitutional origin of the disease. The old clinicians, moreover, always regarded intense photophobia in the absence of a lesion of the cornea as a sign of scrofula, and superficial vascularity coming from the bulbar conjunctiva as the distinctive sign of a strumous affection of the cornea. Experiments with tuberculin corroborate those clinical observations, because in patients suffering from phlyctenular conjunctivitis the von Pirquet cutaneous reaction is always positive, and the Calmette ocular test is exceedingly dangerous. On the other hand, a very minute dose of tuberculin injected subcutaneously frequently exercises a favourable influence in a case 
which, up to that point, has obstinately resisted treatment. Furthermore the experiments of Paton and Nias have demonstrated that the negative phase of the opsonic index is marked by the occurrence of pustules and phlyctens on the surface of the eyeball. It is not surprising therefore that if left alone, or simply treated by local remedies, the ophthalmia often proves to be the very first warning of the imminent danger of tubercular infection. This is especially likely to happen in those patients in whom photophobia is most distressingly persistent, although lesions of the conjunctiva or of the cornea are at first absent, or are of a very trivial character.

It is by no means unusual for the conjunctivitis to alternate with suppuration of the middle ear, or with eczematous eruptions on the head or other parts of the body. Nutrition is seriously interfered with, and the coated tongue studded with large red papillæ shows the irritable condition of the mucous membrane of the whole intestinal tract. There is sometimes a constant craving for food, and almost invariably more is eaten than can be digested; fermentation results, the belly is distended, and the alvine evacuations have an offensive smell and an unnatural appearance. The urine is high-coloured, deposits urates, and often contains indican and traces of sugar. The skin is pale and rough, the flesh hangs loose, the hands and feet are often persistently cold, and the head perspires freely. After a time the system becomes infected by tubercle bacilli, and then the joints, both large and small, are very frequently destroyed by tubercular inflammation of their synovial membrane and caries and necrosis of the bones. Severe ectropion-causing hideous disfigurement-will arise if the bone necrosis occur in the neighbourhood of the orbit; and indelible scars invariably result if there be suppuration of the lymphatic glands in the neck or elsewhere.

Owing to the intense blepharospasm, it is always a difficult matter to examine the eyes of children suffering from phlyctenular conjunctivitis. Every care ought to be taken not to hurt the child unnecessarily, and its eyes may be best examined by placing the head between the surgeon's knees while the nurse holds the arms against the body. After cocain has been instilled, it is easy to depress the lower and raise the upper lid gently by means of retractors, but, as the child persists in rolling the eyes upwards, it is often difficult to obtain a good view of the cornea, unless by the exercise of great patience. The condition of the cornea must, however, be accurately determined before any treatment is begun, and in very bad cases it may be necessary, before making the first examination, to administer a general anæsthetic.

Treatment.-The treatment of phlyctenular conjunctivitis must be constitutional as well as local. It is necessary always to bear in mind that the 
very essence of the disease lies in the mal-assimilation of food, and that most, if not all, cases arise as a result of improper dieting. You may be told that the child eats very sparingly, and that may be quite true as far as the regular meals are concerned; but by careful inquiry you will usually discover that the small appetite at the proper times for eating generally arises from the patient's having been, in response to cries for "a piece," supplied liberally between meals with bread and butter, sweets, fruits, or any other eatable which may have chanced to be at hand. Instead, therefore, of eating too little, these children eat too much; and although they may appear ill-nourished, the mal-nutrition is due to mal-assimilation rather than to insufficiency of food, with resulting auto-intoxication from the intestine. The first thing to be done, therefore, in treating a case of strumous ophthalmia, is to give particular directions regarding the diet. The child must not be allowed to eat whenever it cries for food, but ought to be fed at regular intervals; nor must it get what is among the poor popularly called "the run of the house," but should have meals. specially prepared, and in quantity and quality of such a kind as not to overtax the powers of digestion. Sugar in every shape and form, fruits, pastries, potatoes, etc., must. be rigidly excluded from the dietary; and it is usually advisable to confine the patient wholly to milk and water for the first forty-eight hours of the treatment, and then to add gradually meat-soups, eggs, fish, fowl, rice, etc., as the tongue becomes clean. Simultaneously with the cleaning of the tongue the intolerance of light begins to pass off, and the child opens its eyes, and interests itself in its surroundings. The fretfulness and unnatural irritability both become less, and the necessary treatment is, in consequence, much more willingly submitted to.

In addition to the careful supervision of diet, the free elimination of the toxins, which, as a result of mal-assimilation, have accumulated in the blood, must in every way be promoted. This is to be accomplished by stimulating the action of the kidneys, the bowels, and the skin-more especially the last. Hence the importance of the hot bath, which the child should have every night at bedtime, diaphoresis being further encouraged by the wearing of a warm flannel nightgown. It is usually advisable first of all to administer a dose of castor-oil; but it should be. borne in mind that many of these children do not bear purgatives well, as the intestinal mucous membrane is so irritable that troublesome. diarrhœa is easily induced.

Of drugs, none is so useful as tartar emetic, which should be given in slightly nauseating doses. Its chief virtue depends on its diaphoretic. action, and its efficacy is greatly increased when it is administered with a laxative, $e$. g. powdered rhubarb. If the tongue be brown, a few grains 
of grey powder may be added, with advantage, to the rhubarb and antimony combination. Under this treatment it is wonderful how soon the whole appearance of the patient changes. As the power of digestion becomes greater, the skin improves in colour, the hands and feet keep. warmer; and the child, instead of lying with its face buried in a pillow, and fretting and crying when spoken to, is now quite good-tempered, and runs about the ward and amuses itself with its playthings. Any indiscretion in diet will, however, promptly bring on a relapse, for in these cases the conjunctiva reflects the condition of the gastro-intestinal mucous membrane even more quickly than the tongue. Intolerance of light, blepharospasm, and increased lachrymation speedily show themselves after some forbidden food has been eaten, while the tongue may not become furred till the second or even the third day. The fear of relapse makes it imperative, therefore, that the treatment be continued for at least a fortnight after the recovery seems complete. Apart from simple digestive agents, such as pepsin, pancreatin, Bynin, etc., no medicines are, as a rule, necessary, but most of the patients are benefited by quinine, which, either alone, or in combination with an acid, seems to exercise a favourable influence after the acute symptoms have subsided. Cod-liver oil, compound syrup of the phosphates, syrup of the iodide of iron, etc., are also very valuable remedies, but they should never be prescribed in the early stages, for they then add to the difficulties by still further overtaxing the digestive powers. When, however, all feverishness has disappeared, and the tongue is clean, they are decidedly helpful in improving the general nutrition, and promoting the repair of any local lesions in the eyes themselves. All syrups, however, such as chemical food, etc., must be used with great caution, lest they disturb digestion, and so determine a relapse. Dusart's syrup of the lacto-phosphate of lime and iron is the most easily assimilated; but, as a general rule, preparations in which sugar is replaced by glycerine or malt extract are safer. When there is much enlargement of the glands, a mixture of chloride of calcium and iron is very serviceable.

For the first few days of the treatment the patient should be kept in bed, with the eyes shaded from the light; but the use of all poultices and bandages must be strictly prohibited. Whenever improvement begins, the child ought to be encouraged to run about, and, if possible, sent to the country, preferably to a high, dry, bracing locality, and should live out of doors as much as the weather will permit. The sea-coast should be avoided, as the glare from the water is apt to prove irritating to the eyes, and to cause a relapse.

The local treatment will necessarily vary according to the stage of the disease, but the indications are to subdue irritation, to prevent 
secondary infection, and to promote cicatrisation. The child's face and hands must be washed frequently, and, as far as possible, it ought to be prevented from crying and rubbing its eyes. The eyes themselves should be bathed night and morning-oftener if there be much discharge-with a warm solution of boracic acid, or a lotion containing perchloride of mercury and belladonna, and argyrol in ro per cent. solution instilled three or four times daily. While the symptoms of acute irritation last, atropin and cocain, by soothing the nerves of the conjunctiva, and diminishing the congestion of its blood-vessels, lessen the photophobia. Whenever the pupil dilates, the child usually opens its eyes; but when the superficial irritation is great, the action of the atropin persists only for a few hours, and, in order to obtain full benefit, the drug ought to be re-instilled whenever the pupil begins to contract. As the blepharospasm lessens, the cocain may be omitted from the ointment, and the atropin combined with boracic acid or the red oxide of mercury. Eserin has been recommended instead of atropin, and in obstinate cases it is worthy of trial, but taken over all its usefulness is not nearly so great as atropin employed in the manner just described. Even in cases which have lasted for a long time, leeches or blisters should not be used.

After the acute inflammatory symptoms have somewhat subsided, more stimulating applications are necessary, and calomel and the yellow oxide of mercury are the two favourite remedies at this stage. The former is most serviceable in relieving passive congestions. It must be dried thoroughly, and flicked from a camel-hair brush into the conjunctival sac. Its beneficial action seems due to the fact that the calomel becomes, by the action of the tears, converted into perchloride of mercury. It is therefore necessary to be careful not to use it too freely, or in too coarse powder, for any excess collects in the lower retrotarsal fold, upon which it is apt to have a caustic action; nor must calomel be dusted into the eyes of children who are taking any preparation of iodine internally, lest the biniodide form and irritate the conjunctiva. The yellow oxide of mercury often acts like a charm in cases where there is an efflorescence of pustules upon the bulbar conjunctiva. It is best applied in the form of an ointment of the strength of I or 2 gr. of the oxide to a drachm of white vaseline. The bulk of a split-pea of this is inserted into the conjunctival sac, and the eye gently massaged for a few minutes through the closed eyelids. In order to avoid the occurrence of crystalline particles in the ointment the yellow oxide must be freshly precipitated.

In an ordinary case of strumous ophthalmia no further treatment than that just indicated is required. If a case is not making satisfactory progress, careful inquiry should at once be made as to whether the 
prescribed diet is being strictly attended to, as well as the nightly warm bath.

When the disease has been neglected, complications arise which require special remedies.

1. Swelling of the Eyelids, and Muco-purulent Discharge.-The eyes must be kept scrupulously clean by bathing with an antiseptic lotion; and once a day, or oftener, according to the amount of the discharge, the conjunctival surface of the lids should be everted, and painted with a 2 per cent. solution of nitrate of silver. The presence of a fissure at the external canthus, when such treatment requires to be carried out, adds greatly to the child's sufferings; but as this fissure will never heal until the eyes can be opened voluntarily, there is no help for it but to persevere, every care, of course, being taken not to cause unnecessary pain.

2. Inflammation of Nasal Mucous Membrane.-This membrane is swollen and ulcerated, and secretes a discharge so acrid that its presence produces much swelling of the alæ nasi and the upper lip. If the child be permitted to lie with its face buried in a pillow, an eczematous eruption is sure to appear. The nostrils must be kept clean by douching with an . alkaline antiseptic lotion, and the nasal mucous membrane brushed afterwards with ointment, e.g. a combination of iodol and tannic acid. As in these cases there is always an excess of acidity, alkalis are most serviceable. They should be given in small doses frequently repeated, and there is nothing better than a mixture of calcined magnesia and sulphur, or a combination of sulphate of magnesia with bicarbonate of soda.

3. Pediculi.-In neglected cases this is a very troublesome complication, and one regarding which the nurses must always be warned to be on their guard. In the most filthy cases it is necessary to cut the hair short, and in all the head must be washed with carbolic soap, well dried, and stavesacre ointment or sassafras oil rubbed into the roots of the hair. By this means the pediculi themselves are destroyed; but the nits remain firmly attached to the hairs, so that, after the first thorough dressing, scrupulous cleanliness and daily use of a small tooth comb are necessary in order to prevent the vermin from re-accumulating.

4. Ulceration of the Cornea.-In every case of strumous ophthalmia which has lasted for a long time, the cornea is almost certain to suffer, and whenever ulceration occurs the ulcer becomes the prominent feature, and all treatment is directed towards its cure. Even the small superficial ulcers leave a nebulous opacity after they have healed, and cause much irregular astigmatism. No disease is responsible for more bad sight in adults than phlyctenular conjunctivitis. The aim of all treatment, 
therefore, is to prevent implication of the cornea, and that can usually be accomplished if the case be skilfully treated from the outset. Chief reliance must be placed on the yellow oxide of mercury in I to 2 per cent. ointment either alone or in combination with the alkaloids of atropin and cocain, according to the severity of the photophobia and the lachrymation. 


\section{CHAPTER XV}

AFFECTIONS OF THE BULBAR CONJUNCTIVA-PINGUECULA; PTERYGIUM; LYMPHECTASIA； LIPOMA; DERMOID CYST; TUBERCULOSIS ; SARCOMA; EPITHELIOMA

1. Pinguecula.-A pinguecula is a small whitish-yellow elevation situated most frequently at the inner margin of the horizontal diameter of the cornea. It is triangular in shape, with its base at the corneoscleral margin, and projects above the level of the surrounding conjunctiva. Notwithstanding its name it contains no fat, but is composed of connective tissue and elastic fibres, both of which have undẹrgone hyaline degeneration. It rarely occurs before thirty years of age, but when it has developed it remains permanent, although it seldom increases much in size. It causes no symptoms, and requires no treatment. If, however, the patient is nervous, and complains about the disfigurementslight though it be-excision is necessary.

2. Pterygium.-A pterygium is an affection involving the conjunctiva and the superficial layers of the cornea. It is indeed nothing more than a fold of conjunctiva, which, having become adherent to the cornea, has been drawn up over it. It occurs only in adults, and is seen for the most part in men, who have been much exposed to dust and glare, or to the vicissitudes of weather. The conjunctiva and subconjunctival tissue become hypertrophied to form a fold of triangular shape. The blunt apex of the triangle is directed to the cornea, while the base passes into the bulbar conjunctiva with no sharp line of demarcation between the two. It is always situated at the inner and the outer sides of the eyeball at the level of the palpebral fissure. It occurs most frequently at the inner aspect of the globe, and in this position is much larger than when situated at the outer aspect. It consists of a head which encroaches upon, and is firmly attached to, the cornea; of a body turned towards one canthus or the other and spread out to lose itself in the conjunctiva of the eyeball, with an ill-defined upper and lower border; and of a neck placed at the margin of the cornea. The last is usually red and vascular, and sharply defined, but the body is more or less membranous. Its form and the arrangement of its blood-vessels bear a fancied resemblance to the wing of an insect-hence the name pterygium. 


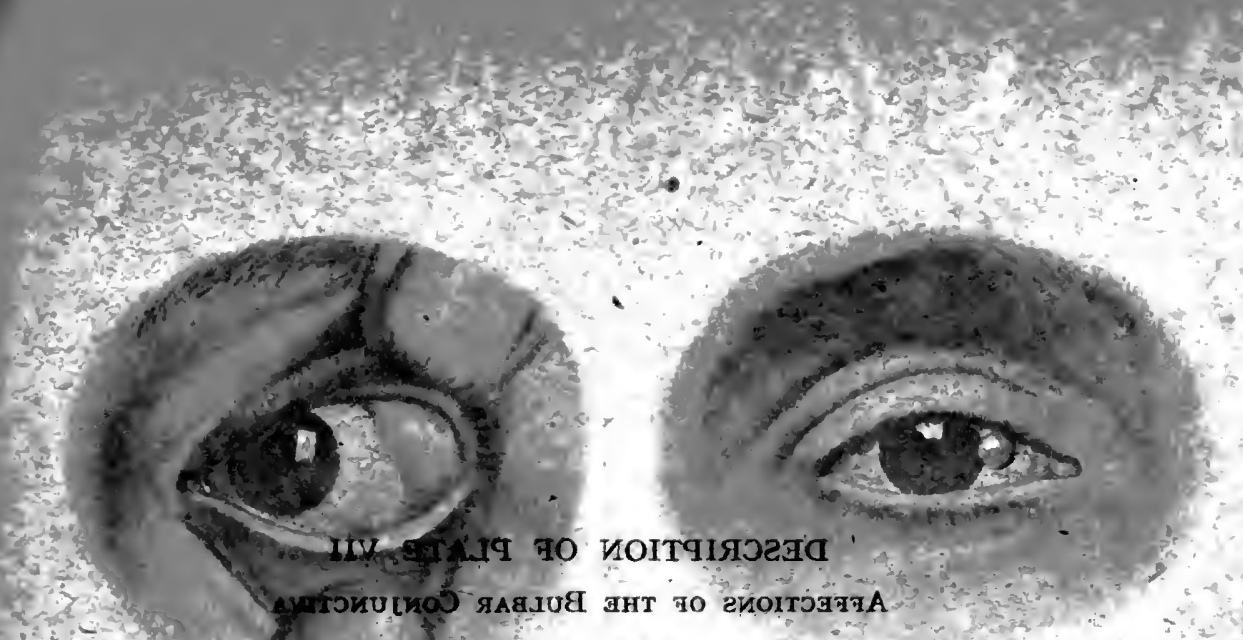

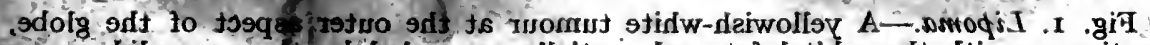

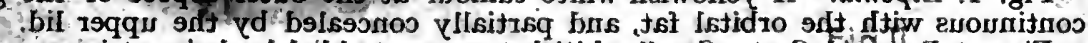

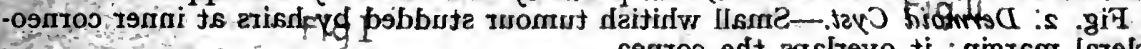
.s9mos onlt zqsligvo fi i rigrom Lstoloz

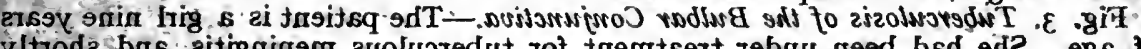

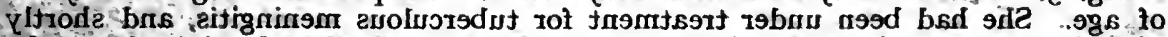

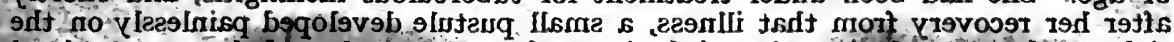
Is79loe-09mos odf of

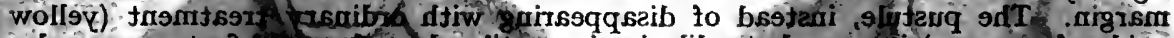
ighnu 9 mso tant

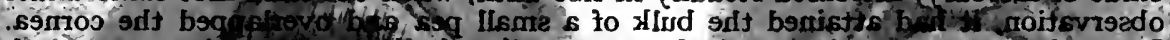

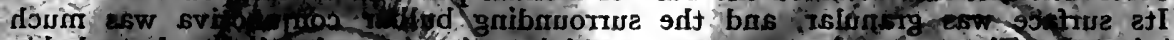

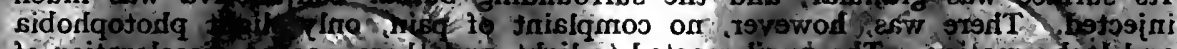

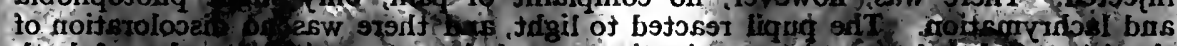

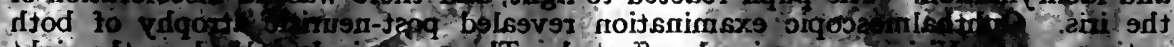
trigit oft ato $b$ ?

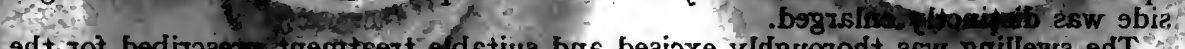

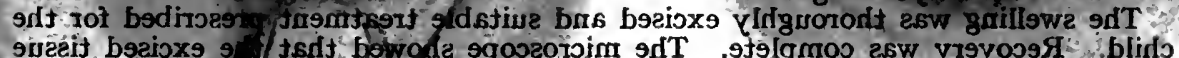

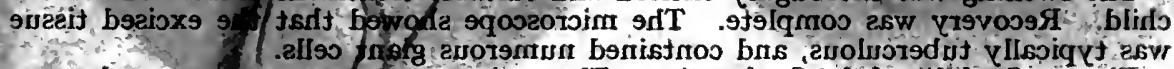

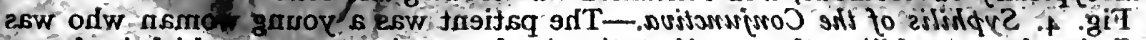

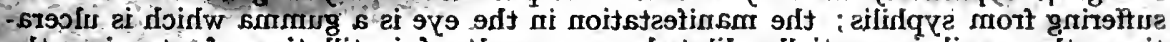

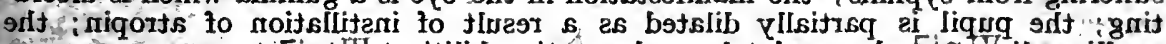

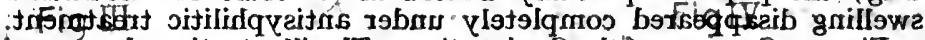

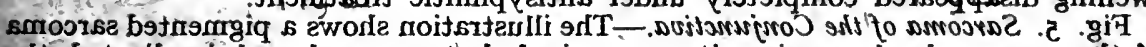

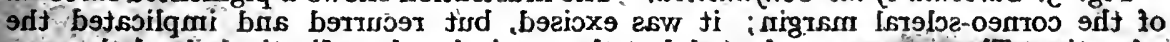

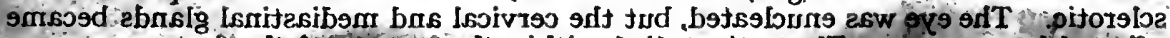

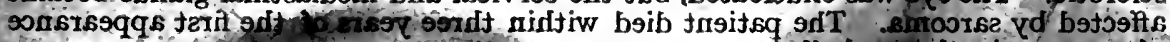

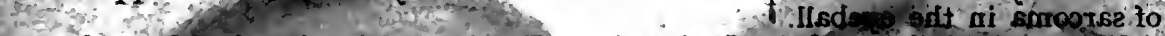

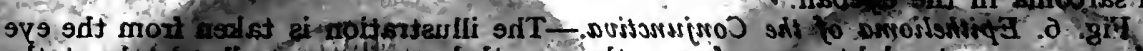

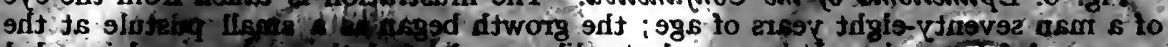

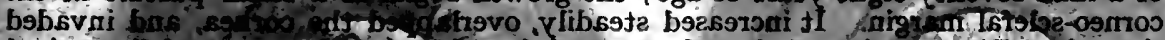

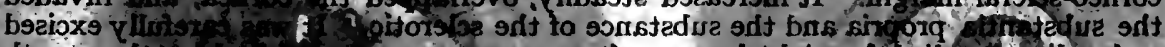

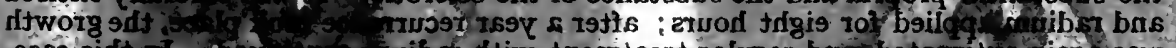

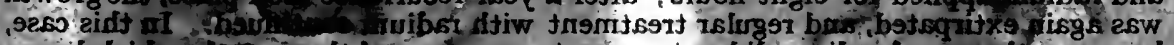

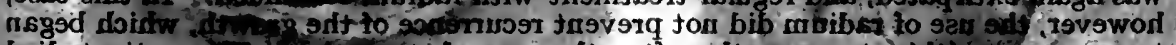

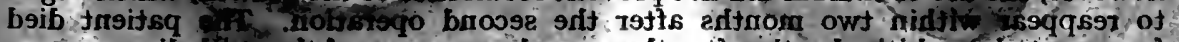

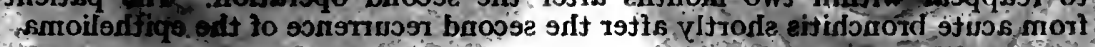

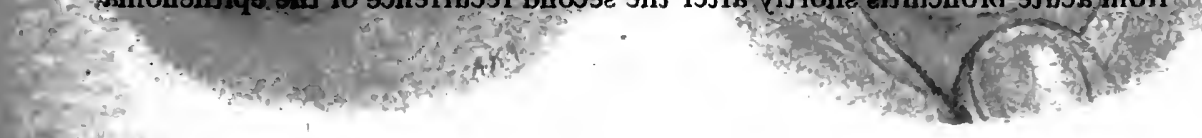




\section{CHAPTER XV \\ DESCRIPTION OF PLATE VII LA PTERYGIUM ; AfFections of the Bulbar Conjunctiva}

Fig. I. Lipoma.-A yellowish-white tumour at the outer aspect of the globe, continuous with the orbital fat, and partially concealed by the upper lid! on

Fig. 2. Dermoid Cyst. - Snall whitish tumour sfudded by hairs at inner corneoscleral margin; it overlaps the cornea.

Fig. 3. Tuberculosis of the Builbar Conjtinctiva. The patient is the girl nine years of age. She had been under treatment for tuberculous meningitis; and shortly after her recovery from that illness, a small pustule developed painlessly on the right eye between the insertion of the internal rectus muscle and the corneo-scleral margin. The pustule, instead of disappearing with ordinary treatment (yellow oxide of mercury) increased steadily in size until, when the child first came under observation, it had attained the bulk of a small pea and overlapped the cornea. Its surface was granular, and the surrounding bulbar conjunctiva was imuch injected. There was, however, no complaint of pain, only slight photophobia and lachrymation. The pupil reacted to light, and there was no discoloration of the iris. Ophthalmoscopic examination revealed post-neuritic atrophy of both optic nerves. Vision was seriously affected. The preauricular gland on the right side was distinctly enlarged.

The swelling was thoroughly excised and suitable treatment prescribed for the child. Recovery was complete. The microscope showed that the excised tissue was typically tuberculous, and contained numerous giant cells.

Fig. 4. Syphilis of the Conjunctiva. L The patient was' a young woman who was suffering from syphilis; the manifestation in the eye is a gumma which is ulcerating; the pupil is partially dilated as a result of instillation of atropin; the swelling disappeared completely under antisyphilitic treatment:

Fig. 5. Sarcoma of the Conjunctiva. The illustration shows a pigmented sarcoma of the corneo-scleral margin; it was excised, but recurred and implicated the sclerotic. The eye was enicleated, but the cervical and mediastinal glands became 1) affected by sarcoma. The patient died within three years of the first appearance of sarcoma in the eyeball.

Fig. 6. Epithelioma of the Conjunctiva. The illustration is taken from the eye of a man seventy-eight years of age the growth began as al small pustule at the corneo-scleral margin. It increased steadily, overlapped the cornea, and invaded the substantia propria and the substance of the sclerotic. It was carefally excised and radium applied for eight hours'; after a year' recurrence took' place, the growth was again extirpated, and regular treatment with radium continted.n In this case, however, the use of radium did not prevent recurrence of the growth, which began to reappear within two months after the second operation. The patient died from acute bronchitis shortly after the second recurrence of the epithelioma af 


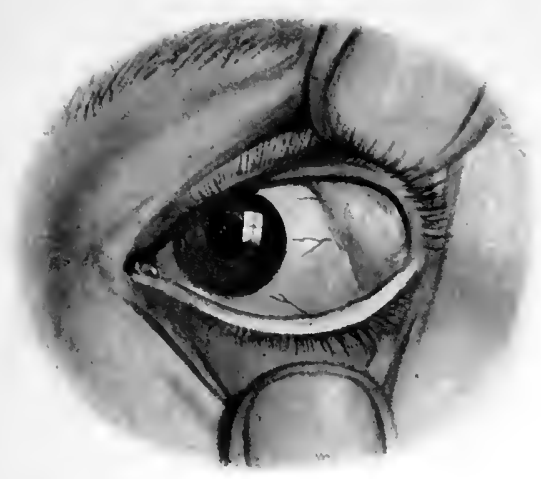

Fig I

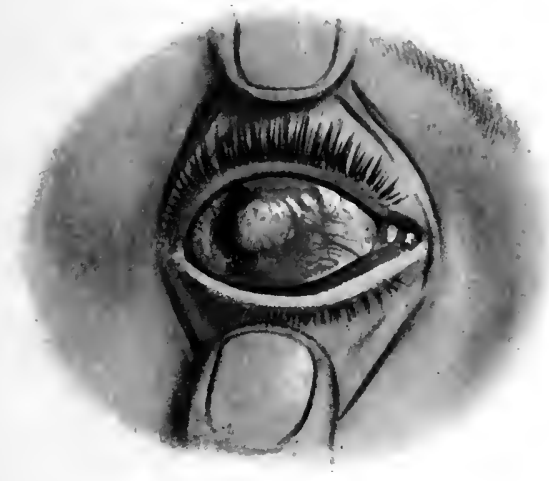

Fig III

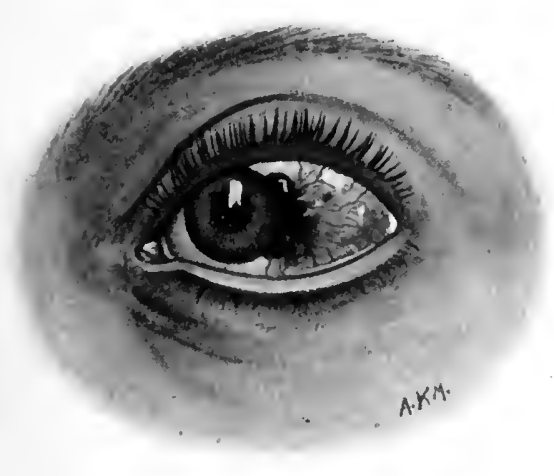

Fig $\nabla$

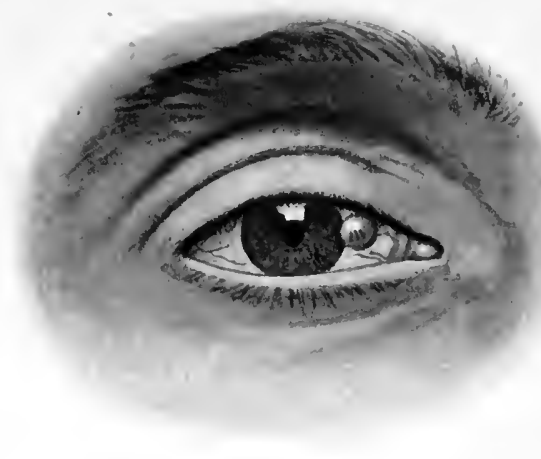

Fig II

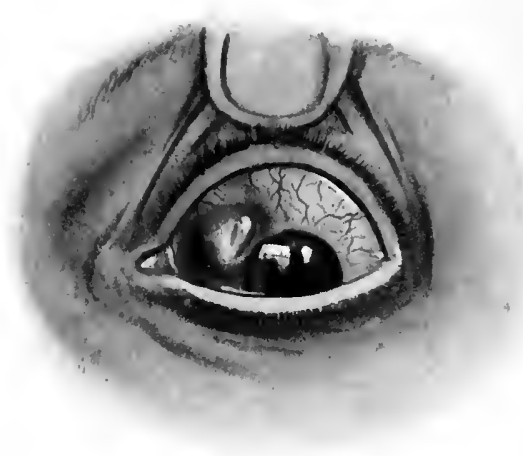

Fig W

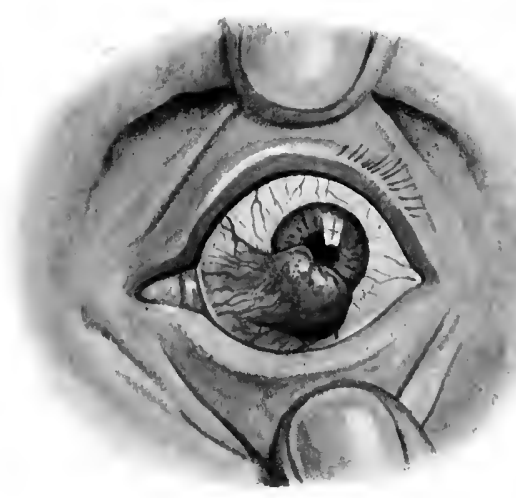

Fig II 

The evolution of the disease is slowly progressive, the tendency being for the head to push its way gradually towards the centre of the cornea. In some cases, however, a pterygium remains stationary, and is distinguished from one that is progressive by its membranous body and the cicatricial appearance of the head; whereas a progressive pterygium has a vascular fleshy body, and a head surrounded by an area of cornea grey, soft, and gelatinous.

A pterygium may cause slight irritation and watering of the eye; but when stationary it produces few subjective symptoms. It is only when the pupil is encroached upon that sight suffers, but from the moment the head reaches the pupillary area of the cornea vision is affected, and the defect increases in direct proportion as the centre of the pupil is invaded. Whenever, therefore, a pterygium is progressive it must be removed, but if it is stationary no interference is necessary. Patients, however, frequently complain of the disfigurement, and in these circumstances operation is required for cosmetic reasons.

A pseudo-pterygium is always cicatricial, and of traumatic origin. It never continues to graw over the cornea, because the head remains fixed to the spot to which the conjunctiva first became adherent.

The treatment of pterygium is purely surgical, and in the majority of instances the patients seek advice solely for the purpose of getting rid of the disfigurement.

3. Lymphectasia.-In this condition the lymphatics become dilated, filled with clear lymph, and form bright vesicles arranged in a beaded chain, although occasionally they dilate into larger cysts filled with limpid milk-coloured fluid. The condition is quite innocuous, and nothing needs to be done unless the patient complains of the disfigurement. In these circumstances, the conjunctiva containing the cysts or the dilated lymphatic vessels should be excised, and the edges of the wound neatly sutured with fine black silk.

4. Lipoma.-A subconjunctival lipoma is always congenital, and forms a yellowish white swelling, situated for the most part at the upper and outer aspect of the globe, between the superior and the external recti'muscles. It occurs more frequently in females than in males, and is occasionally bilateral and symmetrical. It is always solitary, varies in size from a small pea to a large almond, and consists of adipose tissue which is directly continuous with the orbital fat. It is covered by conjunctiva which has become thickened and cuticular-hence the name dermo-lipoma.

As a rule the tumour is so small that it lies hidden beneath the outer canthus, and may therefore easily escape notice at the time of birth. It is however, frequently accompanied by other congenital abnormalities, 
and at once becomes visible when the lids are widely separated and the eyeball turned strongly inward. At other times it is larger, and is readily seen when the patient looks straight forward. In these circumstances it may cause considerable disfigurement, and its removal will become necessary; it sometimes begins to grow as the age of puberty is reached. Except for the disfigurement and the liability to increase in size, a lipoma seldom does any harm, and in the majority of cases it is prudent to leave it alone. If, however, the removal of the tumour become necessary, all that requires to be done is to incise the overlying conjunctiva in order to expose it as thoroughly as possible, and with a pair of strong scissors curved on the flat to cut out as much of the fatty tissue as is visible in the palpebral fissure. It is quite unnecessary to dissect the tumour into the orbit; and in any operative procedure great care must be taken not to injure the superior or the external rectus muscle.

5. Dermoid Cyst.-A dermoid tumour is the most common of all the benign growths which take origin from the conjunctiva. As its name implies, it is composed of skin. It is always congenital, and may be accompanied by developmental anomalies both in the eye and other parts of the body. It lies in the line of the palpebral fissure, is usually situated on the corneo-scleral margin at the temporal side of the eyeball, and always overlaps the cornea, to the superficial layers of which it is firmly attached. It varies in size, is solid, hemispherical in shape, whitish yellow in colour, and presents a smooth surface, which is for the most part studded with hairs. Except for the conjunctivitis to which these latter may occasionally give rise, a dermoid causes no irritation. It usually remains stationary; but now and again it starts to grow at puberty and spreads over the surface of the cornea. It may then cause considerable disfigurement, and in these circumstances its removal becomes necessary. The tumour should be carefully dissected from the cornea, and the edges of the resulting wound in the conjunctiva must be accurately stitched together with fine black silk sutures.

6. Tuberculosis of the Conjunctiva.-Tuberculosis of the bulbar conjunctiva is a very rare disease. It may arise as a result of direct inoculation, from tubercle bacilli entering the conjunctival sac along with dust or other foreign bodies. As long as the epithelium is intact, no harm will be done; but if the epithelial cells are injured, the microorganisms readily settle down and try to effect a lodgment. From the clinical point of view the majority of cases are primary, because it is in many instances very difficult to find evidence of tubercle in other parts of the body; but from the pathological standpoint primary tuberculosis is very uncommon. The diagnosis is sometimes difficult; and it may be necessary to remove a piece of tissue for microscopical examination or for 
inoculation experiments on the guinea-pig, before it is possible to express a definite opinion, The knowledge that in so many instances tuberculosis of the eye is accompanied by tuberculous lesions in other parts of the body, makes the practitioner very cautious in the application of tuberculin tests by hypodermic injection; but the von Pirquet reaction is always available, and is trustworthy in so far that when it is positive it proves that the patient is a tuberculous subject, although it may tell nothing of the nature of the lesion in the eye itself.

The patients are usually young, and many of them are children. As a rule tuberculosis attacks one eye only, is rarely accompanied by pain or any acute symptoms of irritation, and runs a very chronic course. It commences as a pustule on the ocular conjunctiva, but differs from pustular ophthalmia in that it does not disappear as a result of treatment with yellow oxide of mercury ointment. On the contrary, its whole tendency is to increase in size and ultimately to resemble an inflammatory new formation of the granuloma type. It gets astride the cornea, to which it becomes firmly adherent, and if allowed to run its course uninfluenced by treatment, it breaks down, forming a caseating ulcer. Thereafter the cornea becomes invaded by blood-vessels, or affected by ulcerative keratitis. The pre-auricular and submaxillary glands are usually enlarged.

Tuberculosis is apt to be mistaken for syphilis of the conjunctiva; but in the latter the patients are generally adults old enough to be able to give a history of infection. In all doubtful cases, however, a Wassermann test of the blood must be made; but, in many instances, the rapid and favourable results obtained by the use of anti-syphilitic remedies are sufficient to remove all difficulties in diagnosis.

The treatment consists in trying to eradicate the diseased focus by careful excision, the subsequent application of the cautery, and the use several times a day of an iodoform and guaiacol ointment. The patient must be supplied with an abundance of good food, be comfortably housed, and live as far as possible in the open air. A preparation of tuberculin, in very carefully regulated doses, is always helpful; but the injections must never induce a prolonged negative phase, nor excite a severe general or focal reaction.

7. Sarcoma of the Conjunctiva.-Sarcoma of the conjunctiva is a very rare affection. It frequently originates in a nævus, but sometimes appears in the first instance as a pustule, which, however, is always surrounded by an unusual amount of episcleritis. Its favourite site is the limbus corneæ on the temporal aspect; and, while its base of attachment to the conjunctiva remains narrow, it grows in height and spreads like a mushroom over both cornea and sclerotic. In many instances it simply rests on those structures and does not seriously implicate them, because 
the disease has little tendency to penetrate into the interior of the globe. It may be latent for a long time, but sooner or later begins to grow, and may become so large as to fill the palpebral fissure and prevent the lids from closing. It is prone to recur locally after it has been removed by a surgical operation, and as in all other sarcomatous tumours the chief danger is the risk of metastasis to distant parts of the body. It is generally pigmented, and shines through the conjunctiva as a bluish or brownishblack tumour, with a smooth or a lobulated surface. An epibulbar may co-exist with an intraocular sarcoma, in which case its appearance under the conjunctiva is due to an extension of the tumour along one of the veins which perforate the sclerotic. Secondary growths may occur in the glands, when those in front of the ear, at the angle of the jaw, and in the anterior aspect of the neck may all become involved.

The prognosis largely depends upon the degree and the extent of the adhesion of the neoplasm to the sclera or to the cornea. If the tumour can be raised from the surface of the globe, and is only adherent to a limited area of conjunctiva, the outlook is much more favourable than when, even although its actual size be much smaller, the base of attachment is more extensive and includes the superficial layers of the cornea and the sclerotic.

Treatment.-The patient usually hesitates to part with an eye the sight of which is not much impaired, consequently in the first instance every effort should be made to eradicate the disease from the globe. After the tumour has been freely excised, and the wound carefully covered by conjunctiva, radium should be applied in order to reach any trace of tumour tissue which has escaped the knife. The application of the radium ought to be repeated in six weeks. If care be taken to protect the eye properly, radium will do it no harm, and may remain in contact with the lids from eight to twelve hours. If, however, in spite of all that can be done, there is local recurrence of the sarcoma, the eye must be enucleated without delay.

8. Epithelioma of the Conjunctiva.-Epithelioma of the conjunctiva may originate at the corneo-scleral junction, but more frequently extends to the bulbar conjunctiva from the eyelid. It always occurs in elderly patients, and appears as a rose-pink tumour abundantly supplied with large blood-vessels; but although there is always a certain amount of bleeding from its surface, there is seldom any serious hæmorrhage. It causes little irritation, spreads slowly but steadily over the cornea and sclerotic, and is firmly adherent to both of these structures, but has little tendency to penetrate into the interior of the globe. There is often considerable difficulty in diagnosis in the early stages of the disease, but in all cases of doubt a piece of the suspected tissue should be excised, 
and examined microscopically. If epithelioma be present its typical histological structure will be readily recognised.

An epibulbar epithelioma sometimes grows to such a large size as to cover the cornea so completely that the eyeball seems to have disappeared. In many instances, however, it has simply spread over the surface of the globe without having become adherent to it.

Prognosis is always serious, because the disease proceeds relentlessly, and sooner or later the lids and the orbit will be implicated. If, however, the tumour be small an attempt should be made to remove it, and the subsequent application of radium may hold the disease in check. Nevertheless, if the patient lives long enough, local recurrence is sure to occur, and in these circumstances the eye must be sacrificed. The removal of the neoplasm is always difficult, because it is solidly adherent to the cornea and so very friable that it requires careful dissection. The conjunctiva must be cut away far beyond the apparent limits of the disease, and if the preauricular or the submaxillary glands are enlarged they also must be extirpated. 


\section{CHAPTER XVI}

\section{ULCERATION OF THE CORNEA}

Forms of corneal ulceration-Corneal ulcer occurring in catarrhal conjunctivitisIn purulent conjunctivitis-In strumous conjunctivitis-In trachoma-In trichiasis-In cases of abnormal exposure of the eyeball-In diseases characterised by interference with the corneal nerve supply-Marginal keratitisHerpes corneæ-Neuroparalytic keratitis-Keratomalacia-Ulceration due to breaking down of a cicatrix-Methods of examination-Clinical courseComplications-Perforation-Hypopyon-Prognosis-Treatment.

ALL forms of ulceration of the cornea fall under one of three groups.

I. Where the ulcer is primary and local ; and is due to direct injuryphysical, chemical, or bacterial.

2. Where the ulcer is secondary to disease previously existing in the eye-the majority of those are complications of conjunctival affections.

3. Where the ulcer is secondary to a general disease, and is merely a sign of the constitutional state.

For clinical purposes, secondary ulcers may be divided into those which occur: (A) in conjunctival affections; (B) through abnormal exposure of the eyeball; (C) in disease due to interference with the nerve supply, and (D) through the breaking down of a cicatrix.

\section{(A) CONJUNCTIVAL AFFECTIONS}

1. Acute Catarrhal Conjunctivitis.-In this, more particularly in adult cases, there are found, for the most part on the margin of the cornea, small grey crescent-shaped ulcers, generally superficial, which by their coalescence occasionally produce a breach of surface of considerable size. They are the result of thrombosis of the blood-vessels at the limbus corneæ, and, if carefully treated from the outset, rarely cause perforation.

2. Chronic Catarrhal Conjunctivitis.-Ulcer is not common in this form of inflammation of the cornea, which is usually the result of infection by the Morax-Axenfeld diplo-bacillus. In severe and long standing cases, however, shallow infiltrations occur at the margin of the cornea and break down to form transparent superficial ulcers.

3. Purulent Conjunctivitis.-In this, whether in adults or in newlyborn children, there occur sloughy ulcers, which may be either central 
or marginal, those on the margin being sometimes concealed by chemosis of the conjunctiva overlapping the surface of the cornea. They may completely surround the cornea, leaving the centre a sequestrum. They are rapidly progressive, as there is always round them an infiltrated area, which may spread either in depth or along the surface. When the cornea is destroyed down to Descemet's membrane, the base of the ulcer is quite bright, owing to the exposure of the posterior elastic lamina, but this appearance ought never to be mistaken for the clean, smooth surface of a healing ulcer. As a result of intraocular tension, the membrane bulges forward, and perforation takes place.

4. Strumous Conjunctivitis.-In the conjunctivitis of strumous children, many different forms of ulceration are seen. Sometimes there is only a tiny abrasion of the corneal epithelium, which, from the intense photophobia and blepharospasm to which it gives rise, can with difficulty be detected without staining by fluorescin. In other cases there is a transparent faceted ulcer, which looks as if a little bit had been chipped out of the surface of the cornea. Indolent, and always very difficult to heal, this niay become vascular, and persist for many weeks as a red speck: it rarely perforates. Sometimes a vascular papule forms on the limbus corneæ, and, followed by its leash of blood-vessels, moves in towards the centre of the cornea. In such a case its track may persist for a very long time. A round, rapidly perforating ulcer with prolapse of the iris occurs when a pustule situated in the-corneo-scleral margin suppurates, ulcerates, and bursts. At times, especially in unhealthy children, an abscess forms in the centre of the cornea, and, bursting externally, gives rise to a purulent ulcer. Pus forms within the layers of the cornea-onyx-and, accumulating in the anterior chamber, gives rise to hypopyon.

5. Granular Conjunctivitis or Trachoma.-Ulceration is always present, situated for the most part at the progressive margin of the pannus.

6. Ulceration is also present in trichiasis, where misdirected eyelashes irritate the cornea, and in lithiasis, where an exposed chalky particle in either the upper or lower lid scratches the front of the globe with every movement of the eye.

\section{(B) ABNORMAL EXPOSURE OF THE EYEBALL}

In ectropion, facial palsy, advanced exophthalmic goitre, and in tumours of the orbit, when the eye is not properly protected either by day or by night, the corneal epithelium tends to become dry, and ulceration occurs, for the most part at the lower border of the cornea. 


\section{(C) DISEASES GHARACTERISED BY INTERFERENGE WITH NERVE SUPPLY}

The cornea is protected not only by the winking of the lids during the day and their closure at night, but also by its acute sensibility. Whenever, therefore, there is any interference with the function of the fifth cranial nerve, a very trivial injury may determine serious ulceration. The insensitive cornea cannot withstand what would have no injurious influence on the healthy structure. In every case of chronic ulceration, therefore, it is necessary before giving a prognosis to test carefully the sensation of the cornea. The head of a pin sterilised by passing it through a flame can be used to touch the cornea lightly. If sensation be present, it always provokes reflex closure of the lids. Acute sensibility is essential to the life and health of the cornea, and whenever the nerve supply is interfered with or lost, diffuse purulent infiltration quickly takes place. This form of ulceration presents several clinical types.

1. Marginal Ulceration.--In old and debilitated persons minute oval-shaped ulcers, parallel to the limbus and attacking only a small area of the cornea, are said to arise from exposure to cold, but the special vulnerability is more probably due to defective nerve supply. They occur in the absence of blennorrhœa of the lachrymal sac, run a very chronic course, and rarely perforate.

2. Herpes Corneæ.-After influenza, pneumonia, and other diseases accompanied by increase of temperature, little blisters form on the cornea similar to those occurring on the lips and the angle of the nose. They form in groups, and, after bursting, cause ulceration, which occasionally assumes a dendritic shape. Those ulcers are very chronic, and are accompanied by much blepharospasm, intolerance of light, and lachrymation. The cornea is not insensitive as a whole, but sensibility is usually lost in the ulcerated area. In zona ophthalmica the cornea is usually affected when the nasal branch of the trigeminus is involved. There is diffuse infiltration and want of sensation, and the corneal lesion is frequently accompanied by irido-cyclitis. The attack is usually heralded by a feeling of malaise, and acute pain which follows the distribution of certain branches of the ophthalmic division of the trigeminus. After an interval of hours or days the characteristic herpetic eruption appears, but it may be so masked by signs of general inflammation that the disease is frequently mistaken for erysipelas. Zona ophthalmica is always unilateral, and occurs as a rule in elderly persons. The attack itself lasts about three weeks, but pain and anæsthesia of the skin may persist for months, and the ocular symptoms are usually the last to disappear.

3. Neuroparalytic Keratitis.-After extirpation of the Gasserian 
ganglion in the radical treatment of severe trigeminal neuralgia, the eye on the same side occasionally suffers in the manner first described by Magendie after experimental division of the fifth nerve in animals. The corneal epithelium becomes cloudy and peels off, exposing the substantia propria, which, becoming infected, forms a large central ulcer, usually purulent and accompanied by hypopyon. There is considerable circumcorneal injection; but neither pain nor photophobia is present, and the insensibility is so complete that the eye may be enucleated in the absence of either local or general anæsthesia, without suffering to the patient. Other causes of neuroparalytic keratitis are intracranial tumours, basal meningitis, and fracture of the skull; both eyes may be affected.

4. Keratomalacia occurs in the majority of cases in syphilitic children during the first year of life. The cornea becomes dry and infected with nicro-organisms; usually the streptococcus or the pneumococcus. It speedily ulcerates and bursts. The children are always very ill, and frequently die. Both eyes usually are affected.

\section{(D) THE BREAKING DOWN OF A GIGATRIX}

This is seen in cases whether due to traumatism or to disease where a scar having undergone degenerative changes breaks down, becomes infected, and rapidly ulcerates. There is little or no attempt at repair, and the ulcer goes on to perforation, which is followed in many instances by panophthalmitis.

In diseases of the eye in which ulceration of the cornea occurs, the complication is so serious as, for the time being, to overshadow the original disorder. Hence the need for careful examination. It is not enough to suspect the ulcer, it is necessary to make sure of its presence. The cornea must not be glanced at from a distance in the daylight, but ought to be carefully explored by the help of a lens which concentrates the light. If there be any doubt, a drop of an alkaline solution of fluorescein should be instilled, when the surface of an ulcer, if it be present, will be stained bright green. The danger lies in an abraded cornea becoming secondarily infected from the margin of the lids, or from a diseased tear sac, or an inflamed nasal mucous membrane, and becoming a sloughy ulcer, which may destroy the eye in a very short time.

Corneal ulcers may be classified into superficial and deep, central and marginal, progressive and healing. Pain, blepharospasm, intolerance of light, and lachrymation, are much more pronounced in superficial than in deep ulceration, because, in the former, the nerves are exposed and irritated, while in the latter they are destroyed. Photophobia is always 
worse in children than in adults, and pain, accompanied by œdema of the lids, may be regarded as a sure sign that the ulceration is increasing. It reaches its maximum immediately before perforation, but relief sudden and lasting is experienced as soon as the cornea gives way. Deep injection is always present, but varies in degree, and is usually most intense in adults. Chemosis of the ocular conjunctiva indicates that the iris and ciliary body are implicated. The following signs show that an ulcer is progressive: Its surface is uneven, and greyish or yellowish in colour, and its edges are ragged, steep, swollen, undermined, and wholly or in part infiltrated with pus. Both pericorneal injection and subjective phenomena are acute. If, on the other hand, an ulcer be healing, the pericorneal injection and the subjective phenomena gradually disappear. Its surface is clean and smooth, its edges are even and rounded, and the cloudiness of the cornea beyond the ulcer tends steadily to clear up.

A further stage in the healing process is marked by the development of "vessels of repair" running towards the ulcer from the limbus, and preventing extension; but it must be remembered that, although an ulcer which has become vascular does not grow larger, it may increase in depth and ultimately perforate. Immediately after all the necrosed tissue has been removed, there is a rapid development both of epithelial cells to cover the surface, and of connective tissue to fill up the cavity. As a rule, these two processes go on simultaneously, so that by the time the surface of the ulcer has become protected by epithelium, the depression has been raised to the level of the surrounding cornea. Sometimes, however, the surface is completely covered long before the lost substance has been replaced by new tissues-e.g. in "indolent," "transparent," or " corneal-facet" forms.

\section{GOMPLICATIONS}

1. Perforation of Cornea.-In severe ulceration, as for example after the eye has been burnt, the whole substance of the cornea may be destroyed and melt away, with the exception of Descemet's membrane. This, denuded of the other layers of the cornea, escapes the general destruction for the time being, and presents a smooth, shining surface, which must on no account be taken for a sign of the healing of the ulcer. Such an error ought never to arise, because all the other signs and symptoms point to rapid progression; and probably within twenty-four hours Descemet's membrane also will have given way, and the iris will be seen lying exposed and prolapsed. In some cases, however, simple perforation is to be regarded as a favourable occurrence, because after the aqueous has escaped, and all tension has in consequence been removed, the 
disease is arrested, and healing begins. If the perforation be in the centre, the anterior capsule of the lens will come forward against the posterior surface of the cornea immediately after the aqueous chamber is emptied; but if the rupture takes place at any other part, it is the iris that comes forward, and the intraocular pressure is sufficient, on the escape of the aqueous humour, to force it into the gap, and, as a rule, to cause it to prolapse beyond the cornea. In cases where ulceration is extensive and rupture of the cornea occurs, the damage to the eye is so great that the lens becomes dislocated, and sometimes escapes from the globe, followed by a gush of vitreous humour. Such a complication is occasionally accompanied by intraocular hæmorrhage, and may be followed by panophthalmitis.

2. Hypopyon is usually accompanied by inflammation of the iris and ciliary body. The exudate in the anterior chamber is derived, partly from the ulcer through a perforation in Descemet's membrane, and partly from the inflamed iris and ciliary body. In the absence of acute iridocyclitis, a comparatively large hypopyon can become rapidly absorbed; but when the ulcer is complicated by inflammation of the iris and ciliary body, the collection of pus in the anterior chamber tends to persist, and may vary. in amount from day to day. There is great accentuation of all the subjective phenomena; and pain, often markedly nocturnal in character, radiates from the eyeball along the branches of the fifth cranial nerve. The pupil dilates irregularly to atropin, the anterior chamber is deep, and the ocular conjunctiva is raised above the level of the cornea as a fleshy chemosis, the colour of which varies from a bright to a livid red, according to the degree of implication of the uveal tract. It is a wise rule, therefore, before giving a prognosis in a case of hypopyon-ulcer, to instil atropin and watch its effect upon the pupil. The more quickly the iris responds to the influence of the mydriatic, the more favourable will be the course of the disease.

Prognosis.-On the whole a favourable prognosis may be given in all forms of ulceration of the cornea, provided there has been skilful treatment from the very outset of the disease. Each case, however, must be judged on its own merits, and the prognosis will be influenced not only by the nature, size, and situation of the ulcer, but also by the state of the patient's health. When ulcer of the cornea complicates conjunctival affections, the earlier in the course of the disease the ulceration occurs, the worse the prognosis. Simple traumatic ulcers are, as a rule, very amenable to treatment, but if they become infected-for example, by blennorrhœa of the tear passages-the prognosis must always be guarded. In women who are nursing, and in patients suffering from diabetes or albuminuria, suppuration and hypopyon are very prone to occur, and 
such cases are also very liable to be complicated by inflammation of the iris and ciliary body. If the ulceration has invaded the substantia propria of the cornea, and more especially if the healing process has been accompanied by a large development of newly-formed blood-vessels, a permanent opacity is certain to remain, and the degree of the scarring and the permanence of the cicatrix will depend upon the depth of the ulcer and the length of time it has taken to heal, as well as upon the age of the patient and the energy and fidelity with which appropriate treatment has been carried out.

Treatment.-In the numerous cases in which ulcer of the cornea is the result of conjunctivitis, or of blepharitis, or of affections of the lachrymal passages, or of the nasal mucous membrane, the essential part of the treatment consists in the cure of those diseases. The epilation of an ingrowing eyelash may be all that is required to cure a corneal ulcer of long standing; but unless the displaced cilium is permanently destroyed by electrolysis or by other means, the operation will require to be repeated every two or three weeks. The cause, whatever it may be, having been removed, the ulcer in many instances heals spontaneously.

In the treatment of inflammation of the conjunctiva complicated by ulceration, care must always be taken to protect the cornea from the action of the caustics employed, and solutions of nitrate of silver are to be preferred to sulphate of copper. Irritating astringent collyria, containing alum or acetate of lead, ought never to be employed. If, in ignorance, the latter has been applied, a deposit of white lead will form upon the raw surface of the ulcer, and give rise to a staring white opacity, which is permanent.

In the treatment of the ulcer itself, the most important indications are to keep the eye at rest, to protect it from further injury, and to subdue pain. In all severe cases the patient should be kept in bed in a room from which direct sunlight is excluded; and in addition to local means, general constitutional treatment is nearly always necessary. The patient must be suitably fed, and if old and debilitated, ought to have a small quantity of alcohol with the two principal meals; malt liquors if they do not disturb digestion are preferable to any other form of stimulant. In strumous cases it is of the first importance to attend to the kind of food the patient eats, and all forms of sugar, sweets, cakes, fruits, potatoes, etc., must be rigidly excluded from the dietary. Whenever there is purulent infiltration of the ulcer, quinine in full doses does good, and sedatives can be given along with it if there be much pain.

When the ulcer is due to injury, a bandage is necessary, but. when it is secondary either to disease previously existing in the eye, or to a disordered constitutional state, bandaging ought to be avoided, unless in 
very exceptional circumstances, as, for example, when an ulcer is threatening to perforate, when the cornea is insensitive, or the eye not sufficiently protected by the lids. In the great majority of cases atropin plays an important part in the treatment. Its action in dilating the pupil and paralysing the ciliary muscle ensures physiological rest for the eye, while locally it soothes the nerves and diminishes congestion of the bloodvessels. A I per cent. solution of the sulphate is most commonly employed, and in mild cases it is usually sufficient to instil a drop once a day; but as the object of the treatment is to keep the pupil dilated, the solution must be reinstilled just as often as is necessary to maintain full mydriasis. When pain is acute and there is great congestion of the iris, the good effect of atropin is increased by leeching the temple. In some persons, however, owing to idiosyncrasy, atropin produces redness and swelling of the eyelids accompanied by an eczematous eruption of the skin in the neighbourhood of the eye. When that occurs, the use of the drug must be at once stopped, and another mydriatic-duboisin, hyoscin, scopolamin, or daturin-substituted. In certain rare cases also, atropin may cause general intoxication, and induce acute mania. It is always necessary, therefore, to guard against any of the solution finding its way into the mouth: and while it is being instilled, the lid should be drawn away from the eyeball, to prevent it from being sucked into the tear passages. Moreover, when the patient is to employ the drug himself, it is safer to prescribe it as an ointment. The use of atropin is contra-indicated when the ulcer is deep and situated at the margin of the cornea, or when it is threatening to perforate.

When pain is severe and there is much deep pericorneal injection, great benefit is got from the application of fomentations. Moist heat diminishes the engorgement of the blood-vessels, and so, by reducing tension, relieves pain. Fomentations are applied every two or three hours, day and night, but as soon as pain abates, they are used less frequently. They may be made more soothing by the addition of a teaspoonful of Battley's sedative solution of opium to a pint of an infusion of chamonile flowers.

If, in spite of that treatment, the ulcer continues to progress, disinfectants must be applied to its surface; but the general practitioner requires to be careful not to do harm by the use of strong remedies. When he is in doubt, he ought, at the earliest opportunity, to seek help from an expert, and never wait until the eye is obviously very seriously destroyed. Bathing should be carefully done with a warm, saturated solution of boracic acid and borax, but irrigation with large quantities of lotion must be avoided. Argyrol in 20 per cent. solution should be instilled five or six times a day, or a 3 per cent. iodoform ointment can 
be similarly employed. Cauterisation of the ulcer, either by drugs, such as pure phenol and iodine, or by the application of the galvano-cautery, is best left to the specialist. When the ulcer is accompanied by want of sensibility of the cornea, the trophic changes greatly increase the danger, and the application of caustics or the use of the cautery, may precipitate its complete destruction. Nothing that irritates, either mechanically or chemically, must be used. The eye ought to be carefully protected by a bandage, and in the worst cases, the lids should be sutured in their middle third, leaving the outer and inner thirds free. In spite of that treatment, however, the ulceration sometimes progresses steadily, and enucleation becomes necessary. Similar treatment is applicable in those cases where the eye is insufficiently covered by the lids, and no bandage or dressing equals in efficacy the more or less extended tarsorraphy of the palpebral margins. In herpes of the cornea, and in zona ophthalmica, pain is always very severe; and oily. applications of the alkaloids of atropin and cocain with hot applications usually afford relief for the time being. In prolonged ulceration, eserin is sometimes helpful; and occasionally the ulcer is healed quickly by painting it thoroughly with absolute alcohol. In these cases, however, the general treatment is all-important. In the early acute stage the patient must be confined to bed, and treated with alkaline salines, with quinine combined with aspirin, salicylate of soda or other sedatives to relieve pain. Elliot advises Donovan's solution in Io minim doses, gradually increased to 20 or even 30 minims three times a day, three-quarters of an hour after a meal. He believes it to be a "specific for the affection." As soon as possible, change of air and treatment at a Spa, if possible, is advisable, and the patient should be warned that pain is likely to persist for weeks or even for months, and is always aggravated by exposure to wind or to any sudden change of temperature.

Perforation may be averted by reducing the intraocular pressure by fomentations, myotics, or by paracentesis of the anterior chamber. Chief amongst the myotics is the sulphate of eserin, which is employed as a $\frac{1}{2}$ per cent. solution, instilled into the eye every four or six hours. It is especially serviceable where the ulcers are at the margin of the cornea, or when the cornea is threatening to give way. After perforation it aids in preventing prolapse of the iris, by contracting the pupil and so drawing it tightly in front of the lens. As eserin tends to aggravate any inflammation of the iris, its use is contra-indicated in ulceration complicated by iritis. In such cases it is necessary to lower intraocular tension by paracentesis of the anterior chamber, and the subsequent instillation of atropin. Pilocarpin acts less powerfully than eserin, but has the advantage of causing no pain in the eye and brow. It is used in I per cent. 
solution of the nitrate, and at times better results may be obtained by a combination of pilocarpin with eserin, than with either drug separately. When perforation has taken place, the iris may either lie against the posterior surface of the cornea, or may prolapse. In the former case no change requires to be made in the treatment, but in the latter the prolapse, if recent, must be carefully excised. It must first be entirely freed from the perforation in the cornea, then grasped with forceps, drawn out of the opening, and excised while being held on the stretch, and the wound should be covered over with a flap of conjunctiva. If the prolapse, however, be more than two days old, inflammatory exudation will have begun to cover it and to seal it firmly to the lips of the opening, and in this case excision must not be attempted. The most favourable result is obtained when the prolapse becomes converted through cicatricial contraction into a flat scar; but should a constriction form at its base and it become pedunculated and assume a mushroom-like shape, it will not flatten down until it has been punctured repeatedly, or partially destroyed by the galvano-cautery. Needless to say, those cases should never be treated by a general practitioner. After a prolapse of the iris has healed, the pupil is always displaced towards the corneal scar, and if the lesion has been extensive it may be altogether hidden by the leucoma. In such cases the excision of a piece of iris opposite a transparent portion of the cornea will not only improve the sight, but will also avert the onset of secondary glaucoma with which an eye suffering from leucoma adherens is always threatened. 


\section{CHAPTER XVII}

SERPIGINOUS ULCER OF THE CORNEA, AND THE DUTY OF THE GENERAL PRACTITIONER IN ITS PREVENTION AND TREATMENT

Occurrence of infection of corneal abrasions-Pneumococcal ulcer-Clinical course and sequelæ-Prognosis-Pathology-Preventive treatment: attention to tear-passages; first aid-Curative treatment-The use of antiseptics-Ethylhydrocuprein-Vaccines-Cauterisation-Saemisch's operation.

PRIMARY ulceration of the cornea takes place after injuries resulting from violence which has not been great enough to kill the tissues outright - the most familiar examples being burns by molten metal, by mineral acid, or by caustic alkali. The results of such accidents are often most disastrous: in many instances sight is entirely lost, and the eyeball itself may even be completely destroyed.

Serious and distressing as injuries of this kind are, they cause, when taken as a whole, less damage than is occasioned by pyogenic infection of superficial abrasions of the cornea. Pus-producing microbes of many kinds lurk in the tear-passages, on the margins of the eyelids, and in the conjunctival sac; but these micro-organisms do no harm until the local or the general resisting power of the patient is lowered. It is well known that the cornea may be bathed in pus and yet no evil results ensue as long as the epithelial layer is intact. The microbes only become hurtful, and cause infection and ulceration, if there is an abrasion of the corneal epithelium to give them a field on which to work. The clinical signs which follow infection vary, and their severity depends not only on the resisting power of the patient, but also on the virulence of the specific organism. Bacteriology is, therefore, being applied more and more to determine the causation, and to assist in the classification of ulcers of the cornea; but there are still many gaps in our knowledge, and these can be filled up only gradually by patient investigation of unusual types of ulceration. The difficulties in arriving at a bacteriological classification are many, for not only is there a probability of a mixed infection, but different micro-organisms can give rise to, similar clinical appearances, and the same micro-organism seems capable of producing dissimilar appearances in different subjects.

The pneumococcus lanceolatus is a very frequent cause of infection in 
many diseases of the eye. It may indeed be regarded as one of the ophthalmologist's worst enemies. It produces suppuration after an operation or after an accidental wound; it gives rise to a special type of conjunctivitis in children; and in addition to being an ætiological factor in keratomalacia and other destructive forms of ulceration of the cornea, it is invariably associated with ulcus serpens cornea. This last is a very characteristic form of corneal ulcer, which was first described by Saemisch in 1870 . It is one of the most dangerous diseases of the eye, consequently its bacteriology has been very carefully studied. As early as I894, Uhtoff and Axenfeld had proved experimentally that the pneumococcus is the cause of Saemisch's ulcer, and now, in modern text books, the term pneumococcal is frequently substituted for the old clinical name serpiginous.

While pneumococcal conjunctivitis is essentially a disease of children, pneumococcal ulcer of the cornea is almost exclusively confined to adults. The patients for the most part belong to the labouring classes; men are attacked more frequently than women, and it is a noteworthy fact that a large proportion of those who suffer are troubled by chronic suppuration of the tear passages. Dacryocystitis is always a standing menace to the eye, for when it exists a simple abrasion of the cornea is all that is necessary for infection. In a large proportion of the cases the pneumococci come from the tear passages, the edge of the eyelids, and the conjunctival sac; at other times they come from the mouth or the nose through sputum or a soiled pocket-handkerchief ; only very occasionally are they conveyed by the object which has caused the injury to the eye.

In the course of his daily work a coal-miner, a stone-breaker, or an agricultural labourer runs considerable risk of accident to his eyes, and so frequently do foreign bodies scratch the cornea that as a rule little attention is paid to what is considered to be a very trivial injury. The eye feels uncomfortable, waters freely, and shuns the light, but generally, in spite of these discomforts, the man continues at work. After a day or two, however, pain becomes so severe as to compel him to desist, and then steadily increasing and darting from the eye to the top and the back of the head, it interferes with rest and sleep, and at length drives the sufferer to consult a doctor.

At the outset the superficial layers of the cornea are simply infiltrated, but so rapidly is the epithelium destroyed, that usually by the time medical advice is sought, an ulcer, which presents very characteristic features, has formed. As a rule the ulcer is situated towards the centre of the cornea opposite the pupil. It is somewhat crescentic in shape, and while its floor shows only a shallow sloping depression, rough and glistening, its margins are opaque, raised, undermined, and suppurating. The cornea 
appears transparent, but on careful examination fine grey lines are seen passing into its substance, and for the most part radiating from the most densely infiltrated border of the ulcer. One of its most striking characteristics, and that from which it derives its usual name, is the tendency it has to creep over the surface of the cornea. While progressive in one direction, however, it tends to cicatrise along the opposite margin. Its peculiarly infective nature may be early diagnosed from its extreme painfulness, by the occurrence of deposits on Descemet's membrane, and by the formation of a hypopyon in the most dependent parts of the aqueous chamber; while associated with these are intolerance of light, injection of both the superficial and the deep blood-vessels, chemosis of the conjunctiva, and swelling of the lids. Severe inflammation of the iris occurs early, and is one of the most serious and distressing complications. To complete the certainty of the diagnosis, a smear should be taken from beneath the spreading edge of the ulcer, prepared on a cover glass, stained by Gram's method, and examined under the microscope. If the case be one of serpiginous ulcer, pneumococci will be found in greater or less abundance.

The virulence of the infecting organism determines to a large extent the severity of an attack, but in this connection the personal factor in the patient himself counts for much. Pneumococci which in man produce only a mild ulcus serpens may prove to be of high virulence in rabbits and other animals; while, on the other hand, if the resisting power of the cornea, and the patient's general health be lowered from abuse of alcohol or from other causes, the pneumococci find a congenial soil, and the ulceration often spreads with such intense rapidity that in a few days the whole cornea is completely destroyed.

When left to itself serpiginous ulcer rarely heals spontaneously. The micro-organisms do not spread deeply, but superficially between the lamellæ, and so the ulcer creeps over the whole surface of the cornea. As it increases in size the floor bulges, and may become so thin that Descemet's membrane projects. In more severe cases the whole of the epithelium and the substantia propria are destroyed, and only the more resistant posterior layer remains. When at length this perforates, the pneumococci gain access to the interior of the globe. If they propagate in the uveal tract and in the vitreous, intense inflammation is set up and may involve every tissue of the eyeball. Happily, owing to improved methods of treatment, panophthalmitis is not nowadays a common sequel to hypopyon ulcer; but whenever the cornea is extensively destroyed, and especially if perforation and prolapse of the iris have occurred, secondary glaucoma, or partial or complete anterior staphyloma frequently follows. 
Prognosis. -The prognosis of serpiginous ulcer is always serious. Even a mild case requires several weeks to heal ; and as the formation of fibrous tissue always follows every inflammation which has caused partial necrosis, a cicatrix is inevitable. The whiteness and density of the scar depend upon the depth of destruction of the cornea; but as the leucoma is usually situated in front of the pupil, sight is always seriously impaired, and can only be imperfectly restored by operation.

Pathology.-The problems connected with the study of pneumococcal ulcer are of great interest, not only to the eye specialist, but also to the general pathologist. Leber has shown by experiment, that when the cornea is inoculated with micro-organisms the toxins they manufacture are speedily carried by diffusion to the surrounding parts, and set up an irritant action in blood-vessels at a considerable distance from the original seat of infection. Whenever, therefore, pneumococci find a field for action in an abraded cornea, they at once multiply and manufacture toxins, which are diffused in every direction. These toxins rapidly reach the periphery, and cause irritation of the circumcorneal vessels, which, becoming congested, allow blood plasma to exude and white blood corpuscles to migrate through their walls. This exudation of fluid and cellular elements causes chemosis of the conjunctiva, which is obviously an effort of Nature to overcome the disease. The fluid elements, rich in protective bodies, rapidly pass into the substance of the cornea to act on the microbes in such a way that the leucocytes are able to destroy them. The leucocytes also quickly force their way towards the spot where the infection is most acute. The nearer they approach this, however, the more intense becomes the action of the toxins, and at length, overcome by the poison, many perish and form a purulent zone round the ulcer. Those which survive enter the infective area to make war upon the microbes. Phagocytosis, however, depends in great measure on the supply of protective bodies the tissue is able to furnish, and although the cornea shares in the general immunity of the body, yet as it is an avascular structure, the destruction of the germs attacking it is neither so rapid nor so thorough as it is in other tissues possessing a better blood supply. If, therefore, the patient be old and weakly, it is easy to understand why a serpiginous ulcer spreads with great rapidity.

From the point of view just indicated, the cornea is perfectly passive, and the leucocytes wander through it to overcome the action of the microbes, or to be themselves overcome by the toxins as they near the area of infection. The cornea, when attacked by inflammation, is not, however, passive. On the contrary, it is intensely active, and its protoplasmic elements respond at once to the very slightest irritation. Throughout every part of the substantia propria are corpuscles united the one 
to the other by thread-like processes, so that in essence the corneal tissue may be regarded as a sheet of protoplasm, and in consequence one of the most vital structures of the body. In ordinary circumstances these corpuscles proliferate and multiply to provide for the growth and maintenance of the cornea, so it is not surprising to find that under the increased stimulus brought about by disease this physiological activity becomes greatly exaggerated.

Not only are the irritant toxins diffused superficially, they also penetrate to the deepest parts of the cornea. They are absorbed by the aqueous, and very soon induce congestion of the blood-vessels of the iris and ciliary body. . Leucocytes at once migrate towards the focus of infection, but as Descemet's membrane offers a barrier to their farther progress, some adhere to its posterior surface, while others fall to the bottom of the anterior chamber. It is this action of the toxins upon the highly vascular uveal tract that determines the occurrence of hypopyon. At one time it was thought that pus could only be produced by the direct action of the microbes themselves, and consequently it was believed that the pus in the anterior chamber came direct from the ulcer through a perforation in Descemet's membrane. Such a communication between the floor of the ulcer and the aqueous chamber only occurs after perforation of the cornea has taken place. An intact Descemet's membrane forms an insuperable barrier to the passage either of leucocytes or of bacteria from the ulcer to the aqueous, but it readily permits the diffusion of toxins. A hypopyon, therefore, is due to the irritant action of toxins, and occasionally pus can be seen actually rising up from behind the iris and entering the aqueous through the pupil. In many cases also the hypopyon is intimately mixed with fibrinous exudation which can be derived only from the blood-vessels of the iris and ciliary body. At first then the hypopyon is sterile, and so it will remain unless the cornea perforates and the micro-organisms pass through the perforation. It is, therefore, only after the ulcer has burst, and the pneumococci have gained entrance to the interior of the eyeball, that there is any danger of suppuration of the deeper structures of the globe.

Treatment.-The treatment of pneumococcal ulcer is both preventive and curative-

I. Preventive treatment.-There are many diseases of the eye which can be treated by the family doctor with satisfaction to his patient, and no little credit to himself ; but ulcus serpens cornece is not one of them, and whenever it has declared itself expert assistance at once becomes necessary. It must never be forgotten that the pneumococcus, which is the cause of the ulcer, is, when it attacks the eye, as destructive to sight as it is fatal to life when it invades the lung. Prophylaxis is, however 
a different matter, and in this, as in so many other diseases, prevention lies almost entirely in the province of the general practitioner, and can be carried out only with his skilled assistance. If he be well informed as to the ætiology of the disease, he will fully realise his own special responsibility in the matter, and recognise the importance of the following recommendations.

(I) Suppuration of the tear passages, is a frequent source of infection of an abraded cornea, therefore the general practitioner should advise immediate extirpation of the lachrymal sac in all patients suffering from dacryocystitis, if their occupation exposes them frequently to injury of the eye.

(2) The chief sufferers from serpiginous ulcer belong to the labouring classes, who are very prone to neglect slight injuries to the eye; therefore the general practitioner should do all in his power to warn them of the . dangers arising from neglect, and to teach them to seek medical advice with as little delay as possible. It is most important that the workmen themselves be made to understand the serious nature of pneumococcal ulcer, and much good might be accomplished if in the Ambulance lectures delivered to the employees of public works, the lecturer gave some general instructions regarding the care of the eyes, and warned the men that no injury to the eye is so trivial that it can be neglected with impunity.

(3) When infection has occurred, the ulcer may spread with great rapidity, and all chance of saving sight be lost within a few days; therefore, whenever the general practitioner is consulted by a patient suffering from serpiginous ulcer he should restrict himself to giving first aid, and then send the patient to a specialist with as little delay as possible. First aid in such a case consists in washing out the conjunctival sac with a warm solution of boracic acid, instilling a few drops of a 2 per cent. solution of cocain, examining the eye carefully by focal illumination, removing any foreign body from beneath the eyelids or from the surface of the comea, applying an ointment of I per cent. atropin, 2 per cent. cocain, and 3 per cent. iodoform, and covering the eye with a carefully adjusted pad and bandage. The patient ought then to be sent to a specialist, for it cannot be repeated too often or urged too strongly, that the sooner the eye is treated by an expert the more satisfactory will be the result. When left to themselves the patients rarely seek the advice of an ophthalmic surgeon until days or possibly weeks after the onset of the disease, and by that time sight may be irreparably damaged. It is true that many of these patients say that in the interval they have been using a lotion, which may or may not have been prescribed by a doctor, but that cannot in any way be regarded as efficient treatment of such a dangerous disease as serpiginous ulcer. 
The majority of the patients suffer during the most active period of their working life, and as many of them are rendered wholly or partially unfit for their ordinary work, the disease has considerable economic importance. It is difficult to estimate the loss in wages arising from inability to work during the period of illness and from diminished earning power afterwards, but the sum paid in settlement of claims for compensation is more easily calculated. An insurance manager has told me that in the company with which he is connected, serpiginous ulcers of the cornea form from 3 per cent. to 4 per cent. of all non-fatal underground accidents, and the amount actually paid in compensation for these injuries alone in a single year is little short of three thousand pounds. It is unfortunate that the pecuniary alleviation afforded by the Workmen's Compensation Act tends to make many workmen satisfied with diminished earning ability, and content to bear considerable physical inconvenience. Much of the money could be saved, and, what is of more importance, much of the suffering and hardship avoided, if all trivial injuries to the eye were skilfully treated at the time of their occurrence.

2. Curative treatment.-When the patient passes to the expert, a bacteriological examination ought at once to be made to confirm the diagnosis, and the tear passages should be examined carefully so that if any suppuration be detected, treatment may be begun by syringing, and it may be by extirpating the lachrymal sac. When the diagnosis has been established, and risk of infection from dacryocystitis prevented, the indications for further treatment are-

(I) To give the eye complete rest ;

(2) To relieve the pain;

(3) To destroy the infecting micro-organisms;

(4) To protect the eye from further injury; and

(5) To promote the rapid healing of the ulcer.

The patient ought to remain in bed with all bright light excluded from the room, and a calomel purge-3 to Io grains followed in three hours by a saline draught-is necessary in most instances; after the bowels have acted a distinct improvement in the condition of the eye is frequently seen. Atropin must be instilled from the very outset. When pain is acute and there is much inflammation of the iris, the good effect of the atropin is greatly increased by the application of leeches. In the earliest stages free bleeding from the temple always does good for the time being, and in many cases brings about a favourable turn in the course of the disease. Three to six leeches may be applied around the outer canthus, or a Heurteloup's artificial leech may be employed. In either case free bleeding from the wounds should be encouraged. The 
liquid extract of belladonna applied to the brow will sometimes ease pain and cause the pupil to dilate, when instillations of atropin have failed. When the ulcer is about to perforate, the use of eserin is preferable to that of atropin ; but with this exception, the former drug has in serpiginous ulcer a very limited sphere of influence, and almost invariably it is the latter which should be employed.

When the irritation is very severe and there is much pericorneal injection, great benefit is obtained from the application of fomentations; but a fomentation to be effective must be properly applied, and it should be repeated every two or three hours. It may be made more soothing by the addition of Battley's sedative solution of opium, to an infusion of chamomile flowers.

The chief end of treatment, however, is to compass the destruction of the pneumococcii in the substance of the cornea. Many antiseptics have been recommended for that purpose-pure phenol, tincture of iodine, bichloride of mercury, and sulphate or chloride of zinc being the most popular. The ordinary antiseptic, however, expends its energy upon every form of protoplasm with which it comes in contact, and, consequently, may be as destructive to the tissue of the cornea as it is to the invading microbes. For that reason treatment in infective diseases of the eye aims not so much at killing the bacteria directly, as at stimulating the natural defences of the body.

What is wanted is a drug which is at one and the same time poisonous for the pneumococcus and non-poisonous for the tissues of the body, or, as Wright says, it must be monotropic for the protoplasm of the pneumococcus. Professor Morgenroth has introduced such a drug in ethylhydrocuprein, and, as Wright points out, his experiments have furnished the first demonstration of the possibility of preventing and curing a bacterial, as distinguished from a protozoal or spirochætal infection, by the administration of a drug. Morgenroth's researches were undertaken to elucidate the pharmacotherapy of pneumonia. $\mathrm{He}$ experimented with derivatives of quinine, and found that ethylhydrocuprein and its salts exerted a bactericidal influence specifically upon the pneumococcus, and had only a very slight effect upon other microorganisms. Early in the course of his investigations he expressed the opinion that the drug ought to prove of great value in the treatment of serpiginous ulcer of the cornea, and encouraged his ophthalmic colleagues to put it to a clinical test. A. Leber, at the meeting of the Heidelberg Ophthalmological Congress of I9I3, was the first to record successful results ; and, since his communication, Goldschmidt, Schur, Darier, and others, have reported favourably on the drug, which from all accounts now bids fair to dominate the therapy of pneumococcal ulcer of the cornea. 
My personal experience amply confirms the favourable reports already published. Provided the pneumococcus is the infecting organism, cases treated by ethylhydrocuprein run a much shorter and conspicuously more favourable course than did those dealt with in earlier days by the methods formerly in use. After recovery there is only a thin cicatrix marking the site of the ulcer; and, consequently, either iridectomy or tattooing is much less frequently required.

The technique of treatment is simple. Ethylhydrocuprein hydrochloride, also known by the trade name of optochin, a white salt readily soluble in water, is employed in I per cent. or 2 per cent. aqueous solution. Before it is used, the floor of the ulcer should be thoroughly washed with a lotion-containing chinosol ( $\mathrm{I}$ in 4000 ) or quinine hydrochloride (3 grains to I fluid ounce), diluted with an equal quantity of warm waterand the boundaries of the ulcer should be demarcated by staining with an alkaline solution of fluorescein. This done, a swab of sterilised cotton wool-sufficiently large to cover the whole of the ulcerated surface, and saturated with a I per cent. aqueous solution of the optochinshould be applied to the ulcer, and kept in firm contact with its floor and margin for about two minutes. .Should the patient be sensitive, it is well to instil cocain drops beforehand, because the application of the optochin causes sharp burning pain, lasting from five to ten minutes. That irritation, however, soon lessens, and by the end of fifteen minutes there is complete anæsthesia of the cornea, which will last for fully half an hour. To begin with, these applications should be repeated two or three times daily - the good effect being maintained by instilling a few drops of I per cent. solution of optochin every hour during the day, while, during the night, a I per cent. optochin and atropin ointment may be applied as often as possible without interfering with sleep. Ethylhydrocuprein is sometimes applied by simple instillation alone, but the use of the swab and the hourly instillations combined will be found much more efficacious and satisfactory.

The grateful anæsthesia following the first application develops gradually, and is kept continuous by the repetitions. Relief from pain is therefore prompt and effectual; and in two or three days the ulcer is clean, all infiltration having disappeared from its margins, the hypopyon has become absorbed, and the pupil begins to dilate. After this, the applications of optochin need not be so frequent; but as there is, until healing becomes thoroughly established, considerable risk of re-infection, the instillations should be continued, and repeated three or four times a day until all danger is past.

The success of the treatment by ethylhydrocuprein depends upon the fact that the pneumococcus is the infecting organism, and that it is possible 
to kill the microbe in the substance of the cornea without injury to the cornea itself. Preliminary bacteriological examination is, therefore, essential ; because it is very difficult to distinguish, by clinical signs alone, a serpiginous ulcer due to the pneumococcus from one the result of infection by other micro-organisms, and, as has already been said, optochin has little or no effect on microbes other than the pneumococcus. In cases of mixed infection, it is, therefore, well to apply pure phenol; or, if the diplo-bacillus of Morax-Axenfeld be present, a 20 per cent. solution of sulphate of zinc, in addition to optochin, and this combined treatment is often attended by very satisfactory results.

It happens occasionally that healing proceeds very slowly, or improvement may come to a standstill, even in a case in which the pneumococcus has been demonstrated to be the cause of the ulcer. In such circumstances it is well to be sure that the optochin solution is freshly prepared, for the drug loses its efficacy so quickly that solutions more than a week old ought not to be employed.

From what has been said, it will be clear that the two essentials for the success of chemo-therapy in pneumococcal ulcer of the cornea are, first, beginning treatment at the earliest possible moment; and, second, the intensive applications of optochin during the first few days.

In this connection brief reference may be made to specific serum and vaccine therapy. Roemer has introduced and strongly advocates such treatment, and claims very satisfactory results. He employs it in conjunction with the older methods, and advises that it be used at the earliest possible moment, but warns us not to expect it to work any miracle of healing. One of the difficulties lies in the production of a stock vaccine which will be specific against the many varieties of pneumococci which may be present. That might be overcome by the preparation of an lautogenous vaccine; but such procedure would, of course, occupy a considerable time, and in the interval the cornea might be destroyed. Probably the best results will be obtained by injecting, as soon as possible after the patient comes under treatment, a large dose of a polyvalent stock vaccine prepared from pneumococci of high virulence. As the cornea shares but feebly in the general immunity of the body, the patient can be bled, and his own serum, enriched in antibodies by the action of the vaccine, applied directly to the ulcer, the instillations being repeated every two or three hours. By such means the healing of the ulcer can be hastened; and all clinical experience proves that the more quickly an ulcer heals the less will be the scar. In this connection the recent researches of Bonnefon and Lacoste are worthy of attention. These investigators have demonstrated on the eyes of rabbits that regeneration of transparent corneal tissue will follow an injury, provided the cornea 
has not been perforated, the operation has been carried out aseptically, and the wound has been protected from germs by a flap of conjunctiva.

After forty-eight hours of the treatment just outlined, most cases begin to show signs of improvement; but if the pain and other symptoms of irritation are in no way relieved, if the pupil does not dilate, and if the ulceration progresses, the actual cautery should be applied without delay. To be of any service, however, the cautery must be used thoroughly; and as the infection of the cornea extends beyond the apparent margin of the ulcerated surface, operation should not be attempted until the boundaries are demarcated by instilling an alkaline solution of fluorescein. The resulting bright green line then serves as a guide, a fine platinum wire heated to a dull red being applied to the cornea just outside of it, and the burning continued until every part of the infiltrated margin is thoroughly destroyed. As healing will be greatly promoted by the reduction of the intraocular tension, the cornea should be punctured at its lower aspect with a keratome, and the anterior chamber slowly emptied. The evacuation of the aqueous and of the hypopyon is always attended by excruciating pain, owing to the displacement of the iris and ciliary body, and on the surface of the former hæmorrhages are often visible. If, however, a fomentation be at once applied to the eye, and $\frac{1}{6}$ grain of morphia injected under the skin of the temple, the patient's sufferings are mitigated so much that in a short time he falls asleepfor the first time perhaps since the onset of the disease. If the cauterisation has been properly performed, healing begins at once, and proceeds rapidly; and, unless fresh infection take place, the progress of the case will be uninterrupted until cicatrisation is complete, the resultant cicatrix being usually much less than could have been anticipated from the extent and virulence of the ulceration. Fresh infection is always accompanied by recurrence of pain, and is in many instances due to carelessness, either on the part of the patient himself, or of those in charge of his case. Rest in bed, and the careful application of a bandage until the ulcer has healed completely, are essential parts of the treatment; and if, after sepsis has been overcome, these precautions be rigidly followed, there will be no relapse. Rapid healing will be promoted by the protection afforded to the eye by the bandage, or by a shield; and cicatrisation is hastened by the use of 5 per cent. scarlet red ointment, or by covering the ulcer, after it is thoroughly disinfected, with a flap of conjunctiva.

It sometimes happens that through neglect the cornea has been destroyed so extensively before the patient comes under expert treatment that it is not possible to apply the cautery. In these circumstances the cornea ought, as Guthrie and Saemisch recommended, to be freely incised through the ulcer with a linear section knife, to permit the escape 


\section{SERPIGINOUS ULCER OF THE CORNEA}

not only of the hypopyon, but also of the pus contained between the corneal lamellæ. The wound ought to be kept open for several days to facilitate drainage. This treatment has the obvious disadvantage of allowing the iris to become extensively adherent to the wound in the cornea; but its use in desperate conditions is always to be recommended, because some sight may thereby be saved in cases which would otherwise inevitably result in total blindness, if not in complete destruction of the eye. 


\section{CHAPTER XVIII}

\section{OPACITIES OF THE CORNEA}

Causes-Varieties of resulting cicatrices-Prognosis-Treatment-KeratoconusKeratoglobus-Arcus Senilis.

In the great majority of cases, an opacity of the cornea is simply a cicatrix, resulting from an ulcer which has healed. It occurs most frequently and in its most serious form in those who have suffered from purulent conjunctivitis, strumous ophthalmia, or serpiginous ulceration. It is seen, however, after non-suppurative keratitis, or after degeneration of the superficial layers of the substantia propria and the subsequent deposition of lime salts; it may also come on spontaneously with the advance of years unaccompanied by any sign of irritation-arcus senilisor it may be congenital.

The epithelial layer is the only part of the cornea which is replaced without loss of transparency.

An ulcer which has involved the substantia propria always leaves a scar, which is dense and permanent in proportion to the severity of the previous inflammation and the loss of corneal substance. The cicatrix receives different names according to its density, being called a nebula when it is only faintly visible, a macula or an albugo when it is more pronounced, and a leucoma when it is prominent and milky white. Fuchs has pointed out that from the shape and position of an opacity a good idea can be formed of its origin. A macula situated at the margin of the cornea, or forming a faint streak across its surface, is almost certainly due to strumous ophthalmia, while dense, white scars, occupying a large area of the centre of the cornea, are likely to follow purulent conjunctivitis, or serpiginous ulceration. A linear or a punctiform cicatrix is probably the result of an injury or of an operation wound. Linear opacities in the substance of the cornea, and opacities of triangular shape, the base being situated at the periphery, are evidence of a past interstitial keratitis, while bluish-white opacities at the corneo-scleral margin and projecting into the transparent cornea indicate that there has been inflammation of the sclera and the underlying portion of the uveal tract.

The permanence of a cicatrix is largely dependent upon age-the 
younger the patient the more chance there is that the opacity will lessen and the cornea recover its transparency; moreover, a cicatrix following non-suppurative keratitis is much less likely to be permanent than one which is the result of ectogenous infection and suppuration. Time also may work in its favour, but, as a general rule, no change is likely to take place after the lapse of two or three years. The impairment of vision due to a corneal opacity depends not only on its size, its density, and its situation with regard to the pupil, but also on the amount of distortion of the cornea which has been brought about by the contraction of the cicatrix, and the resulting dispersion of the light. Even a slight nebula may interfere greatly with sight; for it so alters the condition of the surface of the cornea, as to give rise to a degree of irregular astigmatism which no lens will correct. The patients usually complain of short sight, and in those whose vision has been seriously impaired in infancy squinting and nystagmus are apt to develop as the child grows older.

When an ulcer goes on to perforation, it is always followed by a leucoma; and in that case the iris is adherent to the posterior surface of the cornea-leucoma adherens-the pupil being at the same time altered in shape and drawn towards the cicatrix. It is very important to determine whether the iris is included in an old corneal scar. A cicatrix of that kind is usually peripheral in situation, round in shape, and made up of a margin of dense white fibrous tissue, and a thin central portion somewhat dark in colour from the presence of the incarcerated iris.

It sometimes bulges beyond the level of the surrounding corneaectasia-and it then is a real source of danger to the eye, because the epithelium covering it readily becomes abraded, and a way is opened through which micro-organisms can enter the interior of the globe and infect the whole uveal tract. The serious menace of panophthalmitis is not, however, the only danger to which such an eye is exposed. The apposition of the iris to the cornea, and the dragging of the pupil towards the scar, cause partial obliteration of the corneo-iridic angle and blocking of the anterior filtration spaces. When that occurs, the damaged eye becomes hard and painful; and, unless means be taken to prevent it, sight will be lost from secondary glaucoma. That distressing sequela rarely, if ever, follows a simple leucoma; but whenever there has been perforation of the cornea, and the iris has prolapsed and become adherent to the scar, increase of intraocular tension is certain to occur if the cicatrix be prominent, or the margin of the pupil is so firmly gripped that there is little dilation following instillation of a mydriatic.

When the destruction of corneal tissue has been very extensive, the iris prolapses after perforation has occurred, and the resulting cicatrix 
is composed chiefly of iris and fibrous tissue. 'In bad cases of purulent conjunctivitis, the rupture of the cornea is so large that it causes dislocation of the parts within the eye, and the crystalline lens may escape. In these circumstances there is retraction of the cicatricial tissue, and the cornea flattens-applanatio cornece-owing to the dense scar tissue obliterating completely all trace of aqueous chamber. In the majority of cases, however, the lens does not escape, and the force of the intraocular pressure is greater than the resisting power of the cicatrix, which consequently bulges forwards to form an anterior staphyloma. When the whole cornea has been replaced by a protuberant cicatrix, the staphyloma is total, in contrast to the cases in which some transparent cornea has been left and an aqueous chamber has been so far preserved. In these circumstarices the staphyloma is described as partial. When there have been several perforations, the staphyloma is lobulated, and the racemose appearance is intensified by the development of firm cicatricial bands between the lobules. In its origin, therefore, an anterior staphyloma is really a prolapse of the iris, which becomes covered with granulation tissue to fill the gap due to the destruction of the cornea. When the parts have become firnly soldered together, the granulations are converted into cicatricial tissue, which subsequently becomes white or slate-coloured, and several large blood-vessels pass from the conjunctiva over its surface. In every staphyloma, therefore, the anterior chamber is obliterated, while the posterior chamber is greatly enlarged. The consequence is that there is always increase of intraocular tension, a very important factor in the growth of the staphyloma, which, always very disfiguring, 'may be so protuberant that the lids will not close over it. There is then danger of ulceration and rupture, followed by intraocular hæmorrhage, irido-cyclitis, or panophthalmitis. In some cases, the sclerotic surrounding the cornea, and the ciliary region becomes affected and gives rise to a general distension of the globe. It occasionally happens that, after perforation, the opening in the cornea does not close properly, and a fistula remains from which the aqueous drains away constantly. If the opening does not close within a short time, the nutrition of the eyeball suffers severely, and the globe softens and shrivels, and undergoes those degenerative changes which are grouped under the term phthisis bulbi.

Prognosis.-In young children an opacity of the cornea may clear in a wonderful manner; and that favourable result is all the more likely to occur if the ulcer has healed rapidly. The absorption of an opacity can be hastened by the use of stimulating applications, but those must never be employed until all signs of irritability have passed away. It is important, therefore, to distinguish between an opacity which is recent 
and one that is of old standing. A recent opacity usually appears grey and dull, with ill-defined borders and evidence of vascularity; whereas an old opacity is bluish-white and smooth, and its borders are well demarcated from the surrounding cornea. Moreover, an opacity never clears to any appreciable extent if it is either depressed beneath the level, or projects beyond the surface, of the surrounding cornea; nor will it recover its transparency if there has been in the course of the ulceration either a hernia or a rupture of Descemet's membrane. Speaking generally, the chance of absorption diminishes with the age of the opacity and the sharp definition of its boundaries; and it will always be permanent if signs of fatty or calcareous degeneration are present, or if, without any apparent cause or sign of irritation, blood-vessels develop in its substance.

Treatment.-The remedies chiefly employed to promote absorption are the yellow or the red oxides of mercury, iodide of potassium, calomel dust, and dionin. Ointments are prescribed with massage to the cornea. A piece the size of a split pea is well rubbed into the eye twice a day, care being taken that the patient looks straight forward, in order that the massage may be applied directly to the cornea through the closed upper lid. If a full measure of success is to attend this treatment, it must be carefully carried out and persevered with for many months, during which time the stimulants employed should be varied, and their strength graduated in such a way as to promote absorption of the opacity without exciting inflammation in the eye. Vision improves as the cornea becomes more transparent, but as its curvature is always altered, carefully adjusted sphero-cylindrical glasses are required to enable the patient to get the full use of his sight. A stenopæic slit will sometimes improve vision to a most gratifying extent, as by directing the light through the transparent portion of the cornea selected, it lessens the dazzling produced by diffusion. If the opacity be situated in the centre of the cornea, and if no further improvement can be expected from the use of drugs or of lenses, an optical iridectomy should be performed. The opening in the iris ought to resemble a slit, and be placed opposite the portion of cornea which is clearest and most regularly curved. Vision, moreover, is improved and disfigurement greatly lessened if the opacity be carefully tattooed with Chinese ink.

If perforation of the cornea has occurred, the chief aim of treatment is to prevent the occurrence of staphyloma. Sometimes, however, chiefly owing to the large size of the perforation, but also to want of proper care on the part of the patient or his medical attendant, the remains of the cornea and iris bulge forward, forming a protuberant cicatrix which partially involves or wholly replaces the cornea. In partial staphyloma 
an iridectomy ought to be performed as soon as possible. The coloboma in the iris is placed to ensure the best sight; but the chief benefit of the operation is that, if properly performed, it lowers intraocular tension and thereby diminishes the dangers incident to secondary glaucoma, and also encourages the formation of a flat cicatrix. In total staphyloma the anterior segment of the eyeball should be excised. This procedure possesses two great advantages over enucleation; it leaves a freely movable stump on which an artificial eye can be adjusted; and in the case of young children it does not interfere with the growth of the orbit. If strict attention be paid to secure asepsis, and the operation be carefully performed, there is little danger of sympathetic inflammation of the fellow eye. When, however, the whole eyeball has become distended owing to inflammation of the anterior portion of the sclera and the underlying ciliary body and choroid, nothing short of enucleation must be attempted. The deformed and sightless globe is not only very disfiguring, but, owing to glaucomatous changes, it is liable to become hard and painful ; consequently, all things considered, the sooner it is got rid of the better for the patient.

Malformations of the cornea also occur where its transparency is retained. This is seen in the conditions described as keratoconus, and keratoglobus

I. Keratoconus, or conical cornea, often appears for the first time about the age of puberty, and is seen most frequently in young women who are in delicate health. The first symptom complained of is short sight, but ordinary concave spherical glasses do not afford much relief. As the disease increases the disturbance to vision becomes very great; everything is surrounded by a halo; polyopia is often very troublesome; and as both eyes are usually affected, the patient may be not only incapable of distinguishing objects only a few feet distant, but also quite unable to read. The development of conical cornea is unaccompanied by any symptom of inflammation; but at the outset those who suffer from it complain occasionally of headache, and of a full, uncomfortable feeling in the eyes themselves, which are often unnaturally brilliant, and present a peculiar lustrous appearance. At this early stage it may not be easy to detect the conicity of the cornea by simple inspection of the surface; but if the patient be placed opposite a window a few feet away, the image will appear misshapen. These changes are more easily observed when the eye is examined with a keratoscope or Placido's disc-a flat disc painted with broad black and white concentric circles, with a hole in the centre through which the reflex on the cornea is observed. In conical cornea the reflex is distorted. If the fundus be examined by the ophthalmoscope, its details will be seen distorted. In advanced cases 
the prominence of the cornea is very evident, whether looked at from the front or from the side: while in nearly every instance where the disease has existed for many years, oblique illumination reveals a minute nebulous opacity at the apex of the cone, and small irregularities in the curvature around its summit. These changes are all due to a progressive thinning of the substantia propria of the cornea, whereby it becomes so weakened as to be unable to resist the normal intraocular pressure.

Treatment.-In the early stages of conical cornea, high-power cylindrical or hyperbolic lenses may improve vision, but after the disease is well established operation is necessary. The most satisfactory procedure is that devised by Sir Anderson Critchett. He applies to the cornea at a very low heat a galvano-cautery with a flat electrode. With that he chars the surface of the cone and an area of tissue round about it; within the charred area he makes a second application at a slightly increased temperature; and within that second zone he burns deeper still, using a smaller point at a dull maroon heat, but stops short of perforation. $\mathrm{He}$ therefore creates two zones and a small central spot, the whole bearing some resemblance to a target, and exercising a graduated but most efficient pressure on the part affected, with the object of producing flattening of the cone. This operation usually results in considerable improvement in sight, and an optical iridectomy is not often required, because, if the cauterisation be carefully performed, the resulting cicatrix is much smaller than that obtained by any other means.

2. Keratoglobus is a condition in which the eyeball is so much enlarged that it resembles the eye of an ox. The cornea is unnaturally large and bulging, and may be perfectly transparent or clouded by opacities. The sclerotic is greatly distended, very thin, and of a dark slate-blue colour. The anterior chamber is abnormally large and deep, and the iris trembles with every movement of the eye. If the increased tension be maintained, atrophy of the optic nerve, and cupping of the disc will ensue, and when the disease has reached an advanced stage, intraocular hæmorrhage is prone to occur.

Treatment.-Keratoglobus, fortunately, is not a common disease, and little can be done for its relief. Sight is ultimately lost through the persistence of increased intraocular tension, and the occurrence of intraocular hæmorrhage. The only operations likely to be helpful are anterior sclerotomy, and trephining at the corneo-scleral margin. In the early stages attempts should be made to correct the error of refraction. Atropin should never be instilled; but the daily use of I per cent. pilocarpin is advisable.

Arcus Senilis, or Gerontoxon Gorneæ.-After middle life is reached, a 
bluish-white opaque ring is often seen encircling the cornea, a narrow transparent strip of which separates it from the limbus. At first it is of semi-linear shape, and is situated beneath the upper eyelid, but by and by a similar opacity appears beneath the lower lid, and the two crescents continue to grow until their horns meet. The arcus thus formed presents a perfectly smooth surface, and its outer edge, towards the limbus, is dense and sharply defined, while the inner is concave, and gradually shades off into the transparent cornea. There is no inflammation, the appearance being due to a deposit of fatty particles in the substantia propria at the periphery of the cornea. It is of no practical importance, and does not in any way interfere with the healing of a wound carried through it, e.g. in the extraction of a cataract. Its presence, however, is sometimes coincident with the occurrence of other changes indicative of the approach of old age, such as grey hair, ossification of cartilage, degeneration of blood-vessels, etc., and. although by no means pathognomonic of that condition, yet it is frequently associated with fatty degeneration of the heart, and of other senile changes in the cardiovascular system. Taken by itself, however, it is of no diagnostic importance. It occasionally develops at a comparatively early age, and is sometimes congenital-arcus juvenilis. 


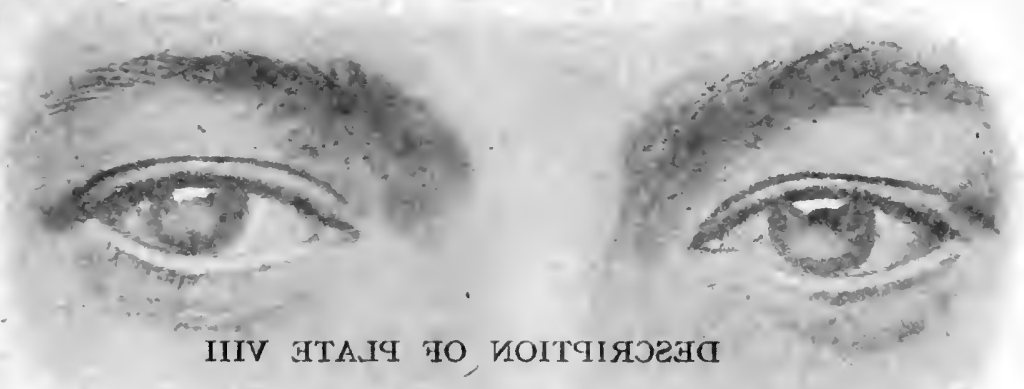

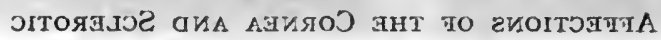

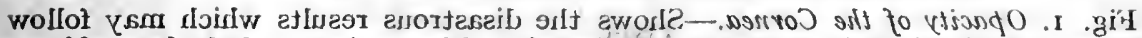

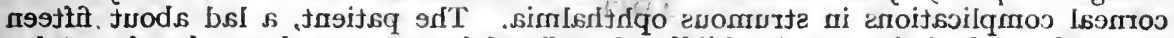

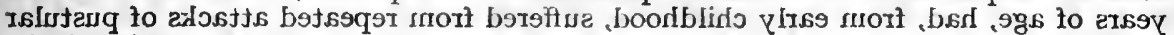

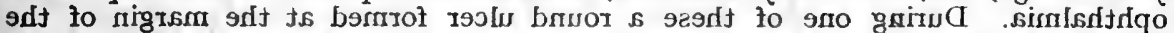

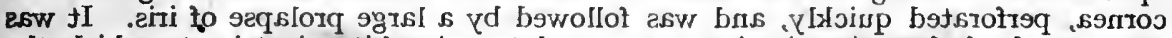

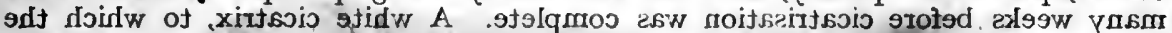

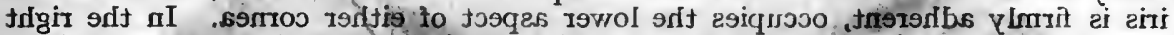

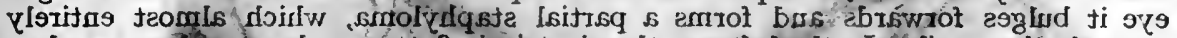

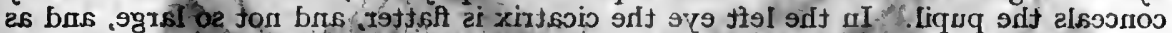

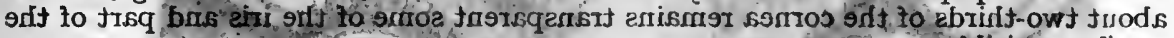

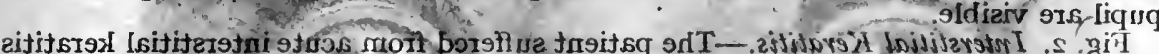

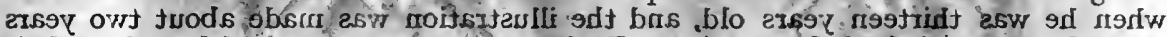

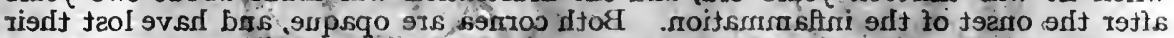

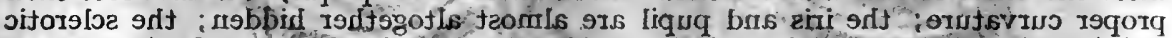

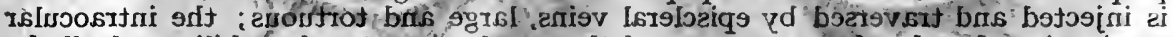

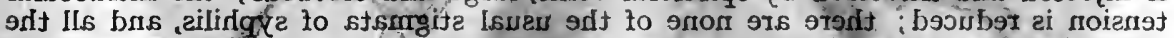

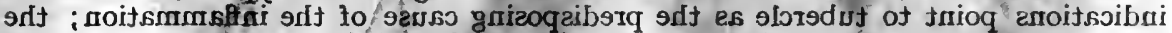

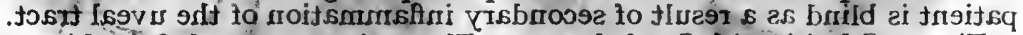

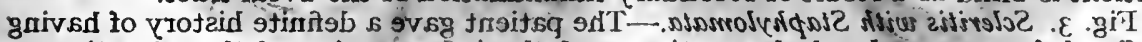

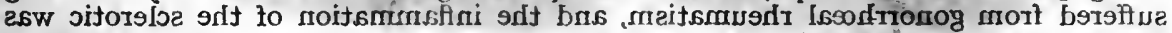
र

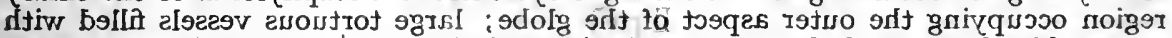
mott rsluggrni liquq

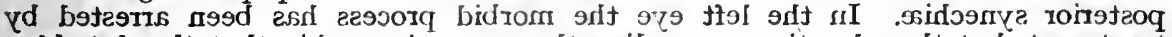

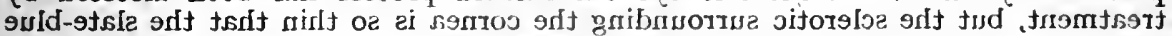

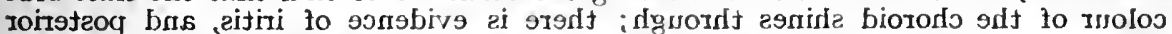
s9odnonog dfiw b9Josłni essw bns sgo to ems97 owt-ү

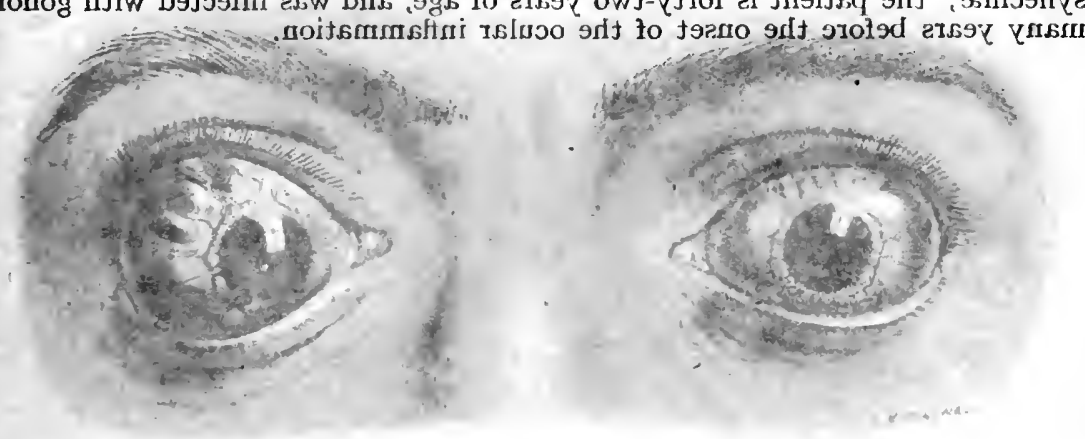


bluish-w 19, "yague ring is nften seen encircling the cornea, a narrow

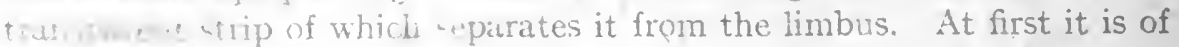

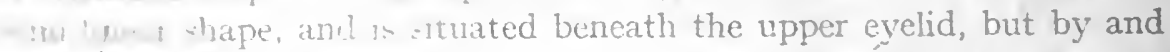

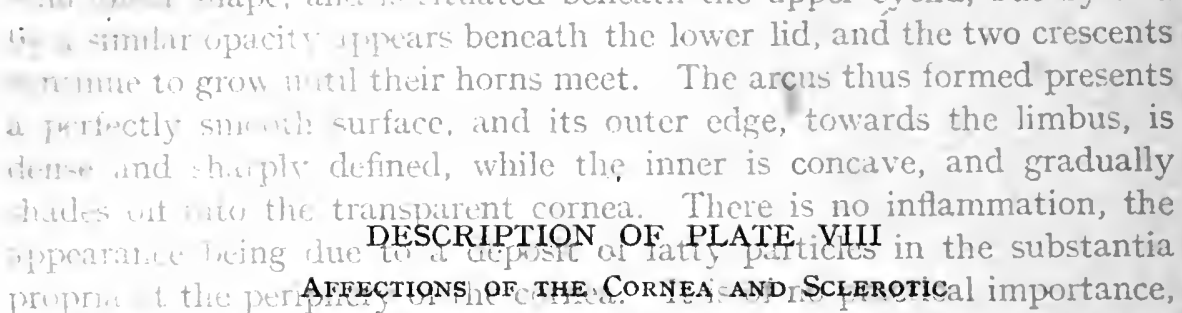

Fig. I Opacity of the Cornea Shows the disastrous rasults which may follow corneal complications in strumous ophthalmia. The patient, a lad about fifteen years of age; had, from early childhood, "suffered from repeated attacks of pustular ophthalmia. CDuring one of these (a roind ulcer formed at the margin of the cornea, perforated quickly, and was followed byia large prolapse of iris:tillt was many weeks before cicatrisation was complete. A white cicatrix, to which the iris is firmly adherent, occupies the lower aspect of either cornea. In the right eye it bulges forwards and forms a partial staphyloma, which almosit entirely conceals the pupil. In the left eye the cicatrix is flatter, and not so large, and as about two-thirds of the cornea remains transparent some of the iris and part of the pupil are visible.

Fig. 2. Interstitial Keratitis. - The patient suffered from acute interstitial keratitis when he was thirteen years old, and the illustration was made about two years after the onset of the inflammation. Both cornea are opaque, and have lost their proper curvature; the iris and pupil are almost altogether hidden; the sclerotic is injected and traversed by episcleral veins, large and tortuous; the intraocular tension is reduced; there are pone of the usual stigmata of syphilis, and all the indications point to tubercle as the predisposing cause of the inflammation; the patient is blind as a result of secondary inflammation of the uveal tract.

Fig. 3. Scleritis with Staphylomata.-The patient gave a definite history of having suffered from gonorrhœal rheumatism, and the inflammation of the sclerotic was clearly of gonococcal origin. In the right eye there is a staphyloma of the ciliary region occupying the outer aspect of the globe; large tortuous vessels filled with venous blood surround the cornea; the iris is inflamed; the pupil irregular from posterior synechiæ. In the left eye the morbid process has been arrested by treatment, but the sclerotic surrounding the cornea is so thin that the slate-blue colonr of the choroid shines through; there is evidence of iritis, and posterior synechiæ; the patient is forty-two years of age, and was infected with gonorrhoea many years before the onset of the ocular inflammation. 

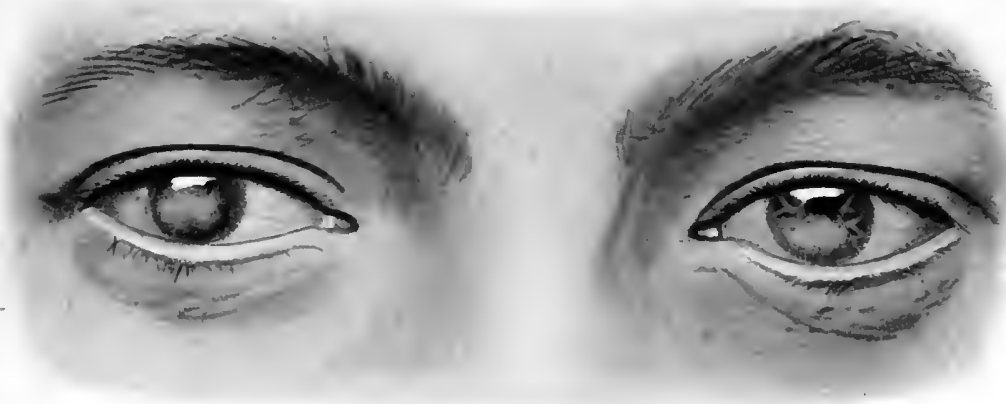

FigI

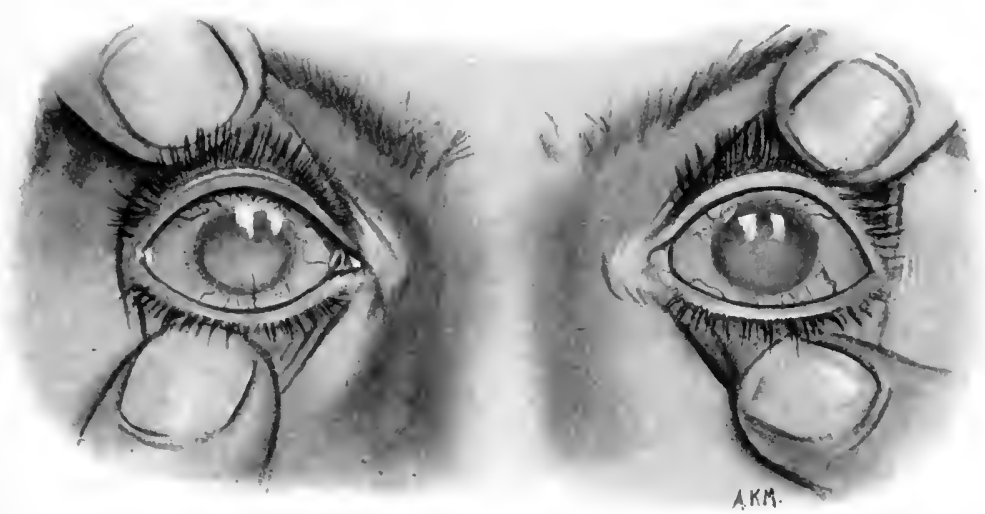

Fig II

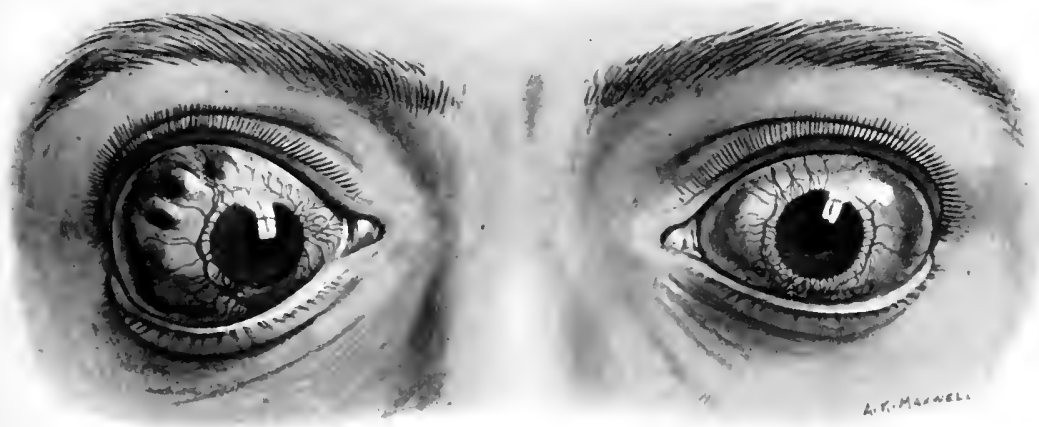

Fig III 



\section{CHAPTER XIX}

\section{INTERSTITIAL KERATITIS-NON-SUPPURATIVE KERATITIS}

Varieties-Signs and symptoms-Complications-Stigmata-Treatment.

INTERSTITIAL or parenchymatous keratitis is an inflammation of the substantia propria of the cornea. It may be circumscribed, when it occurs as an opacity which varies in shape-triangular, bandshaped, annular, etc.-and is usually associated with inflammation of the sclerotic; or it may be diffuse, when the opacity implicates the whole of the middle layers of the cornea, and in these circumstances it is always a sign of constitutional disease. The diffuse form of interstitial keratitis usually attacks those predisposed to it during early life, for the most part between the ages of five and twenty years. It is seen more frequently in girls than in boys; and the onset of an attack is often attributed to an injury, which on inquiry may be found to have been very trivial. The explanation of this special vulnerability is that the disease, in the vast majority of cases, some say 90 per cent., is the result of inherited syphilis. Careful inquiry into the history of the case will often elicit the fact that the patient suffered in infancy from snuffles, cutaneous eruptions, or other signs of congenital syphilis, and that the mother has had one or more miscarriages, or a still-born child, before she gave birth to the patient. The disease occurs, although very rarely, in the course of acquired syphilis, when the keratitis may confine itself to one eye, and although the symptoms may be very severe, yet, with appropriate treatment, recovery may be rapid and complete. Tubercle also is an important ætiological factor, and accounts for the causation of a small percentage of the cases. The inflammation of the cornea is usually secondary to tuberculosis of the iris and ciliary body, and the keratitis, as a rule, commences in Descemet's membrane, from which it spreads to the substantia propria of the cornea. The prominent symptoms may be described as follows-

At first the patient has difficulty in facing the light, and when the eye is examined a few small greasy-looking spots are to be seen in the substance of the cornea, the surface of which is dull, as if it had been breathed upon, but is not as a rule abraded. The inflammation, starting 
from these foci, spreads rapidly, until the cornea loses its transparency, and comes to resemble a piece of ground glass. Through this opacity it is impossible to see the iris distinctly, and in many cases it may be hidden completely. The blood-vessels of the limbus are congested; and the amount of this pericorneal injection, taken along with the degree of photophobia and lachrymation, affords a good indication of the severity of an attack. Fringes of minute blood-vessels not only traverse the surface of the cornea, but also invade its substance, with the result that the ground-glass appearance is lost, and the cornea takes on a rusty colour, and in some cases resembles a piece of red cloth, so great is the vascularity. Since these newly-formed blood-vessels encroach from the margin, there is generally in the centre a non-vascular area of a yellowishwhite colour, somewhat resembling an abscess; but the formation of pus or the occurrence of ulceration is exceedingly rare. In the most severe cases the cornea becomes greatly thickened and bulges forward, so that the eye appears to be in a hopeless condition; but, in spite of those disquieting appearances, the cornea in the end generally clears up and assumes its proper curvature, and useful vision is restored.

When the appearances have reached the stage just described, 'vision is reduced to a perception of hand movements, the intolerance of light being so extreme that, for the most part, the eyes are kept firmly closed; and the blepharospasm and lachrymation contribute to form a fissure at the outer canthus, which causes intense pain whenever the eyelids are forcibly separated. In a time varying from a few weeks to several months, the vascularity lessens, and, with the disappearance of the blood-vessels, the cornea begins to clear, after which it will, in a mild case, regain its transparency in a comparatively short time. The cornea clears from the margin towards the centre, which may remain opaque, although, as a rule, it also recovers after many months. It may be here stated, however, that when a mother is infected at conception, or during the early months of pregnancy, the child will suffer acutely, and, should keratitis ensue, it will always be severe, and may take years to run its course.

Eyes that have been affected by interstitial keratitis are easily recognised by a peculiar lack-lustre appearance of the iris, and the increased depth of the anterior chamber. When the cornea is carefully examined by a magnifying glass, fine brush-like vessels, empty of blood, are seen deep in its substance, and sometimes ophthalmoscopic examination will detect the scars of a pre-existing disseminated choroiditis. The disease is almost always symmetrical, but it is rare for both eyes to be attacked simultaneously, and generally just as the one is recovering the other begins to suffer. Mild cases last on an average from three to nine or 
twelve months, but many run a much more protracted course, in spite of careful medicinal and hygienic treatment. The more severe the attack the denser is the infiltration of the corneal substance, and the less likely it is that there will be a perfect recovery. Permanent nebulous opacities are apt to remain, and these, although so slight as to be visible only on careful examination of the eye, may interfere greatly with vision. Moreover, the curvature of the cornea may be so much altered that a high degree of astigmatism results. In all severe cases the iris becomes implicated, and the inflammation, travelling backwards, involves the ciliary body, choroid, and retina. These complications are all the more likely to occur if during the course of the keratitis any acute illness supervenes. In the most unfavourable cases, the anterior zone of the sclerotic participates in the inflammation, and the adjacent portion of the cornea becomes permanently opaque. At other times the corneoscleral ring becomes stretched, and staphylomata occur, which by their coalescence form a large bulging mass, of a dark slate colour owing to the uveal pigment showing through the thin sclerotic, and surround a part or even the whole of the circumference of the cornea. In the worst cases, the cornea remains more or less opaque, loses its natural curvature, and becomes flattened. When it remains sufficiently transparent, it will be seen that the pupil is contracted and fixed, and blocked by inflammatory exudate and cataractous lens. The intraocular tension is always reduced, and vision only equal to a poor perception of light. Synovitis of the elbow and knee-joints, periosteal swellings on the long bones, enlarged painless lymphatic glands in the neck, perforation of the soft palate, etc., most frequently occur in those cases where implication of any portion of the uveal tract has taken place.

Interstitial keratitis, when due to hereditary syphilis, is always associated with certain peculiarities of physiognomy; and although all these appearances are rarely to be seen in any one patient, yet when even a few of them are found in combination they constitute most trustworthy evidence of the existence of hereditary taint. The conformation of the face is somewhat angular, the features are contracted and drawn, the skin coarse, and the complexion pale and earthy. The forehead is prominent, and the skin covering it is thrown into wrinkles through the frowning incidental to the long suffering from fear of light. The bridge of the nose is depressed, and may be completely sunken, through loss of the bones from syphilitic ulceration: from a similar cause affecting the skin, the angles of the mouth and the alæ nasi are scarred and fissured by white cicatrices. The palate is generally high and narrow, the teeth stunted in their growth, peg-shaped, widely spaced and irregularly placed, and most characteristic of all is the notching of the upper central 
incisors of the permanent set--Hutchinson's test teeth of inherited syphilis.

Some of the patients are abnormally precocious, while others are dull and stupid. In a certain proportion of cases there is suffering not only from defective sight, but also from deafness. This last is sometimes due to disease of the middle ear, connected with ulceration of the throat and perforation of the palate, and then the power of hearing may to a certain extent return as the otitis media improves. When, however, there is inflammation of the auditory nerve the hearing is lost rapidly, sometimes within a few days, and the patient remains stone deaf for the remainder of life. Deafness, a peculiar formation of the teeth, and keratitis form the triad of Hutchinson in the diagnosis of hereditary syphilis; but of these three keratitis is by far the most important.

Treatment.-At the very outset, the general practitioner should warn the child's parents that the disease will run a very protracted course, and that in all probability the second eye will become affected, no matter what treatment is employed. He will also be well advised to ask for a consultation with a specialist, because, as the eye will certainly get worse during the early stages, the parents in all probability will become dissatisfied, and fail to carry out treatment from which they think so little good is resulting.

The treatment must be both local and constitutional, and will vary according to the stage of the disease.

I. During the period of progression.-During this stage all irritants must be avoided, and none but soothing remedies prescribed. The eyes should be douched at regular intervals with a hot infusion of poppy heads and chamomile flowers, and a I per cent. solution of atropin instilled two or three times a day; but the effect of the drug both on the pupil and on the intraocular tension, must be carefully watched. If the pain be severe, it usually means that the uveal tract is implicated, and is consequently a measure of the need for the use of atropin. No bandages are permitted, but the eyes should be shaded from bright light by smoked glasses.

2. During the period of retrogression.-Whenever the irritability and vascularity begin to pass off, absorption of the exudate in the cornea may be stimulated by massage with an ointment containing $\frac{1}{2}$ per cent. freshly precipitated yellow oxide of mercury. The ointment is used twice a day, and if well borne the percentage of the yellow oxide is increased gradually until the limit of tolerance is reached. Its use must be kept up for weeks and months, with regular intermissions during which calomel dust is substituted. A favourite remedy with the older practitioners was red precipitate of mercury, triturated thoroughly with white 
sugar $(3 \mathrm{j}$ to $3 \mathrm{j}$ ) into an impalpable powder, a small quantity of which was dusted from a camel-hair brush on the surface of the cornea or blown into the eye through a quill. Vinum opii, another of the older remedies that ought not to be forgotten, may be used as an instillation once a day, combined with atropin and boracic acid. If there be pain and irritability, dionin, in from 5 per cent. to Io per cent. solution, acts better than mercurial preparations; but, as the eye soon becomes accustomed to the drug, the strength of the solution must be regularly increased, and there is consequently no advantage in its frequent application : no more than one or two instillations are advisable in the twenty-four hours. If there be great intolerance of light and persistent blepharospasm, nothing does so much good as the careful but thorough blistering of the upper lids with solid nitrate of silver. It happens not infrequently that when the œdema due to the blistering subsides, the patient opens the eyes spontaneously, and a turning-point is reached in the course of the disease. When the cornea appears swollen and gelatinous, and threatens to bulge, it may be necessary to support the eye with a bandage, but there can be no doubt that in the treatment of interstitial keratitis the less bandaging the better.

3. General or constitutional treatment.-From the very outset mercury in some form must be prescribed. Small doses of grey powder, combined with sulphate of quinine, can be given night and morning for weeks on end, without fear of the occurrence of ptyalism or any other toxic symptom : on the contrary, children suffering from interstitial keratitis thrive and gain weight while they are taking grey powder and quinine. In the more severe cases mercurial inunction is required. Oleate of mercury ointment is to be preferred on the ground that it is more cleanly than ordinary mercurial ointment. A piece the size of a large bean should be well rubbed into the skin of the inner side of the thighs, the arms, or the axillæ, etc., every day, and the rubbing should last from ten to twenty minutes. A different part of the body must be selected for each inunction, to avoid irritation of the skin. If the child be anæmic, small doses of Fowler's solution in combination with syrup of iodide of iron are always useful, and may be prescribed along with mercurial inunction. At the beginning of the disease an intravenous injection of a medium dose of one of the Salvarsan group of preparations, usually acts promptly in reducing the blepharospasm, but it has no influence in either cutting short the attack, or in preventing the second eye from becoming affected. Sometimes, however, the improvement following an intravenous injection of Salvarsan is rapid and decided; but it should be clearly understood that it only prepares the way for mercury, which must always be regarded as the mainstay of the treatment. In the later stages, cod-liver oil and 
the iodides are of value; but if preparations like the syrup of the iodide of iron are prescribed, calomel dusting must not be employed at the same time. The cure of the patient is greatly promoted by judicious feeding, and regular exercise in the open air. It will be a great advantage if children suffering from interstitial keratitis can be treated not in the town, but in the country, where fresh eggs, butter, milk, and cream will naturally form the principal part of the dietary. 


\section{CHAPTER XX}

\section{THE DIAGNOSIS AND TREATMENT OF IRITIS}

Idiopathic iritis-Clinical description "Quiet Iritis "-Acute Iritis-SymptomsSequelæ-Treatment.

Inflammation of the iris occurs as a complication in diseases of the conjunctiva, the sclerotic, or the cornea, and it may appear in the course of both retinal and choroidal affections, but it also exists as an independent disease. Statistics show that idiopathic iritis occurs in from 2 to 4 per cent. of all ocular disorders, that it attacks men more commonly than women, and that it is met with most frequently in young adults, although no age is exempt. The cause most usually assigned for its onset is exposure to cold, but as the disease depends largely upon certain constitutional states, a predisposing, in addition to an exciting cause must always be sought for when investigating the ætiology of an attack. In some cases inflammation of the iris is due to direct injury, and hence it occurs frequently after a blow, a penetrating wound, or an operation upon the eye, more particularly if the iris has been much bruised and lacerated, and if the lens capsule has been ruptured. Traumatic iritis is usually accompanied by purulent exudation, the suppurative process depending upon the direct action of micro-organisms, upon the toxins manufactured by them, or upon chemical influences such as result from the presence of metallic bodies within the eye.

The following is a brief clinical description of a case of idiopathic iritis. The patient complains that whenever he looks at the light the eye feels tender and waters, and that the sight is blurred. He may also complain that he feels out of sorts, suffers from headache and loss of appetite, and is feverish and restless. The pulse may be quick, the tongue foul, the bowels constipated, and the urine high-coloured and scanty. There is also pain of a neuralgic character, not so much in the eyeball itself as in the forehead, the cheek, and the side of the nose. It is usually more severe at night than during the day, and is often markedly periodic, coming on in paroxysms about the same hour, and very frequently waking the patient from sleep, but no matter how severe the pain may be it is rarely, if ever, accompanied by vomiting. At first a faint pink blush appears round 
about the cornea, but soon inflammatory œedema of the conjunctiva becomes superadded, giving rise to a livid red injection, which may completely conceal the sclerotic. The iris loses its brilliancy, changes colour, and all details of its structure become invisible. The pupil is contracted, not quite circular, and sluggish in its response to the stimulus of light. If it yields at all to the action of atropin, the dilation is irregular, as the iris has become firmly attached to the capsule of the lens by inflammatory exudation-posterior synechia. If the irregular pupil be carefully examined by the ophthalmoscope, the lens capsule is seen spotted with pigment at those points at which the inflamed iris has been in contact, but where the adhesion was not sufficiently strong to resist the action of the mydriatic. There is always more or less fibrinous effusion, the aqueous is turbid, and in some cases the exudation collects in spots over the posterior surface of the cornea, or may, when the disease is very acute, more especially if it be of traumatic origin, form a purulent deposit at the bottom of the anterior chamber-hypopyon iritis. Hutchinson gave the name "quiet iritis" to a group of cases occurring for the most part in elderly people in which the onset and course of the disease are very insidious. There is no pain, or any manifest sign of inflammation, but sight steadily fails as a result of increase in the intraocular pressure. "The attack may be accompanied by so few symptoms that serious loss of vision in one eye is discovered accidentally before the patient becomes aware that there has been anything wrông with his sight. Careful examination, however, will at once detect the presence of posterior synechia, which had been forming over a long space of time, but which had given rise to little or no external sign or symptom to attract attention to the eyes.

The reaction to iritis, therefore, varies in different individuals, and a blood-shot eye unaccompanied by discharge ought always to be regarded with suspicion. Whenever a patient complains of pain which is more severe at night, and says that he has difficulty in facing the light and that his eye waters on the slightest provocation, think at once of iritis, and examine the pupil carefully for evidence of posterior synechiæ. At all times the symptoms vary greatly in severity, being very pronounced when the disease is acute, and only slightly marked when it is chronic. An ordinary attack lasts from four to six weeks, but whenever complications arise its duration is very much longer. Moreover, the patient should always be warned that relapse and recurrence are very frequent, and that both eyes may be affected.

The chief symptoms, then, in every case of acute iritis are pain, intolerance of light, lachrymation, impairment of sight, pericorneal injection, discoloration of the iris, turbidity of the aqueous, irregularity of the pupil, and general malaise. The pathological explanation of all these consists 
in active congestion of the blood-vessels of the iris, and the occurrence of fibrinous exudation derived partly by diapedesis through the walls of the vessels, and partly through proliferation of the cellular elements of the iris stroma. This exudation plays the most important part in the pathological history of the disease, gives rise to all the complications, and is the chief cause of blindness, for the course of an attack depends wholly upon its character and behaviour. It may be slight in amount, be confined to the iris, and disappear completely, or it may pass into the anterior chamber, occupy the pupil, and becoming converted into connective tissue, lead to adhesions between the iris and the lens capsule. The following are the more important complications-

1. Increase of Tension.-In acute iritis the tension of the eyeball is usually unaltered, but it sometimes becomes considerably increased. This is all the more apt to occur if the patient be elderly and gouty, and the ciliary body and choroid be acutely implicated. It is always accompanied by most intense pain, which is usually aggravated, rather than relieved, by the use of atropin. The term glaucomatous iritis is sometimes used to describe this form of the disease, but it is always wise to draw a sharp line of distinction between iritis and glaucoma. No error is more fatal than to confuse the one with the other, and the most reliable guides in diagnosis are the size and shape of the pupil and the depth of the anterior chamber. If the pupil be contracted and irregular in outline and the aqueous chamber normal or increased in depth, the disease is iritis, whereas, on the other hand, if the pupil is dilated and the anterior chamber shallow the increase of tension is almost certainly due to glaucoma.

2. Implication of the Uveal Tract.-The iris and ciliary body together form the anterior division of the uveal tract. They are in most intimate anatomical connection the one with the other, and possess a common blood supply. Moreover, the causes which lead to iritis are similar to those which induce cyclitis, consequently there is little wonder that an inflammation primarily attacking the iris may by continuity of structure implicate the ciliary body. Increase of intraocular tension is often the first indication of the onset of cyclitis, and is soon accompanied ${ }^{\text {o }}$ by rapid diminution of sight, and great tenderness on pressure over the ciliary region. On the other hand, persistent diminution of tension shows that the posterior division of the uveal tract has become seriously involved, and that in consequence the nutrition of the eyeball is suffering so severely that atrophic changes may supervene, leading ultimately to shrinking of the eye.

3. Exclusion and Occlusion of the Pupil.-In every case of iritis there is more or less exudation, which seals the iris to the capsule of the lens and interferes with the natural action of the pupil. Instead of being 
limited to points around the pupillary margin-posterior synechia-the adhesion may be so complete as to preclude the possibility of any circulation of fluids between the posterior and the anterior aqueous chambers -exclusion of the pupil-and, when such a condition exists, there is generally also exudation occupying the area of the pupil-occlusion of the pupil. The amount of exudation on the surface of the lens may be so great as to resemble cataract. When the pupil is " excluded" or "occluded," cyclitis followed by secondary glaucoma occurs, the iris becomes bulged forward from the pressure of the aqueous behind, and the depth of the anterior chamber is diminished except at its central portion-iris bombé, or iridoncosis. In such cases the iris is always discoloured and atrophied, and at one or more points its fibres are so thinned that the uveal pigment shines through as black spots or stripes. The iris stroma proper atrophies, the blood-vessels degenerate, and intraocular hæmorrhage is of frequent occurrence. By and by the whole posterior surface of the iris becomes adherent to the capsule of the lens-total posterior synechia-and the constant dragging upon the ciliary body sets up a chronic irido-choroiditis, whereby the nutrition of the globe is seriously impaired, cataract forms, the tension diminishes, the vitreous shrinks, the retina separates, and the eyeball gradually shrivels-phthisis bulbi.

Inflammation of the iris may be primary or it may be secondary either to some local affection such as infective ulcer of the cornea, or to some constitutional disease. It is met with in three forms, the serous, the plastic, and the purulent. This classification is based upon the character of the exudation, and consequently as the three types tend to merge the one into the other, they must be regarded only as different stages of the same pathological process. In the serous form there is always inflammation of the ciliary body and a muddy exudation is poured out into the aqueous chamber and is deposited in tiny dots on Descemet's membrane. The tension is increased and the anterior chamber is deep, but there is little tendency for the iris to become firmly sealed to the lens capsule, and the pupil is usually semi-dilated. This is all in marked contrast to the plastic form where the whole tendency of the exudation is to glue the iris to the lens capsule, to occlude the pupil, and at times to collect to form excrescences from the surface of the iris or ciliary body. The purulent form is seen only in cases complicated by suppurative keratitis, by penetrating wounds, or by some infectious disease, such as ulcerative endocarditis, rheumatic fever, pneumonia complicated by influenza, endometritis, etc. In any of these a septic thrombus may form in the eye and rapidly destroy it by suppuration. Instead of trying to found a classification upon a pathological basis it is more convenient in practice to divide cases of iritis into certain clinical types. It is true that it is 
not easy to distinguish these types by their anatomical features alone, but experience has shown that they differ from each other in their course, prognosis, and treatment.

Treatment.- The three chief indications to be attended to in the treatment of iritis are, to dilate the pupil, to subdue pain, and to combat the constitutional disorder which is the predisposing cause of the attack. In every case it is important that treatment be begun at the earliest possible moment, in order to prevent the occurrence of those complications which are so fraught with danger to sight. The one great safeguard against these complications is to prevent the iris from becoming attached to the capsule of the lens. Of all local applications atropin is the sheet-anchor. It puts the iris at rest, and by its mydriatic action not only relieves pain and intolerance of light, but also hinders the formation of posterior synechiæ and tends to break down adhesions which may have already formed. The drug may be used in the form of an aqueous solution, an ointment, or a tabloid, in strength from I to 2 per cent. It is usually employed from three to six times a day according to the severity of the symptoms. Whenever atropin fails to give relief, or when the use of the drug is followed by an exacerbation of pain the intraocular tension should be examined, and if it increases pain the drug must be at once discontinued, as its further use will do harm. The occurrence of pain always acts as a new exciting cause of the disease, consequently every means which subdues pain acts beneficially and aids the action of the atropin. In all cases when the symptoms are severe it is good practice to combine cocain and dionin with the atropin, but of even more value than these are the local application of heat or of cold, of leeches, and the use of mercury and anodynes internally. Heat diminishes the engorgement of the blood-vessels, and so, by reducing tension relieves pain. It is usually employed in the form of fomentations, which unless the case be very acute, ought not to be applied continuously, but three or four times daily for periods of from half an hour to an hour. Between the applications the eye must be protected by a pad of cotton-wool kept in position by a bandage. As a rule the temperature of the fomentation ought to be as high as can be borne by the patient, and when pain is very severe the periodic applications must be kept up unremittingly. The fomentation may be made more soothing by the addition of opium, belladonna, or chamomile. Dry heat is sometimes preferred by the patient, and is applied to the eye by masses of heated cotton-wool, the Japanese muff warmer, or by an electric heater, such as that devised by Maddox, embedded in wadding. There are, however, some patients who cannot bear the application of heat, but derive great comfort from cold. The applications are generally made three or four times daily for 
periods varying from a quarter of an hour to half an hour, the methods most frequently employed being an ice-bag, iced compresses, or Leiter's tubes. When the signs of inflammation are very acute, the pain excruciating, and the photophobia intense nothing affords such quick relief as local blood-letting. This is usually carried out either by natural leeches, or by Heurteloup's artificial leech. Leeches are best applied around the external canthus, or over the mastoid region. The amount of blood which should be withdrawn will vary according to the individual case, but, generally speaking, the bleeding should be continued until the pain is relieved. The patient for some hours after the leeching should be kept in bed in a darkened room, and if necessary, the bleeding encouraged by fomentations. Should it tend to become excessive, it may be checked by the pressure of a pad of absorbent cotton-wool dusted with powdered alum, or moistened by adrenalin solution. No one can deny the great value of blood-letting in the treatment of a recent case of acute iritis, for after the application of three or four leeches to the temple the pain lessens, the patient is able to open his eye, and the pupil now yields to the influence of atropin, though previously the drug had been inefficacious, or had even caused increased irritation.

The favourable influence of mercury in all cases of severe iritis is well known. A convenient method of administering the drug is to give 2 grains of calomel combined with I grain of opium in the form of a pill at bed-time. One pill should be given each night until the gums become slightly tender, and the effect afterwards maintained by giving a pill every second or third night as required. After from four to six pills have been taken the mercury, as a rule, begins to affect the gums and teeth, and contemporaneously with this the adhesions between the iris and lens capsule give way, although previously atropin had failed to act.

A patient suffering from iritis ought to be put to bed in a darkened room, kept on simple diet, and the use of tobacco and of stimulants forbidden. At the onset of the disease a sharp purgative should be administered, and during the course of the illness the action of the bowels must be carefully regulated. When the acute symptoms subside exercise may be allowed out of doors in favourable weather, the eyes being protected from light and wind by goggles. The use of all irritating and astringent lotions must be forbidden, for although of great service in conjunctivitis they always do harm when the iris is inflamed.

If in spite of the treatment just described nocturnal exacerbations of pain continue to torment the patient and cause sleepless nights, morphia should be administered by injection beneath the skin of the temple, or I5 grains of aspirin may be given at bed-time and repeated every three 
or four hours as required. Whatever objections may be urged against the use of morphia there are none against aspirin. The drug relieves pain due to iritis and cyclitis as if by magic, and in many instances the relief is lasting. As it is a derivative of salicylic acid-aceto-salicylic acid-it has special value in gouty and rheumatic inflammations, but its use is by no means restricted to those forms of iritis, for it does good in all except those complicated by suppuration. In the great majority of cases within half an hour of the time of its administration the patient is quite free from pain, and where sleeplessness has been a distressing symptom its good results are increased by combining it with trional. A combination of $I_{5}$ grains of aspirin with Io grains of trional is a very reliable remedy to relieve pain and induce sleep in irido-cyclitis. When after the acute symptoms have subsided the disease does not go on steadily towards complete resolution, the application of a blister to the temple will often so modify its course that a favourable termination to the inflammation is brought about in a comparatively short time. A blister is often of great value to relieve pain, and its efficiency can be greatly increased by dressing the raw surface with an ointment containing cocain and morphia.

It happens now and again, however, that all the means above described fail to give more than temporary relief, and it is well from the beginning to draw a distinction between cases where the intra-ocular tension is normal and those in which it is increased. The treatment which in the former group is effective is of no avail in the latter. In spite of it pain increases till it is almost unendurable, the upper lid becomes œdematous, the eyeball is exceedingly tender to touch, the media become more and more turbid, and sight rapidly deteriorates, until the patient can hardly distinguish the light. Under these circumstances pilocarpin or eserin may afford relief, but the contraction of the pupil induced by these drugs is a great disadvantage, as the small open space is very apt to become occluded by inflammatory exudation. In most instances it is wiser to reduce the tension by paracentesis of the cornea. This operation is usually followed by great relief to the patient's sufferings, although he should be warned that he will probably experience some spasms of very severe pain immediately after the aqueous escapes, owing to the forward displacement of the inflamed iris and ciliary body. Fortunately, however, those cases are exceptional, and the rule is that treatment by atropin, leeches, fomentations and calomel and opium speedily effects an amelioration of the symptoms. It is, however, very important to warn the patient not to discontinue the treatment whenever the signs of irritation disappear from the eye, for if he does so there is great danger of relapse. No care should be relaxed until all signs of inflammation have M 
disappeared completely, and afterwards the remedies ought to be used at longer and longer intervals, until at length they can safely be given up altogether. It is, moreover, always necessary to continue the line of treatment prescribed to combat the predisposing cause of the inflammation of the iris, because unless that be done, relapse and recurrence are sure to take place. 


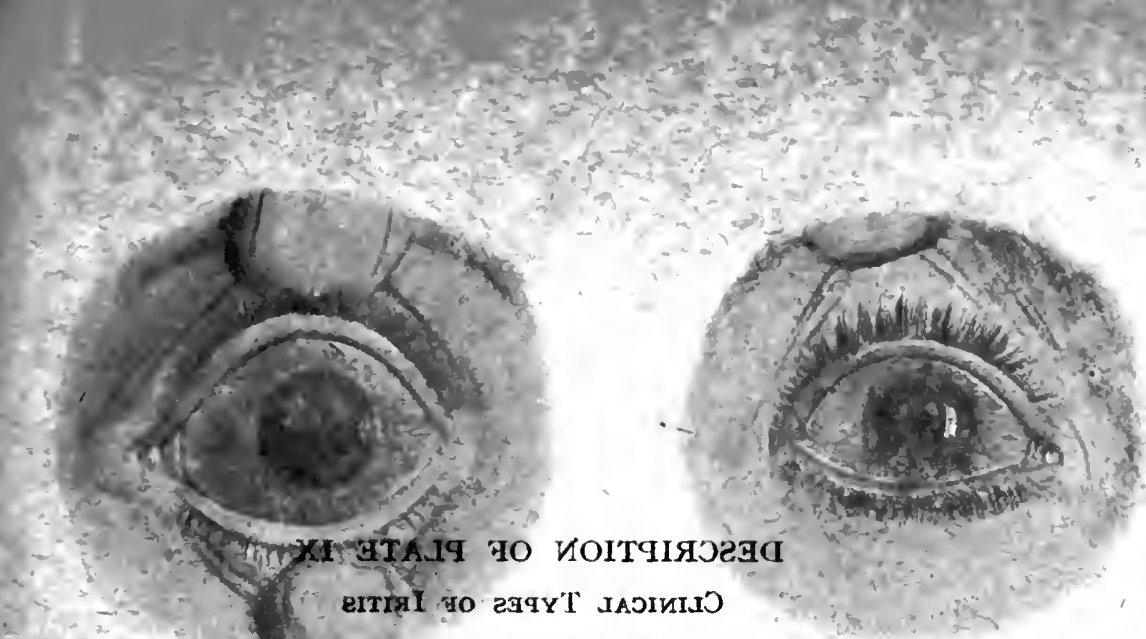

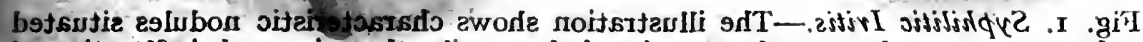

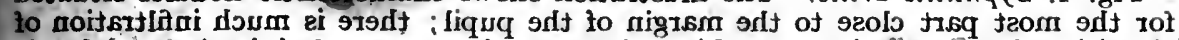

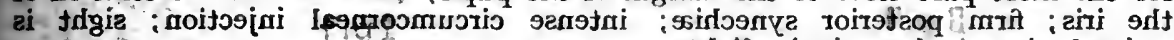
.trgile ei nisq : b9tisqmi үlessorise

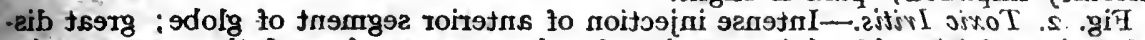
roingteoq no noifsbuxs to efoqe : I9dmsdo toingfas mi boold ; aiti to noitstoloj

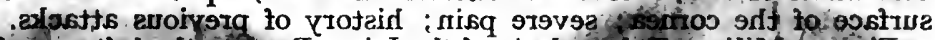
2x69y xie yod s to ovg ttol ody af a q

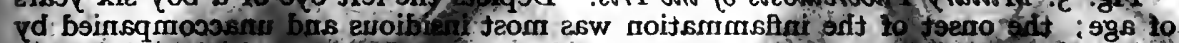

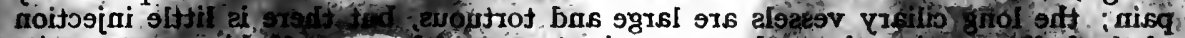

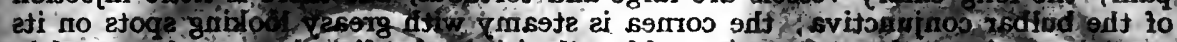

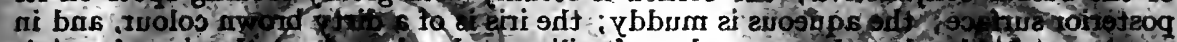

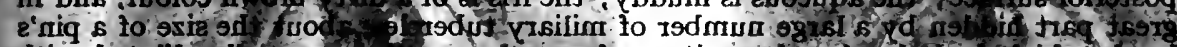

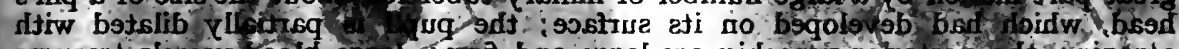

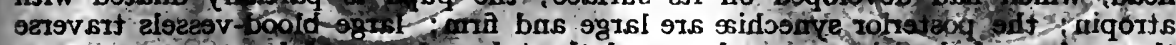

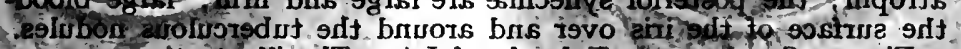

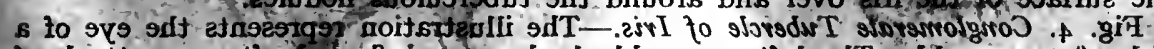

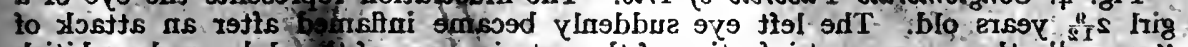

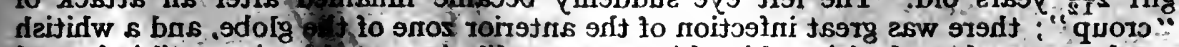

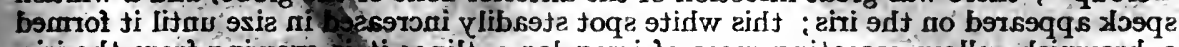

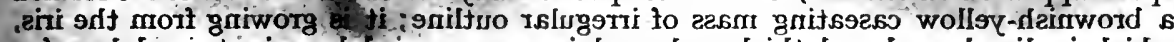

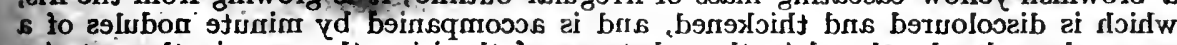

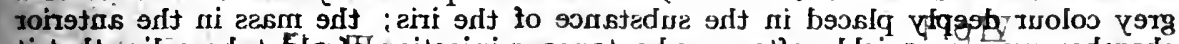

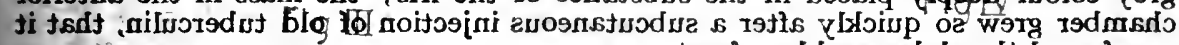
.9fstotisq blew odolg orlt botsot a.sw

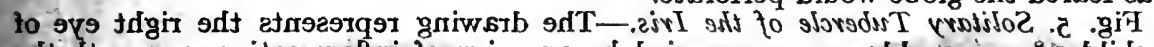

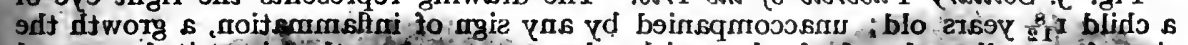

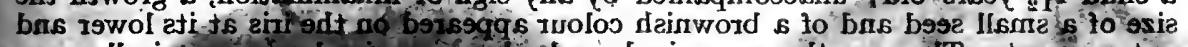

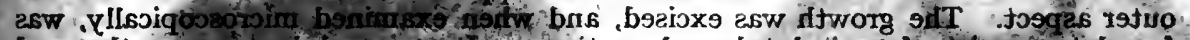

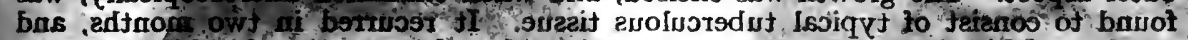

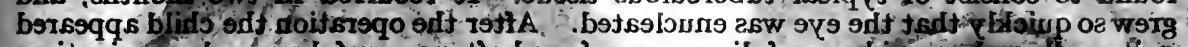

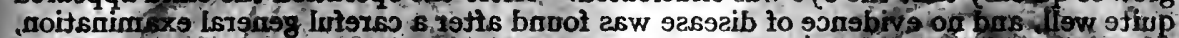

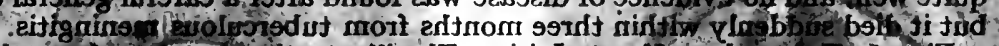

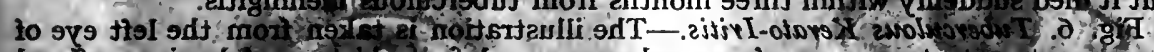

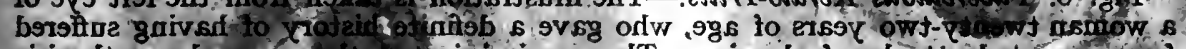

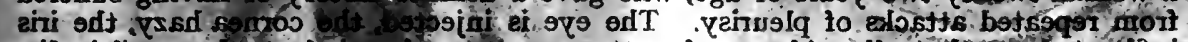

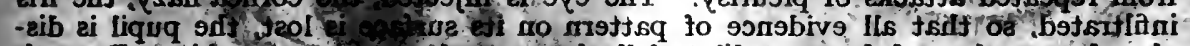

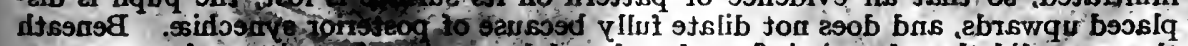

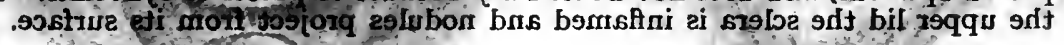
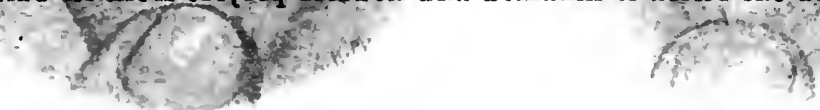

Fig V 


\section{DESCRIPTION OF PLATE IX}

\section{Clinical Types of Iritis}

Fig. I. Syphilitic Iritis.-The illustration shows characteristic nodules situated for the most part close to the margin of the pupil; there is much infiltration of the iris; firm posterior synechiæ; intense circumcorneal injection; sight is seriously impaired; pain is slight.

Fig. 2. Toxic Iritis.-Intense injection of anterior segment of globe; great discoloration of iris; blood in anterior chamber; spots of exudation on posterior surface of the cornea; severe pain; history of previous attacks.

Fig. 3. Miliary Tuberculosis of the Iris.-Depicts the left eye of a boy six years of age; the onset of the inflammation was most insidious and unaccompanied by pain; the long ciliary vessels are large and tortuous, but there is little injection of the bulbar conjunctiva; the cornea is steamy with greasy looking spots on its posterior surface; the aqueous is muddy; the iris is of a dirty brown colour, and in great part hidden by a large number of miliary tubercles, about the size of a pin's head, which had developed on its surface; the pupil is partially dilated with atropin; the posterior synechiæ are large and firm; large blood-vessels traverse the surface of the iris over and around the tuberculous nodules.

Fig. 4. Conglomerate Tubercle of Iris.-The illustration represents the eye of a girl $2 \frac{4}{12}$ years old. The left eye suddenly became inflamed after an attack of "croup"; there was great infection of the anterior zone of the globe, and a whitish speck appeared on the iris; this white spot steadily increased in size until it formed a brownish-yellow caseating mass of irregular outline; it is growing from the iris, which is discoloured and thickened, and is accompanied by minute nodules of a grey colour deeply placed in the substance of the iris; the mass in the anterior chamber grew so quickly after a subcutaneous injection of old tuberculin, that it was feared the globe would perforate.

Fig. 5. Solitary Tubercle of the Iris.-The drawing represents the right eye of a child $I_{1}^{8}$ years old; unaccompanied by any sign of inflammation, a growth the size of a small seed and of a brownish colour appeared on the iris at its lower and outer aspect. The growth was excised, and when examined microscopically, was found to consist of typical tuberculous tissue. It recurred in two months, and grew so quickly that the eye was enucleated. After the operation the child appeared quite well, and no evidence of disease was found after a careful general examination, but it died suddenly within three months from tuberculous meningitis.

Fig. 6. Tuberculous Kerato-Ivitis.-The illustration is taken from the left eye of a woman twenty-two years of age, who gave a definite history of having suffered from repeated attacks of pleurisy. The eye is injected, the cornea hazy, the iris infiltrated, so that all evidence of pattern on its surface is lost, the pupil is displaced upwards, and does not dilate fully because of posterior synechiæ. Beneath the upper lid the sclera is inflamed and nodules project from its surface. 


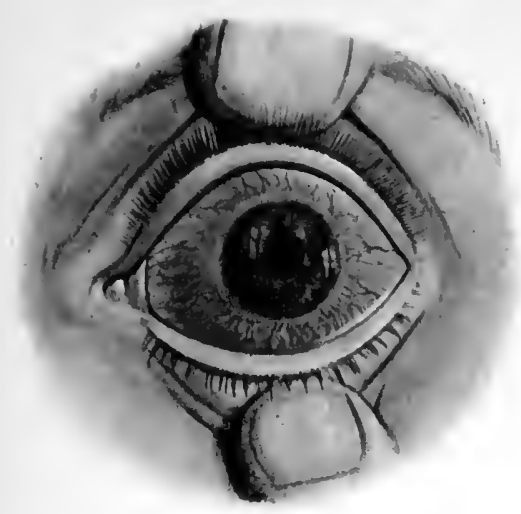

Fig I

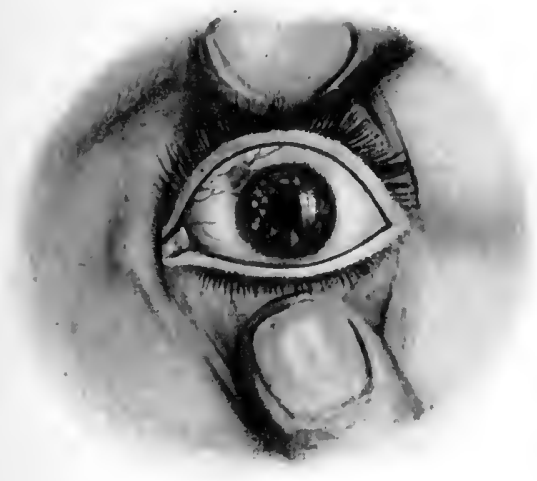

Fig III

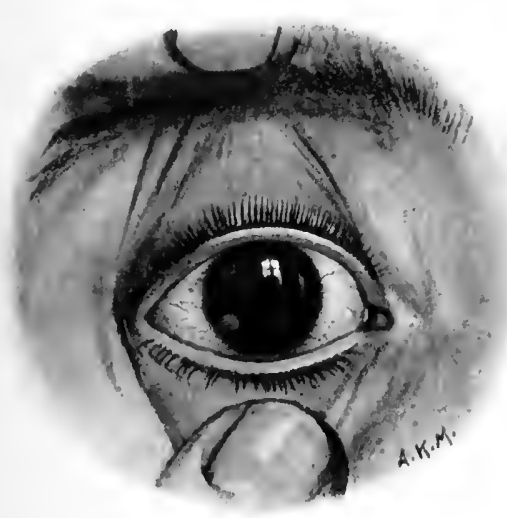

Fig I

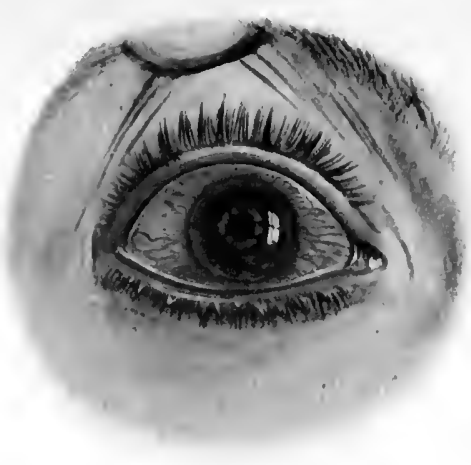

Fig II

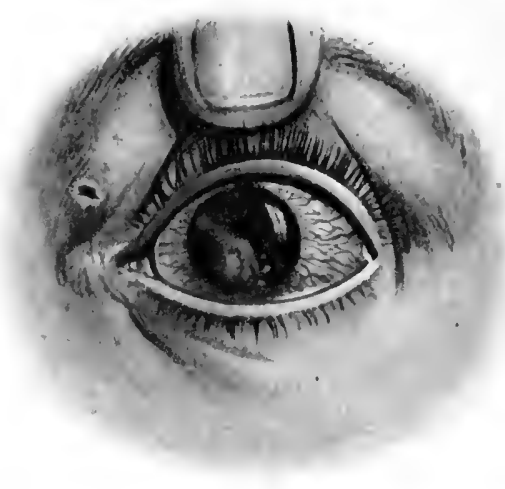

Fig प

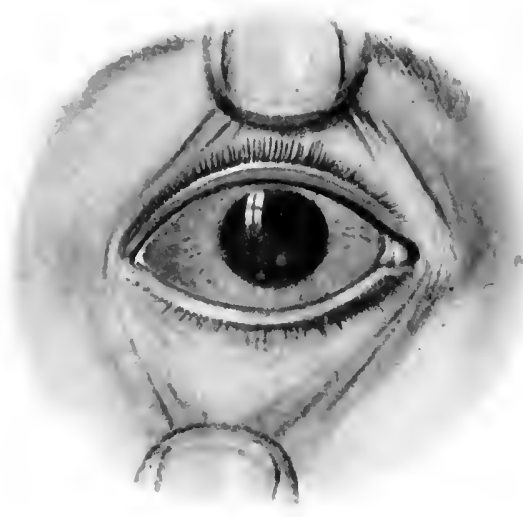

Fig VI 



\section{CHAPTER XXI}

\section{CLINICAL TYPES OF IRITIS AND THEIR TREATMENT}

Syphilitic iritis : treatment-Arsenical compounds-Mercury-Iodides-GeneralToxic iritis : types of-Clinical features-"Recurrent iritis "-TreatmentTuberculous iritis-Differential diagnosis from syphilitic iritis-PrognosisTreatment.

A STUDY of inflammation of the iris affords an admirable illustration of the fundamental truth that in every idiopathic inflammation there is a predisposing as well as an exciting cause. The predisposing cause may in some cases be readily discoverable, and give the inflammation a character so distinctive as to be recognised at the bedside without difficulty: but it is in other cases quite unknown to the patient, and far from apparent on the surface. It must then be sought for diligently, much tact being needed in the quest, for in its proper recognition depends the success or the failure of treatment. It is true that in every case of iritis the indications for the local treatment are similar-to dilate the pupil and to relieve pain. If, however, recovery is to be satisfactory, and relapse prevented, the constitutional state underlying the ocular inflammation must also be remedied. The disease, always serious, tends to linger and relapse; and its gravity is largely due to the nature of the cause which has produced it. Careful observation will furnish many examples of the truth of the old clinical aphorism that the longer a cause has been in operation the more intractable to treatment, and the more liable to recurrence, is the resulting disease. Both prognosis and treatment therefore depend upon a correct appreciation of causation.

It is of great assistance in diagnosis to remember the relative frequency of the various causes of iritis, syphilis, rheumatism, gout, toxic conditions, tubercle, etc. Careful enquiry must always be made into the clinical history of the case. In the majority of instances a Wassermann blood test ought to be made before syphilis is excluded; and the urine should always be examined for signs of renal inadequacy, for the presence of sugar, of indican in excess, of micro-organisms, especially for the bacillus coli. In doubtful cases, evidence of tubercle ought to be sought for by the von Pirquet test or by the subcutaneous injection of a minute dose of Koch's old tuberculin; and the patient should be examined for a history 
or for evidence of gonorrhœa, for pyorrhœa of the gums, or for suppuration of the fauces or tonsils, etc., etc. Such thorough examination makes it possible to place patients suffering from iritis in the following clinical groups-

1. Syphilitic Iritis.-The possible existence of syphilis should be considered in every case of iritis, and in many instances the appearance of the eye at once suggests it as the cause, but in others there is nothing in the ocular inflammation that is specially characteristic. Evidence of other manifestations must therefore always be sought, and the personal history should be strictly scrutinised. The disease may occur in the innocent: the chancre is extra-genital, or the patient, especially a woman, may be infected through no fault of her own, and may have no suspicion of the real nature of the illness. Hence the need for tact in unravelling the personal history, and the importance of trying to form a clear conception of syphilis as it affects the eye.

The patients for the most part are young adults of both sexes, and with few exceptions they suffer from the acquired form of the disease. The iritis appears as a rule within the first year after infection, but rarely, if ever, before the third month. Both eyes are frequently implicated, one being affected as a rule after the other, but it is by no means unusual to find both suffering at the same time. Relapses in the form of frequently recurring attacks are not common; but iritis due to syphilis is liable to be complicated with, or to be followed by, inflammation of the choroid and retina. The objective appearances presented by the eye, however, may give little or no clue to the ætiology of the inflammation, and it should be remembered that the pathological alterations found in an eye are by themselves of no greater diagnostic value than those of the skin, mucous membranes, etc. Clinical experience, however, teaches that iritis is to be regarded as a sign of severe syphilis, and is followed in many instances by grave sequelæ.

The importance of accurate ætiological diagnosis cannot therefore be too strongly emphasised; and the signs and symptoms specially characteristic of syphilitic iritis may be briefly summarised as follows-turbidity of the aqueous, greasy spots on the posterior surface of the cornea, opacities in the vitreous, a degree of dusky red pericorneal injection out of all proportion to the severity of the pain, which is for the most part circumorbital and nocturnal, and last and most important, the formation in the iris of nodules, compared to condylomata or papules of the skin, generally situated close to the margin of the pupil, although they may also occur at the ciliary attachment and in the substance of the iris itself. These nodules vary in size, are usually orange-red in colour, and are surrounded by new-formed blood-vessels; but they may not be visible till after the 
dilatation of the pupil by atropin, when they are seen in the broad firm posterior synechiæ. Those appearances, however, cannot be regarded as pathognomonic; consequently in every case an examination of the blood should be made, and if the Wassermann reaction be positive, the diagnosis is certain. That does not mean that the Wassermann blood test can take the place of clinical observation; because bedside examination and laboratory investigation must always go hand in hand, and whenever it is possible both methods ought to be employed in making a diagnosis.

Iritis is not by any means a frequent accompaniment of hereditary syphilis, but it is met with occasionally. A form of infantile iritis, appearing as a rule about the fifth month, has been described fully by Hutchinson, who regarded it, however, as " amongst the rarest of the symptoms of hereditary syphilis." The disease is also seen in older subjects, in whom it usually takes the form of an irido-cyclitis, and is often accompanied by interstitial keratitis.

Treatment.-In every case of syphilitic iritis the usual local treatment must be carried out efficiently, and an equally thorough constitutional anti-syphilitic treatment must be instituted simultaneously, in order to prevent the complications and sequelæ which are otherwise certain to occur. What is needed is persevering rather than too vigorous treatment ; consequently the patient ought to be kept under strict medical supervision, and be duly impressed with the need for attention to detail. That at once raises the question how much a patiert should be told of the nature of the illness. Fortunately, knowledge regarding venereal diseases is daily becoming more widely spread among the general community; but the situation will always require sympathetic insight, and the exercise of judgment and right feeling on the part of the doctor. Treatment often fails because the patient does not understand the necessity for it, and in consequence discontinues it prematurely.

The ocular tissues are so delicate that they are damaged quickly, and in a very short time sight may be permanently impaired, or even destroyed. It is therefore most important to act at the earliest possible moment with promptness and energy.

The great value of the arsenical compounds lies in their power to destroy the spirochætes before they have had time to produce irreparable damage to the delicate structures of the eye. The rapidity with which those products act is their most outstanding characteristic, and in a recent acute case their effect is little short of magical. This rapid disappearance of clinical lesions must not encourage either patient or doctor to expect too much, for there is good reason to doubt whether by themselves alone they are really curative. They prepare the way for mercury, which in 
the cure of syphilitic iritis is as necessary at the present day as ever it was. The one drug is the complement of the other, and the most trustworthy results are obtained when an arsenical preparation is combined with a course of mercurial treatment.

The arsenical compounds most frequently employed are kharsivan, neo-kharsivan, galyl, and arsenobillon. They are administered either intravenously, subcutaneously, or intramuscularly, and the dose varies from 0.3 grammes to 0.6 grammes intravenously, and 0.45 grammes to 0.6 grammes intramuscularly. Experience in the use of these drugs has shown the need for caution at the commencement of the treatment, and also the necessity for repeating the dose at regular intervals, to insure immunity from recurrence. To give only one or two doses is of very little use.

Valuable, however, as these means are, our chief reliance in syphilitic cases must still be placed on mercury. The aim is to saturate the tissues as rapidly as possible, and at the same time to secure steady and gradual elimination of the drug from the system. Patients vary greatly in their susceptibility to the toxic effects of mercury; consequently every one must be dealt with according to the special indications presented by the particular.case, but, as a general rule, calomel and opium in pill form is the most satisfactory routine treatment. The opium serves the double purpose of relieving pain, and of locking the calomel in the system, and by that means the constitutional effects of mercury are speedily manifested. A pill containing 2 grains of calomel and $x$ grain of opium is administered every night at bedtime, and usually in from four to eight days the patient begins to show signs of salivation. After the appearance of soreness of the gums and an increased flow of saliva, the acute inflammatory eye symptoms begin to abate, and posterior synechiæ, which up to this point had resisted the action of atropin, now give way and the pupil dilates. It occasionally happens, however, owing to individual idiosyncrasy that the pills cause an intense feeling of malaise, with sickness and vomiting. In these circumstances they ought to be discontinued, and the mercury administered by inunction. In urgent and difficult cases, indeed, the latter method of treatment should always supplement the administration of mercury by the mouth, and by this double means rapid and satisfactory results are frequently obtained. Not only, however, must the patient be brought quickly to the border of salivation, he must also be kept under the influence of mercury for weeks, and even for months, after the eye shows no sign of inflammation. All this should be accomplished without inducing active salivation-the patient must never be salivated. It is all-important that the mouth and teeth be kept scrupulously clean by the liberal use of a gargle containing chlorate of potash or 
peroxide of hydrogen; and if care be taken to regulate the dose properly, the beneficial action of mercury can be easily maintained for months without causing injurious results.

After a course of calomel and opium pills, which must be administered at varying intervals according to the constitutional effects which they produce, the preparation of mercury may be changed-bichloride, protoiodide, or grey powder being substituted for the calomel. At this stage also the iodides will render important service. Their therapeutic efficacy is increased if they are combined with sarsaparilla, and iodism is in great measure prevented if one or two drachms of Fairchild's pepsencia be added to each dose. The iodides of potassium, sodium, and ammonium in combination are often more efficacious than any one of these salts by itself. They may be given, well diluted, in a large dose once a day, or from five to ten grains may be repeated three times a day after meals. When the former method is employed, mercurial treatment by inunction, or by intra-muscular injection, should be continued; but in the latter case perchloride of mercury in a suitable dose may be combined with the iodide mixture. The chief drawback to that combination is that it is liable to give rise to pain in the bowels, and occasionally to troublesome diarrhœa, but these unpleasant complications are often completely overcome by the addition of one or two minims of nepenthe to each dose of the medicine.

Treatment by mercury and the iodides, preceded by intravenous or intra-muscular injection of one of the arsenical compounds, is undoubtedly the most important means of combating syphilis of the eye, but, if it is to be successful, it must be steadily continued for at least two years; the treatment all through should be controlled by the Wassermann test of the blood, which ought to be repeated at intervals even after the patient is considered to be quite free from the disease.

If the inflammation tends to spread from the iris and to implicate other portions of the uveal tract, good results are obtained from an open blister, which is usually applied to the temporal region, or over the mastoid process. Continuous counter-irritation is most conveniently kept up by dressing the raw surface night and morning with D'Albespeyre's papier epispastique No. 2.

In addition to antispecific remedies, patients suffering from syphilitic iritis require to be well fed, warmly clad, and comfortably housed, and are much benefited by the use of cinchona, iron, and similar tonics.

2. Toxic Iritis.-This group includes patients who have suffered from gonorrhœa, chronic rheumatism, gout, glycosuria, intestinal toxæmia, typhoid fever, influenza, pneumonia, herpes, the acute exanthemata, infection by the bacillus coli, or from pyorrhœa alveolaris, etc. The 
outstanding clinical feature common to all of them is their tendency to relapse and recurrence. Formerly they were all classified together and called " rheumatic" or "gouty" or " recurrent" iritis.

When inflammation of the iris occurs as a complication of acute rheumatism, the likelihood is that gonorrhœa is the cause both of the iritis and the rheumatism. In these circumstances, the attack is frequently very acute and destructive, and may terminate in blindness. More usually, however, the disease is seen in adults who give a general history of "rheumatism," or who suffer from digestive disturbances, often indicated by a urine scanty in quantity, of acid reaction, deep colour, and high specific gravity, and depositing urates or oxalate of lime crystals after standing for a few hours. Iritis may, however, also occur in those who, although very sensitive to cold and to damp, have never suffered before from any symptom of rheumatism in other parts of the body. It prevails for the most part in the early spring or the late autumn, and although as a rule both eyes suffer, one only is implicated at a time. The onset is usually sudden, the pain intense, the injection brick-red, the lachrymation copious, and the photophobia most distressing: while froth-the so-called "arthritic foam"- - collects along the edge of the eyelids and at both canthi. The discoloration of the iris is not so pronounced, and the amount of plastic exudation is not so great, as in the syphilitic or the tuberculous forms of iritis; but as the disease progresses the pupil becomes gradually closed, and may be contracted to a mere pinpoint from the formation of posterior synechiæ. In the gouty form more especially, intraocular tension becomes increased, and hæmorrhage into the anterior chamber is not infrequent.

In this group ought also to be included-

(a) A large number of cases of chronic iritis, occurring in elderly patients, in which there has been neither pain nor any redness of the eye, but sight has steadily failed-" quiet iritis." Examination of those cases always reveals extensive posterior synechiæ with increased intraocular tension.

(b) A peculiarly insidious and very destructive form of iritis which attacks young adults of gouty parentage. The whole uveal tract is gradually involved, floating bodies form in the vitreous, the lens becomes cataractous, and the extinction of vision is hastened by the occurrence of secondary glaucoma. It is very probable that in these cases the patients suffer from some failure in the internal secretions; but on this subject our knowledge is still very imperfect.

(c) Another very insidious form of iritis is occasionally seen in adolescents, more especially of the female sex. The first symptoms are misty vision and musca volitantes. There is little complaint of pain, but inquiry 
elicits the fact that the eye is somewhat tender and readily waters on exposure to bright light. Careful examination reveals the presence of minute spots of exudation on the posterior surface of the cornea; and although the details of the fundus may be visible on ophthalmoscopic examination, they are seen as through a cloud, and numerous flocculent bodies, mostly of very small size, are detected in the anterior part of the vitreous. At the outset the pupil dilates fully under the influence of atropin, but as the disease progresses, posterior synechiæ form, and a pink zone of congestion surrounds the cornea. This form of inflammation is always very tedious, and is occasionally complicated by attacks of increased intraocular tension. Sight slowly but steadily deteriorates, as the disease is usually persistently progressive. Some of the cases are undoubtedly due to hereditary syphilis, or to tubercle, but many are due to some other form of toxæmia. The rheumatic and gouty toxins accumulating in the blood, induce marked anæmia, which is a characteristic accompaniment of this form of inflammation. There is sometimes a personal history of articular pains, of quinsy, perhaps of pleurisy or of pulmonary inflammation, and, moreover, as a rule, there is a distinct gouty or rheumatic family history.

In the great majority of the cases included in this group, the two most outstanding features are the great severity of the pain, and the history of repeated attacks. The pain usually increases steadily from the onset of the attack, and is, when at its worst, almost unbearable. It radiates from the eye to the forehead, temple, and cheek, and is always most severe at night and during the early hours of the morning. The patient, during an access of suffering, frequently rises from bed and walks about the room, in a vain attempt to find relief; and as a result of the sleeplessness consequent on the suffering, he soon feels very ill. Relapse readily follows apparent recovery, and recurrence takes place so frequently that the disease was formerly described as "recurrent iritis." The cases in this group, therefore, give much trouble in treatment. Many of them are complicated by high tension; consequently atropin-the sheetanchor in the treatment of iritis-must be used with caution, and at once intermitted if an accession of pain follows each instillation of the drug. In these circumstances, dionin is of service, but must be used sparingly, because it is in the so-called gouty and rheumatic cases that its lymphagogue action is most pronounced. Salicin, or preferably aspirin, relieves pain quickly; and a combination of calomel, salicylate of soda, and Dover's powder, seems to act more beneficially in these cases than the calomel and opium pill. Blisters afford much relief, even in the acute stage of rheumatic iritis; but when the patient is gouty, a blister often induces intense inflammation of the skin, while the application of leeches and the 
administration of colchicum afford speedy relief. The danger of relapse is best overcome by the prescription of a prolonged course of the iodides combined with alkalies, and by the avoidance of cold and damp, or the exposure of the eyes to extremes of temperature.

Autogenous vaccines are very useful in suitable cases, and may effect a cure when other methods of treatment have failed. That applies more especially to the gonococcus, and the bacillus coli. Iridectomy may be necessary when posterior-synechiæ are so numerous that increased tension occurs from exclusion of the pupil; and the operation not only does good at the time, but is frequently followed by freedom from recurring attacks.

If, in spite of all that has been done, the inflammation continues, a turning-point may be got after diaphoresis from the administration of $\frac{1}{12}$ to $\frac{1}{8}$ of a grain of pilocarpin hypodermically. Free sweating is promoted by the use of hot packs and warm drinks. In many of those lingering cases, the inflammation is moderately kept up, and relapse favoured, by absorption of toxins from the intestine; constipation therefore must be very carefully avoided. The calomel will be helpful in promoting intestinal antisepsis, but the old-fashioned plan of giving a dose of turpentine and castor oil is often followed by successful results. Turpentine in drachm doses three times a day was highly recommended by Carmichael in the treatment of iritis, and probably any success that follows its administration is due to its antiseptic action on the bowel.

In every case, if the patient can afford it, the proper course is to send him to a Spa, care being taken to select the most suitable locality and the waters best fitted for his individual requirements ; but whether the patient be rich or poor, a proper regimen is a most important factor in the treatment. As a rule those patients eat too much, and take far too little exercise; and consequently their habits and mode of life require very careful consideration. The best guide to suitable treatment is repeated and thorough examination of the urine. The food should be plain and well cooked, and the patients must be on their guard against eating too much, while alcohol in all its forms should be excluded from the dietary.

In diabetic or glycosuric iritis, prudent restrictions in the kind of food that the patient is allowed to take are essential; but that is a matter more for the consideration of the general practitioner than for the eye specialist. Care, however, must always be taken that the prescribed dietary is not too strict, and in every case it must be modified to meet the needs of the individual patient. Daily exercise in the open air, short of fatigue, is absolutely necessary, and the dwelling house, and more especially the sleeping apartment, must be well ventilated. A moderately high altitude, a gravelly soil, and a dry, warm, equable climate, are the ideal conditions for these patients. Nor must they ever depart from that prescribed 
mode of life; for, if they weary of the restrictions imposed on them and return to their old habits, the eyes will in all probability again become inflamed, and each fresh attack is more and more difficult to cure.

3. Tuberculous Iritis.-Tubercle as a cause of iritis is much more frequent than was at one time supposed. It may appear in one eye only, but is often bilateral. It is very doubtful if primary tuberculosis of the eyeball ever occurs. The most careful clinical examination may, and very frequently does, fail to reveal the existence of disease in the chest, in the abdomen, or in any other part of the body; nevertheless the probability is that there is somewhere a hidden focus from which infection has reached the eye. Its appearance there ought therefore to be regarded as a local manifestation of a general infection of the blood; and in some instances the eye symptoms mark the beginning of a series of successive local infections terminating in death, which is frequently immediately preceded by an attack of tuberculous meningitis.

The eye symptoms often appear suddenly after surgical operations upon diseased bones or joints. They may run their course with little pain and end in recovery, but, on the other hand, and more especially when the ciliary body is implicated, the suffering may be acute, and the manifestations of the disease so virulent that the eye is destroyed completely within a few days.

As a rule, however, the onset of the disease is most insidious. The patient is usually run down in health, and says he has caught cold in the eye, which is bloodshot and waters readily when exposed to light. As the disease progresses, the eye becomes more injected, the intolerance of light increases, and the lachrymation becomes copious and constant. Vision is affected, and although there may not be actual pain, there is usually a constant sense of aching, especially at night. The injection is deep and very intense around the cornea ; the anterior chamber is usually increased in depth, the aqueous is muddy, and numerous spots of inflammatory exudate adhere to the posterior surface of the cornea; the iris is infiltrated, much discoloured, and bound down to the lens capsule by several broad firm synechiæ, over which atropin has little or no influence. The actual tubercles occur in the iris in three distinct forms-

(I) Fine miliary tubercles, sometimes so small that they can only be seen by a magnifying lens during life, or by the microscope after the eye has been enucleated. They are yellowish grey in colour, and although scattered all through the iris stroma, are most numerous either at the angle of the anterior chamber, or at the margin of the pupil. In this form the disease is usually " attenuated," and the tuberculous nodules grow very slowly, and after they have reached a certain size, remain stationary for a time, then shrivel, and finally disappear. 
(2) Associated with the miliary tubercles are others of greater size. They are orange-yellow in colour, and rather larger than a pin head. No blood-vessels are visible over their surface; but the capillaries are greatly injected all around their base. They usually occupy the corneo-iridic angle. The true cornea is always implicated; it may become opaque, and soften and caseate, so that if the disease be not arrested by treatment the eyeball ultimately shrinks.

(3) Conglomerate or solitary tubercle of the iris forms a yellowishwhite swelling accompanied by very little inflammation of the iris.' It projects from the surface of the iris, steadily increases in size, fills the anterior chamber, and implicates the cornea. As the disease progresses the eyeball becomes distended; but the softened cornea is soon perforated, and after it has burst the eyeball gradually shrinks-phthisis bulbi.

Ocular tuberculosis may be mistaken for syphilis of the eye, because there is little that is pathognomonic in the appearance of the nodules in the iris. Tubercle, however, occurs for the most part in childhood, at an age when acquired syphilis is very rarely seen. In every case, however, the personal and family history must be thoroughly investigated, and careful examination made to discover any evidence of tubercle in other parts of the body, or to detect any of the stigmata of congenital syphilis. In all doubtful cases the blood should be examined by the Wassermann test; and the diagnosis may not be possible until the patient's reaction to tuberculin is known. The most satisfactory methods are von Pirquet's cuti-reaction or the hypodermic injection of $\frac{1}{40}$ to $\frac{1}{20}$ of a milligramme of Koch's old tuberculin. The latter is the more reliable, and, if tubercle be present, there will be not only a general rise in temperature, but also a local reaction in the eye itself. Calmette's reaction must never be employed if there be any suspicion of ocular tuberculosis; because in these circumstances the instillation of tuberculin into the conjunctival sac will give rise to inflammation which it is almost impossible to control.

The prognosis in tuberculous iritis is always more favourable in the adult than in the child, because in the latter the eye may burst and death ensue from meningitis.

Treatment.-The special treatment for tuberculous iritis resolves itself into care for the health as a whole. Good food, fresh air, and the use of tonics, are all indicated for the purpose of improving general nutrition. In addition to the usual local remedies-atropin, fomentations, and protective spectacles-injections of tuberculin, repeated at regular intervals and continued for months, it may be for years, ought always to be used. The great secret of success is to graduate the dose properly and to persevere faithfully in the treatment. Guaiacol and thiocol, either in the form of local applications to the eye or in cachets prescribed for internal adminis- 
tration, have been strongly recommended, and in some cases encouraging results have followed their use. That treatment should be supplemented by the use of cod-liver oil and other easily digested fats, and by a prolonged course of quinine and grey powder, alternating with a mixture containing quinine combined with the perchlorides of iron and mercury. The value of small doses of perchloride of mercury in chronic tuberculous disease has long been known, and ought not to be forgotten.

After the eye has become hopelessly disorganised, or after it has perforated, enucleation may become necessary; but in such cases there is always the danger that the removal of a tuberculous eyeball may be followed by tuberculous meningitis, or by acute miliary tuberculosis.

Other two clinical groups-traumatic iritis, and sympathetic iritisdepend so much on the nature and consequence of the injury which has given rise to them, that they are specially considered in the chapters on penetrating injuries of the eye, and on sympathetic ophthalmia. 


\section{CHAPTER XXII}

THE COMPLICATIONS AND THE SEQUELE OF IRITIS, AND THEIR TREATMENT

Anatomical considerations-Cyclitis-The clinical importance of the exudateOcclusion of the pupil-Iris bombé or iridoncosis-Secondary glaucomaShrinkage of the globe-Irido-choroiditis-Treatment, medicinal and dietetic - Renal inadequacy as one important cause of chronic uveitis-The use of anti-diphtheritic serum-Operative treatment-Treatment during convalescence.

All the complications and sequelæ of iritis depend upon the amount and the behaviour of the exudation, and the explanation of their occurrence is much more easily understood if three anatomical facts are constantly borne in mind.

I. The different parts of the vascular tunic of the eyeball are not separate and distinct structures, but are continuous the one with the other. 'If inflammation, therefore, attacks iris, ciliary body, or choroid, it does not confine itself to the structure primarily affected, but spreads to the others with ease and rapidity, whenever circumstances are favourable. The two most important and determining factors are the intensity of the inflammation, and the state of the patient's health.

2. The intimate relation between the border of the pupil and the anterior capsule of the lens facilitates the occurrence of adhesion between these two structures, whenever inflammatory exudation is present.

3. The small size of the posterior chamber explains how easily it becomes blocked by exudate, which, accumulating behind the iris, either pushes it forward into the aqueous chamber-inidoncosis-or drags it backwards and seals it completely to the lens capsule-total posterior synechia.

When the deeper parts of the uveal tract become implicated, the ciliary body is involved, and consequently the prognosis becomes much more serious. Cyclitis is readily recognised by great increase of pain and by rapid failure in sight. The eye is acutely tender to pressure, the aqueous humour is cloudy, and minute dots of exudation form on the posterior surface of the cornea-Descemetitis. Moreover, in many of the so-called rheumatic cases the episcleral tissues are implicated, and in more severe forms the sclerotic itself becomes inflamed; consequently in many instances sight is in the end irreparably lost from the occurrence of ciliary or intercalary staphylomata. 


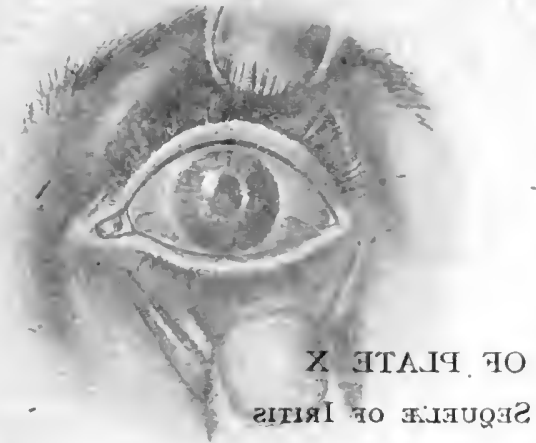

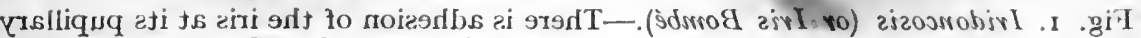

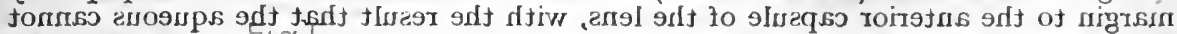

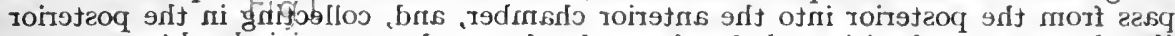

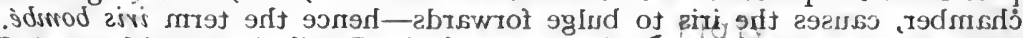

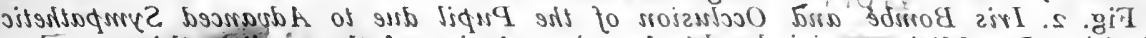

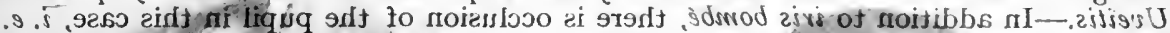

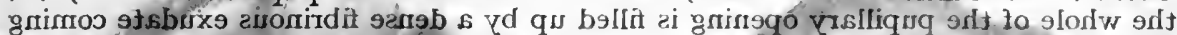

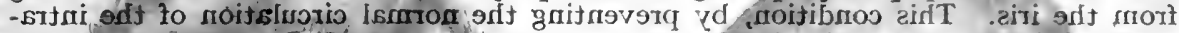

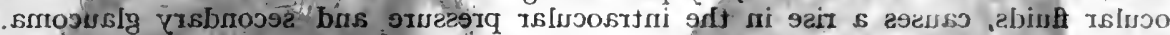

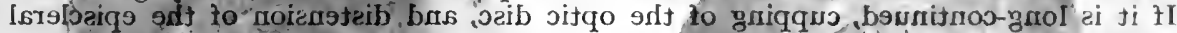

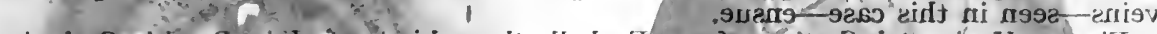

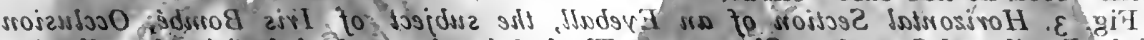

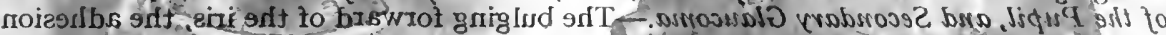

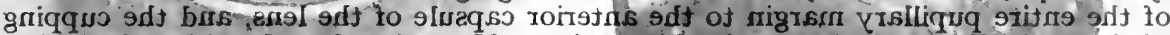

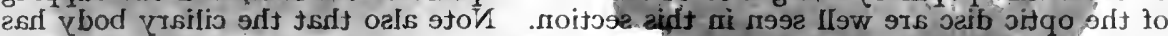

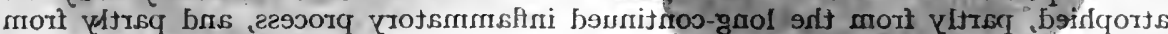

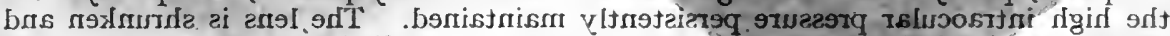

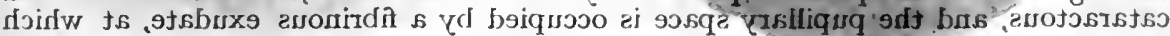

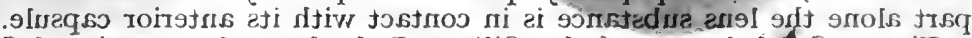

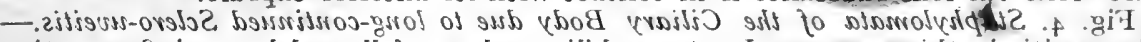
noifsmmsthi ns vd b9wollol zsw bns ailindque of 9ub zsw

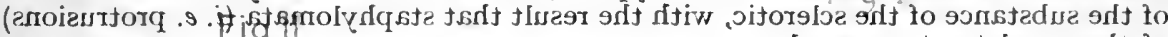

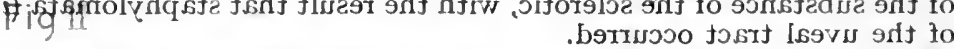

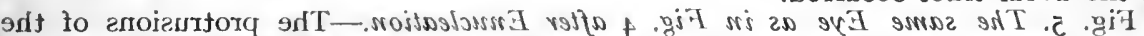

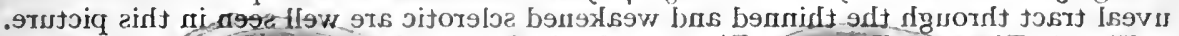

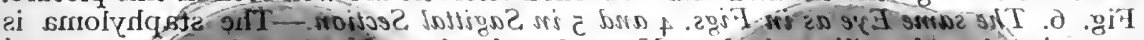

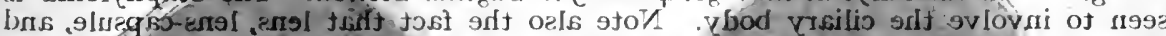

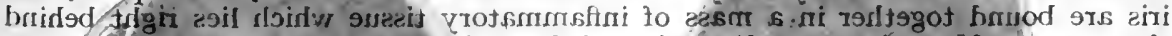

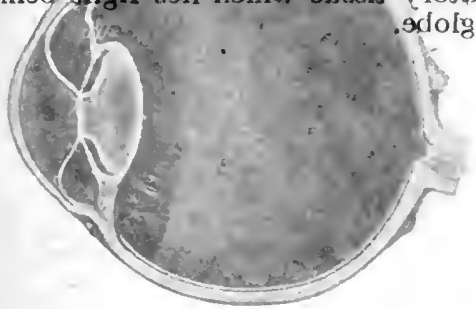

Fig U

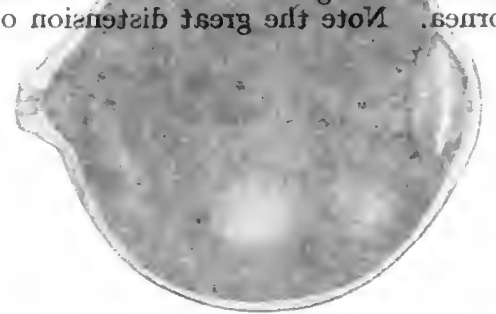

Fig II 


\section{CHAPTER XXII}

\section{DESGRIPTION OF PLATE Xnce of the exudate-} COMPLICATi bombé iridoncosis-Secondary glaucoma-

Fig I Irdoncosis cor Iris Bombert There is af chronic uveitis-The use of margin to the anterior capsule of the pass from the posterior into the anterior chamber, and, collecting in the posterior chamber, causes the iris to bulge forwards-hence the term iris bombé.

Fig. 2. Iris Bombé and Occlusion of the Pripil due to Advanced Sympathetic Uveitis.-In addition to iris bombe, there is occlusion of the pupin in thisrcase, ire. the whole of the pupillary opening is filled up by a dense fibrinous exudate coming from the iris. This condition, by preventing the normal circulation of the intraocular fluids, causes a rise in the intraocular pressure and secondary glaucoma. If it is long-continued, cupping of the optic disc, and distension of the episcleral veins - seen in this case-ensue.

Fig. 3. Horizontal Section of an Eyeball, the subject of Iris Bombe, Occlustion of the Pupil, and Secondary Glaucoma.-The bulging forward of the iris, the adhesion of the entire pupillary margin to the anterior capsule of the lens, and the cupping of the optic disc are well seen in this section. Note also that the ciliary body has atrophied, partly from the long-continued inflammatory process, and partly from the high intraocular pressure persistently maintained. The lens is shrunken and cataractous, and the pupillary space is accupied by a fibrinous exudate, at which part alone the lens substance is in contact with its anterior capsule.

Fig. 4. St phylomata of the Ciliary Body due to tong-continued Sclero-uveitis.The uveitis in this case was due to syphilis, and was followee by lan inflammation of the substance of the sclerotic, with the result that staphylomatah (is protrusions) of the uveal tract occurred.

Fig. 5. The same Eys as in Fig. 4 aften Enucleatidn. The protftisions of the uveal tract through the thinned and weakened sclerotic are well seen in this pilature.

Fig. 6. The same Eye as in Figs. 4 and 5 in Sagittal Section. The staphyloma is seen to involve the ciliary body. Note alse the fact that lens, lens-capsule, and iris are bound together in a mass of inflammatory tissue which lies cright ibehind the cornea. 'Note the great distension of the globe.

$$
\text { capsule--total posterior }
$$

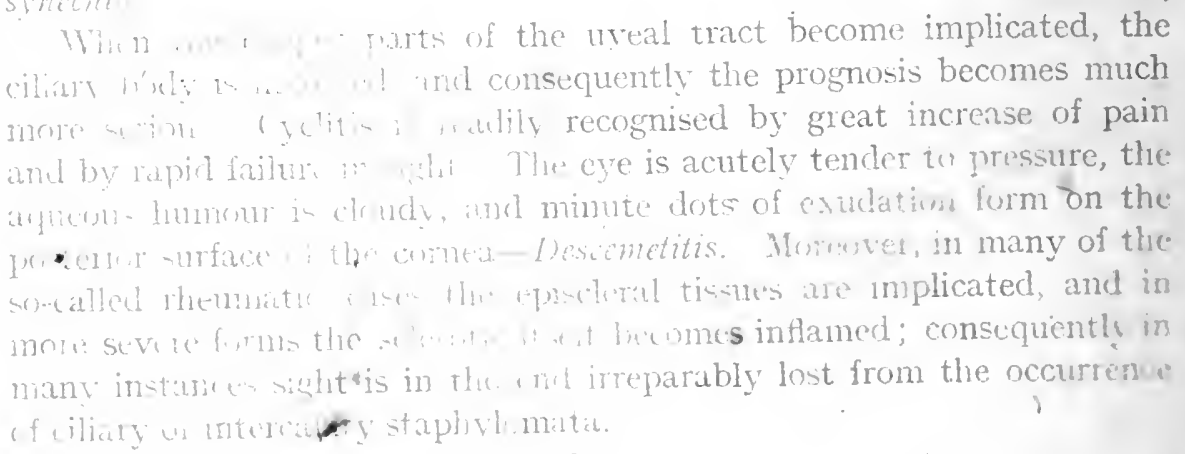




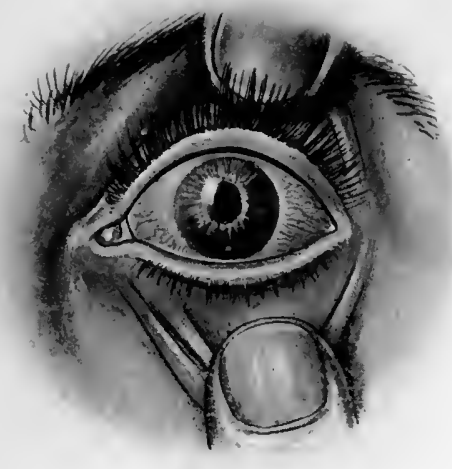

FigI

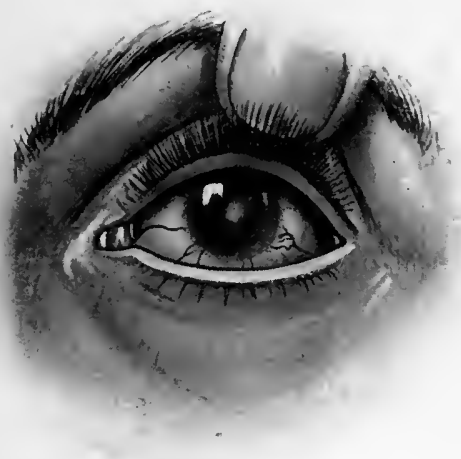

Fig II

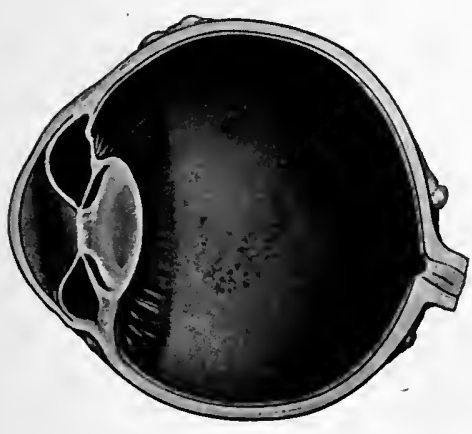

Fig III

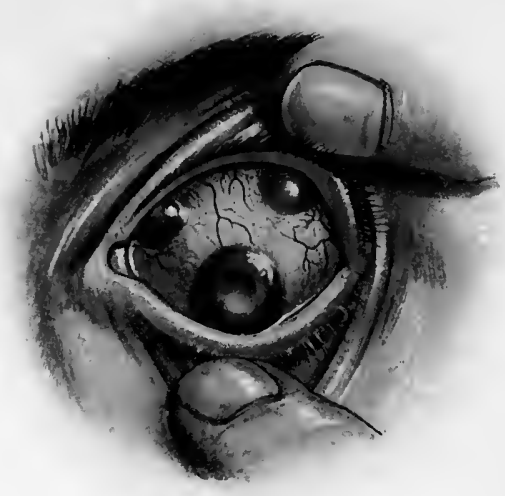

Fig $\square$

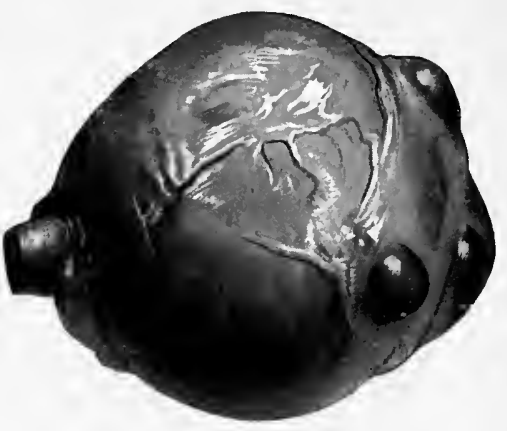

Fig $\nabla$

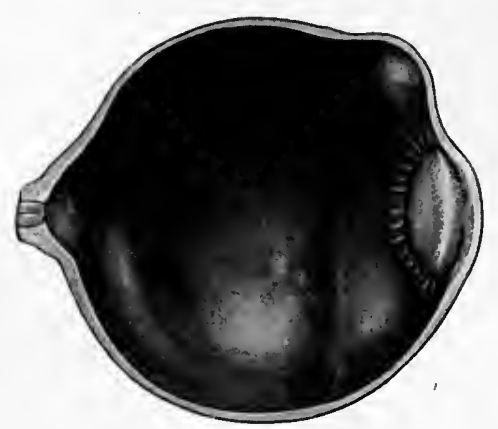

Fig $I I$ 

When inflammation beginning in the iris spreads backwards to the ciliary body and the choroid, it is characterised from the first by pain and all the usual external signs of inflammation. Although at times the patient may be free from suffering, yet vision, instead of improving during these periods of respite, becomes steadily more and more blurred, and in consequence the disease has been described as the despair of both the patient and his doctor. In every case there is more or less exudation ; and, as has already been said, it is the exudation which plays the most important part in the pathological history of the whole morbid process. Instead of being limited to points around the pupillary margin-posterior synechia-the adhesion of the iris to the lens capsule may be so complete as to preclude the possibility of any circulation of fluids between the posterior and anterior divisions of the aqueous chamber-exclusion of the pupil-and when such a condition exists, a mass of fibrin generally fills up the pupillary area more or less completely-occlusion of the pupil. The amount of exudation on the surface of the lens may be so great as to resemble cataract. When the pupil is excluded or occluded, cyclitis followed by secondary glaucoma occurs; the iris bulges forwards from the pressure of the aqueous behind, and the depth of the anterior chamber is diminished except at its central portion-iris bombé or iridoncosis. Glaucomatous symptoms are liable to supervene after the slightest exposure to cold, dust, or glare; after any indiscretion in eating or drinking; or after a sleepless night, or any worry or anxiety. In such cases the iris is always discoloured and atrophied, and at one or more points its fibres are so thinned that the uveal pigment shines through as black spots or stripes; the iris stroma proper becomes atrophic and so completely adherent to the lens capsule, that if iridectomy be attempted, only the superficial layers can be removed piecemeal; and the dense pigment layer remains glued to the lens capsule from the pupil to the ciliary border. The border of the pupil is often deeply pigmented, and there may be ectropion of the pigment layer of the iris. The bloodvessels also degenerate, and intraocular hæmorrhage is of frequent occurrence. By and by, the nutrition of the globe is so seriously impaired that the intraocular tension diminishes, cataract forms, the vitreous shrinks, the retina becomes detached, and the eyeball, now quite blind, gradually shrivels. In the very early stages of a case in this group, where cataract is threatened, it is always right to instil into the conjunctival sac, or to inject beneath the conjunctiva, a I per cent. solution of iodide of sodium, combined with I per cent. solution of chloride of calcium; because there is both clinical and experimental evidence in support of the contention, that cataract may in some cases at the very first start be arrested, or even dispersed, by the use of iodides, more especially the iodide of calcium. 
Where the disease begins in the choroid, the patients usually complain of muscæ volitantes, and of indistinctness of sight; but although an aching in the affected eye and on the same side of the forehead may be mentioned, pain as a rule is not a striking feature of this form of inflammation; sight, however, steadily fails. When the eye is carefully examined, several greasy-looking spots are usually observed on Descemet's membrane; the iris is thickened and presents a lack-lustre appearance, and the instillation of a drop of cocain reveals that it is more or less completely glued to the capsule of the lens by posterior synechiæ. The pupil itself may have a somewhat cloudy appearance, but ophthalmoscopic examination shows it to be quite free from opacity. Floating bodies are, however, usually detected in the vitreous, and signs of inflammation of the choroid may be present. Both eyes, as a rule, are affected, although one may be attacked considerably in advance of the other. Vision gradually deteriorates, and finally may become extinguished; while, from increase of the opacities in the vitreous, it becomes more and more difficult to distinguish any detail in the fundus oculi. These cases have been for the most part regarded as due to syphilis, inherited or acquired; but the recent experiments of Stock upon animals, and the use of injections of Koch's old tuberculin have demonstrated that many of them are tuberculous. Over and above all these, however, a number of cases remain which cannot be attributed either to syphilitic or tubercular infection, and clinical experience shows these to be manifestations of the rheumatic, or even more of the gouty diathesis. Our fuller methods of physical examination, especially the careful investigation of the urine, enable us to make this differential diagnosis; and the truth of it is proved by the gratifying results occasionally obtained by general treatment, $i$. e. by a regimen directed against the arthritic diathesis, carefully suited to the needs of the individual patient.

Treatment.- In the acute cases, all that has been said of the treatment of iritis is doubly true. Both the general and dietetic treatment must be thoroughgoing, and patiently persevered in over a long period of time. The all-important point is to make a searching diagnosis early, so that from the first the treatment may be both local and constitutional. Both are important, and each should always have its proper place. Neglect of either will bring about complications; and, of course, if tissues be destroyed, no amount of medicine of any kind can restore them. In many instances, however, when, as a result of complications, the whole uveal tract has become involved, and the prospects of success are not hopeful, a well-thought-out line of treatment and earnest perseverance will accomplish much, while an ill-considered plan and intermittent effort will altogether fail. It is, too, of the greatest value for the patient 
that the doctor be resourceful. There is no royal road in medicine, and, whatever drugs be employed, it cannot be too strongly urged that, no matter how high the reputation of a remedy, it at once does harm to the individual for whom it has been prescribed, if it interferes with his powers of digestion and assimilation of food. He is as a rule the most successful practitioner who can at once vary the preparation and the combination of drugs to suit the idiosyncrasies of the patient, and at the same time never depart from the line of treatment he has laid down for himself.

As a rule the patient should be put to bed for three or four weeks, in order that any active treatment-leeching, sweating, etc.-may be carried out efficiently. In every case the first thing to do is to attend to the action of the bowels. In the early stages of all diseases of the uveal tract, a mercurial purge followed by an alkaline saline draught stimulates the liver, bowels, and kidneys; and their prescription is usually attended by the most satisfactory results. Many of the patients suffer habitually from constipation; and it happens not infrequently that the bowels cannot be satisfactorily evacuated, until the rectum has been thoroughly washed out with a soap-and-water enema containing a large dose of castor oil and turpentine. Renal inadequacy is always one of the outstanding features of this condition, and the defective elimination is revealed by the high specific gravity of the urine. An increased quantity is frequently accompanied by a rise in the specific gravity, which may continue for several days until what seems to be a urinary crisis is reached; after that the specific gravity falls rapidly, and the quantity of urine passed in the twenty-four hours is steadily maintained. Improvement in the ocular condition is usually synchronous with the occurrence of what has been called the urinary crisis.

The use of salicylate of soda ought not to be forgotten; and in cases due to absorption of microbic toxins from the alimentary tract and elsewhere, pain and other signs of inflammation are frequently relieved with surprising rapidity by the hypodermic injection of 2000 units of antidiphtheritic serum. As to the why or wherefore of the beneficial action of this last, no satisfactory explanation has been given. Modern teaching encourages the belief that both microbic infection and antitoxins are specific, and, therefore, so far as is known, the use of a nonspecific serum can have no scientific basis. Therapeutics being, however, more of an art than of a science, a sane empiricism is always of value to the patient; and of the clinical fact of the power of antidiphtheritic serum, under certain non-specific conditions, to relieve pain I am so convinced that I would rather trust to it than to hypodermic injection of morphia. Its use is, as a rule, unaccompanied by any constitutional disturbance; untoward results have been described by Darier and others, 
such as cutaneous eruptions, backache, and albuminuria, but of these I have up to the present had no personal experience. In one of my cases there was sudden rise of temperature accompanied by a rigor, but the fever lasted only a few hours and was followed by no unpleasant consequences. Although very large doses may be administered with safety, 2000 units are, in my experience, on an average quite enough, and this quantity may be repeated daily, until three or four doses have been given. After that, however, the serum must not be used again, on account of the danger of anaphylaxis. In medicine it is very easy to argue post hoc ergo propter hoc, but the uniformly favourable results which I have obtained at the bedside in dealing with cases of extreme gravity enable me confidently to advise the use of antidiphtheritic serum in those very acute and apparently hopeless cases of irido-choroiditis in which enucleation seems to be inevitable. In this connection it is interesting to read the account of the successful results obtained by Dr. Robin Brown in the treatment of orchitis by antidiphtheritic serum.

Generally speaking, it is wise to begin by prescribing the salts of soda and potash, the therapeutic effects of which are enhanced by combining them with ammonia; the mixture to be well diluted with water and taken between meals. For the gouty, lithia may be added with advantage, and in their case the large amount of fluid taken with the medicine is often of great value, for, as a rule, such patients drink far too little water. If complaint be made of feelings of depression, and of disinclination for, and discomfort after, food, a simple tonic containing tincture of nux vomica and a liquid preparation of pepsin, taken twenty minutes before meals, will very often remove the unpleasant symptoms, and enable the patient to persevere cheerfully in the use of the alkali. The effervescent preparation of piperazine may be given with advantage as an alternative to the alkaline mixture.

In both chronic and acute cases much good is obtained from repeated diaphoresis and the use of alterative medicines. Turkish baths, at regular intervals, and carefully graduated so that they do not produce any after-depression, are most valuable.

The older practitioners had great faith in mercury; and there can be no doubt that a pupil which has stubbornly resisted the mydriatic action of atropin will often begin to dilate synchronously with the slight soreness of the gums which indicates the first sign of approaching salivation. Just that amount of mercurialisation, and no more, should be obtained as quickly as possible; and by carefully regulating the dose, and using a chlorate of potash mouth-wash freely, the patient can be kept gently under the influence of the drug for weeks without the slightest danger. In the later stages, mercury in the form of a Plummer's pill, every night 
at bedtime, or the bichloride combined in a mixture with quinine and iron, three times a day after meals, and alternating with a course of arsenic, are useful methods of carrying out general treatment. A combination of chloride and iodide of ammonium, or of the iodides of sodium and ammonium, is also of great service. In all this medication, however, attention ought to be paid to the caution already given, that the plan must be adjusted and carried out so that nothing in any way interferes with the patient's digestion and assimilation of food.

Subcutaneous injection can be employed to administer any of the remedies likely to be prescribed in dealing with diseases of the uveal tract, and is a valuable means of treatment for patients who are, owing to digestive peculiarities, unable to assimilate medicines when taken by the mouth. The hypodermic use of 25 per cent. iodipin has been very satisfactory. The injections are made between the shoulder-blades, every second or third day, in the usual way, with strict antiseptic precautions. The dose is from one to two drachms, and before being used it should be warmed to about the temperature of the body. Heat, by increasing the fluidity of the iodipin, enables it to pass more readily from the syringe, and it also renders the treatment less painful. The puncture made in the skin should be covered with adhesive plaster.

The essence of treatment is, after all, careful dieting on the lines already indicated in dealing with iritis. The advice to patients should be to eat sparingly, to avoid alcohol, to take plenty of exercise, to have the dwelling thoroughly ventilated and well exposed to the sun, and to live if possible on a gravelly soil in a high, dry, bracing loccality.

Early surgical interference is to be deprecated. The symptoms when the patient is first seen, may, however, be so urgent that delay is not possible. If the pupil threatens to become excluded by posterior synechiæ, and symptoms of subacute glaucoma supervene, iridectomy should be done without any hesitation. It ought to be carefully borne in mind, however, that the necessity for operation arises from the failure of local and constitutional treatment to prevent the formation of extensive posterior synechiæ. Great improvement in sight often follows the iridectomy, and the patient naturally attributes this to the operation. In reality, the cutting out of a piece of iris only removes a mechanical obstruction to the free circulation of the intraocular fluids, and can in no way be looked upon as a substitute for continuous maintenance of the regimen directed against the gout or the rheumatism, or whatever else may be the underlying cause of the whole condition. Operation, therefore, must never be regarded as a cure for the disease. It simply gets rid of an obstacle, and thereby gives general and dietetic treatment a fair chance. 
While, therefore, operation must not be too long delayed, and ought to be resorted to at once, if the intraocular tension is high, and sight is rapidly failing, it ought always, when possible, to be preceded by careful general treatment; and the most lasting results are obtained when an equally careful regimen is steadily followed after the operation has been performed.

In such circumstances, it is always prudent to operate with the patient thoroughly under the influence of a general anæsthetic, because so much depends upon the exactitude of the operation. If much pain follows, the prompt application of an ice-bag to the eye, and the administration of $\frac{1}{6}$ of a grain of morphia subcutaneously, will often insure a restful night-a great help in obtaining a good result, and securing permanent improvement. What has just been said must not, however, be interpreted to mean that the occurrence of pain is always to be prevented. On the contrary, pain may be of the greatest service, for when properly understood it is a danger-signal warning both patient and surgeon that something is wrong. The suffering, however, which follows immediately after an operation is quite different, because it interferes with the patient's capacity for sleep, which, as Sir James Paget insisted, is of the greatest value in giving Nature the chance to encourage healing of a wound, of which she is never slow to avail herself.

The question as to the most favourable time for operation on gouty or rheumatic patients, will also present itself in those unfortunate cases in which the patient's one eye is completely blind and shrunken as a result of advanced irido-choroiditis, and a fully developed cataract is present in the other eye when first seen by the surgeon. If the eye with cataract be free from inflammation and the projection of light good, the temptation to extract the opaque lens is great; but, on the whole, a more favourable result will be obtained if time be given to treatment of the patient and his special disease proclivities before the operation is undertaken.

During convalescence the patients should be sent to the countryto woods and green fields-for a few weeks, and advised to avoid reading, writing, and sewing. They should wear peacock-blue glasses to protect the eyes from bright light; that colour of glass, by cutting out the red rays, is usually found most grateful to those suffering from inflammation of the uveal tract. They must also be counselled to adhere strictly to the regimen prescribed for them. An alkaline chalybeate tonic after meals, and a simple laxative at bedtime, are usually necessary. A recurrence of constipation will, almost to a certainty, be followed by a relapse in the ocular inflammation, which in many instances is attended by the occurrence of intraocular hæmorrhage. 


\section{CHAPTER XXIII}

\section{INFLAMMATION OF THE SCLEROTIC}

Episcleritis: symptoms: episcleritis periodica fugax-Sclerotitis: symptomsSclerotinising keratitis-Treatment: local: general.

INFLAMMATION of the sclerotic usually attacks the part between the equator and the margin of the cornea. It is a comparatively rare occurrence, and may assume one or other of two forms : first, episcleritis, where only the superficial layers are affected, and where, though it causes a good deal of annoyance, it is never serious, and second, sclerotitis, where the middle and deeper layers are affected, and where, owing to complications involving the cornea, iris, ciliary body, and choroid, there is grave danger. to sight. No matter which form be present, while a certain number of cases are the result of syphilis, gonorrhœa, or tubercle, by far the larger proportion are undoubtedly due to gouty or rheumatic predisposition. The disease is generally found in young adults; and women suffer more frequently than men, the attacks often beginning with the onset of a menstrual period, and passing off at its close. When that is so, a recurrent attack may be expected at each subsequent period.

Whether the inflammation be superficial or deep, the form of the lesion is largely explained by the anatomical structure of the sclerotic. Its blood supply is small, but it is in close relation externally to the vessels of the conjunctiva, and internally to those of the choroid, ciliary body, and iris; consequently inflammation of the sclerotic is often secondary to a similar condition in the ciliary body.

1. Episcleritis.-Episcleritis is, generally chronic, the attack often lasting for some weeks; the patient complains of an aching pain in the brow on the affected side. When the eye is examined, a raised patch, of bluishred colour, is seen on the sclerotic, close to the corneal margin, obviously below the conjunctiva, and usually tender to touch. As time goes on, the elevation changes colour, becoming more and more livid, until at length it is slate blue, and then it gradually disappears. Recurrence is, however, very common, either at the same place, or at another, also near the corneal margin; and these relapses may go on until the whole circumference has undergone the inflammatory process. 
A very rare condition, but one which, when it does occur, is always distinctly associated with gout or rheumatism, is that described by Hutchinson as " hot eye," and by Fuchs as episcleritis periodica fugax. It resembles the more chronic forms in its tendency to recur, but the disturbance is much more serious while it lasts, and the persons affected are generally of middle age. The duration of the attack, which may involve both eyes or only one, varies from a few hours to a few days, and when it is over, recovery is complete. The eye presents, on examination, a striking clinical picture : considerable swelling is present, and both conjunctiva and episclera are inflamed. The patient complains of pain, the eye waters, and there is great intolerance of light.

It has been demonstrated that the raised patches in episcleritis are due to the separation of the fibres of the episclera by exudation, and these elevations are thus connected with the subcutaneous nodules found in "rheumatic" subjects. Suppuration never occurs, and cure takes place by gradual absorption of the exudate. The characteristic bluish colour is due to the choroid shining through the thinned sclerotic.

2. Sclerotitis.-Sclerotitis is, as has been already said, a much more serious condition than episcleritis; for, although at first the sclerotic alone is affected, the disease is very insidious, and, later, the cornea and uveal tract are involved. Vision is always impaired and often lost. The patient suffers acute pain, the eye is tender to touch, there is profuse lachrymation, and there is great intolerance of light. Examination shows that the sclerotic is much swollen, and that on it, at intervals, there are thickened patches having the appearance of porcelain. The swelling is not in circumscribed elevations, as in episcleritis, but is more uniform, and may occupy the whole circumcorneal zone, while a few dilated bloodvessels may be seen on the surface. By and by the cornea is involved, inflamed patches seeming to spread to it from the sclerotic-sclerotinising keratitis-there is iritis of a chronic type, and there is serious diminution of vision. With the occurrence of iritis the pupil is contracted and occasionally displaced; and, as a result of posterior synechiæ, the iris may impinge on the posterior surface of the cornea and cause obliteration of the corneo-iridic angle and of the canal of Schlemm, so that secondary glaucoma is a not unusual complication. The pathological change in the tissues is chiefly marked by a deposit of leucocytes in the middle and deep layers of the sclera, accompanied in some cases by a fibrinous exudate, which is afterwards absorbed, leaving a dark-coloured scar. The sclera thus, in the course of cicatrisation, becomes so much thinned that ectasiæ are apt to take place. The weakened sclerotic, unable to resist the increased intraocular pressure, bulges forward to form a staphyloma, which is at first usually partial and is situated immediately behind the corneal margin, 
around which are blue elevations-multiple staphylomata. Should the ectasiæ be situated over the ciliary processes (where they show as dark streaks) they are called ciliary staphylomata; but their usual site is a little farther forward along the zone between the cornea and the root of the iris, and then they are called intercalary staphylomata. As the disease progresses, the increased secretion of fluid into the vitreous causes still greater tension, with the result that the already weakened walls of the globe stretch, and there is a certain amount of apparent exophthalmos. By this time, too, the cornea is opaque and enlarged, and its curvature so altered as to make it seem almost continuous with the attenuated sclerotic.

Treatment.-In both forms of sclerotic inflammation, prolonged and painstaking care is necessary to cure the attack and prevent relapse; and the ophthalmic surgeon has often need of all the medical resources at his command to combat this chronic and tedious affection with success. Treatment should be both local and general : the all-important matter is to deal with the underlying cause, as well as to make use of palliative measures for relief of the actual pain. The ordinary treatment will be the same for both forms of the disease, as they differ only in degree. As far as local measures are concerned, a blister should be applied to the temple, or two leeches to the outer canthus; the former is of much service in a rheumatic case, but may cause great inflammation of the skin of a gouty patient in whom leeches always give prompt relief. The value of atropin used early should be remembered, as it is an important point in the treatment to see that the pupil dilates at once and remains dilated. Should the. instillations of atropin, however, be followed by pain and increase of intraocular tension, they must at once be discontinued, and eserin or pilocarpin substituted. In all inflammations of the eye of rheumatic or gouty origin, the patient is very intolerant of irritating lotions; consequently, in dealing with diseases of the sclerotic, all eye-washes, except the most bland, should be avoided : bathing with warm saline solution, or with borax lotion, will be the best means of affording relief. Fomentations may sometimes be very helpful, but must be used with caution, as the skin of both gouty and rheumatic patients is very prone to eczema. For the relief of photophobia, dark glasses should be worn in bright light, and any error of refraction must be corrected.

Ionic medication has been highly recommended by Leduc, and very satisfactory results have been obtained. Clearly, however, such measures can be successful only if the disease has passed its active stage, and this method is, therefore, of service merely in dealing with the results of the morbid process. A hot solution of chloride of sodium, $x$ or 2 per cent., is prepared, and a compress made of several layers of absorbent lint dipped into it and 
laid upon the affected eye. The lids are closed, and the anode, being applied to any part of the body, the electric current is allowed to pass with a gradually increasing strength up to 5 or 6 milli-amperes. If the increase be very gradual, the patient ought to feel no pain. The application should last for a quarter of an hour each time, and be repeated two or three times a week.

If the symptoms be in the least degree acute. the patient should be kept in the house and in bed; and, indeed, at the commencement of recurrent sub-acute attacks in the more chronic torms, a few days in bed may be sufficient to effect a cure. Cold and damp are very powerful factors in causing a relapse, and exposure to either must, therefore, be carefully avoided. General medical treatment should begin with a brisk purgepreferably calomel followed by a saline draught -and after that the bowels should be kept freely open with some form of salts which has a special action on the liver. Should the family or personal history of the patient show any tendency to gout or rheumatism, it will be very beneficial to prescribe colchicum or salicylate of soda combined with an alkali. Fxamination of the urine must never be neglected; and it is often of great help to take the specific gravity of the whole quantity passed in twenty-four hours and to test carefully for indican in excess. In all these cases the free use of diluents is necessary ; consequently it is very important to know the actual quantity of urine passed in twenty-four hours, and to compare that with the specific gravity of the specimen. It is equally important to exercise careful supervision over all the digestive processes in order to prevent a relapse.

Most important of all, however, is it to obtain strict adherence to a carefully planned regimen, which is the essential part of the treatment of this as of every other form of "gouty " or "rheumatic" disease. A dry soil and bracing air often work wonders; and a person, who, while living on a clay soil in a river valley, is continually subject to annoyance from attacks of inflammation of the sclerotic, may find himself perfectly free from trouble from this cause if he can live on a sandy or gravelly soil, a few hundred feet above sea-level. A period at a Spa, either at home or abroad, has often excellent results, for it has the double advantage of giving the patient appropriate medical treatment, and at the same time of taking him away from his business and other cares. A great many natural mineral waters are now to be obtained in bottle, and may, in this state, do good to those who, from any cause, cannot be sent away from home; but there is no question that residence at the watering-place itself is much more beneficial, partly no doubt on account of the environment, and partly perhaps because the waters lose some of their finer qualities by being put in bottle. At the Spa there are the greatly increased amount of sunshine and fresh air 
that patients enjoy, the brightness of the place and its surroundings, the company, the change from the daily round of home routine, the lack of the wear and tear of work and worry, all of them most helpful. As to the waters themselves, just as the air around us contains some rare constituents, that have only recently been determined, so these products of Nature's laboratory may contain fine gases that escape after their transfer to the bottle; or they may contain minute quantities of other ingredients, which with even brief lapse of time may change their combination, and so cease to exercise the same influence on the living tissue. We know now, too, that many waters have certain radio-active properties, and it is, therefore, possible that there may be, in the process of bottling and keeping, a loss of these powers. Looking at all the conditions, we may be quite certain that the value of natural mineral waters is ever greatest at the source, and that the advantages of the actual well-appointed Spa far outweigh any comfort that may come from having the water in home surroundings. Apart from anything else, the complete regularity of the place and its ways fits thoroughly in with the scheme of inducing the patient to abide strictly by the regimen which has been prescribed for him, and this is, as I have already insisted, the very essence of the cure.

Such methods as I have indicated often bring about a great improvement in general health, but, unfortunately, this may come too late. The prolonged and recurrent attacks of inflammation may already have induced such grave ocular changes, due to complications with the iris and ciliary body, that the nutrition of the eye is constantly imperilled. Increased intraocular tension is a very serious danger, and prompt measures often require to be taken for its relief. When leeches, fomentations, myotics, morphia, etc., all fail, surgical interference ought at once to be resorted to. Probably the easiest and safest method of operating, in the first instance, is to puncture the sclerotic with a broad needle. The puncture should be made as near as possible to the equator of the globe, and the needle should be turned on its axis, so as to permit some vitreous to escape. This causes immediate hypotension, and pain is at once relieved, the respite from suffering being occasionally of considerable duration. When the cornea is extensively involved, and the inflammation is being kept up by large blood-vessels, which enter its substance and traverse its surface from the periphery, the excision of a circumcorneal ring of conjunctiva (peritomy) cuts off the abnormal vascular supply, and so facilitates the disappearance of the corneal opacities. A somewhat similar operation, also at times attended by very gratifying results, is the cauterising of the tissues surrounding the cornea, a ring being burnt down to the level of the sclerotic. When intraocular tension is high, trephining at the corneo-scleral margin is recommended (Elliot). 
Occasionally an iridectomy does good; for not only does it lower intraocular tension, but, if the piece of iris be excised opposite a transparent portion of the cornea, the artificial pupil may greatly improve sight. Iridectomy should, however, never be performed till all symptoms of inflammation have subsided. Premature operation may make matters much worse, and be followed by acute inflammation leading to general distension of the eyeball. On the whole, therefore, scleral puncture and peritomy, either by the knife or by the cautery, are the safest surgical methods, and they have the additional advantage that, provided aseptic precautions be taken, they may be repeated as often as necessary without fear of doing harm. Unfortunately, however, in many instances, while the distress incident to acute attacks may be, to a great extent, relieved, it is not possible to arrest the progress of the disease. In favourable conditions there may be some slight improvement, but in almost every case it is merely temporary, and, in predisposed subjects, the slightest cause induces a relapse. After each recurrence vision deteriorates more and more; and, when sight is entirely lost and the suffering still continues, nothing can be done except to enucleate the eyeball, which by this time is completely disorganised. 

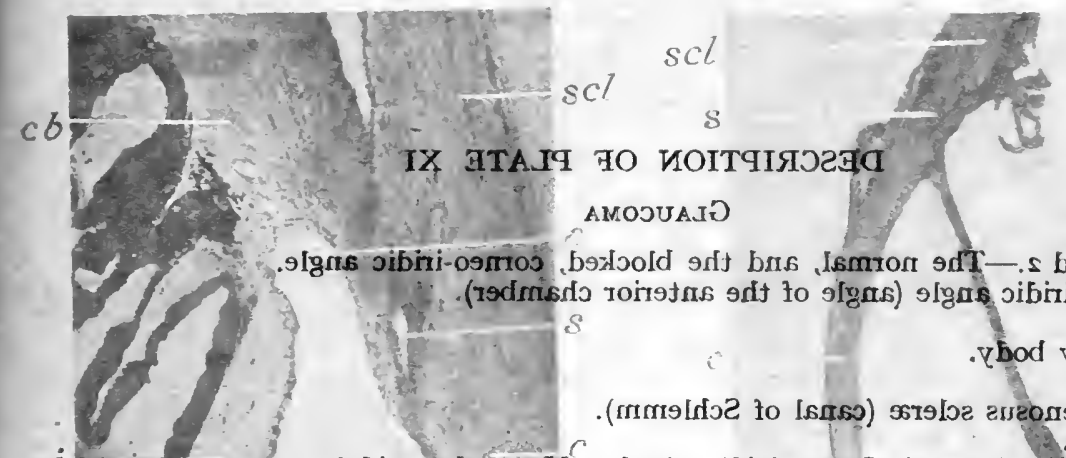

cis

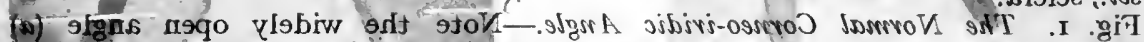

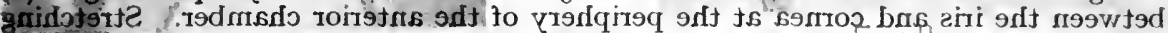

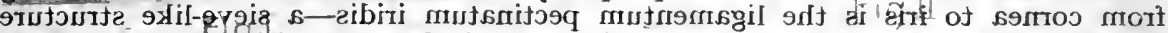

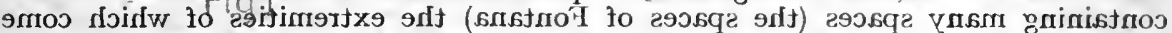

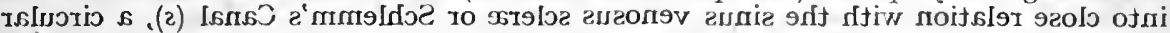

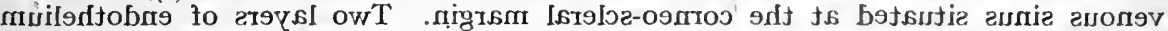

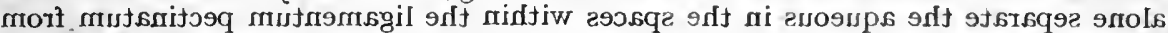

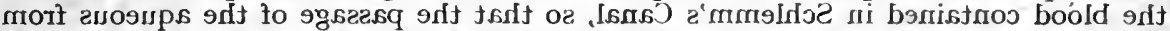

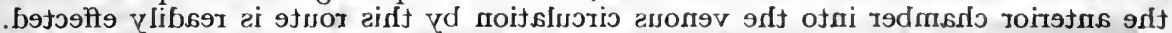

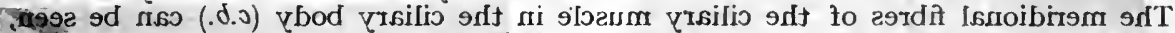

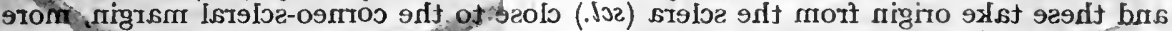

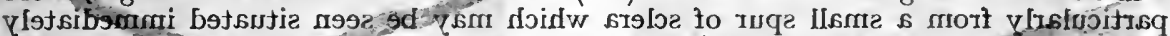

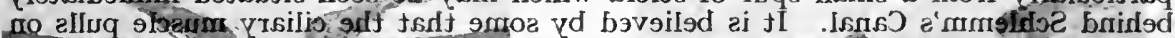

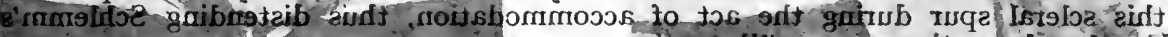

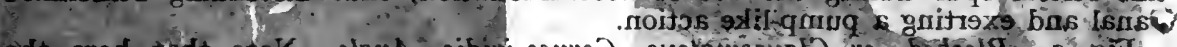

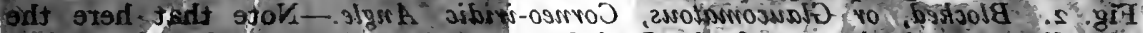

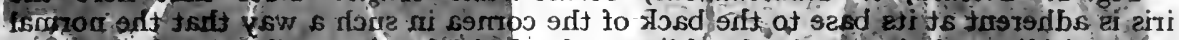

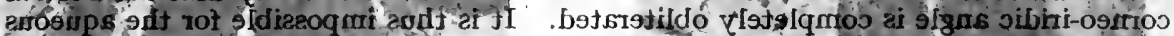

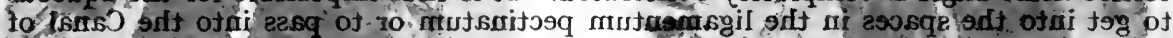
. (2 +5) - armslifo?

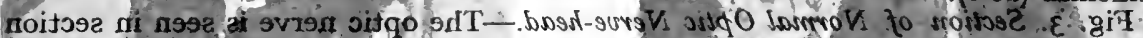

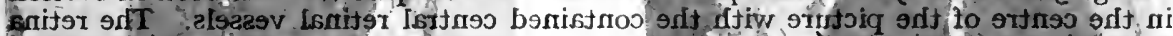

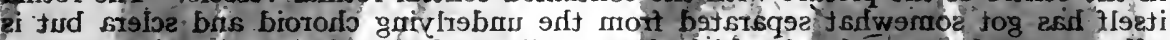

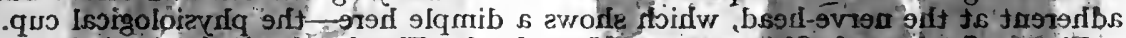

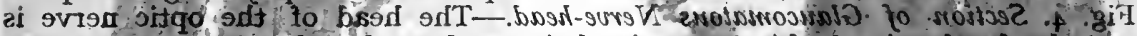

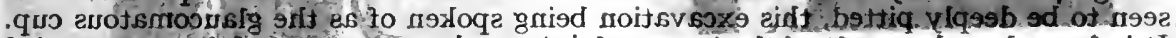

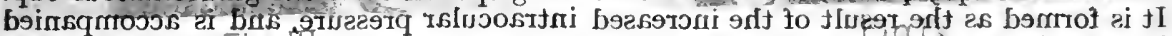

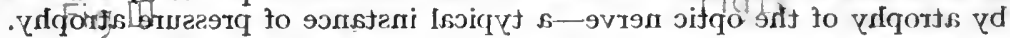


().ernus a d in indectomy does good; for not only does it lower intraor 1 , the the the if the proce of iris be excised opposite a transparent

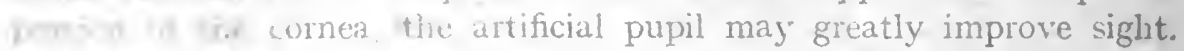

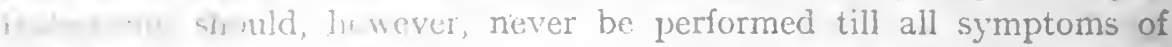
. . un have cubsided. Premature operation may make matters and be followed by acute inflammation leading to general the cyeball. On the whole, therefore, scleral puncture and DESCRIPTION QF PLATE XI $_{\text {re the safest surgical }}$ have the adGiucomadvantage that, provided aseptic

Figs. I and 2.-The normal, and the blocked, corneo-imidic anglessary without $a_{2}$ corneo-iridic angle (angle of the anterior chamber)in many instances, while

c.b., ciliary body. $i$,iris t posable to arrest the progress of the disease. In favourable $s$, sin venosus sclerze (canal of Schlemm). scl., sclera.

Fig. I. The Normaln Corneo-iridic Angle -Note the widelyt open anglet (a) between the iris and cornea at the periphery of the anterior chamber Stretching, from cornea to iris is the ligamentum pectinatum iridis a sieve-like structure containing many spaces (the spaces of Fontana) the extremities ofl which icome, into close relation with the sinus venosus scleræ or Schlemm's Canal $(s)$, a circular venous sinus situated at the corneo-scleral margin. Two layers of endothelium alone'separate the aqueous in the spaces within the ligamentum pectinatum from the blood contained in Schlemm's Canal, so that the passage of the aqueous from the anterior chamber into the venous circulation by this route is readily effected. The meridional fibres of the ciliary muscle in the ciliary body $(c . b$.) can be seen, and these take origin from the sclera $(s c l$.) close to the corneo-scleral margin, more particularly from a small spur of sclera which may be seen situated immediately behind Schlemm's Canal. It is believed by some that the ciliary muscle pulls on this scleral spur during the act of accommodation, thus distending Schlemm's Sanal and exerting a pump-like action.

Fig. 2. Blocked, or Glaucomatous, Corneo-iridic Angle.-Note that here the iris is adherent at its base to the back of the cornea in such a way that the normal corneo-iridic angle is completely obliterated. It is thus impossible for the aqueous to get into the spaces in the ligamentum pectinatum or to pass into the Canal of Schlemm (at $s$ ).

Fig. 3. Section of Normal Optic Nerve-head.-The optic nerve is seen in section in the centre of the picture with the contained central retinal vessels. The retina itself has got somewhat separated from the underlying choroid and sclera but is adherent at the nerve-head, which shows a dimple here-the physiological cup.

Fig. 4. Section of Glaucomatous Nerve-head. - The head of the optic nerve is seen to be deeply pitted, this excavation being spoken of as the glaucomatous cup. It is formed as the result of the increased intraocular pressure, and is accompanied by atrophy of the optic nerve- a typical instance of pressure atrophy. 


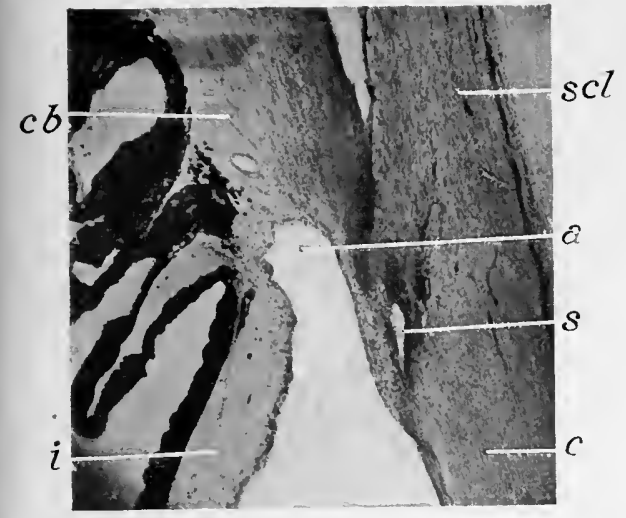

Fig I

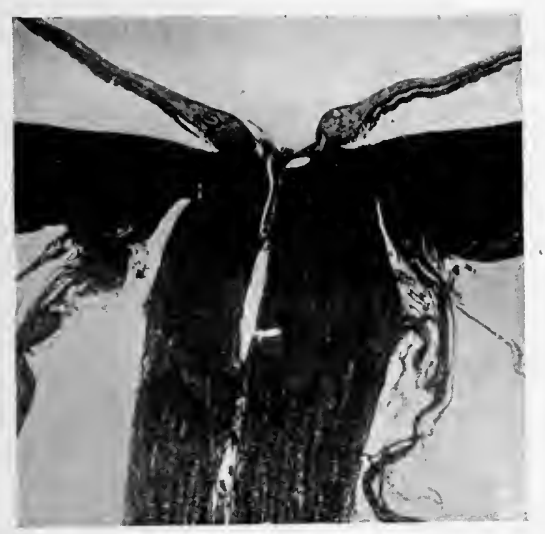

Fig III

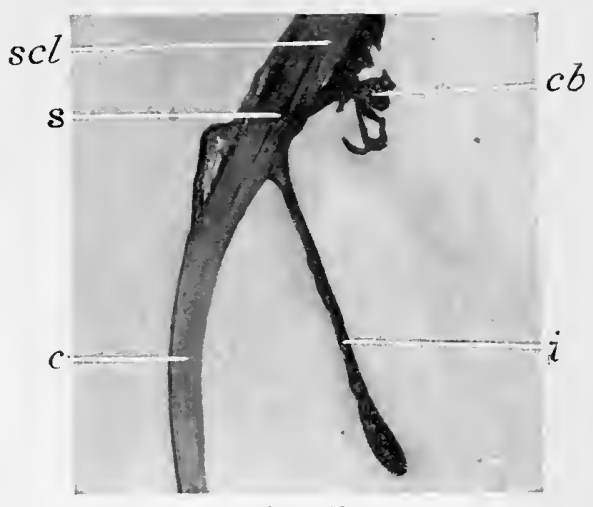

Fig II

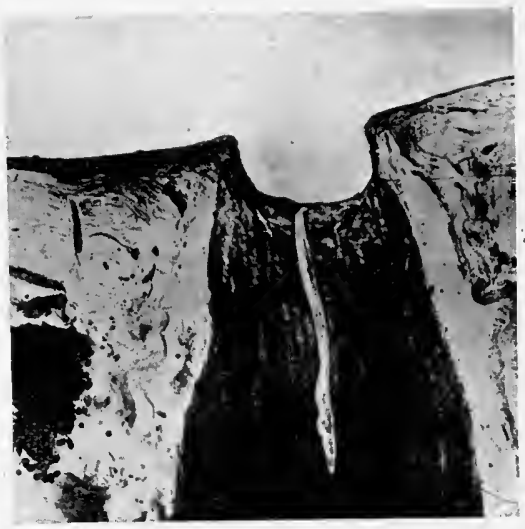

Fig IV 



\section{CHAPTER XXIV}

THE CLINICAL SIGNIFICANCE OF A HARD EYE

Increased tension of the eyeball-Secondary glaucoma-Primary glaucomaCausation-Symptoms, early and late-Alterations in the cornea and sclerotic - Alterations in the uveal tract-Changes in the transparent media-Changes in the retina and optic nerve-Pathology-Non-congestive or simple chronic glaucoma-Symptoms-Glaucoma and cataract-Glaucoma and atrophy of the optic nerve-Congestive or acute glaucoma-Symptoms-Glaucoma and iritis-Hæmorrhagic glaucoma.

\section{THE DIAGNOSIS OF GLAUGOMA}

GLAUCOMA is characterised by increased tension of the eyeball, a physical sign which is frequently observed in inflammation of the uveal tract. The hypertension is due either to an abnormally viscous condition of the ocular fluids, or to some form of posterior synechia, there being in both cases a hindrance to the free excretion of fluids through the filtration spaces at the corneo-iridic angle. It is, therefore, secondary to the disease already present in the eye, but becomes, as soon as it has declared itself, the predominant and most serious feature in the case; and treatment must at once be modified so as to check the fresh complication, and restore, if possible, the normal tension of the globe. A hard eye is always dangerous.

Hypertension occurs, however, also in eyes in which, as far as can be discovered, there was no pre-existing disease. It is then spoken of as primary glaucoma. Even here the disease is not to be looked on as a morbid entity, but as a symptom complex; and its true nature will be all the better understood if one thinks of its acute manifestations as analogous to an attack of angina pectoris.

Its occurrence depends, not only upon the size and the minute structure of the eyeball, but also upon the age, race, and general health of the patient. The small eye of the hypermetrope, for example, is much more liable to be attacked than the larger one of the myope; and so, also, are eyes which are malformed, as, for instance, those in which the iris appears to be congenitally absent. Both eyes are usually affected, but seldom simultaneously, for months, perhaps years, may elapse before the secohd is attacked; and it may be stated generally that the more acute the attack is in the one first involved, the shorter will be the 
interval before the other suffers. On the other hand, if the disease in the first eye is non-congestive, the longer is the second eye likely to escape.

With regard to age, glaucoma is a disease of senility, rarely met with in persons under forty years old, but often seen in those over sixty. It attacks women more frequently than men; and this extra liability of females, more particularly to the congestive forms of the disease, is in many cases intimately connected with the cessation of the menses. Certain races, too, such as the Egyptians and the Jews, seem to have a special predisposition, and the English suffer in greater numbers than the Scotch. This racial proclivity being in great part dependent on heredity and consanguinity, those in whom it is present, or those even who have a strong family tendency, are apt to be affected at an abnormally early age. One of the most striking examples of hereditary predisposition that has occurred in my own practice was a woman of only twenty-seven years of age, both of whose eyes had been attacked by severe congestive glaucoma. I had previously operated on her mother (in whom the first symptoms had appeared when she was thirty-four years of age), also on an uncle. Her grandmother, uncle, and four cousins, all on. the maternal side, suffered from the same disease, every one of them except the uncle having been blind for years. I have recently had to perform a trephining operation on both eyes of that woman's brother, who at the age of thirty showed all the signs and symptoms of advanced glaucoma.

Patients frequently attribute the onset of glaucomatous symptoms to some injury, but there is rarely any sure ground for this assumption. In such cases the eye must be regarded as having been predisposed to attack, for in most of them the injury has been very trivial-so slight, indeed, as to have been forgotten till recalled to mind by the failing sight. Anything that depresses and disturbs the action of the heart predisposes to glaucoma, and an attack may in many instances be clearly traced to some mental distress or moral shock; to the injurious influences of cold or of hunger; or to fatigue, the result of worry or of sleeplessness; while there may be associated anæmia, lithiasis, bronchitis, constipation, or the suppression of some habitual discharge. The most important underlying conditions from an ætiological point of view are certain constitutional states, more particularly the rheumatic and the gouty. In the vascular changes so frequent in patients of arthritic disposition, the ocular blood-vessels do not escape. In eyes predisposed to glaucoma there is probably always an abnormal rigidity of the walls of these vessels, and consequently alterations in the intraocular circulation will take place more readily. A familiar example is seen in the glaucomatous 
attack which occasionally occurs after atropin or other mydriatic has been instilled into the eye of an elderly person. No matter what may have been the determining cause, however, as soon as there is disturbance in the equilibrium between the processes of secretion and excretion within the eyeball, there is at once a rise in the intraocular pressure, and this increase of tension is the essence of the disease; as Priestley Smith puts it, "There is an excess of pressure within the eye, plus the causes and consequences of that excess." The first and most important symptom, therefore, is increase of tension, and from it arise all the other phenomena which go to make up a picture of primary glaucoma. Many instruments have been devised for estimating the amount of the intraocular pressure; but in practice nothing is so satisfactory as the educated finger of the surgeon, and there is always a standard of comparison in the sound eye of the patient, when only one is affected, or in the practitioner's own eye. When, however, frequent observations are necessary in order to make a record of the progress in a particular case, a tonometer is necessary, and the instrument devised by Schiötz is easy to apply and quite trustworthy.

- Once glaucoma has become thoroughly established, there is not much difficulty in its diagnosis. The stages most apt to be overlooked are the early ones; and as successful treatment depends for the most part upon early recognition, it is essential that a clear and accurate conception be formed of the symptoms of which a patient whose eyes are about to become glaucomatous will complain.

The symptoms in the earliest or premonitory stage, whether it be of long or short duration, are transient, and are thus, unfortunately, liable to be disregarded. As one who was suffering from a severe attack of congestive glaucoma, and in whom the premonitory symptoms, lasting over a period of two years, had been very characteristic, said to me, "I thought nothing of them, as they just passed away." That being so, it is the more necessary that the medical attendant should be quick to appreciate, and appraise at their proper value, remarks regarding eyesight, seemingly trivial, but often in reality of the gravest significance, made by a patient to his doctor in a casual manner. In particular, suspicion should always arise : (I) if a patient states that he every now and then suffers from temporary obscuration of vision-that he seems for the time being to see everything through a fog-even although when tested by the ordinary methods the visual acuity is found to be quite up to normal ; (2) if a patient who requires to use spectacles for presbyopia says he has had to change them frequently, and is found to be using glasses much more powerful than ought to be necessary at his age; (3) if he sees coloured rings round a gas or candle flame-an appearance 
which must be familiar to all who have driven in a cab on a frosty night and looked at the street lamps through the steamy windows. Usually while these symptoms last the patient complains of dull pain in the eyes and forehead, and may be conscious that both his central and peripheral vision are defective; but it is only when his medical attendant chances to see him during an attack that any objective signs of glaucoma can be detected. It is then found that the eyeballs are harder than normal, and that the cornea is more or less cloudy. There may be slight dilatation of the pupil, but as a rule there is no diminution in its response to the stimulus of light, and the iris presents a normal appearance. There may, or may not, be some slight pericorneal injection, and overfulness of the episcleral veins. The proper tests will show that the extent of the visual field is contracted, with the limitation most marked on the "nasal side.

In incipient glaucoma Bjerrum's sign will, if sought for with care, be almost invariably found. It may be detected before the development of any symptoms indicating impairment of the functional activity of the eye, and may then be regarded as belonging to the prodromal stage of the disease. It consists of a characteristically shaped relative scotoma, which can always be traced to the blind spot. For its demonstration it is necessary to have a screen of black velvet suspended vertically opposite a window, and a white test object of ivory or cardboard mounted on a long slender black rod. The test objects employed are very much smaller than those used with the perimeter, and in practice a disc $3 \mathrm{~mm}$. in diameter will be found most convenient, the patient being placed six feet in front of the screen. The test is most delicate, for not only does it show the presence of glaucoma in eyes in which the central visual acuity and the peripheral field of vision are normal, but, as Sinclair has pointed out, since the area of relative defect can always be traced to the blind spot, it at once distinguishes glaucoma from atrophy of the optic nerve. In the latter, though Bjerrum's method also reveals the existence of a relative scotoma, this is invariably separated from the blind spot. Ophthalmoscopic examination will disclose congestion, and perhaps increased tortuosity, of the retinal veins; and pulsation in the retinal arteries, if not present, can always be produced by slight pressure upon the eyeball. Attacks such as these, in the intervals between which the eye is to all appearance healthy, may vary in duration from a few minutes to several hours; but the periods between them get gradually shorter, until at length the condition of the eye is one of permanently increased tension with the changes consequent upon it, and glaucoma is then thoroughly developed.

It is characteristic of many cases that this increase of tension is 
intermittent; but whenever it has existed for any length of time a number of changes follow in its train.

1. Alterations in the Gornea and Sclerotic.--Sudden increase in the intraocular pressure is shown by a diffuse cloudiness of the cornea, most marked towards the centre, and specially distinguished by the fact that it passes off almost immediately after the excessive tension has been relieved. To this condition must be attributed the occurrence of the iridescent vision. It is due to œdema; and as the anatomical seat of the fluid which causes this is in the nerve channels in Bowman's membrane, the corneal nerves get pressed upon and paralysed; a condition which explains the more or less anæsthetic condition of the cornea found in nearly every case. Owing to its rigidity, the sclerotic in the adult is not much influenced by pressure, except at its weakest part-the lamina cribrosa-or in old cases of absolute glaucoma where it occasionally happens that staphylomatous projections form at any part where the sclera has been weakened by injury, and in the equatorial region of the ball. When, as a result of intra-uterine disease, or after ophthalmia neonatorum or other inflammatory affection occurring during the first years of life, there is an increase in the intraocular tension, the tissues of the eyeball yield very readily, and the globe may become enormously distended. From the large bulging cornea-in some cases clear, in others opaquethis form has received the special name of buphthalmos, or ox-eye; in reality it is simply the consequence of the glaucomatous process occurring in very early life.

2. Alteration in the Uveal Tract.-Sudden congestion of the choroidal and ciliary blood-vessels leads to excess of pressure in, the vitreous, with the result that the lens and iris are pushed forward at the expense of the depth of the anterior chamber. The blood-vessels on the outside of the eyeball become dilated and tortuous, and the conjunctiva may become œedematous; while in very acute cases serous effusion may form between the choroid and retina, and lead to rapid extinction of vision. In advanced cases the choroid may become thinned (the atrophy being usually most marked at the posterior pole of the eye), and this thinning shows itself, on ophthalmoscopic examination, as a whitish-yellow ring surrounding the optic disc. The increased pressure of the distended ciliary processes upon the base of the iris leads to constriction of its blood-vessels, and the pupil becomes dilated; and if the pressure be more pronounced at one part of the iris circle than at another, the dilatation is unequal, and the pupil tends to assume an oval form. In the earlier stages, at least, there is no real paralysis of the sphincter of the iris, for the pupil will contract with eserin and dilate with atropin; but after the increased pressure has been long maintained, the iris fibres 
lose their elasticity and begin to atrophy, and myotics are powerless to influence the pupil. The ciliary muscle is early affected in a similar manner, and its enfeeblement gives rise to the progressive failure in the power of accommodation, which is one of the most suggestive of the premonitory warnings of the onset of glaucoma. Of course, direct pressure upon the ciliary nerves will also tend to intensify the muscular paresis, while irritation of the branches of the fifth nerve affords an explanation of the neuralgic pain which is an invariable accompaniment of the congestive forms of the disease.

3. Changes in the Transparent Media.-The aqueous tends to become muddy, and the lens and the vitreous to lose their perfect transparency; and these changes, together with the opacity of the cornea, serve to explain the peculiar greyish-green reflex observed in the dilated pupil of a glaucomatous eye, which, being to the ancients the most striking feature of the disease, gave rise to the name.

4. Ghanges in the Retina and Optic Nerve.-The retinal circulation is naturally disturbed, because, as a result of the increased pressure, the arteries are incompletely filled, and the veins congested; indeed, when advanced degenerative changes have taken place in the walls of the blood-vessels, hæmorrhages are not uncommon. On ophthalmoscopic examination, both the arteries and the veins, as they pass over the optic disc, are seen to pulsate, and, as pulsation in the arteries never occurs in a healthy eye, its recognition is of great diagnostic value. The increased pressure leads also to changes of a very characteristic kind in the optic nerve and retina. Brailey has demonstrated that the first change in the optic disc, resulting from the increased pressure, is a backward displacement of the lamina cribrosa; and as this carries along with it the optic nerve fibres, excavation of the surface of the disc begins, and is readily revealed by the ophthalmoscope. As the disease progresses, and the increased tension is maintained, the lamina cribrosa recedes farther and farther, the excavation of the disc becomes deeper, and can, if the case be kept under observation, be easily measured.

A glaucomatous cup requires time for its formation, and is only developed after a considerable period of increased tension; consequently it is not seen after a single acute congestive attack, unless there has been a prolonged premonitory stage. When fully formed, it presents certain appearances which are pathognomonic. It involves the whole disc, and is surrounded by steep, overhanging margins, while its floor has a greyish stippled look, due to the lamina cribrosa. As the retinal vessels are pushed backward along with the nerve fibres, they are abruptly bent at the margin of the disc, and get under cover of the overhanging edges until they reach the floor of the cup, pressed against which they lie until they again dis- 
appear into the vascular canal of the optic nerve. The arteries are usually somewhat reduced in calibre, but the veins are always congested. The bending of the nerve fibres over the margin of the cup, and the pressure to which they are subjected, sooner or later tell on their nutrition, and atrophy sets in. When this commences, the colour of the disc is altered, and, coincidently, sight begins to fail-the failure depending not so much on the actual depth of the cup as upon the stage of atrophy of the nerve fibres, the amount of this being indicated by the degree of the pallor. Observation of the pallor, and of the calibre of the retinal vessels, is, therefore, of the highest prognostic importance. A pale, or, what is worse, a bluish or greenish-white nerve, with small arteries and distended veins, has a much more serious significance than a deeper cup in a disc of a more natural colour.

Along with defects in the visual acuity there is always associated a restriction of the visual field. At first this is confined for the most part to the nasal portion, but after the disease has made considerable progress it involves the temporal region also, and steadily increases till vision is totally lost. It is interesting here to note that colour vision, which disappears so early in primary atrophy of the optic nerve, is retained in glaucoma until a comparatively late period; consequently, in a doubtful case, the results of the examination of the colour-sense may be of very considerable value.

Once established and allowed to run its course unchecked by treatment, the natural tendency of the disease is to take on one of the forms about to be described, and sooner or later to produce complete loss of vision. Even after absolute blindness has set in, degenerative changes proceed. Subjective sensations of light may continue to torment the patient and encourage a delusive hope that sight may yet be restored; or attacks of pain may again and again recur, until consent is readily given to the removal of the eyeball. These degenerative changes affect the cornea in a very striking manner. Its surface may become opaque, with the epithelium here and there raised in blisters; or it may become completely necrosed, and separate as a slough. The iris is atrophied, and forms a narrow rim round its ciliary attachment; the lens is cataractous; and there are staphylomatous projections from the sclera, most frequently in the equatorial region of the globe. Finally, the tension begins to diminish, and complete atrophy of the eyeball takes place; and then, but not till then, will the patient be free from pain and discomfort.

In connection with the pathology of glaucoma there are many points still undecided; but in a clinical work such as this it is not possible to deal with these, except in so far as may be necessary to make the principles of treatment clear. Those who wish to pursue the subject farther are 
referred to the admirable discussion of the experimental and clinical facts to be found in the third volume of Parson's Pathology of the Eye, or to Elliot's text-book on Glaucoma. The tension of the eyeball is kept normal by the maintenance of a constant relation between the. inflow and the outflow of the ocular fluids. In health, increase of the inflow is at once compensated by increase of the outflow; but whenever this ceases to be so, the internal pressure becomes too great for the distensibility of the walls of the eye, and glaucoma occurs. Increase of tension, therefore, is brought about by disturbance of the equilibrium between the secretion and excretion of the ocular fluids-if one may still use the term secretion in this connection, seeing that recent observations favour rather the view that the aqueous is a transudation from the ciliary vessels, and depends on the blood pressure in the arteries. Examination of eyes that have been enucleated for secondary glaucoma shows that, in nearly every case, the corneo-iridic angle and the filtration spaces are closed, on account of the adhesion of the base of the iris to the inner surface of the sclerotic and the cornea; hence the fluids produced by the ciliary body, instead of circulating through the pupil into the anterior chamber, and then passing out of the eye through the spaces of Fontana and the canal of Schlemm, are retained within the eyeball, the tension of which is thus increased. It is true that in irido-cyclitis glaucomatous symptoms may supervene, although the corneo-iridic angle remains wide and open; but in this disease the character of the secretions themselves is changed, and Brailey has demonstrated that, as a result, the spaces become blocked, and hence the filtration function is lowered. It has also been proved experimentally that the injection of oil into the anterior chamber will produce an attack of glaucoma, because the oily fluid closes up the filtration spaces; and from this fact Priestley Smith and others have argued, that in certain inflammatory affections, we have to deal not only with an increase in the amount of fluid, but also with an abnormal fluid, which, owing to its highly albuminous nature, obstructs the excretory channels. The study of cases of secondary glaucoma leads, therefore, to the conclusion that the increased tension is due to the retention of fluids within the eyeball due to hindrance to their outflow.

It has, indeed, been objected to the retention theory, that glaucoma has occurred in eyes from which the lens has been removed, and in others in which the iris was absent. Treacher-Collins has, however, set such difficulties aside by demonstrating that, in cases of glaucoma following cataract extraction, there had been faulty technique, and the corneoiridic angle had been blocked by the entanglement of the capsule, the iris, or the hyaloid in the wound; while in cases in which the iris seemed to be absent, he has proved that there was merely an arrest of develop- 
ment, whereby that structure, instead of appearing in the anterior chamber, remained as a rudimentary stump, which, having become adherent to the periphery of the cornea, had closed the filtration spaces.

Most of the old theories as to the causes of primary glaucoma were, however, based upon hypersecretion as opposed to retention. Mackenzie and von Graefe, starting with this idea, attributed the increased secretion to serous choroiditis, while Donders thought it was due to irritation of the choroidal nerves-a neurosis of secretion. This last theory has been revived in a modified way by Abadie, who, as the result of experiment, concludes that simple glaucoma is due to irritation of the cervical sympathetic nerves. The chief objection to the hypersecretion idea is, that when the existing channels are in good working order, any increase in the inflow of fluids is very soon counterbalanced by an increased outflow, and so the natural tension is maintained. Knies and Weber advanced a retention theory for primary glaucoma, similar to that just described in connection with secondary glaucoma, and the general consensus of opinion now favours their view.

Some authors speak of an anterior and a posterior glaucoma, the former including those cases in which the external or congestive signs are wellmarked, and the latter those which present no congestive symptoms, but in which there is excavation of the optic disc. The bond of connection between such diverse examples lies in the increase of tension, and many cases occur which mark intermediate stages between the one group and the other.

Granted the increase of tension, there is no difficulty in explaining all the symptoms met with in a clinical picture of glaucoma; and, given the blocking of the filtration spaces, either anterior or posterior, it is easy to account for the increase of tension; but the real difficulty lies in finding a satisfactory explanation of the initial changes that lead up to those gross pathological lesions. Eyes in the early stages are not available for pathological investigation; when examination becomes possible, the stage is advanced and the glaucoma absolute, and then of course many of the lesions must be looked on as results rather than as causes.

In explanation of the initial causes of glaucoma, Priestley Smith has shown that the lens continues to grow up to the end of life, while the size of the eyeball, after a certain age, remains stationary. This being so, there must, with advancing years, be a corresponding diminution in the space round about the lens; and as glaucomatous eyes are, according to the same authority, smaller than normal, this disproportion must, in course of time, become all the more marked, until after a certain point the lens becomes too large for the globe, and the slightest congestion 
of the ciliary processes will obliterate the circumlental space altogether. When this happens, the lens is pushed forward by the increased pressure in the vitreous chamber, the base of the iris becomes closely adherent to the posterior surface of the cornea, and the filtration spaces at the corneo-iridic angle are so pressed upon that their excretory function ceases. With the retention of the fluids symptoms of glaucoma begin, and as long as the drainage channels remain closed, the disease will continue to progress.

There are, however, other predisposing factors that require consideration. For example-(I) glaucomatous eyes are usually hypermetropic; and, when the circular fibres of the ciliary muscle are hypertrophied, a still further narrowing of the circumlental space must occur; (2) there is a gradual sclerosis of the pectinate ligament incident to advancing years; and Henderson, in his recent papers on the pathogenesis of glaucoma, attributes to this physiological process a preponderating rôle in the ætiology of the disease; (3) as part of the general loss of elasticity in the tissues so characteristic of old age, the sclerotic becomes more rigid, and will not therefore yield to the normal extent at a time of increased tension; (4) the arteries, from the same cause, lose their natural elasticity, and degenerative changes take place in their walls; their length is in consequence increased, and the size of the ciliary body augmented, with the result that the circumlental space is again encroached upon. Considering, indeed, how closely glaucoma is allied to diseases such as gout, rheumatism, and probably syphilis, in all of which vascular changes are of frequent occurrence; and considering also that the primary form almost invariably attacks both eyes; it would seem as if the root of the evil were to be traced to increased coagulability of the blood, and to degeneration of bloodvessels, reaching its maximum in the hæmorrhagic type of the disease. In the study of individual cases, however, there is often great lack of proper pathological evidence, and the numerous gaps necessarily give rise to much conjecture; but if the conditions observed in the eye can be brought into line with what is known of general pathological processes, the problem as a whole becomes simplified.

Primary glaucoma occurs only in eyes predisposed to attack, and, given the predisposition, as Laqueur says, everything that " dilates the pupil or debilitates the patient" may precipitate a seizure. While the arthritic diathesis, with its attendant arterio-sclerosis, is the constitutional state which most frequently underlies the local manifestations of increased intraocular tension, the great variety in the clinical types of the disease makes it very difficult to formulate a working hypothesis which will include the whole pathology of glaucoma. At first sight, it seems impossible to offer an explanation which will cover both the simple and the 
acute types; but if simple chronic glaucoma be recognised as the typical form of the disease, and all the congestive changes characteristic of the acute and the sub-acute varieties be regarded as complications, the difficulties lessen considerably. In every case, whether it be simple or acute, the lens is too large for the eyeball, and the type assumed by the disease will be mainly determined by the manner in which the circumlental space is encroached upon. If obstruction be brought about gradually by an insidious process of sclerosis, degenerative changes occur slowly, uninterrupted by any great or sudden increase of intraocular tension. In the acute forms, on the other hand, something occurs which at a given moment provokes a rupture in the equilibrium between secretion and excretion, with the result that hypertension occurs rapidly, and is accompanied by all the signs, and symptoms of acute inflammation. True inflammation, however, occurs in no form of glaucoma. The phenomena are similar to those seen in thrombosis of blood-vessels; the sudden blocking of the vascular supply being indicated by acute pain, and followed by congestion, œdema, and infiltration of the tissues. There is both clinical and pathological evidence in support of the supposition that it is thrombosis, more or less extensive, that disturbs the normal balance between the inflow and the outflow of the ocular fluids. Some cases are preceded by thrombosis of the retinal vessels visible on ophthalmoscopic examination, and are so well recognised clinically that they have received the name of hæmorrhagic glaucoma, and are regarded as a distinct type of the disaase. Dr. George Coats, moreover, has demonstrated microscopically the presence of thrombosis of the central vein of the retina in eyes in which the diagnosis was made by the ophthalmoscope, and 11 which enucleation was necessary, owing to loss of sight and severe suffering consequent upon the occurrence of acute glaucoma. Every case of thrombosis of the central vein of the retina, however, is not followed by destructive hypertension; but Coats has also demonstrated that, as a result of thrombosis, the fluids of the vitreous and of the aqueous become more albuminous than normal; and thus his observations are brought into line with the interesting experimental researches of Uribe $\mathrm{Y}$. Troncoso. The latter has demonstrated that albuminous fluids filter with great difficulty through the spaces at the corneo-iridic angle, and consequently are retained within the eye, whose tension gradually becomes increased. In consequence of this abnormal pressure, the circulation of the blood in the vena vorticose, and in the veins of the retina, is hindered, transudation occurs, and œdema of the vitreous supervenes, with the result that the iris is pressed forward, and its base, coming in contact with the posterior surface of the cornea, obliterates the filtration angle. The adhesion is at first slight and does not involve the extreme periphery of the iris, but, 
if the hypertension be maintained, the root of the iris becomes firmly soldered to the posterior surface of the cornea, and the spaces of Fontana and the canal of Schlemm are permanently blocked.

Thrombosis may occur as a result of disease of blood-vessel walls, or in consequence of the circulation of toxins in the blood stream, either or both of which conditions may readily arise in those who suffer from glaucoma. Coagulation of the blood within the vessels is favoured still further by anything that tends to produce stasis in the veins, and, as has already been said, the onset of an acute glaucomatous seizure is usually precipitated by such emotions as grief, anger, or fear, especially in circumstances which depress the action of the heart. The occurrence of thrombosis cannot always be seen by means of the ophthalmoscope, for, granted that the conditions are favourable, there are no reasons why the formation of thrombus should be limited to the veins of the retina. Coagulation of blood is even more likely to take place in the vessels of the choroid, and, occurring there, to give rise to the same alteration in the ocular fluids as Coats has shown to occur after thrombosis of the central vein of the retina. If, then, this alteration in the composition of the ocular fluids due to thrombosis be the cause of acute glaucoma, the extent of the coagulation of the blood within the vessels offers a ready explanation of the varying severity of the attacks-how some are slight and pass off quickly, while others are so serious that sight is permanently lost within a few hours. To recapitulate; glaucoma, either in its simple or in its acute congestive form, can occur only in eyes mechanically predisposed to the disease, owing to narrowing of the circumlental space. In chronic simple glaucoma there is slow and gradual degeneration, and the disease may be regarded as an incident in general arterio-sclerosis. The acute congestive forms are probably always associated with thrombosis of the choroidal or retinal veins, and the severity of the attack will be proportionate to the extent of the thrombosis.

It is convenient to divide all cases of primary glaucoma into the noncongestive and the congestive types, although it must be remembered that congestive complications may occur in any case of the disease.

1. Non-congestive Type, or Simple Ghronic Glaucoma.-The three pathognomonic features of the disease are, moderate and intermittent increase of tension, excavation of the optic disc, and diminished visual acuity. The patients are usually over sixty years of age, and consult the doctor on account of failing sight, and as they enter the room present, in advanced cases, a very characteristic physiognomy. They rarely complain of pain, but careful inquiry will elicit a history of some or all of the prodromal symptoms already mentioned. Frequently the vision of one eye is very defective; but, owing to the absence of any 
active discomfort, the patient has paid little heed to this, and has become alarmed only after the other eye began to fail. The better eye may present a healthy appearance, and the visual acuity may be normal; but, on testing, the field of vision will be found restricted, especially on the nasal side and below, and examination by the ophthalmoscope will reveal characteristic cupping of the optic disc. If no pulsation of the retinal artery be visible, it may be induced at once by slight pressure on the globe. There may be increase in the intraocular tension; but very rarely indeed is the eyeball more than " tight " or " firm," and it is almost never of " stony hardness." In the eye in which the disease is more advanced, in addition to the symptoms just described there will be some injection of the episcleral veins, a lessening of the sensitiveness of the cornea, and dilatation of the pupil, which presents a greenish reflex.

It sometimes happens that this greyish green reflex is mistaken for senile cataract; and such an error in diagnosis is fraught with the most disastrous consequences. I can recall many men and women who came from remote country districts to the hospital for the purpose of having their eyes operated on for cataract. They had been gradually losing sight for a number of years, but the family doctor had told them that as they were suffering from cataract, nothing could meantime be done, and no operation was possible till they had become blind. They accordingly waited till, in one eye loss of vision was complete, and in the other there was not sufficient sight left to enable them to find their way about. The diagnosis had been entirely mistaken. An examination showed that the lenses were perfectly transparent, but that the optic nerves were deeply excavated; while their greenish-white colour told at once how much they were atrophied. Operation was of no avail-the disease was simple chronic glaucoma, which had been allowed to run its course until the vision was all but totally lost, and there was not the slightest hope of recovery. Until the ophthalmoscope is more generally used, such mistakes will continue to be made, though these are just the very cases which Mackenzie distinguished so carefully. They present no striking external symptoms; the pupillary reflex is well marked, and the tension is very variable, and probably at no time very high, so that, unless the ophthalmoscope be employed, accurate diagnosis is impossible.

Simple glaucoma may also be mistaken for atrophy of the optic nerve. Von Graefe himself classed such cases under the title- "Amaurosis accompanied by excavation of the optic papilla;" the pathological distinction between glaucomatous excavation, and cupping of the optic disc from atrophy pure and simple, lies in the fact, already mentioned, that in the former there is backward displacement of the lamina cribrosa, while in the latter that structure retains its normal position. As seen by 
the ophthalmoscope, the blood-vessels in the atrophic condition can be traced from the margin to the bottom of the cup, while in the glaucomatous they are hidden under the steep overhanging edges. In simple atrophy there is also no increase of tension, and no pulsation of the retinal arteries, but there is an early implication of the colour sense.

Würdemann has recommended transillumination as a "valuable corroborative method of examination." He employs the transilluminator devised by himself, and applies it " to the sclera at the outer canthus after application of 2 per cent. alypin or 5 per cent. cocain solution, although the light may be passed through the lids for superficial examination. The pupil is thereby lighted up, and if the eye be looked at obliquely the ciliary body on the opposite side will appear as a dark ring, and the circumlental space as a clear ring at the root of the iris between the rounded margin of the lens and the ciliary processes. This is usually from 5 to 6 $\mathrm{mm}$. wide. If the lens diameter be increased, or if it be farther forward than normal, or if the ciliary processes be thickened by congestion, this space is encroached upon and may be obliterated." That method of examination seems to offer a way of determining during life what is the approximate size of the circumlental space, the condition of which is, as has been said so often, the chief predisposing cause of glaucoma.

2. Gongestive Type, or Acute Glaucoma.-The slow insidious progress of simple glaucoma is in vivid contrast to the rapid and striking outburst of the acute form of the disease. It is true that as a rule premonitory symptoms, more or less pronounced, have for a longer or shorter time been intermittently present, but these unfortunately have in most cases been disregarded, so that the seizure appears to come suddenly and without warning.

The patient may have gone to bed apparently quite well; but is awakened in the early hours of the morning by very severe pain in the forehead and in one eye. Almost immediately after the onset the eyeball becomes acutely congested, and feels as if it were too large for the socket; the eyelids are œdematous; there is profuse lachrymation; and the sight of the eye rapidly becomes dim. The pain, accompanied by feverishness and persistent retching and vomiting, increases, and radiates with agonising severity along the branches of the fifth nerve. In some cases the second eye is attacked a few hours after the first, and the patient's condition becomes critical in the extreme.

The danger is, that the pronounced symptoms so common as accompaniments of an ordinary bilious attack may deceive the doctor, who has probably been called in haste, and the patient himself may add to the misapprehension, for he naturally connects the pain in the head with the vomiting, and attributes the failure of sight to the biliousness. Almost 
invariably, the eyes, if both unfortunately be attacked, are of stony hardness. There are extreme congestion and œdema of the ocular conjunctiva, the pupil is dilated, oval shaped, and irresponsive to light, and the anterior chamber shallow. The cornea shows a smoky cloudiness, and in many cases is so insensitive that it can be touched without the patient being aware of the fact. The media are so hazy that the fundus cannot be illuminated by the ophthalmoscope, but, unless there has been a prolonged period of pronounced premonitory symptoms, the optic disc is not excavated after a single attack. The practitioner must be on his guard not to misinterpret the significance of this form of ocular disease, for to do so is disastrous. The dilated pupil and shallow anterior chamber, associated with the stony hardness of the eyeballs, are unmistakably characteristic, and ought to prevent glaucoma from ever being mistaken for iritis. Unfortunately, however, while that is so, such a mistake is far too common; and as the treatment for the one is exactly the opposite of that for the other, the error is a very serious one. Atropin is instilled, with the result that the symptoms are aggravated, and the patient's chances of recovering sight are materially lessened.

$\because$ The severity of the symptoms varies in different cases. In the large proportion the acute symptoms pass off after a few days, but with the visual function sensibly diminished. The eye is always liable to future attacks; and after each the visual field is more and more contracted, and the optic disc becomes not onl more deeply excavated, but progressively atrophied, from the recurrent hypertension, until at length sight is reduced to a bare perception of light. The most acute cases are described as fulminating; and in them glaucoma is seen in its most tragic form, its onset, course, and termination being the incidents of a few hours; after a single attack, appearing with overwhelming suddenness, vision is totally and irrevocably lost.

Intraocular hæmorrhage is not unfrequently an accompaniment of glaucoma. In certain cases-which according to de Wecker, form about 2 per cent. of the whole-hæmorrhage constitutes such a marked feature that they have been grouped in a class by themselves. Hæmorrhagic glaucoma is, however, simply a local manifestation of a general state. 


\title{
CHAPTER XXV
}

\section{THE CLINICAL SIGNIFICANCE OF A HARD EYE (continued)}

\begin{abstract}
The treatment of glaucoma : medical, general, local-Surgical, iridectomy,
\end{abstract} sclerotomy, sclerectomy-Post-operative complications.

\section{THE TREATMENT OF GLAUCOMA}

AlL experience goes to prove that primary glaucoma, whether in its non-congestive or congestive form, when left to itself, invariably ends in complete blindness; and formerly the disease was looked upon as absolutely incurable. The aim of all treatment is to diminish the increased tension of the eyeball, and the therapeutic measures employed for that purpose are both medical and surgical.

Medical means are either general or local, and in all cases are only palliative. They may delay, but it is doubtful if they ever prevent, the steadily progressive downward trend of the disease. At the best they are merely tentative; and although"the long natural history of a case of chronic glaucoma prevents one from estimating accurately how much good they do, and how much they really prolong the course of the disease, yet it may safely be said that no case was ever cured by their means alone. Whenever a patient complains of symptoms significant of the prodromal stage of glaucoma, means ought to be taken at once to prevent the development of an attack. He must be warned of the gravity of the condition, but the information should be conveyed to him in such a way as not to produce any unnecessary alarm. He should be encouraged to believe that by taking proper care he can do much to prevent the onset of a genuine glaucomatous seizure. His habits and mode of life ought to be scrutinised; he must be cautioned against keeping late hours, and warned to avoid excesses of every kind. Copious repasts of rich food, accompanied by over-indulgence in spirituous liquors, are always injurious, and the excitements incident to the gaming table are especially harmful. Business worries, domestic cares, an outburst of passionate rage, the depression occasioned by grief, or the exhaustion produced by want or over-exertion, have been rightly held responsible for the onset of many an acute attack; consequently the patient must be counselled to live as far as possible free from all exciting 
or depressing emotions. He ought to lead an active outdoor life, and attend with care to his digestion and the regular action of his bowels. The urine ought to be carefully and repeatedly examined, and any abnormal constitutional state rectified as far as possible. Alkalis, alternated with piperazine, salicylate of soda, iodides, and bromides, should be prescribed methodically and for a prolonged period in accordance with the indications presented by the individual patient; and if there be a history of syphilis, hereditary or acquired, antisyphilitic remedies ought always to be given. When the pulse is soft and the heart feeble, there is no doubt of the value of strophanthus, in doses of 3 to 5 minims of the tincture B.P., frequently repeated, as advised by Zimmerman. The refraction also must be accurately estimated, and suitable spectacles prescribed. When an attack is impending, a brisk purge of calomel, followed in three hours by a saline draught, ought to be prescribed; and the instillation of myotics into the conjunctival sac must be begun without delay. The myotics in most general use are eserin and pilocarpin; and their favourable action depends for the most part upon their power of drawing the iris away from the corneo-iridic angle, and so opening up the filtration spaces, and permitting freer circulation of the intraocular fluids; but as Henderson insists, the iris is an absorbing surface, which "varies inversely with the size of the pupil "; consequently the myosis produced by eserin or pilocarpin not only increases the area of the absorbing surface, but also, according to the same authority, opens up the crypts in its loose stroma to allow freer drainage. Myotics are of undoubted service in the early stages, a. premonitory attack being at once brought to an end by the timely instillation of eserin; and their regular use when glaucoma is fairly established may hold the disease in abeyance for a considerable time. The sulphate and the salicylate of eserin, being soluble in water, are the salts most frequently employed; but the pure alkaloid may be used dissolved in sterilised oil. The solution ought always to be freshly prepared, and should be just sufficiently strong to cause the pupil to contract; and the instillations should be repeated as often as is necessary to keep it contracted. The usual strength is from $\frac{1}{5}$ to $I$ per cent., and the instillations require as a rule to be repeated every two or three hours. 'Eserin, however, possesses several disadvantages; its use, even in weak solution, is apt to cause severe pain in the eyeball and forehead, while its continued application frequently gives rise to acute follicular conjunctivitis. Pain may be avoided or mitigated, and the good effects much enhanced, by combining cocain and adrenalin with the eserin, but the myotic must retain the mastery over the pupil. One occasionally has patients with whom both cocain and adrenalin do not 
agree, and when that is so they aggravate rather than alleviate the suffering induced by the eserin. In these circumstances the old-fashioned plan of adding glycerine, in the proportion of ro per cent., to the solution of eserin, enables its use to be continued without much discomfort.

Pilocarpin is not such a powerful myotic as eserin, but the aqueous solution of its salts is more stable. It is employed as a rule in the form of the nitrate, and the usual strength is from I to 2 per cent. It never gives rise to painful contractions of the ciliary muscle, and very rarely causes irritation of the conjunctiva. More recently the hydrobromide of arecolin, and the sulphate of isophysostigmin, in solution containing from $\frac{1}{2}$ to I per cent. of the salt, have been highly recommended. They contract the pupil more quickly than eserin, but the myosis is of shorter duration. A combination of myotics is often more beneficial than the use of any one of them separately.

Mydriatics are just as harmful in primary glaucoma as myotics are useful. An acute attack has followed the instillation of a single drop of atropin solution into a predisposed eye, and it is a golden rule in practice never to prescribe any drug that dilates the pupil to a patient over forty years of age, without first making certain that there is no increase in the tension of the eyeball.

When a glaucomatous attack is threatening, nothing is of greater value in preventing its onset than a soporific-veronal-sodium or chloralhydrate-which latter, from its power of lowering intraocular tension, is especially useful; and very marked benefit follows the use of an ordinary chloral and bromide draught, the patient awaking from several hours of sound sleep apparently quite well. At the beginning of a congestive attack, it is necessary (in addition to using myotics which ought now always to be combined with from 5 to Io per cent. of dionin, and accompanied by gentle massage of the eyeball) to relieve pain by the application of fomentations and of leeches; and a hypodermic injection of morphia in the temple ought to be given at once if the sufferings of the patient are severe. The combination of morphin and of chloral, however, must always be avoided, because the one intensifies the narcotic action of the other. The patient must be kept warm in bed, and 3 to 6 grains of calomel, followed in an hour by a saline draught, should be prescribed, in order that the bowels may be freely moved as soon as possible. If after twenty-four hours the attack shows no signs of abating, surgical intervention should not be delayed, because the congestion at the corneo-iridic angle rapidly becomes greater, and the patient's chance of recovering sight depends altogether on the promptitude with which the eyeball is opened and the strangulation 
relieved. If the practitioner cannot obtain assistance, he ought not to wait, but should at once puncture the sclerotic with a narrow Graefe cataract knife, and allow some of the vitreous to escape. The patient should be told to look inward and upward, and the puncture should be made at the outer side of the eyeball between the external and inferior rectus muscles about $7 \mathrm{~mm}$. from the limbus cornea. The point of the knife must be directed towards the centre of the eyeball in order to avoid wounding the lens, and when the blade is in position the handle must be gently turned to cause the wound to gape sufficiently to permit a small quantity of vitreous to escape. When the tension of the eyeball is sufficiently lowered, the knife must be brought back to its original position and gently withdrawn from the eye. If the eye be thoroughly anæsthetised by holocain, in I per cent. solution, and strict antiseptic precautions taken, this operation can be performed without danger, and will give relief till the services of a specialist can be obtained. If the increased tension be not lowered, impaired sight or even blindness will almost inevitably result, but the hopelessness of the condition is at once removed by the performance of iridectomy: the all-important thing is that action be taken in time.

Up to 1856 glaucoma was regarded as uncontrollable and incurable, but in that year von Graefe announced that the disease could be arrested in its progress, and even permanently cured by the simple operation of iridectomy. No more beneficent discovery has ever been made in the whole realm of operative surgery; but von Graefe arrived at it in a manner purely empirical, and even now, when the operation has stood the test of experience for more than sixty years, and thousands of sufferers from glaucoma have retained their sight by its means, authorities are undecided regarding the exact explanation of its curative action. To be successful, however, the operation must secure a reduction in the intraocular tension; and the rules von Graefe laid down for its proper execution are adhered to, with very slight modifications, by the great majority of operators. Iridectomy for glaucoma, especially in its acute congestive form, is undoubtedly one of the most difficult operations in ophthalmic surgery, and where success is only partial the cause frequently lies in faulty technique. Whenever there is even slight congestion, I prefer to have the patient under chloroform; and before the general anæsthetic is administered I instil I per cent. eserin, 4 per cent. cocain and Io per cent. adrenalin into both eyes. It is a great advantage to have the pupil well contracted, because this makes the iridectomy easier, and may prevent accident to the sound eye, in which such an operation has sometimes determined an attack of acute glaucoma, a result due, not so much to the operation itself, as to the nervous shock which so often both 
precedes and follows surgical treatment. The iridectomy ought to be upwards, so that the coloboma may be hidden by the upper lid; but when the iris is much atrophied, the healthiest part should always be excised. Von Graefe insisted strongly that the incision must be as peripheral as possible, so that the knife enters the cye at the angle of the anterior chamber, thus permitting the iris to be removed up to its periphery. As Berry has pointed out, however, too peripheral an incision is apt to lead to serious accidents-profuse hæmorrhage from wounding of ciliary body, dislocation of the lens, or escape of the vitreous-and consequently the aim must be to make an incision just as peripheral as is compatible with safety. Some insist on the use of a narrow Graefe knife, others prefer a broad keratome. A keen edge is of more importance than the shape of the blade. In every case the incision must be wholly in the sclerotic, usually from I to $2 \mathrm{~mm}$. from the apparent margin of the cornea, and parallel to the limbus. The edges of an incision made with a keratome are smooth, and readily come together, so that the wound has no tendency to gape, and with ordinary dexterity and care there is not much risk of injuring either iris or lens, except in those cases where the anterior chamber is very shallow. In these circumstances it is wiser to use a narrow Graefe knife. The aqueous must be allowed to drain off very slowly, in order to prevent prolapse of the iris, and to avoid the danger of intraocular hæmorrhage. The piece of iris to be excised ought to be grasped by forceps at the margin of the pupil and slowly pulled out of the wound. The loop ought to be kept gently on the stretch and drawn towards one angle of the wound. De Wecker's scissors are then held parallel to the wound with the blades pressed down on the globe, and the iris is cut by a series of snips, the operator making sure that it is removed close up to its attachment. The corners of the cut iris must next be carefully replaced by a spatula in order that no tags may become adherent to the wound. As a rule the excised portion of the iris should include about one-sixth of its circumference. The anterior chamber usually rapidly fills up with blood, but that is of little consequence, as the hæmorrhage will soon stop, and in two or three days the blood will be all absorbed. Both eyes should be bandaged carefully, and the patient confined to bed for at least a week after the operation.

The most successful results are undoubtedly obtained when surgical intervention takes place at a time when the iris is sufficiently healthy to react perfectly to eserin, and when its base is simply in contact with, and not firmly adherent to the posterior surface of the cornea. Whenever the diagnosis of glaucoma is clearly established iridectomy should be performed, more especially if the fellow eye is blind from the disease. If, however, both eyes be affected, but one much in advance of the other, 
the worse eye ought always to be operated upon first, in order to find out how it behaves after iridectomy and thereby to obtain a reliable guide for the treatment of the fellow eye. Undoubtedly the most brilliant results after operations for glaucoma are obtained in the congestive forms of the disease, in which there is considerable increase of tension. In these, iridectomy is speedily followed by relief to the pain, and by improvement in sight, in consequence of reduction in intraocular tension, and clearing of the transparent media.

In chronic simple glaucoma, where the tension is never much above normal, the results of the operation are not so certain. As Risley points out, however, "increased tension of the eyeball is always relative," and probably " mild exacerbations of increased tension coming on, especially in the night, often pass without recognition by the patient." In chronic glaucoma the cupping of the optic disc is the result of hypertension, and consequently that is the best guide in estimating the probable result of an operation. The contraction of the visual field, on the other hand, is a measure of the amount of atrophy of the optic nerve, which obviously must be permanent in spite of any form of treatment. My clinical experience has taught me that in every form of genuine glaucoma the earlier the operation is performed the more satisfactory the result. Delay is dangerous, because every case of simple glaucoma may at any time become complicated by a congestive attack from which in all probability an iridectomy would have protected it. A single congestive attack may, if it be fulminating, destroy vision completely; even in milder cases, where the patient regains sight after a few days, the recovery is never complete, and each succeeding relapse leaves greater visual incapacity than before. The most that we can hope to do is to restore vision to that point at which it was before the occurrence of the acute attack immediately preceding the operation.

Unfortunately, a good result does not always follow an iridectomy for acute glaucoma; on the contrary, in some cases the vision is worse after the operation than before it. In most instances, however, postoperative accidents are the result of faulty technique due to restlessness on the patient's part, and consequently they can in great measure be averted by having him fully under the influence of chloroform. The chief accidents to be feared, are traumatic cataract, or the occurrence of intraocular hæmorrhage. When the anterior chamber is unusually shallow and the lens pressed forward, there is always difficulty in introducing the knife; and if the section is not made with great care and dexterity, the point of the blade may come into contact with, and so rupture, the capsule of the lens, with the result that a traumatic cataract will form. In eyeballs which have become distended, as in buphthalmos, 
the ligament of the lens may be so loose that, when the aqueous escapes, the lens is driven forward with force sufficient to rupture the zonule. The dislocated lens may then present itself at the operation wound, and require to be extracted, if it does not escape of itself. In cases in which vascular degeneration is advanced, hæmorrhage is very liable to occur at the time of the operation. If, as has already been said, the blood flows into the anterior chamber, it may prove a troublesome complication and delay the various steps of the operation; but it is never really serious. It is quite different, however, when the bleeding comes from the retinal or choroidal vessels, for then sight may be destroyed by a hæmorrhage into the macula, or the eye itself may be ruined, in consequence of a large expulsive hæmorrhage which detaches the retina, and may cause both lens and vitreous to be extruded en masse upon the patient's cheek.

One of the gravest dangers in simple glaucoma is when a sudden deterioration of vision takes place soon after the iridectomy. Von Graefe himself noticed that when such a misfortune happened, it was always in cases where the visual field had, before the operation, been contracted nearly up to the fixation point, and became further encroached upon by the constriction that subsequently occurred. Such a result is most disappointing, as the loss of central or direct vision renders a patient practically blind, who before the operation was able to read. The lesson to be learnt from such cases is the necessity of early operation, before the contraction of the field has advanced so far as to encroach near the area of fixation.

The most disastrous complication of all, however, is where the glaucoma is found to be malignant. The percentage of such cases is fortunately small, and the patients are most usually women who are approaching, or have reached, the period of the menopause. Unfortunately this is a complication that cannot be foreseen; but its onset should be suspected if the iridectomy be followed immediately by severe inflammatory reaction. The anterior chamber becomes permanently abolished, the eye acutely painful, and vision is speedily lost completely. The suffering continues for weeks or even for months; and then, as the pain subsides, the globe softens and is destroyed by phthisis bulbi. In connection with such cases, Schweigger says that "the cause of the unsatisfactory results of the operation must be in some obscure structural anomaly inherent in the eye itself," and as a result of his own experience he has laid it down as a rule that "when glaucoma malignum attacks one eye, it follows iridectomy in the other eye, even when the second eye is not affected for years after the first." He also says that " experience teaches us that in all cases of chronic glaucoma affecting both eyes, 
it is advisable first of all to operate upon the worse one, even if it should be absolutely blind. Should this be followed by the normal healing process, the second eye may be operated upon without the least apprehension."

It sometimes happens that the scleral wound, instead of healing firmly and smoothly, bulges to form a cystoid cicatrix, which yields to any sudden increase of intraocular pressure, and permitting aqueous to filter through it, acts as a kind of safety-valve to the eye. Every operator of experience can recall cases where the intermittent relief of hypertension through a cystoid cicatrix was very beneficial. Berry quotes a very interesting case which he has had under observation for more than twenty years, in which the cystoid scar " has its efficacy as a filter increased from time to time by the habit which the patient has acquired of forcibly rubbing his eye when he notices it become obscured. This causes a trickle of aqueous humour to escape, of which the patient is conscious, and which is immediately followed by the disappearance of haze." Such a means of diminishing quickly any sudden increase of intraocular tension is undoubtedly of great value in individual cases; but it must not be forgotten that a cystoid cicatrix brings danger to the eye, in so far as it may readily be the point of departure of ocular suppuration. If, however, it were possible to form a filtration cicatrix which would at the same time be free from all ordinary risk of pyogenic infection, that would undoubtedly be a great gain; for many eyes which now gradually deteriorate as a result of the recurrence of high tension subsequent to iridectomy would be permanently cured. In these circumstances, von Graefe was in the habit of making a second iridectomy diametrically opposite to the first, and thus in many instances succeeded in arresting the disease. It is probable that in the second operation a healthier piece of iris was excised than at first, because undoubtedly, in glaucoma the more degenerate the structure of the iris the less successful is the iridectomy. Henderson has also recently pointed out-and the observation is an interesting and important one-that "After an iridectomy the iris stump shows no signs or vestiges of a cicatrix whatsoever," and from this he argues that " the operation of iridectomy opens up a permanent channel for the intraocular fluids to drain away-a channel which will not be influenced by the state of the pupil, and which will always be effective if the operation is performed before the iris tissue becomes atrophied and degenerated."

De Wecker considered the section of the sclerotic of more importance than the excision of the iris, and devised an operation which he called anterior sclerotomy. It is performed differently by different operators, one of the most important modifications being that of Galezowski, who 
practised what he termed equatorial sclerotomy, but the aim of all is the same, to make an incision in the sclera at the periphery of the anterior chamber, but to leave the iris intact. Sclerotomy is chiefly useful in those cases in which an iridectomy has already been performed with an unsatisfactory result, and here a scleral puncture at the site of the iridectomy may completely relieve hypertension and subdue pain. Moreover, if necessary, the operation may be repeated over and over again.

De Wecker taught that the success of a sclerotomy operation depended upon the formation of a "filtering cicatrix"; but unless there is a distinct rise in intraocular tension, no form of scleral incision will be followed by a permanent permeable scar. Lagrange, therefore, set himself deliberately to excise a piece of the sclerotic from the corneal lip of the operation wound, and to secure thereby a cicatrix which would permanently allow a certain amount of filtration. Fergus and Elliot have both sought to attain the same end by the use of a trephine; and if the technique which Elliot has described be closely followed the operation is not difficult, and the results on the whole are very satisfactory, even in those cases in which iridectomy is known to be dangerous.

Iridectomy has still, however, its own proper rôle in glaucoma. surgery, because in all congestive forms of the disease accompanied by increased tension, it still easily holds the first place; but in simple chronic glaucoma a sclerectomy operation (Elliot or Lagrange) is much more likely to secure permanent benefit. In simple glaucoma, however, general diathetic treatment must be methodically continued after operation. Every means must be taken to avert the progress of general arterio-sclerosis upon which the disease so largely depends. The patient must recognise his limitations, and live well within them.

In eyes in which the pupil is dilated and will not contract with eserin, and the iris is atrophied and the anterior chamber very shallow, posterior sclerotomy, to permit of an escape of vitreous and the formation of a leaking subconjunctival scleral wound, is sometimes attended by much success; and Priestley Smith has advised that when hypertension is great, the sclerotic should be punctured as a preliminary to iridectomy. The combination of the two operations is often of the greatest value, and permits the excision of the iris to be made with perfect safety, in circumstances where otherwise the risk would have been considerable. Finally, when an eye is blind from glaucoma, and continues to be painful in spite of all treatment, nothing but optico-ciliary neurectomy remains, if the patient is to be relieved from his suffering and yet to retain his eye. In these circumstances, however, enucleation is the more prudent course, because the globe, in consequence of the steady progress of glaucomatous degeneration, is sooner or later almost certain to give trouble. 


\section{CHAPTER XXVI}

THE DUTY OF THE GENERAL PRACTITIONER TOWARDS A PATIENT SUFFERING FROM CATARACT-BEFORE, DURING, AND AFTER THE OPERATION

Classification of cataract-Symptoms and signs-Procedure in examinationPrognosis-Treatment in early stage-Conditions necessary before undertaking operation - Preparation of the patient - Choice of operation Instruments-Post-operative treatment-Accidents during and after operation.

CATARACT, in medical acceptation, denotes an opacity of the crystalline lens or of its capsule; and this implies a classification of cataracts into lenticular and capsular, and a further subdivision of the former group into nuclear and cortical. Since the opacity must be in the lens, in its capsule, or in both, this anatomical division has the advantage of including all forms of the disease; but clinically it has been found more convenient to refer to cataracts as hard, soft, or fluid; as white, amber, or black; as congenital, juvenile, or senile; as primary or secondary; as progressive or stationary; as mature or immature.

All forms of cataract depend upon three main factors-the anatomical position of the opacity, the age of the patient, and the condition of the eye. From the time of birth till about the age of thirty, all lenticular opacities are cortical and soft; but after the third decade of life, the nucleus has developed so far that it acquires a predominating influence over both the consistency and the character of a cataract; while after the sixtieth year, the lens becomes so hard that the cataract is spoken of as nuclear and senile. Under ordinary circumstances, therefore, the development of lenticular opacity may be looked upon as an incident due to the advance of years, and except for the loss of transparency in the lens the eye is physiologically sound.

In such a case the cataract is spoken of as primary, in contradistinction to the secondary one which appears in the course of some eye disease, or after an eye injury. For example, ophthalmia neonatorum sometimes leaves on the anterior capsule of the lens an opacity due to inflammatory changes resulting from a central perforating ulcer of the cornea; or a cataract, characterised by rapid development and by a peculiar whiteness, due to inflammatory thickening of the capsule, may arise in choroiditis 
or in detachment of the retina; or, lastly, the lens may lose its transparency, and a cataract, secondary to pathological processes in the choroid, may occur in high degrees of myopia. This last begins, as a rule, in the posterior pole of the lens, is usually brownish in colour, and takes many years to mature. A cataract is also at times spoken of as secondary to some constitutional disorder, the most familiar example being the opacity of the lens which occurs in the course of diabetes.

-As a result of the advance of years, the lens steadily undergoes a process of hardening or sclerosis, which gradually increases the size, and changes the colour, of the nucleus. A lens in this condition possesses in considerable degree the property of reflecting light, and consequently the pupil of an elderly person has rarely the brilliant jet-black appearance characteristic of youth, but is generally dull and grey. This is what is known as the " senile reflex," and must never be confused with cataract, for though the lens is old and hard, it has lost none of its transparency. The alteration is so far purely physiological, and it is very difficult to determine why, in certain cases, superadded pathological changes make their appearance and render the lens opaque. At first, after the diseased condition has shown itself, the cataract, as a rule, develops very slowly; for months, and even years, it may remain seemingly stationary, and then the opacity may, without any obvious cause, rapidly become complete.

The ætiology of cataract cannot be associated with any special disorder of the general health, although, as has been said, the frequency of its occurrence in connection with diabetes mellitus has given rise to the term "Diabetic Cataract." A considerable percentage of cases is associated with albumen in the urine, and with the digestive difficulties and vascular changes which accompany renal cirrhosis. Heredity seems to play an important rôle in the causation; and the disease is often seen in several members of the same family, and can be traced back through successive generations. Senile cataract may occur any time after the fortieth year, although it is far more common in the aged. Statistics prove that certain occupations act as predisposing causes; and the most frequent sufferers are glass-blowers, blacksmiths, and those whose occupations necessitate the exposure of the eyes to strong heat and bright light. Similar conditions account for the great frequency with which cataract occurs in India.

One of the earliest premonitions is when a person who has been using glasses for reading finds that he can dispense with their assistance. Such an one usually puts his spectacles aside saying he has got his "second sight," and can read his newspaper perfectly well with the naked eye; but he fails completely to connect this with the fact that he has now 
difficulty in recognising a friend on the opposite side of the street, and that vision for distant objects is not nearly so keen as formerly. As these changes are due to increased refractive power in the lens, which renders the eye myopic, vision for distance may, at first, be improved by the use of concave glasses; and although this can only be for a time, yet the failure in sight due to increasing myopia must always be distinguished from amblyopia, the result of progressive opacity of the lens. Another characteristic symptom is when a person complains that on looking at a bright light, such as the moon or a star, he sees it doubled or quadrupled. This monocular polyopia is also due to changes in the refraction of the lens, and as it causes the patient much annoyance, may lead him to complain of his sight before the slightest trace of cataract can be detected by means of the ophthalmoscope. Bright light usually causes dazzling; consequently the patients prefer to shade the eyes whenever they have to walk in the sunshine, or to pass from a. place that is dimly lighted to one that is brightly illuminated. Where the eyes have been overtaxed at fine work, the first symptoms are usually eye-strain, accompanied by hyperæmia of the lids and ocular conjunctiva ; and in these cases the patients often complain also of muscæ volitantes, describing the motes as moving with the eye and having at times a resemblance to a cloud of dust or a fine cobweb. The change once begun usually progresses; and as time goes on the transparency of the lens is interfered with more and more, until at length the pupil loses its blackness and becomes occupied by "a cataract.

As the opacity in the lens increases, the vision naturally becomes more and more indistinct; till in the final stages of the disease the patient is only able to distinguish light, and is quite unfit to walk about alone. The gait of a person in this condition is very characteristic; he walks slowly as if afraid to step forward, and holds one hand outstretched to feel his way, while with the other he tries to shade his eyes from the light. This attitude should be specially noted, because it is in such marked contrast to that of one who has lost his sight through atrophy of the optic nerves. The latter shows no sign of fear, but walks boldly onward with head and shoulders erect, and looking straight in front of him.

The chief objective sign of cataract is the opacity of the lens; and $a$ diagnosis of cataract should never be made unless the opacity can be seen. In the earliest stages, this can be recognised by means of the ophthalmoscope with $a+3$ D.S. lens behind the sight hole of the mirror ; but as the disease advances towards maturity, it becomes quite plain to the naked eye. In all uncomplicated cases, the iris presents a normal appearance, the pupils respond actively both to the stimulus of light and in the act of convergence, and the intraocular tension is not increased. 
A shallow anterior chamber indicates that the lens has become swollen, owing to rapid opacity of its cortex; but as maturity is approached the lens again shrinks, and permits the iris to fall back into its natural position, so that the aqueous chamber is restored to its normal dimensions.

When investigating a case of cataract, it is always advisable that the various steps of the examination should be conducted in a definite order. Particular attention should be given to the patient's account of his symptoms; for much may thus be learned of the past history of the eye and the probable duration of the diseased condition, as well as of the state of his general health. The eye should then be examined carefully with the aid of good artificial light and a strong bi-convex lens, for any abnormality in the pupil or iris, for inflammation of the conjunctiva, or of the edge of the lids, and for any inadequacy of the tear passages. The visual acuity should always be tested; although, if the eye be already blind, it will be possible to determine only his power of distinguishing and locating a light held about twelve feet distant in a dark room, the other eye being carefully covered. The functional activity of the retina may be still further interrogated by gently palpating the sclerotic, and noting the presence or absence of phosphenes. That method of examination is, however, very seldom employed.

The eye should afterwards be examined by oblique illumination, and by the ophthalmoscope, the latter being of special value, as its use enables us, when the pupil is dilated, to detect not only the very beginnings of cataract, but also changes in the fundus oculi, more especially when these are situated in the periphery, or in the macular region. Moreover, in a case where the sight is dim and the pupil hazy, ophthalmoscopic examination will prevent the terrible blunder of allowing a patient to become hopelessly blind from glaucoma, while an imaginary cataract is supposed to be slowly maturing.

Even when the existence of cataract has been distinctly made out, the surgeon should give a very guarded prognosis as to the length of time it may take to ripen. As a rule the complete opacity of the lens requires from two to three years; but during its passage through the incipient and progressive stages, many a cataract remains apparently stationary. This is very often the case where it is complicated by myopia ; and in the aged a lenticular opacity may not increase for years. In many cases, indeed, the progress is so slow that the stage of maturity is never reached. On the other hand, a cataract may be left till it is over-ripeMorgagnian cataract. Its cortical portion liquefies, and the ligament of the lens ruptures so readily that a very slight injury causes dislocation. That explains those cases in which a cataract has spontaneously disappeared. 
To say that a person with a few opaque striæ in the lens is suffering from cataract is no doubt perfectly true; but the use of the word at this early stage is apt to convey a false impression, and to create much unnecessary alarm. Many a patient, would, after hearing such a diagnosis, live in constant dread of the onset of blindness which might never come. While it is always prudent to tell a relative, the less said to the patient about a cataract the better, until such time as it is possible to operate in the near future.

While a cataract may be said to be optically ripe, when the vision is reduced so far that the sufferer is unable to distinguish the knife and fork on his dinner plate, it may not then be surgically ripe; and it is important to wait, if possible, for the latter stage before operating, because then the extraction will be more complete. The degree of maturity can always be estimated by sharply illuminating the edge of the pupil, and observing the breadth of the shadow cast by the iris. When the whole cortex is opaque; the iris lies closely against the cataractous surface, and does not form a shadow when a light is held against the eye. Whenever the anterior chamber has regained its normal depth, and there is no shadow, the cataract is said to be surgically ripe. When a patient sees well with one eye, there is no great advantage in removing a cataract from the other, unless the operation be desired for cosmetic reasons, or to get rid of the inconvenience of the blind side.

A patient suffering from cataract, however, requires help in the early stages. Unfortunately, in spite of what may be said to the contrary, there is no means known whereby an opaque lens can be rendered transparent; but during the period of waiting till surgical operation becomes possible, the sight may be improved by the use of mydriatics if the opacity be central, and of myotics if it be largely peripheral. Many aged persons with nuclear cataracts are enabled to see satisfactorily, if they bathe the eyes daily with a very weak solution of atropin, $\frac{1}{4}$ grain to an ounce of normal saline solution. Frequently also concave glasses give relief for a time. All patients should be cautioned against overtaxing the eyes by struggling to see small print, or to do fine needlework; and they should avoid, as far as possible, the exposure of the eyes to bright light and strong heat. In every case, attention must be paid to the state of the general health, and means taken to ensure the efficient action of bowels, kidneys, and skin, and to maintain nutrition as a whole at the highest possible level. Whenever the patient's sight becomes so defective that he has difficulty in following his usual occupation, the question of surgical interference must be considered; and though it is always preferable to delay operation until the cataract is mature, a patient ought in no case to be permitted to become blind. It is not 
common for both eyes to be attacked simultaneously; and consequently the disease is usually more advanced in the one eye than in the other, but when both eyes are equally affected, one ought to be operated upon whenever the patient experiences difficulty in carrying on his work. It may then be necessary to perform a preliminary iridectomy operation a few weeks before extraction is attempted. It is, however, usually quite safe to extract a cataract before the stage of maturity is reached; but as, in that case, a considerable quantity of cortical matter is necessarily left in the eye, the process of healing is apt to be complicated, and demands much skill and care on the part of the surgeon. Before any operation is undertaken, the patient's ability to locate the position of a light in a dark room must be tested, and his field of vision carefully examined with a candle, in order to determine as far as possible that there is no atrophy of the optic nerve or detachment of the retina. The presence of either of these lesions would obviously render the operation of no avail in restoring sight. Careful examination must also be made to negative the existence of chronic conjunctivitis, or of blennorrhœea of the tear passages; and in all doubtful cases there should, without fail, be a bacteriological examination of the fluids in the conjunctival sac. If pyogenic microorganisms be present, there is great danger that after operation the eye will be lost from suppuration; indeed, to operate in these circumstances is simply to court defeat.

Wherever possible, a påtient should be placed in charge of a competent nurse at least forty-eight hours before the time fixed for the operation. He should have a warm bath, put on clean underclothing, and go to bed. The nurse must report if there be any cough, sickness, or unusual restlessness or nervousness. Early on the following morning, a purgative, preferably a half to one ounce of castor oil, is administered, to make sure that the bowels will be freely evacuated. The heart, lungs, and urine should be examined. Twenty-four hours before the operation, the face, the surface of the closed lids, the eyelashes, and the eyebrows should be thoroughly washed with hot water, in which I per cent. of carbo-sapol has been dissolved. While this is being done, the eyelids must be kept tightly closed to prevent any irritation of the conjunctiva. After all the soap has been washed away with hot sterilised water, the conjunctival sac is douched with warm normal saline solution, special attention being given to the outer and inner canthus and the edges of the eyelids. On the morning of the day of operation, the eyelids and surrounding skin are again thoroughly washed, and the conjunctival sac douched, every detail of the process being as carefully attended to as on the previous day. Female patients should have the hair divided and put into two plaits, in order to prevent it from becoming matted or 
entangled in the bandage. On the day itself the patient must not be allowed to eat heartily, and the food should be light and easy of digestion. Whenever it is practicable, the time chosen ought to be in the morning, shortly after the patient has awakened from sleep. For at least an hour before the operation, the eyes are kept covered by a compress soaked in a solution of perchloride of mercury, $x$ in 5000 , or a pad smeared with bichloride of mercury ointment, $I$ in 3000 (White), and this should remain in place until it is removed by the surgeon. It is, however, even more satisfactory to paint the skin of the lids and of the parts for a considerable distance around the orbit with 3 per cent. tincture of iodine, care being taken to sterilise the free edge of the lids. Half an hour before the operation, 30 grains of bromide of potassium or of sedobrol, or a teaspoonful of bromo-soda, should be administered. After the patient is on the operating-table, the perchloride dressing is removed, a towel is adjusted like a turban round the head, and a mackintosh covered by a towel is spread over the chest and tucked closely round the neck. Every instrument and dressing must be thoroughly sterilised, and the hands of the surgeon and of his assistants rendered aseptic.

Of all operations for the removal of a cataract, extraction of the lens in its capsule (Smith's Indian Method) is undoubtedly the ideal; but it requires special skill, and is so frequently accompanied by accidents, that in this country it is not likely to become popular. Simple extraction, when successful, leaves little to be desired. Unfortunately, however, in a considerable percentage of cases, a prolapse of the iris occurs within a few hours after the operation, and causes difficulties and dangers, all of which may, be avoided by performing an iridectomy, either at the time of the extraction, or a few weeks in advance of it. The preliminary iridectomy has many advantages. It helps to ripen the cataract, and saves bleeding from the iris at the time of the operation, and it also tends to equalise the intraocular tension, thereby diminishing the risk of choroidal hæmorrhage, so that almost all authorities are agreed that it is advisable when patients are old and feeble, or have only one eye. Notwithstanding the increased safety, however, which the preliminary iridectomy affords, patients shrink from being operated on twice, and so, if there be no complications, it is the combined operation that is most frequently performed.

The family doctor is frequently requested to be present at the operation; but only very rarely is he expected to assist the specialist under whose care he has now placed the patient.

The instruments required are a speculum, a Graefe linear section knife, iris forceps and scissors, a cystitome, a curette, and a small spatula. They must be thoroughly disinfected, and no instrument must be used 
a second time without fresh sterilisation. The knife should be fresh from the cutler, and after its edge and point have been carefully examined and tested, should be disinfected in absolute alcohol, and just before being handed to the surgeon be dipped for a moment in boiling water, and afterwards cooled in a bath of sterilised water. After the eye has been bathed with warm normal saline solution, and anæsthetised by a 2 per cent. solution of cocain, the lids are separated by the speculum, the limbs of which are extended to their utmost capacity, and then firmly clamped with a binding screw. The patient is instructed to look downwards, and the surgeon, if he is not ambidexter, stands behind him for the right eye and in front of him for the left. The blade of the knife is laid gently across the cornea in order to satisfy the patient that the eye has been thoroughly anæsthetised. A puncture is made, perpendicular to the surface of the cornea, and exactly in the corneo-scleral margin in a line with the upper border of the pupil, and the handle of the knife depressed until the blade lies parallel to the surface of the iris. It is then carried slowly across the anterior chamber to a corresponding point on the opposite side, where the counter-puncture is made. The knife is now pushed slowly through the cornea, right up to the hilt if possible, but care must be taken that the point does not come into contact with the skin at the inner canthus. The section should be wholly in the corneo-scleral margin, and is completed by two or three sweeps. It should not be any larger than necessary, but should always be big enough to permit the lens to escape easily. The forceps are then introduced through the wound, the iris lightly seized at the pupillary border, gently pulled out of the eye, and a portion excised by a single snip of the scissors. The cystitome is now passed into the eye, and directed downwards to the lower border of the pupil. Its cutting edge should be turned towards the lens, and the capsule freely ruptured by an incision from below upwards. At this stage, if there is any bleeding from the conjunctiva or the iris; the blood should be washed away by douching with warm

- saline solution. Pressure is now applied along the lower edge of the cornea, and at the same time counter pressure is made along the scleral lip of the wound; by that means the lens is extracted from the eye, the aim being to leave a jet-black pupil. If any cortical matter remain, it should be pressed out by means of a curette, or by digital manipulation. The "toilet" of the eye must now be attended to. If the iris be prolapsed, it should be carefully replaced by the spatula, and the lips of the wound left in accurate position. After the lens is removed, the patient ought to be able not only to count fingers, but to distinguish the thumb from the little finger.

When the operation is complete, the eye is again bathed with the 
saline solution, and one drop of I per cent. atropin instilled. The eyelids are wiped dry with a pledget of absorbent cotton-wool, and covered by an oval-shaped dressing of wet bicyanide gauze. Over this are laid several layers of sterilised absorbent wool, care being taken to fill up the hollow at the inner canthus so that there will be no unequal pressure upon the eyeball. The dressings are retained in position by a net bandage, which covers both eyes, and is secured by tapes tied behind, above the head, and below the chin. The patient, after being warned to make no exertion, is carried to bed, which has been comfortably warmed by hot-water bottles, the temperature of the room being about $60^{\circ} \mathrm{F}$., and all bright light excluded. He is laid on his back, and told to keep his head as still as possible, and not to rise or get out of bed for any purpose whatever.

Of the accidents which may occur in the course of an operation the chief are: (I) Premature escape of the aqueous. When this occurs, it is not possible to carry the knife across the anterior chamber without wounding the iris. The knife should therefore be withdrawn, the eye bandaged, and the operation postponed until the aqueous has had time to reaccumulate. (2) The iris may fall in front of the knife. When all efforts to disengage the iris are unsuccessful, the edge of the blade should be turned slightly forwards, so that a piece of the iris may be excised during the completion of the section. The piece of iris thus cut off should be picked out of the eye with forceps, and the coloboma forms the iridectomy. (3) Escape of vitreous. This is a much more serious matter, and is always to be feared when the patient, is nervous and unsteady, when the corneal section is too small, or when the vitreous is fluid. When the escape occurs before extraction, the lens is apt to slip backwards and pass out of sight, so that the surgeon may fail to remove it. If, on the other hand, the escape follows the extraction, a small loss of vitreous is not of much moment ; but whenever the loss is large, there is always danger that sooner or later detachment of the retina will occur. (5) Hæmorrhage from the choroid. Happily this is a rare accident, but when it does occur the eye is hopelessly ruined. Usually it comes on without warning. The operation may have been completed successfully, when all at once the patient cries out with pain, and blood escapes through the wound in sufficient quantity to saturate the dressings. In some cases the eyeball retains its shape; but more frequently it shrinks and requires to be enucleated, partly to get rid of pain, and partly lest it should excite sympathetic inflammation of the fellow-eye.

For at least forty-eight hours after the operation, the nurses must keep the patient under continuous observation, see that he lies still and is kept comfortable, and be particularly watchful that the bandage is 
not disturbed. They should report at once if there be much restlessness, coughing, delirium, retention of urine, or complaint of pain; but never under any circumstances should they administer either stimulants or narcotics, unless these have been specially ordered. If all goes well, the eyes are dressed on the second day, every care being taken to keep the parts aseptic. The lids are wiped by a piece of moist gauze and then gently separated, so that the wound may be examined. If by this time the anterior chamber has not reformed, it is probable that there is a piece of capsule or conjunctiva between the lips of the wound, and that in consequence the process of healing will be much delayed; but as eyes in which the incision heals slowly rarely suppurate, this complication need cause no alarm. A single drop of a solution of I per cent. atropin and 2 per cent. cocain is instilled; a dressing similar to the one removed applied, and the eyes thereafter bound up for twenty-four hours. More light is now allowed to enter the room, and on the third or the fourth day the dressings are again removed, the eye examined, and, if the pupil be not dilated, another drop of atropin and cocain solution is instilled. The eyes may now be left unbandaged, but should be protected from light by a pair of London smoked glasses. On the fourth day the patient is allowed to sit up in bed for meals, and if there be no sign of inflammation to leave bed on the ninth day. The practitioner should bear in mind that in aged patients post-operative delirium is not a very infrequent occurrence. It is usually violent-the patient being noisy, restless, and difficult to keep in bed. It is due to feebleness of the circulation and the complete exclusion of light, and is probably considerably influenced by idiosyncrasy to atropin. Whenever delirium threatens, the bandage should be removed, and two or three tablespoonfuls of brandy administered; if, however, delirium is pronounced, a hypodermic injection of morphia, $\frac{1}{6}$ to $\frac{1}{4}$ grain, should be given without delay. After a good sleep the patient usually awakes refreshed and in his right mind, but a severe attack may last for several days.

At first the dietary should consist largely of liquids, so as to avoid as far as possible the movement of the jaws during the act of chewing. On the fourth day some minced meat and potatoes may be added, and if there are no unfavourable symptoms the patient may be allowed to feed himself. If the bowels have not acted, a laxative is given early in the morning of the fourth day.

After successful extraction of the cataract, the patient is not able to see clearly until he is provided with glasses equivalent to the lens which has been removed-usually a + Io D.S. for distance and + I4 D.S. for reading, together with any astigmatic correction that may be necessary. It is not wise, however, to allow him to use spectacles until six or eight 
weeks after the operation, and he should be warned to be very careful in the use of the eye for several months. After a patient has recovered sight in one eye, he frequently becomes anxious regarding the other, and asks when it will be ready for operation. The answer depends on many different circumstances; but in no case is operation on the second eye obligatory, unless the results in the first have been disappointing The patient must state his own wishes on the matter.

Quite a large percentage of patients regain normal vision; but the good results naturally expected are occasionally spoiled through complications that occur during healing. One of the simplest, but most troublesome of these, is inversion of the lower lid. Usually the entropion disappears of itself; but in cases of difficulty it may be rectified by the application of contractile collodion to the skin of the lid, or the edge of the lid may be pulled outwards by a strip of sticking-plaster. The great majority of cataract extractions are followed by inflammation of the iris, but, as a rule, this is so slight that it need cause no alarm. When, however, there is a large amount of cortical matter, the exudation from the iris becomes mixed with the lenticular substance, and seriously interferes with its solution. As a consequence a tough after-cataract remains, and before the patient can regain his sight this requires to be removed by operation. Iritis is always to be feared when, after the first dressing, arthritic foam is seen at the outer and inner canthus, since this may be regarded as indicating that the patient has rheumatic or gouty tendencies. In these cases the inflammation may not confine itself to the iris, but may also involve the ciliary body. Severe irido-cyclitis is always followed by a closed pupil. Usually this can be remedied and sight restored by a subsequent operation; but in some cases-more particularly if the dressings have been disturbed, and the eye thereby injured, or if the patient suffer from chronic disorder of the stomach or bladder-the lens matter assumes a yellowish colour, the conjunctiva becomes thickened and velvety, and the eyeball is tender on pressure. There is then great danger that the intraocular tension will become lessened, and that ultimately the eyeball will shrink. If any capsular matter, or a tag of iris, get entangled in the corneal wound, irido-cyclitis is sometimes accompanied by increase in the intraocular tension. This is a very grave complication, and unless relief is speedily obtained by operation, sight will be lost from subacute glaucoma.

If post-operative hæmorrhage be excluded, the most dangerous aftercomplication is suppuration. The introduction of antiseptic methods, and the care now taken of patients both before and after operation, have done much to diminish the risk, but in spite of everything some eyes are still lost by septic infection. The inoculation is almost always due 
to micro-organisms that have been lurking in the conjunctival sac, or along the margin of the lids and the roots of the eyelashes. They, are probably always present, but become able to work mischief only in unhealthy subjects, especially in those whose tissues have been rendered easily vulnerable by alcoholic excesses and want of proper nutriment. Infection takes place all the more readily, if, during the extraction of the lens, the cornea has been bruised, or an escape of vitreous has taken place.

The lens and vitreous are most favourable culture media for pyogenic micro-organisms; and so suppuration, once started, pursues its course practically uninfluenced by any form of treatment. The application of local antiseptics, or the use of the cautery, avails but little; and the only chance of saving an eye after suppuration of a corneal wound lies in keeping the parts thoroughly clean by copious douching with sterile saline solution, by the immediate injection beneath the skin of 4000 units of antidiphtheritic serum, and by the judicious administration of quinine and stimulants. In every case where there is a history of inflammation of the conjunctiva, and where suppuration is to be feared, the secretions ought to be examined for bacteria ; and as long as any dangerous organisms-more particularly the streptococcus, the staphylococcus aureus, the pneumococcus, or the bacillus coli-are present, operation should not be attempted.

Prior to the days of aseptic treatment, the cases of cataract extraction lost through suppuration were, as now, those in which there had been previous inflammation of the conjunctiva or tear passages, or in which the patient's vitality was low. The tissues might heal in spite of microorganisms present in the conjunctival sac; but where these are in abnormal numbers and pyogenic, or where the vulnerability of the tissues is great, the eye cannot be saved. Antiseptic methods have done much to diminish the frequency of disaster in the former group, by destroying the germs or lessening their number, but the liability to failure in cases of the latter kind remains as before; and the necessity for action along this line suggested by clinical observation is borne out by the results of theoretical and experimental research so ably dealt with in Professor Welch's Huxley Lecture.

"The struggle," says Dr. Welch, "between the bacteria and the body cells in infection may be conceived as an immunising contest, in which each participant is stimulated by its opponent to the production of cytotoxins hostile to the other, and thereby endeavours to make itself immune against its antagonist." When the body is healthy, it possesses the power of producing substances specifically antagonistic to all sorts of micro-organisms, but with this power any morbid state at once 
interferes; and as in all infections, whether local or general, " abnormal metabolism is an essential condition," patients of low vitality will be in such a state that the substances produced may be not only not antagonistic to parasitic organisms, but actually stimulating to their growth.

This being so, one must not only endeavour to render the field of operation sterile, but must also devote the utmost care and attention to what is of equal importance-the physical condition of the patient himself. For some months before the operation he must be well fed, and kept as much as possible in the open air; and a course of salines with iron and quinine is usually attended with the happiest results. Rest and preparation are necessary in every case, no matter what the sufferer's position in life may be, but should he be one who has been, owing to defective eyesight, unfit for work for several months, and through poverty inable to procure a sufficient amount of food, he will require special care, since not only will he be in a condition unfavourable for the rapid healing of any wound, but will also have tissues vulnerable to the slightest infective taint, and suited for the rapid spread of suppuration. While, therefore, every effort should be made to render the field of operation free from micro-organisms, care should equally be bestowed on the state of the patient himself, especially if he be poor and habitually ill-nourished. Were more attention paid to the health of these broken-down subjects prior to cataract operations, the number of failures from suppuration would be very considerably reduced; and it is only by attention beforehand, and by careful preparation, that this much-to-be-desired result can be attained. 


\section{CHAPTER XXVII}

\section{THE CLINICAL SIGNIFICANCE OF EXOPHTHALMOS}

Exophthalmos a cardinal sign of disease of orbit-Simulated proptosis- Etiology of proptosis-Exophthalmos accompanied by signs of inflammation-Tenonitis-Orbital abscess-Cavernous sinus thrombosis-Exophthalmos unaccompanied by signs of inflammation-Graves' disease-Causes of unilateral proptosis-Pulsating exophthalmos-Proptosis due to tumour.

An eye that is unduly prominent at once arrests attention, and merits most careful consideration, on account of its importance and far-reaching significance both in ophthalmology and in the larger sphere of clinical medicine and surgery. In its relation to the orbit the globe may be displaced forwards, backwards, or to either side, and of these abnormal positions the first, which is known as proptosis, or exophthalmos, is not only the most frequent, but also the most important. It is a cardinal sign in affections of the orbit, occurs also in leukæmia and exophthalmic goitre, and is, indeed, to be found in so many different diseases, that the correct interpretation of its significance is often an exceedingly difficult matter. It varies in degree from a prominence so slight as to require the most careful examination for its discovery, to one in which the globe stands out so far that it is said to be dislocated. A trained observer will at once detect any undue protrusion of the eyes by inspection alone, and the detection will obviously be all the more quickly made if the exophthalmos affect only one eye. For more exact observation, however, and especially when it is necessary to keep a careful record of the progress of a case, it is well to employ an instrument of precision, and for this purpose nothing can be better than the exophthalmometer, devised by Hertel, which is simple in construction and accurate in working.

The normal position of the eye in its socket necessarily varies in different individuals, because, while it is largely determined by the control of the eyelids, the extraocular muscles, the capsule of Tenon, and the tarso-orbital fascia, it depends also, to a certain extent, on the amount of adipose tissue in the cushion of fat on which the globe rests. This is the explanation of the prominent bulging eyes of corpulent persons, and the sunken eyes of those who are emaciated.

In genuine proptosis the whole eyeball is displaced forward; and the 224 


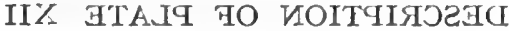

\section{OMJAHTH}

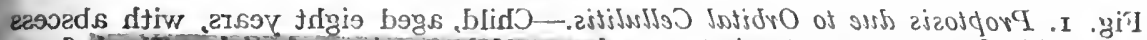

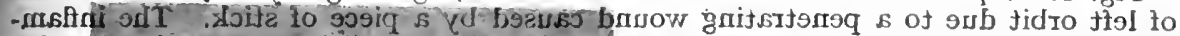

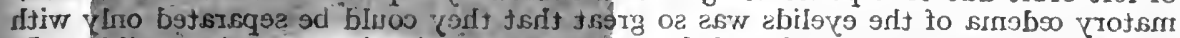

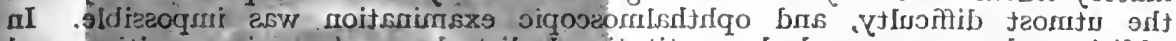

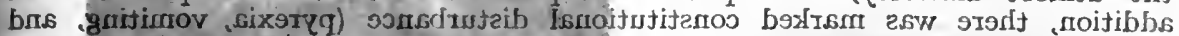

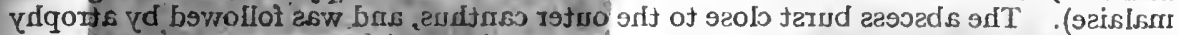

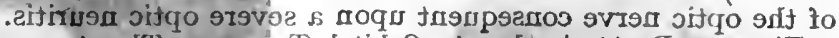

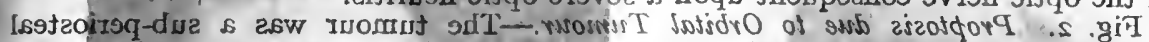
to yrotzir \& 9 vsg frrgijsq $9 r \mathrm{~T}$.

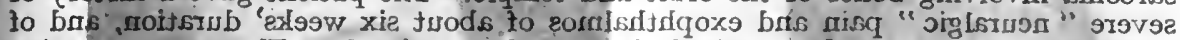

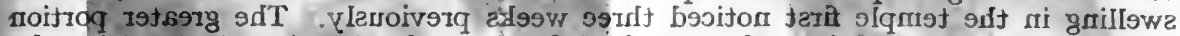

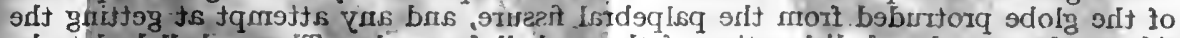

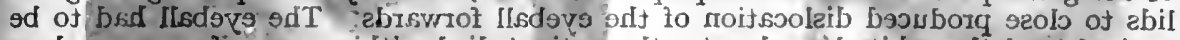

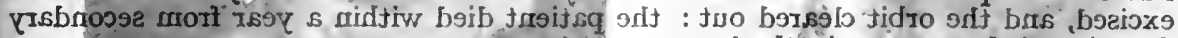

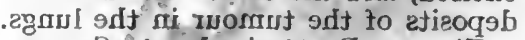

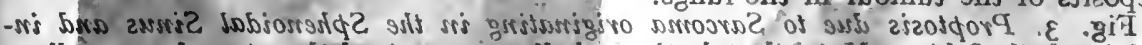

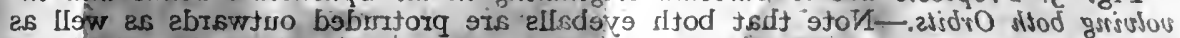

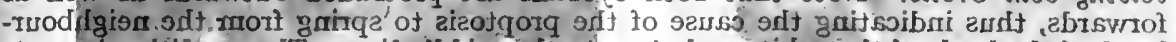

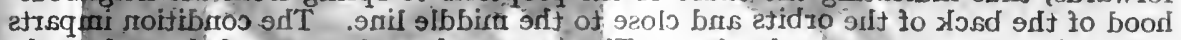

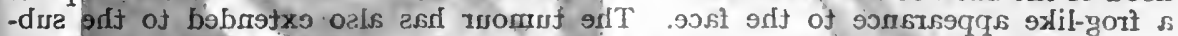

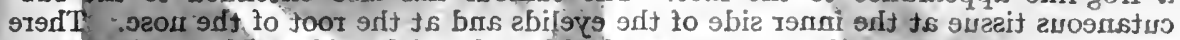

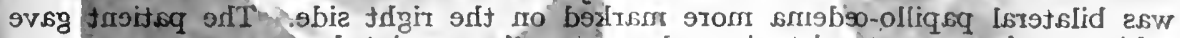

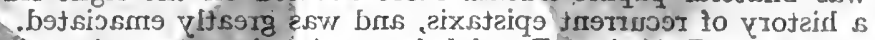

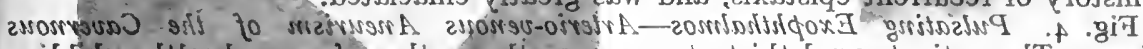

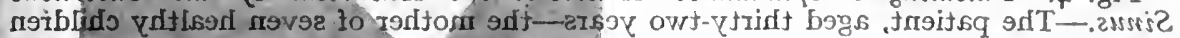

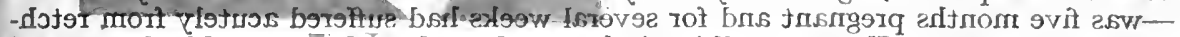

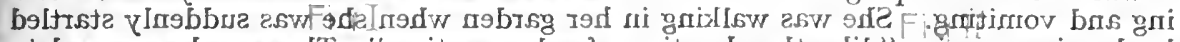

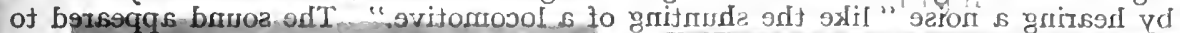

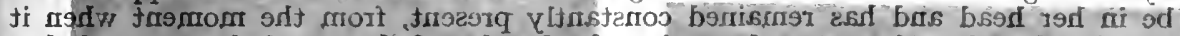

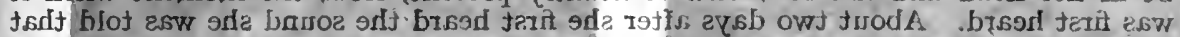

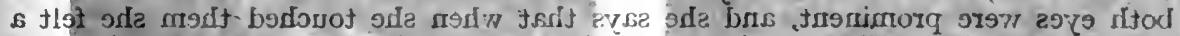

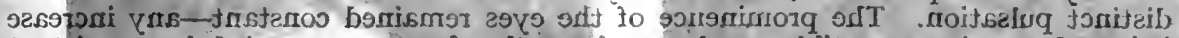

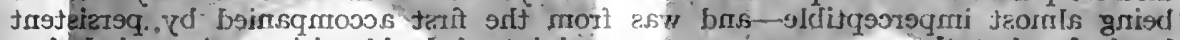

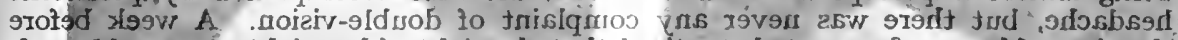
¿bormuld k.6w פy

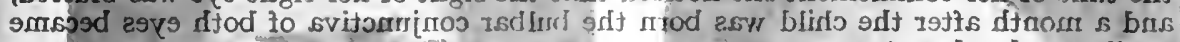

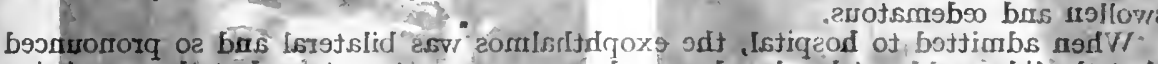

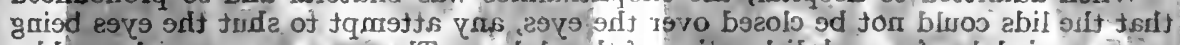

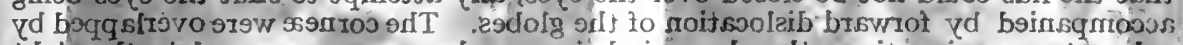
frgir

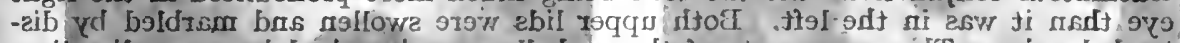

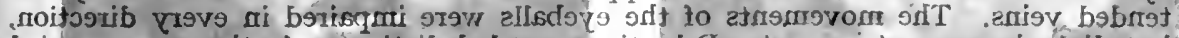

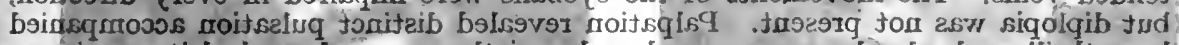

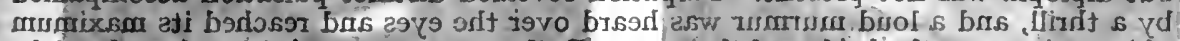

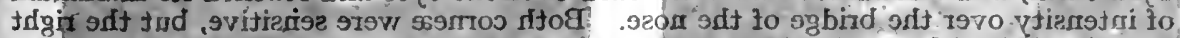

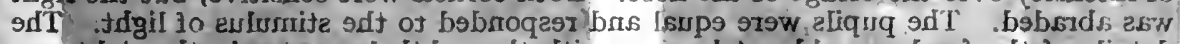

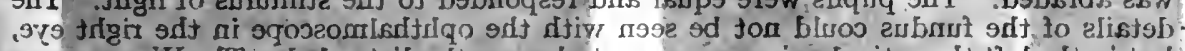

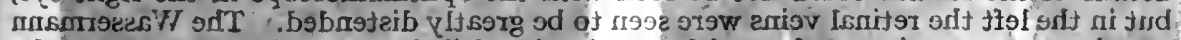

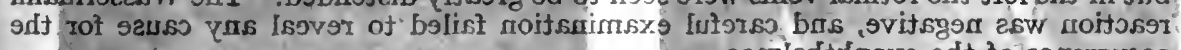

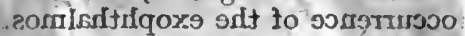

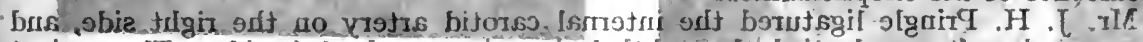

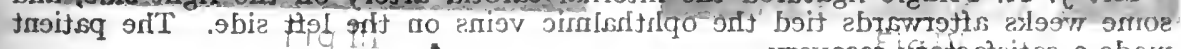

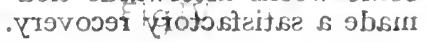




\section{DESCRIPTION OF PLATE XII}

\section{Exophthalmos}

Fig. I. Proptosis due to Orbital Cellulitis.-Child, aged eight years, with abscess of left orbit due to a penetrating wound caused by a piece of stick. The inflammatory œdema of the eyelids was so great that they could be separated only with the utmost difficulty, and ophthalmoscopic examination was impossible. In addition, there was marked constitutional disturbance (pyrexia, vomiting, and malaise). The abscess burst close to the outer canthus, and was followed by atrophy of the optic nerve consequent upon a severe optic neuritis.

Fig. 2. Proptosis due to Orbital Tumour. - The turnour was a sub-periosteal sarcoma involving bones of the orbit and temple. The patient gave a history of severe "neuralgic" pain and exophthalmos of about six weeks 'duration, and of swelling in the temple first noticed three weeks previously. The greater portion of the globe protruded from the palpebral fissure, and any attempt at getting the lids to close produced dislocation of the eyeball forwards. The eyeball had to be excised, and the orbit cleared out: the patient died within a year from secondary deposits of the tumour in the lungs.

Fig. 3. Proptosis due to Sarcoma originating in the Sphenoidal Simus and involving both Orbits. - Note that both eyeballs are protruded outwards as well as forwards, thus indicating the cause of the proptosis to spring from the neighbourhood of the back of the orbits and close to the middle line. The condition imparts a frog-like appearance to the face. The tumour has also extended to the subcutaneous tissue at the inner side of the eyelids and at the root of the nose. $Y$ There was bilateral papillo-œdema more marked on the right side. The patientngave a history of recurrent epistaxis, and was greatly emaciated.

Fig. 4. Pulsating Exophthalmos-Arterio-venous Aneurism of the Cavernous Sinus. - The patient, aged thirty-two years - the mother of seven healthy children -was five months pregnant and for several weeks had suffered acutely from retching and yomiting. She was walking in her garden when she was suddenly startred by hearing a noise "like the shunting of a locomotive.". The sound appeared to be in her head and has remained constantly present, from the moment when it was first heard. About two days after she first heard the sound she was told that both eyes were prominent, and she says that when she touched them shelfelt a distinct pulsation. The prominence of the eyes remained constant-any increase being almost imperceptible-and was from the first accompanied by persistent headache, but there was never any complaint of / double-vision. A week before the time of her confinement she noticed that the sight of her right eye was blurred, and a month after the child was born the bulbar conjunctiva of both eyes became swollen and œdematous.

When admitted to hospital, the exophthalmos was bilateral and so pronounced that the lids could not be closed over the eyes, any attempt to shut the eyes being accompanied by forward dislocation of the globes. The corne were overlapped by œdematous conjunctiva - the chemosis being much more pronounced in the right eye than it was in the'left. 'Both upper lids were swollen and marbled by distended veins. The movements of the eyeballs were impaired in every direction, but diplopia, was not present. . Palpation revealed distinct pulsation accompanied by a thrill, and a loud murmur was heard over the eyes and reached its maximum of intensity over the bridge of the nose. Both cornex were sensitive, but the right was abraded. The pupils were equal and responded to the stimulus of light. The details of the fundus could not be seen with the ophthalmoscope in the right eye, but in the left the retinal veins were seen to be greatly distended. The Wassermann reaction was negative, and careful examination failed to reveal any cause for the occurrence of the exophthalmos.

Mr. J. H. Pringle ligatured the internal carotid artery on the right side, and some weeks afterwards tied the ophthalmic veins on the left side. The patient made a satisfactory recovery. 


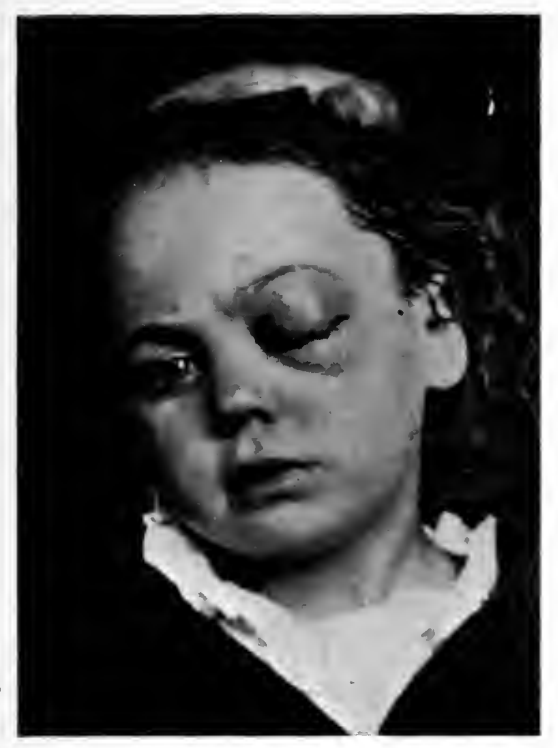

Fig̣ I

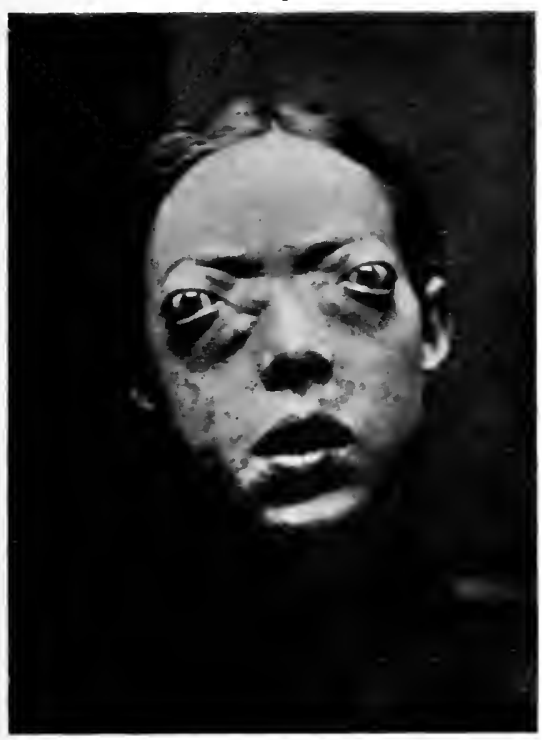

Fig III

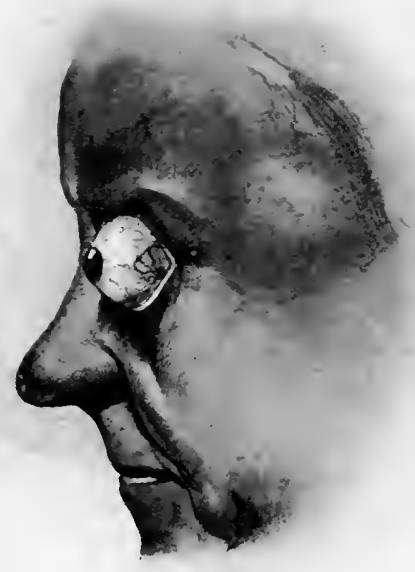

Fig II

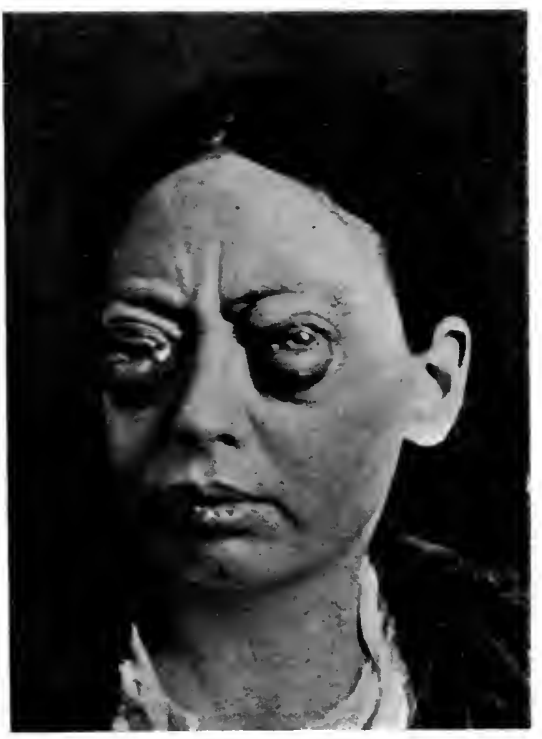

Fig $\mathbb{}$ 
condition is only simulated when the eyes appear prominent owing to increased width of the palpebral fissure, or to abnormal length of the globe itself, as in high myopia, or in buphthalmos. Anything, however, which interferes with the structures which normally hold the eyeball in position will induce true exophthalmos; and that explains the undue prominence seen after tenotomy of an internal rectus muscle when the capsule of Tenon has been freely lacerated.

Proptosis may be caused either by diminution of the normal capacity of the orbit, or by increase in the bulk of its contents. The latter condition is by far the more frequent, and is brought about by inflammation of the orbital tissues, by disease of the orbital blood-vessels, or by the formation of a new growth; while the former is due to deformity of the osseous walls of the orbit, which may be the result of congenital malformation, for example, tower-skull, or of pressure consequent on disease of the neighbouring air sinuses.

The danger to the eyeball depends in great measure on the cause of the proptosis; and in order to arrive at an accurate diagnosis, the exact ætiology of the exophthalmos must be determined with the greatest care. The first thing to do is to obtain from the patient or from his friends a clear history of the onset and progress of the disease; and particular inquiry should always be made about injury, and whether the proptosis occurred suddenly, or came on slowly and gradually. In the clinical examination of a patient inspection naturally comes first. By this means the proptosis itself, any vertical or horizontal displacement of the globe, and any impairment of movement, can all be at once discovered. Limitation of motion in a particular direction must always be carefully noted; and it is very important to observe whether or not the proptosis is accompanied by signs of inflammation. Palpation is of equal value, the little finger being pressed cautiously but deeply into the orbit, so that tenderness, fluctuation, or increased sense of resistance may thereby be detected. Palpation will also reveal at once the presence of thrill or of pulsation. The globe ought also to be pressed gently backwards into its socket, to find out whether the exophthalmos can be lessened or overcome, and whether the pressure causes pain.

The nose, too, should always be carefully examined; because empyema of the sinuses very frequently gives rise to disease of the optic nerve, accompanied or not by displacement of the eye. In obscure cases, in which a slight degree of exophthalmos is associated with severe frontal neuralgia, persistent asthenopia, alteration in the field of vision, or intractable inflammation of the eyeball and its appendages, examination of the nose by a specialist will often reveal diseases of the ethmoidal, sphenoidal, frontal or maxillary air spaces, and thereby clear up the 
diagnosis, and point the way to rational and successful treatment. In addition to expert rhinological examination with speculum and mirror, transillumination ought always to be employed; a skiagram of the bones of the skull should be obtained, and in doubtful cases the blood ought to be examined by the Wassermann test, and the von Pirquet tuberculin cutaneous reaction applied. Lastly, when the exophthalmos is accompanied by signs of inflammation, it is of paramount importance to take the temperature, to count the pulse and the respirations, to examine the tongue, and to test the urine.

The differential diagnosis of a sign common to many diseases is of the first importance; and in proptosis this will be best accomplished by first referring each case to one of two main groups, according to the presence or the absence of inflammation.

1. When the Exophthalmos is accompanied by signs of Inflammation.-When signs of inflammation are present, one naturally thinks at once of abscess of the orbit, but if the patient be rheumatic or gouty, tenonitis should be excluded before a positive diagnosis is made. That disease is also characterised by the occurrence of œedema of the lids and bulbar conjunctiva, and by protrusion of the eyeball; but its differential diagnosis is arrived at by paying attention to the following special characteristics. The inflammation usually occurs suddenly without known cause. The eye is prominent; but the proptosis is not in proportion to the degree of œdema: The bulbar conjunctiva is greatly swollen, and usually projects over the whole circumference of the cornea, but there is no purulent discharge. The movements of the globe are impaired in all directions, and any attempt to turn the eye is accompanied by pain and by a great sense of tension and pressure. When the eyes are allowed to remain at rest, the pain is moderate, but is always apt to be very severe in paroxysms, and is worse at night. In spite of those alarming symptoms, vision remains unaffected, temperature is rarely disturbed, and the disease ends in recovery, although the inflammation may relapse many times before it ultimately runs its course.

Abscess of the orbit may originate either as a direct result of injury, or through metastasis in certain pyæmic infections; while in many cases the inflammation spreads to the orbit from the neighbouring air sinuses. Pain, shivering, feverishness, and occasionally vomiting, are the earliest signs; and, following quickly on their appearance, come swelling of the lids, chemosis of the ocular conjunctiva, and protrusion of the eyeball. The agonising, deep-seated pain extends over the whole head, and is frequently accompanied by constitutional symptoms, at times so violent that they threaten to endanger life. Pus forms rapidly, but distinct fluctuation may be difficult to detect until the suppuration approaches 
the surface. There is great tenderness on pressure, and any movement of the eyeball to the side, or any backward pressure of it into the socket causes intense access of suffering. The patient himself is so conscious of this that he instinctively keeps his eyes as still as possible. As the proptosis increases, the globe gets displaced towards one or other wall of the orbit, and its movements become steadily impaired, until at length it is firmly fixed in the midst of the inflamed tissues. The vision may be unaffected, but that is not always the case, for there may be rapid deterioration arising from the spread of the inflammation to the optic nerve. As the case progresses the lids become more and more livid and swollen, and the bulbar conjunctiva, especially the retrotarsal folds, are turgid, œdematous, and prominent, while the globe may project so far that it cannot be covered by the lids. At length the pus reaches the surface and escapes through the skin of the eyelids, along the margin of the orbit, or it bursts through the conjunctiva at the lower or upper fornix. After the pus has been freely evacuated, the globe sinks by degrees back to its normal position, and recovery usually takes place, but with sight more or less seriously impaired. In unfavourable cases, however, the eyeball may be lost from panophthalmitis ; and until convalescence is well established there is always a danger of cerebral complications, which may lead to a fatal termination from meningitis, abscess of the brain, or cavernous sinus thrombosis.

Of all these complications, cavernous sinus thrombosis is the most dramatic in its onset as well as the most tragic in its results, and the possibility of its supervening must never be forgotten. Following the occurrence of a focus of infection situated on the skin of the face, in the nostrils, the mouth or the middle ear, the patient complains of severe headache, accompanied by shivering and vomiting; and shortly thereafter the eyelids on one side become swollen and discoloured, and the eyeball is observed to be unduly prominent. There may or may not have been a complaint of dimness of vision in the affected eye, as the patient feels so ill and restless from the pain that he may not have noticed the impairment of sight until the other eye is attacked. This generally occurs within a day or two after the beginning of the disease; the onset is sudden, and the proptosis, as well as the lividity and swelling of the lids of the second eye are usually more pronounced than in the one first affected. It is important in connection with diagnosis to remember that in cavernous sinus thrombosis the signs at the commencement of the disease are confined wholly to one side, and that they become bilateral later on. Cellulitis confined to the orbital cavity rarely occurs on both sides simultaneously, but in thrombosis the implication of both orbits is frequent: Indeed, as Sir William MacEwen points out, the side on which the 
symptoms began " may be partially restored, while the other side becomes markedly affected." He regards " this alternation of the seat of symptoms" as an important distinction "between inflammations confined to the orbital cavity and cavernous thrombosis." Ptosis is usually present and is due either to implication of the nerve supplying the levator muscle, or simply to the swollen and often brawny condition of the upper eyelids themselves. The conjunctiva is chemosed, protrudes, and usually discharges a thin irritating muco-purulent secretion. The skin of the brow and the root of the nose is swollen and erythematous. The corneæ are usually insensitive, and when the proptosis is great they are not properly protected by the lids, and, consequently, quickly suffer from exposure. The pupils are dilated and fixed; and when the state of the cornea permits ophthalmoscopic examination, acute neuro-retinitis is frequently observed. The movements of the eyeballs are impaired, and sometimes a wellmarked squint is present. Palpation increases the pain, but fluctuation is rarely detected. The temperature is always high, the pulse quick, small and thready, the respirations increased, and the urine usually contains albumen. Even to an unskilled observer the patient appears to be dangerously ill, and the suffering is obviously very acute, but it is characteristic that the mental faculties remain unimpaired. Indeed, one of the most pathetic features of this disease is the fact that the patient retains consciousness almost to the end.

The course of the illness is steadily downward; for while the eye first affected so far improves that there may even be partial recovery of sight, the other eye becomes worse and worse, and the constitutional symptoms more and more critical. The proptosis increases, the chemosed conjunctiva becomes necrotic, the cornea suppurates, and odema spreads from the eyelids to the brow, temple, cheek, mastoid process, and the upper part of the neck on the same side. The temperature is distinctly pyæmic; the pulse is rapid, and becomes steadily weaker and smaller; the respirations increase in frequency; the tongue is dry and thickly coated; and secretion accumulates on the teeth and in the throat, so that it is difficult to keep the mouth clean and the breath free from a heavy fœtid odour. Anorexia is complete, and often towards the end uncontrollable diarrhœa adds greatly to the patient's discomforts, and rapidly increases his weakness. The disease rarely lasts longer than a fortnight, and a fatal termination is ushered in by rigors, hyperpyrexia, muttering delirium, and loss of control over bladder and rectum.

2. When the Exophthalmos is unaccompanied by Signs of Inflammation.-The first subdivision of this main group is into cases where the proptosis is bilateral, and those in which it is confined to one 
side. It may be stated as a general proposition, that cases with the PROPTOSIS AFFECTING BOTH SIDES are symptomatic of general disease, whereas when one side only is affected, the cause is in all probability local; but to this generalisation there are many exceptions. It is easy to recall cases of Graves' disease, where the exophthalmos was confined entirely to one eye; and in tumour originating in the basi-sphenoid, both eyes are affected sooner or later. The advice of an ophthalmic surgeon may be sought by a patient suffering from Graves' disease, on account of excessive prominence of the eyes, and he can often reduce the disfigurement by diminishing the width of the palpebral fissure. A tarsorraphy operation not only adds greatly to the patient's comfort, but also protects the cornea, and is to be advised in all cases in which the patient is suffering as a result of the exophthalmos. It is true that in Graves' disease ulceration of the cornea occurs very seldom; yet the proptosis, the infrequent winking, together with the diminished sensitiveness of the cornea, all favour the onset of that dangerous complication.

WHEN THE PROPTOSIS IS MONOCULAR, a distinction must be drawn between the cases in which the exophthalmos occurs suddenly, and those in which the onset is slow and gradual. The cases included in the first category are usually the result of accident. The co-existence of pronounced chemosis, exophthalmos, and fixation of the globe following a wound by bullet or shrapnel, is highly. suggestive of the presence of a foreign body lodged in the cavity of the orbit. It is very important to observe whether or not the exophthalmos can be reduced by pressure. By that means a differential diagnosis can readily be made between emphysema and hæmorrhage; for, while pressure has no influence in the latter, it causes the former to disappear completely for the time being, although it returns gradually whenever the pressure is withdrawn. Hæmorrhage into the orbit due to injuries-gunshot or penetrating wound, or blows-is somewhat common, and when the effused blood becomes infected, orbital abscess readily occurs. Spontaneous hæmorrhage, on the other hand, is of rare occurrence. It generally takes place in patients suffering from hæmophilia, from scurvy, or from blood-vessels so diseased that they readily rupture when they are subjected to any sudden extra strain, such as violent coughing or vomiting; and in children it not unfrequently occurs during a spasm of whooping-cough.

Dilatation of the blood-vessels of the orbit-angioma-also gives rise to proptosis, the degree of which can always be modified by pressure. In these cases it is important to observe whether or not there is pulsation, and if any thrill can be felt. Proptosis, pulsation, and thrill are the cardinal signs of a pulsating exophthalmos. This usually occurs as a result of injury which has brought about a rupture of the internal carotid 
in the cavernous sinus. The disease is characterised by marked proptosis of one eye, and in exceptional cases both are affected. The exophthalmos can be reduced by digital pressure; but the eye returns at once to its abnormal position the moment the pressure is removed. Pulsation synchronous with the beats of the heart is visible, and can be distinctly felt when the fingers are laid upon the closed lids, and becomes much more pronounced when the eye is pushed gently backwards into its socket. Palpation does not cause pain; but as a rule the patient complains of severe pain behind the affected eye immediately after he recovers from the first effects of the accident. The pain is accompanied by a whizzing noise in the head, like the sound of rushing water, which may be so loud and constant as to interfere with sleep. If a stethoscope be applied to the lids a loud blowing murmur is heard, and the sound is conducted by the bones of the skull for a considerable distance away from the eye.

After the bruit, pulsation, and proptosis have existed for several months, tortuous enlargements of the superior ophthalmic veins occur at the upper and inner aspect of the orbit. These venous masses are very characteristic. They are rounded, soft, easily compressible, quite painless, and communicate a distinct thrill as well as pulsation when palpated. Vision may be lost from optic neuritis, but as a rule is unaffected, although the ophthalmoscope shows that the veins on the diseased side are larger and more tortuous than they are in the other eye. The symptoms are at once relieved by pressure on the common carotid artery, ligature of which is a recognised method of treatment.

In a case of monocular exophthalmos, which cannot be reduced on pressure, and which is not due to hæmorrhage, orbital tumour should be suspected. Proptosis is not only the chief sign of a tumour of the orbit, but its degree enables an approximation to be made of the size of the new growth, while the date of its appearance gives some indication of the site of the neoplasm. The nearer to the apex of the orbit the tumour develops, the later in the course of the disease will the proptosis appear. The direction in which the eyeball is protruded is a point of considerable importance in differential diagnosis; for, unless the tumour be situated within the cone formed by the recti muscles, the proptosis is not in the line of the axis of the orbit, but above or below, or to one side or the other. In almost every case the movements of the eyeball are impaired, and the greatest restriction is always towards the site of the tumour. The restriction may be due simply to the presence of the new growth, or to the involvement of the muscles, vessels, and nerves in the increase of the neoplasm.

A tumour of the optic nerve as a rule pushes the eyeball straight 
THE CLINICAL SIGNIFICANCE OF EXOPHTHALMOS 23I

forward. It is characterised by its slow progress and by loss of sight, due to atrophy of the optic nerve occurring very early in the course of the disease.

Lastly, the orbit may be invaded by a tumour, which has originated in a neighbouring structure, e.g. the lachrymal gland, the antrum, or any other of the adjoining air sinuses, or in the eyeball itself after an intraocular growth has burst through the sclerotic. 


\title{
CHAPTER XXVIII
}

\author{
SARCOMA OF THE CHOROID, AND GLIOMA OF THE RETINA
}

Sarcoma of the choroid-Tendency to metastasis-Clinical course and stagesDiagnosis by transillumination-Occurrence of secondary glaucoma-Extraocular stage-Metastatic stage-Treatment-Glioma of the retina-Age incidence-" Amaurotic cats' eye "-Metastases-Pseudo-glioma-Treatment of glioma-Description of a typical case.

\section{SARCOMA OF THE GHOROID}

SARComa is by far the most important neoplasm found in the uveal tract. Other tumours also occur in that region, but they are very rare, and although of great interest to the pathologist, are of little clinical importance, because it is only in very exceptional cases that their true nature can be diagnosed until after the eyeball has been enucleated and examined by the microscope. It may almost be accepted as a clinical aphorism, that a malignant tumour of the eyeball, occurring in a patient from forty to sixty years of age, is a sarcoma of the choroid, whereas if it occurs in early childhood it is a glioma of the retina.

Sarcoma may originate in any part of the uveal tract; but its favourite site is the choroid in the neighbourhood of the optic papilla. It is almost invariably unilateral; takes origin in the layer of the larger blood-vessels; is composed either of round or of spindle cells; and is usually pigmented - melano sarcoma-although in a small percentage of cases pigmentation is absent-leuco sarcoma. The differentiation into pigmented and nonpigmented has, however, little clinical value, because both varieties have a similar evolution, the latter indeed being regarded as an early manifestation of a melano sarcoma. It is generally stated, however, that non-pigmented tumours develop more frequently in the anterior portion of the uveal tract, and occur at an earlier age than the pigmented.

Sarcoma of the choroid is almost always primary; appears in an eye which, up to the time of its onset, has been supposed to be perfectly healthy; is steadily progressive, and ultimately destroys the eye. It has, moreover, not only a great tendency to recur locally after it has been removed by a surgical operation, but also to give rise to secondary growths in distant parts of the body. Its chief danger, therefore, consists in the risk of early metastasis; consequently in every case it is very important to make a careful examination of the liver as soon as possible after a diagnosis of sarcoma of the choroid has been established. In the majority 


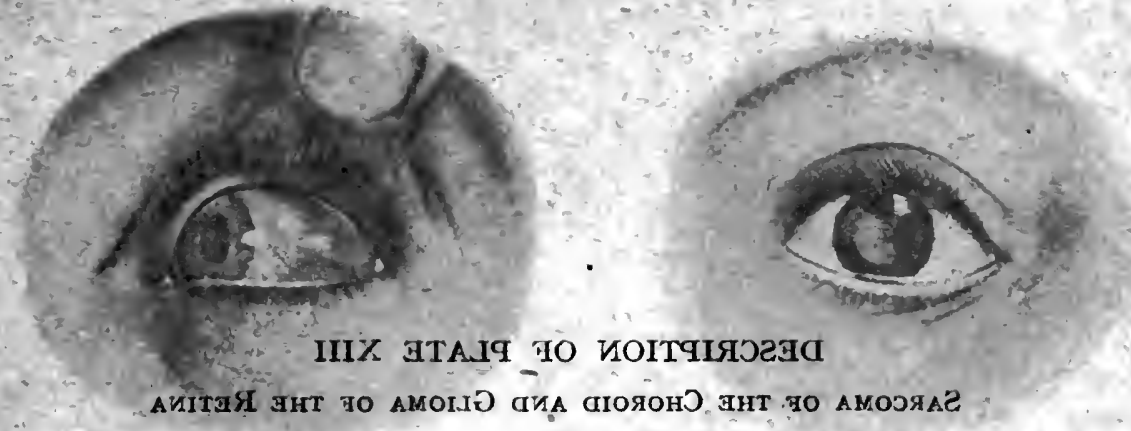

bionodo edt lo smooras .E of I .egi't

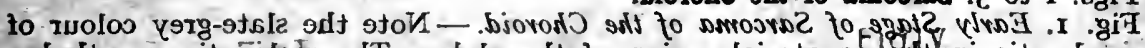

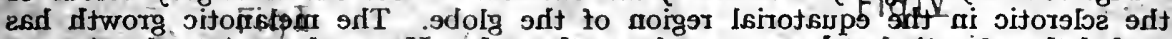

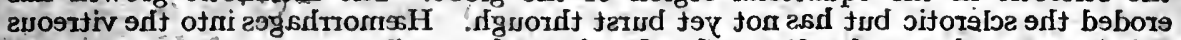

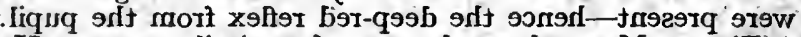

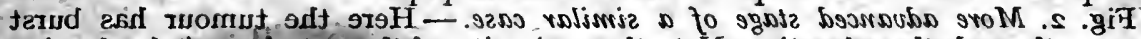

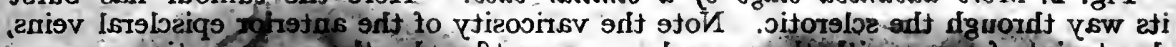

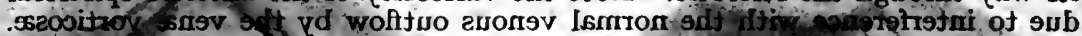

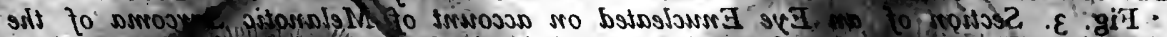

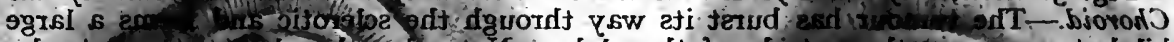

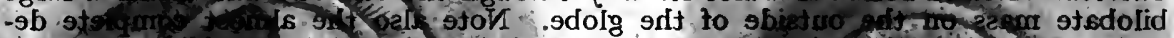

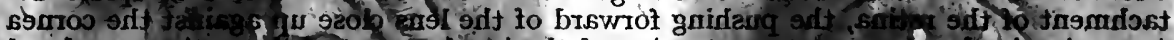

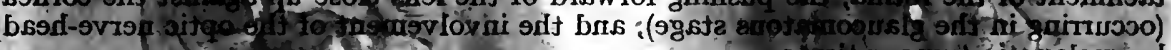

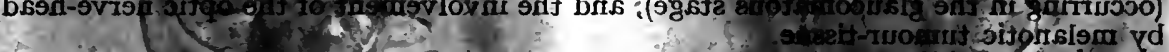

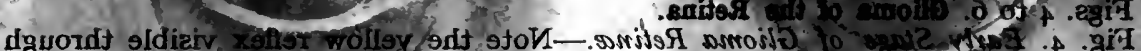

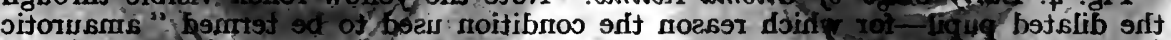

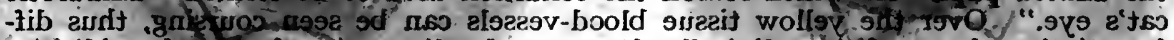

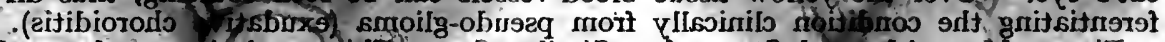

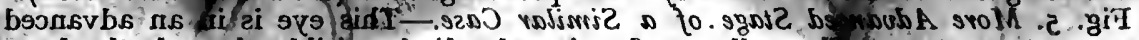

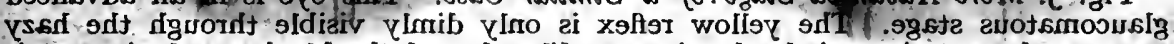

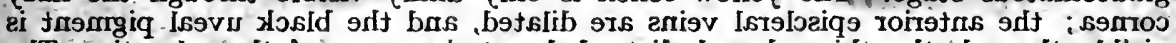

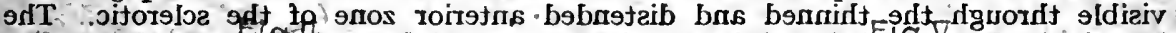

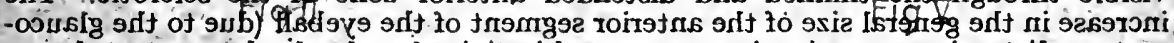

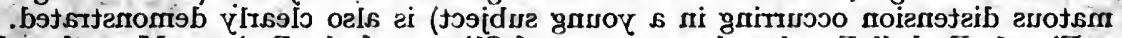

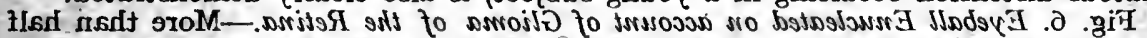

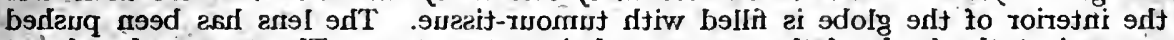

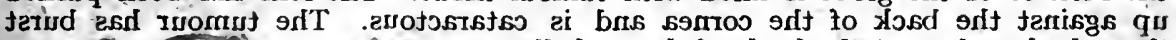
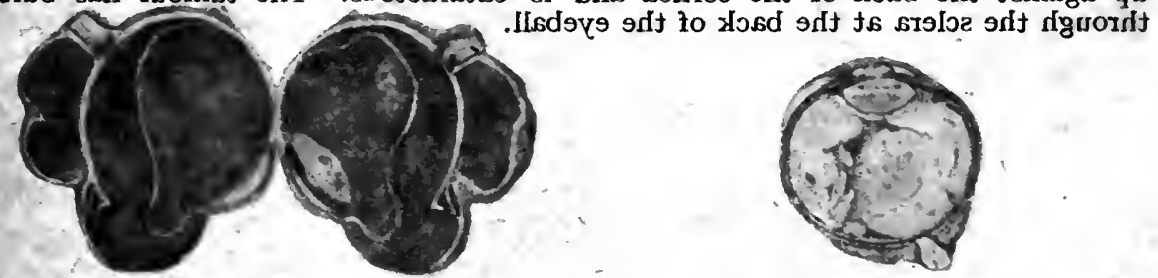

Fig III

Fig II 


\section{HAPTER XXVIII}

TIE (T:CJRID, AND GLIOMA OE THE RETINA

oroud- Lindernes

metastasis-Clinical course and stages-

urrence of sccondary glaucoma-Extra-

reatment-_iona of the retina-Age

DESCRIPTION OF PLATE XIIL do-glioma-Treatment

SARCOMA OF THE CHOROID AND GLIOMA OF THE RETINa

Figs. I to 3. Sarcoms of the choroid.F THE CHOROID

Fig. 1. Early Stage of Sarcoma of the Choroid. - Note the slate-grey colour of the sclerotic in the equatorial region of the globe. The melanotic growth has eroded the sclerotia but has not yet burst throughrio Hemorrhages into the vitreous were present-hence the deep-red reflex from the pupil.

Fig. 2. More advanced stage of a simitar case. Here the fumour has burst its way through the sclerotic. Note the varicosity of the anteriot epischeral veins, due to interference with the normal venous outflow by the venæ vorticosæ.

- Fig. 3. Section of an Eye Enucleated on account of Melanotic Sarcoma of the Choroid. The tumour has burst its way through the sclerotic and formscarlarge bilobate mass on the outside of the globe. Note also the almost complete detachment of the retina, the pushing forward of the lens close up against the cornea (occurring in the glancomatons stage), and the involvement of the optic nerve-head by melanotic tumour-tissue.

Figs. 4 to 6 . Glioma of the Retina.

Fig. 4. Early Stage of Glioma Retince.-Note the yellow reflex visible throutgh the dilated pupil-for which reason the condition used to be termed It amaurotic cat's eye." Over the yellow tissue blood-vessels can be seen coursing, thus differentiating the condition clinically from pseudo-glioma (exudative chorolditis).;

Fig. 5. More 4 dyanced Stage of a Similar Case.-This eye is in an advanced glaucomatous stage. The yellow reflex is only dimly visible through the hazy cornea; the anterior episcleral veins are dilated, and the black uveal pigment is visible through the thinned and distended tanterior zone of the isclerotid. rithe increase in the general size of the anterior segment of the eyeball (due to the glaucomatous distension occurring in a young subject) is also clearly demonstrated.

Fig. 6. Eyeball Enucleated on account of Glioma of tha Retina. More than half the interior of the globe is filled with tumour-tissue. The lens has been pushed up against the back of the cornea and is cataractous. "The tumour has burst through the sclera at the back of the eyeballrequently in the anterior portion

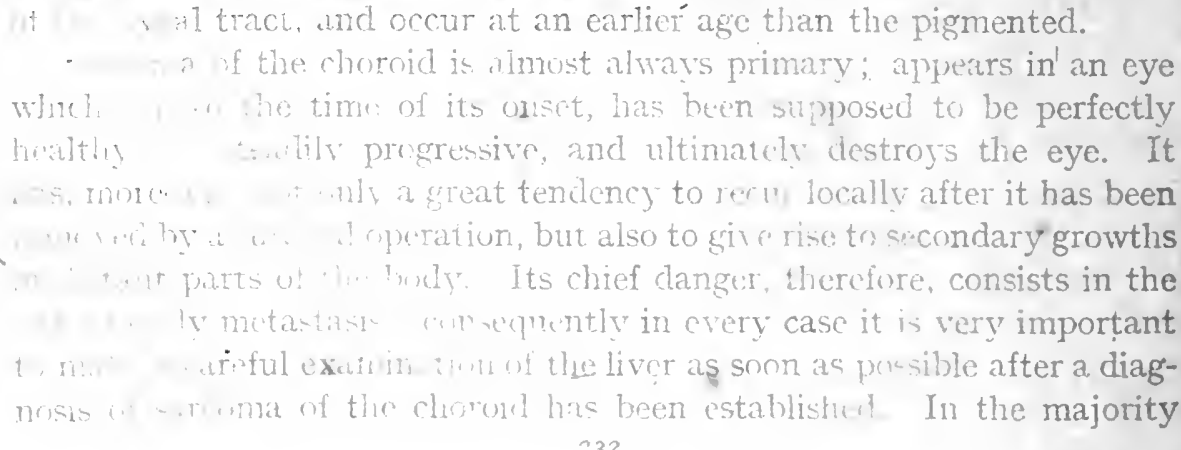




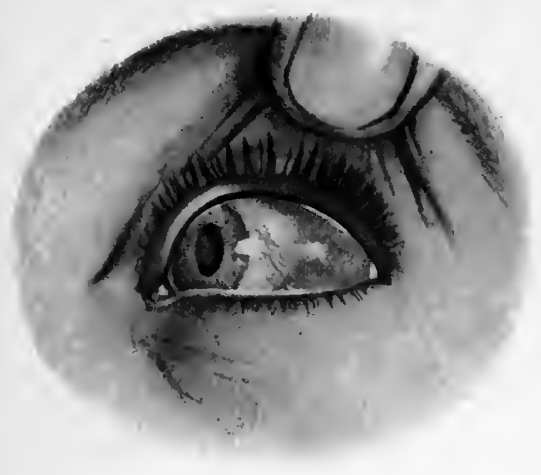

FigI

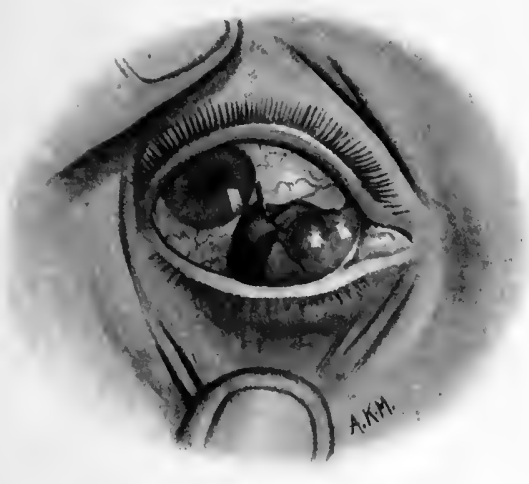

Fig II

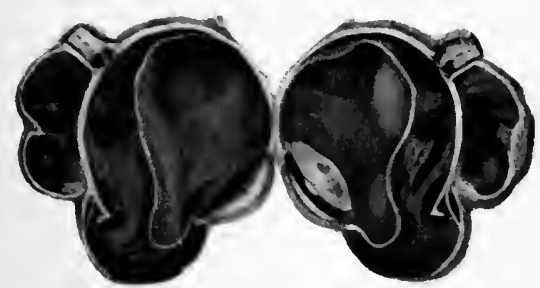

Fig III

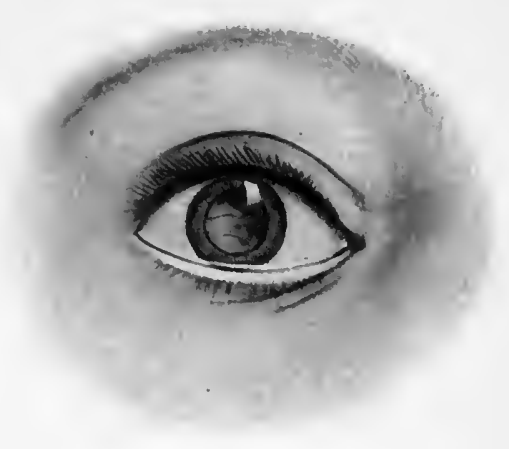

Figl

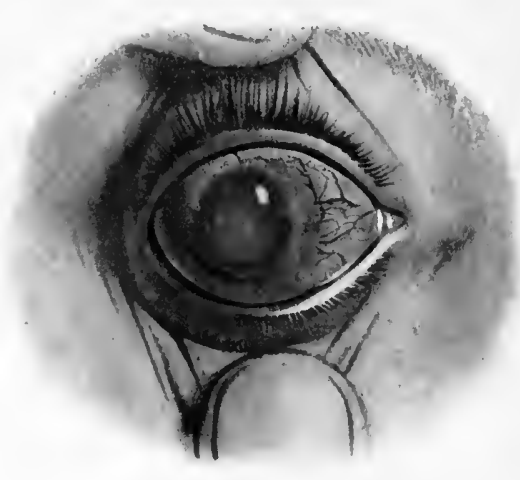

Fig $\nabla$

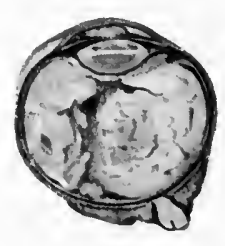

Fig 표 
I

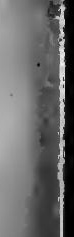


of instances, a fatal termination cannot be averted, even when enucleation of the eyeball has been performed in the earliest stages of the disease.

The progress of a case is usually divided into four periods-

1. The Quiescent Stage-As long as the tumour is small, it may remain unsuspected unless it takes its origin in the region of the macula, when vision will be affected from the very outset. In the great majority of cases, however, failure of sight is the first warning the patient has that something is wrong with his eye. He is conscious of a cloud, of a blank-scotoma-in the field of vision; and an ophthalmoscopic examination reveals a more or less extensive detachment of the retina corresponding to the defect in the visual field. It is usually difficult to determine from the ophthalmoscopic appearances alone, whether the detachment is simple or secondary to a tumour of the choroid. If the patient is seen very early in the course of the disease, the retina is closely applied to the surface of the growth, and in these circumstances its shape, its solid appearance, its brown-black colour, and its peculiar vascularity are all helpful in diagnosis. Very soon, however, serous exudation occurs from the vessels of the choroid, and the retina becomes separated over an area far greater than that occupied by the tumour. In these circumstances, the absence of the more usual causes of detachment-myopia, traumatism, etc.- and the unusual site of the separation, ought at once to suggest that a tumour is present. If the new growth be situated in front of the equator of the globe transillumination through the sclerotic will quickly discover it, because the tumour prevents the transmission of the light, and the pupil is not illuminated by the red glow which is always there when the detachment is simple. When, however, the neoplasm is situated behind the equator of the eyeball, it is out of reach of that method of examination; but if a powerful electric lamp be placed in the patient's mouth, his face covered by a rubber mask with openings for his eyes, in a room absolutely dark, both pupils will be illuminated, and a tumour, if present, will be seen as a shadow in the bright red pupil. At this stage, however, the two most important signs of a tumour of the choroid are increase in the intraocular tension, and dilatation and tortuosity of the anterior ciliary veins.

As the tumour grows, sight steadily deteriorates, as a result of increase in the detachment of the retina, and at length when the separation becomes complete, blindness is absolute. Even at this stage, the eye may show no sign of active mischief on external examination, but if a strong light is focussed on the pupil it is frequently possible to see the tumour behind the lens. In some cases, however, the lens suffers from malnutrition, and a cataract prevents any examination of the interior of the globe. 
After the tumour has been present from twelve to eighteen months, sudden increase of intraocular tension occurs, and the onset of pain and other symptoms, due to secondary glaucoma, mark the commencement of the second stage.

2. The Glaucomatous Stage.-Although, as a rule, this stage is not reached until the tumour has existed for one or two years, yet in many cases secondary glaucoma occurs very much sooner. Its onset always indicates the continuous evolution of the neoplasm, and the danger is that the case may be mistaken for primary acute glaucoma. That mistake happens all the more readily if the patient has not consulted a doctor during the quiescent stage, but is seen for the first time during the progress of the acute symptoms. In all cases, however, the difficulty in diagnosis will be lessened if the following facts be borne in mind-

(I) The occurrence of acute unilateral glaucoma ought always to excite suspicion that an intraocular growth may be the cause of the symptoms.

(2) If an intraocular tumour be present-

(a) The history of the case will show that failure of sight in the affected eye was present before the onset of the glaucomatous seizure.

(b) No rainbow-coloured rings around a light have been noticed by the patient.

(c) Careful examination reveals no sign of glaucoma in the other eye.

In these circumstances, the probabilities are that the loss of sight is due to detachment of the retina, and the combination of detachment of the retina with increased tension occurs only in intraocular tumour.

From the commencement of the second stage the patient's sufferings are continuous, the eyeball is constantly congested, and on one or other side of the globe the ciliary veins are large and varicose. In the majority of cases, when a diagnosis of tumour is given the patient is glad to sacrifice a blind eye in order to get rid of his pain. If, however, he is unwilling to submit to operation, the disease will take its natural course, and as the neoplasm increases in size the sclerotic covering it becomes thin, and the uveal pigment shines through it. A slate-blue discoloration of the sclerotic is thus brought about; and at length the sclera becomes so weak that it cannot resist the pressure from within, and bulges outwards. The end of the second stage has now been reached, and its duration is, as a rule, much shorter than that of the first.

3. The Extraocular Stage.-After the tumour perforates, it grows very rapidly, and forms a bluish-grey mass projecting from the sclerotic. Its surface is usually nodular, but is continuous with the curvature of the sclera and is covered by conjunctiva. That covering, however, soon gives way, and the tumour, now free from all restraint, grows rapidly. 


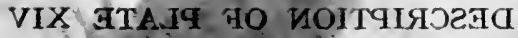

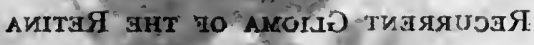

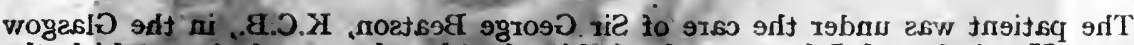

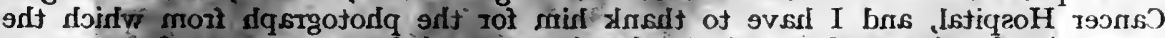

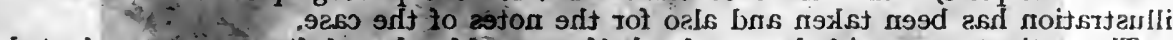

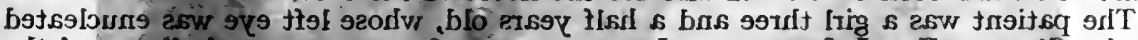

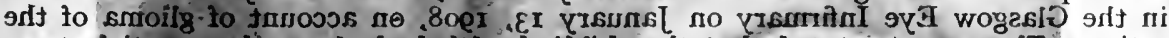

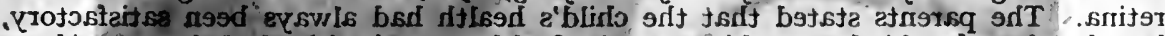

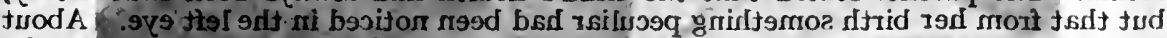

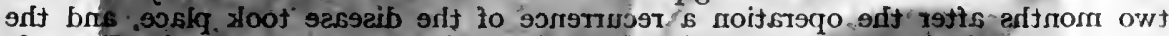

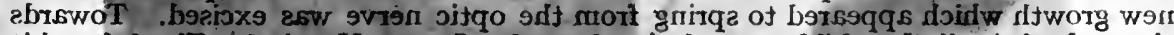

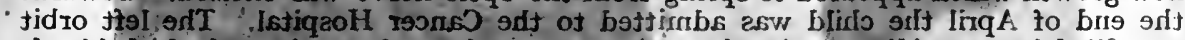

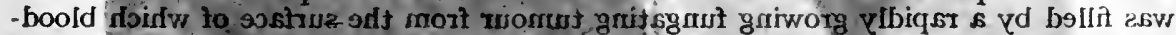

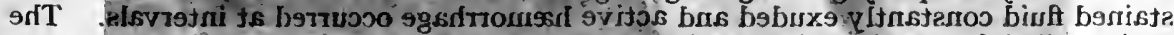

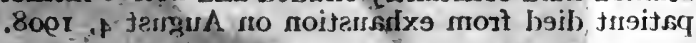

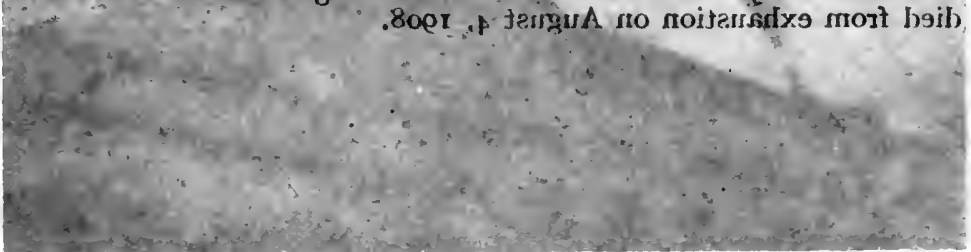




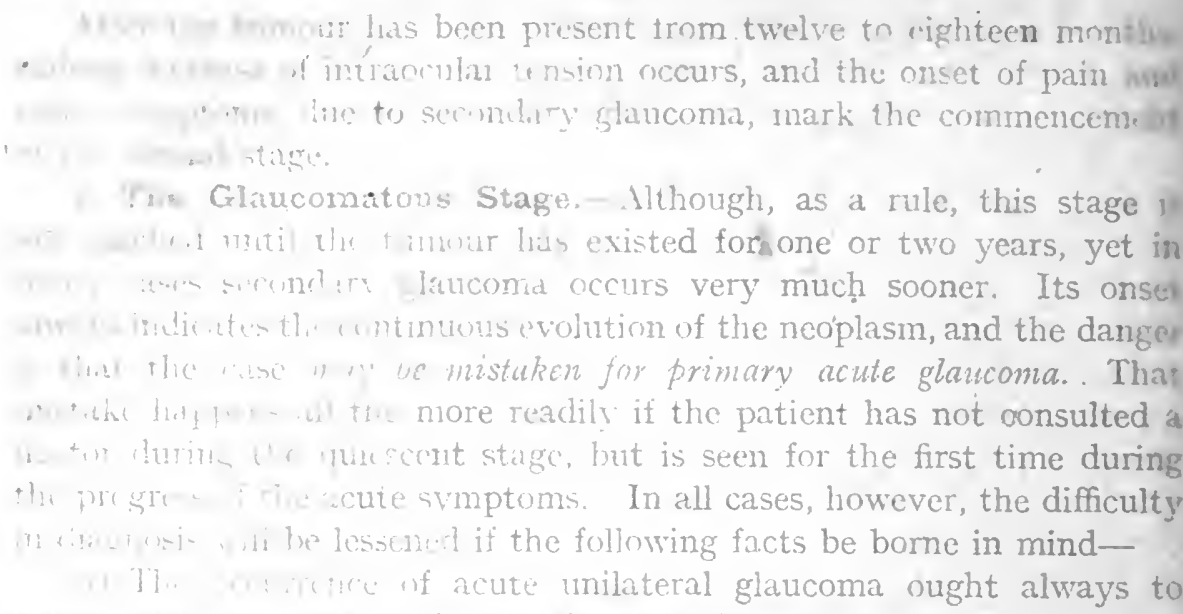
DESCRIPTION OF PLATE XIV . be the cause of the Recurrent Glioma of the Retina

The patient was under the care of Sir George Beatson, K.C.B., in the Glasgow the Cancer Hospital, and I have to thank him for the photograph from which the illustration has been taken and also for the notes of the case.

The patient was a girl three and a half years old, whose left eye was enucleated he in the Glasgow Eye Infirmary on January 13, 1908, on account of glioma of the retina. The parents stated that the child's health had always been satisfactory, but that from her birth something peculiàr had been noticed in the left eye. ot Abput two months after the operation a recurrence of the disease took place, and the new growth which appeared to spring from the optic nerve was excised. Towards the end of April the child was admitted to the Cancer Hospitalin The left orbitent was filled by a rapidly growing fungating tumour from the surface of which bloodstained fluid constantly exuded and active hæmorrhage occurred at intervals. The patient died from exhaustion on August 4; 1908.nd stage the patient's sufferings

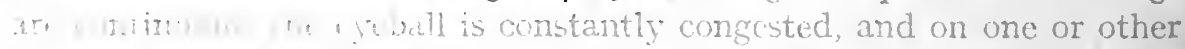
wh illur veins are large and varicose. In the majority (hetgine is of tumour is given the patient is glad to sacrifice vlo to get rid of lis pirin. If, however, he is unwilling will take its natural course, and as the wo the sclerotic covering it becomes thin, and the tlirnugh it. A slate-blue discoloration of the ahrut; and at length the schar becomes so weak pressure from within, and bulges outwards. las now been reached, and its duration is, wh of the first.

3. The Extrue 2... Sige. After the tumulur perforates, it grows mass projecting fom the sclerotic. intinucu wit? the curvature of the What in ring, however, soon
frum all icstraint, grows rapidly. 


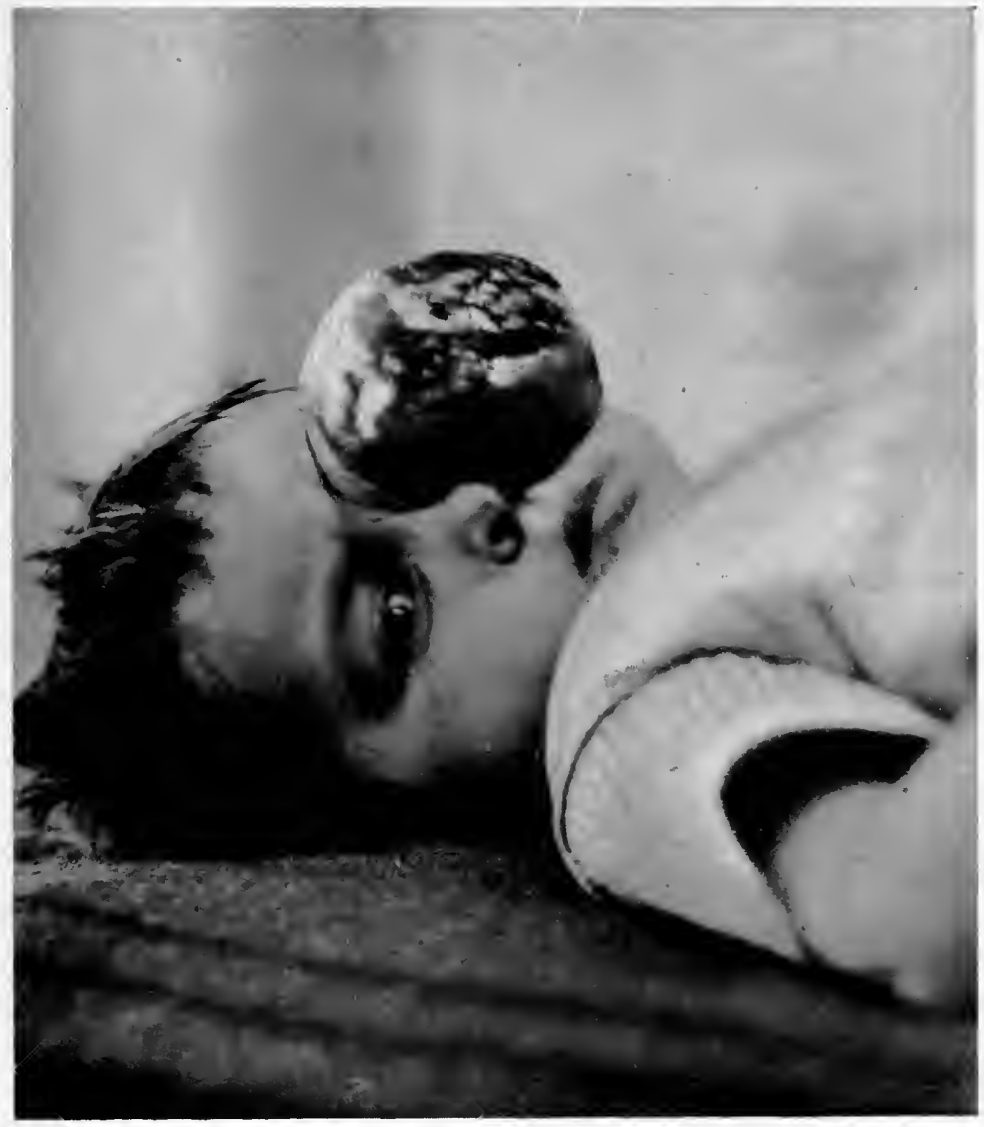




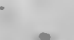

I

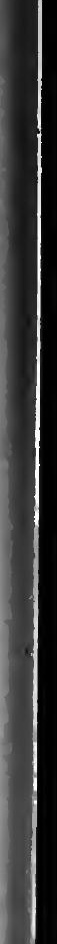


If it burst through the posterior part of the globe it grows into the orbit, and pushes the eyeball forwards. In some cases this proptosis is so great that the lids cannot be closed and the eye is left unprotected.

Although in this stage pain is much less, yet the patient is tormented by foul-smelling discharge, and constantly recurring hæmorrhages from the ulcerating surface of the tumour. The lymphatic glands are not affected; but the disease spreads forward to attack the lids and the surrounding skin, or backwards to implicate the bones of the orbit, and may reach the base of the brain through the sphenoidal fissure or the optic foramen.

At other times a sarcoma, instead of fungating externally to the globe, destroys the eye, and then undergoes a retrogressive metamorphosis. In these circumstances, it is found within a shrunken eyeball, which has been enucleated for cosmetic reasons, or for the relief of pain. If its presence be unsuspected, and no urgent symptoms occur to require enucleation, sooner or later the tumour will again become progressive, and not only will develop locally, but, in all probability, kill the patient from metastatic growths in the liver or some other internal organ.

4. The Metastatic Stage.-From the moment that an intraocular sarcoma appears, there is danger of metastatic infection; but that risk is very much greater after the extraocular stage has been reached. Metastasis in remote organs occurs through embolism. The blood current detaches, cells from the tumour, and carries them into other parts of the body, where they develop into independent growths. Any part of the body may, therefore, be attacked; but the liver is the most frequently affected, and the great majority of cases of sarcoma of the choroid terminate fatally from metastatic disease of that organ.

Treatment.-The only treatment for sarcoma of the choroid is enucleation of the eyeball; and the operation must be performed as soon as possible after the diagnosis is made. If the extraocular stage has been reached when the patient comes for treatment, the tissues of the orbit will be infected, in which case enucleation of the globe will not suffice : total exenteration of the orbit is necessary. It is almost impossible, however, to prevent local recurrence, or to avert the danger of metastatic infection.

\section{GLIOMA OF THE RETINA}

Glioma is the only neoplasm which occurs in the retina, and although in structure it closely resembles a round-celled sarcoma, yet its clinical features are so characteristic, that it is convenient to give it a distinctive name and describe it as a separate disease. It occurs only in young 
children, for the most part between two and four years old, and is seen very rarely after twelve years of age. Many, however, regard it as a congenital affection, which has remained latent for a time. Both eyes may be affected, the one quite independently of the other; and several members of the same family may suffer. At its outset glioma causes neither pain nor irritation; and as the patient is usually too young to make spontaneous complaint of defective sight, the first intimation the parents have that the eye is affected is the appearance, in certain lights, of a peculiar yellow reflex from the pupil. That, as a rule, at once excites alarm, and causes them to consult the family doctor, who must be careful not to try to calm the parents' fears by giving an optimistic assurance regarding the condition. Nothing ought to be said until the child's eye has been thoroughly examined, after the pupil has been fully dilated with a mydriatic. If glioma be present, a strong light condensed on the eye, and the use of the ophthalmoscope, will reveal a pale yellow nodular mass traversed by blood-vessels, and more or less deeply situated in the vitreous chamber. There may be more than one tumour, and in addition small white disseminated swellings are usually seen in the retina in the neighbourhood of the principal growth.

Glioma usually originates in the outer or the inner granular layer of the retina, and, in consequence, is classified into glioma exophytum and glioma endophytum. The former grows outwards, separating the retina from the choroid, while the latter grows inwards, filling up the vitreous chamber. The tumour is made up of closely set small round cells, arranged in characteristic fashion around blood-vessels, and very prone to degenerate. Its growth is always rapid; and in a few months from the time it is first noticed, the bright coloured reflex becomes so obvious that the disease received from the older writers the name " Amaurotic cat's eye "- "Amaurotic" because the eye is blind, and " cat's eye" because it shines like cats' eyes in the dark.

If left to itself, glioma passes through the same stages as sarcoma of the choroid-quiescent, glaucomatous, extraocular, and metastatic.

The second stage is characterised by the occurrence of inflammation, and by increase in intraocular tension; and from now onwards the child's sufferings are constant. The optic nerve becomes affected early in the course of the disease, which, once it has got a foothold, steadily spreads by direct continuity to the brain. At other times, after the glioma has filled the vitreous chamber, it bursts through the ocular tunics, and invades the structures in the orbit. It grows so rapidly that a large tumour forms and displaces the eyeball, which, by this time, has become greatly enlarged. In advanced cases, the exophthalmos is so extreme that the eye projects between the lids in the centre of a large fungating, 
bleeding mass-fungus hamatodes. The neighbouring lymphatic glands become implicated, and the bones of the face and the cranium are attacked. In a few months, the child becomes worn out by its sufferings and dies from exhaustion, from some intercurrent septic inflammation, or from the development of a cerebral tumour. In some instances the eye, instead of enlarging and perforating, gradually shrivels, and the disease appears to undergo spontaneous cure; but such cases are very uncommon, and are liable at any moment to light up afresh and become progressive.

Whenever, in a young child, the pupil is observed to be dilated, to be irresponsive to light, and to present a yellowish reflex, the likelihood is that it is suffering from glioma. There are several conditions, however, also occurring in young children giving rise to similar appearances, and those may cause considerable difficulty in diagnosis. They have all been grouped together under the general term pseudo-glioma, and as in all of them the eye affected is blind, it is always prudent in a doubtful case to sacrifice it, rather than run the risk of the danger to life which is always to be dreaded in every case of glioma. Pseudo-glioma includes many different conditions, but all of them can be grouped as follows-

I. Exudative choroiditis, which sometimes occurs in children as a metastatic complication of acute infectious diseases-meningitis, pneumonia, influenza, measles, scarlet fever, etc.

2. Tubercle of the choroid.

3. Congenital defects, due to the persistence of part of the fibrovascular sheath on the posterior surface of the lens.

In the majority of instances, pseudo-glioma can be differentiated from true glioma, by the clinical history of the case-previous acute illness, by the age of the patient-glioma is very uncommon after five years of age, whereas pseudo-glioma may develop much later in life-and by the appearance of the eye affected-signs of past iritis or irido cyclitis, retraction of the base of the iris, diminution of intraocular tension, and occasionally shrinking of the globe.

Although glioma is one of the most malignant diseases, yet, in its earliest stages, it admits of a more favourable prognosis than is possible in sarcoma of the choroid; because, if enucleation of the eyeball be performed before the optic nerve is infected, the chances are that the disease will be completely eradicated. Recovery can take place even after both eyes have been removed; and, as a general rule, it may be said that the child's life is out of danger if no recurrence has taken place within three years after the operation. The all-important thing is that the eye be enucleated before the optic nerve has become affected.

The only treatment for glioma of the retina is enucleation, which 
ought to be performed if possible during the quiescent stage, and while the tumour is confined within the ocular tunics. Care must always be taken to divide the optic nerve far back in the orbit; and the cut end of the nerve ought at once to be examined for any sign of the disease. When the optic nerve is affected, or when the tumour has perforated the sclerotic, simple enucleation is not enough, and the whole of the tissues of the orbit must be removed. Even after such an extensive operation, however, there is always a danger that the disease has not been eradicated, consequently chloride of zinc or other caustic, radium or X-rays should always be applied for the purpose of reaching any diseased tissue which has escaped the action of the knife.

The following case is a striking illustration of the terrible nature of glioma, when the natural course of the disease has not been interrupted by treatment during its earliest stage: The patient was a girl four years of age, who was seen for the first time towards the end of August Igog. The mother stated that for more than a year she had observed " something peculiar" about the left eye, but as she did not think "it was serious" she paid no attention to it, until the child began to complain of pain. She then noticed that the left eye was enlarged, and much more prominent than the right. When the child was brought to hospital, it was seen that the anterior segment of the left globe was much distended; the sclera around the cornea presented a slate-blue colour, and large tortuous veins coursed over its surface; the anterior chamber was filled by semi-solid material of a rusty brown colour; the iris and the pupil were not visible, and palpation revealed that the eye was slightly tender to touch, and the intraocular tension greatly increased. The child was pale and emaciated, was obviously suffering pain, and was said to be liable to recurrent feverish attacks. The left eye was at once enucleated; and although the optic nerve was cut far back, its appearance showed that it had already become infected; consequently the contents of the orbit were removed, and chloride of zinc paste applied to the socket. Healing took place without any complication, and for a time the child appeared to improve. In about six weeks, however, it was seen that the glioma had recurred in the orbit, which was soon filled by a rapidly growing tumour. The lymphatic glands of the neck and at the angle of the jaw became enlarged, and a soft swelling appeared over the left frontal eminence. A similar swelling was detected over both lower maxillary bones in front of the ramus; the right eye became divergent, owing to paralysis of the internal rectus muscle, and the right eyelids were much swollen. The growth in the left orbit increased steadily, and extended to the right side behind the nose. By the middle of December, i.e. rather more than three months from the date of the 
operation, the right eye was surrounded by tumour tissue which had displaced the globe forwards and outwards, although no intraocular disease could be detected. The left superior maxilla became affected, and additional swellings appeared over the right frontal eminence, the left temple, and the occiput. There were frequent hæmorrhages from the recurrent growth in the left orbit, and foul-smelling discharge constantly exuded from its ulcerated surface. The child was now obviously suffering from septic absorption-sickness, vomiting, diarrhœea, rapid pulse, and high temperature-pallor and emaciation were increasing; and pain and general restlessness were always present, except when the child was under the influence of an opiate.

From this time onwards the progress of the disease was rapid and relentless. In quick succession, new growths appeared at the right inner canthus, the bridge of the nose, and the right lower jaw, while the glands of the neck increased to such a size that they interfered both with deglutition and respiration. There was complete loss of hearing on the right side. Both lungs became implicated, from the apices downwards; and the liver was so much enlarged that its surface and border could be distinctly palpated. The sclera of the right eye became affected and burst, and soon all that remained of the eye was a shrivelled mass half hidden in the midst of a reddish purple tumour. The growths from the jaw displaced the teeth and fungated into the mouth, out of which foetid blood-stained discharge constantly dribbled. The child's condition was pitiable in the extreme, and it always seemed impossible that it could live more than a few hours, but it was not until the beginning of January that death put an end to its sufferings. 


\section{CHAPTER XXIX}

GENERAL PRINCIPLES UNDERLYING THE TREATMENT OF INJURIES TO THE EYEBALL

General considerations-The protective mechanism of the eyes-Methods of preventing infection of the wound-Prophylaxis of ocular injuries.

THE exigencies of general practice accustom a medical man to act with promptness and decision in a case of emergency. He learns in all circumstances to trust to his experience for guidance; but even an experienced practitioner may feel anxious when called in haste to attend to some critical condition in connection with the eye. When he arrives at the house the patient may be in great agony, and both he and his relatives all much excited lest blindness or impaired vision is to be the result of the accident. Unless the medical man keep cool and collected, know well what he is about, and be possessed of a fair measure of common sense, he is prone to become infected by the atmosphere of nervousness around, and to err in being either too optimistic or too pessimistic. In the former case, knowing nothing of the conditions, he may assure the patient that all will be right in a day or two, and may thereby arouse hopes that can never be fulfilled. On the other hand, by being too pessimistic, he may, with equal ignorance of the case but having more regard for his own reputation, occasion much needless suffering by giving a grave prognosis, when, with fuller knowledge, he might have been able to do much to relieve the patient's suffering, and to quiet the alarm of all concerned.

Unguarded expressions of medical opinion are always to be deprecated, and can be avoided only when a case is approached with full professional knowledge, and with the care that comes of deliberate consideration; for it must be admitted that want of care is just as common a cause of mistakes in practice as is want of knowledge. If a general practitioner does not feel confident in his own ability to make a thorough diagnosis, he ought to have no hesitation in expressing a wish for aid by consultation; and when he is in doubt, he ought never to accept the responsibility of advising the patient to wait for a few days, on the chance that the difficulty will pass over. An opinion begotten of hope rather than 
based on experience is always baneful, for it lulls a patient into a false sense of security, and does irremediable harm by allowing time to be wasted which tuller knowledge and larger experience might have used to effect a cure.

In every large industrial centre, accidents resulting in impairment of sight and loss of eyes are of daily occurrence. It is usually to the general practitioner that the patients come first for relief, and few cases give him greater cause for concern, inasmuch as, no matter how trivial an eye injury may seem, there is always the risk of disastrous after-results. The fate of the damaged eye depends indeed on the treatment first adopted; and at all times the sense of responsibility is intensified by the knowledge that a wound of one eye may be followed by sympathetic inflammation of its fellow, and that this may be so severe as to cause complete loss of vision.

Moreover, if in any other part of the body a wound heals perfectly, the presence of a scar is not, as a rule, of much consequence; but in the eye, if the functional integrity of the organ is to be perféctly preserved, there must be no scar at all. A cicatrix, situated in the central area of the cornea, may be so thin and nebulous as to be detected only after very careful examination; but such an apparently trivial lesion is quite sufficient to cause serious impairment of eyesight; and it is because injuries to the eye are so often followed by deterioration or loss of vision, that these accidents give rise more frequently perhaps than any others to legal proceedings, both civil and criminal. As therefore the surgeon who has examined the case may be cited to give evidence in a court of law, and be examined as to the loss to the earning ability of the patient -the result of the accident-this fact must not be overlooked; and it is of the utmost importance that the examination of the patient be conducted in a methodical and systematic manner, and notes for future reference taken at the time. When, moreover, the results of an examination are to be embodied in a medical report, it is always necessary to distinguish clearly between lesions that have previously existed in the injured eye, and those which are directly the result of the accident. When an expression of opinion has to be given regarding the probable progress and ultimate issue of the case, it must be remembered that an injury, otherwise trivial, may become very serious when complicated by a blennorrhœa of the tear passages, and that the healing process will always be retarded by such general diseases as diabetes or albuminuria, either of which may per se give rise to grave disturbances of vision.

When, indeed, we consider the exposed and prominent position of the eyes, the wonder is that they are not accidentally injured much oftener than is the case. Their escape is entirely due to the admirable provision 
nature has made for their protection. Every ophthalmic surgeon could cite from his own experience numerous cases of eye-injuries where the full force of the violence had fallen upon the appendages, while the ball itself had escaped unhurt. The whole anatomical formation of the eyes and their surrounding structures seems specially designed to secure the greatest possible safety for the delicate tissues composing the organ of vision. Lodged in the cavity of the orbit, the rounded shape of the eyeballs facilitates their movements, and the fat in which they are partially embedded prevents the globes from coming roughly in contact with the bony walls of their sockets. In addition to this, there is a special provision made by the capsule of Tenon, which gives all the security of a ball-and-socket joint. By means of prolongations attached to the orbital margin, and called outer and inner check ligaments, this capsule maintains the eyeball in its normal position. When these are ruptured, the globe may be dislocated completely forwards (exophthalmos) or driven backwards into the socket (enophthalmos). The shape of the orbital cavities, and their sunken position within the skull, render the eyeballs fairly secure against external injury, but great additional protection is afforded by the formation of their margins, more especially the overhanging frontal border, the projection of the nose, and the malar eminence. The eyebrows and eyelashes also contribute to safety by directing drops of perspiration, etc., away from the globe, and by entangling small particles of dust, and preventing them from impinging upon the conjunctival surface; while the busy winking of the eyelids keeps the surface of the cornea constantly clear and bright. On the slightest warning of the approach of danger the eyelids close instinctively; and in association with this movement the ball rolls upwards, bringing the cornea wholly under cover of the upper lid. If, however, a foreign body manages to evade all these natural guards, and gain entrance to the conjunctival $\mathrm{sac}$, the amount of irritation which it excites is in itself a source of safety, because the fifth cranial nerve not only endows the cornea and conjunctiva with sensibility, but also supplies the lachrymal gland; with the result that when a particle of dust falls upon the eye, a reflex flow of tears at once occurs, sufficient in most instances, provided a little patience be exercised, to wash the irritant towards the inner canthus, where it floats on the surface of the lacus lachrymalis until it is washed out. Again, the ease and rapidity with which the balls can be turned in every direction, and the perfect co-ordination of the nervous and muscular arrangements for the maintenance of binocular vision, widen the visual field, and so diminish the risk of danger approaching unawares, by enabling one eye to act as the guardian of the other. It is well known that a workman, who has lost the sight of an eye, is, from that very 
circumstance, more liable to suffer injury of the other. A missile approaching from the blind side will be all the more likely to strike the sound eye, because it comes unseen, and consequently no time is given for defence against its assault. It is true that a person who has been so unfortunate as to be born blind of one eye, or to have lost one in infancy or early childhood, grows up practically unconscious of his defect; but it is far otherwise when such a misfortune happens in adult life. There is then always present the consciousness of a contracted visual field, of the need to turn the head in order to see all round about, and of difficulty in estimating the distance and the size of objects. These difficulties certainly to a very large extent disappear in time, and one-eyed labourers learn to see easily, and to estimate distance correctly; and the courts have therefore decided that the loss of an eye, provided its fellow be normal, in no way interferes with a man's wage-earning capacity; nevertheless the loss of binocular vision should never be under-estimated. The proper treatment of ocular injuries is, therefore, a matter of great moment for the whole community. All experience teaches that the greatest anxiety arises, in most instances, not from the actual physical damage, but from infection of the wound; and so, in each case, the diminution, or prevention, of sepsis ought to be the guiding therapeutic principle. While the researches of Pasteur, and their practical application by Lister and his followers, gave a great stimulus to all surgery, ocular surgery included, it must never be forgotten that the antiseptic treatment of wounds of the eye has to be carried on under conditions peculiar to this special organ. The conjunctival sac probably always contains germs which are, or may readily become, pathogenic; and if by chance it is at any time swept clear of microbes, it is speedily reinvaded through the tear passages by bacteria which come from the nasal cavities and their adjoining sinuses. To destroy these germs is practically impossible. It would be useless to kill the micro-organisms, if the bactericide employed caused so much structural damage that the transparency of the cornea was impaired. While, therefore, strong antiseptics cannot be used, weak are quite ineffective; and consequently it is wisest to avoid the risks of ordinary. chemical disinfectants, and to douche with a bland fluid, for example, sterile physiological salt solution, or a saturated solution of boracic acid, or of biborate of soda.

Happily, however, as may be seen every day in connection with cataract and other operations, absolute freedom from germs is not a necessary condition for primary union of a wound of the cornea. For years past a systematic bacteriological investigation has been made of the fluids in the conjunctival sacs of all the patients with cataract on whom I have operated; and it has been demonstrated over and over again 
that the wound heals perfectly in the presence of those germs which are found most frequently in the normal conjunctival sac, provided the patient be in sound health, and the microbes are not present in overwhelming numbers. The risk of suppuration is always greatly increased if there has been previous inflammation of the edges of the eyelids, of the conjunctiva, or of the lachrymal sac and tear passages, or if the patient's vitality is low. Collateral conditions are indeed always of considerable importance; for while the intense germicidal action of a certain chemical can readily be demonstrated by experiment in the laboratory, the results there obtained are but seldom borne out in actual ophthalmic practice. The great amount of attention which has been given to the study of micro-organisms has tended to divert attention unduly from the soil in which the bacteria grow. All clinical experience proves, indeed, that suppuration occurs much more rapidly in some patients than in others, and that general malnutrition, by lowering the resisting power of the tissues, favours the onset of sepsis. That being so, one must not only pay every attention to strict antiseptic precautions, but must also devote the utmost care to what is of equal importance-the giving the patient himself increased power of resistance. Good food, fresh air, rest, and every means of strengthening digestion are all essential.

While on the other hand, therefore, the most trivial ocular injury may, through neglect or unskilful treatment, have the most disastrous results; the natural power of recuperation of the eye is, on the other hand, so great that damage which in former days would have been treated by enucleation, can now be repaired and sight preserved, by following as far as possible from the very outset the practice of strict antisepsis.

Moreover, inasmuch as occupation plays a very important part in the occurrence of injuries to the eye, it is most expedient for every medical practitioner to be competent to advise regarding the best means for the prevention of such accidents. Prophylaxis of ocular injuries is of firstrate importance, because so many of them are preventable. Carelessness. in regard to well-recognised precautions is to blame for much loss of sight. The great majority of trivial accidents to the eye could be prevented, if the workmen would wear an eye-protector, of which many varieties are on the market. They are, however, but little used; as men have a great reluctance to wear them, and complain that the glasses soon become blurred by dust and perspiration, and that they are hot and uncomfortable. The disinclination is largely due to prejudice, but is none the less difficult to overcome. The workers prefer to take the risk of injury rather than wear anything that is unusual or uncomfortable, even although they may be prepared to admit after an accident that it would not have occurred had proper precautions been taken to prevent it. 'If, then, the 


\section{TREATMENT OF INJURIES TO EYEBALL}

use of a protector be made obligatory, care must be taken that it is efficient for its purpose, that it is strong and cheap, and that it does not cut off too much light, or press uncomfortably on the face or heat the eyes. All these conditions are fulfilled by Crooke's glass No. I or No. 2, mounted in a frame that will not rust, and furnished with sides of gauze so fine in the mesh that the most minute particle of grit or metal will not pass through. 


\section{CHAPTER XXX}

\section{THE TREATMENT OF SUPERFICIAL INJURIES TO THE EYES}

Importance from an industrial standpoint-Foreign body beneath the upper lid -Foreign body in the cornea-Dangers of infection of cornea in cases in which tear passages are diseased-Illustrative cases-Recurrent traumatic Keratalgia.

THE treatment of superficial injuries to the eyes is a subject of great importance, on account of the large number of trivial accidents occurring daily to the eyes of industrial workers. In most instances the actual physical damage is slight, and the patient will be able to resume his duties in a few hours, or at most in one or two days, if only the injury to the eye be promptly and skilfully treated. If, on the other hand, the injury be neglected, or if it be treated by any one who is unskilful or careless, sepsis will almost certainly occur; and all experience teaches that infection of the wound is a far greater danger than the actual physical damage to the ocular structures. The occurrence of sepsis at once transforms a very trivial injury to the cornea into a suppurative keratitis, which may run a prolonged course, lead to more or less impairment of sight, and in serious cases even destroy the eye.

The case of a man, under my care at the time of writing, is an apt illustration of what I desire to emphasise. $\mathrm{He}$ is a munition worker, who received a "spark" in his left eye, but thought little of the injury, and asked a fellow-workman to remove the foreign body from the cornea. His friend used the point of a pin for the purpose, and after some difficulty succeeded in dislodging the offending particle. By this time the eye had become so painful that the man felt unable to continue at work. He went home and applied some domestic remedies, but getting no relief, he, at the end of three days, consulted a doctor, who prescribed "drops" and "lotion." In spite of that treatment the eye became slowly but steadily worse, and when I saw the patient for the first time, five weeks after the accident, he was suffering from suppurative keratitis. The attack was not severe, but it will be at least a month before the man is able to resume work. Here, then, is a case where a worker's time is lost for two months, on account of an eye injury which, had it been properly treated at the time of its occurrence, 
would have been healed in two or three days. Doubtless this is an extreme case; but it is not by any means an isolated example of what occurs through neglect of a trival injury to the eye; and the importance of prompt and efficient treatment in relation to time-keeping and earning ability cannot be over-estimated. In every factory where such accidents are of frequent occurrence, there ought to be a rule that injuries should be reported at once, and provision should be made whereby " first-aid" may be given with the least possible delay. Such an arrangement would not be difficult to carry out; and timely first-aid would effect a cure in the mild cases, and prevent as far as possible extension or increase in severity of the injury in those that were more serious.

When any foreign body manages to get inside the lids, it often becomes lodged beneath the upper eyelid, and, in order to effect its removal, it is necessary to evert the lid. The doctor should invariably make a careful examination of the conjunctival surface of the upper lid, and thoroughly explore the superior fornix. Eversion of the upper lid is a simple matter if it be gone about in the right way. Seat the patient in a good light, ask him to look downwards, grasp the eyelashes with the thumb and index finger of the left hand, pull the eyelid forwards and downwards, lay a probe or the edge of a coin along the upper margin of the tarsal cartilage, and turn the eyelid upwards. To expose the upper retrotarsal fold, all that is necessary after the upper lid is everted is to push the lower lid upwards against the eye and gently press the globe backwards into its socket. This simple manœuvre causes the folds of the upper fornix to stand out, and any foreign body lodged there can be at once detected.

When a patient complains that he has received an injury to his eye, his doctor should invariably make a careful examination of the conjunctival surface of the upper lid; to overlook a foreign body in this position is certain not only to cause much unnecessary pain to the injured person, but also to bring a great deal of discredit on the medical man concerned. Always suspect the presence of a foreign body if the patient complains of pain in one eye of sudden onset, aggravated by every movement of the globe, but relieved when the eye and lids are at rest. Among farm labourers, particles of straw or chaff frequently become so deeply lodged in the upper conjunctival cul-de-sac, that they may be hidden from view, even when the upper eyelid is everted. It is necessary, therefore, if it be suspected that a foreign body is present, not only to evert the eyelid, but also to make a careful exploration of the cul-de-sac with a small probe. It is all the more needful to bear this in mind, because though the smallest particle of dust within the eye usually gives rise to intolerable uneasiness, yet larger bodies may be 
tolerated for weeks in the loose folds of this cul-de-sac, without their presence being indicated by more than inflammatory symptoms, accompanied by swelling of the conjunctiva, and puro-mucous discharge. Such an inflammation, however, is not at all influenced by the usual remedies, and subsides only after the removal of the exciting cause.

Particles of dust, etc., etc., are usually found lying loosely upon the conjunctiva, and are readily removed with the point of an unused toothpick, a new camel-hair brush, or the edge of a small spud. A loop of platinum wire fixed to a handle is a convenient instrument for that purpose, and can be thoroughly sterilised before use by heating it in the flame of a spirit-lamp. A drop of 2 per cent. solution of hydrochloride of cocain renders the operation quite painless; and after the foreign body has been removed, the conjunctival sac should be thoroughly washed with normal saline or boracic acid solution, and when the eye has been dried by a pledget of cotton-wool, a few drops of 5 per cent. chloretone oil should be instilled. A case of that kind very rarely gives any further trouble.

Foreign bodies of larger size, the husk of bird's seed, the wing of a small insect, etc., usually adhere to the cornea at the limbus; and as in a few days newly formed blood-vessels appear, the condition may simulate a keratitis; but careful examination of the eye in a good light will discover the foreign body, whose removal will be speedily followed by the disappearance of all symptoms.

When the foreign body is embedded in the substance of the cornea, its removal requires more skill and more care. The workmen themselves speak of such a foreign body as a "fire." It is, in the majority of cases, a minute particle of metal which flies from a tool or a grinding-wheel, at a white heat, and burns its way into the cornea. As the heat has rendered it aseptic, it would in all probability rarely cause any serious subsequent damage to the eye, but it gives rise to so much pain that the workman at once appeals for relief. It is most important that the person to whom he applies for aid should be skilful and careful, because rough and persistent efforts to remove the foreign body, with an instrument which is not surgically clean, will cause much more damage to the eye than the "fire" itself. The great danger lies in the abrasion of the cornea becoming infected; consequently in every case treatment must be carried out in such a way that risk of sepsis is diminished or altogether prevented.

The sooner a "fire" is skilfully removed the better; and the instruments usually employed for the purpose are a cataract needle or a spud; but I much prefer to use the point of a small Beer's knife. The instillation of a few drops of a 2 per cent. solution of cocain renders the 
operation perfectly free from pain; and in cases where the foreign body is very minute, material aid is afforded by the introduction of a drop of a solution of fluorescin. This, by colouring the abraded epithelium green, enables the lesion to be more readily seen, and the foreign body more easily removed. It is also very important to have the light accurately focussed on the cornea by a lens.

If the patient does not present himself to the surgeon until two or three days after the injury has been received, and if he has not allowed any one to meddle with his eye in the interval, it will be found that the embedded substance is surrounded by a whitish ring of softened corneal epithelium, which a very slight touch with a spud will cause to separate from the parts beneath. In fact, if the sufferer had waited a day or two longer, the probability is that nature alone would have brought about the same result, by casting off the softened corneal tissue, foreign body and all, leaving a breach of surface of greater or less depth.

When dealing with cases of a foreign body embedded in the cornea or in the conjunctiva, seat the patient in a good light, with his head firmly supported by the back of a chair, or preferably by the hands of an assistant. Separate the lids with the thumb and index-finger of the left hand, and press backwards so as to fix the eyeball; then pass the point of the small Beer's knife behind the " fire," and lift it from the cornea. This little operation requires skill and experience, but it presents no real difficulty unless the foreign body has penetrated deeply. There is then danger of pushing it into the aqueous chamber. In such cases it may be necessary to pass a broad needle through the cornea behind the particle of metal, thereby supporting it and preventing such an untoward accident from occurring. When the foreign substance is embedded in the conjunctiva, it should be grasped with forceps and cut out with a pair of curved scissors.

After the foreign body has been removed, the patient experiences an immediate sense of relief; and as a rule nothing more requires to be done, except to bathe the eye with normal saline solution, to apply an ointment of iodoform, cocain, and vaseline, and to carefully adjust a compress and bandage, which ought to be worn until all signs of irritation have passed away. Lotions containing lead must never be used, as, whenever there is a breach of the surface of the cornea, a white precipitate is formed giving rise to a permanent opacity.

If the patient suffers from disease of the tear passages, the risk of infection of the cornea is very great, and he ought to be sent to an expert as soon as possible after he has received first-aid. Suppuration of the tear passage is a frequent source of infection of an abraded cornea, and extirpation of an infected lachrymal sac constantly discharging pus 
is advisable in those whose occupation exposes them frequently to risk of injury to the eye. The workmen themselves are very prone to neglect slight ocular injuries, and rarely seek medical advice until after infection has occurred. By that time the condition is fraught with danger, because the ulcer may spread with great rapidity, and all chance of saving sight be lost within a few days. In such circumstances a prudent general practitioner will restrict himself to giving first-aid, and will then send the patient to a specialist with as little delay as possible. When, by accident, a particle of an aniline pencil enters the conjunctival sac, the white of the eye rapidly becomes stained purple. The discoloration causes great alarm, but gradually passes off, and recovery may be hastened by free douching with a weak solution of alcohol and glycerine (Werner).

It often happens that, owing to the extreme sensitiveness of the structures implicated, an accident to the eye seems much more serious than it really is. Indeed, the pain and symptoms of distress are often in inverse proportion to the gravity of the injury. Here, again, it is very difficult to make a satisfactory examination; for the pain and intolerance of light set up blepharospasm so intense that it is almost impossible to separate the lids. The accident often occurs in the simplest manner possible. A lady while dressing her hair strikes the cornea with the brush or the curling tongs; a gentleman is lighting a cigar when a spark flies from the head of the match and enters the conjunctival sac; or a nurse is playing with an infant, and receives a scratch on the eye from the baby's finger-nail. These are only a few examples from many that might be cited. In nearly every case the patient believes that serious damage has been done. He speaks of the pain as agony; and is quite unable to open the eyelids. As a rule, however, a few applications of a 2 per cent. solution of cocain overcome the surface irritability, and permit the surgeon to separate the lids and to examine the cornea. A small abrasion is usually detected, and if this be treated by an antiseptic lotion, an ointment containing cocain, and a compress and bandage, healing takes place rapidly. If, however, the breach of surface becomes infected by micro-organisms, suppuration ensues, and what was, to begin with, a very insignificant injury becomes serious, and a grave menace to sight. It must never be forgotten that no injury to the eye is so trivial that it can with safety be neglected, or so insignificant as not to require the most skilful treatment.

In cases of corneal abrasion, therefore, the greatest danger is in the risk of septic infection. Micro-organisms, some of which may be pyogenic, are always lurking in the conjunctival sac, but as long as the epithelium remains intact they do no harm. The corneal epithelium 
forms an insuperable barrier to the entrance of microbes; but, let it be abraded ever so little, and at once they find their way through the breach and immediately begin to produce toxins, whose irritant action extends to the deeper as well as along the superficial ocular tissues. This infection may occur at the time of the accident, or may take place later from inoculation of the wound by secretion from the conjunctiva, from diseased tear passages, or from the nasal mucous membrane. Some cases run a peculiarly virulent course; and not only is the destruction of the cornea rapid and complete, but the whole of the tissues of the globe may become implicated, and the result be acute panophthalmitis.

I remember once seeing a carter, who stated that he had, the day before, got some dust from the road into his eye. Intense inflammation followed, and within twenty-four hours the whole cornea had suppurated. Bacteriological examination revealed the presence of a streptococcus. Treatment was of no avail : panophthalmitis speedily followed, and in less than a week the patient was seized with erysipelas, beginning in the eyelids, and extending all over the head and neck.

Another case I remember well was that of a woman who was under treatment for blennorrhœa of the tear sac. A doctor when washing out the lachrymal passages accidentally scratched the cornea, and next morning there was a rapidly spreading ulcer, with evidence of infection of the deeper tissues. In a couple of days the eye was hopelessly destroyed. Fortunately, however, such virulent and intractable cases are of rare occurrence; and in most instances the resources of modern ophthalmology can prevent the onset of sepsis, or successfully combat it, if it be in progress.

Although in the great majority of cases an abrasion of the cornea, when properly treated, heals quickly, leaves no trace, and causes no further trouble; yet it occasionally happens that some days or weeks after the eye has apparently recovered, the patient suffers acute stabbing pain in the eye that had been injured. The onset of the pain is sudden, comes without warning, and usually occurs on first opening the eyes in the morning. When the cornea is examined an abrasion, or one or more small blisters are seen. The use of a borax lotion and an ointment containing holocain is usually followed by speedy relief; but the attacks are apt to occur over and over again for months. The cause of the recurrence of the erosion is a loose attachment of the epithelium to Bowman's membrane, which renders it very liable to be torn when the lids are opened after sleep. The disease as a rule passes off sooner or later; but it may persist, and be accompanied by increased intraocular tension which in the long run may destroy sight.

Patients suffering from recurrent traumatic keratalgia ought, therefore, 
to be carefully supervised, and the intraocular tension tested at every examination. Atropin must be used with great caution; and both cocain and adrenalin should be avoided. The patient's chief complaint is of pain, which is as a rule relieved promptly by a draught containing a combination of salicylate of soda and antipyrin, and by applying a non-irritant ointment to the conjunctival sac every night at bedtime.

Wounds of the conjunctiva usually heal rapidly, and, unless extensive, do not require to be sutủred. It 'is sufficient to keep the part thoroughly clean with a weak antiseptic solution, and to protect it by means of a compress and bandage. Should a fungoid granulation develop at the site of the injury, it must be snipped off with a pair of scissors. It will not disappear spontaneously; it acts as an irritant, and is usually the source of persistent muco-purulent discharge.

When a wound implicates the margin of the eyelid, the surgeon must be very careful to bring the edges accurately together with a suture; for if he neglects' to do so, a gap will remain in the lid margin, and it is likely that one or more cilia may become inverted, and, scratching the cornea with every movement of the lids, prove a constant source of discomfort. If the injury be situated at the inner canthus, there is a great risk that the tear passages will be wounded; and unless the condition be at once recognised, and a road for the escape of the tears opened up, complete obstruction will take place in the process of cicatrisation, and the patient will suffer from persistent watery eye. These precautions appear obvious, but they are often neglected, and what might have been a very trivial injury becomes, through inefficient treatment at the outset, a cause of great trouble and annoyance. 


\title{
CHAPTER XXXI
}

\author{
BURNS OF THE EYE
}

Necessity of a guarded prognosis-Clinical course-Symblepharon-Anchylo- blepharon-Treatment-Operations for symblepharon-Electric ophthalmia$\mathrm{X}$-ray and radium burns-Burns from warfare gases.

OF all injuries to the conjunctiva, none is more disastrous in its results than a burn, especially one due to the action of any chemical irritant, such as quicklime, sulphuric acid, etc. In all cases of this kind the prognosis must be very guarded, as the action of the chemical extends far beyond its point of application; and what seems at first sight only to have caused a greyish haze over a part of the surface of the cornea, will probably, by the end of a fortnight, have brought about the total destruction of that structure, and the consequent extinction of vision.

After a burn from ammonia, the eye may not appear seriously damaged for several days; and, because immediately after the accident the cornea appears transparent, the general practitioner may be tempted to give a favourable prognosis, which, however, will be completely falsified later when the cornea becomes white like porcelain. On the other hand, after a burn from hot metal ${ }_{t}$ the immediate results are most alarming, because from the very outset the cornea is covered by a whitish-grey pellicle. In such circumstances it would be natural for the doctor to give a grave prognosis; but in a considerable proportion of the cases the cornea will regain its transparency in from twenty-four to forty-eight hours, and no cicatricial opacity remain as a result of the accident. In the former case, the ammonia becomes diffused throughout the whole substance of the cornea, which it kills outright; whereas in the latter it is only the epithelial layer that has been scorched. It is not, therefore, the burns that look worst to begin with that necessarily have the most unfavourable termination. The surface extent depends upon the size and the nature of the burn; while the occurrence and the degree of a permanent opacity depends upon the depth to which the corneal tissue has been destroyed. Hence the danger of a burn in which particles remain in the eye to continue the work of destruction, lime or mortar, for example; or in which an acid or an alkali is diffused through the substance of the cornea, to continue its escharotic action at a distance from the original 
site of the injury. A grave prognosis must always be given when the cornea is insensitive, or when there are signs that the sclera will be perforated in the neighbourhood of the limbus corneæ; and the dangers always to be feared are opacity of the cornea, and cicatricial contraction of the conjunctiva. In the majority of cases it is the lower portion of the conjunctival sac that is most severely damaged, and particles of molten metal or of pitch may adhere so firmly to the conjunctiva that their removal is sometimes very difficult.

In all burns or scalds of the eyeball the general symptoms are the same, and their severity depends upon the nature of the irritant, the length of time it has remained in contact with the eye, and the extent of the structural injury it has produced. After a burn, from whatever cause, the pain in the eye is very severe, and the inflammatory reaction is acute and violent. The eyelids are usually injured, but whether they are or not, rapidly assume a dusky red colour, and become so swollen that the eye can be seen only with difficulty. There is chemosis of the ocular and palpebral portions of the conjunctiva, and the cornea becomes hazy over greater or less part of its surface. In from twenty-four to fortyeight hours, the burnt surfaces are clearly mapped out, and separated from the other tissues by a well-defined zone in which the inflammatory reaction, the result of the injury, is most acute. Pus now begins to be discharged, and the tissues killed by the burn separate as sloughs; after which, if the injury to the eye has not been too great, recovery more or less complete commences. Should the case, however, be severe, the inflammatory process goes on, the conjunctiva becomes thickened and fleshy, pus forms in the anterior chamber, the cornea suppurates and bursts, and the eye ultimately shrivels. In cases where the cornea does not give way, a dense leucoma remains; or when from the thinning of the parts, due to the separation of the sloughs, the cornea is unable to resist the intraocular pressure, it bulges forward, and a staphylomatous projection is produced. In this connection it may be noted that very severe causes do not always produce proportionately severe results. When molten metal, for instance, gets into the eye, it might be expected to, and very often does, cause serious structural damage; yet; on the other hand, there are cases where the injury is remarkably slight. The explanation is that, on the first entrance of the metal, the great heat causes the moisture in the eye to pass into what physicists call its " spheroidal state," and the spheroids of water, acting as a non-conductor, protect the eye from injury.

In all severe burns, both ocular and palpebral mucous membranes are injured; and the raw surfaces left adhere in the process of healing, so that the eyelid becomes firmly fixed to the eyeball. This condition, 
which is known as symblepharon, will, no matter how much care has been taken to prevent it, certainly occur in every case where the retrotarsal fold of conjunctiva has been destroyed ; and if it is extensive, it not only interferes with sight, but causes intolerable discomfort from double vision, as well as from the sense of dragging which it produces with every movement of the eye. It is unfortunately but little amenable to treatment, and may be complicated by union of the eyelids-anchyloblepharon.

After a burn the healing process is slow. The vitality of the tissues has been lowered by the injury; and as the cicatrix goes on contracting, the eyelids may either become inverted so that the eyelashes rub upon the eyeball, or they may be everted, with the result that the face is frightfully disfigured, and the original damage to the cornea augmented by the constant exposure of the globe.

Treatment.-Burns of the eye are treated in the same way as an acute suppurative inflammation; and where the injuries have been caused by chemical irritants, special precautions have to be taken from the very first. In the case of quicklime, the severity of the burn is due to the fact that the lime usually remains for a considerable period in contact with the tissues, and its powerful escharotic action has thus time to take full effect. The slightest particle of it entering the eye gives rise to the most intense irritation, leading to spasmodic closure of the eyelids and a copious secretion of tears-a combination of circumstances which is most favourable in ordinary cases for the extrusion of a foreign body, but which in this case is most unfortunate, because by the firm closure of the eyelids the lime is pressed against the eyeball, while the tears slake any portion which is yet unslaked, and the heat consequently evolved increases the rapidity of the work of destruction. When, therefore, unslaked lime has caused the injury, any particles remaining should be at once removed, and after the eyelids have been everted, the conjunctiva should be thoroughly douched by a stream of cold water, or by a weak acid solutionvinegar-if this be available. On the theory that sugar will dissolve the lime and so cause its thorough removal, it has been recommended that the eye should be douched with a solution of sugar; but the rapidity with which the lime destroys the tissues is so great that the mischief is probably done before the surgeon sees the case; and as the solvent effect of saccharine solutions on slaked lime is very gradual, it is better to waste no time, but at once to make sure that the conjunctival sac is thoroughly freed from any of the particles which may be adhering to it. After a burn from an acid-for example, vitriol-throwing - an alkaline douche is of course preferable-3 per cent. solution of bicarbonate of soda; but in all cases, after the eye has been washed, castor oil and 2 per cent. cocain, or preferably a 5 per cent. solution of chloretone in oil, should be applied 
to the conjunctiva, an iced compress laid over the closed eyelids, and the patient put to bed. In order to combat the inflammatory reaction which is sure to follow, the ice-cold compress should be continued, and saline purgatives administered; while the pain must be subdued by the application of leeches, and by the administration of full doses of opium, or by hypodermic injection of morphia in the temple. The use of these anodynes has the additional advantage of inducing sleep, and soothing the nervous system generally. After forty-eight hours, warm applications are usually found to be more grateful to the patient than the iced compress, and the sedative effect of these fomentations may be increased by poppy heads and chamomile flowers; while much additional relief may be obtained by smearing the eyelids, forehead, and temples, with a mixture of extract of belladonna and glycerine. When the acute symptoms have subsided and the sloughs have begun to separate, antiseptic lotions should be used; and much benefit is often obtained from the daily instillation of a 2 per cent. solution of nitrate of silver, or, preferably, by the frequent application of a 2 per cent. solution of picric acid. A lotion containing 5 per cent. to 20 per cent. ammonium tartrate is recommended after burns by lime, but it is very doubtful if it has any specific action. Any iritic complications must be treated by the judicious use of atropin ; but in this connection it may be mentioned that as burns of the corneo-scleral margin are apt to give rise to glaucomatous symptoms, the use of the atropin must be at once stopped if the patient complains of increased pain.with sudden dimness of vision; and an endeavour must then be made to reduce the increased tension of the eyeball by the energetic use of eserin or pilocarpin, or by paracentesis of the aqueous chamber.

During the cicatricial stage the greatest attention must be devoted to overcoming the tendency to symblepharon. The adhesions must be diligently broken down with a probe, and the eyelids drawn away from the eyeball, so as to permit a layer of vaseline to be instilled between the two raw surfaces. If the eyeball and eyelid be dried carefully, they may be kept apart by applying a layer of melted paraffin-ambrine-to the retrotarsal fold. The lids are then closed by strips of plaster to keep this paraffin mould in position. When this device is successful, healing goes on under cover of the layer of paraffin, and the result is occasionally quite satisfactory. Another method well worthy of trial is to use dentist's wax for the mould, and to cover it with a Tiersch's graft. The mould and the graft are placed accurately in the retrotarsal fold, and kept in position by a bandage. In spite of all one's efforts, however, symblepharon will, in bad cases, occur; and although much ingenuity has been displayed in devising mechanical apparatus to keep the lid separated from the eyeball, these, as a rule, fail to accomplish their purpose, because as 
cicatrisation progresses they get displaced, and the adhesions form again and again with a pertinacity that is most vexatious. Nevertheless it is on the whole easier to prevent the occurrence of a symblepharon than to cure one after it has formed. Of the many operations that have been proposed for accomplishing the same object, the one first described by Teale is most likely to give satisfactory results. The lid must be dissected from the eyeball as far as the fornix, and then a flap of conjunctiva taken from either side of the globe. One of these is utilised to cover the raw surface of the lid, and the other is taken to fill up the gap in the bulbar surface. The flaps must not be put too much on the stretch, and are kept in position by fine silk sutures. When the symblepharon is too extensive to be satisfactorily dealt with by Teale's method, flaps of mucous membrane may be transplanted from the lip, from a frog's mouth (Leslie Paton), or from a rabbit's conjunctiva; and if proper precautions are taken; considerable success may follow these transplantation operations. If an area of cornea has retained its transparency, vision may be improved by an optical iridectomy. If, however, no improvement is to be looked for from that operation, it may be necessary to remove the disfiguring eyeball, and replace it by a glass shell, or it may be possible to cover it by a very thin prothesis. In many cases, however, neither of these means, so desirable for cosmetic reasons, will be feasible until the conjunctival sac has been restored by a plastic operation.

Electric Ophthalmia. - This is occasionally observed in those engaged in electric-welding operations, and usually appears about eight hours after the eyes have been exposed to the glare, whose evil effects are due in great part to the predominance of ultra-violet rays. The eyes at first feel hot and pricking, and every now and then acute darting pains shoot through the globes. The pains become more and more intense, and are accompanied by erythematous swelling of the skin of the lids and face, similar to what is seen in severe sun burning, while tears gush continuously from the eyes in large quantity. The symptoms gradually subside after one or two days, and vision is unaffected, although for a considerable time the patient is unable to work freely by bright electric light. Much relief is obtained by frequent instillation of 2 per cent. solution of cocain, and the application of iced compresses to the lids; but it is even more important to remember that when engaged in working with strong arc lights, the eyes ought always to be protected by smoked or coloured glasses-Crookes' No. 2.

The therapeutic use of radium or of the $X$-rays occasionally gives rise to burning; and the eyelids and the delicate structures of the globe may suffer severely. The burns may be deep, are always slow to heal, and may 
give rise to disastrous results. The possibility of their occurrence must, therefore, never be forgotten, and when those means of treatment are employed, every precaution must be taken to avoid the risk of such an unfortunate accident.

Burns of the Eye from Warfare Gases.-Of the various poison gases employed by the enemy during the recent war ethyl sulphide $\left(\mathrm{C}_{2} \mathrm{H}_{4} \mathrm{Cl}_{2}\right) \mathrm{S}$, or mustard gas, is the one that does most damage to the eyes. It is a vesicant, and may exert its irritant action either as a vapour in low concentration in the air, or by direct contact from splashes of the liquid. One of its special peculiarities is that there is always a delay of from two to six hours before any irritant effects are experienced. There is therefore no warning, as is the case with other poison gases. In the early stages the patient complains of photophobia, increased lachrymation, a sense of the presence of foreign bodies in the eye, heaviness of the upper lids, headache, and asthenopia. As the action continues there is great swelling of the lids and conjunctiva, and tears gush from the eyes. Pain, photophobia, and blepharospasm increase, so that it becomes very difficult to examine the globe. After the lids are separated, there is seen great chemosis of the bulbar conjunctiva, the iris is congested, the pupil contracted, and in the worst cases the cornea is steamy and clouded in the area opposite the interpalpebral space. Its epithelium appears rough, and minute opacities appear in its substance. The patient suffers great pain, and cannot face the light. He lies with his face covered by the bedclothes in his efforts to get relief from the photophobia. The skin of the lids and face becomes swollen and excoriated from the constant irritation of the tears. Secondary infection takes place, and the secretion becomes purulent, collects in the corners of the eyes, and forms a crust along the edge of the lids.

In spite of the violence of the symptoms, there is usually a good recovery in the end; for after reaching its height, the inflammation gradually subsides in about three to four weeks. Relapse, however, is very prone to occur again and again, and many patients are thereby disabled for months. Some disturbance of vision due to astigmatism may persist after recovery, and occasionally an opacity of the cornea remains. In very severe cases the eyelids are blistered, and the interpalpebral conjunctiva burnt, by the caustic action of the poison liquid. Serious burning of other parts of the body may also occur.

Treatment.-The patient ought at the beginning of the attack to be put to bed, and the eyes protected by dark glasses. The worst cases may require a protective bandage for a few days; but as a rule the less the eyes are covered, the quicker recovery will take place. The eyes must be bathed frequently with a hot solution of borax, and an ointment 
containing a weak alkali applied freely to the conjunctival sac. The pain and photophobia are relieved by instillation of cocain 2 per cent. and adrenalin solution ( $\mathrm{I}$ in IOOo) 5 per cent.; when the pupil is much contracted, a drop of atropin solution I per cent. ought to be instilled and repeated as often as is necessary to secure a medium dilatation. If the secretion is purulent, the everted eyelids should be painted with a 2 per cent. solution of nitrate of silver; and after the discharge becomes less, a solution containing sulphate of zinc and boracic acid ought to be instilled three or four times a day. Whenever the acute symptoms subside, the patient should be encouraged to get out of bed, and walk about the ward. He must be warned against rubbing his eyes, because the irritation thereby induced always determines troublesome relapse. In the event of relapse occurring repeatedly, a sinapism to the nape, and full doses of bromide of ammonium, are often helpful; but the chief means to prevent relapse and to promote recovery is to encourage the patient to go about and occupy himself as much as possible in the open air, and to discourage the use of local anæsthetics to the eyes, and the wearing of dark glasses, unless the symptoms are acute.

McDonagh recommends' a 5 per cent. solution of colloidal manganese given by intravenous injection, but I have had no personal experience of that method of treatment. 


\section{CHAPTER XXXII}

\section{CONTUSION-INJURIES OF THE EYE}

The effects of a blow on the eye-Blows on the surrounding tissues-A black eyeEmphysema of lids-Injuries to optic nerve-Ophthalmoplegia-Blows on the eyeball itself-Commotio retinæ-Hæmorrhage, sub-conjunctival and intraocular-Lesions of iris and ciliary body-Lesions of choroid-Lesions of retina-Detachment of retina-Dislocation of lens-Traumatic cataractRupture of eyeball-Treatment.

THE effect of a blow on the eye is immediate, but its results are frequently remote, and vary greatly both in severity and in character. When first struck the patient usually suffers severe sickening pain and feels faint and stunned, even in cases where the actual damage done to the eyeball is slight. In proportion to the severity of the injury, the eyeball may be simply concussed; its coats may be flattened for the moment at the place of impact; the deeper structures may be dislocated or ruptured; or the sclera may be burst, and through the rent the contents of the globe may more or less completely escape. Such injuries may be caused by any blunt object whatever; and every ophthalmic surgeon could cite instances where the eye had been damaged by a blow from a fist, a stone, a cricket or a tennis ball, by the impact of a cork suddenly expelled from a bottle of aërated water, by the rebound of a stick while wood is being chopped, or by an injury from a cow's horn, etc., etc. Of any sharp, sudden shock the structures of the eye are peculiarly intolerant; and many persons have lost their sight from such an apparently trivial accident as the flick of a whip-lash, a sail, a rope, or a branch of a tree. Observation of warfare injuries has also demonstrated the fact that injuries of the face or cranium caused by bullet, shrapnel, or pieces of shell, are capable of producing at a distance serious and irreparable damage to the eye; and in many instances damage has been done, although the projectile has not actually struck the patient. The displacement of the air-" windage "-produced by the explosion of a shell is of itself sufficient to cause serious lesions; and in many such cases, although the eye at first may seem to be uninjured, yet sight is impaired, and examination with the ophthalmoscope reveals opacity of the lens, or hæmorrhage at the posterior pole of the globe.

On the other hand, a heavy blow, falling as it does for the most part 260 
on the bony walls of the orbit, may be unaccompanied by any damage to the eye itself. For instance, in a street fight where the combatants face one another, eyes are not often seriously damaged, although the amount of swelling and discoloration of the lids may be extreme; but, on the other hand, the would-be peacemaker, going in from the side, is very apt to have his eyeball burst as a result of a blow at the outer canthus, where there is practically no protection.

Contusion injuries therefore divide themselves, naturally, into two classes-

I. Where the full force of the violence falls on the lids and surrounding parts.

2. Where the eye itself is more or less seriously damaged.

FIRST.-Where the full force of the violence falls on the lids and surrounding parts.

The simplest and most familiar example of an accident of this kind is a black eye-ecchymosis of the eyelids. The circumorbital tissues are so lax that a blow at once induces rapid swelling of the lids, and the parts surrounding, all of which speedily become discoloured from the effusion of blood. This would of itself pass away in about a fortnight; but the disagreeable ideas so often associated with a black eye, and the unsightly appearance it presents, make patients extremely desirous to get rid of all traces of this form of injury as quickly as possible. The swelling and discoloration may be kept in check by the application of cold and a pressure bandage. Nothing is more efficacious than a pocket-handkerchief dipped in cold water and tied tightly over the eye. Many socalled specifics have been recommended-arnica, hazelene, a raw beef steak, a slice of raw ham, etc.-but it is doubtful if any of them is of the slightest use in hastening the natural process of absorption. If it be very important to get rid of the disfigurement at once, the expedient of painting the parts must be resorted to. The application of leeches, or puncturing the skin is not to be advised, and the practice of sucking out the effused blood is objectionable, and not without danger. I have known a chancre of the eyelid result. In severe contusions in which the eyelids are more or less lacerated, suture should be inserted to bring the parts approximately into proper position as soon as possible after the accident.

When the injury is more severe, the bones at the inner side of the orbit may be fractured, and communication with the frontal and ethmoidal sinuses opened up, thereby allowing air to infiltrate the surrounding cellular tissue-emphysema of the eyelids. This is usually accompanied by epistaxis, and the nature of the soft swelling is readily diagnosed by its crackling to the touch, and increasing rapidly in size when the patient 
blows his nose. Here, again, the best method of treatment is the application of a pressure bandage. The patient must also, of course, be warned not to blow his nose, or to sneeze, lest air be forced under the skin and interfere with the process of healing.

All ophthalmic surgeons are aware how frequently an injury to the head and a fracture of the skull are followed by loss of vision on one side. Indeed, since the time of Hippocrates, it has been known that blows on the frontal or malar region often result in blindness of one eye. In many instances the traumatism appears to have been slight, and the trivial nature of the injury may have given rise to the old idea that the loss of sight is the result of reflex action through the fifth cranial nerve. The usual explanation of the occurrence of monocular blindness following diffuse violence to the skull, is that there has been a fracture through the optic foramen. The optic nerve is thereby torn or compressed, and vision is immediately extinguished. Such an explanation, however, applies only to a limited number of the cases; because it has been demonstrated both in civil and in military practice, more especially by Pringle and Lagrange, that fractures of the optic foramen or of the sphenoidal fissure do not occur as a result of diffuse violence applied to the cranium at a distance from the orbit. Those authorities both agree that when fracture occurs it is due to direct violence, and that in the great majority of cases of head injury followed by blindness of one eye, the optic nerve has been compressed by hæmorrhage into its sheath. The intravaginal hæmorrhage may be associated with fracture of the optic foramen and of the roof of the orbit, but in a large number of cases it occurs independently of either. Unfortunately, hæmatoma of the nerve sheath cannot be diagnosed by means of the ophthalmoscope at the time of its occurrence; but as the hæmorrhage becomes absorbed, the blood pigment migrates and forms a brownish ring on the temporal side of the atrophied optic disc. Lagrange attaches considerable importance to that ophthalmoscopic appearance, and regards this pigmented ring as " a sign of a hæmatoma of the optic nerve of old standing."

Many examples might be given of the mode of occurrence of this form of monocular blindness. The three which follow are quite typical. In the first the patient fell when stepping off an electric car while it was in motion; in the second the injury was the result of a bicycle accident; and in the third the lad fell from a scaffold. In every one of these the loss of sight in the affected eye was complete. Of course, after an injury of this kind, the general symptoms of concussion first occupy the attention of the medical attendant, and it is usually several days at least before any definite opinion can be formed regarding the state of the eyes. It is only after the patient comes to himself that any complaint is made of 
loss of sight ; and in the three cases referred to no.proper examination of the eyes was made for some days after the accident. Even then in none of them was there anything abnormal in the external appearance; and although the blindness was absolute, yet the ophthalmoscope revealed no pathological changes in the fundus oculi. The reaction of the pupil, however, showed that the optic nerve had been injured; for although the consensual reflex was present, and the pupil of the blind eye responded to the stimulus of light, yet the contraction was only partial and momentary, and full dilatation occurred while the light was still being held in front of the eye. The serious nature of the condition could not be misunderstood : and in a few weeks it was easy to detect complete atrophy of the optic nerve by means of the ophthalmoscope. Here obviously no treatment is possible. Skiagrams are of little use; indeed, the $\mathrm{X}$-rays are not in such cases of so much assistance in diagnosis as one might imagine. Pringle's important observation that so many cases of monocular traumatic amaurosis are due to hæmorrhage into the sheath of the optic nerve makes the prognosis more favourable. Occasionally partial recovery of sight occurs, and that small percentage of cases ought to encourage the surgeon to advise operation at the earliest possible moment after the blindness is discovered. It is neither a difficult nor a dangerous operation to open into the orbit by removing its outer wall-Krönleinto expose the optic nerve, and evacuate any blood found in its sheath; but if such an operation is to have any chance of success, it must be done without delay-before the compression of the blood-clot has had time to cause irreparable damage to the nerve.

It happens occasionally that after a blow on the head there follows complete paralysis of all the muscles of the eyeball-ophthalmoplegia externa and interna. In such a case the lesion is at the sphenoidal fissure through "which the third, fourth, and sixth nerves enter the orbit. In addition to the motor paralysis, there is also anæsthesia of the parts supplied by the ophthalmic division of the trigeminus. When the nerves are simply compressed by blood-clot or by inflammatory exudation, more or less complete recovery may be expected as absorption takes place; but when the bones are fractured and the nerves torn, the paralysis remains permanent.

SEConD.-When the eyeball is itself damaged.

When an eye is struck, the patient usually experiences severe pain and a sudden "sparking" of the eye, and may for the moment feel stunned. Examination usually reveals slight abrasion of the cornea, injection of the conjunctiva, congestion of the iris, contraction of the pupil, and more or less dimness of sight. The pupil does not readily dilate under the influence of atropin, which may have to be instilled 
several times before full dilatation is produced. Even then the expansion is often irregular, and it is characteristic that the shape frequently becomes oval before assuming the natural circular form. The eye waters, and is so intolerant of light that it may be difficult to make an ophthalmoscopic examination; and if this is possible, as a rule the details of the fundus are indistinct, as if viewed through a cloud of smoke. If, however, the injury has been more severe, the optic disc is obviously congestedits outline is soft and indistinct as if seen through a mist, and between it and the macula the retina presents a greyish swollen appearance. This condition has been described under the name of commotio retince by Berlin, who has demonstrated that in addition to œdema of the retina, there are also minute hæmorrhages between the choroid and sclerotic.

These instances of concussion of the eyeball differ only in degree; and, although spontaneous recovery after a few days is the rule, it is not well to trust to a purely expectant line of treatment, but at once to adopt antiphlogistic measures. As soon as possible after the accident, put the patient to bed, instil atropin, leech the outer canthus freely, apply a cold compress, and administer a purgative.

In blows of much severity, the symptoms just described as due to concussion are complicated by hamorrhage, which may be sub-conjunctival or intraocular. Sub-conjunctival hæmorrhage may be limited to the spot where the eyeball was struck, or, as. sometimes happens, may completely cover the sclerotic. The effused blood forms a clot, visible through the transparent conjunctiva, and bounded in front by the corneal limbus. Now and then, however, these limits are overstepped, and blood finds its way for a short distance into the substance of the cornea. It always more or less completely hides the sclerotic; and this changed appearance of the eyeball is at once noticed by the patient or his friends, and usually causes much anxiety. Gradually the clot becomes absorbed, passing through varying shades of brown and green and yellow, until the sclerotic again becomes visible. The time required for the disappearance of the blood varies from a few days to several weeks; but in all cases in which the hæmorrhage has been extensive, a yellowish staining of the white of the eye remains for a much longer time. When sub-conjunctival ecchymosis occurs immediately after an accident, it is of very little significance, and Nature alone will do all that is required for its removal, so it is rather with a view to calming the fears of the patient than with the idea that the process of absorption will be in any way hastened, that a lotion containing arnica or hazelene is prescribed. It must, however, be remembered that if the hæmorrhage make its appearance after a lapse of several days, the prognosis is very serious; because it is then one of the most trustworthy indications of fracture through the anterior 
fossa of the skull. This sign is therefore of great value as indicating a grave condition, which may be followed by death; so that it is always very important in every case of injury to the head to note whether subconjunctival ecchymosis is present, and to inquire whether it appeared immediately after the accident or not for several days. Rollet has laid down the following rules which are of great assistance in diagnosis: (I) Ecchymosis due to fracture of the roof of the orbit spreads by degrees; (2) it is limited to the conjunctiva and the palpebral folds; (3) it produces only a moderate amount of tumefaction; whereas the ecchymosis and œdema following direct contusion of the soft parts are rapid in onset, spread quickly to the face, and readily assume large proportions.

Intraocular hæmorrhage is always serious; but the gravity of the condition is much less when the blood is in the aqueous, than when it is in the vitreous, chamber. It may indicate not only rupture of bloodvessels, but also rupture of some of the internal structures of the eyeball. As long as the hæmorrhage persists, it is, of course, impossible to say how much damage has been done; and it is only after the clot disappears that the true nature of the injury becomes apparent. If the effusion be confined to the aqueous, the bleeding comes from the vessels of the iris or ciliary body; but if it be into the vitreous, it comes from the vessels of the choroid or retina.

The iris frequently suffers. As a result of a contusion, both iris and ciliary body may be paralysed; and, though occasionally either or both of these conditions may persist, yet, in most instances, the dilatation of the pupil, and the loss of the power of accommodation pass away in a few days. Structural lesion, whether it occur at the pupillary border or at the ciliary margin, is permanent, although in its slighter forms it does not necessarily interfere with sight. A small clot arising from simple rupture of blood-vessels may easily be mistaken for a structural tear-an error that time will soon show-as the blood will quickly disappear. Lacerations of the pupillary border, so small that they can be detected only by careful examination with a corneal loupe, give rise to permanent dilatation of the pupil, and if a distinct rent be visible, the pupil will also be irregular in outline. When the iris is separated from its ciliary attachment -irido-dialysis - the point of lesion is marked by a black crescent, and the pupil loses its rounded form, being flattened on the injured side. If the aperture be at first small, it will, in course of time, become less; but if it be large, it will always remain visible, and when light is transmitted to the eye by the ophthalmoscope, a red reflex is obtained through the rupture as well as through the pupil. Monocular diplopia may be produced by this injury. In severe cases there may be more than one lesion; and occasionally the whole iris is separated, and may either be seen lying 
loose, or disappear from sight in the bottom of the anterior chamber. In the latter case the traumatic aniridia resembles total inversion of the iris, where, as a result of injury, the whole iris turns backwards on itself and disappears from sight by becoming flattened against the ciliary body. More commonly the inversion is partial and gives rise to an apparent coloboma, which, however, is readily distinguishable from surgical or congenital coloboma, by the fact that the fibres of the suspensory ligament and the ciliary. processes cannot be seen.

Hæmorrhage from the vessels of the choroid is always a very serious matter, because the blood will be effused either between the sclerotic and the choroid, or between the choroid and the retina. The former condition involves separation of the two structures concerned, and the latter more or less extensive detachment of the retina. The history of the accident, the suffering of the patient, the appearance presented by the eye, and the diminution of the intraocular tension, would lead one to suspect rupture of the choroid, even although the presence of the blood renders it impossible to see the lesion. When, after a time longer or shorter, according to the size of the blood-clot, the media become sufficiently transparent to permit the fundus to be seen, there will be found in the choroid, usually to the outer side of the papilla, one laceration, possibly more than one. The rupture shows itself as a narrow streak, thick in the middle, pointed and sometimes branched at the extremities, and bordered by pigment, the white colour being due to the fact that the sclerotic is visible through the tear. As time goes on, the inflammatory symptoms subside, and the outline of the lesion is more sharply defined, while in many instances the posterior pole of the eye becomes distinctly pigmented. When hæmorrhage into the vitreous is large, it may be seen by oblique illumination if the pupil be dilated. It usually becomes more or less completely absorbed; but occasionally the clot becomes organised and gives rise to retinitis proliferans.

Rupture of the retinal vessels through violence is not nearly so common; and when it does occur, the hæmorrhage takes place, as a rule, between the retina and the hyaloid, giving rise to a large disc-shaped clot situated, in most instances, in front of the macular region. That the retina itself is not seriously implicated in this bleeding, is clear from the fact that although the patient may not be able to distinguish any object, yet when he looks at a gas or candle flame it appears red, this being due to the blood coloration. Such clots become, in most cases, gradually absorbed; and, if the eye has not been otherwise injured, the sight will in time be completely restored. A "hole" in the retina, at the macula, is a rare lesion after a blow on the eye. It forms a circular opening of deep red colour, frequently studded by minute glistening spots. 
Where detachment of the retina is the result of violence, the prognosis is much more favourable than when it is due to disease; but under any circumstances the condition is always most serious. If the detachment is localised, it will be distinctly visible on ophthalmoscopic examination, and its extent may be mapped out by the loss of a part of the visual field as measured by the hand or by the perimeter. In such cases much may be accomplished by suitable treatment; but if the separation be complete there is no hope of recovery of sight.

Lagrange gives the following useful classification of cases of traumatic detachment of the retina-

I. Detachment by sub-retinal hæmorrhage.

2. Detachment by post-traumatic retraction of the vitreous body.

3. Detachment which results from the cicatricial contraction of a wound.

4. Detachment which follows a loss of vitreous.

5. Detachment which results from concussion at a distance with or without laceration of the retina.

It must also be remembered that detachment of the retina is very prone to occur in a myopic eye; consequently in the short-sighted a very trivial injury may be the immediate cause of its appearance.

A contusion so severe as to produce the injuries just described will also almost certainly cause rupture of the zonule, and dislocation of the lens. The displacement may be partial or complete. In the former case the lens remains in the posterior chamber, its black outline being readily visible on ophthalmoscopic examination; and, if the vitreous be fluid, it is seen to swing up and down, and the iris, deprived of its support, trembles with every movement of the eye. In complete dislocation the lens may be forced into the vitreous, and may lie at the bottom of the chamber quite removed from the line of vision. In other cases, more especially if it be small, it escapes through the pupil and falls into the aqueous. In this situation it acts like a foreign body, and may give rise to much trouble by setting up secondary glaucoma. After dislocation the lens may remain transparent, but more usurally it becomes cataractous, and in old-standing cases undergoes calcareous degeneration, when its bulk may be so reduced that it slips backwards and forwards through the pupil without any difficulty.

When cataract is due to a blow on the eye, the capsule of the lens is usually ruptured, and the opacity appears within a few hours, just as it does after a penetrating wound which implicates the ocular tissues and the crystalline lens. It may or may not be accompanied by subconjunctival ecchymosis and swelling of the lids. The cataract follows quickly after the injury, and only affects the eye that was struck. More- 
over, its progress is so rapid that the lens becomes swollen, pushes the iris forward into the aqueous chamber, and causes increase in the intraocular tension. The rent in the capsule in such cases does not require to be large, and it may not always be visible, even with the help of the ophthalmoscope. When cataract is due to concussion, the injury to the lens is indirect, and the opacity, which affects both eyes, may not occur for months after the accident. Moreover, the progress of the cataract is slow, and unaccompanied by swelling of the lens, or by increase in the tension of the globe.

When a cataract follows an injury, which has not caused rupture of the capsule of the lens, its occurrence must be explained by analogy rather than by direct proof. It is well known that the transparency of the lens depends upon the integrity of the sub-capsular epithelium, and if this be damaged, either by blood changes or by physical injury, loss of transparency gradually takes place. A true concussion cataract usually occurs in the young and healthy, and generally is the result of an explosion injury so severe as to render the patient unconscious. Neither degenerative nor blood changes are likely therefore to contribute to the causation of the cataract. The damage is more likely to be physical, and to have occurred at the time of the accident. It is a well-established practice among ophthalmic surgeons, in a case where an ordinary senile cataract is slow in ripening, to draw off the aqueous and gently massage the anterior capsule of the lens. After that operation the cataract usually matures rapidly; and the explanation is that the massage has injured or destroyed the epithelial cells lining the posterior surface of the anterior capsule, and has also broken up their connection with the lens fibres. The aqueous humour is thereby permitted to infiltrate the substance of the lens, which, in consequence, loses its transparency. It seems, therefore, reasonable to suppose that a concussion so violent as to cause loss of consciousness, may also at the same time originate changes in that layer of delicate epithelium which lines the capsule of the lens, and thereby lead later on to the formation of cataract.

When the injury is more severe, the tissues of the eyeball may be ruptured, and the lens escape beneath the conjunctiva. The sclerotic usually gives way at its upper or inner aspect, just beyond the apparent corneo-scleral border, where it is naturally weak owing to the presence of the canal of Schlemm. It is easy to distinguish the lens as a little swelling covered by conjunctiva. At the site of the rupture the iris seems wanting, owing to its having been driven back into the vitreous at the time the lens escaped. Here, as in the other instances that have been described, the true nature of the injury is at first often obscured by the occurrence of hæmorrhage, followed later by considerable inflammatory 
exudation. Although the upper and inner aspect of the globe is the favourite site for rupture of the sclerotic, the eye may, through direct violence, be burst at any point. Where the blow is a very severe one, the rent in the tissues is so large that the clear structures escape, and the choroid and retina prolapse through the wound. The globe becomes filled with blood, which may infiltrate the substance of the cornea, and form a thin layer in front of the iris, giving rise to the appearance of a pupil although no pupil is there. There is always great chemosis of the conjunctiva, and swelling and discoloration of the lids.

Treatment.-In the treatment of these severe conditions one must necessarily be guided by circumstances; and good results can only follow if the structural damage has not been extensive. Blood is readily absorbed from the aqueous, but the clot is not easily removed from the vitreous. It breaks up in course of time, but is apt to persist in the form of little black flakes which disturb sight. It may, however, as a general rule, be said that the antiphlogistic line of treatment indicated when concussion of the retina was described, is indicated in all cases in which there is any chance of recovery. Atropin, however, must be used with caution, on account of the risk of increased intraocular tension.

The consequences of a severe blow on the eye are usually marked at the outset by intraocular hæmorrhage. Much time is required for the disappearance of the blood clot; but instillation of 5 per cent. dionin and careful massage (Ormond) help to promote absorption. A combination of iodide of potassium and chloride of ammonium, taken three times a day after meals, and continued for several weeks, is frequently prescribed in the hope that it may help to cause the blood-clot to disappear; but it must be admitted that all internal remedies are of very little value. In all recent cases the patient must be kept in bed.

When any part of the uveal tract is ruptured, nothing can be done to mend the rent. As a rule the patient should be kept at rest, atropin instilled, and the eyes protected by a compress and bandage, until the acute symptoms have subsided.

The treatment of traumatic detachment of the retina consists of rest in bed, instillations of atropin, careful applications of a pressure bandage, and a sub-conjunctival injection once a week of ten to twenty drops of a solution containing chloride of sodium, bicyanide of mercury and dionin. The solution should be used at blood-heat, and injected very slowly. Time is an important factor in the cure, consequently the duration of the treatment must not be less than a month; and the larger my experience becomes the more am I inclined to prolong the period of rest in bed, because relapse, prone to occur at all times, takes place more readily in patients who have remained in bed for only two or three weeks. If, after 
three weeks in bed, no sign of betterment can be detected, further treatment is useless, for the case under these circumstances is certainly incurable.

It is very desirable that, after the course of treatment, the patient should be sent to the country for a few weeks, in order that the general health be brought up to the highest possible standard. Occasionally it happens that considerable improvement occurs during this period of convalescence. The patient must always be warned to carefully avoid all acts which tend to cause straining, or congestion of the head and face, and to pay particular attention to secure regular action of the bowelsconstipation seems to play a most important part in bringing about a relapse.

In dislocation of the lens there should be extraction as soon as the operation becomes possible. It is easy to remove a lens from the aqueous chamber, provided it can be kept there till the operation is completed; but should there be any chance of its slipping back into the vitreous chamber, it should first be fixed by passing a needle behind it, before the operation of extraction is begun. When it is floating free in the posterior chamber operative interference is undesirable, unless there be some special reason. Sub-conjunctival dislocations are easily dealt with by slitting the conjunctiva over the lens and allowing it to escape; but the operation should never be attempted till several days after the accident, so that the wound in the sclerotic may close before the conjunctiva is disturbed. This delay prevents any risk of the lens slipping back through the wound into the eye, and diminishes the danger of septic infection. When an eyeball is burst, enucleation is the best possible treatment, and the sooner the better in order to anticipate the onset of inflammation and suppuration, and so save the patient much needless suffering. 


\section{CHAPTER XXXIII}

\section{PENETRATING WOUNDS OF THE EYE}

Classification of penetrating wounds-Importance of estimating tension-Wounds of the cornea--Wounds of the sclerotic-Involvement of the lens-SepsisTreatment by the general practitioner-Specialist treatment-Recent prolapse -Excision and formation of conjunctival flap-Prolapse complicated by adhesions-Administration of antidiphtheritic serum-Treatment of panophthalmitis-Prothesis.

Penetrating wounds of the eyeball may be divided into lacerated, incised, and punctured. The first are the result of a blow which causes rupture of the sclerotic, and have already been described. Incised and punctured wounds are inflicted by some sharp instrument, for example a knife, scissors, a fork-prong, a pen, a piece of glass, a chip of metal, a bullet, etc., etc. When a bullet strikes the eyeball in warfare it rips it up into numerous leaves, and eviscerates its contents more or less completely. The most distressing cases are those where the bullet has passed transversely through both orbits, and in its course has completely destroyed both eyes. The eye is frequently injured by the bursting of a bottle, and the lids are nearly always severely implicated, and swelling up, conceal the globe. Under these circumstances, great care must be taken not to overlook a wound of the cornea and sclerotic-an error in diagnosis which might obviously be followed by most disastrous results. The great danger, when a wound has perforated the globe, is that the instrument which caused it may at the time have infected the eyeball. Aseptic wounds are to be found only after carefully performed surgical operations ; all other wounds may be regarded as septic. The injury may, in a case of puncture, be very slight-so slight indeed as to be hidden by subconjunctival ecchymosis-yet micro-organisms may have been conveyed into the interior of the ball, and in a few days the eye be entirely destroyed by suppuration. The difficulty in the diagnosis of these seemingly trivial cases will be considerably lessened if care be taken to examine the tension of the globe. Whenever there is an opening in the cornea or sclera, the intraocular tension is diminished.

Incised wounds frequently implicate both cornea and sclerotic, and are usually self-evident in themselves. They are often accompanied by prolapse of the iris, ciliary body, or choroid, by injury to the lens, and by 
escape of the vitreous. If they be extensive, and more particularly if the ciliary region be involved, and the escape of vitreous be large, the eye will almost certainly be lost; because, even although the wound heal, there is always subsequent inflammation of the uveal tract, which leads to shrinking of the globe, and possibly to sympathetic inflammation. Indeed, the chances of preserving a serviceable eye after a penetrating wound will depend very largely upon the site and extent of the injury, the amount of the loss of vitreous, the risk of septic infection, and the treatment prescribed during the first forty-eight hours after the accident.

Wounds of the cornea admit, as a rule, of a more favourable prognosis than those involving the sclerotic; not because the cornea is less easily infected, but probably because the aqueous humour in escaping washes any micro-organisms away from the lips of the wound. The progress of a case is governed, however, more by the depth of a wound than by its surface extent ; hence it is that punctured wounds are always so disastrous, for they not only involve the cornea or sclerotic, but generally also implicate the iris, ciliary body, and lens. When the last-named structure is wounded it rapidly becomes cataractous, and, swelling up, acts as a foreign body, greatly augmenting the damage done to the eye. This is the case especially when the patient is an adult; the more favourable prognosis in the child depending on the fact that, although the lens may swell up rapidly after injury, it is in early life so soft that the cataract quickly dissolves, and becomes absorbed by the aqueous, provided always that no septic infection has occurred. Simple wounds of the cornea heal very rapidly. Almost immediately after an accident the cut edges become covered by a new development of epithelial cells; and if this reparative process be not arrested, the wound within a very few days heals by first intention. This is seen to best advantage after incisions for surgical purposes, for then the union is oftentimes so perfect that the scar is visible only after careful examination. It is of the utmost importance, therefore, that the lips of a corneal wound should come into accurate apposition; but after an accident the iris very frequently prolapses and keeps the edges apart. Prolapse of the iris is always serious, and the fate of the eye depends in most instances on the skill with which it is treated. In the majority of cases the aqueous quickly reaccumulates, and the anterior chamber reforms; but the iris itself remains exposed, and constitutes the real source of danger, because the prolapse interferes with the healing of the wound, and lays the whole uveal tract open to infection, which may not only destroy the eye that has been injured, but also lead to sympathetic inflammation of its fellow. A recent prolapse may readily be overlooked by the inexperienced, for the exposed iris, owing to its grey colour, may be difficult to see. In every case, however, in which 
the iris is prolapsed, the shape of the pupil is altered. It becomes displaced and pear-shaped, and the narrow part always points to the prolapsed iris.

The sclerotic, unlike the cornea, when cut, does not unite of itself. The most recent pathological researches have shown, that in the process of healing the sclera may be regarded as passive, union being effected by reparation changes which take place both in the episcleral tissue and conjunctiva, and in the choroid subjacent to the wound. Moreover, wounds of the sclerotic always tend to gape, and generally implicate the choroid and retina. They may heal quickly in favourable circumstances; but they are often followed by detachment of the retina, and by chronic inflammatory changes, which may terminate in shrinking of the globe. Wounds of the corneo-scleral region-the so-called danger zone-expose the ciliary body to infection, and are of all penetrating injuries the most liable to cause sympathetic inflammation. A wound of the sclera, situated close to the cornea, may not heal firmly, but simply become covered by conjunctiva. A fistulous opening remains, and affords a passage through which aqueous humour escapes, and, collecting beneath the conjunctiva, distends it like a bladder. This bulging cicatrix is very liable to secondary infection, and is, therefore, a constant menace to the eye.

When the lens is injured, opaque lenticular substance escapes into the aqueous chamber, and always excites a certain amount of inflammatory reaction, accompanied by increase in the intraocular tension, which, unless speedily relieved, will cause loss of sight from secondary glaucoma. Lens substance is, moreover, very liable to become infected, and give rise to a plastic or a purulent irido-cyclitis; and it is the liability to sepsis after traumatic cataract that makes such injuries so dangerous, both in the adult and in the child.

Whenever sepsis becomes pronounced, pain is always severe, deepseated, and throbbing in character; the lips of the wound become infiltrated, the conjunctiva œdematous, and the iris discoloured. Unless the suppuration can be checked by treatment, pain persists, and is always characterised by periods of exacerbation during the night. The eyeball is exceedingly tender to touch, the iris is intensely inflamed, deposits of pus form in the ciliary body, whence septic micro-organisms migrate to the vitreous, which rapidly becomes purulent. Meanwhile, the cataractous lens is infiltrated with pus, and assumes a yellow colour; a ring abscess forms in the cornea, concentric with the corneo-scleral margin; pus appears in the anterior chamber; and within a few days the eye is destroyed by panophthalmitis.

Treatment.-The treatment of penetrating wounds of the eye forms one $\mathrm{T}$ 
of the most important chapters in ophthalmic surgery. In the great majority of cases the patient in the first instance consults the family doctor; and the fate of the injured eye largely depends upon his judgment and skill. The management of such a case will tax his powers to the utmost; and unless he has had special training and experience in the diagnosis and treatment of eye diseases, he will be well advised to ask for a consultation with a specialist, if possible within the first twenty-four hours after the accident. In many circumstances, however, there must be considerable delay before the patient can be seen by an expert; and the general practitioner ought to be able to tide the patient over that period of waiting. He ought to know what to do and what not to do until the consultant arrives. It is important to find out how the accident happened. Much valuable information is got by the knowledge that the eye has been cut by a knife, or punctured by a hat-pin, or struck by a large blunt object, in contradistinction to being hit by a chip of metal flying from a workman's hammer, or a shot pellet fired from a gun. The eyelids should be inspected carefully, to see if they are cut or lacerated, or simply bruised and swollen. Particular attention ought always to be paid to the inner canthus, to find out it there has been injury to the tear ducts. Sepsis is the danger most to be dreaded after every injury to the eye; consequently the doctor must be particular to disinfect his hands thoroughly before he touches the patient. Much can be learnt of the state of the globe by palpation of the eyelids. When the eyeball is felt to be of normal tension, it is not likely that there is a penetrating wound; but on the other hand, if intraocular tension is diminished, the eyeball has either been very seriously contused, or its coats have been perforated; and generally speaking the softer the eye the larger is the wound. In some cases the globe can hardly be felt through the closed lids, because it is so much collapsed from the extent of the injury and the large escape of its contents. The eyelids should now be separated; but no force must be used, because any rough manipulation may cause further loss of vitreous. The instillation of a few drops of 2 per cent. cocain solution enables the examination to be made without pain; but in children it may be necessary to administer a general anæsthetic, before the eyeball can be properly seen. The upper lid ought to be raised by a retractor, for fear of accidental pressure on the globe; and a convenient instrument for the purpose, at all times obtainable, is a bent hairpin which has been sterilised by boiling. When the cornea is exposed, it must be carefully examined to find out whether it has been cut or punctured. The all-important question to decide, is whether or not the injury is a penetrating wound. If the anterior chamber is empty, the cornea has been penetrated; although a minute, but fatal, puncture may have taken place without escape of aqueous. Another 
sign of much value that ought always to be looked for, is the presence of blood in the anterior chamber-hyphama, because that indicates rupture of the vessels of the iris and ciliary body. Particular attention should be paid to the pupil. If it be round and active, the eye is not likely to have sustained much damage, provided, at the same time, there be little diminution of the intraocular tension; but if the pupil be displaced and pear-shaped, there is a penetrating wound, complicated by prolapse of iris; and if the pupil appear grey instead of black, there is in all probability injury to the lens and commencing cataract. In severe cases a large gaping wound is visible, and there can be no doubt about the diagnosis. The conjunctiva is then always swollen and suffused with blood; and if the injury is in the sclera, vitreous is usually seen projecting between the lips of the wound. In all injuries to the sclerotic, the doctor should note whether they implicate the ciliary region, or are behind that danger zone.

When, therefore, a general practitioner is called to a patient suffering from a penetrating wound of the eye, he must avoid all rough manipulation, and everything that might cause secondary infection of the wound; and he should never attempt any surgical interference, unless he has considerable experience in operating on the eye, and possesses suitable instruments for the purpose. The sole function of the family doctor is to give first aid, in such a way as will not aggravate the patient's condition. After the eye has been carefully examined, the skin of the lids and the parts surrounding should be painted with a 3 per cent. tincture of iodine, cocain be instilled to relieve pain and facilitate the removal of any superficial foreign bodies that may be in the conjunctival sac; but free douching of the eye must be avoided. No powerful antiseptics ought to be used, but a few drops of ro per cent. argyrol and I per cent. solution of atropin should be instilled. Afterwards, a sterile compress and bandage ought to be adjusted, and the patient either put to bed to await the arrival of the consultant, or sent to an eye hospital with as little delay as possible.

The treatment that the specialist will prescribe will depend altogether on the extent of the injury, and the interval that has elasped since the time of the accident and his examination of the patient. Too much emphasis cannot be laid upon the importance of acting without hesitation or delay, because treatment, likely to be successful within the first fortyeight hours after the injury, may be quite powerless after some days have elapsed. Many eyes can be saved by prompt and careful treatment, but both eyes may be lost by delay.

In a small wound of the cornea, no further treatment than that just described is necessary; but in a large proportion of cases the injury is complicated by prolapse of iris, and injury to the lens. The proper 
treatment of prolapse of the iris is one of the most important problems in ophthalmic surgery. The method to be adopted in any particular case, and the success or failure of the treatment, depends in large measure on the interval of time that has elapsed since the occurrence of the accident. If the prolapse is quite recent, there is little difficulty in knowing what to do; but if several days have been allowed to pass before the patient is sent to a specialist, the prolapsed iris will be sealed to the edge of the corneal wound by inflammatory exudation. The longer the time these adhesions have had to form, the greater is the difficulty for the surgeon, who must now be guided by experience whether to interfere or to leave the case to Nature. The expectant line of treatment is often the most prudent course to follow, for in many instances the result is quite satisfactory; but the patient will require constant supervision, and the surgeon will not be free from anxiety until the wound is securely healed.

The first step in the consideration of treatment, therefore, is to distinguish between the cases that are of quite recent origin, and those that are of more than forty-eight hours' standing.

(a) A prolapse that is recent. - The first indication for treatment is to try to draw the iris out of the wound back into the anterior chamber; and for that purpose atropin is used if the wound be towards the centre of the cornea, eserin if it be in the periphery. Although the use of these drugs is always recommended, it is only on rare occasions that they are successful; and there is constant risk that even although the visible prolapse may disappear, an anterior synechia will follow, and give trouble in the future. The whole secret of successful treatment is to avoid the formation of an anterior synechia. Moreover, in the majority of cases, the iris is bruised and has lost its elasticity, so that even if by means of drugs aided by a spatula it is put back into the anterior chamber, it has not sufficient contractile power to enable it to remain in place; consequently, it is almost certain to fall between the lips of the wound in the cornea, even if it does not prolapse again. There is, too, always the risk that, in replacing an iris that has prolapsed, infection may be carried inside the eye. All things considered, therefore, the best treatment for a recent prolapse is careful excision.

Infection by pyogenic micro-organisms is the great primary danger; and failure will follow the most skilfully performed operation, if there has not been strict attention to every precaution for the avoidance of sepsis. It is necessary, therefore, in order to avoid risk of infection from these sources, that the tear passages, the edges of the eyelids, and the conjunctival sac should be made as clean as possible before the eyeball is touched. The skin of the eyelids and the surrounding parts should be painted with 3 per cent. tincture of iodine immediately before the 
operation. A general anæsthetic is not necessary, unless the patient is a child or an exceedingly nervous adult; but care must be taken to obtain perfect local anæsthesia, by the free use of cocain and adrenalin solution. The lids are separated by a spring speculum-one that keeps the eyelashes out of the way is to be preferred. A good light is indispensable. The iris will now be seen exposed on the surface of the wound. It is to be caught firmly by forceps, and while kept on the stretch, cut by a single snip of De Wecker's scissors, the blades being held parallel to the corneo-scleral margin, and pressed backwards against the eyeball. If the iris be kept tense in that manner, the cut edges retract within the anterior chamber, and the coloboma resembles an ordinary surgical iridectomy. It is very important that no tag remain adherent to the wound in the cornea. After the iris has been completely excised, the lips of the wound must be brought into accurate apposition, by means of a small spatula.

The risk of further infection is avoided by covering the wound with a flap of conjunctiva, which is an essential part of the modern treatment. The flap is formed by dividing the conjunctiva at the corneo-scleral margin in the neighbourhood of the wound. The part of the conjunctiva which has been thus separated must be freely undermined so that, with the minimum of tension, it can be drawn over to cover the wound perfectly. It is then fixed in position by one or two sutures. After the parts have been carefully dried, a coating of paraffin is applied, to seal the edge of the flap to the cornea and give additional protection to the wound. The paraffin employed is a substitute for ambrine. It is non-irritating to the eye, and is readily applied in the liquid state by a medium-sized camel-hair brush. It becomes solid almost immediately. After a drop of I per cent. atropin solution has been instilled, the speculum is removed, and the lids closed. The skin is painted again with the iodine solution, and both eyes are carefully bandaged. The patient is put to bed; and if no untoward symptoms occur, the dressings are not removed from the eyes for forty-eight hours. Pain and restlessness seriously interfere with healing, and ought at once to be overcome by a sedative draught, or subcutaneous injection of morphia. The patient, as a rule, however, makes little complaint after being put to bed; and unless infection has taken place at the time of the accident, the eye is quiet and the pupil dilated when the dressings are removed. Any discharge should be wiped away from the edge of the lids, a drop of atropin instilled, and the bandage reapplied to both eyes. In a week or ten days, the sutures holding the flap give way, and the conjunctiva falls gradually back into place, leaving the wound of the cornea securely healed. The eye may now be regarded as free from danger. If sepsis occurs, means must be taken to overcome it; but unless the infection has been acute, the chance of a 
successful termination to the case is greatly increased by the protection afforded by the flap of conjunctiva.

When the wound implicates the sclera as well as the cornea, it gapes, and is accompanied by loss of vitreous; and the prolapse includes not only the iris, but also the ciliary body and the choroid. The immediate danger in these circumstances is further loss of vitreous humour, consequently the spring speculum is to be avoided : there will be less risk of accident if the upper lid is held by an assistant with a strabismus hook or a light retractor. The bulbar conjunctiva should be separated from the posterior lip of the wound, and undermined freely for a considerable distance around the seat of injury. The whole of the prolapsed uvea must now be carefully excised, and the lips of the wound approximated by drawing the conjunctiva over the cornea like an apron, and fixing it securely in its new position by as many sutures as are required-the fewer, however, the better. No attempt ought to be made to stitch the sclerotic.

(b) A prolapse that has existed for several days. Here the prolapsed iris will have become sealed to the lips of the wound of the cornea by inflammatory exudation; and before any attempt at excision can be made, those adhesions must be carefully broken down. The longer the interval that has elapsed since the injury occurred, the firmer the adhesion, and the greater the difficulty in separating them from the prolapsed iris. If active infection has taken place, no attempt to reduce the prolapse must be made until the inflammation has subsided; and in all cases the greatest care in manipulation is necessary, because, after the iris is glued by exudate to the lips of the wound, rough or unskilful interference may readily open a path for the entrance of fresh sepsis to the interior of the eyeball. When the prolapse is small, it is wisest to leave it alone: it will heal in a few weeks, forming a dense white scar, which seals the wound, and binds iris and cornea firmly together. The cicatrix thus formed is, however, not free from danger; because, apart altogether from its being a probable cause of secondary glaucoma, it is, long after the injury, liable to infection, which may set up a suppurative irido-cyclitis, that may terminate in panophthalmitis.

Whenever, therefore, it is possible to deal surgically with a prolapse, it is prudent to do so; but the technique of the operation must be carried out with great care and consideration. The iris must be separated thoroughly from the lips of the wound, by breaking down the newly formed exudate with a small spatula or a sharp probe; and when the case is of long standing, it may be necessary to enlarge the wound on either side of the prolapse with a keratome. After the iris lies free in the wound, it should be grasped by forceps, pulled gently on the stretch, and completely 
excised with De Wecker's scissors, the blades being pressed firmly against the globe. As soon as the prolapse has been cut off, a small spatula should be passed into the anterior chamber, to make sure that no part of the edge of the coloboma remains in the wound. If any tags of iris tissue be discovered, they must be removed by fine forceps and scissors. 'If the edge of the wound show signs of septic infection, they ought to be touched with the galvano-cautery, and afterwards covered by a flap of conjunctiva. In those cases, it is my practice to give 4000 units of antidiphtheritic serum subcutaneously before the patient is removed from the operating table, and in twelve hours to repeat half that dose. In medicine it is easy to argue post hoc ergo propter hoc; but, having seen at the bedside the favourable results that follow the administration of serum, I advise its use confidently in all cases where the eyeball is infected. As a rule, it gives rise to no constitutional disturbances. When the lens has been wounded, the cataract frequently swells so rapidly as to cause increase in the intraocular tension, which must be promptly relieved by operation. In children the opaque lens substance may be dissolved spontaneously by the aqueous humour; but in adults surgical interference is required in the majority of cases. Whenever the injured eye becomes red, painful, and hard, the cornea should be incised, and the lens substance washed out of the anterior chamber by copious irrigation with warm sterile saline solution. The point of the irrigator, rendered thoroughly aseptic by boiling, is carefully introduced into the anterior chamber through the wound in the cornea, and the stream of fluid so directed that it passes inside the capsule. By that means the great bulk of the cataract is got rid of, and at once the increased tension is relieved. Nevertheless, there is always danger of inflammation of the iris and ciliary body after that operation; consequently means should be taken to prevent it by careful asepsis, by the regular use of atropin, and by a sharp calomel purge. A 5 per cent. solution of dionin in addition to the atropin is to be recommended. It quickly relieves deep-seated pain, and hastens the absorption of lenticular substance remaining in the anterior chamber.

After the wound has healed, the opaque capsule of the lens, to which the iris is always more or less adherent, blocks the pupil and interferes seriously with vision. It is usually necessary therefore to perform a capsulotomy operation. An incision is made with a keratome in the cornea, and a pair of Carter's or De Wecker's scissors introduced into the anterior chamber through the opening. The blades of the scissors are now opened, and one passed behind and the other in front of the constricting band of capsule, which is then divided. If the operation be successful, the iris at once springs open to form a pupil. Each case must be judged on its own merits; and as a rule the principal incision must be made where 
previous examination has shown the iris to be most tightly stretched. The opening thus formed can be made larger by one or more cross cuts. In this operation great care must be taken to avoid escape of vitreous; and as a rule it is safest, if the patient is nervous, to administer an anæsthetic, or preferably, to give omnopon-scopolamin or trivalin by intramuscular injection an hour and a half before the operation. Whenever sepsis becomes pronounced, pain is always severe, and is deep-seated and throbbing in character. In such circumstances, the suppurative process may be held in check by freely irrigating the conjunctival sac with hot sterile saline solution. This mechanical cleansing washes away all discharge, and diminishes the number and the activity of the microorganisms. The ocular congestion is lessened, and pain relieved, by snipping the chemosed conjunctiva with scissors, and encouraging the bleeding by douching with warm salt solution. A collargol disc ought to be placed in the conjunctival sac every two or three hours; and the first sign that the infective process is being overcome is when the collargol gelatine is seen to cling to the lips of the wound. The eye must be kept covered by antiseptic fomentations, and the pain relieved by the judicious use of hypodermic injections of morphia. The patient ought to be kept in bed, well fed, and skilfully nursed.

When the interior of the eyeball becomes infected, the process of suppuration goes on unchecked by the treatment just described. Even in such desperate circumstances, an attempt ought always to be made to save the eye. The wound in the cornea must be opened up, and the cautery applied thoroughly to its edges. The anterior chamber should be irrigated freely with saline solution, and any pus thoroughly washed away. A few drops of a 25 per cent. solution of argyrol should then be injected carefully into the interior of the eyeball, or a little rod of gelatine impregnated with collargol may be introduced into the aqueous chamber, where it gradually dissolves. My experience makes me more favourable to the use of argyrol and collargol in this way than to that of iodoform. In most instances, however, the infective action will have penetrated to a. point too deep to be reached by any antiseptics; and the probability is that the eye will be lost from panophthalmitis, or from chronic iridocyclitis. Whenever, therefore, it is evident that sight is to be lost, it is far the wisest course to enucleate at once. If there is acute suppuration of all the tissues of the globe, an element of danger attends enucleation, for there is then always a risk that the operation may be followed by purulent meningitis. Under such circumstances, it is wiser to eviscerate than to enucleate. In order to get the rapid healing which usually follows enucleation, and at the same time to avoid the danger of meningitis, Colonel Lister has recommended the following modification of excision of the 
eye, in the presence of orbital cellulitis or panophthalmitis. After the cornea has been removed the contents of the globe are eviscerated, great care being taken to remove every trace of retina or choroid. The ocular muscles are then divided in the usual way, the sclerotic pulled well forward, and divided far back, "leaving a frill of about four or five millimetres in radius round the intact optic nerve."

When the injury is extensive, and the eyeball has collapsed through loss of vitreous, the wisest plan is to enucleate at once; in order that the patient may be saved much useless suffering, and the risk of sympathetic inflammation avoided.

Ophthalmic surgeons have suggested many means whereby the deformity produced after the removal of an eyeball may be lessened, and a more natural appearance given to the prothesis. Mules suggested evisceration, and the implantation of a glass or silver ball in the sclerotic; and the cosmetic results following the successful performance of this operation leave little to be desired; but it has the disadvantages that the whole of the eyeball is not removed, that there is a tendency for the glass ball to escape from the sclerotic, and that healing after the operation is often accompanied by much inflammatory œedema of the orbital tissues, and very severe suffering for several days. In order to get rid of these disadvantages, I have employed an injection of paraffin into. Tenon's capsule to form a stump for an artificial eye. The paraffin simply lies in the tissues and gives rise to no irritation. Others have employed a mass of fat, or a piece of costal cartilage, or a graft of the sclerotic, etc., etc. The four recti muscles are stitched by catgut sutures to the cartilage or to the graft of sclerotic, and the resulting stump is very satisfactory. The advantage of these modifications depends not so much in the greater mobility of the prothesis, as in the minimising of the flat, sunken appearance of the upper lid, which nearly always follows a simple excision of the globe.

After explosion or warfare injuries, the laceration of the lids and the infiltration of the tissues of the orbit cause much scarring and cicatricial contraction of the socket, and in such cases plastic operations are necessary to form new eyelids or to enlarge the socket, so that it may support an artificial eye. 


\section{CHAPTER XXXIV}

\section{PENETRATING WOUNDS OF THE EYE WITH RETENTION OF A FOREIGN BODY}

Importance of determining the presence of a foreign body in the eye-Siderosis bulbi-Localisation of the foreign body-Application of the magnet-Fate of the injured eye-After-treatment-Injuries from dynamite explosions and their treatment.

THE presence of a foreign body in a penetrating wound of the eye greatly increases the gravity of the prognosis, not only because of the added risk of infection, but also because of the special danger of sympathetic inflammation. It is true that a small foreign body may become encysted within the globe and remain quiescent for many years; but such cases are very exceptional; and all experience goes to prove that in such circumstances destructive inflammation may arise at any moment. A particle of metal may be carried about in the eye for forty years or more, and then, suddenly and inexplicably, there occurs an attack of iridocyclitis so severe that sight is quickly destroyed, and the safety of the fellow eye threatened by sympathetic inflammation. On the other hand, the exceptional cases just mentioned, and the experience of warfare injuries have shown that the presence of one or more foreign bodies within the eye does not always necessitate immediate enucleation of the injured globe. Nevertheless, the possibility of the occurrence of destructive inflammation after many years, emphasises the fact that, so long as any foreign body remains within the globe, the safety of the eyeball cannot be regarded as permanently assured.

It is only very occasionally that a foreign body can be seen by the naked eye, or by the aid of the ophthalmoscope. In most instances, it rapidly becomes concealed by hæmorrhage, or hidden by increasing opacity of the lens, or by an outpouring of inflammatory exudation. It is therefore of the first importance that the eye be thoroughly examined at the earliest possible moment after the accident, and careful inquiry made regarding its nature and all the circumstances that accompanied it. If those facts be fully elicited, much valuable information will be obtained, because obviously an eye that has been penetrated by the prong of a fork, or cut by a knife, is not at all likely to contain a foreign. body; whereas, when the accident has been due to a blow from a chip of steel 
of glass, or stone, or a lead pellet shot from a gun; or where it has been the result of an exploding cartridge or bursting shell, the risk that the missile has become lodged in the globe is exceedingly great. The appearance presented by the eye is of great value in aiding a diagnosis of the presence or absence of a foreign body. If one be present, there is usually a penetrating wound of the cornea or sclerotic, the iris is torn, the aqueous stained by blood, and the lens cataractous. A hole in the iris is almost certain proof that a foreign body has penetrated the eye and lodged within the globe. It is often remarkable how slight is the suffering immediately after such a serious accident. The patients rarely make any complaint of pain, and are usually wholly concerned about the impairment in sight. In a few days, however, there is marked congestion of the circumcorneal blood-vessels, and considerable tenderness on pressure over the ciliary region. The degree of inflammatory reaction subsequent to the injury depends not only upon the size and shape of the foreign body, and the position which it occupies within the globe, but also upon the changes which it is capable of originating, as a result either of direct sepsis, or of siniple chemical reaction between the foreign body and the tissues in which it is embedded. Chips of metal, for example, nearly always set up severe inflammation; whereas splinters of glass, if introduced into the eye aseptically, may produce but little disturbance. Leber has shown that copper is particularly dangerous, even when it is quite free from micro-organisms; for it always sets up purulent inflammation as a result of chemical reaction. The inflammation, however, differs from that due to microbic infection, in that it is most pronounced in the immediate vicinity of the foreign body, shows no tendency to spread, and is not likely to cause sympathetic ophthalmia. Particles of iron are also acted upon chemically by the fluids of the eye, and sooner or later cause the condition known as siderosis bulbi. This is caused by impregnation of the ocular tissues with iron, and is recognised clinically by a rusty deposit on the anterior capsule of the lens, by a reddish-brown discoloration of the iris, and by a degeneration of the retina through which sight is ultimately lost. Other metals, more especially lead, are better tolerated by the eye. A shot pellet may become encapsuled and remain quiescent for years. It happens, though rarely, that when the cornea is penetrated, eyelashes are driven into the eye and imprisoned in the anterior chamber. Their presence in that unnatural position tends to keep up inflammation, and may form the starting-point for the development of an implantation cyst of the iris.

The history of the accident, the position and character of the wound, and the subsequent history of the case, may all afford strong presumptive evidence that the eye contains something extraneous; but this suspicion 
can only be confirmed by the actual demonstration of the presence of a foreign body. If the media be transparent, the chip of metal, glass, stone, etc., may be seen, either by the unaided eye, or by means of the ophthalmoscope; but care should always be taken not to mistake a small blood-clot, or a minute rent in the iris, for a particle of metal. In the majority of instances, however, the media have lost their transparency by the time the patient seeks advice. In these circumstances it is indispensable to employ the sideroscope, the magnet, or the $\mathrm{X}$-rays, to complete the diagnosis. If the foreign body is a chip of iron or steel, its presence can be determined by the aid of Hirschberg's sideroscope, or by bringing a powerful magnet close up to the eye. When the substance which has penetrated the globe cannot be attracted by the magnet, a skiagram ought to be taken. In many cases the Röntgen rays afford most valuable assistance; for not only do they demonstrate the presence of a foreign body whose existence would otherwise be doubtful, but give the still greater assistance of determining its exact localisation. The principles underlying the method by which this is accomplished were first described by McKenzie Davidson. Stereoscopic photographs are of great assistance in localising foreign bodies by means of skiagrams, but in some cases localisation is not nearly such a simple matter as might be imagined. More than one foreign body may be present, and it sometimes happens that a chip of steel, or a shot pellet, passes right through the eye and lodges in the orbit.

Treatment.-Even after a foreign body has been carefully localised, its extraction from the eyeball is always extremely difficult, unless it be a chip of iron or steel, which can be attracted by a magnet. In every magnet operation the great secret of success lies in prompt intervention. If the case be dealt with within twenty-four hours of the accident, the patient should be laid flat on a table with the head and shoulders comfortably supported by pillows. The eye should be thoroughly cleansed, the pupil dilated as fully as possible, and the conjunctiva made perfectly insensitive by instillations of a 4 per cent. solution of cocain. The magnet ought to be brought close up to the eye, when the patient will at once experience sharp pain, owing to the metallic particle being attracted from the deeper parts of the eyeball towards the surface. A general anæsthetic is necessary only in the case of a very nervous, troublesome patient, because an injection of novocain and adrenalin deep into the tissues of the orbit renders the operation painless.

If the wound is in the cornea, an attempt ought to be made to extract the chip of metal through it; but if, as so often happens, it is situated at the corneo-scleral junction, or in the sclera further back, it is safer practice to try to guide the metal from the vitreous chamber into the 
aqueous, rather than run the risk of injuring the ciliary body and choroid still further, and of disorganising the vitreous, by dragging the foreign body through the perforation caused by the wound. Of course, every now and then a case occurs in which the wound in the sclerotic is so situated that the splinter may be most readily extracted by enlarging the cut, if necessary, and applying the point of the magnet to its lips; but, as a general rule, the eye will be damaged less, if the magnet be used in such a way as to draw the foreign body to the aqueous chamber - along the path of least resistance. If, then, the point of the magnet be held against the centre of the cornea, and the current gradually increased by means of a rheostat, the patient will, as a rule, suddenly give a sharp cry of pain, and at that moment the metallic particle will be seen to pass in front of the lens and cause the iris to bulge forwards:

If, as sometimes happens, there is difficulty in attracting the foreign body, the operator must exercise patience, and apply the current in such a way as to act on the metal by a series of jerks. The splinter reaches the aqueous by passing behind the lens and perforating the zonule; and, provided that the lens has escaped injury at the time of the accident, it is very seldom injured during a skilful attempt to extract a piece of metal.

It is most important that the field of operation be properly illuminated, so that every movement of the foreign body may be quickly seen, and all damage from tearing of the iris prevented. The current ought at once to be shut off as soon as the splinter reaches the aqueous.

The operator must be guided by experience as to further procedure. If the splinter possesses sharp edges which have caught the iris, the safest plan is to incise the cornea and cut out the piece of iris overlying the metal : but if the iris be not involved, an attempt ought to be made to draw the chip gently into the pupil, and to keep it there until the anterior chamber is opened. The particle can then be removed without iridectomy.

In practice, it is well to have a small magnet at hand as well as the giant magnet; because if the rough edge of a chip of metal becomes entangled in the iris, there is great danger that a large, powerful magnet will extract it so rapidly and forcibly that a considerable portion of the iris will be dragged out at the same time. It is better, indeed, after the foreign body has been displaced from the deeper parts of the eye into the aqueous chamber, to complete the operation for its removal with the less powerful instrument.

If, on the other hand, the patient be not seen till the wound in the cornea or sclerotic has had time to become healed, the site of the splinter 
must be determined as accurately as possible, and an incision made through the sclerotic in its immediate neighbourhood. The point of the magnet can then be introduced within the lips of the wound, and the metal extracted. After the operation, the cut in the sclera must be carefully covered by conjunctiva to prevent infection, and the patient should be kept in bed for at least a fortnight. If no means be at hand for accurately localising the foreign body; the large magnet should be applied to the centre of the cornea, and the chip of iron or steel attracted and drawn into the anterior chamber.

The size and the nature of the foreign body, and the speed at which it was travelling when it struck the eye, are all of great importance in prognosis. In every case, however, the sooner it is extracted the greater the chance of preserving sight; and if the lens has escaped injury, obviously the prognosis is so much the more favourable. In such circumstances, a piece of metal of considerable size may be removed from the vitreous chamber, and the patient be not much the worse.

In many cases, however, even although the operation has been performed with promptitude and apparent success, sight may afterwards be lost owing to detachment of the retina; or the globe may in the end be completely destroyed by plastic cyclitis, the result of microbic infection. The eye has indeed been irreparably damaged from the first. The shock, the large size of the foreign body, and the great liability of the vitreous to . become infected by septic organisms, all contribute to bring about a disastrous result. In every case where the eye is shattered at the time of the accident, it is wiser to remove it at once, rather than to attempt to extract the foreign body; because, in these circumstances, the eye is sure to be infected and damaged beyond all possibility of recovery, and as time passes it will become painful and dangerous to its fellow.

Hirschberg divides foreign bodies according to their weight; the small, weighing from twenty to thirty milligrammes, can usually be successfully extracted, while the medium, weighing from fifty to one hundred and fifty milligrammes, imperil the globe in proportion to their size, and raise the question whether it would not be wiser to enucleate than to make any attempt to save the eye. If the foreign body be one which is not attracted by the magnet-wood, stone, glass, non-magnetic metals, etc.-all that can be done, after accurately localising its position within the eyeball, is to cut down upon it and attempt to extract it with forceps. It is not often, however, that those efforts are successful, and the operation is usually completed by enucleation; indeed, in such circumstances the surgeon ought always to take the precaution to have 
previously obtained the patient's consent to remove the eye, if he considers it necessary to do so. Failure may sometimes occur, although the foreign body be one which the magnet will attract, for it is not at all unusual for a particle of metal to become so embedded in the ocular tissues as to be completely insulated; and the more minute the particle the greater this danger.

Although it is not always possible to preserve the function of an eye after a perforating injury, or after the successful removal of a foreign body, yet modern surgical technique and the advances of physical science enable the surgeon, greatly to the satisfaction of the patient, to preserve its form. Unfortunately, however, it sometimes happens that, after an apparently successful result, degenerative changes supervene, and these give cause for much concern regarding the fate of the injured eye, and the safety of its fellow. Roughly speaking, these retrograde processes result either in shrinking, or in distension of the injured globe, and of these the former is by far the more frequent.

An eye may seem for several weeks to be recovering quite satisfactorily, and the patient may even be able to see with it; when the cicatrix of the wound begins to retract, and at the same time the sight at first becomes weaker, and then disappears altogether, owing to detachment of the retina. The intraocular tension gradually diminishes, the cornea gets flatter and smaller, and the anterior chamber becomes so shallow that the iris at length lies closely against the posterior surface of the cornea. The globe loses its spherical shape, and assumes a somewhat quadrilateral form, owing to flattening of its walls at the insertion of each of the recti muscles; and the upper eyelid tends to droop and fall in. Hæmorrhage frequently occurs from the vessels of the iris and ciliary body, and the iris becomes much altered in colour from absorption of blood pigment. All this time there is tenderness on pressure over the ciliary region, the eye readily flushes and waters on exposure, and there may be complaint of sympathetic irritation.

Obviously, enucleation is here the proper treatment; but should the patient, as is sometimes the case, refuse to allow the eye to be removed, there will be recurrent attacks of inflammation, accompanied frequently by much suffering, and lasting for many months. After they subside, the stump, now shrunken to a very small size, may remain quiescent for years; but as long as it remains, it is unwise for the patient to wear an artificial eye, because the prothesis may cause irritation sufficient to precipitate the onset of sympathetic mischief. These shrunken globes are the seat of various pathological changes: deposits of lime take place in the ciliary body and lens, and the choroid gradually becomes converted into a shell of bone. 
Distension of the eyeball, which is more likely to follow a punctured than an incised or a lacerated wound, is due to general inflammation of the uveal tract. The cornea and the sclera may become uniformly distended; but more usually staphylomata form in the ciliary region and at the equator. The vitreous becomes quite fluid, and frequently contains numerous cholesterine and tyrosine crystals-synchysis scintillans-the intraocular tension is increased, there is always liability to the occurrence of pain and inflammation from recurrent glaucomatous attacks; and sooner or later, owing to detachment of the retina, sight is completely lost. Such an eye is not only very disfiguring, but is also a serious menace to its fellow.

It cannot be insisted on too strongly that every eye, which is blind and painful, ought to be enucleated; for not only is it a constant source of trouble to its possessor, but it may, at any time, give rise to sympathetic ophthalmitis, or possibly become the seat of a malignant growth.

Dynamite Explosions.-Injuries to the eye from dynamite explosions form a class by themselves, and usually occur in healthy labourers, who, through carelessness in firing a shot of dynamite, receive part of the charge in the face. The skin is burnt and impregnated with grains of powder and sand. The lids are usually so much swollen that they cannot be opened voluntarily, and are separated by retractors only with difficulty. The surface of the cornea and sclerotic is bespattered with grains of powder, particles of grit, etc., and there is always more or less sub-conjunctival ecchymosis. There are great pain, intolerance of light, and lachrymation ; and in serious cases the iris and lens are injured through foreign bodies having penetrated into the interior of the globe. There may be rupture of the cornea and sclerotic, and in all cases the eye suffers severely from shock. Superficial lesions gradually clear up, but sight is often more or less permanently impaired from opacities of the cornea. Whenever the globe has been perforated, the foreign bodies which have been driven into the eye set up irido-cyclitis which may go on to panophthalmitis. Obviously under such circumstances there is no hope of saving the eye; and after a dynamite explosion, the prognosis ought always to be guarded, as, even although there may not be much apparent physical damage to the eye, the degree and effect of the shock cannot at first be fully appreciated.

Treatment.-The treatment consists in bathing the eyes thoroughly with hot sterile water, removing as far as possible all foreign particles, and instilling castor oil in which atropin and cocain are dissolved, into the conjunctival sac. The patient ought to be put to bed as soon as possible, antiseptic fomentations applied to the eyes, and starch poultices 
to the face; a smart calomel purge administered, and anodynes given, if necessary, to relieve pain and procure sleep. If there be a large lacerated wound of the cornea and sclerotic, it is wisest to enucleate at once. After all inflammation has completely subsided, it may be necessary, when vision is impaired from corneal opacities, to make an artificial pupil and tattoo the scars; at other times a cataractous lens requires to be extracted. 


\section{CHAPTER XXXV}

\section{SYMPATHETIC OPHTHALMIA}

Conditions in which sympathetic ophthalmia is likely to occur-Time of onsetSympathetic irritation-Sympathetic inflammation-Theories as to paths by which infecting agent travels from one eye to the other-Intractability of the disease in the sympathising eye-Treatment: prophylactic and curative.

THE true nature of sympathetic ophthalmia, the most dangerous disease to which the eye is exposed, is still obscure; but recent investigations have proved that it is due to micro-organisms; which have infected the injured eye. It is usual to speak of the injured eye as the "exciter," while the one secondarily affected is called the "sympathiser." The morbid changes in the former which are likely to give rise to sympathetic inflammation may be classified as follows-

I. Penetrating wounds of the ciliary region, accompanied by prolapse of the iris and ciliary body, are, above all others, the most likely to cause sympathetic disturbance, and all the more readily if the wound is lacerated and the instrument with which it was inflicted surgically unclean.

2. Foreign bodies lodged within the eyeball, more particularly if they lie near the ciliary processes, are a constant source of danger, since they keep up inflammatory reaction in the whole uveal tract.

3. Degenerative changes in an eye previously injured are always accompanied by a certain amount of irido-cyclitis; and consequently an atrophied globe, tender and irritable through calcification of the lens and ossification of the choroid, is a menace to the sound eye.

4. Corneal ulcers which have perforated may form the starting-point for a sympathetic ophthalmitis, but it is interesting to remember that an eye which has burst from within is not nearly so dangerous as one in which perforation has occurred from without. Moreover, all clinical experience goes to prove that after panophthalmitis the danger of a transference of infection from one side to the other is very slight.

5. Sarcoma of the choroid, or dislocation of the lens accompanied by plastic irido-cyclitis, may also induce sympathetic inflammation; but these are probably the only instances in which the disease arises apart from the existence of a perforating lesion of the "exciter."

The kind of eye which most frequently endangers the safety of the 
other is one which, in consequence of perforation by injury or ulceration, has been attacked by plastic irido-cyclitis, and has become soft and tender to touch. The length of time which may intervene between the injury and the onset of sympathetic inflammation is very variable. It probably never occurs earlier than a fortnight; but there is, after that, practically no limit to the interval which may elapse before it declares itself. On an average, it develops most frequently five or six weeks after the accident; but it is not wise to wait longer than a fortnight, unless there are signs of progressive amelioration of the symptoms in the injured eye. If the second eye escape until the one injured has healed, it will probably not suffer at all, unless fresh inflammation or degenerative changes occur in the exciting eye. In the latter case the attack may not take place for many years. If an injured eye heal quickly without much irritation it is not likely to set up sympathetic mischief.

The insidious nature of the onset of sympathetic ophthalmia is one of its most outstanding characteristics; for, although it is occasionally ushered in by severe pain and other symptoms of acute inflammation, it usually develops in a manner so treacherous that serious results are not anticipated until the disease is thoroughly established. It may make its appearance at any age, but is more frequent in the young than in the old, and particularly in children who are naturally delicate and of a highly neurotic temperament. When it has started it is extremely difficult to arrest. Even under the most favourable conditions, it runs a protracted course; and though recovery occasionally takes place, the result is very often total blindness. The destruction of the sympathising eye is, indeed, often more complete than that of the one which received the injury; and the knowledge of this fact adds greatly to the responsibilities of the surgeon, when he is called upon to treat a case in which sympathetic inflammation has fully developed, and where the exciting eye still retains a fair amount of sight.

It is always necessary to draw a distinction between sympathetic irritation and sympathetic inflammation; for the former is simply a neurosis, and passes off without leaving any organic changes; but the latter is plastic, and in the long run involves the whole of the internal structures of the eye.

1. Sympathetic Irritation is usually an early symptom, but is often met with in cases where the injured eye has been blind for a long time, and is undergoing degenerative changes. Its characteristics are: The patient feels that his eye gets soon tired; has difficulty in reading small print; and after prolonged work suffers from transitory attacks of dininess of vision when he looks at distant objects, or it may be from momentary total blindness. He feels uncomfortable in a bright light, which 
may cause neuralgic pains to dart through his head, and induce injection of the conjunctiva, accompanied by copious lachrymation.

If the field of vision be at this stage carefully examined by Bjerrum's screen, the blind spot in the sympathising eye will, in many instances, be found to have assumed a spindle-shape; and, without wishing to insist too strongly on the value of this objective sign in the diagnosis of impending sympathetic mischief, it seems highly probable that it denotes active congestion of the optic disc, which in a case of infected wound or of degenerative changes in one eye, may be regarded as a danger-signal heralding the approach of sympathetic disturbance in the other. At this stage, too, the patient sometimes is found to suffer from a low degree of myopia; and the co-existence of both signs greatly strengthens the diagnosis. This temporary myopia may be due to spasm, but it sometimes persists under atropin; when it can be explained only by supposing that an altered state of the media has been brought about by congestion of the choroid, whereby the refractive index has been increased. Whatever be the true significance of these signs, it is certain that both at once disappear after removal of the exciting eye, and that when for a time after enucleation, the blind spot persists in the form of a spindle, the patient also continues to complain that his vision is weak and uncertain, and that everything looked at appears "wavy " and " unsteady"; but all these symptoms pass, away as soon as the blind spot assumes its normal shape. Associated with the signs just mentioned, ophthalmoscopic examination commonly reveals congestion of the optic disc, and increased fulness and tortuosity of the retinal blood-vessels. It is, however, always a difficult matter to determine by the ophthalmoscope alone, when there is no healthy fundus for comparison, whether the optic disc is or is not congested; hence the value of the confirmatory evidence afforded by the coexistence of all these signs.

2. Sympathetic Inflammation.-This may or may not be preceded by the symptoms just described; failing sight usually is the first warning that the patient receives of the development of the disease. When the eye is examined, a zone of pink hair-like vessels is seen surrounding the cornea ; the iris is dull; and the pupil is small and sluggish, and dilates irregularly after the instillation of atropin. Even at this early stage there may be neuro-retinitis, with floating bodies in the vitreous; but more frequently there is difficulty in seeing the details of the fundus, owing to haziness of the media incidental to inflammation of the uveal tract. The cornea also becomes inflamed; spots, characteristic of infection of the ciliary body, form in its posterior surface-keratitis punctata; the aqueous is turbid, and the anterior chamber deep. The corneal signs are all the more marked when the inflammation assumes the serous 
type-serous irido-cyclitis-but the plastic form is by far the more frequent and more serious. Blood-vessels now develop upon the surface of the iris, whose substance thickens and bulges into the anterior chamber, the exudation filling up the pupil, and later on gluing the whole posterior surface of the iris to the capsule of the lens-complete posterior syncchiamatting the ciliary processes together, and implicating the choroid so extensively that the nutrition of the eye is gravely affected. As a result, the iris is retracted at its ciliary attachment; the tension diminishes; the lens becomes cataractous; and the shrinking vitreous causes detachment of the retina and of the anterior portion of the choroid. Up to this time there has been perception of light; blindness now becomes total. The eye is liable to recurrent attacks of irido-cyclitis; its blood-vessels degenerate, and rupturing, give rise to intraocular hæmorrhage; it steadily shrivels; and the final result is phthisis bulbi.

Mackenzie was the first to demonstrate that traumatic irido-cyclitis of one eye is capable of inducing destructive inflammation of its fellow; and he considered that the noxious influence was transmitted from the one to the other by the blood-vessels, by the ciliary nerves, or by the union of the optic nerves. The last hypothesis was the one which claimed his special favour, and he believed it "extremely probable that the retina of the injured eye is in a state of inflammation which is propagated along the corresponding optic nerve to the chiasma, and that thence the irritation which gives rise to inflammation is reflected to the retina of the opposite eye, along its optic nerve." Since Mackenzie's day many speculations have been advanced, and numerous experiments undertaken, to explain the pathogenesis of sympathetic ophthalmia. The most outstanding of these is the work of Leber and of his pupil Deutschmann. The former advanced the theory that sympathetic inflammation was the result of a septic infectious process in the injured eye, and that the microorganisms travelled along the optic nerves to the sound eye; the latter set himself to prove these theories by experiments conducted on rabbits. He injected fluid containing staphylococci into the sheath of an optic nerve, and traced the micro-organisms across the chiasma in their course along the optic nerve of the one eye to reach the optic nerve of the other. $\mathrm{He}$ asserted, further, that when the staphylococci reached the sound eye they set up in it a typical sympathetic inflammation. For a time the difficulties of explaining the origin of ophthalmia migratoria, as the disease was now called, seemed to be ended; but, unfortunately, subsequent investigation failed completely to verify Deutschmann's observations.

Both laboratory experiment and clinical observations go to support the theory that infection is necessary before a wound of one eye can be the cause of inflammation in the other. Indeed, if it were possible to 
avoid sepsis, sympathetic ophthalmia would be altogether prevented. Difficult though it be, it is desirable to relate the pathogenesis of sympathetic inflammation to the present-day knowledge of the pathology of inflammation as a whole, and if possible to place the one in line with the other. At the very outset, however, the peculiarities of structure and of function existing between the two eyes must be emphasised; for it seems probable that many of the difficulties incident to such an endeavour will lessen considerably, when due importance is given to the close nervous and vascular connections subsisting between the one eye and the other. Of all the paired organs of the body, the eyes are the most closely related, and so it is that the slightest injury to the one is almost immediately followed by sympathetic irritation of the other. Numerous experiments have demonstrated that irritation of the ciliary nerves of one eye produces dilatation of the blood-vessels of the other; but it is doubtful if any such irritation can increase the amount of albumen in the aqueous humour. Moreover, such nutritional disturbances, although they favour, will not of themselves set up, inflammation; consequently there must, in addition to vaso-motor changes, be another factor present before true inflammation is excited. There can be no doubt that this other factor is the micro-organisms which have entered the eye at the time of the injury, or subsequently through the perforation in the eyeball due to the accident. It is supposed that there is a micro-organism so minute that it has escaped detection up to the present time, but which is specific for the eye, and more especially for the uveal tract. Every day operation wounds are made in the eye, which, if aseptic, heal without the slightest trouble, and give rise to no risk of sympathetic inflammation. When, however, an operation wound is infected, it is as liable as any other to set up destructive sympathetic irido-cyclitis; and should the wound be in the ciliary region, disaster happens all the more readily. Up to this point the problem is quite simple; the real difficulty lies in demonstrating the channels of communication between the two eyes along which this micro-biotic factor finds its way. There are three such passages, the blood-vessels, the ciliary nerves, and the optic nerves. Some weeks usually intervene between the occurrence of the accident and the onset of the secondary trouble; and if there has been acute suppurative inflammation of the injured eye, there will probably be no sympathetic inflammation at all. This would imply that the condition of the "exciter" played a part in originating inflammation in the "sympathiser," apart from the presence of micro-organisms. The kind of eye most likely to excite sympathetic inflammation is one in which the acute symptoms have become quiescent, but which is tender on pressure and beginning to shrink. If such an eye be enucleated, and, after hardening in preservative 
fluid, be bisected, it is seen that there has been a great outpouring of exudation from the iris and ciliary region, and that this has distributed itself behind the lens capsule to form in some cases a complete partition between the vitreous and the aqueous chamber. The terminations of the ciliary nerves are embedded in this exudation, which, as time goes on, begins to become organised, and, contracting, compresses and drags not only on the embedded nerves, but also on the ciliary body and the anterior part of the choroid and retina. In very acute cases, the retina becomes detached completely, and extends like a fibrous cord between the inflammatory exudation in front and the optic nerve entrance behind. In some cases the ciliary nerves themselves show signs of neuritis, and in all a constant irritation is kept up by the traction produced by the shrinking exudation. As a result of this, vaso-motor disturbance is set up and maintained in the second eye, and this renders it all the more vulnerable. The " exciter" produces this disturbance in the "sympa-thiser " partly through ciliary neuritis, but mostly through reflex action; and the destruction of the ciliary nerves in panophthalmitis explains, in part at least, why no such change occurs after acute suppurative inflammation. Fuchs has given a careful description of the histological appearances in eyes affected with sympathetic inflammation, and has claimed to be able to diagnose the disease from the examination of a microscopical section. He has pointed out that this type of inflammation differs from that caused by ordinary pyogenic micro-organisms, in so, far that the uveal tract is infiltrated with lymphocytes, large endothelioid cells, and occasionally giant cells. This infiltration leads later on to great infiltration of the whole uveal tract.

Gradle has described certain changes in the blood to which he attaches a diagnostic importance; an excess of lymphocytes and especially of large mononuclear corpuscles. These blood changes are similar to those found in protozoal infections; but other observers have not confirmed the importance of those blood changes in the diagnosis of sympathetic ophthalmia.

It now remains to trace the course of the infection, which may be either the micro-organisms themselves, or the toxins which they have manufactured. Obviously, the most direct road for microbes to pass from one eye to the other is along the optic nerves connected by the chiasma, and along the lymph channels surrounding them; but that they take this road has not so far been proved by experiment, and clinical experience does not bear it out. In abscess of the orbit, where both optic nerve and lymphatics are implicated, sympathetic inflammation never occurs. Moreover, if the infection travelled along the optic nerve, the occurrence of papillitis ought to be the first sign of the disease in the 
sympathising eye; but that is the exception to the rule in sympathetic inflammation: it is the ciliary region which is first attacked. It would appear, then, that the infecting influence, be it microbe or toxin, reaches the sound eye through the blood stream; and that its virulence is concentrated locally upon the eye owing to the special vascular arrangements, and to the lowered resisting power of the ocular tissues. This last is due, in the sympathising eye, to vaso-motor disturbance in the vessels of the uveal tract brought about by the irritation of the ciliary nerves of the injured eye.

That there is a general toxæmia in cases of sympathetic inflammation cannot be doubted, when one observes the disease as it is usually seen in children. Previous to the onset the child is dull, listless, drowsy, and disinclined for food. It is fretful, although not suffering pain. The tongue is foul, the skin pale and somewhat clammy, and the pulse quick. All these symptoms, however, disappear after enucleation of the injured eye; and within forty-eight hours the whole appearance and demeanour of the child are completely altered. Such general symptoms are also, though rarely, seen in the adult. Elschnig has recently propounded the theory that sympathetic inflammation is an anaphylaxis, due to absorption of broken-down pigment cells of uveal tissue of the injured eye sensitising the uveal tissue of the sound eye, and that the anaphylactic reaction is set going by gastro-intestinal intoxication. That theory, however, is open to many objections.

Why is sympathetic inflammation so intractable, and why does it proceed after enucleation of the "exciter"? It is true that once sympathetic inflammation has begun, it pursues its destructive course quite uninfluenced by the injured eye. The part sympathetically attacked is usually, in the first instance, the ciliary processes, and it is upon the integrity of these that the nutrition of the eyeball for the most part depends. Whenever, therefore, they are seized in the grip of a plastic inflammation, the whole plan for the nourishment of the eye becomes upset, and the disease necessarily goes from bad to worse. The close anatomical and functional relation between the two eyes, and the delicate and peculiar anatomical structure of the ocular tissues, have much to do with the clinical peculiarities of sympathetic inflammation; for, after all, a morbid process is the same, whichever part of the body it attacks, and the results of a morbid process, while no doubt determined to a certain extent by the specific nature of the exciting cause, are also largely dependent on the anatomical structure of the organ affected. The clinical appearances usually associated with sympathetic inflammation are also seen in general uveitis, the result of tubercle or of auto-intoxication. Although different cases may present somewhat different clinical 
pictures, the special characters of the disease as regards onset, progress, and termination, are the result of ordinary pathological processes working in organs united to each other in the most intimate manner, and whose individual anatomical structure is wholly unlike that of any other organ of the body.

Treatment.- - It is no wonder that a disease so insidious in its onset, and so destructive in its progress, should prove most intractable to treatment ; and it must be admitted that after sympathetic inflammation has fairly begun, practically nothing can be done to check its progress, or to repair the damage which it has caused. The most effective treatment then must be prophylactic; and we know that in the stage of irritation, and even in the early stage of serous irido-cyclitis in the sympathiser, the removal of the "exciter" is followed by most beneficial results. Much of necessity depends upon the manner in which the injured eye has been treated immediately after the accident; and in the chapter on penetrating injuries of the eyeball a full description has been given of how eyes seriously injured may be saved, and sympathetic inflammation prevented. In many cases, however, enucleation is inevitable, and the following rules may serve as a guide--

I. Enucleate at once: when the injury is so severe that the exciting eye is destroyed hopelessly from the beginning.

2. Enucleate at once: on the slightest sign of sympathetic irritation, should the vision of the exciting eye only amount to a perception of light and darkness.

3. Enucleate at once: if a foreign body is present in, and cannot be removed from, the exciting eye, more especially if the eye is inflamed and tender on pressure.

4. Enucleate at once: when an injured eye is blind and suffering from recurrent attacks of acute inflammation; or when it is tender and irritable as a result of the onset of degenerative changes, e.g. ossification of the choroid.

5. Do not enucleate: when there is still sight in the injured eye, and when there is no sign of sympathetic disturbance in its fellow.

6. Do not enucleate: when sympathetic inflammation is in progress, and there is still sight in the injured eye; for under these circumstances the removal of the "exciter" will have no beneficial influence, and the probability is that in the end all the sight the patient will possess will be in the primarily injured eye.

Optico-ciliary neurotomy, simple evisceration, and, as suggested by Mules, evisceration and the implantation of a glass ball within the sclerotic, have all been proposed as substitutes for enucleation; but undoubtedly the last is the safest operation from the point of view of prophylaxis. 
Nevertheless, a period of four weeks must elapse before it is certain that the removal of the "exciter" has averted the onset of sympathetic inflammation.

In the treatment of the sympathetically affected eye, no operation ought to be attempted until all acute symptoms have subsided. The temptation to relieve the acute suffering during a glaucomatous seizure in the course of sympathetic inflammation is great; but any attempt to perform a sclerotomy, or to excise a piece of the iris, even if successful at the time, is followed by an accentuation of the symptoms, and by increased outpouring of inflammatory exudation. At the outset, we must rely on the treatment suited to cases of chronic irido-cyclitis. The eyes must be kept at rest, and this is best accomplished by shading them from the light and instilling atropin: If the inflammation be accompanied by great intolerance of light, lachrymation, and tenderness on pressure over the ciliary region, repeated leeching is useful in the early stages, but in the later, blisters on the temple and behind the ear are of more value. Pain, usually due to increased intraocular tension, ought to be relieved by the local application of heat, and by the judicious use of opiates, aspirin, or other sedatives. Turkish baths are always helpful, but the number and frequency of these must be regulated by the patient's ability to stand them. Large doses of salicylate of soda are strongly recommended (Gifford); but here again great care must be taken that they do not depress the patient unduly. On the theory that the infecting organism is a protozoon, neo-kharsivan by intravenous injection has been advocated, and its use is often followed by encouraging results. In all cases the drug to rely on is mercury, and this must be given until its physiological effects become apparent. It may be administered by the mouth, by subcutaneous or sub-conjunctival injections, by the calomel vapour bath, or by inunction. Of all these, the last is probably best, and $\frac{1}{2}$ to 2 drachms of mercury-vasogen should be rubbed well into the skin of different parts of the temple and brow every night. The action of the mercury is intensified by iodide of potassium, which may be given in Io or 20 grain doses once a day after meals. It is very important, however, during this severe antiphlogistic treatment, to attend carefully to the patient's general health. He must be well fed, and appetite promoted by tonics, especially those containing quinine and iron. In a few cases recovery of sight may take place as a result of this treatment; but as a rule the most that can be hoped for is that the active symptoms will subside, leaving the iris firmly adherent to the capsule of the lens, which in all probability is now cataractous. After a sufficient time-a year or eighteen months-has elapsed, and if, during that interval, there has been no recurrence of inflammation, the intra- 
ocular tension has not diminished, and the patient's perception of light is satisfactory, operative interference may be considered. If it be resolved on, the iris should as far as possible be left alone. The lens must be got rid of, either by needling in the manner suggested by Critchett, or, in more favourable cases, by drawing it off with a curette after the toughened capsule has been divided with a knife. Once the cataract has been removed, an iridotomy may open up the pupil sufficiently to allow light to enter the eye, and enable the patient to see as well as the damaged state of the retina will permit. In operating on such eyes, it must always be remembered that the vitreous is quite fluid and escapes readily; therefore it is important that all incisions should be as small as possible and made wholly in the cornea. After operation, the eye must be carefully bandaged and the patient kept quiet in bed in a dark room; while the local application of ice, and the administration of sedatives internally, will do much to prevent the occurrence of inflammatory reaction. 


\section{CHAPTER XXXVI}

EYE INJURIES, WITH SPECIAL REFERENCE TO THE WORKMEN'S COMPENSATION ACT, I906

The Workmen's Compensation Act of I906-Necessity of careful examination and note-taking-Pre-existing or acquired disease as a complication-Estimation of the damage to sight from an economic point of view-The visual acuity -The field of vision-Binocular vision-Muscular defects-Sympathetic ophthalmia as a complication-Questions involving operation-Malingering.

THE beginning of what may be termed modern State regulation of the relations between employer and employed dates from the beginning of the nineteenth century, when an Act was passed imposing on employers certain obligations for the better housing, clothing, and education of apprentices. From this small beginning legislation has, in more recent years, advanced with rapid strides, and the duties and responsibilities of employers have not only been more explicitly defined, but have been greatly extended. The Employers' Liability Act of 1880 merely extended the general principle that fault or negligence rendered a master liable in damages for any injury thereby sustained by an employee, by depriving the master in certain cases of the defence that the fault was the fault of a fellow servant. It was, therefore, quite a new departure when, under the provisions of the Workmen's Compensation Act of 1897 , the employer was made liable for compensation for personal injuries by accident to an employee, though no one could be held to be at fault. The benefits of this Act were first restricted to certain specified trades, but in Igoo were extended so as to include agricultural labourers; and the Workmen's Compensation Act of I906, which repealed the other two and took their place, is so wide in its scope that it practically affects or may affect, in some way or other, every class of the community.

For the successful working of the Act the co-operation of medical men is indispensable; and it is therefore important that every doctor should make himself familiar with its chief clauses. Briefly put, the main provision is, that if an employee receives an injury " arising out of and in the course of the employment," the employer is legally bound to pay him half his weekly wages and to continue this payment until he is able to resume work. The Act merely provides compensation for loss of earnings. It, as the late Sheriff Glegg pointed out, does not guarantee 
or ensure re-employment after injury: Unless the workman be disabled for a week, he cannot make any claim; but if the results of his injury inçapacitate him for more than that time, he can claim compensation from the day on which he was hurt. The Act also covers disablement arising from certain scheduled diseases, termed industrial diseases, among which may be mentioned miner's nystagmus and bottle-maker's cataract. If a workman contract one of these in the course of his employment, he can claim compensation in exactly the same way as if his incapacity had been caused by an accident. All employers are liable under the Act; and all workers (sailors, agricultural labourers, and domestic servants included) are entitled to compensation, provided that in the case of those who are not manual workers the total sum of their weekly earnings does not amount to more than $£ 250$ in the course of a year. The Army, Navy; and the Police Services are excluded from the benefits of the Act, as also are outworkers-i.e. persons to whom articles or materials are given out to be made up, cleaned, washed, etc., in their own houses, or in other premises not under the control of the person who gives out the material.

The design of the framers of the Act was, undoubtedly, to minimise the need for legal procedure; but their purpose in this respect has not been achieved, for no statute of recent times has been more provocative of litigation. Injuries to the eye are frequent causes of dispute, because, on account of the great delicacy of the ocular tissues, they are so often followed by deterioration or loss of vision. If a wound heals perfectly, the presence of a scar in any other part of the body is not, as a rule, of much consequence; but in the eye, if the functional integrity of the organ is to be perfectly preserved, there must be no scar at all.

In the cases that come before the courts the issue is frequently decided on the medical report; and as the surgeon who has examined a case may be cited to a court of law to give expert evidence, it is of the greatest importance that he carry out his examination from the very first in a methodical and systematic manner. He ought also to take full notes at the time, and preserve them carefully for future reference; because it is the original notes, and not a copy, that he will be allowed to use to refresh his memory in the witness-box. The patient ought to be examined as soon as possible after the accident, before he has had time to recount his symptoms to companions, who may tempt him to exaggerate, or even to deceive. It is always well in the first place to ascertain clearly all the facts of the case-exactly how the accident happened, what caused the injury, and, if the cause be a body projected from without, the nature of the missile, the direction and distance from which it could have come, and the probable velocity with which it was travelling. The tension of 
the eyeball ought next to be estimated, and the vision tested. Afterwards there should be a careful examination, in regular order, of the orbital margins, the eyelids, the conjunctiva, and the cornea; and diligent search should also be made for any sign of perforation of the globe. The colour and general appearance of the iris, and the position, size, shape and mobility of the pupil, should be noted and contrasted with those of the sound eye. Unless the presence of blood in the anterior or posterior chamber prevent it, an ophthalmoscopic examination should never be omitted; for by this means any alterations in the lens and vitreous, and in the deeper structures of the eyeball, can at once be detected. It will be of the greatest advantage to the surgeon to acquire some knowledge of the trade of the workman on whose injuries he is requested to report. It is all-important for him to be sure of his facts, and to be in a position to make his examination as exhaustive as possible; for the penetrating art of cross-examination may find out omissions and discrepancies where none were supposed to exist. It is usually the aim of the opposing counsel to show up imperfections and contradictions in the medical testimony; and the conflicting nature of expert evidence is often alluded to by lawyers. An eminent sheriff, now deceased, once told me that he frequently added to the note to his judgment that the " medical evidence in the case was, as usual, very unsatisfactory." In this connection, however, it must be remembered that a medical opinion cannot be exact in the sense in which a legal one is, for in law there is no personal equation. In medical matters, on the other hand, every one will admit that one person may be much more seriously affected by any given injury than another. There can never be absolute certainty. An expert opinion can only be founded on the doctor's knowledge of the facts, and his personal experience of similar cases; and whenever several causes may contribute to bring about a result, a dogmatic statement on oath is impossible. The doctor can only say what in his opinion is likely to be the case; he cannot be certain. Moreover, the difference in the statements in medical reports is often more apparent to the layman than it is to a doctor, whose professional knowledge may enable him to reconcile statements which at the first glance seem contradictory. The unsatisfactory character of medical evidence would,-therefore, be less frequently commented on, if medical men were careful to make sure of their facts, and to write their reports in clear, simple language, avoiding as far as possible all technical terms. If they would merely, for example, describe a man as having a black eye, and not write of him as suffering from subconjunctival ecchymosis or of extravasation of blood beneath the cuticle. The object of a medical witness is not to win a case for the one side or for the other, but to state the truth, the whole truth, and nothing but the 
truth, in order that by his testimony strict justice may be meted out to. master and servant alike.

The liability of the employer is so well defined by the Act that, after an accident has been duly reported, the recognised amount of compensation is usually paid at once, and continued regularly till the workman is able to resume his employment. It is only after he is thought to have recovered from the effects of the injury, and refuses to go back to work; or when, after six months' incapacity, a dispute arises over the amount of the lump sum the employer is entitled by the Act to offer in lieu of a continuance of the weekly payments, that the services of the Court or of an arbiter are necessary. The medical expert may then be called upon to say whether the injured man is fit for work; and, if not fit, to give an opinion as to whether the results of the injury are likely to be temporary, or to lead to permanent incapacity. He may also be asked to say what kind of occupation he thinks the workman can follow, or to determine the loss to his earning ability which has resulted from the accident. In this connection, however, it must be remembered that a man's capacity for work is not synonymous with his ability to earn wages. A man may be physically quite fit for work, but unable to find employment. His earning ability, therefore, depends not only upon his health, but also upon his technical skill, and upon the state of the labour market. The assessment of compensation for partial incapacity is really a matter for the county court judge or the sheriff; but, naturally, he expects guidance from the medical expert regarding not only the kind of employment the workman can undertake, and the conditions under which he can' work with safety, but also the degree to which his technical skill has been lessened in consequence of the injury, and what probability there is that he will in time recover his former capacity. The surgeon will also be expected to say how far the results of an injury have been modified by pre-existing disease. He must distinguish clearly between lesions that have previously existed in an injured eye, and those which are directly the result of an accident.

A man may have an eye the sight of which is congenitally defective, and yet know nothing about it until dimness of vision makes its appearance after some injury which in itself may have been very trivial. In these circumstances, careful examination may reveal the existence of a high degree of astigmatism or of myopia, or of pre-existing disease of the optic nerve; and so all doubt as to the real cause of the defective sight will be completely removed. Then again, an injury, in itself trivial, may become very serious when complicated by blennorrhœa of the tear passages; and the healing process will always be retarded by such general diseases as glycosuria or albuminuria, either of which may of itself give 
rise to grave disturbance of sight. If, then, a workman suffering from either of these disorders, happens to meet with an injury which is followed by defective vision, he will naturally attribute the failing sight to the injury, and claim compensation for a condition arising from disease and not from the accident.

The following are a few personal experiences of the difficulties that may present themselves to the medical expert in writing a report, or in giving evidence in a case where the effects of an injury have been modified by acquired or constitutional disease. I have known a chancre of the lid to be caused by the well-intentioned efforts of a workman suffering from syphilis to remove with his tongue a foreign body from the eye of a companion. I have seen a man lose an eye through gonorrhœal infection following a comparatively trivial injury; and in patients affected by hereditary syphilis it is by no means uncommon to find severe interstitial keratitis supervene on some slight wound. In high myopia the results of accident are often much more serious than they would be in a normal eye. I remember a man, on whose case I was asked to report, who said that when stooping to get something out of a box, the right side of his forehead accidentally came in contact with a projecting shelf. The injury in itself was exceedingly slight, but the patient was highly myopic, and the result was a detachment of the retina, which gradually increased and gave rise to permanent blindness of the right eye. Malignant disease is frequently attributed to injury, and a sarcoma of the choroid is often supposed by the patient to be of traumatic origin. The medical expert, however, in such a case would have to point out that the condition could not arise from the injury alone, which at most could only be an exciting cause in a person predisposed to the disease. Lastly, I recently saw a man in whose case an injury to the forehead had been followed within a day or two by complete ptosis, and later by paralysis of all the branches of the oculo-motor nerve on the same side. The patient naturally attributed the symptoms to the injury; but careful examination revealed the presence of Argyll Robertson pupils, atrophy of the optic nerves, and absence of the patellar-tendon-reflex on his right side. In this case the injury at most accelerated the onset of the oculo-motor paralysis, which was really merely a sympton of pre-existing tabes dorsalis.

In recent years, various attempts have been made by different authorities to express by means of a mathematical formula the loss of the earning ability occasioned by visual defects; but, as Evans justly observed, " no formula, however complicated, could possibly include such important factors in the estimation of compensation as the intelligence and perseverance of the patient, the work, the state of the labour market, the prejudices of employers and employed, etc.; " and yet, if a just 
decision is to be arrived at, these are factors which cannot be overlooked.

In the estimation of the damage to sight from the economic point of view, the most important factors to be considered are the acuity of vision, the field of vision, binocular vision, and muscular defects.

The visual acuity is most important. This is measured by the patient's ability to read standard test types at a given distance. This distance is fixed at 6 metres, or 20 feet, because the rays coming from an object are then, for all practical purposes, parallel, and the examination is thus not complicated by the accommodation. It is often more convenient, however, in testing those with very defective sight, to employ a simpler test object; for example, to ask the patient to count the outstretched fingers held against a black coat. If he cannot do so, the surgeon should try if he can distinguish hand movements; and if he again fail, trial should be made, if a light can be distinguished when held in front of the patient's eyes. In carrying out the test with Snellen's types, any error of refraction must first of all be corrected, since an ametropic eye without glasses gives the relative and not the absolute acuity of vision, and consequently the result is no measure of its general usefulness. The examination of the visual acuity is completed by asking the patient to read small type, and, if necessary, spectacles suitable for his age and refraction must be used.

It is a great convenience to express visual acuteness in a fractional form $\left(\mathrm{V}=\frac{d}{\mathrm{D}}\right)$, for this method is employed and understood by ophthalmologists all over the world. When recording visual acuity in a medical report, however, it is very misleading to look on this form as an ordinary vulgar fraction, and to say, for example, that six-twelfths or six-twentyfourths is equal to a half or a fourth of the normal. A layman, reading such a statement as that, would inevitably come to the conclusion that the patient's vision for his work was much worse than was actually the case, for it should be remembered that what is regarded scientifically as normal vision, is determined according to a higher standard than that required for efficient working or wage-earning acuity. The farmlabourer can perform his duties quite satisfactorily although he has much less sight than would be necessary for a skilled mechanic. It would simplify matters if a list of employments could be drawn up, with a corresponding list of the minimum visual acuity required for each, similar to the visual requirements published for the Army and Navy; but, obviously, this is not practicable. We must content ourselves, therefore, by dividing all workmen into two main classes-first, those whose avocation demands a high degree of visual acuity-for example, $\mathrm{x}$ 
professional men, skilled mechanics, etc., and, second, those who from the nature of their employment can work satisfactorily with a less degree of visual acuity-for example, carters, labourers, etc. Even for the first of these, however, the visual standard required is lower than that acknowledged as the normal scientific standard. Würdemann has suggested that the visual demands of the first class should be fixed at from three-fourths to one-seventh of the scientific standard, and of the second class at from one-half to one-twentieth; but even if one accepts this wide range as a working basis, individual exceptions will constantly be found. The minimum acuity must always vary within wide limits. Fergus has cited a number of cases where men with a very low degree of visual acuity have earned full, or nearly full, wages, as colliers, ironturners, boiler-makers, blacksmiths, engine-fitters, etc.

I remember a miner who was so blind from choroido-retinitis that he had to be led by his son to his work; yet he earned full wages. This, however, was before the Act of I906, and, undoubtedly, the man exposed himself to risks which were unjustifiable. Every practitioner must know of cases where the eyes have been damaged in early childhood, and yet the persons concerned, with a very inferior visual acuity are doing work, which, according even to Würdemann's classification, should be beyond their power. After all is said, however, it must not be forgotten that, in the case of a young person determined to follow a certain avocation, the patient's age, his brain-power, his character, and his perseverance are factors which are specially important; but in the case of a man who, accustomed to work with good sight, suddenly suffers marked deterioration of vision as the result of an accident, the conditions are quite different, and the same factors do not have the same value.

The field of vision can be mapped out accurately only by the help of - a perimeter; but the surgeon can obtain much information by the simple means of measuring the field roughly with his hand, and comparing that of the patient with his own. When, however, a case is likely to be taken into court, the medical expert should make a full chart, which can be produced if necessary, and demonstrated to the judge. The entire field of vision is divisible into a central, and a peripheral portion. The former is represented by a very small part of the whole, and its size corresponds roughly to that of the thumb-nail held at arm's length. If, however, this small area be destroyed, the patient is quite unable to read, although he may be able to see his way about with comparative ease. An injury, therefore, which would ruin a clerk or an engraver, might leave the unskilled labourer little damaged so far as his working capacity is concerned.

Dehenne and Bailliart have pointed out, that peripheral restriction 
of the visual field is relatively of more importance to coachmen, labourers, etc., than to those who, like skilled mechanics, require a higher standard of visual acuity for their work. This fact should be borne in mind when one is trying to estimate the loss of earning capacity. Another fact of equal importance, in the same connection, is that two-thirds of the binocular field belong to both eyes, so that even if one eye were blind, the restriction in vision would amount to only about one-sixth of the whole. Loss of the temporal or nasal portions of each field does not interfere much with working capacity; but homonymous hemianopsia impairs the earning ability according to the patient's occupation, and according to whether it is accompanied or not by diminution of the visual acuity, or by loss of binocular vision.

Binocular Vision.-The value of the two eyes together is very much greater than the sum of the value of each separately. Dehenne and Bailliart have classified all workmen according as they require good binocular vision or an extensive field of vision; and place in the first category all those who, when they become presbyopic, require glasses for their work. On binocular vision depend, to a large extent, our ideas of solidity and distance; and in many occupations it is, therefore, of the first importance. The simple test devised by Roche, and the diaphragm test described by Bishop Harman, afford very simple methods of demonstrating the presence or absence of binocular vision; but there are other means, such as Snellen's test, or the ordinary stereoscope, which may be employed. Binocular vision must clearly be at once lost when a patient becomes blind of an eye; and its economic value has been much discussed in connection with those cases where a workman has lost an eye through accident. The Workmen's Compensation Act does not, as has been already said, indemnify a worker for the loss of his eye, or, indeed, for any physical damage he may sustain, but merely makes adequate pecuniary provision for him during the time he is unfit for his employment. The question, therefore, which is frequently debated in the Law Courts, is whether a man with one eye is as fit for work as a man with binocular vision. The just answer to that question depends both upon the man himself, and upon the nature of his work; and, consequently, each case must be considered on its own merits. In every trade numerous examples of one-eyed men earning full wages are to be found; but all of them will admit that, to begin with, they experienced more or less difficulty in estimating the position of objects, and in calculating distances. To some workmen, the adapting of themselves to the altered circumstances seems almost like a new apprenticeship; some, owing to their temperament and perseverance, overcome the difficulties in a very short time; while others require considerably longer; and a few, owing to nervous- 
ness and faults of character, never obtain proficiency under the new conditions. The difficulties must obviously always remain greater if a man has to work among machinery, or on scaffolding, or underground, or where the light is insufficient. From the employer's point of view, the man with one eye does undoubtedly run more risk of injury than the one with two; and consequently, in estimating the wage-earning ability of the one-eyed man, this prejudice on the part of the master ought always to be taken into account. Provided the workman has possessed two good eyes, the accidental loss of one in no way impairs the visual acuity, and only slightly restricts the visual field. The diminution of working capacity, therefore, depends almost wholly on the loss of binocular vision.

Würdemann says that one year is a sufficiently liberal allowance of time to give to any one for the adaptation of monocular vision to the demands of his work; and states that " approximately a one-eyed person has lost 30 per cent. of his earning ability for the first year after the accident, and 20 per cent. afterwards for the higher class of trades, and for the lower class the proportion would be 27 per cent. for the first year, and I\& per cent. thereafter." If blindness of one eye develop gradually, the workman has time to adapt himself to the changes incident to the loss of binocular vision, and if he has lost an eye in early childhood, he grows up practically unconscious of his defect; but it is a very different matter when the loss of sight on one side occurs suddenly in adult, or more especially in advanced life; and naturally these are the cases which most frequently give rise to claims for compensation.

A question occasionally arises as to the relative value of one eye over the other. Statistics prove that the left eye is the one more frequently injured, and workmen themselves attach more importance to the right eye, probably, as Evans has suggested, because most of them are righthanded. A carter, however, would form an exception, because when his cart is loaded he always leads his horse on the near side, and naturally turns to the left when he hears vehicles approaching from behind, hence the loss of the left eye is a more serious matter for him than the loss of the right would be.

Double Vision.-Paresis or paralysis of an ocular muscle causes double vision, and occasionally arises from accident. As long as the diplopia persists, the patient is unfit for work; and if he tries to adjust matters by covering up one eye, he puts himself in exactly the same position as if he were a one-eyed man.

Penetrating Wound.-An accident involving a penetrating wound of the eye is always a matter of concern, because the doctor's ordinary sense of responsibility is intensified by the knowledge that the injury 
may be followed by sympathetic inflammation of the other eye, and that this may be so severe as ultimately to cause blindness. The medical expert has rarely anything to do with the treatment of the patient; but, both in his report and in his evidence, he is expected to give an opinion regarding the amount of damage to sight which is likely to result from the accident; and the prognosis in connection with penetrating wounds can, in most instances, neither be certain nor satisfactory, as long as there is danger of sympathetic disease.

Sympathetic Inflammation.-When sympathetic inflammation has fairly begun, practically nothing can be done to check its progress or to repair the damage which it has caused; treatment, to be effective, must be prophylactic; and we know that in the stage of irritation, and even in the early stage of serious irido-cyclitis in the previously healthy eye, the removal of the one that has been injured is followed by most beneficial results. Much of necessity. depends on the manner in which the eye has been treated immediately after the accident, for we know that by skilful treatment eyes seriously injured may be saved, and sympathetic inflammation prevented. In many cases enucleation is inevitable. It may happen, however, that a workman threatened with sympathetic mischief refuses to have the injured eye enucleated, until it is too late to save sight. In these circumstances, the employer may be placed in the position of having to make provision for life for an employee; whereas, if the man had followed the surgeon's advice and submitted to enucleation, he might have been able to resume his work as a one-eyed man. It is hard, legally, to compel any one to part with an eye; but if, as a result of such a refusal to follow a surgeon's advice, sympathetic mischief ensue, then the workman, or, if he be a minor, his guardian, ought to sign a written declaration accepting full responsibility for the decision.

When such a document is produced in court, the judge should have power not to penalise the employer for his servant's wilful disregard of competent advice. Under such circumstances, the medical evidence would turn largely on what is authoritatively considered the recognised treatment of the case; and the expert requires to be very wary lest the counsel for the workman entrap him into saying anything which appears to cast discredit upon a fellow practitioner. He will also be expected to give his opinion whether the workman was acting reasonably or unreasonably in refusing operation; whether the operation was likely to be successful; and whether any, and if any then how much risk to life or danger to health would attend it. In no case should a medical opinion be given, unless the doctor has had an opportunity of making himself absolutely sure of the facts. 
Traumatic Gataract.-The question of operation may again arise if the workman suffer from traumatic cataract. The medical expert may be asked in court whether operation would be desirable, and whether the extraction of the cataract would restore the man to his former efficiency. The answer will depend on the amount of damage to the other tissues of the eye, as well as upon the occupation of the workman. If binocular vision be necessary for the satisfactory performance of his duties, surgical interference will be of little value to him; because, even although the operation were quite successful, full vision could only be obtained by wearing strong convex spectacles. Consequently single vision with both eyes open would no longer be possible. If, on the other hand, the workman depend on a wide visual field, extraction of the cataract will be of decided advantage to him. It will remove the blind side; and obviously to a coachman or to a carter this must be a great gain. It is well known that bottle-makers suffer from cataract more frequently than other persons; and when this affection was added to the list of industrial diseases for which compensation could be claimed, it was explicitly stated that " the compensation should be made payable only in cases where an operation is undergone, and for a period not exceeding six months." A dispute may arise as to whether the cataract is really due to an injury or not, and the medical expert will be expected to settle the question. If the fact of an injury can be proyed, and more especially if the patient be young and the cataract confined to one eye, the probabilities are all in favour of its traumatic origin; and this will become a certainty, if the iris of the affected eye is tremulous, or there is a scar on the cornea, or any other sign of injury to the globe.

Malingering.-In every case in which a patient complains of failure or loss of sight, but in which there is no sign of injury to either the superficial or deep structures of the eyeball, a very guarded prognosis ought to be given. In the absence of any visible lesion, the surgeon is wholly dependent upon the patient's testimony, which may be true or false. In such circumstances, he ought to examine the eyes repeatedly, in order that he may either be assured of the truth of the statements made to him, or able to put an end, as promptly as possible, to any attempt at malingering. The framers of the Act doubtless foresaw that the liberal privileges which it conferred might tempt some to try to obtain the benefit for an undue length of time; therefore they inserted a clause making it incumbent on the workman to submit himself for examination by a duly qualified medical practitioner, provided and paid by the employer. They protected the rights of the master still further by making it legal for him, in the event of the workman refusing to submit himself to such an examination, or putting obstructions in the way of its being made, to 
suspend payment of the weekly compensation until the medical examination had taken place. The workman may refuse to be examined by the doctor employed by the master if he thinks fit; but he cannot both object to be examined, and at the same time enforce the weekly payments. When a medical expert is consulted in these circumstances, he requires to take great care not to cast doubt on the veracity of a patient who is really suffering from defective sight. Cases of traumatic amblyopia undoubtedly occur; and it may be years after the accident before degenerative changes in the optic nerve can be detected. While, however, the surgeon should always be anxious to give the workman the benefit of the doubt, he must not shut his eyes to the fact that there are people who are unable to resist the temptation to make the most of an accident, and who, in striving to obtain greater pecuniary gain, make statements regarding their eyesight which they know to be false. To detect such deception is one of the most obvious and important duties of the surgeon. When the compensation provided by law is supplemented by sick allowance from one or more Friendly Societies, the workman may find himself so comfortable in idleness that he is in no hurry to resume his work. Prolonged idleness is good for no one; and in the case of the workman it is utterly demoralising. He becomes inclined to lounge and smoke all day, with the result that compensation is occasionally being paid when the defective sight is due, not to the injury, but to tobacco amblyopia. At times, in order to deceive the doctor, he applies irritants to the conjunctiva, or tries to make him believe that lesions of old standing are the direct result of a recent injury. It is in the interests, not only of the employer, but also of the employee, that work be resumed as soon as possible. The performance of regular duties has always a distinct therapeutic value; and even although partial incapacity persist, it is better for the man to engage in some light occupation than to remain idle. If a man has lost an eye, or has had the sight of one eye seriously and permanently damaged, he, as a rule, refuses to return to work, unless he receives a lump sum in lieu of the compensation to which he considers himself entitled. The Act, however, allows nothing in the nature of a solatium - an employer is not responsible for the results of an accident, except to the extent to which it produces physical incapacity. Much litigation would be avoided if a specified sum, varying in amount according to the workman's occupation, were fixed as the compensation to be paid in the event of the loss of an eye. A considerable proportion of the cases that now occupy the time of the courts would then be settled without legal procedure. Even in ordinary circumstances, the capacity for selfdeception, especially in things medical, is great with most people; consequently, it is not strange that the mental strain incidental to any 
medico-legal inquiry connected with the settlement of a claim for damages, tends to make those who have no wish to deceive give an exaggerated account of their symptoms. No sooner, however, in such cases, has the amount of compensation been fixed than recovery begins. The medical expert must, therefore, always be on the alert, and by close inquiry and careful examination he will rarely fail to arrive at a just decision. It is not always so easy to deal with the real malingerer. In many instances, he is well acquainted with all the usual tests of vision, and is an adept in the art of deception. When the pretended blindness is said to exist in only one eye, the diaphragm test previously referred to is of great value in exposing the fraud. The certainty of the diagnosis depends, however, upon the degree of blindness which is simulated; and when a malingerer pretends to be blind in both eyes, or possesses only one eye, tests are not of much assistance; and the deceit can be found out only by keeping a careful watch over the pretender, so as to catch him when he is off his guard. 


\section{CHAPTER XXXVII}

LONG SIGHT, SHORT SIGHT, AND ASTIGMATISM, AND THE PRESCRIBING OF SPECTACLES

Apparatus required-The pinhole test-The optical structure of the eye-Accommodation-Presbyopia-Failure of accommodation due to disease-Hypermetropia as a cause of disability for near work-Its diagnosis and treatment -The use of cycloplegics-Myopia : its diagnosis and treatment-Spasm of the ciliary muscle causing low myopia-Astigmatism: varieties, diagnosis, and correction-High hypermetropia-Insufficiency of convergence.

THE public have an idea that they must either consult a specialist or go to an optician when they require spectacles, but there is no reason why a general practitioner should not be able to prescribe for a large number of his patients who suffer from simple errors of refraction. Doubtless he will prefer to send the difficult cases, and the patients who are suffering from nervous and other symptoms due to eye-strain to an ophthalmic surgeon; but he should himself be able to treat cases of ordinary hypermetropia and myopia, and moderate degrees of astigmatism. If, however, he wishes to undertake that work, he must provide himself with an ophthalmoscope, a small box of lenses, and a set of standard test types. Such an equipment does not cost much, and can be obtained from any reliable optician. The following list of the apparatus required is not sufficient for one who may be called upon to deal with any and every kind of error of refraction, but it will be found sufficient for any one who wishes to determine whether symptoms complained of by a patient are due to the need for glasses.

Apparatus required-

I. An ophthalmoscope. It is wisest to get a good one (Morton's). It will last a lifetime. If an electric light be fitted to the instrument, most of the difficulties in using it are avoided.

2. Card of test types (Snellen's)-for use at twenty feet or six metres. It is advisable to have more than one set, because some patients learn the letters very quickly.

3. Test types for examining vision at the ordinary reading distance.

4. A box containing a double set of convex and concave spherical lenses from $50 \mathrm{D}$. to $6 \mathrm{D}$. : single convex and concave cylindrical lenses from 25 D. to 2 D. : a prism of 6 degrees; a black disc perforated by a 
pin-hole and another with a stenopæic slit; an opaque disc; a red and a green glass; a Maddox rod; and a double grooved trial frame.

A practitioner with a little leisure and provided with that simple equipment ought to be able to treat errors of refraction, with great satisfaction to his patients and not a little credit to himself. The initial difficulties are most easily overcome if a short course of instruction be taken at an eye clinic, but if a doctor be on the outlook for those cases he will soon gain considerable experience among his ordinary patients.

When a patient complains of defective sight, the first question that arises is whether the failing vision is due to a structural defect or to an error of refraction. That, of course, can at once be determined by an ophthalmoscopic examination; but the following simple test, within the reach of all, is sufficient for practical purposes. First discover, by testing each eye separately, which line of the test types the patient can read; then give him the black disc perforated by a pinhole from the test case, and ask him to hold it closely to one eye, the other being closed. If, when he looks through the pinhole, sight is improved, he is certainly suffering from an error of refraction, for had there been any want of transparency of the media, or any defect in the retina or the optic nerve, sight would not be improved, but in all probability made worse by the pinhole test. Patients who suffer from an error of refraction may be conveniently divided into three classes: (I) Those who see well at a distance, but who have difficulty in seeing near at hand; (2) those who can see quite easily to read or to sew, but cannot see in the distance; and (3) those who have difficulty in seeing both near at hand and in the distance.

In order to determine what is wrong with a patient he has placed in any of those three categories, the physician must recall a few simple facts bearing on the eye considered as an optical instrument. The eye may be regarded as a living photographic camera, provided with a dark chamber of a definite length, but with an adjustable lens system, and a diaphragm whose opening varies in size according to the brightness of the light. If vision be clear on the horizon, when the eye is in a state of rest parallel rays are brought to a focus on the retina, and the condition is called emmetropia; whereas, if parallel rays come to a focus behind the retina, the condition is hypermetropia; and if in front of the retina, myopia. The cause of an error of refraction, therefore, is in the great majority of cases due to the length of the eyeball. The emmetropic eye being regarded as normal, the hypermetropic is too short, and the myopic too long. Obviously alteration in the curvature of the cornea, of the refractive index of the media, or of the position of the retina, or the absence of the crystalline lens, will also produce an error of refraction; but these are only the exceptions to the general rule, and no further 
reference need be made to them at present, except to point out that unequal curvature of the cornea always gives rise to astigmatism. The normal or emmetropic eye, moreover, can not only see distant objects distinctly without effort, it can with equal ease see to read or to sew. To meet the needs of near vision, the refractive power of the lens is increased. That inherent focussing arrangement is called the power of accommodation, and is brought into play by the action of a small muscle in the interior of the eye called the ciliary muscle. When it contracts, the lens becomes more convex, and consequently its power of refraction is increased. By that means, therefore, every eye possesses the power of adding to its static refraction a definite amount of dynamic refraction, by which it is enabled to see at varying distances-from the horizon to the nearest point of distinct vision. This near point, which may be regarded as the ordinary reading distance, $i . e$. about ten to twelve inches from the eye, is always in the same position in all emmetropic eyes of the same age. The greatest power of accommodation which can be exercised is spoken of as the amplitude of accommodation, and as it steadily decreases as age advances the near point gradually recedes from the eye. By the time an emmetropic patient is forty-five to fifty years of age, the near point is so far distant that he is quite unable to read, although he can still see distinctly on the horizon. It is this diminution of the range of accommodation as a result of age, and the difficulty in seeing small print at the ordinary reading distance, which are the distinguishing marks of presbyopia, or aged sight.

x. Those Who SEe Well in the Distance, but have Difficulty IN SEEING NEAR AT HAND.

It is common knowledge that sight for near objects fails with the advance of years; nevertheless presbyopia often reveals itself somewhat suddenly, and patients are considerably alarmed when they first discover that they can no longer read fine print or thread a sewing needle. It is usually, however, not difficult to reassure those patients, because in a simple case of presbyopia, distant vision, tested by Snellen's types, is found to be up to standard, i.e. 6/6; and a weak convex glass at once restores the power to read the smallest print. The spectacles make up for the deficiency caused by the failing power of the ciliary muscle, and the hardening of the lens from age. It is very important that they should not be too strong; for if so, the patient will have to hold his book very close to see distinctly, and will grumble at this inconvenience; he will also suffer much discomfort from the strain thrown on the internal recti muscles in their efforts to keep up the excessive convergence necessary for such a short focus. It is always wise to determine the ordinary reading or working distance of the individual patient, and to adjust the spectacles 
accordingly. Reading glasses should never magnify the print, for if they do so they are too strong; all that is wanted is that the type is clear and sharp in outline when held from ten to twelve inches from the eyes.

A presbyopic patient needs stronger glasses as time goes on, and as a rule will require to change them every four or five years, about + I $D$. being added each time. When, however, a patient feels the necessity to change his glasses more frequently, the tension of the eyeball should be examined, because in an elderly person rapid increase of presbyopia is one of the premonitory signs of glaucoma. In these circumstances, the patient should be referred at once to a specialist, who will fail greatly in his duty if he does not submit him to a thorough examination, in order that, if glaucoma be found to exist, every means may be taken at the earliest possible moment to check its progress.

The amplitude of accommodation may fail at any age as a result of severe illness; in which case the temporary use of a convex glass will enable the patient to read during convalescence and thereby promote recovery. Paralysis of the accommodation as a sequela of diphtheria is the most familiar example. Although the patient can see as well as ever he could in the distance, he is quite unable to discern near objects distinctly until a convex lens is placed in front of his eye. This lens is much stronger than would be required in ordinary circumstances, taking the age of the patient into consideration, together with the static refraction of his eye. The loss of the power of accommodation after diphtheria always affects both eyes, and is frequently accompanied by paralysis of the soft palate; but the immobility of the iris and the dilatation of the pupil are not so great as in ophthalmoplegia interna, which is usually the result of acquired syphilis. The patient, moreover, will almost invariably volunteer the statement that all objects appear much smaller than they should be; the increased effort of accommodation conveys the impression that an object is nearer than it really is, but as the visual angle remains the same, the final idea is that of a smaller object-micropsia. Diphtheritic paralysis passes off spontaneously in three or four months, but recovery is hastened by instilling a few drops of a I per cent. solution of nitrate of pilocarpin regularly twice a day.

When presbyopia occurs prematurely, the cause in the great majority of cases is hypermetropia. The patient usually says that he sees fairly well in the distance, but that he can only read for a very short time. His difficulties are always increased if he tries to do close work in artificial light. The eyes soon get tired, the head aches, and the print becomes so confused that the effort to see has to be given up. After a rest the symptoms pass off, but no sooner is near work resumed than they reappear. 
If the patients can give up all near work and lead an open-air life, the eyes will give no trouble; but if circumstances compel them to follow the occupation of clerk, engraver, seamstress, etc., the symptoms of strain become constant; there is a feeling of weight in the eyelids, which are opened with great difficulty on first awaking in the morning; and in many cases the edge of the eyelids and the conjunctiva are chronically inflamed. If the patient be a child, convergent strabismus is not infrequently the first symptom noticed, and reveals the presence of hypermetropia which, up till the appearance of the squint, had in all probability been quite unsuspected.

The family doctor is usually consulted in the first instance, and unless he is able to recognise the true significance of the symptoms, he will fail completely to relieve the patient. The existence of an error of refraction ought at once to be suspected; and that suspicion will be confirmed if on asking the patient to read the smallest type, it is found that he holds the book further from his eyes than he ought to do at his age. The effect of weak convex lenses on distant vision is now ascertained, each eye being tested separately. If a convex lens, no matter how weak it is, does not impair the sight, hypermetropia is present, and the following objective tests can then be applied-

I. Retinoscopy with a plane mirror gives a shadow moving with the mirror, and the better defined the shadow and the more quickly it seems to move, the lower the degree of the hypermetropia.

2. Indirect ophthalmoscopic examination with a concave mirror and a convex lens - the optic disc appears larger than usual, and diminishes in size as the lens is withdrawn from the eye.

A hypermetrope having always been in the habit of using his power of accommodation in order to see clearly in the distance, does so involuntarily, consequently he is unable completely to relax the ciliary muscle. It is a good plan, therefore, in testing, to begin with a strong lens, gradually reducing the strength until the $6 / 6$ line of test-types is read correctly. After having found the strongest convex lens with which standard vision is obtained with each eye separately, both eyes should be used together, when it frequently happens that a stronger glass gives equally good results. When the strongest glass has been found with which vision is still normal, its strength is only the measure of the manifest hypermetropia, because some accommodation is still being used, and consequently a certain amount of latent hypermetropia has yet to be revealed. In order to find out the total hypermetropia-i.e. the manifest plus the latentthe ciliary muscle must be paralysed with homatropin and cocain drops. In young persons in whom spasm of the accommodation frequently occurs, I per cent. atropin must be used three times a day for three or four days 
There are, however, two serious objections to the use of atropin in refraction work: (I) it takes nearly a fortnight for its effects to pass off, during which time the patient cannot see near objects distinctly; and (2) there is always danger in persons over forty years of age that the wide dilatation of the pupil may induce an attack of acute glaucoma. It is, however, always advisable to use a cycloplegic to determine the refraction in children and those under thirty years of age; because, as a rule, if only the manifest error be corrected, too much latent hypermetropia will remain and perpetuate the patient's discomforts. It is a good rule to correct all the manifest hypermetropia and one-third of the latent; but if the patient is to wear the glasses constantly, care must be taken that the lenses do not interfere with the distinctness of vision in the distance. Age is the chief determining factor after the degree of the error of refraction has been estimated. If the patient is an adult, and the hypermetropia is not more than two to three dioptres, he will probably see quite well in the distance and be much more comfortable without spectacles, which, however, must always be worn for reading and all close work. If the hypermetropia be more than three dioptres, the glasses must be worn constantly. As a general rule, children should wear their spectacles both for distance and for reading; but school-boys should be told to take them off before joining in games such as football, or in any play in which the glasses might readily be broken. If the patient is over thirty years of age, or if he complains of symptoms of eye-strain, he will require to wear the glasses which correct the manifest hypermetropia for distance, and use a second stronger pair for reading and all close work. In such circumstances, bifocal lenses are most convenient; the upper part of these lenses is adapted for distant vision, while the lower part is used for reading, etc. Some have difficulty in wearing bifocal lenses; but if the optician has taken care to adjust them properly, much may be done by perseverance in their use to lessen and ultimately to remove all discomforts; the more the patient becomes accustomed to them the less likely is he to complain.

2. Those who Cannot see in the distance, but who Can see QUITE EASILY TO READ OR TO SEW.

The patient suffers from short sight-simple myopia. He has very indifferent vision for distance, but can read the smallest print when he holds it near enough to his eye. Vision for distance is immediately improved by the use of a suitable concave glass; and the following objective tests rapidly demonstrate the presence of myopia-

I. Retinoscopy with the plane mirror-the shadow moves "against," and with a concave mirror "with," when the observer is seated one metre from the patient. 
2. Ophthalmoscopy. (a) Indirect method. The optic nerve appears smaller than normal, but becomes larger as the lens is withdrawn from the patient's eye.

(b) When the large ophthalmoscope mirror is used without a lens, the fundus is easily seen in high myopia; and when the mirror is moved, the image of the disc or the retinal blood-vessels moves in the opposite direction.

(c) Direct method. The fundus cannot be distinctly seen until concave lenses are rotated behind the sight hole of the mirror.

The manner of a short-sighted person is characteristic. Whenever he wishes to see anything distinctly, he brings his eyelids together to narrow the palpebral opening. The troubles of the myope are not connected with the power of accommodation, but are due to the fact that he brings objects so near his eyes that he taxes his power of convergence. The problem presented to the doctor, therefore, is how to enable the patient to see distinctly at the greatest possible distance.

When testing a short-sighted person, it is convenient to find out in the first place the furthest distance at which he can see small objects distinctly. Having found the far point, measure its distance from the eye; that measurement will give the focal length of the lens with which vision in the distance will be distinct. When the patient is seated before the test types, concave lenses are placed successively in the trial frame to find out the weakest lens that gives most distinct vision with each eye separately; but when both eyes are used together, it will often be found that the patient sees as well, and feels more comfortable, with a lens from .25 to 50 dioptres weaker. In dealing with young patients, it is always necessary to paralyse the ciliary muscle. Having ascertained the strength of the lens necessary to correct the myopia when the eyes are under a cycloplegic, glasses may be prescribed at once for children; who must be told to wear them constantly, except when at play. In the case of adults, it is better to test the glasses again after the effect of the mydriatic has passed off, because it may be found that a slightly stronger lens gives more distinct vision. If the myopia be over 4 dioptres, it will be necessary to give a glass $I^{\prime} 5$ to 2 dioptres weaker for reading. There is sometimes considerable difficulty in persuading the patient to wear glasses for reading or for any close work. He says he sees much better without them, and complains that the spectacles "draw" his eyes. That is all the more likely to be the case, when the patient has been allowed to reach adult life before beginning to wear glasses. He has accustomed himself to read at his far point or just within it, and has in consequence never used his ciliary muscle. The normal relation between accommodation and convergence has become disturbed; and when the appropriate 
concave glass is worn, a demand is suddenly made on a ciliary muscle weakened from disuse. These difficulties will soon lessen, however, if the spectacles be suitable and the patient persevere in wearing them. He must be made to understand that the glasses are prescribed, not to improve his vision for reading, but in order to increase the distance from his eyes at which he can hold the book. The younger the patient, the more likely will he be to adapt himself quickly to the new conditions. A myope, like every one else, becomes presbyopic as age advances, consequently a short-sighted person who has worn correcting glasses of the same strength both for distance and for close work, will find as he reaches his forty-fifth year that he cannot see to read so clearly with his glasses as he did formerly, and is constantly tempted to remove them and look at time-tables and small objects with the naked eye. His discomforts will be removed at once if he gets bifocal glasses, the reading or lower part of which is weaker than the distance or upper part, by the strength of the convex lens which would have been required at his age had he been emmetropic.

As a rule, it will be prudent for the general practitioner to send adults who suffer from high degrees of myopia, and children in whom myopia is progressive, to consult a specialist, because the danger of serious complications occurring in such cases makes the treatment of those patients a very serious responsibility.

Young persons, who have to use their eyes at fine work, engraving, embroidery, close study, etc., for many hours on end, frequently complain that they cannot see in the distance. In them, however, the myopia is only apparent, and is due to spasm of the ciliary muscle. It is important to recognise those cases, because, if the true nature of the symptoms be misunderstood, the patient's discomforts will become steadily aggravated, and the apparent myopia may become real and progressive. Spasm of the accommodation ought always to be suspected, when a very weak concave spherical lens, e.g. $50 \mathrm{D}$. improves the patient's sight far more than one would naturally expect, e.g. from $6 / 18$ or less to $6 / 6$. Moreover, the condition is usually accompanied by contraction of the pupil, twitching of the lids, congestion of the conjunctiva, headache, and other symptoms characteristic of eye-strain. There is, however, no fear of overlooking spasm, if the precaution be taken to paralyse the ciliary muscle before prescribing spectacles. The moment the accommodation is relaxed, vision for distance is restored, and both subjective and objective tests will, in all probability, revea a low degree of hypermetropia or of hypermetropic astigmatism.

3. Those who CanNot SEe Distinctly Either in the Distance or NEAR AT HAND. 
This class can be sub-divided into three groups-

(a) Those who suffer from astigmatism.

(b) Those who suffer from a high degree of hypermetropia.

(c) Those who suffer from "insufficiency" of convergence.

The correction of the error of refraction in all of these cases requires so much care, patience, and experience that, as a rule, the general practitioner will prefer to send the patient to a specialist; but the following brief description may be helpful in enabling him to recognise such cases when he meets them.

(a) Those who suffer from astigmatism.-When different meridians of the cornea possess different curvatures, the rays of light do not all meet at one focus, with the result that the eye is astigmatic. The bowl of a spoon is a familiar example of an exaggerated astigmatic surface; the curve from side to side being much greater than that from the tip to the handle. Two varieties of astigmatism are described-irregular and regular.

1. Irregular Astigmatism.--The irregular form is usually the result of previous ulceration of the cornea, and is the cause of the defective sight of those who have suffered in childhood from phlyctenular keratitis. It also occurs when the cornea becomes conical. The treatment by spectacles of all forms of irregular astigmatism is, however, so very unsatisfactory, that the general practitioner will be well advised not to attempt to prescribe for those patients himself.

2. Regular Astigmatism. - In this form the meridian of greatest, and that of least refractive power are always at right angles to each other. The vertical meridian is generally that of the maximum, and the horizontal that of the minimum curvature. In these circumstances, the astigmatism is said to be "with the rule"; whereas when the horizontal meridian has the maximum curvature, the astigmatism is "against the rule"; and if the chief meridians are at an angle of about $45^{\circ}$ and $135^{\circ}$, it is "oblique." In every case the chief seat of astigmatism is in the cornea, but in many cases there is also a very small amount present in the lens. The lenticular astigmatism is for the most part opposite to that in the cornea, and must be regarded as an effort to correct it. That is the reason why low degrees of astigmatism, capable of correction by the eye itself, are so often accompanied by headache and other symptoms of eye-strain, and why it is always necessary to paralyse the accommodation completely before attempting to rectify small errors.

In the great majority of cases, astigmatism is congenital; but in a few instances it is acquired, the curvature of the cornea having been altered by the incision in an iridectomy or a cataract operation, or by the pressure of a tumour in the eyelid. 
The following varieties of regular astigmatism occur-

r. Simple-hypermetropic or myopic.

2. Compound-hypermetropic or myopic.

3. Mixed.

The accompanying diagrams illustrate the optical conditions in those different eyes.

I. Simple Hypermetropic Astigmatism.-One meridian is normal in refraction and the other hypermetropic; corrected by a suitable convex cylindrical lens whose axis is usually vertical. + D. Cylindrical.

2. Compound Hypermetropic Astigmatism.--Both principal meridians hypermetropic, but one more so than the other. Corrected by $+\mathrm{D}$. Spherical lens $\approx+D$. Cylindrical-a + D. Cylindrical of the strength corresponding to the difference between the two meridians.

3. Simple Myopic Astigmatism.-One meridian normal, and the other myopic-corrected by a suitable concave cylindrical lens, whose axis is usually horizontal. - D. Cylindrical.

4. Compound Myopic Astigmatism.-Both principal meridians myopic, but one more so than the other. Corrected by $-\mathrm{D}$. Spherical $\approx-\mathrm{D}$. Cylindrical corresponding to the difference between the two meridians.

5. Mixed Astigmatism.-One principal meridian is hypermetropic and the other is myopic. The difference in refraction between the two meridians equals the amount of the astigmatism; consequently a cylinder is required with its axis at right-angles to the meridian to whose sign it corresponds, and a spherical lens of the strength of the meridian whose sign is the opposite of the cylinder. Whenever it is possible, the concave surface of the lens ought to be placed next to the eye, because, as a rule, that ensures the best visual result.

- There is always a certain amount of astigmatism in the normal eye, but it is usually so slight that it may be regarded as physiological. Whenever the physiological limits are overstepped, however, the patient begins to complain; but very rarely does he say anything about those signs which enable the practitioner to diagnose the particular error of refraction from which he is suffering. As a rule, he will only complain that he cannot see properly either to recognise a friend on the street or to read, and that whenever he overtaxes his eyes he suffers from headache. There are rarely any physical appearances that indicate astigmatism, unless it be the asymmetry of the face, which is sometimes detected when the error is very great and confined to one side. If, however, the patient be observed to hold his head sideways while he is reading the test types, oblique astigmatism ought at once to be suspected; and the suspicion will be confirmed if he confuses certain letters, e.g. D and $\mathrm{O}$, or $\mathrm{W}, \mathrm{E}$, and $Z$, and $\mathrm{S}$ and $\mathrm{B}$; and if, although he can see several letters in the 


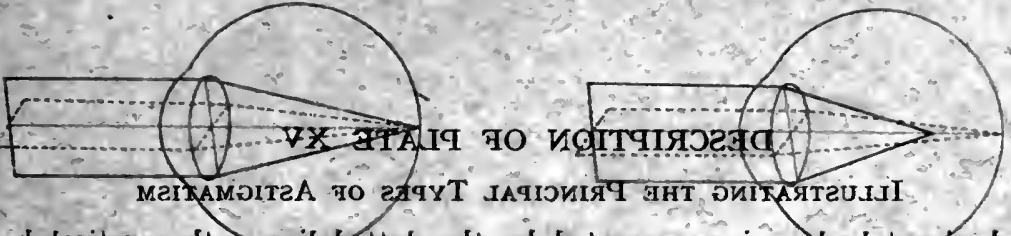

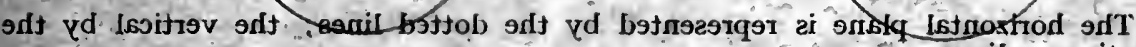

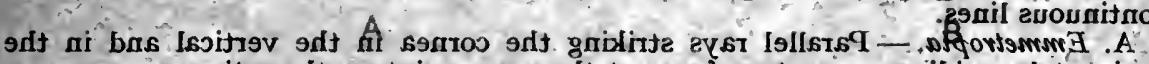

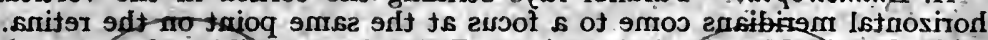

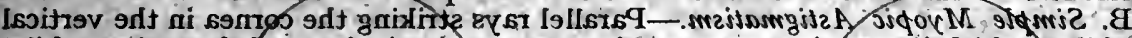

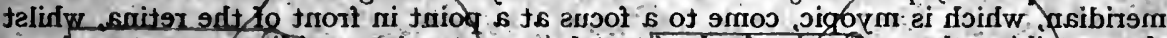

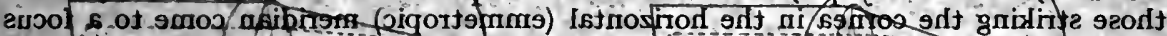

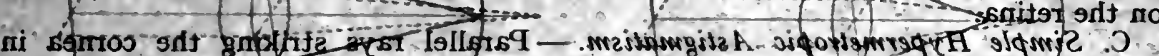

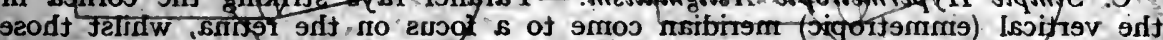

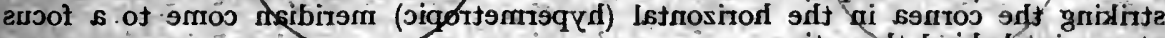

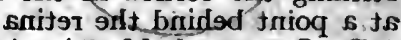

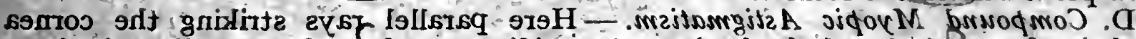

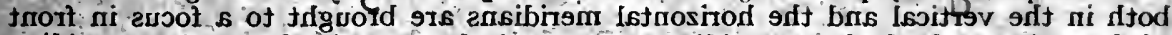

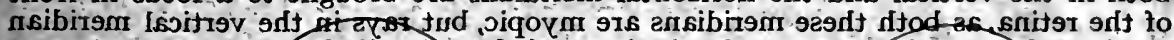

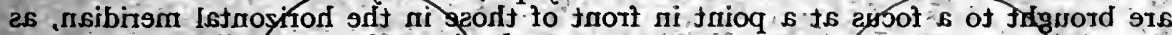

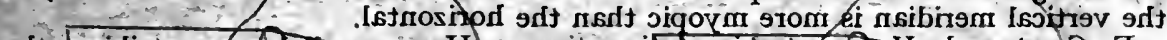

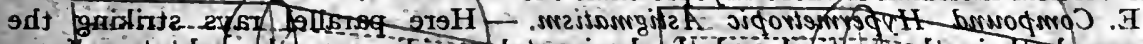

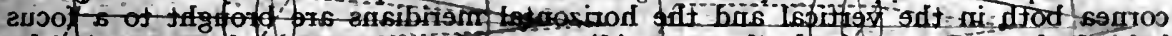

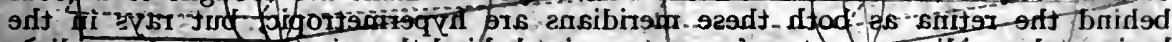

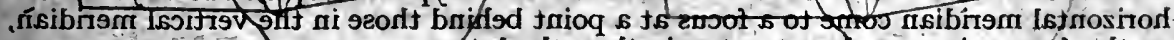

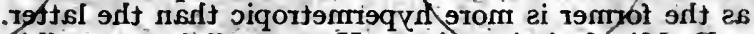

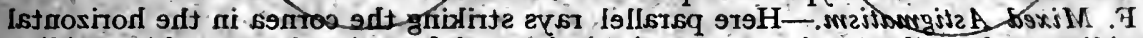

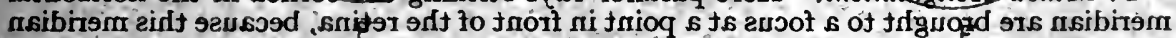

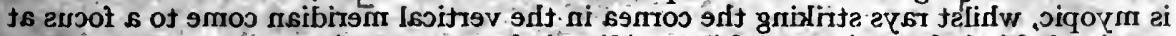
. Jiqotfomig

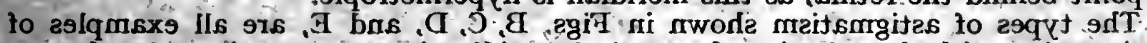

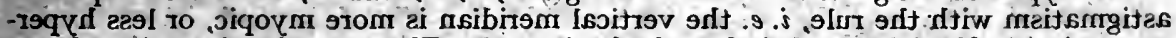

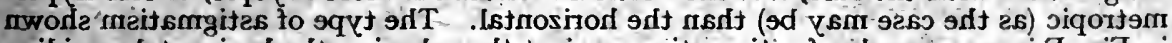

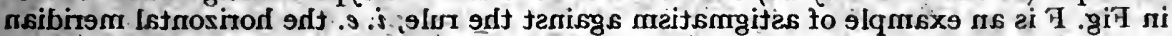
. Isoitrov - 9 dt nsdt jiqopm '9tom ai 
The horizontal plane is represented by the dotted lines; the fvertical by the continuous lines.

A. Emmetropia.-Parallel rays striking the contea in the vertical and in the horizontal meridians come to a focus at the same point on the retina.l, and the

B. Simple Myopic Astigmatism. - Parallel rays striking the cornea in the vertical meridian, which is myopic, come to a focus at a point in front of the retina, whilst those striking the cornea in the horizontal (emmetropic) meridian come to a focus on the retina.

C. Simple Hypermetropic Astigmatism:- Parallel rays striking the cornea in the vertical (emmetropic) meridian come to a focus on the retina; cwhilst those striking the cornea in the horizontal (hypermetropic) meridian come to a focus at a point behind the retina.

D. Compound Myopic Astigmatism. - Here parallel rays striking the comea both in the vertical and the horizontal meridians are brought to a focus in front of the retina, as both these meridians are myopic, but rays in the vertical meridian are brought to a focus at a point in front of those in the horizontal meridian, as the vertical meridian is more myopic than the horizontal.

E. Compound Hupermetropic Astigmatism. - Here parallel rays striking the cornea both in the vertical and the horizontal meridians are brought to $a$ foc is behind the retina as both these meridians are hypermetropicilbut trays in the horizontal'meridian come to a focus at a point behind those in the vertical meridian, as the former is more hypermetropic than the latter.

$\mathrm{F}$. Mixed Astigmatism.-Here parallel rays striking the cornea in the horizontal mericlian are brought to a focus at a point in front of the retina, because this meridian is myopic, whilst rays striking the cornea in the vertical meridian come to a focus at a point behind the retina, as this meridian is hypermetropic cisogical. Then - The types of astigmatism shown in Figs. B, C, D, and E, are all examples of astigmatism with the rule, $i$ e the vertical meridian is more myopic, or less hypermetropic (as the case may be) than the horizontal. The type of astigmatism shown in 'Fig. $F$ is an example of astigmatism against the rule, $i . e$. the horizontal meridian is more myopic than the trertical. 


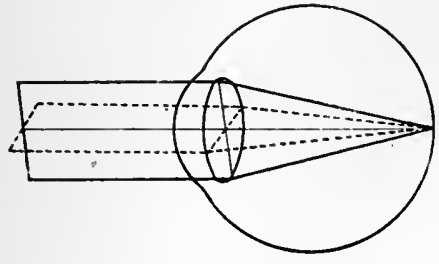

A
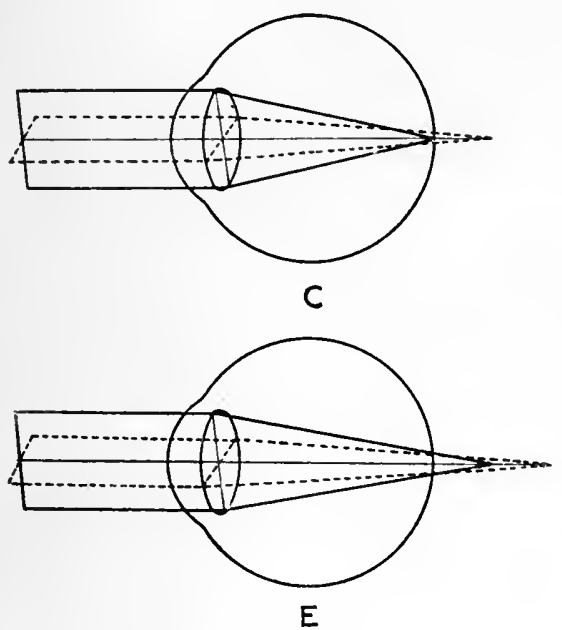
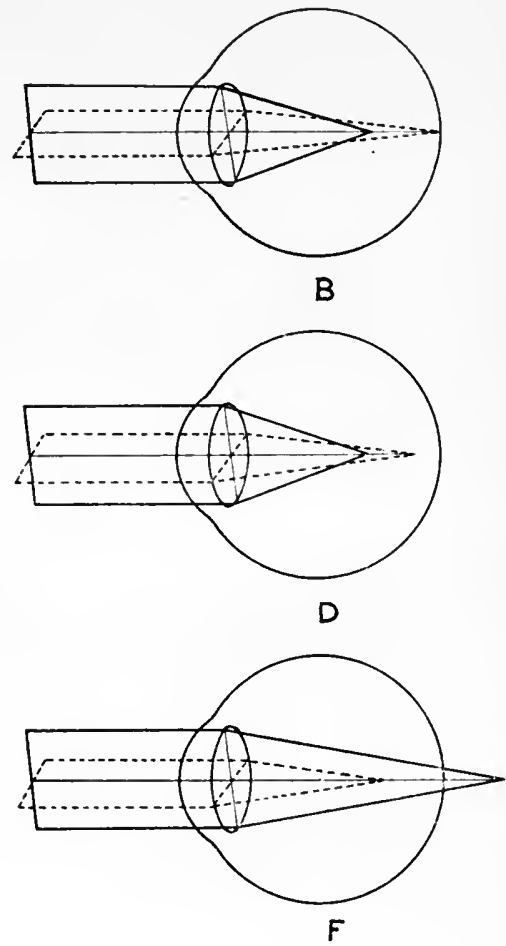

$6 / 9$ or even the $6 / 6$ line, he makes mistakes when reading much larger letters. In these circumstances, the crucial test for astigmatism ought to be applied; when it will be found that at the same distance vertical and horizontal lines are not seen with equal distinctness.

The ruile, therefore, for the correction of astigmatism may be briefly expressed as follows: Use a stenopæic slit, and if necessary make one of the principal meridians emmetropic; then add cylindrical lenses, whose axes are placed in the same direction as the corrected meridian, until both principal meridians are emmetropic. It is well, however, not to waste too much time on purely subjective tests, because astigmatism can be measured more accurately and much more quickly objectively. The chief objective tests are by retinoscopy and the ophthalmometer. The ophthalmoscope by the indirect method is also very useful; because when astigmatism is pronounced, the optic disc appears oval, and its elongation is in the meridian of least curvature. It also seems to change its shape when the convex lens is withdrawn from the eye.

(b) Those who suffer from a high degree of hypermetropia. These patients are usually children who are thought to be short-sighted, because they cannot see the blackboard at school; but they are not myopic, because they are quite unable to see a printed page, or discern near objects distinctly. The same condition of things is also found in an adult who is hypermetropic and has become presbyopic. There is not much difficulty in prescribing satisfactorily for those patients. The children all require instillations of atropin for at least three days before any serious attempt is made to adjust spectacles. Convex lenses before the eye rapidly improve vision; and the need for more than one pair of spectacles is determined by the age of the patient.

(c) Those who suffer from "insufficiency" of convergence. The patient has difficulty in maintaining near vision when both eyes are used together, and usually seeks relief from his discomforts by closing one eye.' This preponderating action of the abductor over the adductor muscles may be met with in emmetropia, and even, although more rarely, in hypermetropia; but it is far more frequent in myopia. One of the advantages of wearing concave glasses for near work is that they help to restore the balance between accommodation and convergence, and thereby lessen strain : but sometimes a prism, base in, must be prescribed along with the lenses which correct the error of refraction, before the patient is quite comfortable. Treatment by prisms, however, is not curative. They simply act as a crutch to help the eye over a difficulty, and in a small proportion of cases operation on the recti muscles may be required. This subject is, however, considered more at length in the chapter on Myopia and its treatment. 


\section{CHAPTER XXXVIII}

\section{MYOPIA AND ITS TREATMENT}

Myopia a product of civilisation-Symptoms-Dangers of myopia-CausationAim of treatment-Importance of attention to general health-Operative treatment-Prophylaxis.

A SAVAGE is very rarely short-sighted. Myopia is really a product of civilisation. As men's wants become more numerous, the use of the eyes for near work increases in importance: clear vision for reading and writing is more in demand than keen sight in the distance. In order to adapt the eye for work under the new conditions, nature has by increase of length somewhat altered its shape. Roughly speaking, an increase of I millimetre in the axial length of the eyeball represents 3 dioptres of refractive power.

The myopic eye may be regarded, therefore, as in some ways an evolution from the emmetropic, but more particularly from the hypermetropic form. In no sense, however, can it be looked upon as more perfect. The visual advantages claimed for the short-sighted are more apparent than real, and a state of myopia can no more be considered perfection, than can a heart, hypertrophied to overcome an obstruction in the circulation, and thereby enabled to maintain compensation, be looked upon as more perfect than the normal heart. Both organs rise to the occasion and overcome a difficulty, but each becomes liable to dangers and accidents to which it is not exposed in the normal condition. The myopic eye contains within itself elements of disease, and must always be looked upon with suspicion until time has proved it to be physiologically sound and trustworthy. This notwithstanding, popular belief has always credited myopia with many advantages, and has persistently declared that short-sighted eyes are good; that they improve as age advances; and that at no time will they require spectacles for reading. Never were more fallacious statements made, and unfortunately -in spite of the bitter regret of many who have been deluded into believing them-they are still being repeated to a credulous public. No doubt there are many cases in which myopia amounts to nothing more than an error of refraction, and in which, apart from the inability to see distinctly in the distance without glasses, it causes no serious trouble; 
but, on the other hand, the myopia may steadily progress until the deeper structures of the eyeball are overtaken by incurable disease. No sharp line separates the two groups of cases. The one passes by gradual and insensible gradations into the other; nor is it easy to determine why it is that in one person short-sight remains stationary, while in another it increases to an alarming extent and terminates in blindness.

Symptoms.--The degree of myopia, considered in relation to the age of the patient, is probably the best guide we possess in forecasting the probable course to be followed in an individual case; and the arrangement of the short-sighted into groups, according as the myopia is low, moderate, high, or very high, possesses many clinical advantages. In myopia of low degree, the far point of distinct vision may be anywhere between the horizon and 20 inches from the eye; of moderate degree, somewhere between 20 inches and Io; of high degree, between Io inches and 4; of very high degree, any distance nearer than 4 inches. Those who suffer from myopia in its higher degrees generally present a very characteristic appearance. They have large and prominent eyes with dilated pupils, and an inattentive, dreamy expression. When they enter a room full of people they have difficulty in recognising their friends, and, in order that they may see more distinctly, screw up their eyelids and incline the head forward. Myopes at all times require to make a conscious effort in order to see clearly; and, when they are engaged in prolonged close work, the excessive degree of convergence necessary to secure distinct vision brings about fatigue of the internal recti muscles, which, sooner or later, become unequal to the duty imposed upon them. At first, sight is quite good, but soon the eyes begin to feel drawn and uncomfortable, the print appears unsteady, and the letters seem to dance about the page. This state of matters is followed by an aching pain in the brow and back of the head, and by a feeling as if the eyes were being pushed violently forward, and had become too big for their sockets. The symptoms grow worse and worse until double vision is induced, and then the patient, if he wishes to continue his work, has to close one eye. Usually, by this time, however, the eyeballs are suffused with tears, and, in consequence, vision is so indistinct that it is impossible to persist long in the effort.

The diplopia is obviously due to the inability of the internal recti muscles to maintain the excessive convergence demanded; and, as a rule, Nature by and by steps in and puts a stop to the work of one of the eyes-generally of that which is the more deficient. This produces relief, but at the cost of sacrificing binocular vision, and of inducing the deformity of a divergent squint. Nor in myopia is it only the nerves 
and muscular arrangement concerned in the maintenance of binocular fixation which suffer; for pathological changes, leading to very serious consequences, are apt to occur in the interior of the eyeball itself. Intraocular mischief should always be suspected when, in addition to the symptoms previously described, the patient suffers from recurrent inflammation of the lids and conjunctiva, and from a sense of fulness and burning heat in the eyes themselves; and danger is usually imminent when there are also complaints of muscæ volitantes, and of subjective flashings of light.

Dangers of Myopia.-Ophthalmoscopic examination shows that all these changes are due to inflammation of the choroid, at the posterior pole of the eye. In most cases of myopia, the optic disc is bounded on its temporal side by a typical crescent; and, when the disease is non-progressive, this is, as a rule, surrounded by a well-defined line of black pigment. Whenever the short-sightedness begins to increase, the ophthalmoscopic picture is altered-the crescent loses this welldefined border, and merges itself in a general thinning of the adjacent choroid. These inflammatory changes lead to weakening of the overlying sclerotic, which, yielding to the force of the intraocular pressure, bulges backwards to form a posterior staphyloma, the mechanism of whose production is in all respects identical with an ectasia in the ciliary region due to prolonged anterior sclerotico-choroiditis. The enlargement of the eyeball, once started, knows practically no bounds; for, unless it be arrested by treatment, the choroiditis is steadily progressive. The myopia then passes from its innocent to its pernicious form; and its further course is marked by the occurrence of intraocular changes, all more or less destructive to sight. If the progress of such a case be watched by the ophthalmoscope, atrophic and pigmented patches become visible in steadily increasing numbers, while retinal hæmorrhages are of frequent occurrence. These changes are at first most marked in that part of the fundus situated between the optic disc and the macula; but, by and by, they become general.

The most scrious damage to sight, however, occurs when the macula itself is implicated. When this important region is threatened, the patient complains that objects appear smaller than normal, and afterwards that their outlines are broken and irregular, and that the faces of friends are so distorted that it is painful for him to look at them. Metamorphopsia is always of serious omen.. It is due to disturbance of the regular arrangement of the cones by inflammatory exudation; and its appearance is usually the forerunner of a complete loss of direct vision, the result of increasing choroiditis or of retinal hæmorrhage. Scotomata, similar, but of less consequence, and due to the destruction 
of localised areas in the layer of rods and cones, occur also in other parts of the field of vision. Such extensive implication of the choroid leads to serious impairment in the nutrition of the eyeball. Floating bodies appear in the vitreous, which, by and by, loses its gelatinous consistency, and becomes fluid. The development of the staphyloma goes on apace, and, as it bulges backward, it drags and twists the optic disc; while the retina, displaced by inflammatory exudation from the choroid, and deprived of its natural support, owing to the fluidity and shrinking of the vitreous, becomes detached. Nothing more serious can befall an eye than detachment of the retina, and, unfortunately, in pernicious myopia this is not uncommon. It may occur suddenly, during a fit of coughing or of vomiting, or may follow a very trivial accident; but, as a rule, musca volitantes and metamorphopsia, accompanied by flashes of light, give premonition of its onset.

The lens suffers also. It may become cataractous; and, as a result of the stretching of its ligament, it may be dislocated by any slight injury. When it falls into the vitreous chamber the results are not nearly so serious as they are when the dislocation occurs forward into the aqueous. In the latter case secondary glaucoma is prone to follow; and, unless operation to extract the lens is successful, there is grave danger that sight may be lost, and enucleation may be necessary to relieve pain. In the course of ordinary choroiditis the appearance of cataract sometimes marks a favourable turn in the disease, and its extraction may be followed by the happiest results; but in high myopia cataract begins, as a rule, in the posterior pole of the lens, is of dark colour, and matures slowly; and operation for its removal is attended by considerable danger, as the fluidity of the vitreous, and the diminished intraocular tension, indicate a special vulnerability of the parts concerned.

Gausation.-In considering whether anything can be done to arrest the progress of pernicious myopia, it is necessary to take into account the causes that contribute to its production.

1. Influence of Heredity.-It is sometimes said that it is safer to inherit short sight than to acquire it ; and undoubtedly there are families, many of whose members are myopic, and yet suffer no further inconvenience from their eyes than the necessity of wearing glasses to see distant objects. Their eyes are large from before backwards, but, apart from an increase in size, show no signs of structural defect. The influence of heredity, however, does not confine itself to simply determining the size and shape which an eyeball will assume, but extends to the ocular tissues themselves, endowing them with certain characteristics, whereby they are either strong and resist the inroads of disease, or weak and succumb readily to its attack. If that were not so, it would be difficult 
to explain the occurrence of progressive myopia among those who, being illiterate, have never used their eyes for any kind of near work, or who have spent their lives vegetating in the country. Whatever may have been the determining cause in such cases, it is very probable that there has been a strong hereditary predisposition, rendering the ocular tissues so intrinsically weak that they readily yielded to the slightest strain.

2. Influence of Eye-strain.-It is a matter of common observation that to overtax the eyes for long periods with fine work leads, sooner or later, to ocular troubles, if not to serious defects of sight. The evil seems to be due, in most cases, to excessive convergence; for those who, like watchmakers, work with one eye only rarely make complaint, while those working less constantly, it may be, but employing both eyes, are frequently affected. The amount of risk connected with the performance of any kind of minute work requires us, if we are to arrive at a correct opinion, to take into consideration not only the refraction of the eyes, but also the general health and strength of the patient. Nor is this a matter of mere theory; statistics clearly establish that myopia is induced by close and continuous application of the eyes for near work. The examination of a large number of school children has shown that myopes, although present in all schools, were far more numerous in high-grade than in elementary schools, and that in the former the percentage of short-sighted scholars was much greater among the seniors than among the juniors.

3. Influence of Age.-During the period of active" growth the eye should increase in size proportionately with the body; but slight variations may take place, with the result that an eyeball, which was in infancy slightly hypermetropic, may at the age of puberty be emmetropic, and, when full growth is reached slightly myopic. Myopia, indeed, is rarely detected before the eighth year, and, as a rule, makes its most rapid advances between the twelfth year and the eighteenth. Influence of age, however, must always be considered in relation to the degree of the short-sightedness, because, as a general rule, it may be said that the higher the myopia the more likely it is to be progressive. Consequently, what might in an adult cause no anxiety, should, in the child, occasion serious concern.

4. Influence of Health.-If the chief anatomical causes of the difficulties incident to progressive myopia are fatigue of the internal recti muscles, and a want of resisting power in the ocular tissues, it may be taken for granted that a powerful influence, either for good or for evil, is exercised by the state of the general health. Common experience teaches that myopia, especially in children, often becomes progressive 
subsequent to an attack of measles or scarlet fever; that in adults any chronic disease or other debilitating influence may đetermine malignancy in a case which, earlier in life, was supposed to be of an innocent nature; and that women, whose general health is for the time being disturbed by the occurrence of the menopause, are the worst sufferers from the complications of pernicious myopia.

All these influences may be intensified by other causes, which may be looked upon as subsidiary. Of these may be mentioned-

(a) An improper position when at work.-No one ought to be allowed to read or write with his body thrown over the desk, and his nose almost touching the paper; for such an attitude causes congestion of the bloodvessels of the head, and, in consequence, encourages these very changes in the choroid which we are most anxious to avoid.

(b) Want of proper light.-One of the boasts of the myope is that he can see with less light than other people; and, consequently, he is often found reading in the twilight, or sitting on a rug before the fire with no more light than comes from the flickering coals. Acts such as these must certainly encourage a tendency to progressive myopia.

(c). Astigmatism.-If a young child, fond of books, suffers from a low degree of hypermetropic astigmatism, prolonged reading is very likely to give rise to spasm of the ciliary muscle, and, in addition, to the symptoms summed up in the word asthenopia; there is indistinct vision in the distance. This short-sight, however, comes and goes, is always worse after continuous use of the eye for near work, and is obviously due to the increased convexity of the crystalline lens brought about by contraction of the ciliary muscle. If any doubt exists as to the diagnosis of this condition, it will at once be removed by the use of atropin, the effect of which is to restore the static refraction of the eye. If, on the other hand, this myopia be unattended to, or, what is worse, if it be wrongly interpreted, and the patient advised to wear concave glasses, it then becomes a most important factor in the causation of true myopia. The constant irritation kept up in the choroid by the over-action of the ciliary muscle necessarily leads to congestion, and this is further aggravated by the habit which the child soon acquires of holding its book close up to the face.

These are just the conditions under which myopia is most likely to become progressive; and as they come into play when the tissues of the eyeballs are weak from rapid growth, there is little wonder that changes are set up whereby the hypermetropic child becomes short-sighted to a greater and greater degree as its development advances. A similar condition of matters is brought about by the irregular astigmatism due to nebulous opacities of the cornea, the result of past ulceration; or in cases 
in which, from malnutrition, the cornea has changed from the spherical to the conical shape.

Treatment. - In the treatment of myopia, two indications must always be kept in view: First, to enable the patient to see distant objects; and, second, to prevent the onset of choroiditis, or to arrest its progress if the disease has already begun. The aim of all therapeutics is to restore the abnormal to the normal; and although the surgeon cannot change the shape of the myopic' eye, he can, by means of lenses, alter the conditions under which it works. In the emmetropic eye, images of objects at infinite distances are accurately focussed on the retina without any effort of accommodation. It is only when reading or writing or any kind of near work is engaged in, that the action of the ciliary muscle is required. The myopic eye differs from the emmetropic in having no power within itself to see objects at infinite distance; and as its far point is in most cases within the working distance, it does not require any effort on the part of the ciliary muscle to enable it to adjust itself to reading or writing. The clear therapeutic indication, then, is to provide the myopic eye with a glass of sufficient power to enable it to see at a distance when in a state of rest, and also to render it necessary for it to exercise its accommodation in reading or writing. A glass which will enable a short-sighted person to see in the distance must be one which will render parallel rays divergent, and thus make the myopic eye optically equivalent to the emmetropic eye. As to the use of spectacles for this purpose, the surgeon has, as a rule, the hearty co-operation of his patient, who, now, perhaps for the first time, realises what it means to see in the distance. When, however, it is explained that the glasses are to be worn also when reading and writing are engaged in, objections are frequently raised, because no difficulty has ever been experienced in seeing near at hand. Indeed, if the lens prescribed corrects the myopia, it may, in the first instance, cause difficulty when it is used for reading; up till now there has been no need to exercise the power of accommodation, and the ciliary muscle, weakened from disuse, resents this call to action. Moreover, as concave glasses diminish the size of objects, myopes invariably say that when they wish to see anything minute they take off the glasses and hold the object close to the eyes. In this lies the crux of the whole matter, and it must at once be explained to the patient that he is asked to wear glasses for reading, not that he may see the print better than he can with his naked eye, but that he may be enabled to hold his book at a proper distance from his face. In short, the only object aimed at in prescribing spectacles for reading is to prevent the patient from sitting with his head bent down over the paper, and his eyes closely converged on the print. 
This faulty attitude is a great evil, and, if the danger of progressive myopia is to be met and overcome, must be counteracted.

It is always necessary to explain this fully to parents, many of whom have a deeply-rooted prejudice to the use of spectacles by their children, and often urge all manner of objections against.it. In truth, however, the earlier a myopic child begins to wear glasses the better; and if it wears them constantly, as directed, it is not nearly so liable to suffer from the consequences of myopia, as another who has worn spectacles only intermittently, and never at all for reading. Their use for distant objects helps the child, by permitting it to see what is going on in the world around it, and thereby enabling it unconsciously to improve its education; and yet, important as this is, it is still more important that, in order to counteract at as early a stage as possible all tendency to excessive convergence, the spectacles should also be worn for near work. It is always a difficult thing for those who are grown up to begin to wear concave glasses for the first time, because the new conditions under which the ocular muscles are called upon to work are sure to give rise to much discomfort. Such persons are startled by the distinctness with which everything is seen, and speak of objects as being painfully bright and near.

For adults suffering from high myopia, usually complicated by typical changes in the choroid, it is very difficult to prescribe helpfully. Their story is that the short-sight has existed since childhood, but that their parents would not allow them to wear glasses; and that they have only at last sought help on account of increasing short-sightedness, and of inconvenience experienced from indistinctness of sight, especially when they are away from home. Acuity of vision, usually less in the myope than in the emmetrope, is in such cases so markedly reduced that it is impossible, even when the macula itself is not implicated, to bring the vision up to the normal standard. The glasses which give the best results are prescribed; but probably in a few weeks the patient will return to say that the spectacles are of no use, as he sees fully as well without them. It is with our brain as well as with our eyes that we see; and these patients have become so accustomed, from long practice, to interpret blurred images conveyed to the brain from the retina that, when, by the help of glasses, distinct images are formed, the effort to understand them confuses the brain and causes mental discomfort. When, however, such a person is removed from his familiar surroundings, he appreciates better the help which the glasses afford him; but, as a rule, he never admits that he feels comfortable when he wears them, and consequently, in most instances, just goes on as he was doing, regretting more and more that he was prevented from wearing glasses at a time of 
life when eyes and brain alike would readily have adapted themselves to their use.

It would be well for parents, and for the public generally, to realise that no harm, but, as a rule, much good follows the proper correction of an error of refraction. In children, the weakest concave glass that enables them to see clearly in the distance ought also to permit them to read and write; but when advice is not sought until adult age has been reached, the ciliary muscle has from disuse become weak, and the power of accommodation insufficient to permit of the use of the same glasses for reading and for looking at distant objects. In such cases, it is necessary to prescribe two pairs of spectacles, a stronger for more distant, and a weaker for nearer, work. In the short-sighted, as well as in others, the advance of age brings with it a lessening power of accommodation; and so, as presbyopia develops, the myope with one pair of glasses finds that he requires to diminish their strength for near work, just as an emmetrope of the same age requires to wear convex glasses. The inconvenience of having to wear two pairs of glasses is very considerable; and a bifocal lens which serves for both distant and near work is a distinct advantage. When diplopia is complained of, or when, in the course of examination, heterophoria is detected, the use of prisms may afford relief ; but it must always be remembered that the occurrence of diplopia indicates that the ocular muscles are fatigued. In most cases, it is wise to interpret the double vision as a danger signal, set up by nature to warn us to examine thoroughly into the whole condition of the patient's health and strength.

The treatment of myopia cannot, however, be summed up in a prescription for spectacles. It resolves itself largely into a question of the best means of promoting the general health. In the management of a young myopic adult, the eye specialist must call to his aid the careful exercise of all the best qualities of a wise physician. Every one of the troubles and dangers incident to the disease owes its origin to, and is explained by, the occurrence of choroiditis; and as this may be influenced by constitutional causes, as well as by the mechanical and other effects due to the condition incident to myopia, a careful estimate should, at the very outset, be made of the state of the patient's health as a whole, and if any constitutional disorder be discovered, it should be dealt with by appropriate remedies. Everything must be done in addition, by means of good food, fresh air, and nerve and blood tonics, to increase the patient's strength; and there is nothing of more value in checking the progress of myopia than a well-regulated course of medical gymnastics. Much attention has been given to this subject, and many good manuals describing exercises have been published. 
In the case of the young, school work ought to be strictly supervised, and all protracted use of the eyes prohibited. Periods of work must be alternated with periods of play, and the play ought to be much in excess of the work. Great watchfulness is necessary to see that the child wears its glasses constantly; that it sits erect at its work, holding the book at a proper distance from the eyes; and that it never requires to read or write in an insufficiently lighted room, or to use badly printed books. If there be any threatening of choroiditis, all close work must be given up, and the eyes protected from strong light by smoked glasses, every encouragement being given to the child to spend as much of its time as possible in open-air pursuits. Such children tend far too much to interest themselves in sedentary amusements, and are too little inclined to join their companions in outdoor recreations; consequently there is a great probability that they will stoop over their work and become "roundshouldered."

Lastly, it is most essential, in every case where the myopia is suspected to be progressive, that the child's eyes be examined at stated intervals; for only in that way is it possible to ascertain with certainty whether the short-sight is, or is not, increasing. In addition to this, however, the inflammation of the choroid requires to be treated actively, because it is rare to find a myopia which has commenced with pathological symptoms get well of its own accord. If congestive and inflammatory changes be acute, the patient must be made to rest in a darkened room, leeches applied to the temples, and the bowels and liver acted upon by saline laxatives, and an occasional dose of calomel. Cases of this kind are often benefited by vapour baths; and when intraocular tension is high, and the patient complains of pain and discomfort in the eyes themselves, speedy relief is often obtained by the use of eserin or pilocarpin, or by paracentesis of the aqueous chamber. Such acute cases are not, however, often met with. It is more usual to find that choroidal inflammation has wrought considerable damage, without having produced any symptom acute enough to arrest the attention either of the patient or of his friends. It is more likely that the fundus oculi will, if the patient be an adult, present changes indicative of inflammation of a chronic type; and in such cases a combination of iodide of potassium and nux vomica has always been a favourite remedy, while saline and alkaline laxatives, such as the waters at many of the mineral Spas, are usually to be recommended.

These are the cases in which the macula becomes involved. A patient, formerly able to read and write, may suddenly find himself, on account. of the twisted and distorted appearance of the letters, totally unable to. do so; or, if the macula has been destroyed by hæmorrhage, the spot at 
which he looks directly may be a perfect blank. These cases are always of unfavourable prognosis, but many of them are considerably benefited by the use of sub-conjunctival injections of sterile solutions-salts of mercury or chloride of sodium. The injections are repeated two or three times a week, and the strength of the solutions is gradually increased.

There is also an operative treatment for myopia. Where, after reading, the patient complains of the symptoms described in the earlier part of this chapter, or where the difficulty in reading has become very extreme and has not been relieved by glasses, it may be necessary to try to bring about an improvement by operating on the ocular muscles themselves. This may be done either by tenotomy of the stronger muscles, or, preferably, by advancing the tendon of the weaker. No such operation, however, must be undertaken, until every opportunity has been given for the recovery of the balance of muscular power by the promotion of an improved condition of general strength.

It has long also been known that short-sighted people who suffer from cataract are sometimes, after the lens has been extracted, able to see without glasses. This suggested a quick and easy way to restore vision in high myopia; many years ago, therefore, it was proposed, in cases of extreme short-sight, to diminish the refractive power of the eye by the removal of the lens. It is one thing, however, to extract a cataract which is mature, and quite a different thing to interfere with a transparent lens.

The operation was strongly opposed by such high authorities as von Graefe and Donders; and practically nothing had been heard of it for many years, until Fukala brought it again into notice by publishing a series of successful cases, and since then it has been looked upon as a recognised procedure in ophthalmic surgery. Obviously, this operation is suitable only in myopia of very high degree-20 D. and upwardsand in cases where the changes in the choroid have not already been such as to seriously interfere with sight. When successful, it is undoubtedly a great boon to those who cannot get spectacles strong enough to help them, or who are so situated that it is inconvenient for them to wear glasses. It is most likely to succeed in young adults where the lens is soft, and can never be attempted in persons over forty years of age without very grave risk. Even in cases in which the result of the operation appears to be quite successful, sight may be lost later on from detachment of the retina; and it is indeed very questionable whether the risks and the dangers attendant upon surgical interference in high myopia do not outweigh the advantages.

Prophylactic treatment in its broadest and most general sense is always of the greatest value, and if begun in the early stages of the disease 
is likely to be successful in checking its progress. The serious intraocular changes which accompany progressive myopia often make considerable advances, however, before they indicate their presence by any subjective symptoms which arouse the patient's concern; and in this silent progress lies the greatest danger. In the interests of the rising generation, therefore, there is nothing within the range of ocular hygiene that more deserves the attention, not only of medical practitioners, but also of parents and of teachers, than wise attention to those under their care who suffer from short-sightedness. 


\section{CHAPTER XXXIX}

\section{CONCOMITANT CONVERGENT SQUINT OCCURRING IN CHILDREN}

Strabismus-Binocular vision-Paralytic squint-Concomitant squint-Differential diagnosis-Classification of squints-Convergent strabismus- EtiologyLatent strabismus-Apparent strabismus-Examination of patient suffering from squint-Treatment of squint-Non-operative and operative.

Strabismus or "cross-eye" can be recognised at a glance; but a squint is something more than a muscular deformity, and consequently, its study demands an investigation into the processes underlying causation, as well as the observation of results.

When a person looks straight at an object he fixes it with both eyes, but if he squints only one eye fixes at a time, and the other deviates, because its visual axis is not directed to the object looked at. In these circumstances binocular vision is impossible. The ability to see single with both eyes open, requires not only accurate co-ordination of the movements of the two eyes, but also the power to fuse the retinal images. This fusion faculty is developed in the visual centres of the brain. It does not exist at birth, but is formed gradually during the first year of life, and in ordinary circumstances reaches its full development before the end of the sixth year. After the fusion sense is well developed, binocular vision occurs automatically, and controls and co-ordinates the ocular movements when both eyes are open. Binocular vision may be lost, either from paralysis of an ocular muscle, or from want of development of the fusion centre in the brain. In both cases squint appears-in the former it is spoken of as paralytic, and in the latter as concomitant.

The diagnosis between paralytic and concomitant strabismus rarely presents any difficulty. A paralytic squint usually appears suddenly, occurs for the most part in adults, is always accompanied by double vision, the deviation is greater when the patient looks at an object with the paralysed eye than when he fixes with the sound eye, and there is always limitation in the movement of the globe corresponding to the sphere of action of the paralysed muscle. A concomitant squint, on the other hand, usually.comes on slowly, occurs most frequently in children, is not accompanied by spontaneous diplopia, the deviation is the same irrespective of the eye that is used for fixation, and there is no limitation 
of the movements of the eyeball in any direction. A paralytic squint usually exists in one eye only, whereas concomitant squint affects both at the same time; either eye taken by itself is normal in its movements, but there is a disorder in the co-ordinated action of the two. As a matter of convenience, one eye is spoken of as the "fixing " eye, and the other as the "deviating" eye, but a concomitant squint in reality implicates both. There is no defect of mobility of any kind in a typical case of concomitant convergent squint occurring in a child.

A squint may be classified according to the direction of the deviation of the visual axis; convergent when the squinting eye turns in towards the nose, divergent when it turns outwards, sursumvergent when it turns upwards, and deorsumvergent when it turns downwards.

For convenience strabismus is said to be : constant, and to belong to the right or left eye, according as the deviation is always present in one or the other; alternating, when either eye squints indeterminately; occasional, when it occurs intermittently, e.g. in near vision, on looking downwards, or under the influence of excitement, fear, anger, fatigue, or disorders of the digestion.

Of all the varieties of squint, convergent strabismus is much the most common. It occurs most frequently in children from three to four years of age-that is about the time when a child begins to amuse itself with picture-books and toys. At first the squint is noticed only occasionally, more especially when the patient is tired or out of health; but as time goes on, it becomes more and more frequent, and at length is always present. The child now squints with one eye, and looks straight with the other, but parallelism of the visual axes is never observed. Examination shows that there is nothing wrong with the muscles themselves; but they have lost the guidance of the nerve centres, and over-convergence has developed through want of a strong impulse for binocular fixation. If squint be judged by its appearance, the natural explanation is that the internal rectus being too strong has pulled the eye towards the inner canthus. On that hypothesis, Stromeyer and Dieffenbach performed tenotomy for the cure of strabismus.

Donders was the first to advance a theory which diverted attention from the muscles. He observed that the great majority of convergent squints are accompanied by hypermetropia; whereas divergent squints are, for the most part, associated with myopia. Having made that generalisation, Donders proceeded to formulate his theory of the causation of squint- " accommodation theory." He pointed out that in emmetropia there is a fixed association between accommodation and convergence. The functions are always performed together- " associated "; but this normal relationship is at once disturbed by an error of refraction. In 
hypermetropia, a certain amount of accommodation is necessary to obtain clear vision in the distance; consequently, the amount of accommodation a hypermetrope requires for reading or other close work is much in excess of the amount of convergence available. Unless accommodation and convergence act harmoniously together, sight is indistinct ; so the hypermetrope makes an effort to turn both eyes inwards to a degree sufficient to balance the excessive action of the ciliary muscle. The attempt, however, is unsuccessful, and gives rise to much discomfort, because the eyes have been made to converge to a point nearer than the ordinary reading distance. There are only two ways in which the patient can get rid of the discomfort caused by over-convergence. He must either hold the book further away-in which case he will not be able to see to read-or else he must sacrifice binocular vision, and thereby put one eye out of action. If he uses one eye only he will suffer little inconvenience, but while he reads with one eye he squints with the other.

All hypermetropes do not squint, consequently other circumstances must co-operate before convergent strabismus can develop in a hypermetropic eye. Anything which lessens the value of binocular vision predisposes to squint; for example, when the two eyes cannot act together owing to a great difference in their refractive power-anisometropiawhen there are opacities of the cornea the result of ulceration in infancyor when there are congenital defects of the transparent media or of the fundus oculi. Moreover, a squint often develops after a fright following an accident to the eye or to some other part of the body; and it is frequently observed for the first time during convalescence from measles, scarlet fever, or whooping-cough.

Latent Strabismus.-In a large number of cases strabismus remains latent. In ordinary circumstances binocular vision is maintained; but owing to muscular insufficiency, the parallelism of the visual axes is easily disturbed, and suitable tests at once reveal a squint. All instances of heterophoria belong to this group; and as the effort to keep the eyes straight is much greater than in normal conditions, the patients frequently suffer severally from muscular asthenopia.

Apparent Strabismus. - This is due to the angle gamma, which is the angle formed between the visual axis and the antero-posterior axis of the eyeball. The line of vision usually lies to the nasal side of the optic axis, and the angle gamma is then said to be positive. 'In rare cases the visual axis is in the temporal side of the optic axis, and in these circumstances the angle gamma is said to be negative. In emmetropia, and more especially in hypermetropia, the angle gamma is positive; consequently, a deceptive appearance of divergent squint may be pro- 
duced, or a slight convergent squint may be hidden. On the other hand, in myopia the angle gamma is usually negative, so that there is an apparent convergent squint. It is easy to distinguish an apparent from a real strabismus, because in apparent squint the visual axes of both eyes are directed to the same point, and no movement of either eye occurs when the other is covered; whereas, in true squint, there is always a movement of the eye towards the object looked at when the cover test is applied.

Every case of squint ought to be carefully examined. It is desirable to know the age of the child when the strabismus was first noticed; whether it began as an occasional squint, or was constant from the outset; whether it followed an illness-measles, whooping-cough, etc.- -a blow on the head, a fright ; if its onset was accompanied by fits ; and if any hereditary tendency to squint existed either on the paternal or the maternal side. As far as the age of the child makes it possible, the vision of each eye should be tested separately. It is often a great surprise to the parents to discover that one eye is for all practical purposes blind. The fixing eye may have good sight, but amblyopia has developed in the eye which is never used. The defect in sight is usually greatest in a squint in which the deviation is constant and confined to one eye, and is never so pronounced in a squint that alternates. It is exceedingly important to observe if the deviating eye wanders; because, if it does, central fixation is in all probability lost, and that has a great influence both on prognosis and treatment. The movements of each eye separately are easily tested, by covering one and asking the patient to follow with the other the movements of a pencil or some bright object as it is carried upwards, downwards, inwards, or outwards. Defective movement in any direction is readily noticed. The angle of deviation should always be measured, and for the general practitioner the method suggested by Hirschberg is very convenient. The patient fixes a candle, or preferably the mirror of an ophthalmoscope, held straight in front of him about a foot from the eyes. The degree of deviation is estimated by the position of the corneal reflex. Hirschberg divides the cases into five groups-

(I) When reflex is nearer the centre than the margin of the pupil the angle is about ro degrees.

(2) When reflex is at the margin of the pupil $(3 \mathrm{~mm}$. diameter) the angle is about $\mathrm{I} 2$ to $\mathrm{I} 5$ degrees.

(3) When reflex is between the centre of the pupil and the margin of the cornea the angle is about 25 degrees.

(4) When reflex is near the corneal margin the angle is about 45 to 50 degrees. 
(5) When reflex is on the sclerotic between the margin of the cornea and the equator of the eyeball the angle is about 60 to 80 degrees.

When accurate measurements are necessary-for example, before and after operation-other methods are employed, i.e. Priestley Smith's tape, Maddox's tangent'scale, Worth's deviometer, etc. The refraction should be examined by retinoscopy, after atropin I per cent. has been used in both eyes for a week. The condition of the fusion faculty is easily examined with Worth's amblyoscope; but as the general practitioner is not likely to possess those special instruments, nothing more need be said regarding them.

Treatment of Squint.-The principal indications in the treatment of squint are-

(I) To prevent deterioration of vision in the deviating eye.

(2) To restore vision if possible to the eye which has become amblyopic from disuse.

(3) To develop, train, and strengthen the fusion sense.

(4) To obtain parallelism of the visual axes.

In some cases of concomitant squint the eyes become straight as the child grows older, and that circumstance is too often made the excuse for putting off treatment when the strabismus is first noticed. It is said that the patient is too young and that he will outgrow the squint. Delay is always to be deprecated, because the development of the fusion sense, which is the basis of all rational treatment, can only be accomplished while the child is from three to six years old.

The one object of all treatment is to put the eye of a squinting child into a natural condition, and afterwards to develop binocular vision.

(I) Correction of any error of refraction.-A mydriatic must always be used for a week before the eyes are examined by retinoscopy, and should not be discontinued until the spectacles ordered are supplied. The glasses must be worn constantly, except when the child is in bed. The optician ought to supply special frames for very young children, and lenses large enough to prevent the child from looking over the top of them.

(2) Occlusion of the fixing eye.-If one eye has squinted constantly for a considerable time it is sure to be amblyopic, and the surgeon's chief aim is to restore sight in it as far as possible. Continuous occlusion of the fixing eye, whereby the child is compelled to use the deviating amblyopic one, ought always to be employed. This is a very successful method of treatment, but it must be carried out efficiently, and the pad and bandage adjusted in such a way that the child cannot pull it off, or see out below or to the side of it. Improvement in vision is usually manifest in about three weeks; but if no improvement has taken place at the end 
of two months, it is little use to continue the treatment longer. As sight improves the bandage need not be worn continuously, but adjusted for an hour twice' a day. A convenient method is to bind up the fixing eye while the child is at meals.

(3) Instillation of atropin into the fixing eye, but not into the eye which deviates.-By that means the child is forced to use the fixing eye for distance, and the deviating eye for things near at hand. He must use it in order to see his toys, and this constant exercise prevents the development of amblyopia ex anopsia. The treatment ought to be continued for several months, and afterwards used intermittently. The atropin should never be instilled into both eyes, because if the ciliary muscle is paralysed in both, the fixing eye will always be used; and the sole object of the treatment is to make the child use the deviating eye.

(4) Training the fusion sense.-This is only of value during the first six years of life, and is most successfully carried out by means of Worth's amblyoscope. If begun when the child is about three years old, and faithfully continued, very gratifying results are obtained, but after six years of age it is not worth while to spend time upon it. It is no use to try to re-educate a function that has never existed.

(5) Operation.-A large number of cases require operation, partly because so many squints are neglected at the outset-at the very time when treatment to establish binocular vision is likely to be successful. It will be obvious, therefore, that in nearly every case operation is undertaken after all hope of establishing binocular vision must be given up. Early operation is not advisable. As long as the child is wearing suitable glasses, and regularly exercising the deviating eye, there is nothing to be gained by operating before ten or twelve years of age. It is inadvisable to try to lay down rigid rules, but it is very important to remember that the main purpose of the operation is to remove the deformity; it is not at all likely to restore binocular vision.

Probably the two most important factors in determining not only the time to operate, but also the choice of the operation, are the age and the visual acuity of the patient.

The choice of operation lies between tenotomy of one or both internal recti, and advancement of one or both external recti. The advantages of tenotomy are its simplicity, and the rapidity with which the result is attained, but the operation has many drawbacks. It ought never to be performed on young children. If it is not very carefully performed it leaves a staring appearance of the eye, and sinking of the caruncle; its results are uncertain; the power of convergence is weakened; and the eye after some years may become divergent. Advancement of an external rectus, on the other hand, keeps the eye back in the grip of the muscles, 
and rotates it exactly to the required amount; the results obtained are permanent, so that there is no fear of divergence occurring later on; the power of rotation of the advanced muscle is increased; while the rotation of the eye in the opposite direction is not weakened.

All operations on the ocular muscles demand special skill and experience, and the family doctor will be well advised to hand the patients over to the care of an ophthalmic specialist. 


\section{CHAPTER XL}

\section{EYE-STRAIN AND ITS CONSEQUENCES}

The importance of eye-strain as a cause of constitutional troubles-AsthenopiaThe mechanism of eye-strain-The use of cycloplegics-A small error of refraction most apt to produce eye-strain in a neurasthenic patient-The importance of exercise and general treatment in dealing with cases of eyestrain-Co-existence of functional disorders of the liver-Medicinal treatment -Eye-strain due to psychical causes.

Although it is now widely recognised that serious constitutional troubles may originate as a result of uncorrected errors of refraction, most of the text-books on general medicine make very slight reference to the subject, and, consequently, an ocular examination, instead of being one of the first steps in diagnosis, is frequently undertaken only as a last resort. For that reason, ophthalmic surgeons must be grateful to Dr. George M. Gould for the forceful way in which he has written on the subject. In the highly suggestive and most interesting volumes, entitled Biographic Clinics, this author has sought to prove that the continued ill-health of De Quincey, the Carlyles, Darwin, Huxley, George Eliot, Browning, and many others, was the result of eye-strain. $\mathrm{He}$ endeavours to show that all the patients whose histories he describes suffered because of near use of the eyes, and obtained relief only when they put reading or writing aside, and took long walks or horseback exercise in the country; whenever they resumed literary or scientific work, the headaches, the anorexia, the sleeplessness, the irritability of temper, and the depression of spirits, which affected them all so greatly, at once returned. It is easy to say that Dr. Gould has failed to substantiate his contention, and that he writes as one possessed by a fixed idea, but every impartial critic must admit that there is much to be said in his favour. He has, at all events, by his strong statements, awakened the mind of the profession anew to the injurious effects of eye-strain, and has, by his teaching, made it more and more apparent that in many cases of dyspepsia and nervous prostration the advice of an ophthalmic surgeon may result in the rapid restoration of the patient to health.

It must, indeed, be admitted that some of our American confrères have shown a tendency to exaggerate the importance of ocular defects, and have set down uncorrected errors of refraction as the cause of migraine, 
epilepsy, chorea, asthma, anæmia, constipation, drunkenness, and many other ailments. Such special pleading has never found favour in medicine; but the loose and irresponsible statements of a few ought not to be made the excuse for ignoring the essential truth of Gould's contention, that low degrees of ametropia are responsible for headache and many other nervous troubles, and that careful examination of the eyes ought to be a first step in rational therapeutics. A great deal of suffering might be prevented were it always remembered that ocular disturbance may be a possible cause of the mischief, and that this is none the less the case though the error of refraction be so slight that it does not in any way interfere with vision. Indeed, it often happens that when eyestrain is suggested as a cause of persistent head-pain, the patient at once replies that there can be nothing wrong with his eyes, because he sees perfectly well. Such a statement should, however, never be accepted as a reason for neglecting to use the ophthalmoscope. It cannot be too often repeated, or too strongly urged, that neglect to examine the fundus oculi may deprive the physician of a knowledge of the very facts necessary to enable him to form an accurate diagnosis. All experience, indeed, teaches that it is the low rather than the high degrees of refractive error which induce the greatest amount of reflex disturbance, and that ametropia or muscular imbalance may, on careful examination, be discovered in cases where the existence of any defect was quite unsuspected by the patient or by his friends. A high error of refraction is not likely to cause peripheral symptoms, because no effort on the patient's part can overcome it; whereas when the error is slight the patient, in order to obtain clear vision, is always unconsciously correcting it by the over-action of the ciliary muscle, and that is the cause of all the trouble.

Gould and his followers, however, weaken their position by underestimating the important part which other causes play in the origin of head-pain, visceral disturbance, and other functional nervous disorders; for, if symptoms suggestive of serious disease of the brain, kidneys, etc., may be simulated by the disturbance due to an error of refraction, so also may troubles arising from constitutional conditions mimic those resulting from eye-strain. There is nothing pathognomonic in the reflex symptoms arising from refraction errors; and although the patient's account of his symptoms may suggest eye-strain as a probable cause, yet a thorough diagnosis requires careful examination, not only of the eyes themselves, but also of every other organ of the body. If, therefore, there be a tendency on the part of the general practitioner to estimate too lightly the influence of eye-strain in causing, or aggravating, headache and other functional disorders, the specialist on his part must be exceedingly careful to avoid attributing to an error of refraction, symptoms 
which are in reality the outcome of some morbid constitutional state. The all-important thing to remember is that eye-strain may be the unsuspected cause of much suffering for which it is difficult to offer a satisfactory explanation.

In'many cases, however, the symptoms are referred in the first instance to the eyes themselves, and are collectively spoken of as asthenopia. The clinical picture presented by this condition is well known. Mackenzie described it as "that state of vision in which the eyes are unable to sustain any continued exercise upon near objects, although the patient on first viewing such objects generally sees them distinctly, although he can employ his sight for any length of time in viewing distant objects, and although the eyes appear sound." Every attempt to read is attended by a sense of confusion-the print first becomes unsteady and blurred, and then gradually fades from view. It becomes visible again after the eyes have been rested and gently rubbed for a few minutes, but persistence quickly brings about exhaustion, and it is, at length, impossible to see any minute object distinctly. Complaint is usually made of a dull aching pain (sometimes accompanied by twitching of the lids and facial muscles) behind the eyeballs, which feel tight and hot, and may be unduly sensitive to light. The margins of the lids are inflamed; the ocular conjunctiva is injected, suffused, and bleary; the ciliary region may be tender on pressure, the choroid congested, the retinal vessels tortuous and full, and the pupil contracted. At other times the local manifestations may be little marked, or even absent altogether, but whenever the patient reads or writes he complains of headache. The pain may be dull and throbbing, or acute and neuralgic; it may come on while the eyes are being used, or may not be felt until the following morning on first waking; it may be constant or periodic; it is frequently frontal, just above the eyebrows, but is also often occipital or in the nape, radiating down the back and along the arm, and at still other times is felt most severely in one or both temples. In this last form, especially when unilateral, it is often accompanied by nausea and vomiting, and closely simulates typical migraine. Occasionally every attempt to use the eyes for close work is accompanied by diplopia, speedily followed by giddiness and sickness, sensations similar to those experienced by some when objects are moved rapidly before the eyes-e.g. on looking at the landscape from a carriage window while travelling by an express train. Some patients also complain of palpitation of the heart, of general nervousness, of irritability of temper, of sleeplessness and of numerous other functional disorders which, although purely reflex in origin, may so closely simulate organic disease as to make diagnosis very difficult.

The mechanism of eye-strain is not difficult to follow, and when it 
is understood the reflex disturbances to which it may give rise are easily explained. The normal eye is one adapted to receive on the retina a distinct image of a distant object when the ciliary muscle is relaxed, and of a near object when that muscle is contracted. This adaptation to variations of distance is spoken of as " accommodation," and is brought about by the action of the ciliary muscle.

In emmetropia distant objects (for practical purposes twenty feet is looked upon as a sufficient distance in which to obtain parallel rays) are seen distinctly when the eye is in a state of rest, and by the exercise of accommodation clear vision is easily obtained of objects at varying distances. It will be remembered further that a very constant association exists between the acts of accommodation and convergence-the nerve impulse conveyed to the ciliary muscle being accompanied by a similar impulse proceeding to the nerves supplying the internal recti. This ideal condition is at once disturbed by any change in the refraction of the eyeball. The hypermetropic eye is too short, and consequontly in a state of rest, images of distant objects will be formed behind the retina, and only a blurred picture perceived, until by contraction of the ciliary muscle the lens is gradually made convex enough to focus accurately upon the retina the object looked at. The nearer the object, the more powerful is the effort of accommodation required to enable it to be seen distinctly; consequently, in order to relieve the accommodation and the convergence power, the natural tendency is to hold things as far away from the eyes as possible. In myopia, on the other hand, this state of matters is entirely reversed, and the strain is thrown, not on the ciliary muscle, but on the internal recti. The myopic eye is too long, and consequently the image of a distant object is formed in front of the retina. If, therefore, it is to be seen distinctly without the help of a concave lens, the object must be brought nearer and nearer to the eye, and thus a short-sighted person holds things close to his eyes, thereby over-taxing the power of convergence. If astigmatism co-exists with hypermetropia or myopia, the strain on the ciliary muscle and the internal recti muscles will be all the greater; and if, in addition, there be a difference in the near point for each eye-anisometropia-the effort to maintain binocular vision under such disadvantageous conditions will bring about exhaustion all the more quickly.

It is well known that for comfortable sight it is necessary to have from one-third to one-half of the accommodation power, and nearly two-thirds of the convergence power, in reserve. Only for a very short time can the full energy of any muscle be exerted without fatigue, and if the muscle is to be used continuously for any length of time, there must always be a large reserve store of power. The ocular muscles form 


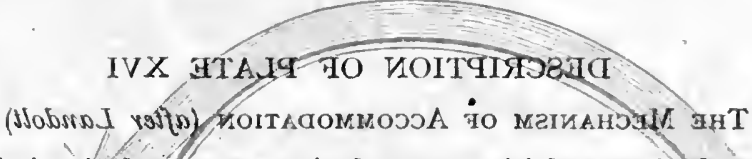

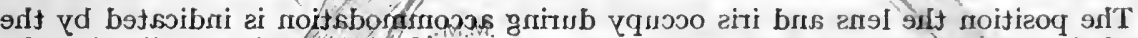

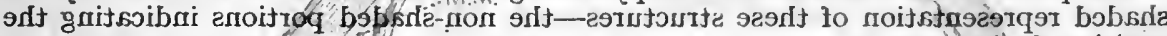

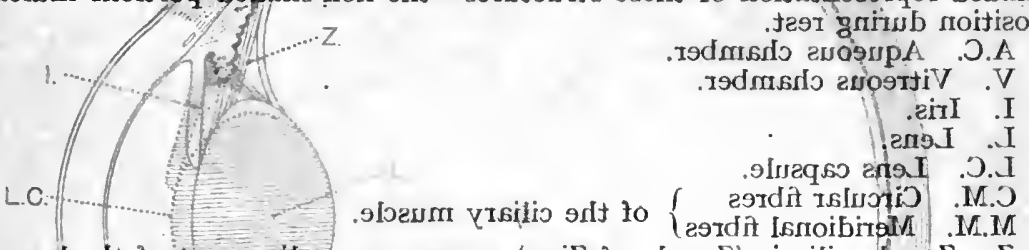

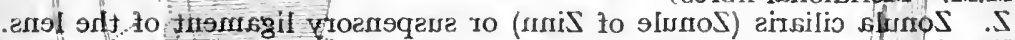

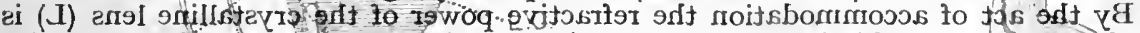

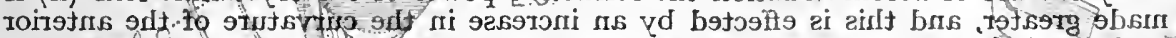

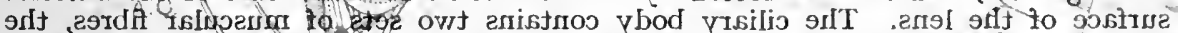

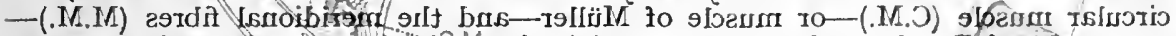

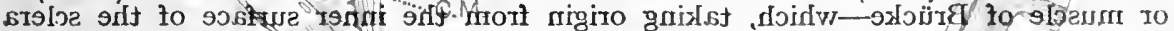

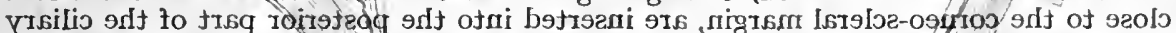

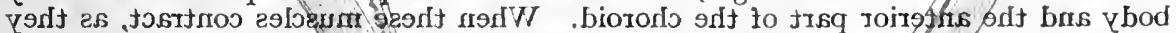
silunoS to enis

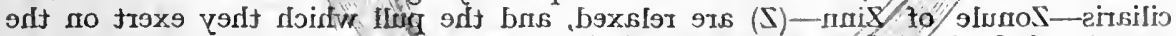

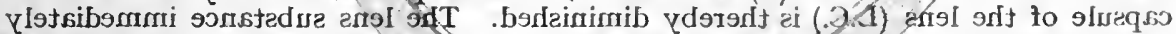

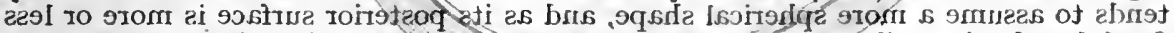

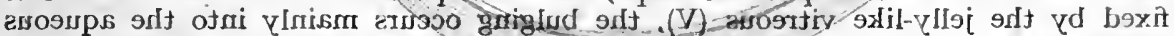
97om asmosgd angl

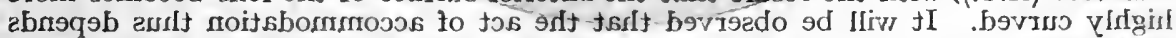

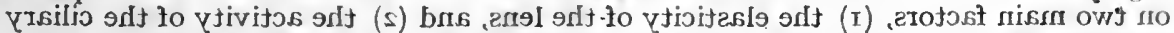

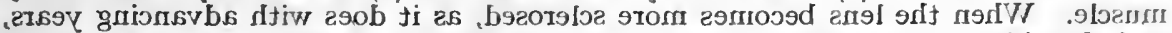

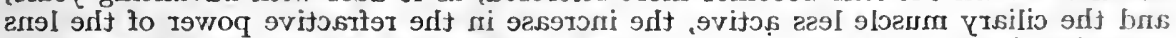

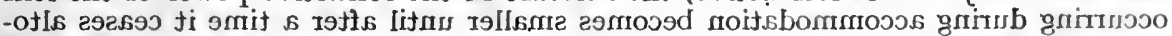

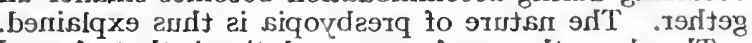

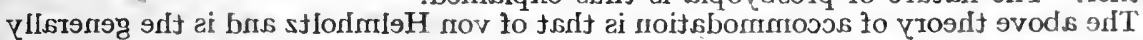

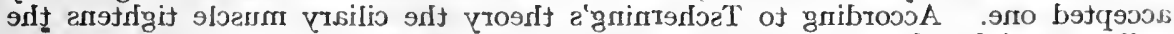
- тoit9fกs

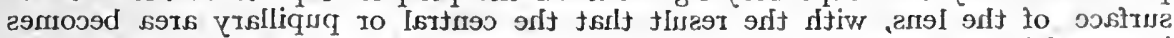

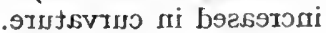



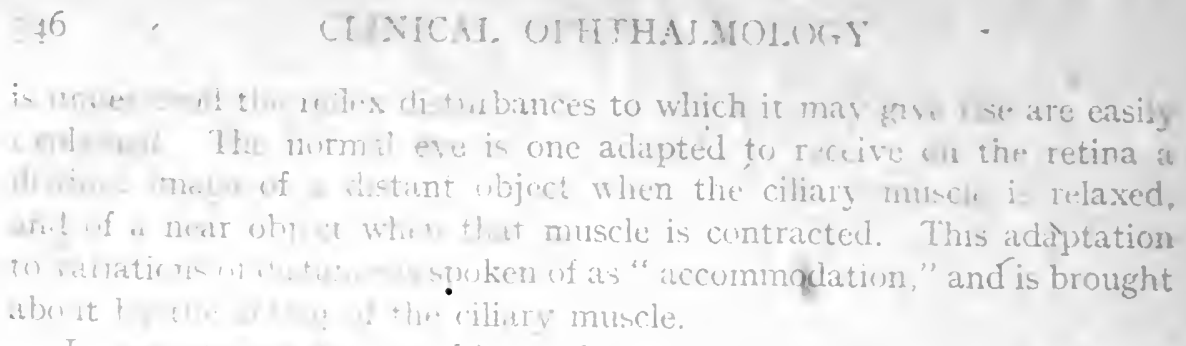

btdin parallel rays) are

\section{DESCRIPTION OF PLATE XVI by the exercise of \\ THE MECHANISM OF ACCOMModTion (after Landolt)s at varying}

The position the lens and iris occupy during accommodation is indicated by the shaded representation of these structures the non shaded portions indicating the position during rest.

A.C. Aqueous chamber, the ciliary muscle being accompanied by a

V. Vitreous chamber. $111 \mathrm{~g}$ to the nerves supplying the internal recti.

I. Iris.

L. Lens.

L.C. Lens capsule.

C.M. Circular fibres

M.M. Meridional fibres of the ciliary muscle. Will be former behind the

Z. Zonula ciliaris (Zonule of Zinn) or suspensory ligament of the lens. of the

By the act of accommodation the refractive power of the crystalline lens $(I)$ is made greater, and this is effected by an increase in the curvature of the anterior surface of the lens. The ciliary body contains two sets of muscular fibres, the circular muscle (C.M.) - or muscle of Müller-and the meridional fibres (M.M.) or muscle of Brücke-which, taking origin from the inner surface of the sclera close to the corneo-scleral margin, are inserted into tlie posterior part of the ciliary body and the anterior part of the choroid: (When these muscles contract, as they do in accommodation, the fibres of the suspensory ligament of the lens or Zonula ciliaris-Zonule of Zinn- $(Z)$ are relaxed, and the pull which they exert on the capsule of the lens (L.C.) is thereby diminished. The lens substance immediately tends to assume a more spherical shape, and as its posterior surface is more or less fixed by the jelly-like vitreous (V), the bulging occurs mainly into the aqueous chamber (A.C.), with the result that the anterior surface of the lens becomes more highly curved. It will be observed that the act of accommodation thus depends on two main factors, (I) the elasticity of the lens, and (2) the activity of the ciliary muscle. When the lens becomes more sclerosed, as it does with advancing years, and the ciliary muscle less active, the increase in the refractive power of the lens occurring during accommodation becomes smaller until after a time it ceases altogether. The nature of presbyopia is thus explained.

The above theory of accommodation is that of von Helmholtz and is the generally accepted one. According to Tscherning's theory the ciliary muscle tightens the pull exerted by the suspensory ligament on the peripheral parts of the anteriorsurface of the lens, with the result that the central or pupillary area, becomes increased in curvature. 


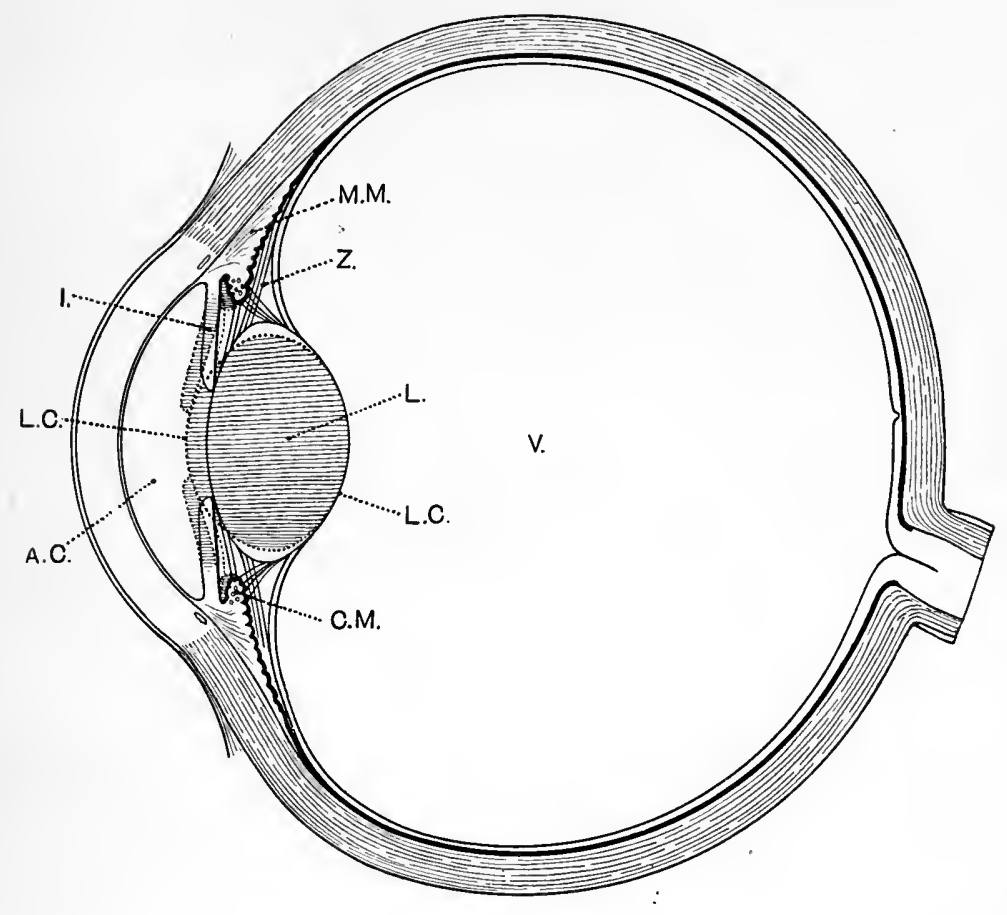



no exception to this law, which is the true key to the proper understanding of the various signs and symptoms associated with eye-strain. The aim of the surgeon in every case of ametropia or of abnormal motility of the ocular muscles, is to re-establish emmetropia or orthophoria by the prescription of suitable spectacles, or by operation on the recti muscles; and in most instances, whenever that is accomplished, muscular fatigue and nervous exhaustion are speedily relieved. The waste of nerve energy has been stopped.

Eye-strain may, however, occur in emmetropia-e. g. during convalescence from acute illness, etc.-and, as has been already said, more commonly along with low, rather than with high, degrees of refraction error or of muscular imbalance. It follows, therefore, that relief of headache, sleeplessness, giddiness, and other reflex disorders, even when these are wholly the result of asthenopia, can be expected only after perfect correction of the optical errors. As a rule, minute defects cannot be detected except when the eyes are examined with the aid of a cycloplegic; and in children, a mydriatic should be habitually employed to relieve spasm of the ciliary muscle, to diminish congestion of the choroidal and retinal vessels, and to reveal the total amount of ametropia. The older the patient the less need is there to paralyse the ciliary muscle; but in every case where the result obtained is not satisfactory, a cycloplegic ought to be employed, and any risk of the occurrence of glaucoma counteracted by instilling a drop of I per cent. solution of eserin, or preferably pilocarpin, immediately after the examination of the eyes is completed. Gould and Ernest Clarke strongly insist that, in many cases, eyes at first pronounced perfect have been demonstrated by careful measurement under cycloplegics to be ametropic, and that subsequently the patients obtained complete relief by the use of suitable glasses. It may be accepted as an axiom that when headache or other nervous affections are due to eye-strain, the patients will get great benefit from complete paralysis of the accommodation; consequently in these circumstances the use of homatropin and cocain is a great help in diagnosis.

Donders stated that as long as astigmatism does not essentially diminish the acuteness of vision, the condition of the eye may be regarded as normal; but obviously, although that may be quite true in regard to the majority of people, it may not be true in regard to those of highly nervous temperament. The indirect effects of anomalies of refraction vary greatly in different individuals. It is common experience that many never complain at all, althoughi on examination the eyes are found to be very defective optically; while others, in whom an error of refraction can be discovered only after most careful examination, complain of symptoms of extreme severity. Neurasthenia makes the use of glasses 
imperative if any error of refraction exists; and in such cases the use of spectacles is a valuable therapeutic measure, because the cure of the patient is aided by the removal of one cause of irritation to the nervous system. It goes without saying, however, that if good results are to be obtained, thoroughness and exactness in the measurement of the refraction are absolutely essential. Without thoroughness there can be no efficient treatment; and it is well known not only that it is from the ranks of the astigmatic children that the short-sighted are recruited, but also that unsuitable spectacles are a very frequent cause of eye-strain. As Gould says, "It is the little, the inconsiderable thing, in refraction work," upon which depends the relief of reflex symptoms.

The prescription of spectacles, although essential and all-important, must not, however, be regarded as the whole treatment. It is not enough, more especially when one is dealing with the young, simply to adjust glasses suitable for the correction of the error of refraction. A quick estimate must at the same time be made of the patient's general strength; for it is most important to remember that though the demand for work made upon the ciliary and the extraocular muscles may not be unreasonably great in itself, yet it may be altogether excessive in respect of the individual in whom it is made. While, therefore, the error of refraction must always be carefully corrected, so that the ciliary muscle may do its duty under the best possible conditions, every effort should also be made to develop and strengthen the whole muscular system, by sufficiency of proper food, by drill, by exercise on the bicycle, or preferably on horseback, and by an active outdoor life.

Among opticians are some who call themselves "Refractionists," or " Optologists," and they of late have tried to promulgate the idea that " spectacles concern the optical part of the eye only and have nothing to do with disease." There can be no greater fallacy than that, for under no circumstances can the living eye be treated simply as an optical instrument. It is not only in the body, but it is also of the body, and the proper treatment of its optical defects requires the physician's skill just as much as does the treatment of its diseases. It must never be forgotten that in the eye, just as in any other part of the body, the limits of muscular capacity must be respected, and the amount of, work to be done regulated accordingly. Children are brought every day by their parents to an eye hospital "to get spectacles." In nearly every case an error of refraction exists, but in many it is so slight that in all probability it would cause no trouble were the general health satisfactory. Many of these children are underfed, live in insanitary houses, are the victims of unwholesome habits, and are, in consequence, physically unfit to bear the strain of continuous school-work. Obviously, in these 
circumstances, it is wiser to prescribe rest for the eye, than to supply glasses which, by the relief they afford, encourage the child to struggle, only to have afterwards, under the stress and strain of school work, a more serious breakdown. There is no doubt whatever that there are many children attending school who are, from want of proper nourishment, physically unfit to bear the mental strain they have to undergo, and who, instead of receiving intellectual gain, suffer actual physical damage in the attempt to instil knowledge into their minds. It is in circumstances such as these that eye-strain is developed, and those structural changes set a-going which determine that variety of short-sight, which is so apt to be progressive, and to lead to permanent impairment of vision in later life, from detachment of the retina, from hæmorrhages, from choroiditis, or from atrophy and degeneration of the macular region.

Nor is the over-taxing of the eyes confined to children. Adults are also at fault, and seem to forget that, of all the muscles of the body, it is the little ciliary muscle which is most constantly in use. The hypermetropic eye is never at rest, but is always straining more or less intensely to form on the retina a clear picture of external objects. Some occupations tax the eyes almost continuously-e.g. the work of students, clerks, typists, seamstresses, etc. - and the accommodation is often strained over fine sewing, drawn-thread work, embroidery, miniature painting, engraving, and similar tasks. Injurious as such occupations are to the eyes at any time-and Motais has said that typists are, from spasm of the ciliary muscle, often more myopic in the evening than in the morning - they are doubly so when they are carried on in a bad light, or when one is physically tired. How often do patients suffering from lack of nerve energy try to rest and read at the same time-for example, when invalids read in bed. They are attempting the impossible. The aching eyes and headache of which they usually complain are clearly due to nervous and muscular fatigue; and when there is any error of refraction the ciliary muscle, already weary, becomes quite incapable of meeting the call on it. Among the commonest accompaniments of eye-strain, more especially in the neurotic and the neurasthenic, are lithiasis and oxaluria; and in the treatment of the symptoms associated with persistent asthenopia the possible co-existence of functional disorder of the liver ought never to be overlooked. In such cases, although the appearances presented are very difficult to describe, and even more difficult to depict, the ophthalmoscope reveals a very characteristic picture. The whole background of the eye is too red, and the retinal vessels, more especially the veins, are turgid, tortuous, and rough in outline. There is a lack of normal transparency, and the whole fundus is distinctly pigmented, as if very fine black pepper had been dusted over it, and then 
rubbed in so as to partially conceal it from view. As a rule, the optic disc is pink, from congestion of its capillaries and smallest blood-vessels. The macula is deeper in colour than the parts around, and is in consequence very easily seen.

Patients who suffer from such forms of eye-strain and its effects are usually sent to the ophthalmic surgeon by the family doctor, or consult him for the headache on the recommendation of a friend, but they rarely come spontaneously to complain of defective sight. Almost invariably, however, careful examination discovers a low degree of ametropia, and perhaps in addition some latent inadequacy of the extraocular muscles. The head-pain is often accompanied by sleeplessness, is, as a rule, unilateral, usually very severe, and always aggravated by any steady use of the eyes-e.g. in reading, sewing, shopping, looking at pictures in a gallery, watching the stage at a theatre, etc., etc. In a few instances the intense headache is preceded by the occurrence of coloured spectra, or by a transient attack of hemianopia, due to spasm of the retinal vessels. It would seem that although the ocular flaw is latent, yet it is capable of inducing sufficient irritation to determine a "nerve storm" in a patient predisposed to such disturbance owing to natural instability of the nervous system. Obviously, the first step in treatment ought to be to adjust glasses to restore both the refraction and the motility of the eyes to the normal; for until the primary cause of the nervous disturbance is removed, there is little chance of the symptoms being relieved by any medicines.

The important thing is to try to estimate the working power of the ciliary muscle. The response it will make to any demands made upon it depends upon many things. It is hypertrophied in the hypermetropic, but usually atrophicd in the myopic, and its strength is influenced by general fatigue during convalescence from illness; and, in short, by anything that tends to weaken muscular strength as a whole. Patients suffering from asthenopia often ask the doctor how long they can read or sew at one time, but that is a question which is not very easily answered. The response that the ciliary muscle will make to demands made upon it will depend almost entirely upon the general state of the health. If a patient stood while he was reading and stopped when he felt inclined to sit down, that would probably be a safe guide, but one that would not be easily followed.

While, however, the ocular causation should always be kept in mind, it ought also to be remembered that many factors may contribute, if not to cause, certainly at least to perpetuate, the irritability of the nervous system; and if treatment is to be rational and thorough, it must not under-estimate the importance of any of them, but take each and 
all into careful consideration, and so look at the whole condition in due perspective. The urine ought in every case be to examined both chemically and microscopically, for the results thus obtained will often afford valuable therapeutic suggestions. As evidence of defective metabolism is very frequently found, it is usually necessary to give careful directions regarding diet. These, however, should not be stereotyped, but be modified to suit the requirements of the individual patient. What is one man's meat may be another man's poison-many people diet themselves to the verge of starvation. It is equally essential to promote free elimination of waste products, and in practice excellent results are obtained from the phosphate or sulphate of soda combined with potash or lithia, administered every morning, or night and morning, for several weeks. These salts may be prescribed in the effervescing form, and taken in a tumberful of either hot or cold water. The dose should be sufficient to act on the bowels, but must never purge. In neurotic patients the efficacy of the salines is greatly increased by combining them with a bromide, such as bromide of ammonium, the hydrobromide of caffeine, or Warner's preparation of bromo-soda. Lauder Brunton speaks highly of the good results obtained by the use of salicylate of soda with bromide of potassium-the former " to clear out waste products," and the latter "to quiet the nervous system."

There is another group of cases in which the patients, who are generally women, complain that every attempt to use the eyes is accompanied by intense discomfort. The symptoms, one of which is usually great intolerance of light, are so distressing that the patient is quite unable to read or write, and in many cases abandons the attempt to take any active part in life, and thinks and speaks of little else than her ocular troubles. One frequently finds that health has originally been impaired by illness, by prolonged sick-nursing, or by some serious mental or moral shock, and that the asthenopia has come on during the period of convalescence. As a rule, the patient has consulted many ophthalmic surgeons, and has fallen a ready prey to the prescribing optician, but all in vain. She will often produce numerous pairs of spectacles, and in a calm, firm voice declare that not one of them is of the slightest use. Examination may detect an error in the refraction and motility of the eyes, and the patient may show signs of the gouty, or of the rheumatic, diathesis; but neither the use of carefully adjusted glasses, nor the most appropriate and painstaking treatment, affords the slightest relief. On the contrary, on each succeeding visit, the patient will express herself as feeling no better, and without any evidence of emotion will again tell a tale of endless suffering. She is always on the outlook for an opportunity to prove the doctor wrong and herself right; and although she 
may be most punctilious in taking her medicines, she has no belief whatever in their power to do her good. Obviously, in these cases the asthenopia is due to psychical causes, but it must not on that account be concluded that the distress is imaginary. On the contrary, I believe it to be very real and very intense; for just as consciousness of pain may be completely inhibited by psychical causes, so in a similar way it may be greatly intensified. Here, obviously, mental influences are among the most important means of cure, and the success obtained in any given case will be in direct proportion to the amount of faith the patient has in her doctor. The reality of the suffering makes it most difficult to withdraw the mind from it, but unless this can be accomplished, treatment will be of little avail. Change of air and scene, the use of galvanism and high-frequency currents, baths, and massage are most helpful; but, after all, it is the personality of the physician that counts for most. In short, it is the patient herself who requires treatment more than her disease; and successful therapeutic results can only be obtained if the doctor can inspire her with hope, and by and by convince her that she is being gradually and completely restored to health. If he is able to do this, he will accomplish all that is necessary. 


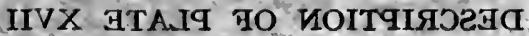

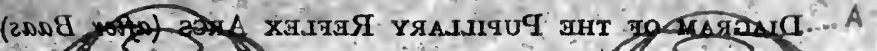

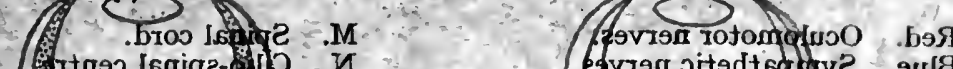

xง

.aftinsoinumos in

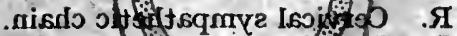

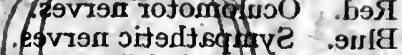
.ani bas Ilsdoy A $A$ 89vion visidip trod? : :

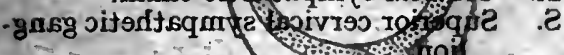
- V1951spitors istotaI $\cdot T$

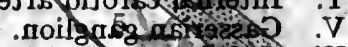

Isaimggint to moizivib qimlef tad $W$ moilgoss/rrailio :D

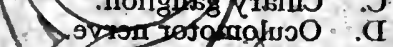

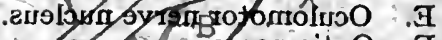

$1-F$ - stran jitqO osmesiñ .H ovion vis lis gta. 3h in jo - Absupsjogrootogging we enlusilloj

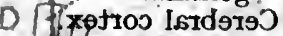
stganoldo sIlubaM

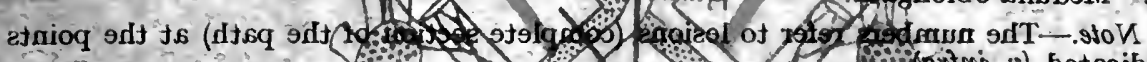

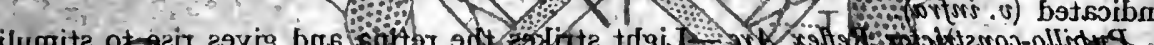

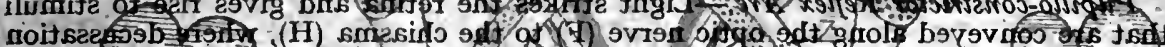
to 2tos

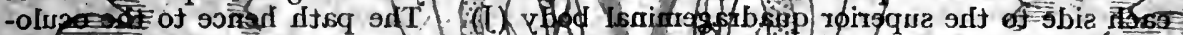

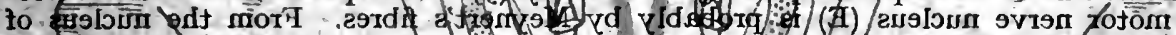

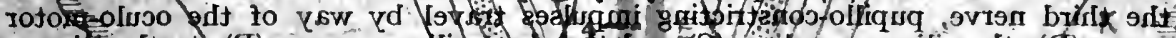

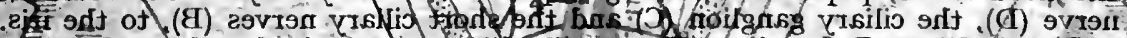

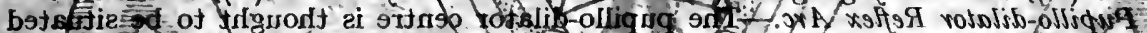

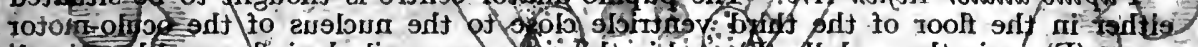
ilumide d

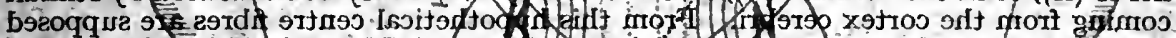

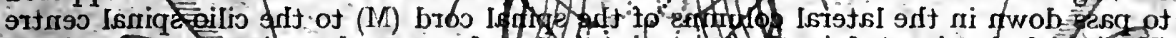

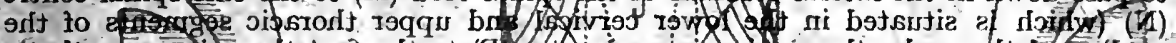

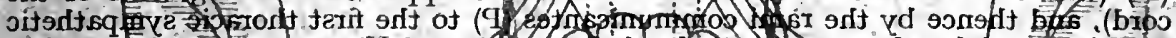

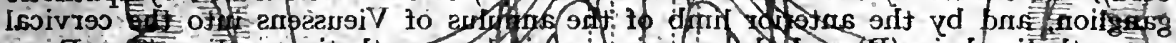

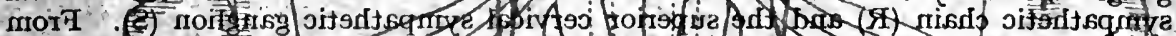

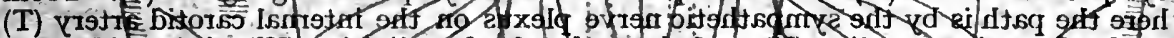

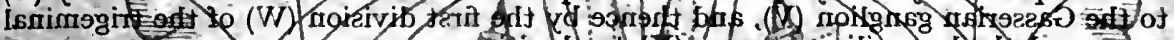

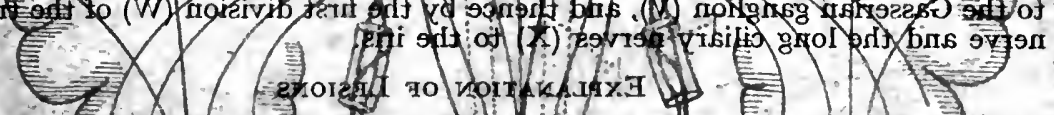

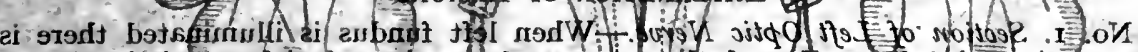

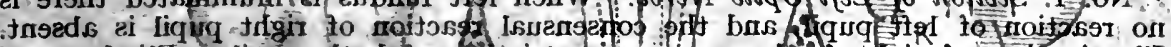

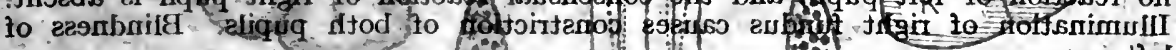

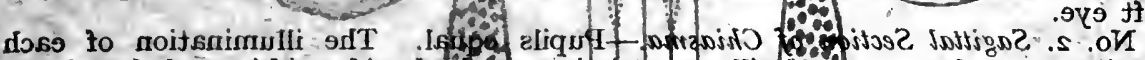

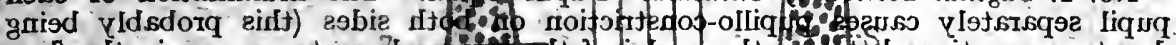

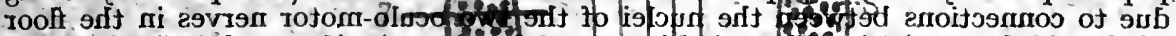

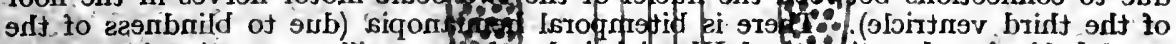

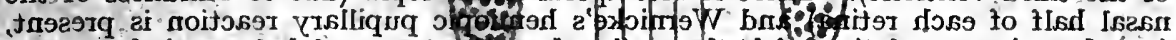

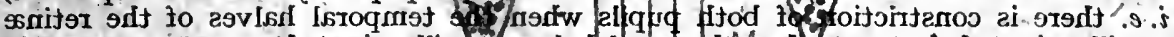

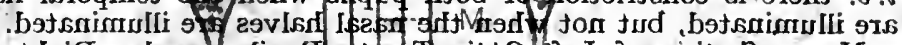

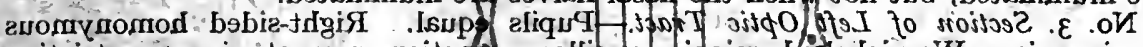

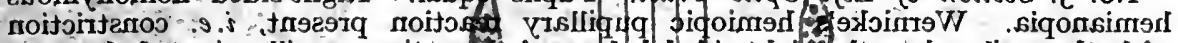

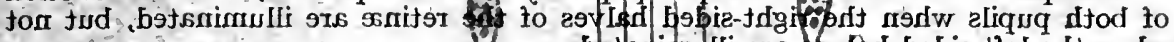

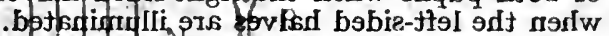

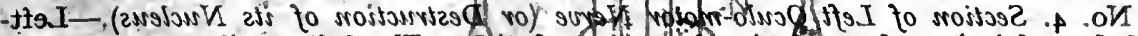

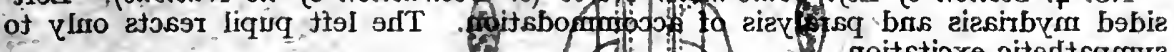

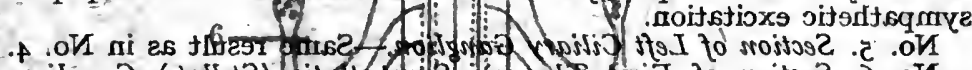

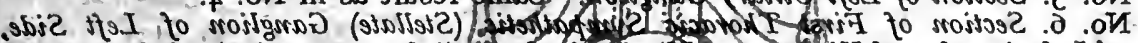

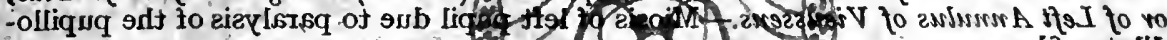

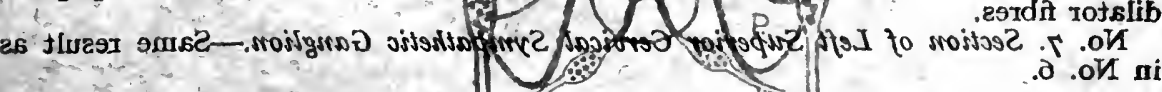




\section{DESCRIPTION OF PLATE XVII}

Diagram of THE PUPILLARY REFLEX ARCS (after Baas) whe aithe

Red. Oculomotor nerves.

Blue. Sympathetic nerves.

A. Eyeball and iris.

B. Short ciliary nerves.

C. Ciliary ganglion.

D. Oculomotor nerve.

E. Oculomotor nerve nucleus.

F. Optic nerve.

H. Chiasma.

J. Colliculus superior of corpora quadragemina.

K. Cerebral cortex.

L. Medulla oblongata.

Note.-The numbers refer to lesions (complete section of the path) at the points indicated (v. infra).

Pupillo-constrictor Reflex Arc.-Light strikes the retina and gives rise to stimuli that are conveyed along the optic nerve $(F)$ to the chiasina $(H)$, where decussation of the fibres takes place. Hence stimuli are conveyed along the optic tracts of each side to the superior quadrageminal body $(J)$. The path hence to the oculomotor nerve nucleus (E) is probably by Meynert's fibres. From the nucleus of the third nerve, pupillo-constricting impulses travel. by way of the oculo-motor nerve (D), the ciliary ganglion (C) and the short ciliary nerves (B), to the iris.

Pupillo-dilator Reflex Arc.-The pupillo-dilator centre is thought to be situated either in the floor of the third ventricle close to the nucleus of the oculo-motor nerve (E), or in the medulla (L), and in this way may easily be influenced by stimuli coming from the cortex cerebri. From this hypothetical centre fibres are supposed to pass down in the lateral columns of the spinal cord (M) to the cilio-spinal centre (N) (which is situated in the lower cervical and upper thoracic segments of the cord), and thence by the rami communicantes (P) to the first thoracic sympathetic ganglion, and by the anterior limb of the annulus of Vieussens into the cervical sympathetic chain (R) and the superior cervical sympathetic ganglion (S). From here the path is by the sympathetic nerve plexus on the internal carotid artery (T) to the Gasserian ganglion $(V)$, and thence by the first division (W) of the trigeminal nerve and the long ciliary nerves $(X)$ to the iris.

\section{Explanation of Lesions}

No. I. Section of Left Optic Nèrve.-When left fundus is illuminated there is no reaction of left pupil, and the consensual reaction of right pupil is absent. Illumination of right fundus causes constriction of both pupils. Blindness of left eye.

No. 2. Sagittal Section of Chiasma.-Pupils equal. The illumination of each pupil separately causes pupillo-constriction on both sides (this probably being due to connections between the nuclei of the two oculo-motor nerves in the floor of the third ventricle). There is bitemporal lemianopia (due to blindness of the nasal half of each retina) and Wernicke's hemiopic pupillary reaction is present, $i$.e. there is constriction of both pupils when the temporal halves of the retina are illuminated, but not when the nasal halves are illuminated.

No. 3. Section of Left Optic Tract.-Pupils equal. Right-sided homonymous hemianopia. Wernicke's hemiopic pupillary reaction present, i.e. constriction of both pupils when the right-sided halves of the retina are illuminated, but not when the left-sided halves are illuminated.

No. 4. Section of Left Oculo-motor Nerve (or Destruction of its Nucleus).-Ieftsided mydriasis and paralysis of accommodation. The left pupil reacts only to sympathetic excitation.

No. 5. Section of Left Ciliary Ganglion.-Same result as in No. 4 .

No. 6. Section of First Thoracic Sympathetic (Stellate) Ganglion of Left Side, or of Left Annulus of Vieussens.-Miosis of left pupil due to paralysis of the pupillodilator fibres.

No. 7. Section of Left Superior Cervical Sympathetic Ganglion.-Same result as in No. 6. 


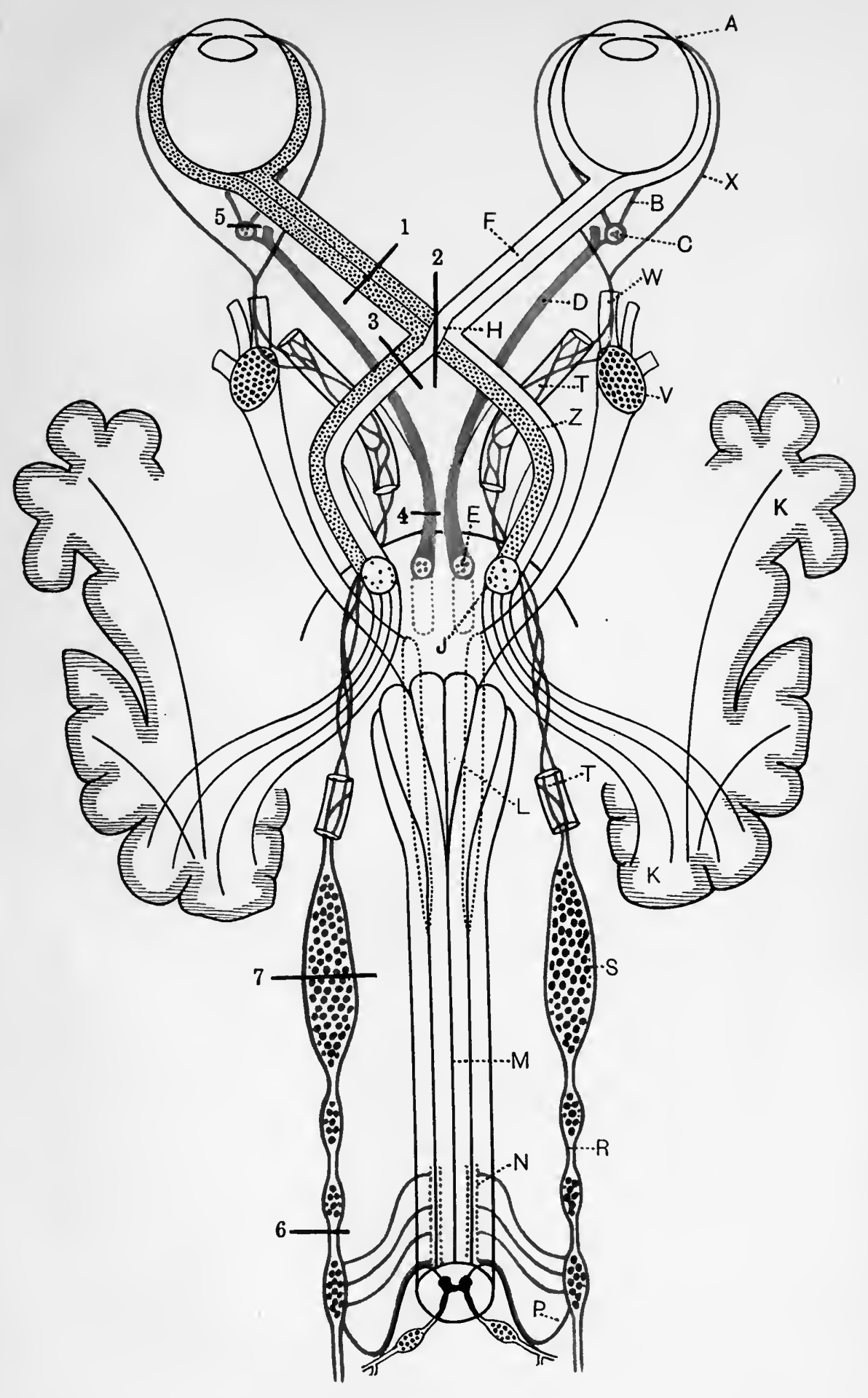





\section{CHAPTER XLI}

THE PUPIL IN HEALTH AND DISEASE

Abnormalities in the shape and colour of the pupil-Factors influencing the size of the pupil-Myosis : as an accompaniment of an ocular affection; occurring in the course of acute and of chronic general disease-Mydriasis : as an accompaniment of an ocular affection; occurring in cases of injury to the skull or orbit; occurring in the course of general disease-Inequality of the pupilsMethod of testing the pupillary reflexes-Hippus-The paths of the pupillary reflex arcs-Abnormalities of pupillary reaction-the Argyll Robertson phenomenon-Wernicke's hemiopic pupillary reflex-Irido-dilatation reflex.

THE practitioner, when he makes an examination of the pupils, ought carefully to observe if they are large or small, if they are equal or unequal, whether or not they react normally to light and in the act of convergence, and if they dilate fully, or if the margin is adherent in any part of its circumference to the anterior capsule of the lens.

In the normal condition the pupil is circular in shape, and jet black in colour; but variations occur both in its form and in its appearance as a result of disease. Abnormalities in the shape of the pupil may be due to congenital malformations, to the results of disease, or to surgical operation. A congenital coloboma is the result of incomplete closure of the choroidal fissure, is usually placed downwards, and in general accompanied by a coloboma of the choroid; whereas a surgical coloboma is as a rule made in an upward direction, in order that the gap in the iris may lie under cover of the upper lid. The pupil may be displaced by the iris having prolapsed through a penetrating wound or a perforating ulcer of the cornea, and been afterwards firmly caught in the scar during the process of healingleucoma adherens; or its border may present irregularities in outline due to the presence of posterior synechice. It is sometimes necessary to instil a mydriatic before the points of adhesion of the pupillary margin of the iris to the anterior capsule of the lens can be seen. In glaucoma the pupil is usually dilated, and somewhat oval in shape.

The jet-black appearance of the pupil is at once altered by the occurrence of inflammation of the iris. It presents a characteristic greenish colour in glaucoma, and a dull grey appearance-senile reflex-in old age; but neither of these conditions should be mistaken for lenticular cataract, because in both the lens remains transparent and the fundus oculi can be seen with the ophthalmoscope. When the lens is dislocated

$$
\text { A A }
$$


into the anterior chamber the appearance resembles a drop of oil in the pupil.

The size of the pupil in health varies as a rule between $2.5 \mathrm{~mm}$. and $5.5 \mathrm{~mm}$., but in exceptional circumstances the measurement may be anything between I $\mathrm{mm}$. and Io $\mathrm{mm}$. and still remain within the limits of health. The diameter of the pupil is always smaller at the two extremes of life than in youth and middle age ; but time does not act upon the pupil in the same way that it affects the ciliary muscle and the power of accommodation. It is generally believed that the pupil is smaller in those with blue irides than in those with dark eyes, especially if they are hypermetropic; but it is very doubtful if either the colour of the iris or the refraction of the eye has any influence on the relative size of the pupil. Other factors, however, have a definite influence, and the following are the most important : the intensity of the illumination, the part of the retina on which the light falls, the adaptation of the eye (convergence and accommodation), sensory stimuli from various parts of the body, psychical changes or emotional states, the action of drugs both general and local, etc. It is, however, interesting to observe that while light is the only stimulus capable of causing reflex contraction of the pupil, every sensory stimulus -cutaneous, visceral, or psychical-can induce dilatation. The size of the pupils is also dependent on the intraocular tension and on the blood pressure. A sudden fall in intraocular tension, such as occurs after perforation of the cornea with escape of the aqueous humour, is followed by great distension of the blood-vessels of the iris, accompanied by contraction of the pupil; whereas, a sudden fall in blood pressure brings about rapid diminution in the cubic capacity of the vessels of the iris, and a corresponding dilatation of the pupil.

An accurate knowledge of the physiology of the movements of the pupil is essential to a proper understanding of its action in disease. Paralysis of the oculo-motor causes mydriasis, while irritation of the oculomotor produces myosis. Paralysis of the sympathetic causes myosis, and irritation of the sympathetic gives rise to mydriasis. Mydriasis, a dilatation of the pupil, is therefore either paralytic or spasmodic; and a similar classification is applicable to all cases of myosis or contraction of the pupil. It is not difficult in an individual case to determine whether the dilatation or the contraction of the pupil is paralytic or spasmodic. The one thing to examine carefully is the direct light reflex. If it be absent, there is a lesion of the oculo-motor nerve-paralytic if there is mydrias.s, spasmodic if there is myosis; whereas if the direct light reflex be present, there must be a lesion of the sympathetic-paralytic if there is myosis, spasmodic if there is mydriasis. The action of mydriatics on a contracted pupil is also helpful in diagnosis; because, whereas atropin 
dilates the pupil fully in spasmodic myosis, it has little or no action on a case of paralytic myosis. Cocain on the other hand causes dilatation in paralytic myosis, but does not act on the pupil in spasmodic myosis; the explanation being that the one drug paralyses the constrictor pupillæ, while the other stimulates the dilator.

A. Myosis.-The pupil is abnormally small, i.e. the diameter of the pupil is less than $2 \mathrm{~mm}$. in ordinary light. It arises either from spasm of the sphincter of the iris (oculo-motor), or from paralysis of the dilator (sympathetic). Myosis, therefore, is either spasmodic or paralytic. In the former the pupil dilates on the application of sensory or psychical stimuli ; in the latter the dilator reflex is absent. When spasmodic myosis gives place to mydriasis, the change always indicates serious danger to life, e.g. opium poisoning, chloroform narcosis, meningitis, etc.

From the clinical standpoint cases of myosis may be divided into three groups-

.r. When there is a coexisting affection of the eye itself.

2. When it occurs in the course of acute general disease.

3. When it occurs in the course of chronic general disease.

I. WHEN MYOSIS OCCURS AS AN ACCOMPANIMENT OF AN OCULAR AFFECTION. It is necessary to enquire in the first place whether dropseserin or pilocarpin - have been instilled, or if opium or morphia has been taken internally. In the great majority of other general intoxications dilatation of the pupil is the rule. The anterior segment of the globe should also be examined for keratitis, iritis, or cyclitis, for a penetrating wound with escape of the aqueous humour, or for a foreign body hidden beneath the upper eyelid or embedded in the cornea itself. In all these instances, the contraction of the pupil is accompanied by symptoms of irritation-pain, pericorneal injection, lachrymation, and photophobia-and in the great majority of cases the condition is unilateral.

2. When Myosis oCCURS IN THE COURSE OF ACUTE GENERAL DISEASE. The contraction of the pupil is bilateral; the patient's general condition is profoundly affected, and as a rule he is unconscious. When bilateral myosis occurs suddenly, it is usually the result of poisoning by opium, morphia, chloral, or chloroform, or an overdose of alcohol ; but it may also be due to uræmia or to apoplexy, especially when the hæmorrhage is in the pons. In these circumstances an examination of the urine ought always to be made.

3. WHEN MYOSIS OCCURS IN THE COURSE OF CHRONIC GENERAL DISEASE. Paralytic myosis is the result of paralysis of the sympathetic in the neck from injury, or from the pressure of enlarged glands or of a new growth. It is always accompanied by preservation of the light reflex, and by signs 
of interference with the sympathetic nervous system, e.g. moderate ptosis and loss of sweating on the same side of the face. If, on the other hand, the myosis is accompanied by loss of the direct light reflex, it is due to irritation of the oculo-motor, and is one of the most trustworthy signs of syphilis of the cerebro-spinal system, more especially of general paralysis or of tabes dorsalis. If the patient is blind, the loss of sight is in all probability due to tabetic atrophy of the optic nerves.

B. Mydriasis.-The pupil is abnormally large : i.e. the diameter of the pupil is greater than $5 \mathrm{~mm}$., and the dilatation persists in bright light. It may result from paresis or paralysis of the sphincter of the iris, from a lesion of the pupil-contracting centre, or in the course of the oculo-motor nerve-paralytic mydriasis. It may also occur, although much less frequently, from irritation of the sympathetic nerve-spasmodic mydriasis. It is, therefore, necessary in every case to examine the light reflex, to observe whether the mydriasis is bilateral or confined to one side, to enquire if double vision exists, and to test the vision both for distance and for near at hand. By that means the patients can at once be divided into three classes-

I. Those who are suffering from an ocular affection.

2. Those who are suffering from injury to the skull or to the orbit.

3. Those who are suffering from general disease.

I. MYdRIASIS THE ACCOMPANIMENT OF AN OCULAR AFFECTION.

(a) The patient can see in the distance, but is unable to read. Two most likely causes are that the patient has been using atropin, cocain, or other mydriatic, or that he has recently suffered from sore throat. Diphtheria, as is well known, may be followed by paralysis of various muscles, voluntary or involuntary, and of the latter the ciliary muscle is one of those most frequently affected. The pupil contracts on exposure to light, but the convergence reflex is lost.

(b) The patient complains of failing sight; glaucoma is the most likely cause : the dilatation of the pupil is accompanied by deep injection of the front of the eye, by pain, not only in the globe but also around the orbit, and by increase in the intraocular tension. In chronic simple glaucoma there are no signs of congestion. It may also occur in orbital cellulitis, in tumour of the orbit, in embolism of the central artery of the retina, in thrombosis of the central vein, in massive retinal or pre-retinal hæmorrhages, or in detachment of the retina. It is also present after a blow on the eye as a result of paresis of the iris, or of dislocation of the lens.

(c) The patient complains of serious loss of sight which in many instances amounts to blindness. Bilateral mydriasis with blindness is usually due to atrophy of both optic nerves, or to serious disease of the 
retina or choroid. It is not difficult to arrive at a correct diagnosis. Bilateral blindness is nearly always accompanied by mydriasis; but to that rule there are two exceptions, viz. :-tabetic atrophy of the optic nerves, and disease or injury of the visual centres in the occipital lobes. In the former the pupils are usually contracted; in the latter they are as a rule of normal size.

2. MYDRIASIS THE ACCOMPANIMENT OF INJURY TO THE SKULL OR THE ORBIT. A blow on the forehead is frequently followed by blindness of the eye on the same side as the injury; it is the result of injury to the optic nerve, either from pressure of extravasated blood, or as a result of fracture through the optic foramen. The light reflex is always abolished in the injured eye, and the consensual reflex in the other; which gives the clue to the real nature of the case at a time when ophthalmoscopic examination shows no pathological changes in the fundus oculi. The oculo-motor nerve may be directly implicated, and give rise to squinting and double vision.

3. Mydriasis THE ACCOMPANIMENT OF General DISEASE.

(a) When the patient appears in every other respect to be in good health, dilatation of the pupil occurs in these circumstances as an isolated sign; but if properly understood it is really a danger-signal of great value, because, in the majority of instances, it not only indicates that the patient has suffered from syphilis, but also that he will in all probability develop in the near future signs of tabes dorsalis, or of general paralysis of the insane. Before any opinion is given, or any treatment begun, a Wassermann test of the blood should be made, in order that the diagnosis of syphilis may be confirmed. The mydriasis is frequently the result of a lesion in the nuclear origin or in the course of the oculo-motor nerve, and is accompanied by external and internal ophthalmoplegia. The allimportant fact to remember is that, probably nine times out of ten, paralytic mydriasis indicates that the patient has been infected by syphilis and that he is now threatened by serious disease of the nervous system.

(b) When the patient is obviously ill, the mydriasis is usually bilateral, and occurs in the course of meningitis, of abscess, or of tumour of the brain, or as a result of a lesion implicating the nuclear origin of both third cranial nerves, e.g. botulism or alimentary toxæmia. In diphtheria the ciliary muscle is always more seriously affected than the iris, consequently, whereas all power of accommodation is lost, the pupil retains a certain amount of response to the stimulus of light. It niay appear also, with startling suddenness, in many acute intoxications, and in these circumstances is a sign of grave danger to life, $e$. g. in poisoning by chloroform.

(c) The pupils are unequal. Inequality of the pupils is of more importance than either bilateral contraction or bilateral dilatation. In rare instances it is congenital, and is then of no pathological significance; but 
its detection ought always to arouse suspicion, and demand the most careful investigation of the pupillary reflexes. In no case can the size of the pupil be separated clinically from a study of its reflexes. When the inequality of the pupils is congenital, the reflexes are as a rule normal; but whenever it can be confidently demonstrated as a new fact, it is the result of causes which have produced myosis or mydriasis in a single eye, or contraction or dilatation has occurred unequally in both. The inequality of the pupil may escape observation if the eyes are examined together, but is detected at once if they are examined separately. The discovery ought to put the practitioner on his guard, and prevent him from falling into the error of considering nervous symptoms to be functional when they are in reality the result of organic causes. Moreover, the size of either pupil taken separately may not be outside the range of the normal : it is the inequality that has the real clinical significance. It is of course always necessary to eliminate the cases that are due to an affection of the eye itself, e. g. iritis, glaucoma, etc. ; but if that be done, acquired inequality of the pupils may be regarded as one of the earliest signs of disease of the nervous system, or it may indicate that there is pressure in the cervical sympathetic from aneurism, tumour, pulmonary disease, etc.

The Mobility of the Pupil, or the Pupillary Reflex.-When the reflexes of the pupil are being examined, the practitioner should tell the patient to look in the distance, and the test is best carried out in ordinary diffuse daylight. It is usual to examine the direct and consensual light reflexes in the first place, and afterwards to test the convergence reflex. The latter can be carried out in a blind person by asking him to converge on his own finger pressed against his nose. In disease, the mobility of the pupil is of the first importance, and consequently a test of the pupillary reflexes ought to form a routine part of a clinical examination.

In the normal state the size of the pupil is continually changing, in consequence of the action of light, accommodation, convergence, and of sensations proceeding from the skin, the viscera, or the mind. All these movements are due to reflex action and quite independent of the will. Hippus, or " the unrest of the pupil," is the perpetual but very minute and irregular fluctuations in the size of the pupil due to the ever-varying sensitive and psychical reflexes which are thus constantly manifesting themselves; hence in delicate, nervous, excitable people the pupils are often much and habitually dilated.

A. Light Reflex, direct and consensual.-In the optic nerve there are both visual and pupillary fibres; and as the former are finer and more delicate than the latter, they usually suffer first in disease. The reaction of the pupil to light is exceedingly sensitive, and is employed with great advantage to determine objectively whether an eye has perception of light 
$-e . g$. in very young children, in those suspected of malingering, in patients suffering from cataract, disease of the optic nerve, etc.

It is always necessary to bear in mind the path of the reflex arc, which is conveniently divided into three portions-

(a) Centripetal or afferent portion-optic nerve, optic chiasma, optic tracts, and fibres to the cerebral cortex.

(b) Meynert's fibres-or the connecting fibres between the corpora quadrigemina and the nuclei of the oculo-motor nerves.

(c) Centrifugal or efferent portion-nucleus of sphincter of iris, oculomotor nerves, ciliary ganglion, and the nerves which terminate in the sphincter muscle of the iris.

B. Convergence or Accommodation Reflex is due to the intimate association of the central innervation of the sphincter muscle of the iris, the ciliary muscle, and the internal recti muscles.

C. Reflex to sensory stimuli or to psychical impressions-e.g. pain, emotion, etc. The following is the reflex path for the sensory dilatation of the pupil: Peripheral sensory nerves, cerebral cortex, oculo-motor nucleus (whose tonus is inhibited), ciliary ganglion, and the short ciliary nerves to the sphincter pupillæ. The sympathetic nerve reaches the iris through the Gasserian ganglion, the ophthalmic division of the trigeminus, and the long ciliary nerves.

Abnormalities of Pupillary Reaction.-When a healthy eye is exposed to light the pupil contracts, but at once dilates in the shade - direct reflex. Moreover, the contraction takes place simultaneously and to the same extent in both eyes, even although only one has been directly exposed to the light-consensual reflex. In disease, variations from the normal occur in the behaviour both of the direct and the consensual reflex. The lesion which causes the abnormality may be situated. either in the centripetal or in the centrifugal pathway of the reflex arc.

I. THERE IS AN INTERRUPTION OF THE CENTRIPETAL SIDE OF THE REFLEX ARC IN FRONT OF THE CHIASMA. It is important in every case to ascertain whether or not sight is affected. In either case the pupil on the side exposed to the light does not contract, and no consensual reaction occurs in the other side; but when light is thrown into the second eye, it causes simultaneous and equal contraction of both pupils. It always indicates a serious lesion of the fundus or of the optic nerve, and sight is always more or less affected. An examination with the ophthalmoscope will generally reveal the cause, $e$. g. embolism, thrombosis, atrophy of the optic nerve, etc.; but a lesion of the optic nerve behind the place of entrance of the central artery of the retina is at the outset unaccompanied by changes that can be seen in the fundus, and it is the state of the pupil that gives the clue to the diagnosis. 
When one eye is said to be blind, malingering must be suspected if the light reflex is present and no pathological changes can be detected on ophthalmoscopic examination. If, however, blindness of both eyes be alleged, the lesion may be situated in the occipital cortex or in the optic radiations of both sides; and as such a lesion is outside the reflex arc, the light reflex is unaffected. In these circumstances, however, other signs of cerebral disease are usually present which enable the practitioner to make a diagnosis of true cortical blindness. A patient should not therefore be regarded as a malingerer, or as simply suffering from hysteria, until all the other possible conditions have been carefully excluded.

The loss of the direct light reflex on one side, accompanied by loss of the consensual reflex on the other, may occur without any complaint of failing sight, and in these circumstances it is always symptomatic of disease of the nervous system. When such a condition of matters is found, the convergence reflex ought to be tested. Almost invariably the pupils will contract. The loss of the light reflex with preservation of the convergence reflex, is known as the Argyll Robertson pupil, and is of very great value in diagnosis. The pupil, moreover, does not dilate on sensory or painful stimulation. It is frequently accompanied by paralytic.myosis, and the outline of the contracted pupil is sometimes irregular. The pathological basis for the Argyll Robertson pupil is not yet accurately determined, but its clinical significance is thoroughly established. It is probably never present except in patients who have suffered from syphilis, acquired or inherited. Moreover, it usually occurs years after infection, and marks the commencement of degenerative changes in the nervous system. It never occurs, therefore, in the absence of organic disease; but it may exist as an isolated symptom for periods ranging from five to eight years or longer, before any other objective signs of nervous degeneration make their appearance. It always develops slowly, and may be further advanced in one eye than in the other, or may be wholly confined to one side; consequently inequality of the pupils is present in a considerable number of cases. It is found in from 70 to 90 per cent. of the patients suffering from tabes dorsalis, but somewhat less frequently in those affected by general paralysis of the insane.

A special variation of the light reflex has been described by Wernicke. In certain patients, who suffer from hemianopsia, light falling on the blind half of the retina causes no contraction of the pupil, whereas light falling on the seeing half of the retina produces a prompt and decided response. It is further claimed that by means of this test a distinction can be made between cases of hemianopsia due to a lesion within the reflex arc, and those which result from a lesion situated in, or just beneath, the cortex. The test, however, is a very delicate one, and difficult 
to apply whth certitude: it is therefore disregarded by many reliable observers. In any case it is a doubtful sign and requires special methods of examination.

2. THERE IS A COMPLETE INTERRUPtion OF THE CENTRIFUGAL SIDE OF THE REFLEX ARC. There is neither direct nor consensual reflex of the pupil of the eye affected, whereas the reflexes of the unaffected eye are normal. The sign is usually accompanied by paralysis of accommodation, and by failure of the pupil to dilate on sensory stimulation. One or both eyes may be effected. When unilateral, the pupil of the affected eye is moderately dilated and larger than the normal pupil, but the inequality disappears in the dark. The sign indicates that there is a peripheral lesion of the nerves of the pupil of the affected eye. The paralysis of the sphincter may be due to atropin or other mydriatic, and the possibility that "drops " have been used to dilate the pupil should never be overlooked. It may also form a part of paralysis of the third cranial nerve when it is accompanied by squint and double vision, and it is sometimes the result of a lesion of the ciliary ganglion. In the latter instance it is complicated by anæsthesia of the cornea. In the great majority of cases it is a sign of acquired syphilis.

3. IRIDO-DILATOR REFLEX. All painful cutaneous sensations produce reflex dilatation of the pupil; and the presence of this irido-dilator reflex serves to distinguish anæsthesia of the skin due to organic causes from a want of sensibility, the result of hysteria. In the former the reflex is absent, whereas in the latter it is always present. 


\section{CHAPTER XLII}

\section{PARALYSIS OF AN OCULAR MUSCLE-THE CLINICAL SIGNIFICANCE OF DOUBLE VISION}

Anatomy and physiology of the ocular muscles-Homonymous diplopia-Crossed diplopia-Signs and symptoms of paralysis of an ocular muscle-How to examine a patient who is suffering from diplopia-Causation of diplopiaTreatment of diplopia.

A PATIENT, suffering from diplopia, experiences so great inconvenience that he rarely puts off much time before he consults the family doctor. He often, however, has considerable difficulty in describing his symptoms. He may complain that his sight is confused, that a shadow appears beside everything he looks at, or that two objects seem to be present, although he knows that there is only one. Such cases are often a puzzle to the general practitioner, for the simple reason that he does not know how to examine them. If the examination be conducted in a systematic manner, there is no difficulty, provided some elenentary facts concerning the anatomy and physiology of the ocular muscles are borne in mind.

The eyeball is moved by six muscles, the superior, inferior, internal, and external recti, and the superior and inferior obliques. These muscles are supplied by the third, fourth, and sixth cranial nerves; the external rectus by the sixth, the superior oblique by the fourth, and the otherssuperior, inferior, and internal recti and the inferior oblique-by the third. They act in pairs to move the eyeballs inwards and outwards, upwards and downwards. The external rectus draws the eye outwards, and the internal rectus pulls it inwards. The superior rectus and the inferior oblique turn the globe upwards, and the inferior rectus and the superior oblique downwards. The superior and inferior recti also rotate the eye inwards, while the superior and inferior obliques rotate it outwards. When, however, those muscles act together, the inward rotation of the recti is so exactly counterbalanced by the outward rotation of the obliques, that the eye moves upwards and downwards without any inclination of the vertical meridian. The six muscles can, therefore, be divided into two groups:(I) Adductors-superior rectus, internal rectus and inferior rectus-and (2) abductors-external rectus and superior and inferior obliques. The innervation of the muscles is arranged in such a manner that, in addition to the action which has just been 


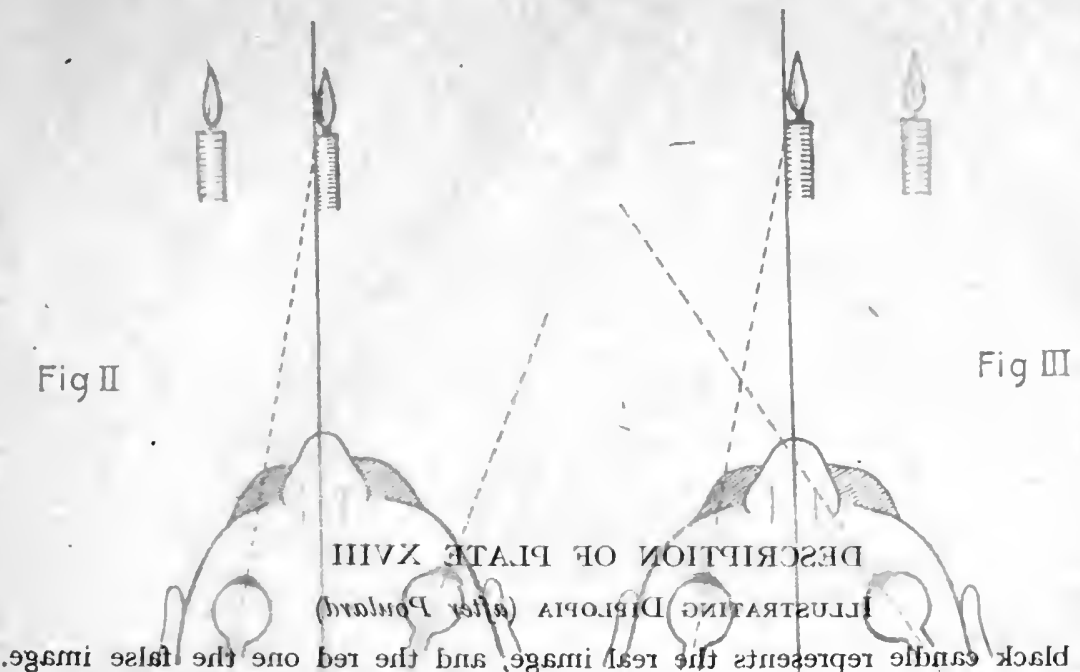

.

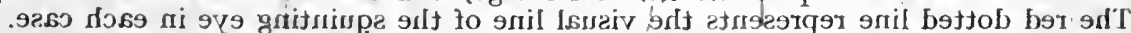

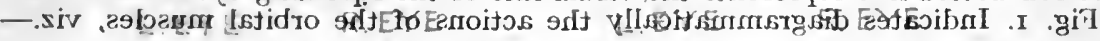

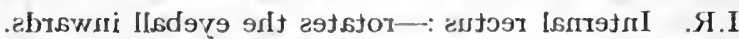

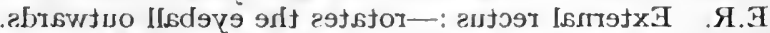

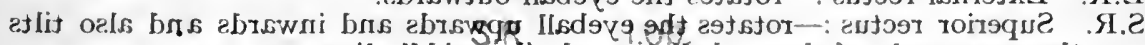

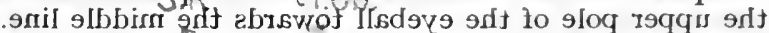

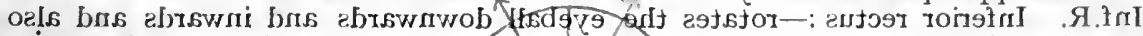

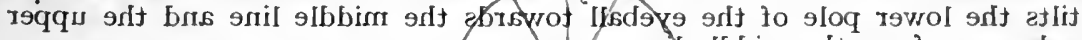

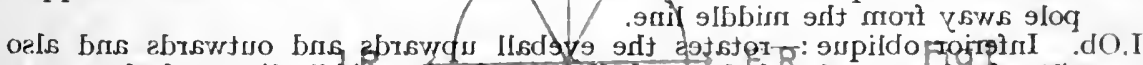

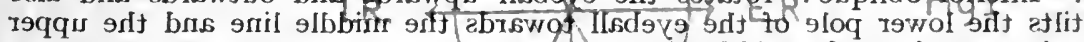

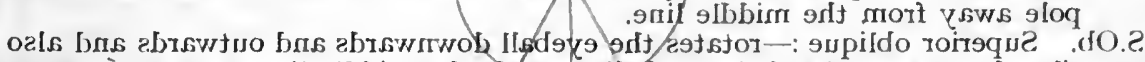
.

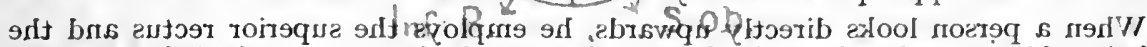

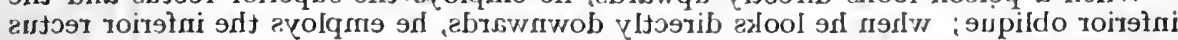

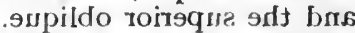

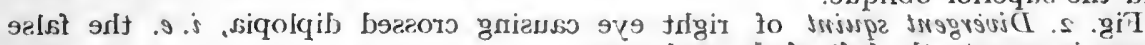

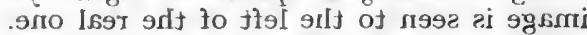

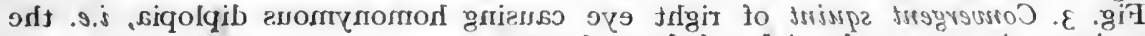

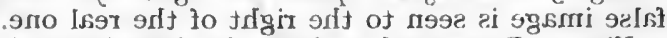

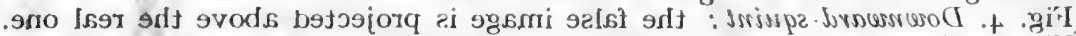

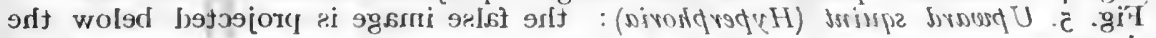
.9110 (597

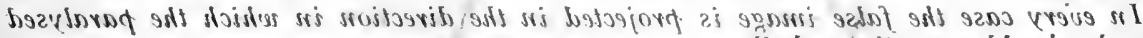

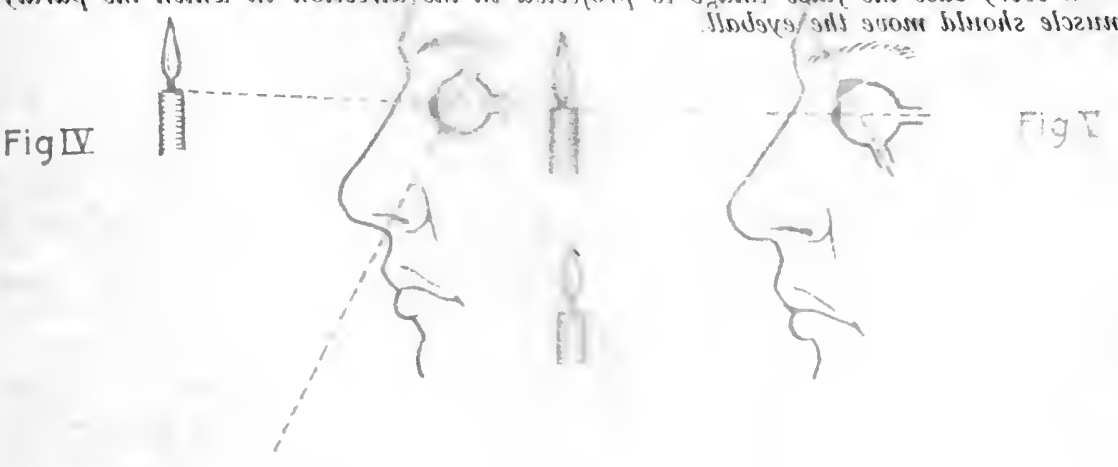




\section{CHAPTER XLII}

\section{Holngy of the ocular mascles-Homonymous diplopia-Crossed DESCRIPTION OF PLATE XVIII Causation of diplopia- Illustrating Diplopia (after Poulard)}

The black candle represents the real image, and the red one the false image The red dotted line represents the visual line of the squinting eye in each case,

Fig. I. Indicates diagrammatically the actions of the orbital muscles, viz.ctor.

I.R. Internal rectus : - rotates the eyeball inwards. describing his symptoms.

E.R. External rectus :-rotates the eyeball outwards. hat a shadow appears

S.R. Superior rectus :- rotates the eyeball upwards and inwards a nd also tilts the upper pole of the eyeball towards the middle line.

Inf.R ${ }^{1}$ Inferior rectus : - rotates the eyeball downwards and inwards and also tilts the lower pole of the eyeball towards the middle line and the upper pole away from the middle line.

I.Ob. Inferior oblique :-rotates the eyeball upwards and outwards and atso tilts the lower pole of the eyeball towards the middle line and the upper pole away from the middle line.

S.Ob. Superior oblique :-rotates the eyeball downwards and outwards and also tilts the upper pole of the eyeball towards the middle lineferior, internal,

When a person looks directly upwards, he employs the superior rectus and the inferior oblique; when he looks directly downwards, he employs the inferior rectus

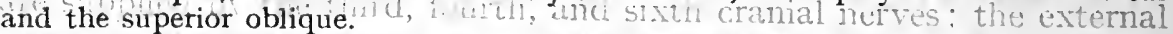
$r$. Fig. 2. Divergent squint of right eye causing crossed diplopia, i. $e$, the false image is seen to the left of the real one.

Fig. 3. Convergent "squint of right eye causing homonymbus diplopia, i.e. the false image is seen to the right of the real one teballs iminards and nutwards, Fig. 4. Downward squint: the false image is projected above the real one.

Fig. 5. Upward squint (Hyperphoria): the false image is projected (below the real one.

In every case the false image is projected in the direction in which the paralysed muscle should onole the eyeball.

The strperior and inferior recti also rutate the ent, hiperior and inferior obliques rotate it nut-

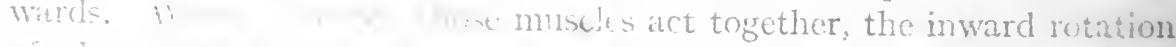
(): the les t anterbalanced by the outward rotation of

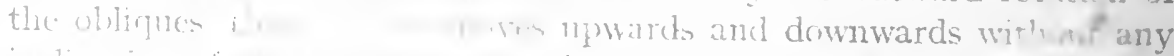

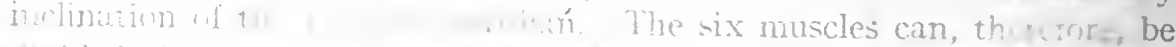

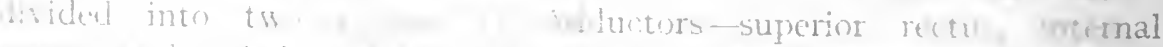

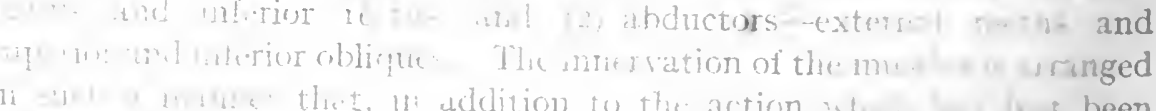



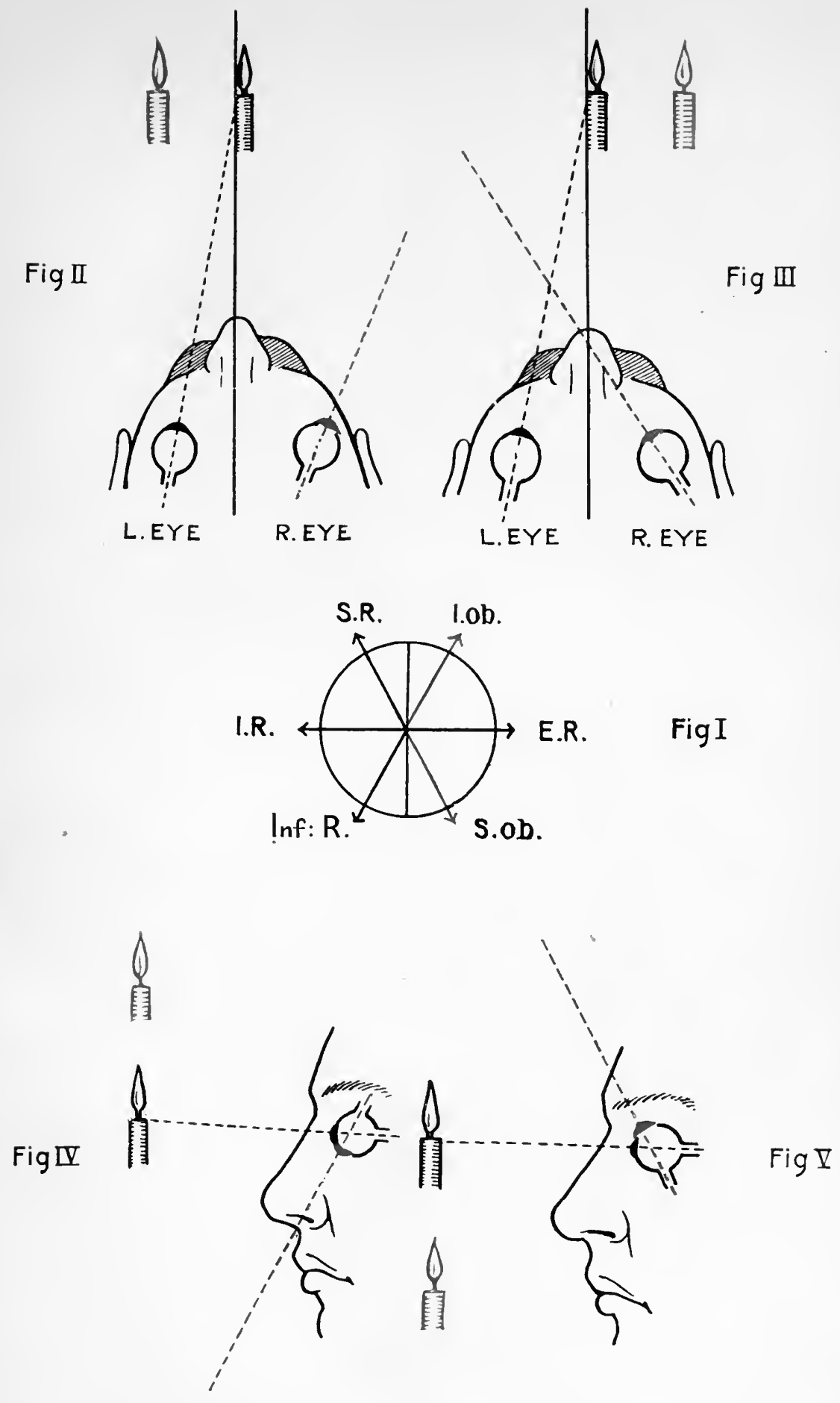

described, the internal rectus of one eye acts along with the external rectus of the other, so that the eyes can turn together from side to side, and when both internal recti act together the movement of convergence is brought about. It is obvious, therefore, that when both eyes are open, single vision can only be secured by perfect co-ordination in the action of the ocular muscles. Whenever the eyes gaze aimlessly into the distance, perfect nerve control is lost as a result of want of attention, and double vision occurs from the disturbance of muscular equilibrium. That is easily demonstrated by a simple experiment. Place a lighted candle about twenty feet distant from the eyes. As long as the eyes are fixed on the candle it is seen singly, but if the forefinger be held in a line with the candle, and the gaze directed to it, two candles will appear, situated side by side. If the experiment be reversed, and the eyes are fixed on a spot on the wall, while the lighted candle is held about two feet distant, a double image of the candle will again be seen. In the second experiment the right-hand image of the candle belongs to the left eye, and vice versa, which means that the diplopia is "crossed"; whereas in the first experiment the right-hand image belongs to the right eye and the left to the left, in other words the diplopia is "homonymous." The explanation is that when the eyes converge to look at a near object, the convergence is excessive in relation to the distant object: homonymous diplopia, therefore, always means excess of convergence. When, on the other hand, the eyes are fixed on a distant object the divergence is excessive in relation to the near object: crossed diplopia, therefore, always means excess of divergence. The results obtained from those two simple experiments form the foundation for the study of all varieties of double vision arising as a result of disease.

The desire for binocular vision is an important factor in maintaining a well-balanced association of the extraocular muscles, and diplopia occurs whenever there is the slightest want of co-ordination in their action. Paresis of an ocular muscle is not, however, the only cause of double vision. Monocular diplopia occasionally occurs after an injury which has caused dislocation of the lens, or in incipient cataract, and sometimes in astigmatism. When, therefore, a patient complains of double vision, each eye must be tested separately; if he is suffering from binocular diplopia the double image at once disappears when one eye is closed.

Paresis of an ocular muscle may be self-evident from the appearance of a squint; the impaired muscle having wholly or partially lost its power, the opponent exercises greater power, with the result that the eyeball is turned away from the weak muscle. Testing shows that the 
movement of the eye is restricted in the direction opposite to the deviation. The most convenient test of amplitude of movement in adduction and abduction, is to observe the relation of the border of the cornea to the inner and the outer canthus. When the enfeeblement of the affected muscle is great, the occurrence of strabismus not only explains the cause of the double vision, but also shows at a glance which muscle is affected. In the majority of patients, however, no squint is apparent. If the patient be observed carefully, it will be noticed that he holds his head in a peculiar manner, and he says that he does so because he has found that when he rotates his head the double vision is less troublesome. The experienced practitioner will observe that the patient is trying to supplement the deficient rotation of the eye by a rotation of the head; and from the position in which the patient is holding his head he may be able to say which of the ocular muscles, or which group of muscles, is affected. All those objective signs-strabismus, impaired movement of the affected eyeball, and rotation of the head-may be absent, and in these circumstances the investigation of the case resolves itself into a study of the diplopia. By means of such study it is possible not only to diagnose paresis, but also to find out which of the ocular muscles is affected. At times the paresis is so slight that the strong natural desire for single vision makes the patient suppress, and consequently disregard, the second image; and although he may complain of confusion of sight, yet he will not admit double vision. The examination must be conducted carefully and systematically, if those slight cases are to be properly diagnosed. The patient should be seated from ten to twenty feet distant from a lighted candle held on a level with his eyes. The head and body must be kept perfectly straight, facing the candle. A red glass should be placed in front of the right eye. The patient now sees two candles-one red, the other white-the difference in colour enabling him the more easily to distinguish and locate the double image. If he be asked to stretch his arms out towards the red and the white candles, the nature of the diplopia-homonymous, crossed, or vertical-will at once become apparent. The double images are spoken of as "true" and "false." The latter is seen by the eye which is at fault, and is projected in the direction in which the affected muscle would normally act.

When a case is being investigated, the first thing to determine is which is the paralysed eye. "The affected eye is at once recognised by the fact that the diplopia increases when the direction of vision is turned towards its image" (Landolt). The false image belongs to the affected eye, and is always the one situated farthest away from it. If, for example, the double vision is greatest on looking to the right, the image situated more to the right is the false one, and is formed by the affected eye; if 
on looking upwards the higher image is the false one; and so on with regard to all the directions in which the eyeball is moved. Having found out which eye is affected, the next point to determine is which is the paralysed muscle. There is no difficulty if the normal action of the individual muscles is remembered. Double vision will be worst in one of the four principal directions of movement, and the muscle paralysed is one whose function it is to move one of the eyes in that direction. The patient can usually say in which direction the double vision is most pronounced, if he is asked to follow the movements of the candle as it is carried upwards, downwards, to the right, and to the left. By that means the group of muscles affected is at once determined. To fix upon the individual muscle affected, it is only necessary to remember that the paralysed muscle is the one which would have placed the eye in the position and direction of the false.image. If, for example, the patient says that the double vision is most pronounced when he looks to the right, obviously it must be one of the muscles which moves the eyes to the right that is affected-right external rectus or left internal rectus. On further inquiry it is found, by means of the coloured glass, that the image further to the right belongs to the right eye; the diplopia, therefore, is homonymous, which means that there is excess of convergence. If the candle be moved further to the right, the patient says the images become further apart, and the separation becomes greater the more he tries to look towards the right, that is, in the direction of the normal action of the right external rectus muscle, which must, therefore, be the one affected. It is sometimes a little more difficult to find out the muscle affected in vertical diplopia, because the patient cannot give precise statements regarding the lateral deviation of the images. It will be remembered that the eye is turned directly upwards by the combined action of the superior rectus and the inferior oblique, and downwards by the inferior rectus and the superior oblique. The recti, however, turn the cornea inwards-adductors-and the obliques turn the cornea outwards-abductors. Interference with the subsidiary action of those muscles produces obliquity of the double images, and from the patient's description it may be difficult to make sure whether the diplopia is homonymous or crossed. The real practical test, when it comes to make a decision between a rectus and an oblique muscle, is that of the increasing difference in height between the two images when the candle is placed in one of the two (upper or lower) lateral positions. The explanation is that, owing to the angle formed by the obliques and the recti with the antero-posterior axis of the eye, the former fulfil their chief functions best when the eye is looking inwards, while the latter act best when the globe is turned outwards. To make a differential diag- 
nosis, therefore, between paralysis of an oblique and of a rectus (superior or inferior), it is sufficient to observe the difference in height between the two images when the eye is turned inwards, as compared with the position of the image when the eye is turned outwards. For example, a patient complains of double vision when he looks downwards: the muscles normally concerned in that movement are the inferior rectus and the superior oblique: the false image is farthest away from the left eye, therefore that is the one affected; the vertical diplopia increases when the eye is turned inwards, to the right or towards the sound eye, therefore the superior oblique is the muscle that is paralysed. If, on the contrary, the vertical diplopia had increased when the eye was turned outward, to the left or towards the affected side, the inferior rectus would have been the muscle that was paralysed.

To recapitulate-when examining a case of diplopia, all'that is necessary is to remember the three following rules-

I. The group of muscles implicated is at once determined by asking the patient to say in which direction the diplopia is most pronounced.

2. The eye affected is the one from which the false image is farthest away when the patient looks in the direction in which double vision is greatest.

3. The muscle affected is discovered by remembering that the false image is always deviated exactly in the direction of the normal sphere of action of the muscle that is paralysed.

When the paralysis affects several muscles, the study of the diplopia is more complicated; but the problem can usually be satisfactorily solved by separating the defect into its component parts, and finding out the muscle responsible for the vertical diplopia, and after that has been done, determining the muscle responsible for the lateral displacement. In these circumstances, however, the objective signs of paralysis -strabismus, impaired movement of the eyeball, and inclination of the patient's head-are usually so apparent that the diagnosis is evident.

If the patient has been in the habit of using the non-paralysed eye for fixation, he is unable to locate an object in its proper position if he tries to do so with the paralysed eye, the other being covered. The false projection is always to the side to which the paralysed muscle would have turned the eye. The greater the paralysis, the greater the efforti.e. the expenditure of nerve energy - to turn the eye towards the object to be located; and Landolt has pointed out that the amount of the false projection is usually in direct relation to the degree of paralysis. Patients who suffer from double vision frequently complain of an apparent movement of objects, especially when they look towards the affected side. That causes nervousness and giddiness, which may be so great as to 
induce headache, nausea, and vomiting: those disagreeable symptoms at once disappear when one eye is closed.

The occurrence of double vision is of great interest to the general practitioner, because of its frequent association with syphilis of the cerebro-spinal system. An attack may be so transitory that the patient thinks little of it; nevertheless, it is a danger-signal warning of the commencement of serious disease of the brain or spinal cord. It is, therefore, very important that the symptom should not be allowed to pass unnoticed, and that its value should be properly assessed.

The causes of diplopia may be arranged in four groups-

I. Infections - diphtheria, influenza, eruptive fevers, zona ophthalmica, acute articular rheumatism, syphilis, tuberculosis, etc.

2. Initoxications-acute or chronic. Among the former are all autointoxications; chief among the latter are alcohol and lead. When diplopia occurs in diabetes, it is usually the result of paresis of the sixth cranial nerve; whereas in tabes and in syphilis the third is most frequently affected.

3. Diseases of the circulatory apparatus-arterio-sclerosis, often associated with cirrhosis of the kidney; aneurysm, or other disease of the cerebral blood-vessels, are the most frequent causes of nuclear lesions of the third, fourth, and sixth cranial nerves.

4. Traumatic lesions-fracture of the orbit, or of the base of the cranium. After all injuries to the head, the ocular muscles should be carefully examined. Paralysis of the external rectus is not uncommon, owing to the frequency with which the sixth cranial nerve is injured as it passes over the petrous process of the temporal bone; its occurrence after an injury to the skull usually indicates that there is a fracture of the base. It is sometimes seen in an infant, when it is due to a hæmatoma which presses on the nerve, in which circumstances the paralysis disappears after the blood becomes absorbed; but when the nerve has been destroyed, as is nearly always the case in the adult, the paralysis is permanent.

The treatment of diplopia consists, for the most part, in excluding the false image by covering the affected eye; but the cause of the muscular paralysis must always be sought for diligently, and suitable treatment prescribed. In all cases of doubt, antisyphilitic remedies should be administered. When the deviation of the paralysed muscle becomes permanent, operation is necessary-advancement of the affected muscle, and tenotony of its opponent-to lessen the deformity and re-establish, if possible, single vision in at least part of the field of vision. 


\section{CHAPTER XLIII}

\section{THE CLINICAL SIGNIFICANCE OF NYSTAGMUS}

Congenital nystagmus-Hereditary form of the disease-Acquired nystagmusAssociated with disease of the ear and with disease of the nervous systemOccupational nystagmus-Description of miners' nystagmus-Prognosis.

NystaGmus is characterised by involuntary rhythmical movements of the eyeballs. The movements are regular, short, rapid oscillations about a central point, and vary both in character and direction.

They may be to and fro like a pendulum, or slow to one side with a rapid return to the other (resilient), while in direction they may be horizontal, vertical, oblique, rotatory, or mixed.

They are nearly always to both sides (bilateral), associated, and synchronous-or the eyes may in some instances swing rapidly toward and away from each other-or the movements may be confined wholly to one side (unilateral).

Very exceptional cases of nystagmus occur in which the movements in the two eyes are dissimilar both in direction and extent.

Nystagmus is arrested during sleep, but is increased by emotion, self-consciousness, effort, turning the eyes in some special direction, extreme convergence, or by covering one or both eyes. Some persons can induce nystagmus voluntarily, but it is usually quite independent of the will.

The different varieties of nystagmus are divided into two classes, according to the presence or absence of ocular lesions.

1. Congenital Nystagmus.-Infantile nystagmus is occasionally hereditary, and is always a sign of defective sight, even when no pathological changes are visible on ophthalmoscopic examination. It occurs in several generations of the same family, and males suffer more frequently than females. Hereditary nystagmus sometimes becomes less pronounced as age advances, and occasionally disappears altogether as adolescence is reached. In the majority of cases congenital nystagmus develops during the first few months of life, and is due to some optical defect which has caused considerable impairment of sight-opacities of the cornea (especially those due to ophthalmia neonatorum), congenital cataract, intra-uterine choroiditis, retinal hæmorrhage of the new-born, 368 
congenital malformations, albinism, colour-blindness, etc. Errors of refraction when not complicated are very seldom accompanied by nystagmus. Amblyopia, which is the most frequent cause of nystagmus, has little effect upon the movements of the eyes, unless the optical conditions causing it have developed during the first few months of life. If it sets in after the eyes have learned how to perform fixation, nystagmus does not occur. Moreover, if amblyopia is unilateral, it causes a squint very much oftener than it produces nystagmus, and if a child becomes blind in early infancy nystagmus will not develop.

The chief clinical characteristic of congenital nystagmus is, that except for the,amblyopia, it causes no symptoms. There is no apparent movement of objects resulting from the unsteadiness of the eyes, and giddiness, headache, or photophobia are absent.

2. Acquired Nystagmus. - This differs from the congenital form in so far that ocular lesions are absent, and in all cases the patient is very conscious of the movement of external objects corresponding to the displacement of their retinal images. It is symptomatic of disease of the internal ear, of the central nervous system, or of strain of eyesight the result of conditions incident to the patient's occupation.

(I) Nystagmus due to disease of the ear.-Aural nystagmus is a reflex, usually slight and transitory, but occasionally more pronounced and persisting for months or years. It frequently originates after surgical manipulations on a diseased ear, syringing, insufflation, catheterisation, etc., in affections of the labyrinth, increase of pressure causing disturbance in equilibrium. The appearance of nystagmus in the course of disease in the middle ear is a valuable sign of implication of the labyrinth.

(2) Nystagmus due to nervous diseases.-Nystagmus occurs frequently in multiple sclerosis, and in disease of the cerebellum. It may be slight and apparent only when the eye is strongly turned to one side or the other, and may disappear when the eyes roam aimlessly in space; but it returns at once whenever the eyes are fixed attentively on an object. Tabes dorsalis is never accompanied by nystagmus, although it is present in the majority of cases of Friedreich's ataxia.

(3) Occupational Nystagmus. - The commonest form of occupational nystagmus occurs in coal miners, and is closely connected with the defective illumination of the pits. Conditions of work and errors of refraction act as predisposing causes. It is one of the scheduled diseases under the Workmen's Compensation Act, and consequently its chief features ought to be familiar to every doctor who practices in a mining district. The patients are usually from forty to sixty years of age, and have wrought in the pits since they were fourteen or fifteen years old. The majority of them have been employed in mines where the safety-lamp is used; 
those who work with the naked light suffer less frequently. The first symptom is usually failure of sight-especially in a dull light-and the disability is at times so great that the man may have to ask one of his companions to guide him to his work. The pit lights dazzle his eyes, and later the lamps appear to wheel round and round. All objects appear unsteady, and the sense of everything moving around him gives rise to a feeling of mental confusion and giddiness, which is so overpowering that the man is compelled to sit down. Those distressing symptoms may lessen and pass off, and the man may be able to resume work; but at other times they persist, increase in severity, and are accompanied by headache, vertigo, and intolerance of light. Work is now impossible, and it happens not infrequently that the symptoms become so acute that the man has to be led home. The majority of the patients complain of night blindness - they say they cannot see in the evening light, and that the continuous oscillation of the street lamps causes them so much distress that they are compelled to remain indoors. They are equally incapacitated by bright light; when out of doors they wear a cap pulled down well over the eyes to protect them from glare, and walk about with the head thrown backward--an attitude which is very characteristic. The movements of the eyeballs are rotatory, and the rapidity of the oscillations makes it impossible to see anything distinctly.

The nystagmus is aggravated by excitement or exertion, and is made worse when the patient looks up; but lessens, and may even cease altogether for the time being, when he looks down. Muscular tremor and spasm of the eyelids are often present, making the examination of the eye very difficult, if not impossible. The lid movements may exist without oscillation of the globes, and this sign is considered so characteristic that it is scheduled as an occupational neurosis, for which compensation can be claimed.

The rotatory movements of the eyeballs may be absent when the patient is at rest; but if he is really suffering from nystagmus, the oscillations at once appear when he is asked to follow a finger or a pencil gradually raised above the horizontal level of the eyes, his head being held quite steady. This test is usually sufficient (especially if carried out in a darkened room) to demonstrate nystagmus; but if it fail, the patient should be asked to stoop or to place himself in any constrained position he may have to assume at his work, and the eyes examined after he has resumed the upright position. Another test is to hold a mirror below his head and examine the eyes while the stooping posture is maintained. Sometimes nystagmus is detected with the ophthalmoscope, when the oscillations of the globe are too fine and rapid to be seen by the naked eye. A peculiar tremor of the eyebrows, and involuntary twitching of the lids, 
are very characteristic of miners' nystagmus, and may persist after the oscillations of the eyes have disappeared.

The disease is always latent for months or years before it becomes manifest, and an attack may be determined by an accident to the eyes, head, or any other part of the body. At other times a miner is unaware that there is anything wrong with his eyes, but becomes incapacitated after having been told by a doctor-whom he was consulting for a disorder quite unconnected with sight-that he is suffering from nystagmus.

Manifest nystagmus may be slight where the patient continues at work and makes little complaint; moderate where underground work must be discontinued, but surface work can be done, and severe where there is complete incapacity. It is not always the amount of actual nystagmus that determines the severity of an attack. Many a man, with what seems very severe nystagmus, is quite fit for his ordinary work; whereas another, in whom the oscillations of the eyes are scarcely to be seen, is wholly incapacitated. It is the neurosis which is the predominating factor in determining whether or not a man with miners' nystagmus is fit for underground work. The personal equation always has a preponderating influence on the course of the disease.

Slight cases usually recover quickly; moderate or ordinary cases may require twelve or eighteen months before they are well; severe cases are often incurable. The one thing necessary in treatment is that the man leave the pit and work for a time on the surface. He ought to work whenever he is able, and in most instances he can do anything that does not require much stooping. Nothing is worse for those patients than idleness; because if they get into the habit of walking about doing nothing, it is very difficult to persuade them to engage in any form of work. Doubtless the severe cases are quite incapacitated, but they also should be encouraged to think of doing some light work as soon as the acute symptoms disappear. Many of the men return to the pit of their own accord, but the majority relapse and are unable to continue at work underground. A considerable number, however, make a good recovery and are able to go on working at the coal face.

It is a mistake to suppose that because a miner has suffered from nystagmus, he is on that account incapacitated from ever working again at the coal face. Each case requires to be considered separately on its own merits, and much depends on the working conditions in the pit in which the man is employed:

The prognosis is also influenced by the following important factors :-

I. Age.-The old and the young are much less likely to recover completely than the man in middle age.

2. Length of time the disease has existed.-The longer the disease has 
been manifest before work has been discontinued, the greater the chance of complete recovery.

3. Vision.--The better the vision, the better the prognosis; high errors of refraction are always a predisposing cause of relapse.

Better lighting, better ventilation, and better working conditions generally in the pits, will do much to lessen the frequency of miners' nystagmus; in this disease, as in so many others, "prevention is better than cure." 


\section{CHAPTER XLIV.}

\section{THE CLINICAL SIGNIFICANCE OF FAILING SIGHT}

General considerations on the use of the ophthalmoscope-Points to be borne in mind in testing vision-Determination of the visual acuity, the pupillary reflexes and the extent of the visual field.

Loss of central vision with preservation of peripheral vision-Scotomata-Hæmorrhage into the macula-Retrobulbar neuritis-Choroido-retinitis-Metamorphopsia, micropsia, and macropsia-Toxic amblyopia-Hereditary optic neuritis.

Loss of peripheral vision with complete or partial retention of central visionOptic atrophy-Retinitis pigmentosa-Chronic glaucoma-Hysterical amblyopia-Retinal detachment-Intraocular tumour-Hemianopsia.

Loss of vision throughout the visual field-Profuse intraocular hæmorrhageTraumatic asphyxia-Embolism of the central artery of the retina-Thrombosis of the central vein-Acute retrobulbar neuritis-Primary optic atrophy -Uræmia.

THE causes of defective sight being so many and so various, it is little wonder that the general practitioner often feels puzzled when consulted by a patient complaining of failing eyesight. The difficulties are, however, more apparent than real. If the doctor can use the ophthalmoscope, he will quickly arrive at a definite diagnosis of the pathological condition actually present in the fundus oculi. He may discover a hæmorrhage, a white patch of exudation, a pigmented spot, an inflammation, or an atrophy of the optic nerve, retina, or choroid; but if those lesions are considered merely as isolated facts, they will possess little clinical value. The morbid appearances revealed by an ophthalmoscopic examination must be assessed in relation to the patient's general health before they can be helpful in diagnosis; and their ætiology must be known if they are to serve as a guide in treatment. Identical tissue changes do not necessarily imply similarity of causation, because one and the same pathological process can give rise to many dissimilar signs of disease. It is not enough, therefore, to be able to recognise a lesion in the fundus oculi; its clinical significance must also be interpreted, and before that can be done, it is necessary to know which. structures of the eye are affected, what is the cause of the disease, and what relation it bears to the other symptoms from which the patient may be suffering. The real value of the ophthalmoscope lies, not in its power to discover disease, but in the help it gives in the interpretation of equivocal signs 
and symptoms. A child, for example, may have been suffering for weeks from headache and vomiting - the cause of which had remained obscure until the eyes were examined with the ophthalmoscope, when the discovery of double optic neuritis enabled a positive diagnosis of intracranial tumour to be established.

The use of the ophthalmoscope has many limitations in clinical medicine, and it is well to recognise them. The instrument is of incalculable service in diagnosis; but it must supplement, and never supersede, a thorough clinical examination of the functional activities of the eye. The family doctor ought to be able to include the eye in the routine examination of a patient; and if he possess a good working knowledge of its anatomy and physiology, he will have little difficulty in interpreting the meaning of the signs and symptoms he may discover. The tendency to divorce the eye from the other parts of the body-and to consider it as a thing apart, only to be examined and treated by a specialist-is greatly to be deprecated; because no true progress can be made in ophthalmic medicine, until it is recognised that it is not a special pathology, but the broad principles of general pathology, that govern the morbid processes occurring in the eye. It will be a great gain therefore when, on the one hand, the specialist co-operates more closely with the family doctor, and tries to keep himself informed of the subsequent medical history of patients who have consulted him for disease of the fundus oculi; and when, on the other, the general practitioner brings his medical knowledge, experience, and common sense to bear on all the ophthalmological problems with which he may be confronted in the course of his daily work.

Unless the general practitioner is an expert in the use of the ophthalmoscope, he is likely to learn more from a functional examination of the eye, than from the use of an instrument with which he is not quite familiar. A specialist is more likely to be able to interpret what he sees in the fundus oculi; but, if he is wise, he will not express an opinion of the clinical significance of the ophthalmoscopic appearances, until he has made a thorough examination of the eye, and has heard all that the family doctor is able to tell him of the medical history of the patient.

While, therefore, the general practitioner is encouraged to make full use of the ophthalmoscope in the same way that he employs the stethoscope, the sphygmograph, the clinical thermometer, and applies chemical tests in the examination of the urine and other excretions; it is more important that he should know how to make a careful functional examination of the eye. If he is to form an opinion regarding the condition of a patient who is complaining of failing sight, he must be able to test the sensibility of the optic nerve and retina. 
A difficulty often occurs at the very outset, owing to the fact that a patient may give a very vague account of his visual troubles. The first thing to do, therefore, in every case, is to-

1. Make sure that the Patient is not Suffering from Double Vision.-Each eye must be tested separately.

2. Eliminate Errors of Refraction.-Ask the patient to read test types through a stenopæic slit, or a pin-hole in a visiting card. If he is suffering simply from hypermetropia, myopia, or astigmatism, vision will be improved; whereas, if his eye is diseased, sight will be made worse by the test.

3. Exclude Opacities of the Transparent Media.-Examine carefully in a good light-with the help of a lens and the large mirror of the ophthalmoscope-the cornea, aqueous, pupil, lens, and anterior portion of the vitreous humour. Any loss of transparency of those structures will be quickly discovered.

After diplopia, an error of refraction, or a loss of transparency of the media has been excluded, the important fact is established that the failing sight of which the patient complains is the result of a lesion in the fundus oculi, or in the visual pathway in some part of its course from the retina to the centres of vision in the cortex of the brain. The nature and the site of the lesion must now be discovered.

Each eye must be examined separately to determine the following points-

1. The Visual Acuity.-The patient should be asked to read the test types in a good light, and the measure of his acuity is determined by the last line he is able to read. It is expressed in the form of a fraction-the numerator of which represents the distance the patient is placed from the test card, and the denominator the distance at which the line he has read can be seen by a normal eye.

\section{The State of the Pupillary Reflexes.}

(I) Reflex to light.-The reflex to direct light can be tested either in diffuse daylight, or by artificial light in a dark room; but whichever method of examination is employed, each eye must be tested separately while the patient is looking into the distance.

(2) Consensual reflex.-The physiological movements of the pupils occur simultaneously in the two eyes. The light stimulus to which one eye is exposed passes by the centripetal pathway to a centre from which it is carried along the centrifugal pathway to the other eye. If, therefore, a lesion occurs in either of those pathways, the consensual reflex cannot take place.

(3) Reflex in the act of convergence and accommodation.-The patient is first asked to gaze into the distance, and then quickly to look at a 
finger held about ten inches from his eyes; this should induce contraction of both pupils.

3. The Extent of the Visual Field Measured by the Hand.From the functional, as well as from the anatomical point of view, it is necessary to distinguish a central and a peripheral part of the retina. The former corresponds to the macula-the latter includes all the rest of the retina. The macula itself is very minute, but is the most highly sensitive portion of the retina. Visual acuity depends upon the healthy condition of the macula, which is a good illustration of the general aphorism that in any structure a high degree of functional activity always carries along with it a greatly increased liability to disease. The peripheral portions of the retina are very much less sensitive than the macula, and correspond to what is known as the "field of vision." The eye can see distinctly the object directly looked at, but perceives alsoin an indefinite manner-objects situated in a wide. space around the line of fixation. The function of the retina can therefore be divided, allocating inspection to the central, and discovery to the peripheral portion.

For clinical purposes the field of vision can be tested satisfactorily by the hand; but when more accurate measurements or consecutive records are required in a particular case, an instrument called a perimeter must be employed. The general practitioner is not likely to possess a perimeter, but ought never to neglect measuring the patient's visual field with his hand, and there is no difficulty in making this examination. The patient should stand with his back to a window, and the surgeon face him at a distance of two feet. The patient is told to cover his right eye with the palm of his hand, and to look fixedly at the surgeon's right eye. The surgeon closes his left eye, and keeping his hand midway between himself and the patient, moves it from the periphery towards the centre of the visual field. The moment he sees his finger coming into view, the patient also should see it, provided his field of vision is normal. The movements of the hand are repeated in different meridians of the field until it has all been explored. This is a simple and trustworthy method. The surgeon compares the patient's field of vision with his own, and any differences in their extent are at once detected. Moreover, as he is continually watching the patient's eye, any wandering from the point of fixation is quickly observed and corrected. Each eye must be tested separately. The extent of the field of vision is influenced by the following factors-

(I) INDividual PeCUliarities of the FaCE FORMation-prominence of the bony margins of the orbit-shape of the nose, drooping of the upper lid, size of the pupil. 


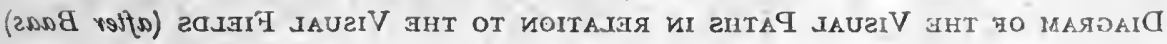

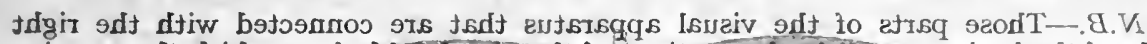

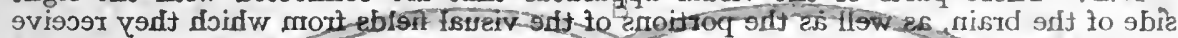

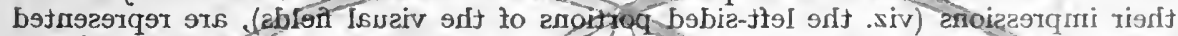

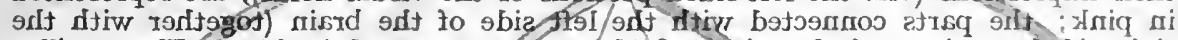

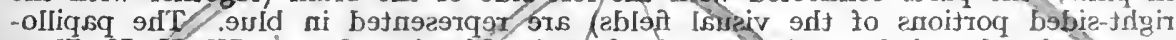

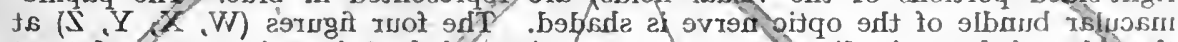

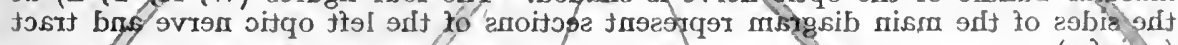

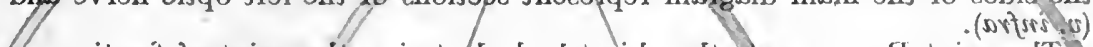

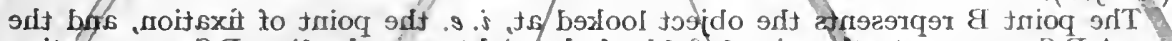

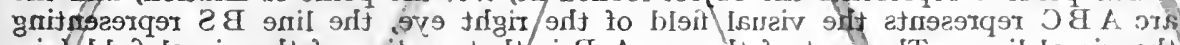

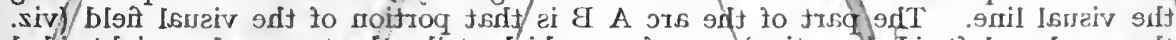

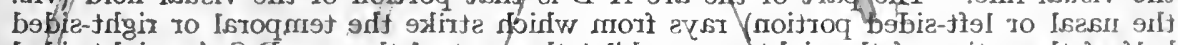

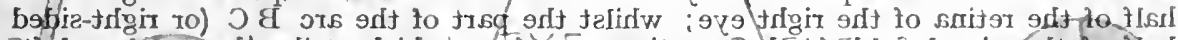

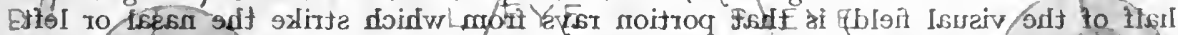

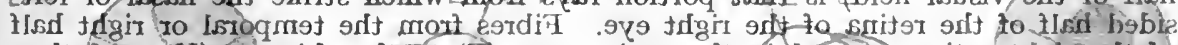

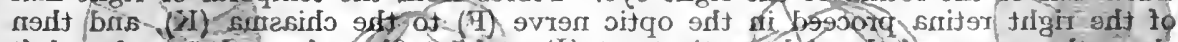

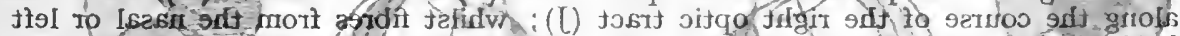

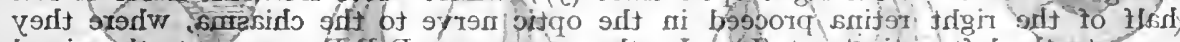

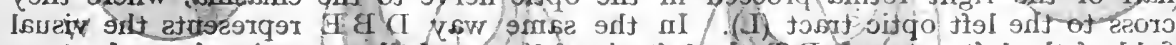

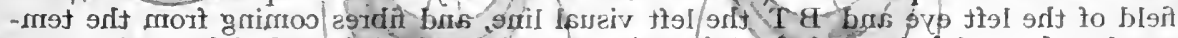

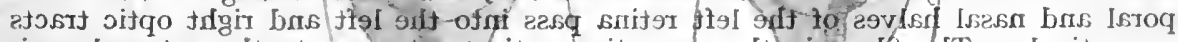

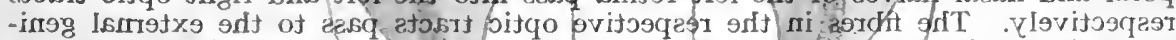
.

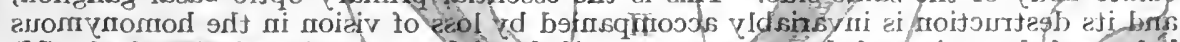

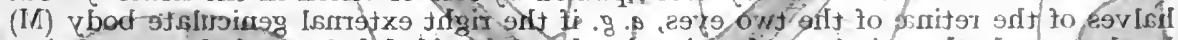

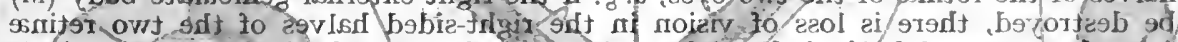

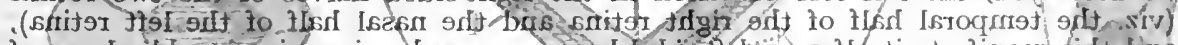

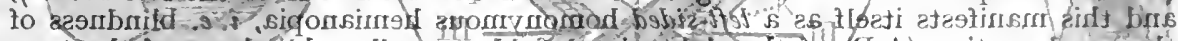

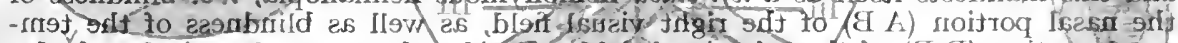

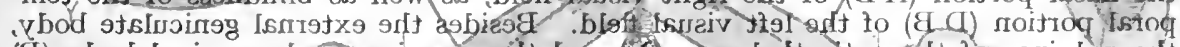

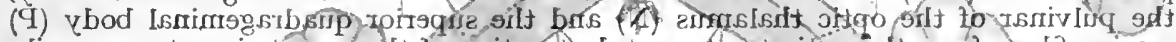

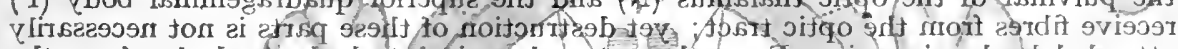
ort mort oels bras) v bod of

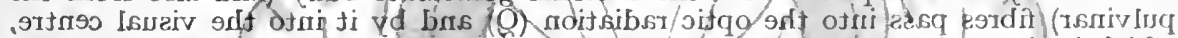

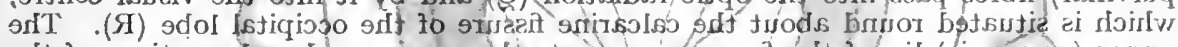

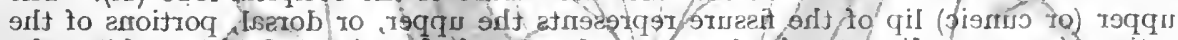

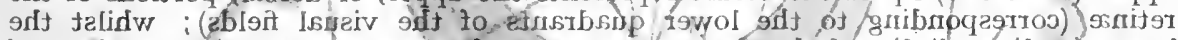

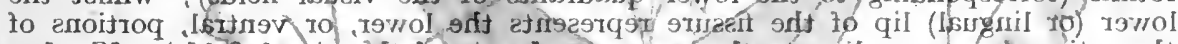

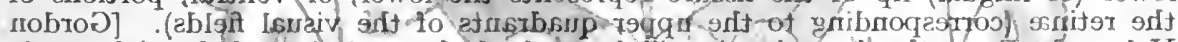

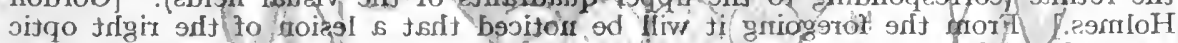

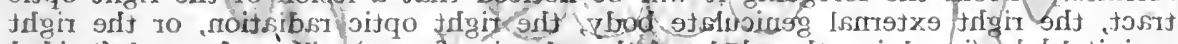

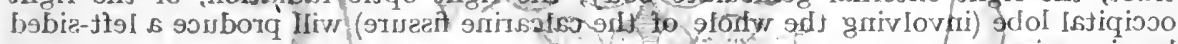

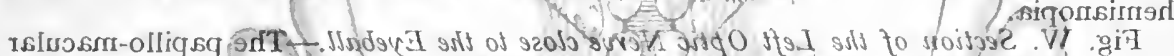
.

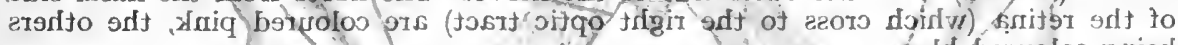
a suld botwolos grrigd

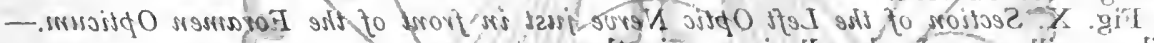

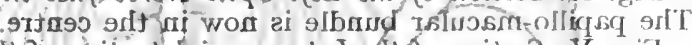

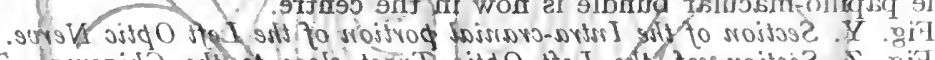

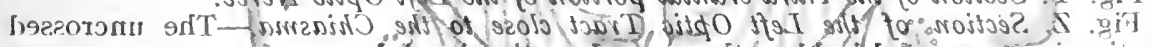

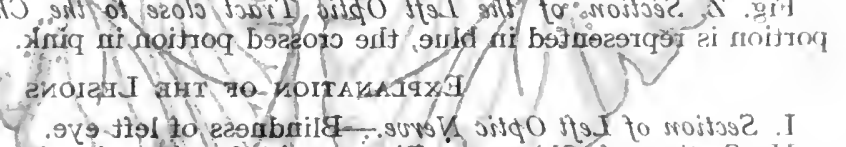

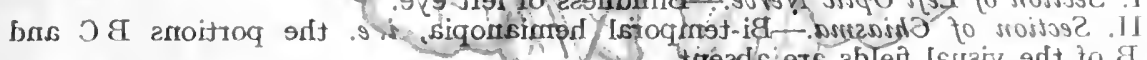

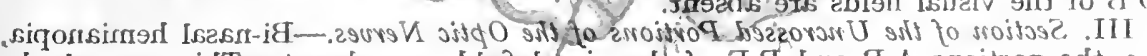

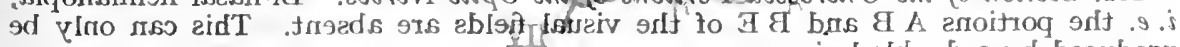

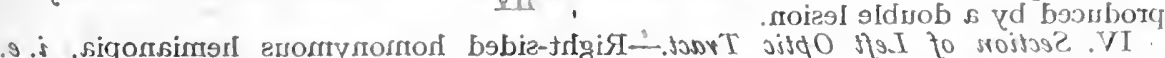

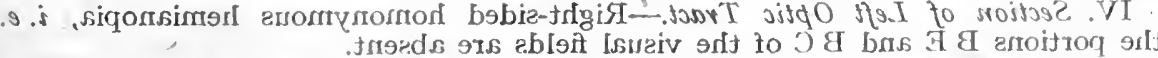

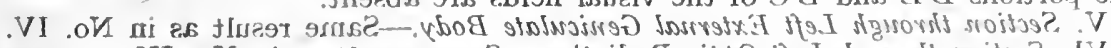

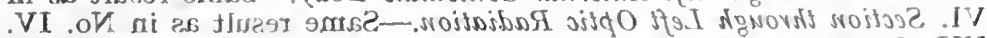

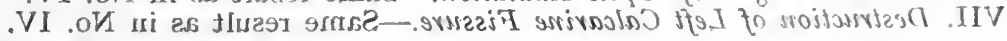


N.B.-Those parts of the visual apparatus that are connected with the right side of the brain, as well as the portions of the visual fields from which they receive their impressions (viz. the left-sided portions of the visual fields), are represented in pink, the parts connected with the left side of the brain (togetheriwith the right-sided portions of the visual fields) are represented in blue. The papillomacular bundle of the optic nerve is shaded. The four figures $(W, X, C, 2)$ at the sides of the main diagram represent sections of the left optic nerve and ract (v. infra).

The point $B$ represents the object looked at, $i$. $e$. the point of fixation, and the arc $A B C$ represents the visual field of the right eye, the lineh $B S_{1}$ representing the visual line. The part of the arc A B is that portion of the visual field (viz. the nasal or left-sided portion) rays from which strike the temporal or right-sided half of the retina of the right eye; whilst the part of the arcn $B, C$ (or right-sided half of the visual field) is that portion rays from which strike the nasal or leftsided half of the retina of the right eye. Fibres from the temporal or right half of the right retina proceed in the optic nerve (F) to the chiasmal (K), and then along the course of the right optic tract $(\mathrm{J})$; whilst fibres from the nasal or left half of the right retina proceed in the optic nerve to the chiasma, where they cross to the left optic tract (L). In the same way D B E represents' the visual

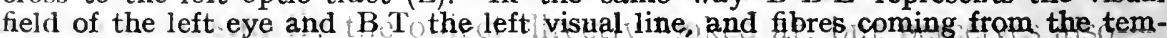
poral and nasal halves of the left retina pass in to the left and right optic tracts respectively. The fibres in the respective optic tracts pass to the external geniculate body of the same side.t This is the essential primary optic basaliganglion, and its destruction is invariably accompanied by loss of vision in the homonymous halves of the retinx of the two eyes, $e . g$. if the right external geniculate body ${ }^{7}(\mathrm{M})$ be destroyed, there is loss of vision in the right-sided halves of the two retinæ (viz. the temporal half of the right retina and the nasal half of the left retina), and this manifests itself as a left-sided homonymous hemianopia, $i$. $e$. blindness of the nasal portion (A B) of the right visual field, as well as blindness of the temporal portion (D B) of the left visual field. Besides, the external geniculate body, the pulvinar of the optic thalamus $(N)$ and the superior quadrageminal body $(P)$ receive fibres from the optic tract; yet destruction of these parts is not necessarily attended by hemianopia. From the external geniculate body (and also from the pulvinar) fibres pass into the optic radiation (Q) and by it into the visual centre, which is situated round about the calcarine fissure of the occipital lobe (R),inThe upper (or cuneic) lip of the fissure represents the upper, or dorsal, portions of the retinæ (corresponding to the lower quadrants of the visual fields); ; whilst the lower (or lingual) lip of the fissure represents the lower, or ventral, portions lof the retinæ (corresponding to the upper quadrants of the visual fields). [Gordon Holmes.] From the foregoing it will be noticed that a lesion of the right optic tract, the right external 'geniculate body, the right optic radiation, or the right occipital lobe (involving the whole of the calcarine fissure) will produce a left-sided hemianopia.

Fig. W. Section of the Left Optic Nerve close to the Eyeball. -The papillo-macular bundle (shaded) is on the outer side of the nerve. The fibres from the nasal side. of the retina (which cross to the right optic tract) are coloured pink the others being coloured blue.

Fig. X. Section of the Left Optic Nerve just in front of the Fordmen Opticum.The papillo-macular bundle is now in the centre.

Fig. Y. Section of the Intra-cranial portion of the Left Optic Nerve.

Fig. Z. Section of the Left Optic Tract close to the Chiasma.-The uncrossed portion is represented in blue, the crossed portion in pink.

\section{Explanation OF THE LESIONS}

I. Section of Left Optic Nerve.-Blindness of left eye:

II. Section of Chiasma.-Bi-temporal hemianopia, $i . e$. the portions B C and $D B$ of the visual fields are absent.

III. Section of the Uncrossed Portions of the Optic Nerves.-Bi-nasal hemianopia, i. e. the portions A B and BE of the visual fields are absent. This can only be produced by a double lesion.

IV. Section of Left Optic Tract--Right-sided homonymous hemianopia, i.e. the portions $\mathrm{BE}$ and $\mathrm{BC}$ of the visual fields are absent.

V. Section through Left External Geniculate Body.-Same result as in No. IV.

VI. Section through Left Optic Radiation.-Same result as in No. IV.

VII. Destruction of Left Calcarine Fissure.-Same result as in No. IV. 


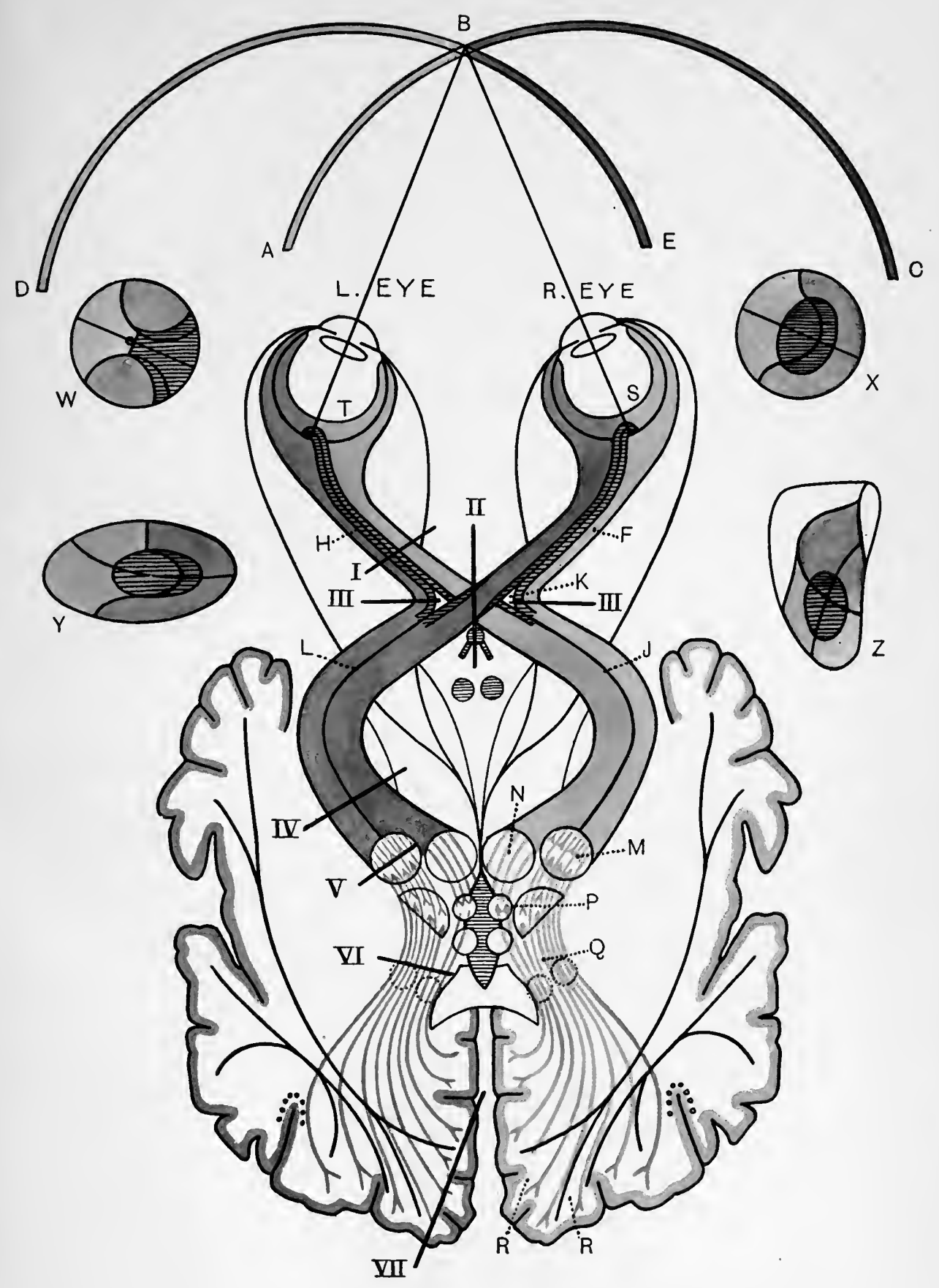



(2) Amount OF Light in THE ROom in which the examination is made.

(3) State of the patient's health, weakness, tiredness, exhaustion, etc.

(4) General intelligence, attention, or mental obtuseness of THE PATIENT, who may or may not understand what the surgeon wishes him to do.

The routine examination just outlined, enables the practitioner to divide patients complaining of failing eyesight into three groups-

(A) Those in whom the defect is in the centre of the field of vision, the peripheral portions being intact.

(B) Those in whom the defect is in the peripheral portions of the field of vision, while the centre is little if at all inpaired.

(C) Those in whom both the central and peripheral portions of the field of vision are affected.

\section{A. LOSS OF GENTRAL VISION WITH PRESERVATION OF PERIPHERAL VISION}

A blank in the field of vision is called a scotoma; and if this is central, the patient is unable to read, to thread a needle, or to recognise the face of a friend on the other side of the street, even although not short-sighted. In every case of central scotoma there is diminution of the acuity of vision; and, in the majority, an object looked at directly is seen worse than one in the periphery of the visual field. The scotoma is said to be relative, and is due to a lesion situated either in the macula, or in the papillo-macular bundle in the optic nerve. In other cases, the patient complains that when he tries to read he cannot see the word he looks at, because it is covered by a dark veil; or if he looks at the sky, or at a white wall, he is conscious of a black patch or cloud straight in front of him, occupying the centre of the field of vision. Such a scotoma is positive, and is caused by something which interrupts the passage of light from an object to the layer of rods and cones, e. g. a retinal hæmorrhage. The distinction between relative and positive scotoma is of considerable clinical value, because the former always implies a lesion of the nervous system, i. e. the retina or the optic nerve; whereas, in the latter, the retinal elements subjacent to the lesion are intact.

In the examination of a patient who complains of failure of sight confined to the centre of the field of vision, ascertain first-

When the Impairment of Sight was First Noticed.-It is of considerable importance to determine the date of onset of the defective vision, because it is often congenital, although the patient may have 
been quite unaware of its existence. The fact that one eye sees much better than the other is frequently discovered accidentally, and the discovery causes so much anxiety that the family doctor is consulted without delay. He ought, first of all, to test the pupillary reflexes, and if possible examine the fundus oculi with the ophthalmoscope; bearing in mind, however, that when a central scotoma is congenital and confined to one eye, it is usually accompanied by squint; whereas, if bilateral, it is frequently associated with nystagmus.

The next points to ascertain are-

Is the defect in sight confined to one eye, or are both affected ? and Did the failure of vision occur suddenly or come on gradually?

\section{One eye only is affected, or the defective sight is very much worse in one eye than in the other}

1. Sudden Failure of Vision.-Hæmorrhage into the macula; or partial retrobulbar neuritis.

(x) Hamorrhage into the Macula.-The bleeding sometimes occurs during the night, and the defect in sight is discovered on first waking in the morning; but more frequently it comes on during the day after some unusual exertion-a fit of coughing, straining at stool, etc. The patients are usually elderly, and show signs of arterial degeneration and of high blood pressure.

The scotoma may be positive or negative. In the former case the patient complains that he sees a black spot which covers every object he looks at directly, or that he sees a black patch in the line of fixation, when he looks at the sky or at a white wall, etc. In these circumstances the layer of rods and cones must be intact; the patient sees the hæmorrhage, and the fact that the spot always remains fixed in one position distinguishes it from a floating body in the vitreous, or from muscæ volitantes. If the patient complains that all lights appear red, the hæmorrhage must be large, and lie beneath the hyaloid-sub-hyaloid. In such cases prognosis is fairly good, because when the blood becomes absorbed sight returns, and the ultimate result depends upon freedom from recurrence.

If, on the other hand, the scotoma is negative or relative, the patient does not describe the obstruction to his vision as a black spot, but says that he cannot see an object straight in front of him. When, for example, he looks at a friend's face, he cannot make out the features-or when he tries to read, he cannot see the word he is looking at. The object in the line of fixation is a blank, although he can see on all sides of it. In these circumstances the hæmorrhage is intraretinal and implicates the bacillary layer. The prognosis in those cases is always unfavourable; 
because, as a large number of cones are certain to be destroyed by the effused blood, recovery of sight will at the best be partial, and a scotoma will be permanent. Moreover, intraretinal hæmorrhage is always the result of degeneration of blood-vessels - or of a diseased state of the blood-and is always very prone to occur in eyes which are highly myopic.

(2) Retrobulbar neuritis.-This causes a central negative scotoma when it affects the papillo-macular bundle in the orbital portion of the optic nerve. It may be caused by a toxic condition, e.g. "rheumatism" -by the extension of inflammation from the sinuses adjacent to the orbit-by a blow on the head-or it may occur as part of a general disease of the nervous system, more particularly insular sclerosis. The ophthalmoscope will reveal little that is pathognomonic; but the behaviour of the pupil is quite characteristic. The direct light reflex is present in the affected eye; but while the light is still held in front of it, the pupil dilates, and the consensual reflex is either feebly present or altogether absent.

Those patients must be very carefully examined for disease of the nervous system, and in all obscure cases the X-rays should be employed, and the blood tested for the Wassermann reaction.

\section{Gradual Failure of Vision.}

Choroido-retinitis.-Although the disease is usually bilateral, the eyes are very rarely affected simultaneously, in point of time one being far in advance of the other. The first thing the patient complains of is, that when he looks at a word with the affected eye, the letters are not all on the same level, but are displaced irregularly-metamorphopsiaand while some are apparently squeezed together and seem smaller than they should be-micropsia-others are separated from each other and seem too large-macropsia. As time passes, reading becomes more difficult, and at length impossible. The onset of metamorphopsia always indicates that the macula is diseased-it is very prone to occur in those who suffer from short-sight. It is well-known that even in very high degrees of myopia, the smallest print can be read if the book be held close enough to the eye; but when inflammatory changes occur in the macula, reading and all fine work are impossible. If, therefore, the patient is myopic, his ability to read the smallest print ought always to be tested, in order to ascertain whether or not the macula is affected. As syphilis is a common cause of choroido-retinitis, the Wassermann test of the blood ought to be made in all doubtful cases.

\section{Both eyes are affected}

Toxic Amblyopia.-The patient is generally a man from forty to fifty years of age, who, for some weeks or months, has found that 
the sight has been gradually failing. He has, in all probability, tried to get spectacles from an optician, but found no material improvement in vision from the use of any glasses. Two symptoms are characteristic of those patients: (I) they see best in a dull light; and (2) they are unable to distinguish a small disc of red or green, although they can recognise both colours if the area shown them is large enough to overlap the point of fixation. The papillo-macular bundle in the optic nerve is affected, and the most frequent cause is overuse of tobacco or of alcohol. The ophthalmoscope reveals very little change in the appearance of the fundus oculi.

(2) Hereditary Optic Neuritis (Leber's disease).--Loss of sight affecting both eyes, usually simultaneously and symmetrically, and occurring about the twentieth year. The disease is transmitted through the mother to her sons, daughters being very rarely affected. In the majority of cases the central scotoma remains permanent, and while in some improvement may occur-in others the disease terminates in blindness. Fisher believes that this form of optic neuritis is caused by the pressure of the pituitary gland, which usually becomes enlarged about the age when this disease makes its appearance.

\section{B. THE DEFECT IS IN THE PERIPHERY OF THE FIELD, GENTRAL VISION BEING RETAINED IN WHOLE OR IN PART}

\section{The Contraction of the Field of Vision is General.}

(a) The oUtLINe IS Very IRREGUlaR.

Atrophy of the optic nerve.-The pupillary reflexes are abnormal. Colour vision is greatly disturbed; and concomitant symptoms of disease of the cerebro-spinal system are present. The ophthalmoscope reveals pallor of the optic disc, and changes in the retinal blood-vessels.

(b) THE OUTLINE IS QUiTE REGULAR.

Retinitis pigmentosa.-Night blindness is always complained of ; and, in advanced cases, the patient feels as if he were looking through a narrow tube. The ophthalmoscope reveals waxy pallor of the optic disc, accompanied by characteristic pigmentation of the retina.

2. The Gontraction of the Nasal area of the Field of Vision is more pronounced than the Temporal.

Chronic Glaucoma.-The patients are elderly, failure of sight has been gradual, the pupil is somewhat large and sluggish, the anterior chamber shallow, the intraocular tension increased, and the optic disc cupped.

3. The Contraction of the Field of Vision is General and very Pronounced, but is apt to vary from Day to Day. 
Hysterical amblyopia.-The pupillary reflexes are normal, and no pathological changes are seen on ophthalmoscopic examination of the fundus oculi. One eye only may be affected, while the other remains quite healthy; and the patient never seems to suffer inconvenience as a result of the great contraction of the visual field, as he would from a similar degree of contraction due to atrophy of the optic nerve or to retinitis pigmentosa.

\section{The Contraction of the Field of Vision is Partial.}

(a) THE BLANK GeNerally is IN THE UPPER PaRT OF THE FIELD.

Detachment of the retina.-The patient is usually myopic, sight very defective, the onset sudden, and usually preceded or accompanied by flashes of light, the intraocular tension is reduced, and the use of the ophthalmoscopic mirror shows the pupil sometimes red and at other times dark, according to the movements of the eye. The detachment is easily seen with the ophthalmoscope.

(b) The blank in the field of vision has an unusual situation.

Intraocular tumour.-Always suspect an intraocular tumour when the affected eye is hard-the episcleral vessels congested locally-and all signs of disease absent in the other eye.

(c) HALF OF THE FIELD OF VISION IS LOST IN BOTH EYES.

Hemianopsia.-This is usually homonymous, and confined either to the right or to the left side. The patient complains that he sees only part of an object. He has a blind side-either right or left-and is astonished at the suddenness with which a hand or other object, approaching from the blind side, comes into view. He has great difficulty in dividing a line into two equal parts, and makes the portion on the blind side much the larger. Homonymous hemianopsia always means that there is a cerebral lesion implicating the optic tracts, on the side of the brain opposite to the hemianopsia. Other varieties of hemianopsia may occur, and are referred to in the description of the plate. There is rarely any change to be seen on ophthalmoscopic examination.

(d) There ARe one or MORE blanks iRregularly situated iN THE PERIPHERY OF THE FIELD OF VISION.

Choroiditis and Chorio-Retinitis.-The patient complains of frequent flashing of lights, of a cloud or a shadow passing before the eyes, of muscæ volitantes, of blanks in the field of vision, and, when the disease affects the macula, of difficulty with all fine work. The ophthalmoscope is necessary before a definite diagnosis can be made. 


\section{THE WHOLE OF THE VISUAL FIELD IS INVOLVED- GENTRE AS WELL AS PERIPHERY}

When the whole of the visual field is lost, it always means that something serious has gone wrong with the optic nerve, or the retina, or with the main blood supply of the eye.

\section{The Onset is Sudden.}

(I) Profuse intraocular hamorrhage.-This occurs both in the old and in the young, and may be spontaneous, or may follow an injury or any violent exercise. In the elderly it is usually associated with arteriosclerosis and other signs of vascular degeneration, and is always of grave. significance. It may precede a cerebral hæmorrhage, and even in the most favourable circumstances recovery of sight is never complete. In the young-and especially about the period of adolescence-profuse hæmorrhage may occur into the vitreous, without the slightest warning or any symptom of ill-health. Recovery of vision is usually rapid after a first attack; but recurrence is almost certain, and ultimately sight is lost. Both eyes may be affected.

(2) Traumatic asphyxia (after an injury).-When loss of vision is complete, blood is effused into the sheath of the optic nerve. The absence of the consensual reflex is a sure guide in diagnosis.

(3) Embolism of the central artery of the retivia.-Without any warning, and in an instant, blindness occurs in one eye. External examination reveals no abnormality, but the patient cannot distinguish day from night. The ophthalmoscope shows the characteristic cherry-red spot in the macula, surrounded by an area of œdematous retina. Very rarely the embolism can be seen blocking a branch of the retinal artery, in which case the loss of sight will be partial, and confined to the area of the retina supplied by the vessels affected.

(4) Thrombosis of the central vein of the retina.-The patients are usually elderly, and frequently suffer from cardiac or renal disease. Several premonitory seizures precede the attack which destroys the sight. The ophthalmoscope shows the veins engorged with blood and extremely tortuous, and the retina covered by large flame-shaped hæmorrhages. Secondary glaucoma follows in a considerable number of the cases; and the eye, which is quite blind, has to be enucleated to relieve pain.

(5) Acute retrobulbar neuritis.-This is a more aggravated form of the retrobulbar neuritis characterised by the sudden onset of a central scotoma, and referred to on p. 379. It is for the most part unilateral, and symptomatic of disease of the sinuses adjacent to the orbit; but is said to occur also in " rheumatic" subjects, after exposure to cold. The patient suffers pain when the eye is turned from side to side, and when 
it is pressed backwards into its socket. It can usually be distinguished from other forms of blindness of one eye by careful investigation of the reactions of the pupil. The pupil of the affected eye reacts to light, but, instead of remaining contracted, dilates while the light is still held in front of it.

2. The Onset is Insidious.

(I) Optic neuritis and post-neuritic atrophy.

(2) Primary atrophy of the optic nerve.- The latter is incurable, and progresses relentlessly, in spite of treatment, to blindness. The ophthalmoscope is always required for the diagnosis.

(3) Urcemia.-Both eyes are affected, and blindness is accompanied by albuminuria, with headache, vomiting, convulsions, coma, etc. Sight returns in one or two days, if the patient recover from the uræmic seizure. The pupillary reflex to light stimulus is present.

In some instances the patient is blind, yet the ophthalmoscope shows no pathological changes in the fundus oculi. In these circumstances great care must be taken not to attribute to hysteria symptoms which are due to organic disease. The great safeguards to accurate diagnosis depend upon thorough examination of the reactions of the pupil, and of the field of vision. Want of attention to those points might readily lead to error on the part of the doctor, with disastrous results to the patient. 


\section{CHAPTER XLV}

\section{THE OPHTHALMOSCOPE IN GENERAL MEDICINE}

Anatomical structure of the fundus and its individual components-Papillitis or choked disc-Post-neuritic atrophy-Primary optic atrophy-Causes and treatment of optic neuritis-Uræmic amaurosis-Retinitis albuminuricaFundus changes in arterio-sclerosis-Prognosis in albuminuric retinitis, in relation to sight and to life-Treatment-diabetic retinitis-Choroiditis disseminata.

IT is recorded of von Graefe, that when he first saw the fundus of the living eye, his face flushed with excitement, and he exclaimed, "Helmholtz has unfolded to us a new world! What remains to be discovered?" Such enthusiasm may well be pardoned, for few things in medicine impress the student more than his first clear view of the background of the living eye. To begin with, however, he has considerable difficulty in understanding what he sees, and only comprehends the picture fully after he has learned to correlate the ophthalmoscopic appearances with the microscopic anatomy of the parts rendered visible. Each tunic of the eyeball contributes something towards the ophthalmoscopic image of the fundus. The sclerotic may be regarded as the white canvas on which the picture is painted; the choroid, with its vessels and pigment cells, contributes largely to produce the red colour; while the retinaquite transparent and therefore invisible-gives, nevertheless, a peculiar lustre to the picture. When the interior of the eye is lighted up by the ophthalmoscope, the contained structures at once come into view in a manner impossible in any other part of the body. There are under direct observation the termination of an artery and the commencement of a vein, and it is easy, from the colour of the blood contained in each, to distinguish the one from the other. There is also the termination of the optic nerve, which is seen as a disc, and forms the most conspicuous object in the red background of the eye.

This appearance as a disc requires a few words of explanation, since, under the microscope, the nerve entrance appears as a distinct papilla. At the level of the lamina cribrosa, the medullary sheath of the fibres stops; and, the fibres of the optic nerve changing both in form and colour, only naked axis cylinders enter the eye to form the retina. These being transparent, the ophthalmoscopic observer looks through the papilla 


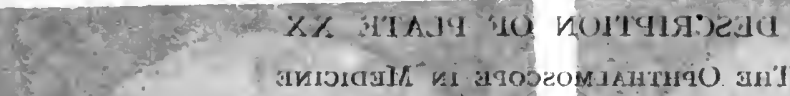

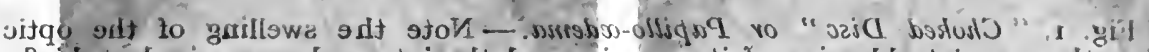

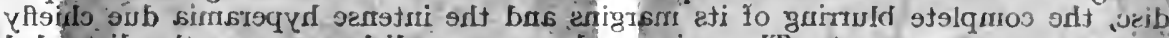

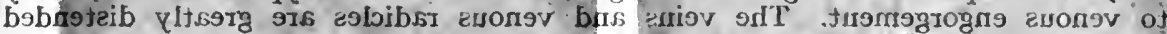

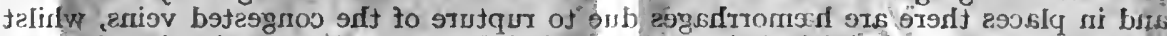

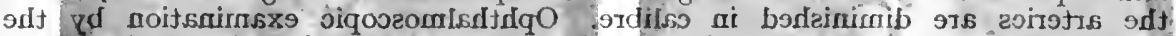
noigat glbbim

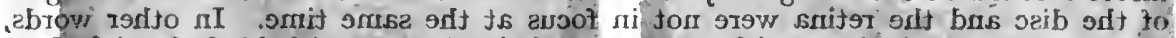

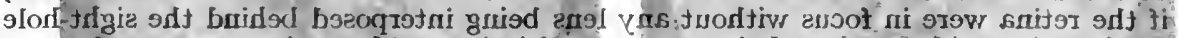

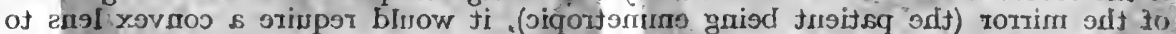

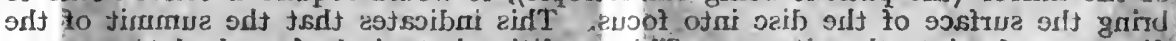

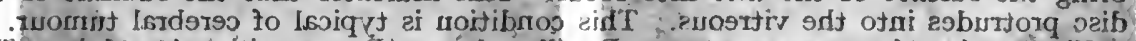

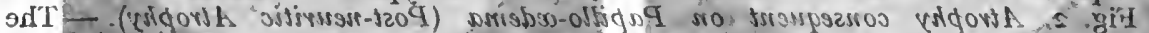

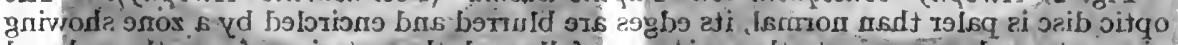

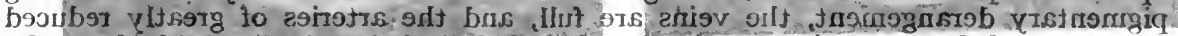

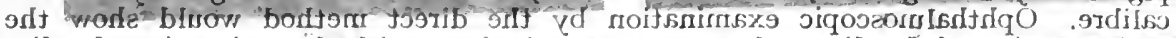

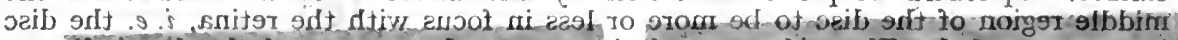

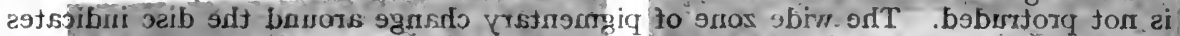

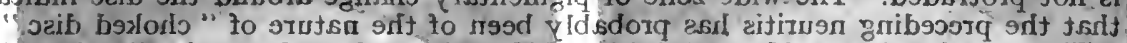

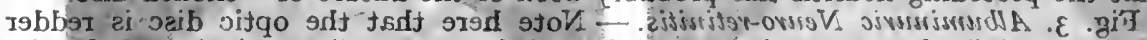

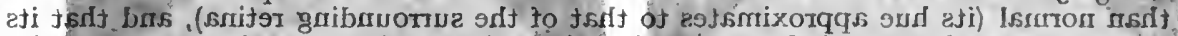

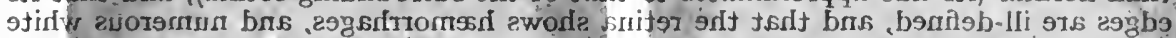

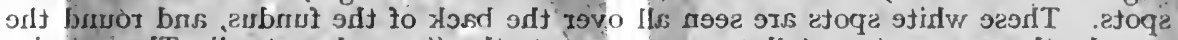

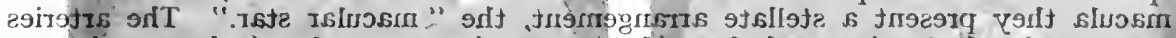

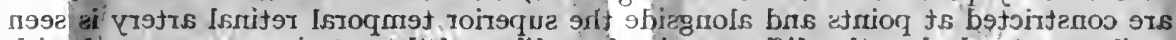

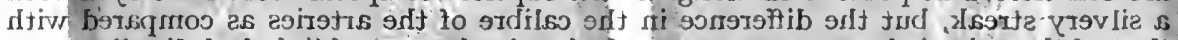

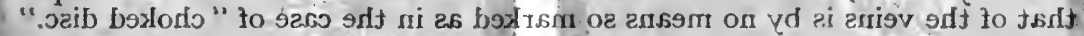

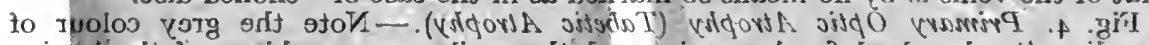

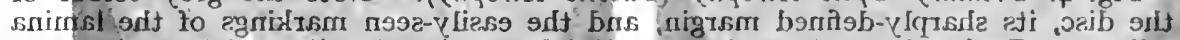

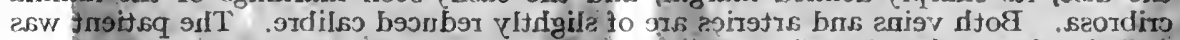
. ailserob agdst nuot grimgitue

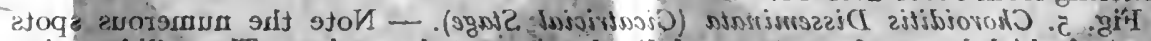
noitioq billsq onT .entigzsn b9tasmrgiq YIq

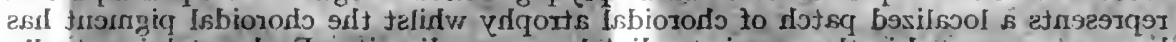

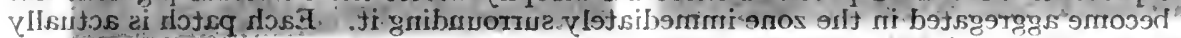

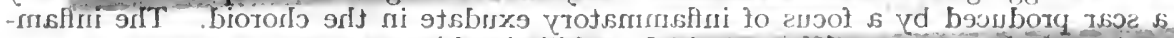

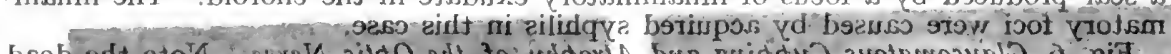

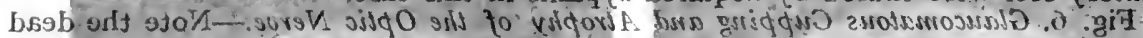

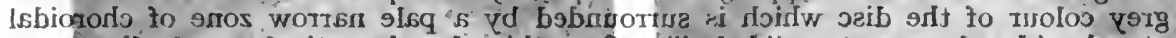

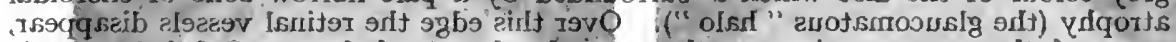

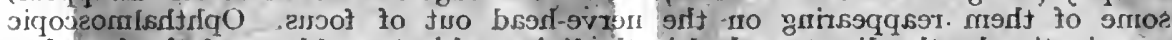

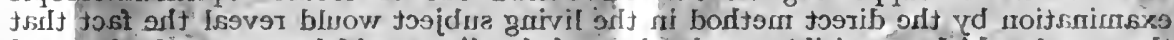

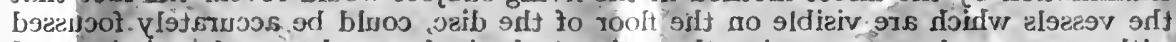

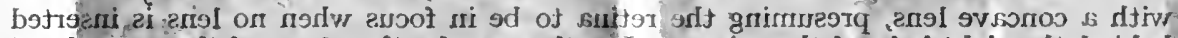

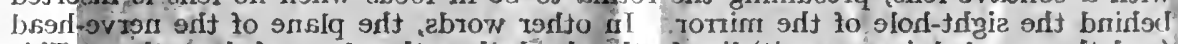

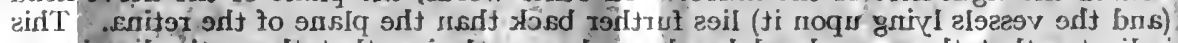

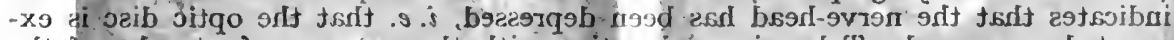

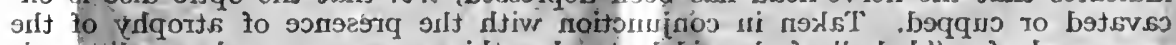

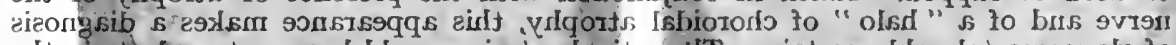

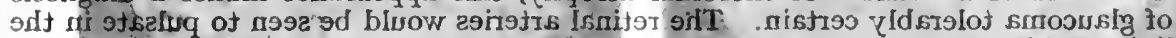

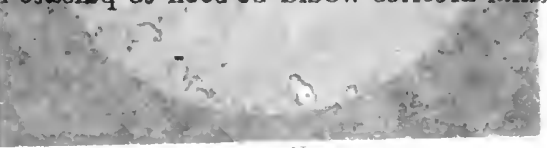
.Josidua gूnivil 


\section{DESCRIPTION OF PLATE XX}

\section{The Ophthalmoscope in Medicine}

Fig. 1. "Choked Disc" or Papillo-oedema. - Note the swelling of the optic disc, the complete blurring of its margins and the intense hyperamia due chiefly to venous engorgement. The veins and venous radicles are greatly distended and in places there are hæmorrhages due to rupture of the congested veins, whilst the arteries are diminished in calibre. Ophthalmoscopic examination by the direct method in the living subject would reveal the fact that the middle region of the disc and the retina were not in focus at the same time. "In other words, if the retina were in focus without any lens being. interposed behind the sight-hole of the mirror (the patient being emmetropic) it would require a convex lens to bring the surface of the disc into focus. This indicates that the summit of the disc protrudes into the vitreous. This condition is typical of cerebral tumour-

Fig. 2. Atrophy consequent on Papillo odema (Post-neivitic utrophy).tis The optic disc is paler than normal, its edges are blurred and encircled by a zone showing pigmentary derangement, the veins are full, and the arteries of greatly reduced calibre. Ophthalmoscopic examination by the direct method would show the middle region of the disc to be more or less in focus with the retina, inencthe disc is not protruded. The wide zone of pigmentary change around the disc indicates that the preceding neuritis has probably been of the nature of " choked disc.

Fig. 3. Albuminuric Neuro-retinitis. - Note here that' the optici disc (iseredder than normal (its hue approximates to that of the surrounding retina), and that its edges are ill-defined; and that the retina shows hæmorrhages, and numerous white spots. These white spots are seen all over the back of the fundus, and (round the macula they present a stellate arrangement; the " macular star." The Tharteries are constricted at points and alongside the superior temporal retinal artery is seen a silvery streak, but the difference in the calibre of the arteries'as compared with that of the veins is by no means So marked as in the case of "choked disc." with

Fig. 4. Primary Optic Atrophy (Tabetic.Atrophy). - Note the grey colour of the disc, its sharply-defined margin, and the easily-seen markings of the lamina cribrosa. Both veins and arteries are of slightly reduced calibre. The patient was suffering from tabes dorsalis.

Fig. 5. Choroiditis Disseminata (Cicatricial Stage). - Note the numerous spots most of which have pale centres and deeply pigmented margins.clsthe pallid portion represents a localized patch of choroidal atrophy whilst the choroidal pigment has become aggregated in the zone immediately surrounding it. Each patch is actually a scar produced by a focus of inflammatory exudate in the choroid. Thecinflammatory foci were caused by acquired syphilis in this case $e_{\mathrm{r}}$ is lighted th

Fig. 6. Glaucomatous Cupping and Atrophy of the Optic Nerve.-Note the dead grey colour of the disc which is surrounded by a pale narrow zonelof choroidal atrophy (the glaucomatous" "halo"). Over this edge the retinall vessels disappear, some of them reappearing on the nerve-head out of focus. Ophthalmoscopic examination by the direct method in the living subject would reveal the fact that the vessels which are visible on the floor of the disc, could be accurately focussed with a concave lens, presuming the retina to be in focus when no lens is. inserted behind the sight-hole of the mirror. In other words, the plane of the therve-head (and the vessels lying upon it) lies further baek than the plane of the retina. This indicates that the nerve-head has been depressed, i.e. that the optic disc is excavated or cupped. Taken in conjunction with the presence of atrophy of the nerve and of a "halo" of choroidal atrophy" this la ppearance makes a diaguosis of glaucoma tolerably certain. The retinal arteries would be seen to pulsate in the living subject. 


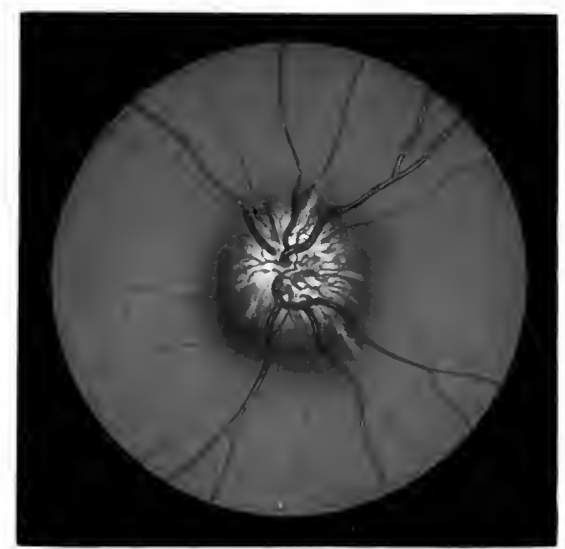

Fig I

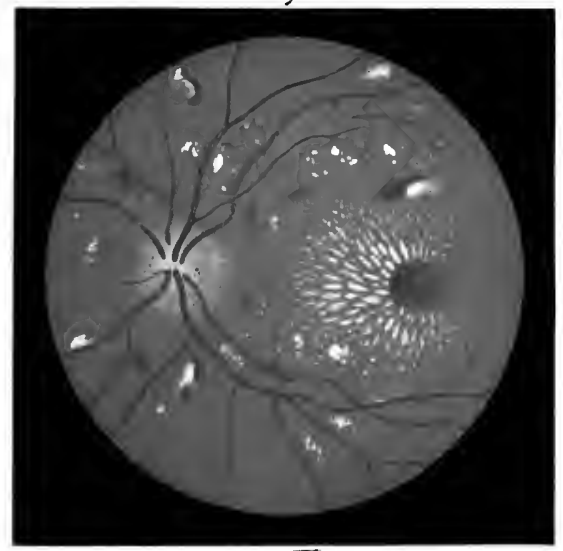

Fig III

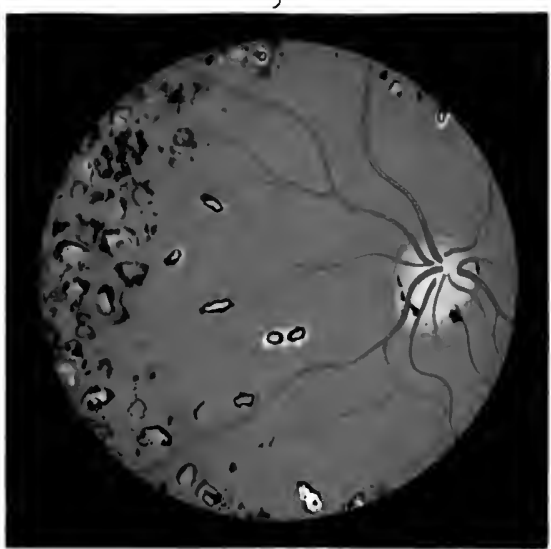

Fig V

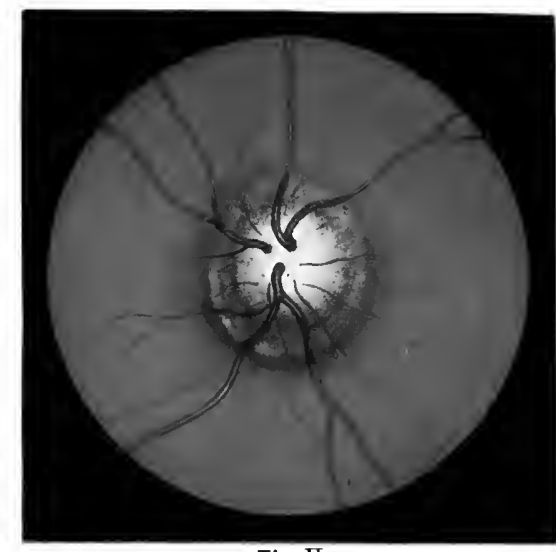

Fig I

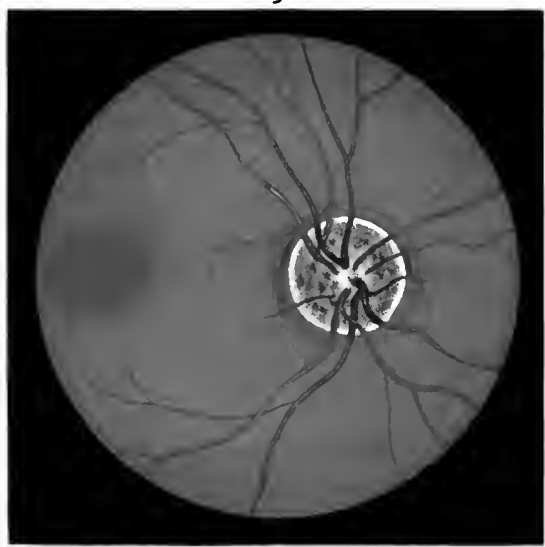

Fig IV

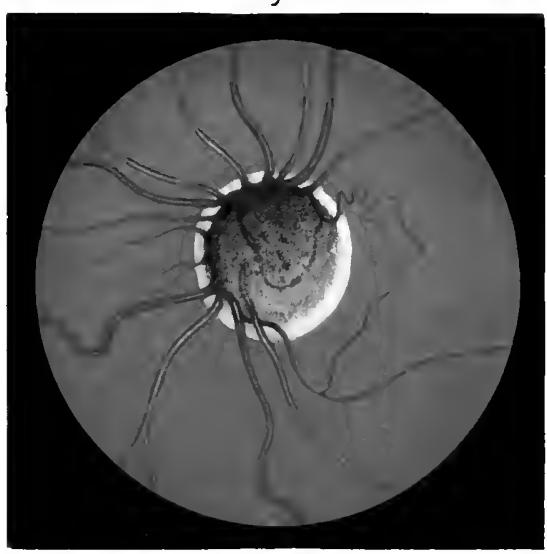

Fig II 

as if it were glass down to the level of the sclerotic-the dark line, bordering more or less completely the margin of the disc, being the edge of the opening in the choroid through which the optic nerve enters the interior of the globe. Occasionally the axis cylinders enter the eye clothed in their medullary sheaths; and, as they then reflect light strongly, are seen as a white patch in the neighbourhood of the optic disc. These opaque nerve fibres are liable to be confounded with inflammatory exudation, but are easily distinguished by the shape and position of the patch, by its brilliant white colour, and by its peculiar brush-like appearance.

From an embryological point of view, the retina may be regarded as the extracranial portion of the cerebral cortex, while the stalk of the nerve is homologous with the association fibres in the brain. Its sheaths are formed by prolongations of the cerebral meninges, and enclose spaces directly continuous with the subdural and subarachnoid spaces around the brain. The arteries are terminal branches of the cerebral arteries, and the veins in part empty themselves into the cavernous sinus. Owing to this intimate brain connection, intracranial disease has a powerful effect on the shape and colour of the optic disc; and it had long been known that intracranial disorder led often to impairment of sight or even to total blindness. It was only, however, in $x 860$, that von Graefe, in calling attention to the frequency of inflammation of the optic nerve in the course of cerebral tumours, gave a clear description of the intraocular changes which cause the defective vision. Later on, Hughlings Jackson drew attention to the fact that pronounced optic neuritis may be present, and yet the patient make no complaint of failing sight. He may complain that a cloud seems to pass momentarily over the eyes, but that is all. It is necessary, therefore, if the optic neuritis is to be detected, that an ophthalmoscopic examination be made in every case; for even careful testing of the field of vision, as well as of the visual acuity, is of no value until the later stages, and then inflammation is passing off and giving place to atrophy. In all likelihood the patient will say that he "sees well enough," and so mislead the unwary physician; for the presence or absence of optic neuritis can be determined only by the use of the ophthalmoscope.

The picture presented-although it admits of many variations-is, on the whole, fairly distinctive. The earliest and most important sign is swelling of the papilla-papillcedema; which, owing to odema, loses its natural transparency and becomes clearly visible. It may attain a height of $2 \mathrm{~mm}$. or more. The congenital cup is soon obliterated, and the outlines of the optic disc blurred with exudation, which may extend into the surrounding retina and give rise to neuro-retinitis. The disc loses its form, and is represented by a reddish-grey mass of a soft, woolly C C 
outline-frequently bespattered by hæmorrhage. In an advanced case, the position of the disc is made out only by tracing the blood-vessels which converge towards the papilla, and become more or less completely buried in the exudation. The arteries are somewhat smaller than normal; and are, in parts of their course, concealed by exudation. The veins are large, turgid, and tortuous, and as they dip down over the swollen papilla, their course appears interrupted as they pass through the clouded retinal tissues. In addition to the hæmorrhage over the surface of the papilla, narrow flame-shaped extravasations occur in the nerve fibre layer of the retina in its immediate neighbourhood.

Cerebral Tumour.-Optic neuritis is one of the most important signs of cerebral tumour; and, according to Gowers, occurs at some period or another of their course in four-fifths of the cases. It derives much of its importance from the fact that it can be seen by the physician, and is not therefore like headache or giddiness, dependent upon the statements of the patient-or like vomiting, which is symptomatic of so many different morbid states. It requires, however, to be looked for; because, as has already been pointed out, the patient is not at all likely to make any complaint of failing sight.

The neuritis is generally bilateral, and it is usually said that the tumour is on the side on which the inflammation is more intense (Horsley); but such a conclusion is untrustworthy (Leslie Paton), and Hughlings Jackson has recorded two cases in which the papillitis was unilateral, and on the side opposite to that on which the cerebral tumour was found. The important point to remember is that it may be the only objective sign of intracranial disease, and that for a time it may be unaccompanied by any symptoms.

The site of the tumour, and the degree of distension of the ventricles to which it gives rise, are the main factors in causing swelling and œedema of the papilla, choked disc, or Staunng's papilla. It is due to increase of intracranial pressure, through part of the cerebro-spinal fluid squeezed out of the cranial cavity by the growth of the tumour finding its way into the spaces around the optic nerve; and these in consequence becoming greatly distended immediately behind the eyeball.

If the fluid absorbs toxins from the tumour, as Leber supposes, it will be all the more capable of inciting inflammation; while in other cases, such as basal meningitis, the inflammation is propagated by direct transmission from the brain to the optic nerve; and so a tumour, by inciting meningitis, may give rise to an inflammation which descends to. the optic nerve, and thus induces papillitis.

Should the neuritis arise from direct pressure of a tumour or a distended ventricle on the optic chiasma, the signs of inflammation 
are of very brief duration, and are succeeded quickly by pronounced atrophy.

Optic neuritis, although such an important general symptom of cerebral tumour, is of no localising value whatever, because it may occur in connection with a tumour in any part of the cranial cavity. "It is," according to Osler, " constant in tumours of the corpora quadrigemina, present in 89 per cent. of cerebellar tumours, and absent in nearly twothirds of the cases of tumour of the pons, medulla, and corpus callosum."

Its absence cannot be said to negative the existence of a growth, although it ought to cause re-consideration of the diagnosis. Moreover, as it may-although absent at the beginning-yet occur at any time during the course of a case, repeated ophthalmoscopic examinations must be made at regular intervals. Occasionally, it does not appear till long after other symptoms have left no room for reasonable doubt of the accuracy of the diagnosis; and under such circumstances, its onset isas Gowers has pointed out-usually significant of impending death. It may occur in connection with any form of new growth, and therefore gives no hint whatever regarding the nature of the tumour. The ophthalmoscope reveals the inflammation, but it can do nothing more. It is, like the stethoscope, a clinical instrument; and, except in the rare instances where tubercle or sarcoma of the choroid are seen, can give no pathological information. Nor is the degree of the neuritis any criterion, generally speaking, of the rate of progress of the cerebral lesion; for, although tumours running an acute course are nearly always associated with acute papillitis, similar ocular changes may be found in a case characterised by great chronicity. This much, however, can be said; that if, while a case is under observation, a sudden increase in the optic neuritis occurs, there is usually at the same time a rapid development of the tumour in the brain. The tendency, however, is for the inflammation to reach its height, and then to subside gradually. Whatever may have been the condition of the vision previously, from this point sight steadily deteriorates, and may fail altogether as the nerve passes into a state of post-neuritic atrophy. This must be carefully distinguished from the so-called primary atrophy of the optic nerve, which occurs so frequently in diseases of the spinal cord. In the former, the optic papilla always shows signs of past inflammation-at first of a greyish-red colour; it sooner or later becomes pure white. Its outlines are irregular and somewhat veiled, and in the terminal stage the whole disc appears shrunken. The retinal arteries are contracted, the veins distended, and tortuous, and both are accompanied by white lines-most apparent in the neighbourhood of the disc-and due to inflammatory exudation.

In primary atrophy, on the other hand, the change in colour is the 
most striking ophthalmoscopic feature; there is steadily progressive greyish pallor of the optic disc, the outlines of which are very sharply defined. The lamina cribrosa is, owing to cupping from wasting of the nerve fibres, distinctly seen, and there is complete absence of the small vessels usually present on the surface of the disc. Even though sight be lost, the retinal blood-vessels do not appear to be much altered in calibre. This progressive form of atrophy is not at all likely to be overlooked, as from the outset the patient complains of failing sight, and testing quickly reveals contraction of the visual field and disorder of the sense of colour. Although the eye troubles are, in most instances, merely an accompaniment of a general affection of the spinal cord, they may be so far in advance of any other symptoms that the patient does not consult a physician, but comes direct to an ophthalmic surgeon. Now, if, in a case with appearances suggestive of cerebral tumour, the physician be in danger of overlooking the optic neuritis; so, in a case of optic atrophy, the specialist must be keenly alive to the possibility of general mischief. The state of the pupils and of the ocular muscles must be carefully examined; and, whenever possible, a consultation with a physician ought to be obtained. In insular sclerosis, atrophy of the optic nerves is a frequent occurrence, but the patient rarely loses his sight completely; whereas in tabes dorsalis the atrophy invariably ends in total blindness.

The practitioner ought not to be content with a diagnosis of atrophy -he should not rest satisfied until he has, if possible, discovered its cause, and found out what other signs accompany it. Moreover, from the standpoint of prognosis, it is important to distinguish partial from complete atrophy. The former is usually post-neuritic, and any sight that remains after the inflammation has subsided may be expected to be permanently preserved; whereas the latter is primary, affects both the peripheral and the central parts of the field of vision, and is characterised by early interference with the colour sense, and by the fact that sight is affected from the outset. When, moreover, the optic atrophy is accompanied by signs of tabes dorsalis-myosis, Argyll Robertson pupil, loss of patellar tendon reflex, etc.-blindness is inevitable.

Pressure on the optic nerve, either within the skull or in the orbit, causes atrophy which cannot be differentiated from primary atrophy by ophthalmoscopic examination alone. In the latter, however, the pallor of the disc is discovered simultaneously with the onset in the. failure of sight; whereas in the former, although vision may be quite gone, no change can be seen in the papilla for several weeks; because it takes a considerable time for the degeneration of the nerve fibres to travel from the site of the injury to the papilla. In such cases a careful examination of the pupillary reflexes-loss of direct light reflex in the affected eye, 
and loss of consensual-reflex in the other-is of more importance at the outset than an inspection with the ophthalmoscope.

Optic neuritis ought never to be regarded as a disease per se; it is always a sign of serious disease in some other part of the body. When it is bilateral, the underlying disease is usually constitutional; whereas, when an inflammation is limited to one side, a local cause is as a rule responsible. The most frequent causes of bilateral optic neuritis-if intracranial disease be excluded-are syphilis and nephritis; consequently a Wassermann blood test, and a chemical examination of the urine, should be made in every case. Some other causes are diabetes, basal meningitis, and acute infectious diseases-influenza, pneumonia, scarlet fever, cerebro-spinal meningitis, etc. The commonest causes of unilateral optic neuritis are purulent inflammation of the orbit, of the m:ddle ear, or the accessory sinuses; and to these may be added abscess of the brain and malformations of the skull-tower skull.

Treatment.-The treatment of optic neuritis must be regulated by the nature of the disease which has caused it, but antiphlogistic measures are always required. The eyes themselves must be protected from bright light by suitable goggles; and free leeching over the mastoid process, the wet pack, and pilocarpin to promote diaphoresis, and the administration of mercury, iodide of potassium, and-in suitable cases-neo-kharsivan, galyl, or other of the arsenical preparations.

It must, however, be remembered that choked disc is not so much an inflammation proper, as an inflammatory œedema, the result of increased intracranial pressure. Vision, although unaffected at the outset, always suffers severely in the later stages; because the cellular exudation becomes organised to form connective tissue within the papilla, and the shrinking due to cicatricial contraction which follows produces atrophy of the optic nerve. The danger of blindness is therefore present in every case of choked disc; consequently Horsley advocates early trephining of the skull to bring about permanent reduction of intracranial pressure, which relieves the strangulation of the optic papilla, and thereby induces subsidence of the neuritis, with corresponding improvement in sight.

Disease of the Kidneys.-The clinical association of defective sight with disease of the kidneys did not escape the observation of the older physicians. Bright, in his Records on Medical Cases, makes special mention of the connection, and in some of those he recorded, eye symptoms were among the earliest-as well as the most striking-features. After the invention of the ophthalmoscope, knowledge of the subject became more and more exact. In 1859 Liebreich published a detailed description, illustrated by drawings of the ocular changes in Bright's Disease. The optic disc is reddish, swollen, blurred in outline, and surrounded by a 
diffuse opacity of the retina, spotted here and there with red patches due to hæmorrhage. Radiating from the macula in the form of a star are numerous small white shining dots; and scattered over the fundus are larger white patches-the result either of the partial absorption of a blood-clot, or of an exudation of leucocytes. The arteries are generally attenuated and accompanied by white lines, and the veins are distended and tortuous.

This is the type, but there are many variations. There may be associated with Bright's Disease very strongly-marked visual defects unattended by any gross lesions in the fundus oculi ; and cases of dimness of sight connected with diseases of the kidney characterised by albuminuria may, therefore, be divided into two classes-

(I) Urcemic amaurosis, where the ophthalmoscope reveals no gross lesions in the retina.

(2) Retinitis albuminurica, where marked retinal changes are present.

Uramic amaurosis occurs most frequently in those cases of Bright's Disease in which cerebral symptoms predominate. It may exist alone, but is more frequently accompanied by headache and vomiting, and an attack is often preceded by a convulsive seizure. Without discussing the various explanations which have been put forward to account for uræmia, it may be said that it is due to blood-poisoning, brought about by retention of waste products that ought to have been eliminated from the system by the urine. The resulting blindness is usually bilateral and complete, but the pupillary reflexes to light are unaltered; and though, as a rule, the attack passes entirely off after some hours, yet, in exceptional cases, it may last for days.

This is in very marked contrast to what is often seen in the second group-retinitis albuminurica, in which retinal changes may be discovered by the ophthalmoscope, and yet the patient be quite unaware that there is anything wrong with the eyes.

The fact that such a serious state of matters can exist without the knowledge of the patient, is yet another proof of the need for, and the great value of, careful ophthalmoscopic examination in every case when the general symptoms indicate any disturbance in the function of the liver or kidneys. Whenever retinitis is the first sign of disease to be detected, the renal affection has been latent, and although present for a long time, has caused the patient no inconvenience until sight became blurred.

In albuminuric retinitis the cases, according to West, naturally divide themselves into two groups, according as the lesions in the fundus oculi are inflammatory or degenerative. The inflammatory form is characterised by the occurrence of œdema, hæmorrhage, and inflammation, 
and is usually found associated with dropsy, and with the presence of albumen in considerable quantity in the urine. It must not, however, be supposed that the retinal lesions are dependent either upon dropsy or upon the amount of albumen. The more acute the nephritis the greater is the former, and the larger the quantity of the latter; but it is rare indeed to find albuminuric retinitis present at all during a first attack of acute parenchymatous nephritis. The eye-changes occur most commonly when the acute attack supervenes on previously existing chronic nephritis. In that case the kidneys have for a long time been doing their work inadequately; and the blood has, in consequence, become charged with waste products, which by their toxic influence have excited inflammatory changes in the optic nerve and retina. Hence the neuroretinitis comes on suddenly, and runs a violent course. Still, notwithstanding this, it may, under favourable conditions, pass off leaving no trace-as is well illustrated in the phenomena observed in retinitis albuminurica during pregnancy. Here the onset is acute, and progress rapid, and sight lost more or less completely-yet the majority of these cases, apparently so hopeless, admit of a favourable prognosis.

The degenerative form has its origin in pathological changes in the retinal arteries; and we are indebted to Mr. Marcus Gunn and to Mr. Foster Moore for an admirable description of the ophthalmoscopic appearances. The arteries are irregularly contracted, abnormally tortuous, and even the very smallest exhibit a brightness of the central light streak which is very characteristic. The artery loses its natural translucency, and comes hard and rigid; and, consequently, when it crosses a vein it not only conceals the latter from view, but also mechanically exerts so much pressure upon its walls that the blood-flow is hindered. The result may be hæmorrhage, and on account of œdema of the retina, more especially in the neighbourhood of the optic nerve and macula, the fundus does not present a bright red reflex, but appears grey and hazy. In addition to these vascular changes-the hæmorrhages and the œdema of. optic disc and retina-there are always present minute white dots, whose stellar arrangement around the macula, and bright shining appearance, are perhaps the most characteristic feature of the ophthalmoscopic picture. The anatomical changes which explain these lesions of the fundus oculi were investigated by Brailey, and are similar to those described by Gull and Sutton as "arterio-capillary-fibrosis." They are found for the most part in association with the contracted granular kidney; but it is important to note that they do not necessarily indicate renal disease, for in many instances they are but a part of a widespread general disease of the blood-vessels. They creep slowly but steadily along the walls of the arteries (their insidious progress being for 
a time marked by no symptoms), and remain quite unsuspected until, little by little, they reach an advanced stage. In this lies the importance of routine ophthalmoscopic examination in all cases of suspected granular kidney in which the urine, though it be copious and of low specific gravity, may contain albuimen only intermittently and in minute quantity.

Marcus Gunn has said that "ophthalmoscopic examination is one of the most ready clinical means for the early detection of important arterial changes"; and de Schweinitz has insisted upon the value of ophthalmoscopic examination of the fundus oculi to detect persistent high arterial tension. The latter lays great stress on the existence of a corkscrew-like appearance of the smaller arterial vessels, of the flattening of a vein where it is crossed by an artery, and of a general congestion of the optic disc; but while he looks upon these three signs as almost pathognomonic of the early stages of arterio-sclerosis, he very wisely urges that the ophthalmoscopic evidence ought, if possible, to be confirmed by examination of the heart and the pulse, and by the accurate measurement of arterial tension by a sphygmomanometer. The clinical importance of such a thorough examination as de Schweinitz insists upon will be at once apparent, when one remembers the intimate connection between the retinal lesions and the state of the blood-vessels in the brain; for in not a few instances the patient dies from cerebral hæmorrhage. Retinal hæmorrhages always mean increased arterial pressure acting on diseased vessels, and are frequently premonitory of apoplexy. It sometimes happens that inflammatory changes become superadded to the ordinary signs of degenerative albuminuric retinitis, and this always indicates that an attack of acute or subacute nephritis has supervened upon one which has been for a time chronic.

Although it is convenient to speak of kidney disease as divisible into two groups characterised by the presence or absence of dropsy, and to connect with each of these groups a definite form of albuminuric retinitis, it must be remembered that the divisions so merge into one another that it is not possible to distinguish different varieties of renal disease by the ophthalmoscopic appearances associated with them. Retinal changes may occur in any form of Bright's Disease, though they are most frequently associated with chronic renal cirrhosis. Both eyes are, as a rule, affected; and while the neuro-retinitis is usually more advanced in one than in the other, one of the most remarkable features is the symmetry of the lesions. In exceptional cases, however, more particularly in the retinitis associated with the albuminuria of pregnancy, one eye may remain perfectly normal.

To diagnose a case of albuminuric retinitis by the ophthalmoscope 
- is much easier than to appraise its true significance. According to Terson, a great preponderance of white spots indicates nitrogen retention; great œedema of the retina, retention of chlorides, and frequent hæmorrhages, high blood pressure. Whether or not that be so, it is generally admitted that the occurrence of retinitis adds additional gravity to the prognosis; and if the results of hospital statistics were accepted, the outlook would be gloomy in the extreme. There is, however, a great difference between the hospital patient and the private patient; and the régime which is applicable in dealing with the one requires, in most instances, to be considerably modified in the case of the other. The main point to remember is that albuminuric retinitis is a late manifestation in the course of renal disease; that, as a rule, it is associated with the phenomena that are attendant upon high arterial tension; and that its onset may be determined by general toxæmia, by vascular degeneration, or by these conditions combined. Prognosis will obviously be more favourable in the inflammatory group (i.e. those due to scarlet fever or to any acute infection), than in the degenerative-because in the former the toxic elements may be removed from the blood. That is why recovery is so frequent in albuminuric retinitis of pregnancy; and why, when eye symptoms occur early-during the first six monthsand are threatening to destroy sight, premature labour should be induced with the least possible delay. The mother risks not only her vision but also her life. Many cases of recovery from albuminuric retinitis are on record; but their interest depends not so much upon the disappearance of the eye-changes, as upon the removal of the cause of the albuminuria. Prognosis must therefore be considered not only in relation to sight, but also in relation to life.

1. Prognosis in Relation to Sight.-Speaking generally, sight is not usually lost through albuminuric retinitis alone; and the degree of amblyopia present depends upon the amount of destruction due to degeneration, and to the position of the retinal hæmorrhages. When the ophthalmoscope clearly reveals changes in the blood-vessels arising from arterio-capillary fibrosis, it will be safe to predict that vision will become steadily impaired; because the vascular degeneration is likely not only to be progressive, but also to lead to local malnutrition and further hæmorrhage. As has already been observed, however, hæmorrhagic retinitis may be present, and yet no complaint be made of defective sight. The fundus may be spotted with flame-shaped blood-clots, but so long as these are peripheral, not only may the patient be quite unconscious of their presence, but subjective examination may fail to detect anything wrong. It is very different, however, when the bloodclot occupies the macula; or when its round shape shows that it is 
situated deep in the substance of the retina; for then the sentient layer is implicated, and the consequent disturbance of the rods and cones, if it does not produce a blank in the field of vision, will certainly induce metamorphopsia. A blank in the central area of the field of vision, due to hæmorrhage in the macula, interferes completely with the patient's power of reading; and, although the blood-clot may become absorbed in course of time, it has probably caused so much disturbance in the regular arrangement of the sentient elements, that objects are seen with their outlines seriously distorted. As a patient once expressed it, " everything seems done in rustic work."

Metamorphopsia is always a very painful symptom, and although it may pass off, the prognosis is more unfavourable because the macula has been seriously involved. When in the course of a chronic attack of albuminuric retinitis, acute symptoms, marked by oedema, exudation, and hæmorrhage, become superadded, an attack of acute parenchymatous nephritis has, as a rule, occurred; and the onset of such retinal changes, although causing greater anxiety at the time, may yet improve the prognosis of the disease as a whole, by placing the case in the category of those where the kidney lesion is for the most part a local affection, rather than a sign of a degenerative "change in the blood-vessels as a whole.

2. Prognosis in Regard to Life.-Albuminuric retinitis is rarely found with acute inflammation of the kidney, except when the acute symptoms are superadded to previously existing chronic disease; and the main points to be attended to are the occurrence of uræmic seizures; the degree of œdema of the internal organs as evidenced by dyspnœa, vomiting, or purging; and the onset of acute inflammation of the serous membranes, or of persistent hæmorrhage. In cases associated with pregnancy the prognosis is usually favourable; but in them also danger becomes imminent if eclamptic seizures are frequent and severe. Formerly, when retinitis albuminurica was discovered in the course of chronic renal cirrhosis, it was regarded as of such serious import that practically a death sentence was passed upon the patient. It was found, however, that although many did die within a few months of the retinal changes having been detected, others, who were more comfortably circumstanced, and consequently better able to take care of themselves, lived for several years. It is not wise, therefore, to disturb the general perspective of the disease by assigning to the eye symptoms a position of importance out of all proportion to the others. They ought to be regarded as part and parcel of the renal disorder; and when a prognosis as to life has to be given, they must be looked upon in their due relation to the whole. An unfavourable opinion must always be expressed, 
when retinitis albuminurica is associated with steadily failing strength, due to a large daily loss of albumen; with a weak heart and a low-tension pulse; with diminished excretion of urine; with the occurrence of uræmic symptoms; and last, but probably most important of all, with progressive anæmia. It is the anæmia that kills.

Treatment.-The treatment resolves itself very largely into a question of how a kidney, which is failing adequately to discharge its proper function, can be aided and protected. For the ocular changes themselves very little can be done, except to advise the patient to avoid straining the eyes over fine work, and to protect them from exposure to bright light. There can be no doubt that exposure to glare is a potent exciting cause of recurrent hæmorrhage from the retinal blood-vessels; hence the urgent necessity of protecting the eyes from bright light. The ordinary Londonsmoke goggles accomplish this; but they do so by diminishing the light, and materially altering the appearance of things. Of this patients complain; but if amber, or chlorophyll, or Crooke's tinted glass be prescribed, the difficulty is overcome; because by this means the highly irritating actinic rays are cut off, and the patient gets a sensation of restfulness and brightness without glare. The general treatment consists chiefly in flushing out the kidneys by copious draughts of water, and in the use of such simple remedies as the citrates or acetates of potash, soda, and ammonia. In cases such as those described by de Schweinitz and others, in which the retinal vessels present the appearance of degeneration indicative of generalised arterio-sclerosis, the iodide and nitrite of sodium administered in a hot saline draught are often very helpful.

Good can be effected only by improving the condition of the blood; and this may be accomplished by calling on the skin and the intestine to aid the kidney in eliminating waste products. Of these the skin is the more important, as skin and kidney present many points of similarity. Both have a common origin, for it was from the ectoderm that the true kidney was originally developed, and the uriniferous tubules and capsules bear a striking resemblance to the coils of the sweat glands. Owing to their community of descent, kidney and skin have a wonderful sympathy with each other, and one of the most familiar facts in clinical medicine is the manner in which renal inadequacy is compensated by increased cutaneous secretion. To promote free action of the skin is one of the first principles of treatment ; and, as a rule, this is best accomplished by means of the vapour bath, and by hypodermic injections of pilocarpin. The dose must be a very small one to begin with, and its effects watched carefully, for fear of the recurrence of cardiac depression, or even of a syncopal attack. While, however, every effort is made to promote free elimination on the one hand, care must also be taken on the 
other to supply the patient with food of such a kind as will throw little work on the kidney. Skimmed milk is the ideal substance for this purpose; but all means should be taken to avoid too limited views about diet. The patient must be considered, as well as the disease from which he is suffering. To maintain strength is essential; and whatever tends to increase anæmia does harm.

It follows, then, that there can be no specific remedy for albuminuric retinitis. At one time fuchsin was largely prescribed, with the result that, thongh there was in some cases a diminution in the amount of albumen passed, there was no real and lasting improvement in the patient's health. Nor could it be otherwise, for the albuminuria-like the retinitis-is merely one of the symptoms, and not the disease itself. A rational therapeusis can be arrived at only by careful study of the natural history of the disease; and the proper treatment for all patients suffering from albuminuric retinitis is to protect the eyes, as far as possible, from glare and strain; to provide proper food, warm clothing, comfortable surroundings, and plenty of pure water and fresh air; and to make judicious use of simple eliminants, and of tonics containing iron.

Glycosuria.-It is often very difficult indeed to make a differential diagnosis with the ophthalmoscope alone, between hæmorrhagic retinitis associated with albuminuria, and that associated with glycosuria. It is generally stated that one distinction is, that the glistening white spots in the macular region in retinitis associated with glycosuria are not arranged so regularly in the form of a star as they are in retinitis associated with albuminuria; and also that the hæmorrhages in the former are numerous, scattered all over the fundus, and more usually punctiform than flameshaped; but it would be rash, even with those differences in mind, to give a decided opinion without examination of the urine: This should always be made, and will afford the right clue, not only to ætiology, but also to the prognosis and treatment of the condition.

In retinitis accompanied by albumen in the urine, the life of the patient is, as a rule, more gravely threatened than is his sight; whereas, when the urine contains sugar, the immediate danger is loss of sight from intraocular hæmorrhage. From the clinical standpoint, therefore, the prognosis in albuminuric retinitis is quite different from that in diabetic retinitis-danger to life in albuminuric retinitis, and danger to sight in diabetic retinitis.

The regimen of the patient with glycosuria will, of course, differ from that suitable for one with albuminuria; but here also the essence of the treatment consists in proper dieting, and in the use of means to promote the free elimination of toxins by kidneys, skin, and bowels. At the same time, careful watch must be maintained over the patient himself, 
lest a too strict dietary, or too vigorous means of elimination, induce blood poverty or muscular weakness, with consequent cerebral failure or syncope.

Arterio-sclerosis may show itself very early in the retinal bloodvessels, and give rise to hæmorrhage long before there is any manifest renal inadequacy. It is prudent, however, to regard those cases as pre-albuminuric; because, by regulating the patient's diet, and wisely supervising his whole manner of living, much may be done to prolong life as well as to preserve vision.

If the examination of the urine gives no clue to the ætiology of the retinitis, an examination ought always to be made of the blood. By that means anæmia or leucocythæmia may be discovered, and afford an adequate explanation of the ocular condition.

Hæmorrhages detected in the fundus oculi may, of course, be local in origin; but their presence ought always to suggest the propriety of carefully examining the heart, kidneys, and blood pressure; of treating any pronounced diathesis-such as the gouty or rheumatic-and of rectifying constipation or any menstrual irregularities.

Inflammation of the Ghoroid.-The choroid also is liable to pathological changes, which are seen by the ophthalmoscope as white spots and pigment deposits. Those changes derive their chief importance from the fact that they are very frequently the result of syphilis; while in a case of 'suspected tuberculosis, the detection of small white isolated spots in the choroid affords strong confirmatory evidence of the accuracy of the diagnosis.

At its outset, non-suppurative choroiditis is one of the most insidious of diseases-it causes no pain, and gives rise to no external signs of inflammation. The fact, however, that the bacillary layer of the retina derives its nourishment from the choroidal blood-vessels, explains the serious impairment in sight which invariably occurs after inflammation of the choroid has become established. There is always diminution of the acuity of vision; and when the macula is affected, a central scotoma makes reading and all fine work impossible. The outlines of objects may appear distorted. This distortion may become so extreme that as the patient walks along the street some of the lamp-posts seem irregularly crooked, while others farther off look as if they had. been broken across-while the faces of passers-by are so misshapen as to be unrecognisable. Dazzling may be very pronounced, and be accompanied by flashes of light of various colours, so vivid that they cause much distress. At other times, black specks in large numbers float in front of the eyes; and stationary spots of larger size, or a veil hanging in front of the line of vision, are the chief cause of the defective sight. The patient sees 
much worse when he enters a room that is badly lighted, although in those conditions the sense of dazzling is less; but that is only a respite for the time being, for it returns-apparently worse than ever-when the eyes are exposed to bright light. Those subjective phenomena are due to implication of the retina, and to clouding of the vitreous with inflammatory exudate.

An attack of choroiditis takes months to run its course, and is always followed by patches of atrophy, which are easily seen with the ophthalmoscope, and can be demonstrated as blind spots irregularly placed in the field of vision.

Treatment.-Syphilis is the chief cause of inflammation of the choroid : consequently mercury is the sheet-anchor in its treatment, which requires to be faithfully carried out for months and years. The arsenical preparations are of great value as adjuvants to mercury, but little reliance can be placed on them alone. A blister applied to the nape, and kept open by d'Albespeyre's paper No. 2, is a great help in the treatment of all forms of deep-seated inflammatory diseases of the eye-more especially when these are of syphilitic origin-and when proper care is taken with the dressings, they cause the patient little discomfort. The eyes themselves must be protected from light by dark goggles.

From what has been said, it will be seen that the routine use of the ophthalmoscope will indeed often afford unexpected aid in determining the true nature and probable consequence of a disease. It is easy to say that in the majority of instances a correct diagnosis can be arrived at without it; but its use has often saved a doctor from serious error-and incidentally from much discredit-by putting him in possession of important facts unobtainable by any other means, at a time when the nature of the patient's disease was a matter of considerable uncertainty. 


\section{CHAPTER XLVI}

\section{AMBLYOPIA AND AMAUROSIS}

Definitions-Amblyopia: congenital, hysterical, traumatic, and toxic-Colour. scotomata occurring in toxic amblyopia-Treatment of toxic amblyopia.

IN former days any deterioration or loss of sight that could not be explained after careful external examination of the eye was diagnosed to be either amblyopia or amaurosis. The introduction of the ophthalmoscope, however, made more accurate diagniosis possible, and cases which had previously been all grouped together, were divided into diseases of the choroid, retina, or optic nerve. Moreover, the great advance in the treatment of errors of refraction has enabled many patients to enjoy perfect sight, who, before Donders' great work, were condemned to partial blindness. The elimination of all diseases of the fundus oculi discoverable with the ophthalmoscope, and of all errors of refraction which can be corrected by lenses, has greatly reduced the number of patients who in pre-ophthalmoscopic days would have been said to suffer from amblyopia or amaurosis: Sometimes sight is much impaired as a result of a cortical lesion of the brain; and in such cases no abnormality can be discovered in the fundus oculi, and no material improvement in sight is obtainable from the use of glasses; but concomitant symptoms-hemianopia, etc.-are usually present, the recognition of which enables an accurate diagnosis to be made.

Amblyopia and amaurosis are found under different circumstances, and the following is a convenient classification-

I. Congenital amblyopia.

2. Hysterical amblyopia.

3. Traumatic amblyopia.

4. Toxic amblyopia.

1. Congenital Amblyopia.-A mother brings her infant to a doctor, saying it does not notice, and examination shows that it stares straight in front of it and never attempts to follow a light or any glittering object held before its eyes. In these circumstances there may have been hæmorrhage into the retina at the time of birth, or the child may suffer from congenital syphilis, evidence of which can be seen with the ophthalmoscope in the fundus oculi-choroiditis (intrauterine), pathological 
changes in the macula or in the optic nerve, etc. In a large proportion of such cases, however, no abnormal changes are detected in the fundus oculi, and the reaction of the pupils shows no departure from health; but occasionally congenital defects can be seen in other parts of the eye - coloboma of the iris, microphthalmos, nystagmus, etc. Many of the cases seem simply instances of delayed development, and the child gradually begins to see as it grows older.

It is much more common to find the amblyopia in one eye only. In these circumstances the abnormality is seldom discovered until the child is about three years old, when attention is drawn to the eyes by the development of a squint. Examination usually establishes the fact that amblyopia is present in the eye in which the abnormal deviation is fixed. Such cases are usually described as amblyopia ex anopsia, and thought to be due to want of use of the deviating eye, which has been thrown out of action by the squint. It is, however, very difficult to know whether the amblyopia is the cause or the result of the strabismus, because many patients who have one defective eye do not squint, while others who squint see equally well with either eye. An error of refraction exists almost invariably, and sight can usually be improved by means of suitable lenses, aided by regular exercise of the defective eye while the other is covered by a bandage. The danger of the onset of

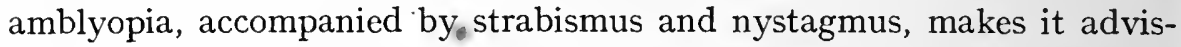
able to operate as early as possible on infants who suffer from congenital cataract, and not to postpone operation until the child is several years old.

2. Hysterical Amblyopia.-Cases in which there is diminution of the acuity of vision and contraction of the visual field-unilateral or bilateral-and which are not explained by the existence of either a peripheral or a central lesion, are described as hysterical amblyopia. The contraction of the field of vision is often very extreme, although it appears to cause the patient very little inconvenience. As a matter of fact, real contraction of the field of vision such as occurs in atrophy of the optic nerve, chronic glaucoma, or retinitis pigmentosa, causes great disability, as any one can prove for himself by trying to walk about looking through a tube; but to the patient suffering from hysterical amblyopia the greatly restricted field of vision appears to be a matter of indifference, for he goes about using his eyes apparently without suffering any discomfort. Even when both eyes are affected, and the amaurosis is so pronounced that the patient says he cannot see light, he is able to avoid obstacles placed unexpectedly in his way, and when he thinks he is unobserved can pilot his way about a room without knocking up against the furniture. In short, although he says he is blind, he does not behave like a blind person. In the majority of cases 
the blindness is unilateral, and usually dates from an injury, which as a rule is very trivial. At other times the amblyopia follows some unusual excitement, grief, or moral shock. When the eyes are examined, the pupillary reflexes and the fundus oculi are always found to be normal; the corneal and conjunctival reflexes are usually preserved, although in certain circumstances they may be lost; peculiar colour anomalies sometimes exist, accompanied by inversion of the colour field-the field for red-being more extensive than that for blue or for white.

Hysterical amblyopia chiefly attacks young people, and the majority of the patients are females, although males are by no means exempt. When children suffer, one of the most striking features is their absolute unconcern about the loss of sight, which is said to be so complete that it is impossible to tell the difference between light and darkness. Other ocular stigmata of hysteria are frequently present-monocular polyopia, want of proper appreciation of the sizes of objects, blepharospasm, partial ptosis, etc. Hemianopsia is sometimes present; but its occurrence ought always to arouse suspicions of the existence of organic disease, and ought never to be looked upon as a manifestation of hysteria, without very careful examination of the patient's general condition.

In hysteria blindness is seldom complete; because the patient manages to preserve, as far as possible, the essential functions, and loses only a part of the vision. The symptomatology of hysterical amblyopia therefore forms a very striking example of the dissociation of function that is so characteristic of all forms of hysterical disease. Moreover, both the acuity of vision and the extent of the visual field vary considerably, according to the patient's general feeling of well-being or otherwise at the time of the examination.

Treatment.- The prognosis is usually favourable, but the disease may last for years. The treatment must be suitable for the general condition, supplemented by any means considered to be specially helpful for the eyes themselves-subcutaneous injections of strychnine in the forehead or temple, massage, the application of electricity, etc. Success or failure will depend entirely on the confidence the patient has in the doctor.

3. Traumatic Amblyopia.-The cases included in this group are very closely allied to hysteria, but the frequency with which amblyopia and amaurosis have occurred as a result of the bursting of high explosive shells in warfare, makes it desirable to consider them in a class by themselves. The following is a brief description of a typical case. A man is knocked down, thrown into the air, or partially buried, by the explosion of a shell. He may be rendered unconscious for a time, and usually arrives at a dressing-station in a dazed condition. He suffers from such intense blepharospasm and lachrymation that the lids are opened with D D 
great difficulty. The man is found to be quite blind; and sometimes the blindness is accompanied by loss of hearing, speech, and smell. The signs of irritation gradually pass off, and the eyes open so that a more careful examination is possible, with the result that no definite organic lesion is to be found either in the eyes themselves or in the visual paths. The pupils may be large and sluggish, but all their normal reactions are present; ophthalmoscopic examination discovers nothing amiss in the fundus oculi; the field of vision is usually contracted. In the majority of cases the patient quickly recovers his speech, smell, and hearing, and whenever he begins to open his eyes freely he regains perception of light. He can grope about the ward by himself-feeling his way with his hands -and, as is characteristic also of hysterical amblyopia, he rarely comes into collision with his fellow patients, or stumbles against beds, chairs, or other obstacles that are in his way.

Treatment.-Recovery of sight is slow, but in the long run complete. The patients, however, always require a great deal of encouragement, and their treatment must be conducted with much patience and perseverance. Many of them are mentally upset, shaken, and battered, liable to burst into tears on the slightest provocation, and greatly perturbed at the prospect of having to return to duty. Although no organic lesion can be discovered to account for the symptoms, and although the behaviour of the patient may sometimes excite suspicion of shamming, those men are in no sense malingerers. Many of them are of very high character, and keenly desirous to do their duty; but by constitution and temperament, they are quite unfit for the ordeal of battle, and have broken down completely under the strain. It is obvious therefore that great care must be taken to treat those patients wisely lest injustice be done to them. They are best dealt with in a hospital for neurasthenics, but the treatment of each individual patient must be wisely planned, and carefully and perseveringly conducted.

4. Toxic Amblyopia.-Of all forms of amblyopia the toxic is of most interest to the general practitioner, and must therefore be considered at greater length than has been thought necessary in dealing with the three groups which have just been described.

Every one is familiar with the indistinctness of sight which so frequently accompanies any functional derangement of the stomach and liver, and this failure of visual power is undoubtedly the result of defective elimination of waste products.

It has long been known that the excessive use of tobacco and alcohol may give rise to defective sight; and, when toxic amblyopia is mentioned, these agents are always thought of as the cause. In many obscure forms of disordered or suspended visual function, however, whose symptom- 
atology is in almost every respect identical with what is recognised as typical tobacco amblyopia, the patient may never have used either tobacco or alcohol. The ætiology of the disease, therefore, in these circumstances, must be ascribed to some cause other than-smoking or drinking to excess, and such a cause is to be found in the defective elimination of waste products due to perverted metabolism. Provided that the digestive function be perfect, and all the eliminating organs doing their work properly, many persons seem to be able to use both tobacco and alcohol very freely without suffering any physical discomfort; but. on the other hand, where digestion is poor and elimination imperfect, more especially where sugar is present in the urine, a very small quantity of. either may do a great deal of harm. They seem to accentuate the already perverted metabolism, and to cause great increase in the toxicity of the blood.

The clinical picture of all forms of chronic toxic amblyopia is vert much alike, and the diagnosis is made not from any pathognomonic ophthalmoscopic appearances, but rather from careful examination of symptoms. Indeed, the contrast between the gravity of the symptoms, and the absence of any marked lesion that ophthalmoscopic examination can detect, is, one of the most striking characteristics. The patients may be strong and healthy in appearance-and often say they have never been ill-but one finds on inquiry that they frequently suffer from digestive disturbances. They often, moreover, tell you that in consequence of domestic cares, or of business difficulties, they have been subjected for many months to much mental anxiety, and themselves in many instances attribute the onset of the defective vision to indigestion and worry. The onset of symptoms, though sometimes sudden, is usually so slow and gradual that patients have difficulty in determining the exact date of commencement. They complain that in bright light they are easily dazzled, and that a whitish or yellowish mist rises like a cloud in front of their eyes and prevents them from seeing clearly. Towards sunset, however, this sense of dazzling disappears, and in consequence they tell you that they can always see best in the evening. By and by small print can be read only with difficulty, and stronger spectacles are procured, but with no good result; while at last, the inability to read even ordinary sized type becomes quite decided.

They then complain also that they do not readily distinguish their friends as they walk along the street, and may volunteer the information that when they look at a person's face they have difficulty in seeing the eyes, and that all healthy colour seems absent from the cheeks. In. pronounced cases they may tell you that the flame in a street lamp seems suddenly to go out, but again becomes visible as it is approached, while 
another then disappears. The dimness of vision is, as a rule, as pronounced in the one eye as in the other; and this symmetry, being a strikingly characteristic symptom, is of great value in distinguishing this form of amblyopia from diseases of the choroid, retina, or optic nerve, in which both eyes are also frequently affected, but in which the diseased condition of the one is generally much in advance of that of the other. Indeed, when a person from forty to sixty years of age is found on examination to see in the distance only the largest letters of Snellen's test type, and, near at hand, only No. I6 or No. I9 Jaeger, and the one eye is as defective as the other, toxic amblyopia ought always to be suspected. The diminution in the visual acuity varies according to the stage of the disease; but in every case careful examination demonstrates the failure of sight to be due to defect in the centre of the field. The patient, however, is not conscious of this, as he would be if the disease were due to a lesion in the transparent media of the eye. The scotoma is therefore " negative" or "relative." It is transversely oval, and usually extends from the point of fixation to the blind spot. It is remarkably constant in size and shape, but may occasionally be of large dimensions and surround the fixation point. These variations in size and shape seem to depend more upon the amount and effect of the toxic agent than upon the particular kind of poison. The more profound the amblyopia, the larger the scotoma.

A central scotoma is not, however, pathognomonic of toxic amblyopia, for a similar blank in the centre of the field of vision occurs in dissemi-

- nated sclerosis, in hereditary optic atrophy, and in some cases of inflammation of the orbit where there is pressure on the optic nerve in the neighbourhood of the optic foramen. In every case a differential diagnosis is cone to through the collateral symptoms, and in toxic amblyopia a pathognomonic sign is that even when a white object can still be recognised, a scotoma for colour exists in the centre of the field. Green appears grey or white, pink blue, and red brown. One patient, a gardener, said that the first indication he had of failing eyesight was inability to distinguish the red colour of a strawberry. Before the fruit was ripe he could recognise the berries. but whenever these became red he failed to see them unless he were looking closely at the plants. Another patient complained that he had to give up playing billiards because in certain positions he could not see the red ball. As the disease progresses, these colours assume a metallic lustre, green looks like silver, while red glitters like gold. The patient may have considerable hesitation in distinguishing gold and silver coins, and is not able to tell half a sovereign from a sixpence until he holds them very closely up to his eye, or until he takes them in his hand and feels the difference in weight. As, however, the 
eccentric parts of the visual field are normal, the patient may be altogether unaware of his colour-blindness, and herein lies a grave danger to the community. He can see a large surface of red or green with perfect distinctness, but not the small circle of colour used as a test object; and consequently, unless he be properly examined, he may occupy a post on a railway engine, or be placed on the lookout at sea, although quite unfit to distinguish the red or the green signal, or to tell the port from the starboard light. In some cases blue is a strikingly predominant colour. One patient told me that one of the first things he noticed amiss was that the flames in the street lamps were blue; while another realised his visual disability when sitting in the dress-circle of a theatre and feeling puzzled to know why every chorus girl was dressed in some shade of blue. It is difficult to explain this, but the symptom is very often associated with the presence of oxalate of lime crystals in the urine. At length comes the difficulty of distinguishing a white object in the centre of the field, and in the most extreme cases even a candle flame cannot be seen. The scotoma is now absolute.

In striking contrast to the changes in the visual field, and the marked disturbance of the colour sense, are the results of ophthalmoscopic examination. The appearances are by no means characteristic, but in the early stages the retinal veins are usually turgid and somewhat tortuous, and the optic disc may be hyperæmic and less transparent than normal. As the disease progresses, however, the natural colour of the nasal third of the papilla contrasts strongly with the greyish whiteness of the temporal two-thirds.

Retrobulbar Neuritis.-Some group all cases of toxic amblyopia under the heading of retrobulbar neuritis; but, as Berry has insisted, there are good reasons for doubting in many instances the existence of actual inflammation. In a typically toxic case, the symptoms often pass off with great rapidity during a holiday on the moors or at sea, provided the regimen is simple and the night's rest is undisturbed; while on the other hand, a colour scotoma can be increased by fatigue. In both functional and organic cases, the position of the scotoma corresponds to the area of the retina which is supplied by the papillo-macular fibres, and there is both experimental and pathological evidence in support of the view that the retina is the structure primarily affected. Both functional and organic forms have a conmmon ætiology in defective elimination, the difference between them being one more of degree than kind. What at first seems to be simply a functional disorder, may, if misunderstood, go on to become a definite inflammation. When that occurs, symptoms develop which are not present in the purely functional cases. A well-marked case of retrobulbar neuritis usually occurs suddenly after exposure to cold, and 
is characterised by pain on movement of the eyes, and tenderness when the eyeballs are pressed backwards into their sockets; the central scotoma is not constant in size and shape ; and the ophthalmoscopic picture is usually characterised by distinct redness and even by more decided inflammatory change in the optic disc. The pupil reacts both to light and in the act of convergence; but in acute cases, as was pointed out by Marcus Gunn, a very characteristic sign (seen quite readily on careful examination) is that, although it contracts at once when exposed to a bright light, if the exposure be prolonged the myosis is not maintained, but slowly gives place to full dilatation. The amblyopia is often more pronounced in one eye than in the other, or may be wholly confined to one eye; and there are often intolerance of light and complaint of aching pain in the circumorbital region. In a typical case of the toxic form, the fairly constant shape of the scotoma, its symmetry, and the similarity of the degree of amblyopia in both eyes, point rather to the existence of a vascular lesion than to structural inflammation. From their anatomical position, the papillo-macular fibres at the optic foramen, where they enter the nerve stem, are those which are most abundantly supplied with b'ood, on account of the very fine capillary meshwork which surrounds them, and are consequently those that will be most liable to nutritional disorder when any toxic agent exercises an irritating action in their neighbourhood. It is probable, therefore, that in the simpler forms there is only a functional derangement of the macular fibres, while in severe cases anatomical changes in the nerve become superadded. An interstitial neuritis, limited to the fibres of the papillo-macular bundle in their course through the nerve stem from the optic foramen downwards towards the optic disc, was demonstrated by Samelsohn in I882, and his observations have been confirmed by Nettleship, "Uhthoff, and others.

Prognosis.-The prognosis is undoubtedly favourable in all cases both of toxic amblyopia and of retrobulbar neuritis, which are definitely due to the imperfect elimination of waste products from the blood consequent upon perverted metabolism. The true guide is to be found in the examination of the urine, rather than in any signs or symptoms presented by the eyes themselves. Even in the most favourable circumstances progress is slow; and, except in very mild cases, six or nine months-or even longer-are required before the patient is able to use his eyes for near, work. It is the slow progress which disappoints and perturbs both the patients and their relatives, and if the physician be not confident and steadfast, grave trouble may arise. In severe cases the general pallor of the discs, the large size of the central scotoma, and the consequent impairment of vision and its prolonged duration, 
regarded as eye symptoms per se, and considered away from their proper relation to the patient's whole general condition, render a diagnosis of atrophy of both optic nerves a very natural one to arrive at, if the case be considered only from the standpoint of an ophthalmic specialist. As Paget said, however, long ago, "no disease can be properly understood unless it be considered in its whole pathology." Whenever, therefore, a diagnosis of optic atrophy is made without due consideration being given to the cause of the atrophy, errors in treatment are very apt to arise. Strychnine, phosphorus, and other nervine tonics are of great value in the treatment of certain states; but they are likely to do much more harm than good, unless, prior to their use, the patient's special disease proclivities have been properly recognised and thoroughly treated. If the gout or rheumatism which determined the amblyopia in the first instance be allowed to progress, structural damage will undoubtedly occur in consequence of prolonged irritation. This process of inflammation leads to proliferation of the connective tissue surrounding the optic nerve fibres, which, suffering compression, will undoubtedly in the end become atrophied, whenever the new-formed tissue begins to undergo cicatricial contraction. The ophthalmoscopic appearance of the optic disc is not of itself, however, a sure guide in prognosis, for the mere colour of the disc conveys very little information regarding its structural condition. When, therefore, there is an absolute scotoma associated with well-marked pallor of the optic disc the visual field ought to be carefully taken with a perimeter. If any peripheral contraction be discovered, a guarded opinion ought always to be given, and the patient further examined very thoroughly for any signs which might indicate incipient disease of the spinal cord. Even if such exhaustive investigation prove negative, caution must be exercised until sufficient time has elapsed to permit the efficacy of the treatment employed to be tested, because peripheral contraction of the visual field always indicates structural disease of the nerve, and atrophic changes in the optic disc, once set a-going, are difficult to arrest and very prone to become progressive. It is very important not to confuse a case of toxic amblyopia with one of tabetic atrophy. A patient suffering from tabetic atrophy sees worse in the evening, rarely suffers from central scotoma, but always shows pallor of the optic disc, which indicates progressive atrophy. $\mathrm{He}$ inevitably becomes blind if the optic nerves are affected; whereas one suffering from toxic amblyopia will in all likelihood make a complete recovery. Repeated examination of the urine affords the surest guide both in the prognosis and treatment of these doubtful cases. In this connection it should be mentioned that in patients suffering from glycosuria, there may be a rapid onset of central scotoma, coincident with the 
disappearance of the sugar from the urine, under the influence of treatment by eucalyptus or by nitrate of uranium. When that occurs, the loss of the power to read is likely to be permanent, although no pathological changes in the fundus can be detected on ophthalmoscopic examination. In these cases also the patients usually die within a comparatively short time after the onset of the visual troubles.

Treatment.-In the treatment itself the first step should always be to find out the patient's habits and mode of life. The possibility of any infection, such as syphilis, must be excluded from the outset ; and the chance of poisoning by quinine, methyl alcohol, etc., ought not to be forgotten. The use of tobacco and alcohol must be entirely prohibited, because it must ever be borne in mind that the result is determined not so much by the amount smoked, or the quantity of spirits consumed, as by the capability of the patient to resist their injurious influence; where sugar is present in the urine, for example, a very slight exciting cause is sufficient to bring on an attack of amblyopia. A proper regimen must at once be prescribed, and the patient advised how to live the healthiest life possible in whatever circumstances he may be placed. After all, disease, when rightly understood, is only a perversion of natural processes, and prophylaxis is the highest form of therapeutics. The food supplied must be of proper quality, and in sufficient quantity to satisfy the needs of the individual patient. If, however, the nutritive value of the blood is to be maintained at normal, it is imperative that all waste products be removed at the earliest possible moment; and to this end nature has made provision in the bowel, the liver, the kidneys, the skin, and the lungs. The constant and free activity of these channels of excretion is essential to health; and if, by reason of illness, the function of one is temporarily interrupted, the others are always ready to undertake vicarious duty. A very short clinical experience will, however, prove that the full functional activity of any one, for example the skin, is, in certain circumstances, of more value than the combined activities of all the others. A mercurial purge, followed by alkaline saline draughts, is of great value in the early stages of disease of the uveal tract; and free diaphoresis and bracing air are equally valuable in toxic amblyopia and retrobulbar neuritis. In the treatment of these diseases the influence of soil and of climate is of paramount importance. A high altitude, a gravelly soil, and dry bracing air are sure to be helpful ; whereas, a lowlying locality, with a clay soil and a moist relaxing atmosphere, are equally certain to hinder progress and encourage relapse.

Many of these patients do well at a Spa ; but they should, when they go, be provided with an introduction to one of the resident physicians, in order that their case may be viewed in its right perspegtive, and treat- 
ment prescribed accordingly. The dose of the waters, and the general regimen, must be adjusted to meet the requirements of each individual. One of the advantages of a watering-place, which ought never to be under-valued, is that it generally provides a patient with a restful holiday and freedom from worry, and in consequence does as much as medicine to promote his comfort and improve his general well-being.

Copious draughts of hot water taken at regular intervals throughout the day flush out the kidneys, and sometimes determine what may be called a urinary crisis, so that the blood is relieved of much of its toxicity; and it is noteworthy, as Dr. Wright Thomson, following Bouchard, has demonstrated, that the urine of patients suffering from tobacco amblyopia is, when injected into the blood of a rabbit, much more poisonous after this increased diuresis, than it is before it. Alkalis and alkaline salines are always beneficial, and their efficacy is greatly increased by free dilution. Diaphoretics also play a distinct part in the treatment, and a Turkish bath regularly once or twice a week rarely fails to do good. The bowels must be carefully attended to, as constipation is always a fresh exciting cause of the trouble. If necessary, a mild laxative should be given every night at bedtime; and an occasional blue pill, followed next morning by a hot draught of alkaline purgative water, is most helpful in promoting assimilation and in clearing waste products out of the system. Systematic exercise in the open air is essential, and its results are invaluable, the great aim being to make sure that there is just sufficient expenditure of physical energy to properly balance the amount of food eaten. Exhaustion will thus be avoided on the one hand, and defective excretion on the other. The eyes ought not to be used for near work, and the dazzling in bright light is much relieved by the wearing of dark glasses.

In acute cases, leeches or blisters on the temple or the mastoid are indicated; but local therapeutics, unless in exceptional circumstances, play but a small part in the treatment either of toxic amblyopia or of retrobulbar neuritis. Reliance must be placed entirely on general treatment, wisely planned and patiently carried out.

When the state of the urine shows, on examination, that assimilation is beginning to improve, the iodides may be given, either alone or combined in a mixture with nux vomica; and strychnine, phosphorus, and arsenic may then also be administered with advantage, and their use continued intermittently for many weeks. If the patient be anæmic, some preparation of iron may be given cautiously after meals. Electricity in the form of galvanism or high-frequency currents, hydrotherapy, and Swedish gymnastics, may all be employed to aid in completing a cure. 
The great benefit obtained from a sea voyage is often very striking. Sea air has undoubtedly a powerful influence in quickening metabolism; but, before the patient is allowed to embark on any prolonged cruise, careful inquiry should always be made as to whether he is likely to be affected by sea-sickness-protracted mal-de-mer is sure to be harmful. 


\section{THERAPEUTIC NOTES AND FORMULÆ}

\section{LOCAL APPLICATIONS.}

\section{A. COLLYRIA-EYE LOTIONS.}

USED for the most part in cases of inflammation of the conjunctiva. Appended to Formula I is a general direction for the use of all collyria. Instead of ordinary distilled water, any of the aromatic waters, e.g. rose water, fennel water, elder-flower water, camphor water, etc., may be used as a menstruum; and when there is pain or itching, cherry-laurel water is specially recommended.

The eyes should be carefully dried, wiping the lids from below upwards, after being bathed with the lotion, and, for a short time, the patient ought not to expose himself to the open air. As the conjunctiva soon becomes accustomed to the use of a remedy, it is necessary, in order to obtain the best therapeutic results, to vary the lotions from time to time.

Pharmaceutically, collyria should be prepared with great care, and, with the one exception noted, filtered through paper : kaolin should be used if necessary.

\section{ALKALINE LOTIONS.}

Alkaline lotions are used for softening and facilitating the removal of encrusted discharge in blepharitis marginalis, lachrymal catarrh, etc. Before any definite treatment can be begun, all crusts must be removed.

\section{Alkaline Lotion (Simple).}

R Liquoris Potassæ Tincturæ Catechu $\quad . \quad \cdot \quad \cdot \quad \cdot \quad \cdot$ Aquæ Laurocerasi $\quad \cdot \quad \cdot \quad \cdot \quad \cdot \quad z^{1 j} \quad 60 \cdot 0$ Aquæ Rosæ . . . . . . ad ऊviij ad $250 \cdot 0$ Misce.

Sig.-Equal parts of the lotion and boiling water to be mixed in a tea-cup previously warmed. With a piece of absorbent cotton, or soft sponge, bathe the eyelids with the mixture for a few minutes; and then incline the head back to allow the solution to flow in upon the eye from a large eye dropper or an undine. Do this thrice a day, or more frequently if necessary.

\section{Sodium Bicarbonate Lotion.}

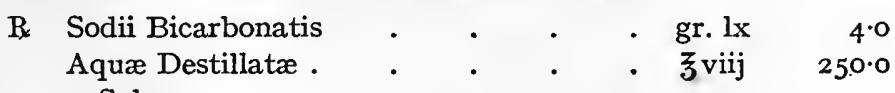

Solve.

Note.-Useful where the eye has been injured by an acid, or for dissolving crusted secretions from the edge of the lids. 


\section{THERAPEUTIC NOTES AND FORMULA}

\section{Sodium Bicarbonate and Coal-Tar Lotion.}

$\mathrm{R}$ Sodii Bicarbonatis gr. $\mathrm{lx}$
Liquoris Carbonis Detergentis (Wright)
Aquæ Destillatæ .
Misce et cola per gossypium.

Note.-Useful in gouty and eczematous inflammations of the eyelids.

\section{Sodium Salicylate and Borax Lotion.}

\section{$\mathrm{B}$} Sodii Sal
Pulveris
Glycerin
Aquæ F
Solve.

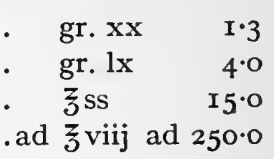

Note.-Lotions 3 and 4 are useful in "watery eye," and Formula 4 is recommended in follicular conjunctivitis. If the lotions have to be injected into the tear passages, care must be taken not to employ too much force, as the lachrymal sac may rupture and the fluid extravasate into the surrounding tissues.

\section{Sodium Sulphate and Hyoscyamus Lotion.}

R Sodii Sulphatis . . . . . gr. $\mathrm{xvj}$

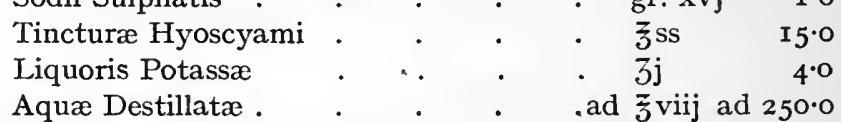

Misce.

Note.-Useful as a mild stimulant in conjunctivitis with opacity of the cornea.

\section{ANTISEPTIC LOTIONS.}

Long before anything was known of bacteriology, the favourite drugs employed in the treatment of inflammation of the conjunctiva were nitrate of silver, sulphate of zinc, and perchloride of mercury. All these have powerful antiseptic action, and that property explains the favour in which they were held by the older ophthalmic surgeons, who, however, employed them wholly as a result of extensive clinical observation.

It was known that nitrate of silver was of special value in purulent ophthalmia, that sulphate of zinc was almost a specific in certain forms of catarrhal conjunctivitis, and that perchloride of mcrcury was generally useful in inflammation both of the lids and of the conjunctiva. A study of bacteriology affords a ready explanation, for it has been proved that salts of silver are fatal to gonococci, sulphate of zinc to the bacillus of Weeks; and perchloride of mercury inimical to all forms of microbic life. This is a good example of how a method of treatment arrived at in the first instance by clinical experiment has been amply justified and confirmed by increase of knowledge.

In the case of the eye, however, there is a difficulty in carrying out full antiseptic methods; owing to the extreme delicacy of the structures it is not possible to employ antiseptic solutions strong enough to kill the micro-organisms without at the same time injuring the conjunctiva. In ocular surgery, therefore, the field of operation is prepared by freely flushing the conjunctival sac with bland solutions. 
By that means the germs are mechanically washed away, and their numbers consequently so reduced that they are unable to exercise any injurious influence over the healing of a wound.

Antiseptic lotions are used for cleansing the conjunctiva, eyelids, and surrounding skin, before operations on the eye; and in all inflammations accompanied by purulent discharge. The patient's friends ought to be informed that eye diseases accompanied by discharge are contagious, and that cleanliness is the most important factor in the treatment. It is necessary, therefore, that the patient should have his own towels and washing utensils.' In adults suffering from purulent ophthalmia the healthy eye should be protected by a Buller's shield. Every patient suffering from severe inflammation of the eye ought to be kept in bed, and, while there is profuse purulent discharge, a nurse ought to be in constant attendance. The whole success of the treatment depends upon the fidelity with which the eyes are cleansed.

\section{Acriflavine Lotion.}

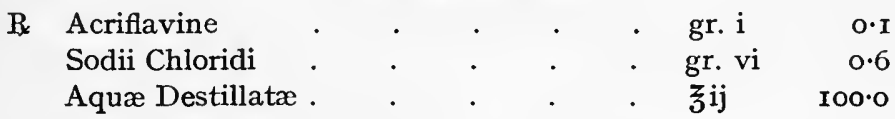

Note.-Acriflavine is the ethyl chloride of diamino-acridin (Browning). It is a powerful antiseptic, whose bactericidal action is enhanced rather than hindered by admixture with serum. A solution I in rooo prevents sepsis, and is recommended in advancement operations and whenever sutures are required. If the solution of acriflavine is to be used for several days $I$ in 4000 is to be preferred, as it does not in that strength interfere with healing. It is very useful in the disinfection of septic wounds, but is less valuable in the treatment of conjunctivitis or ulceration of the cornea.

\section{Boric Acid Lotion.}

$\begin{array}{llllllr}\text { Re Acidi Borici } & . & . & . & . & \text { gr. } l x & 4 \cdot 0 \\ \text { Aquæ Destillatæ . } & . & . & . & . & \Xi \mathrm{viij} & 25^{\circ} \cdot 0\end{array}$

Solve.

Note.-The safest and most generally useful of all eye lotions. Barff's boroglyceride in 5 per cent. solution forms a convenient vehicle for many collyria.

\section{Calomel Lotion.}

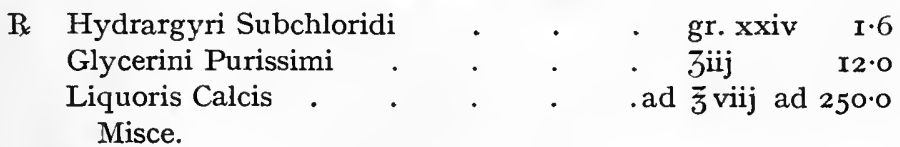

Note.-Commonly known as "Black Wash"; useful application for syphilitic sores, and foul ulcers of the eyelids.

\section{Carbolic Acid Lotion.}

$\mathrm{R}_{*}$ Phenol Purissimi . . (5 per cent. solution in water).

Note.-It is important to use pure phenol for all lotions to be applied to the conjunctiva. Ordinary preparations of commercial carbolic acid cause considerable irritation. 


\section{Chinosol Lotion.}

\section{R Liquoris Chinosol . . . . . ( $\mathrm{r}$ in 4000)}

Note.-Chinosol, a bright lemon-yellow powder soluble in water, is a powerful antiseptic, and is strongly recommended for use in cases of serpiginous ulceration of the cornea.

\section{Chlorine Lotion (Labarraque).}

$\mathrm{R} \quad$ Liquoris Sodæ Chlorinatæ . . . $\quad \bar{z}_{\mathrm{j}} \quad 30 \cdot 0$ Aquæ Destillatæ . . . . . . ad $\overline{3} \mathrm{vijj}$ ad $250 \cdot 0$

Note.-Berry recommends the injection of chlorine water into the vitreous in purulent inflammation of the globe. It ought always to be prepared immediately before it is used. Eusol is a trade name for a freshly prepared solution of hypochlorous acid (Lorrain Smith). It is a powerful antiseptic, extremely potent against micro-organisms and their spores, but causes little harm to the tissues. It can be applied to the eye as a lotion or a fomentation, and if it causes pain it should be diluted with warm water until it can be used without discomfort. As Eusol rapidly deteriorates it ought to be prepared immediately before it is required. The two powders, boracic acid and chloride of lime, used in the preparation of the solution, must be kept dry, as they are useless if they become damp.

Chloramine-T (Dakin) is more stable, exerts more prolonged antiseptic action, and is less irritating than the hypochlorites. It is non-toxic, and for eye infections a $0^{*} \mathrm{I}$ per cent. solution is recommended. It is also prescribed as an oil solution.

\section{Formalin Lotion.}

R Liquoris Opii Sedativi (Battley) . . 3vj $\quad 22.5$ Liquoris Formalin ( $\mathrm{I}$ in 2000) . . ad $\overline{3}$ viij ad $250 \cdot 0$ Misce.

Note.-Causes considerable irritation even in weak solution, but is sometimes of great value in hypopyon ulcers, septic wounds of the cornea, and ophthalmia neonatorum.

\section{Mercury Lotions.}

(a) $\mathrm{B}$ Solutionis Hydrargyri Perchloridi . (I in 5000)

(b) $\mathrm{R}$ Solutionis Hydrargyri Biniodidi . ( $\mathrm{I}$ in 20,000)

Note.-The former is the solution most commonly employed, but the latter is strongly recommended by Panas. The following is a convenient approximate formula-

R Hydrargyri Biniodidi . . . . . gr. $\frac{1}{4}$ 0.I

Potassii Iodidi . . . . . . . gr. j 0.5

Spiritûs Rectificati . . . . ऊ ऊij $340 \cdot 0$

Aquæ Destillatæ . . . . . . ad $\zeta x i j$ ad $2000 \cdot 0$

Solve.

\section{Methylene Blue Lotion.}

B Methylene Blue. . . . .

Sodii Chloridi Puriss. . . . . . gr. vj 0.6

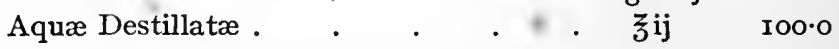

Note.-A very reliable application to clean a dirty socket and prevent it from discharging. The methylene blue solution must be thoroughly applied with a 
swab of sterilised cotton-wool to the conjunctiva lining the socket, and the application repeated two or three times a day.

\section{Phenosalyl Lotion.}

$\mathrm{R}_{\mathrm{x}}$ ' Phenosalyl . . . . I per cent. solution in water.

Note.-Consists of a solution of carbolic, salicylic, and lactic acids, with menthol and eucalyptol. It is a more powerful antiseptic than phenol, and in $x$ per cent. solution does not irritate the conjunctiva. Its application frequently prevents the recurrence of styes.

\section{Potassium Permanganate Lotion.}

R Potassii Permanganatis . . . gr. iv 0.25 Aquæ Destillatæ . . . . . J J viij $250 \cdot 0$

Solve. colour.

Note.-Should always be freshly prepared, and never used after it has changed

12. Quinine Lotion (Tweedy).

R Quininæ Hydrochloridi . . . gr. xxiv $\mathrm{I} \cdot 6$

Aquæ Destillatæ . . . . . . ad 弓viij ad $250 \cdot 0$

Solve.

Note.-Useful in 'diphtheritic and croupous conjunctivitis, and in serpiginous ulcer of the cornea.

\section{Resorcin Lotion.}

$\mathrm{R}$ Resorcin . . . . . . gr. $\operatorname{cxx} 8 \cdot 0$

Acid. Borici Puriss. . . . . gr. $\operatorname{lxxx} 6 \cdot 0$

Aquæ Destillatæ. . . . . ऊviij $250 \cdot 0$

Note.-Is a powerful antiseptic; a serviceable lotion in many forms of conjunctivitis.

14. Zinc Chloride Lotion.

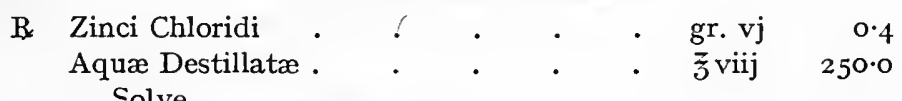

Solve.

Note.-Useful in purulent ophthalmia; but, as it is a powerful irritant, it must be employed with caution.

\section{ASTRINGENT LOTIONS.}

Astringent lotions are used in inflammations of the conjunctiva, but are never employed when the deeper structures of the eyeball are involved. Such a mistake, which might be attended with disastrous results, may be easily avoided if it be remembered that, in deep-seated inflammation, the conjunctival injection is, for the most part, circumcorneal, that the pupil does not react readily to the stimulus of light, and that the pain complained of is characteristically throbbing, markedly nocturnal, and usually circumorbital. 


\section{Alum and Boric Acid Lotion.}
$\mathrm{R}$ Pulveris Aluminis . . . . . gr. $\mathrm{xx} \quad \mathrm{I} \cdot 3$ Acidi Borici . . . . . . gr. lx 4.0 Glycerini Croci (Squire) . . . . 3 ij $8 \cdot 0$ Aquæ Rosæ . . . . . . . ad ろviij ad 250.0 Solve.

Note.-When used too freely, or in too concentrated solution, alum causes the conjunctival discharge to coagulate and adhere to the inner surface of the eyelids, thus giving rise to appearances suggestive of croupous conjunctivitis. In ulcer of the cornea, alum is contra-indicated, as it tends, by dissolving the cement substance, to cause the ulceration to increase.

\section{Chrome Alum Lotion.}
R Chrome Alum • • • • . $\quad$ gr. $x v j \quad I \cdot 0$
Aquæ Destillatæ . . . . . 3viij $250 \cdot 0$
Solve.
Note.-Useful in chronic conjunctivitis.

\section{Lapis Divinus Lotion.}

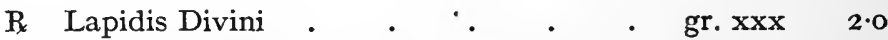
Vini Opii (sine Aromat.) $\quad . \quad$. $\quad 3$ iij $12 \cdot 0$
Aquæ Destillatæ . . . . . . ad §ँviij ad $250 \cdot 0$

Solve.

Note.-Useful in follicular and in granular conjunctivitis.

Alibour water is a valuable application in trachoma. It contains copper sulphate $2 \frac{1}{2}$ drachms, zinc sulphate 9 drachms, camphor I drachm, powdered saffron $\frac{1}{2}$ drachm, water 40 ounces.

\section{Lead Acetate Lotion.}

R Plumbi Acetatis . $\quad$ • $\quad$ • $\quad$ - gr. $\mathbf{x x} \quad \mathbf{I} \cdot 3$ Spiritûs Rectificati $\quad$. $\quad . \quad$. $3 \mathrm{ij} \quad 8 \cdot 0$ Aquæ Sambuci . . . . . . . ad 弓viij ad $250 \cdot 0$ Solve.

Note.-Can be used only if the cornea be sound, as the lead is readily deposited on any abraded surface, and leaves a permanent opacity-white lead.

\section{Lead Acetate and Opium Lotion.}

R Plumbi Acetatis . . . . . gr. $x x x v j ~ 2 \cdot 4$ Pulveris Opii Puri . . . . gr. xlviij $3 \cdot 2$ Aquæ Bullientis . . . . . . $\overline{3}$ viij $250 \cdot 0$

Misce.

Note.--Useful in contusions of the eyelids. 


\section{Mercuric Chloride Lotion (Simple).}

Bk Hydrargyri Perchloridi . . . gr.

Ammonii Chloridi . . . . . gr. vj 0.40

Púlveris Cocci Cacti . . . . . . gr. ij $0 \cdot 13$

Spiritûs Rectificati . . . . . $3 \mathrm{j} \quad 4^{\circ} \mathrm{oo}$

Aquæ Destillatæ . . . . . . ad 弓viij ad $250 \cdot 00$

Solve.

Note.-This is generally known as Mackenzie's Eye Lotion.

7. Mercuric Chloride and Belladonna Lotion (Compound).

Same as 6 , with $3 \mathrm{ij}$ of Tincture of Belladonna added to the formula, the Cochineal and Spirit being omitted.

Note.-Useful in all forms of conjunctivitis, but as the perchloride of mercury sometimes irritates, Formula 7 is to be preferred. If, however, the belladonna cause inconvenience by dilating the pupil, it may be reduced in quantity, or omitted altogether, and 3iij Battley's Sedative Solution of Opium substituted.

\section{Tannic Acid Lotion.}

R Glycerini Acidi Tannici

Pulveris Boracis . . . . . gr. $\operatorname{lxxx} 5 \cdot 0$

Glycerini Purissimi . . . . $3 \mathrm{ij} \quad 8 \cdot 0$

Aquæ Camphoræ . . . . . ad 弓 viij ad $250 \cdot 0$

Solve.

Note.-Useful in chronic conjunctivitis, more especially in the later stages of the follicular and granular forms.

\section{Witchhazel Lotion.}

R Liquoris Hamamelidis Destillatæ . . Jiv ${ }_{15}{ }^{\circ}$

Solut. Adrenalin Chloridi ( $\mathrm{I}$ in IOoo) . $3 \mathbf{j} \quad 4^{\circ} \mathrm{O}$

Aquæ Destillatæ . . . . . 3 viij $250^{\circ} 0$

Note.-Useful in conjunctivitis and simple epiphora.

10. Zine Sulphate Lotion (Simple).

$\mathrm{P}$

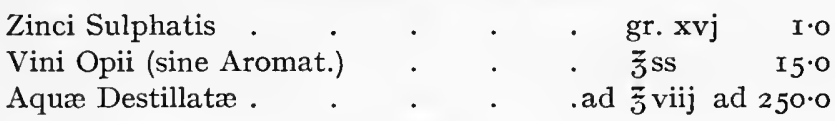
Solve.

\section{Zine Sulphate Lotion (Compound).}

R Zinci Sulphatis . . . . . . gr. xij

Ammonii Chloridi . . . . . gr. vj $0 \cdot 4$

Spiritûs Rectificati . . . . . 3iij I2.0

Glycerini Croci (Squire) . . . . 3 ij $8 \cdot 0$

Solutionis Boroglyceridi ( 5 per cent.) in

Aquâ Camphoræ . . . . ad ǰviij ad $250 \circ 0$

Solve.

Note.-Formulæ Io and II are favourite lotions in simple catarrhal conjunctivitis. No. II is a modification of Horst's eye water, and is of the greatest service in many cases of chronic inflammation of the conjunctiva. 


\section{EVAPORANT LOTIONS.}

Cold applications are of much service in relieving pain after operations upon the eye, or immediately after injuries involving its deeper structures. Ice-cold compresses should be applied only for ten or fifteen minutes every hour, as their continuous use tends to lower the vitality of the part.

\section{Arnicated Lotion.}

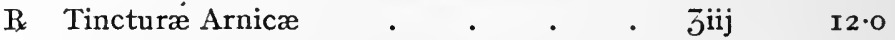

Liquoris Ammonii Acetatis . . . $\jmath^{\mathrm{j}} 30^{\circ} \mathrm{o}$

Aquæ Sambuci . . . . . . . ad $\check{3}$ viij ad $250 \cdot 0$

Misce.

Note.-Arnica is a favourite application in contusions of the eye, but it is doubtful whether it is of any real value in promoting absorption of effused blood.

Da Costa has suggested the following paint to prevent ecchymosis of the eyelid after injuries-

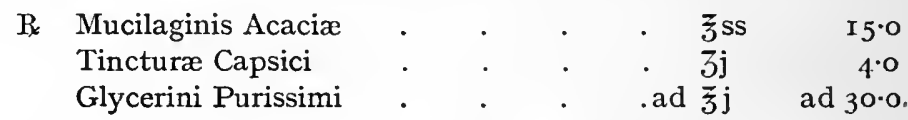

Note.-In applying either of these, care must be taken not to allow them to come in contact with any part over which the skin is abraded.

\section{Evaporant Lotion (Simple).}

Spiritûs Ætheris Nitrosi

Aquæ Rosæ

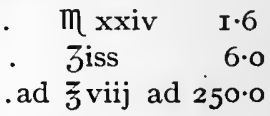

Misce.

Note.-Pour oüt half a wine-glassful of this fluid : with a small piece of clean soft sponge dipped into it, and gently wrung, bathe the eyelids, side of the nose, eyebrow, forehead, and temple for a few minutes, and then allow these parts to dry of themselves. Repeat this three or four times daily, or as often as the eyes eel painful, hot, or weak on exposure to light. The fluid does not require to go i nto the eye. May also be used as a spray. Dilute acetic acid I or 2 drops to $\zeta_{j}$ distilled water is recommended in the treatment of spring catarrh. A teaspoonful of vinegar in a glass of warm water is a convenient lotion in a burn of the eye from quicklime or other caustic alkali.

\section{Lead Subacetate and Spirit Lotion.}

R Liquoris Plumbi Subacetatis dilutæ $\quad$. $3 \mathrm{j}$ Spiritûs Rectificati . . . . . $\varlimsup^{\mathrm{ij}}$ $4 \cdot 0$ Aquæ Destillatæ . . . . . . ad $\lesssim$ viij ad $250 \cdot 0$ Misce.

Note.-Useful with a compress after contusion injuries and in chronic blepharitis marginalis, but must be avoided if there be any ulceration of the cornea. 
V. MISCELLANEOUS LOTIONS.

The uses of these collyria are indicated by notes appended to the.formulæ.

\section{Alumnol Lotion.}
$\mathrm{B}$ Alumnol . . . . . . gr. $\dot{\mathrm{xx}} \mathrm{I} \cdot 3$
Boroglyceridi . . . . . . 3iij $12 \cdot 0$
Aquæ Rosæ . . . . . . . ad $\overline{3} \mathrm{viij}$ ad $250 \cdot 0$

Solve.

Note.-Antiseptic and astringent. Used in chronic conjunctivitis.

\section{Belladonna Lotion.}

B Extracti Belladonnæ . . . . gr. cxx 8.0

Aquæ Bullientis . . . . . ऊviij $250 \cdot 0$

Solve.

Note.-Useful with a fomentation to relieve pain.

\section{Calcium Iodide Lotion.}

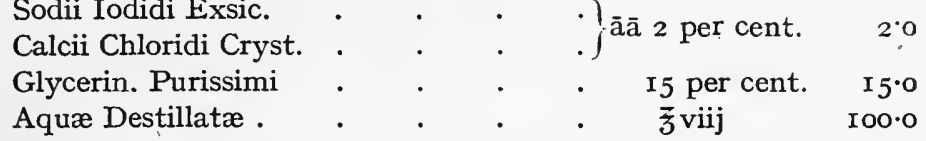

Note.-This lotion is recommended in incipient cataract, and should be used with an eye bath diluted with an equal quantity of hot water. The lotion ought to be applied for ten minutes twice a day (Dor.).

\section{Phyșostigmine Lotion.}

R. Extracti Physostigminæ Spiritûs Rectificati Aquæ Destillatæ . $\quad . \quad . \quad . \quad . \operatorname{ad} \zeta$ viij ad $250 \cdot 0$

Misce.

Note.-Useful in cases where the intraocular tension is increased, but inferior in its action to eserin eye drops (see Formulæ 2 and 3, page 428); a valuable stimulant when applied with a fomentation in indolent ulcer of the cornea.

\section{Potassium Chlorate Lotion.}

R Solutionis Potassii Chloratis Saturati . jँ viij

Note.-Recommended by Bergeon in the treatment of rodent ulcer.

\section{Saline Lotion (Physiological).}

R Sodii Chloridi (Purissimi) . . . . gr. $\operatorname{lxxx} 6 \cdot 0$ Aquæ Bullientis . . . . . . Oj rooo

Note.-Used as a sterile saline solution to wash the eye before and during operations, and in the irrigation of the anterior chamber to facilitate the removal of pus, lenticular matter, etc. Saline solution is even less irritating to the conjunctiva than sterilised water, and in ophthalmic operations is much to be preferred to the mercurial and other antiseptic solutions in ordinary use. 


\section{B. GUTTæ-EYE DROPS.}

Eye drops contain the remedy in more concentrated solution than collyria, and are conveniently dispensed in Chalk's drop bottles, from which the solution can be easily instilled into the conjunctival sac by means of the pipette. As these solutions rapidly decompose, they should always be freshly prepared with sterilised distilled water; or a mild antiseptic may be added, and the solution thereafter carefully filtered. Camphor water is a favourite menstruum, but the preservative recommended by Bishop Harman is more reliable : Tincture of Iodine $m i j$, Oil of Gaultheria $m \mathrm{ij}$, Menthol gr. ij, Aqua $\mathrm{Oj}$. To be kept in a stoppered bottle, and used as a stock solvent. In the prescriptions which follow, the above preservative may be substituted for distilled water or normal saline solution.

\section{ANESTHETICS.}

Anæsthetics are " remedies by means of which the sensations of pain are dulled or abolished." They are divided into two classes, local and general.

(a) Local ancesthetics.-Of these, cocain is facile princeps. It was introduced by Köller in I884, and its use has been an inestimable boon to ocular surgery. If any one has had a foreign body removed from his cornea without cocain, and has had also, at a later period, the same operation performed with the eye anæsthetised by means of that drug, he will realise to the full the benefit of a local anæsthetic. The use of cocain has, however, certain drawbacks. It dilates the pupil, disorders the accommodation, and acts injuriously upon the corneal epithelium, producing in some cases actual inflammation. These disadvantages may for the most part be avoided if the solution employed be weak. Never for any ordinary purpose use a greater strength than 2 per cent., and always make the patient keep the eye closed after the drops hâve been instilled. If the operation has been prolonged, take the additional precaution of instilling a. few drops of 5 per cent. chloretone oil into the conjunctival sac immediately before applying the bandage. In the case of a tarsal cyst, or in cauterising an ulcer of the cornea, however, a small quantity of solid cocain may be applied directly to the part about to be operated upon. The use of the drug in this way seems never to be followed by any bad result. If it be necessary to reach the deeper parts of the eye, for example, the ocular muscles or the iris, a few drops of the 2 per cent. solution may be injected beneath the conjunctiva.

The drawbacks mentioned have, however, led some to discard cocain for holocain, which in a I per cent. solution is a very trustworthy anæsthetic, though, as it possesses highly toxic properties, it cannot without danger be injected beneath the skin or conjunctiva, or along the tear-passages. Holocain acts more slowly than cocain, but penetrates more deeply; and is a most valuable addition to that drug in all operations in which it is necessary to cut the iris. A reliable combination for this purpose is cocain hydrochloride 2 per cent., holocain hydrochloride I per cent., dissolved in solution of adrenalin chloride, I in Iooo, freshly prepared immediately before being used. The employment of this mixture enables an operation for cataract to be performed without pain. Sometimes an oily solution is preferable to an aqueous one, but in that case the pure alkaloid must be used, and not a salt.

Adrenalin is one of the most important of the additions that have been made 
in recent years to the resources of ophthalmic therapeutics. Its marvellous power of contracting blood-vessels enables the surgeon to carry out most operations on the eyeball almost bloodlessly, while the exsanguine condition of the conjunctiva which it produces facilitates the absorption of such drugs as cocain, atropin, and eserin, and so renders their action more powerful. Stovain, eucain, novocain, and acoin are less toxic than cocain, and are all of considerable service in practice.

(b) General ancsthetics.-In ophthalmic surgery, the use of local anæsthetics has to a large extent removed the need for general anæsthesia, but when dealing with a young child, or with a nervous refractory adult, or when operating on an inflamed eye, or enucleating the globe, a general anæsthetic is still necessary. In these circumstances I prefer chloroform; but the patients must be brought thoroughly under its influence, for if they are only partially anæsthetised there is a risk of vomiting, or of awkward movements of the eye at a critical point of the operation, and in enucleation of the eyeball there is also the risk of dangerous collapse at the moment the optic and ciliary nerves are divided. For short operations where a general anæsthetic is deemed advisable, I prefer to employ chloride of ethyl, preceded by nitrous oxide gas. The use of this combination produces a profound quiet anæsthesia, lasting from one to two minutes. The chloride of ethyl is, however, most suitable for women and children, for occasionally it causes men to become greatly excited before they pass thoroughly under its influence. Nitrous oxide gas with oxygen and ether is a combination which is often very useful in operations on the eye, but it requires to be administered by a skilled anæsthetist.

\section{Alypin Solution.}

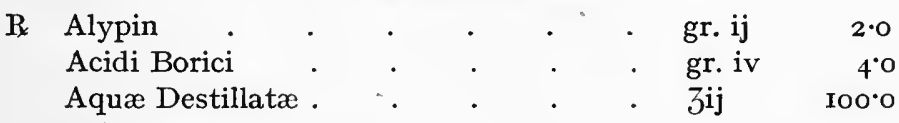
Solve.

Note--Alypin (benzyl-tetramethyl-diamino-ethyl-di-methyl carbinol hydrochloride) is a white crystalline powder, very soluble, and not precipitated by the alkaline fluids of the body. It is equal to cocain hydrochloride in anæsthetising effect, but causes neither mydriasis, nor derangement of accommodation.

\section{Cocain Hydrochloride Solution.}

$\begin{array}{llllllr}\text { Re Cocainæ Hydrochloridi } & . & . & & \text { gr. ij } & 2 \cdot 0 \\ \text { Acidi Borici } & . & . & . & . & \text { gr. iv } & 4^{\cdot 0} \\ \text { Aquæ Destillatæ . } & . & . & . & . & 3 \mathrm{ij} & \text { I00.0 }\end{array}$

Note.-Used chiefly to induce local anæsthesia before operations upon the eyeball and palpebral conjunctiva. The solution should be freshly prepared, as a fungus is always present when it is too long kept. If used too freely, or in too strong solution, it is apt to cause dryness of the corneal epithelium, and even slight keratitis. If the conjunctiva be inflamed, the anæsthetic action of cocain is much less perfect, but is greatly aided by the use of adrenalin solution.

The addition of 20 minims of pure glycerine to any of the prescriptions for eye drops is recommended. 


\section{Cocain and Morphin Hydrochloride Solution.}
$\mathrm{R}$ Cocainæ Hydrochloridi . . . gr. vj 6.0
Morphinæ Hydrochloridi . . . gr. iij $3 \cdot 0$
Aquæ Destillatæ . . . . . $3 \mathrm{ij} \quad$ I00.0
Solve.

Note._-Used to relieve pain-a good dressing after a blister.

\section{Dionin Solution.}

R Ethyl-morphinæ hydrochloridi . $\quad$ gr. iij to $\mathrm{v} 3 \cdot 0$ to $5^{\circ} \circ$ Aquæ Destillatæ . . . . . $3 \mathrm{ij}$ Iо0・o

Solve.

Note.-Dionin (ethyl-morphin hydrochloride) is a white crystalline powder soluble in $\mathrm{I}-\mathrm{IO}$ water, I-8 of alcohol ( 90 per cent.). In 5 per cent. solution is one of the most valuable agents for the relief of deep-seated pain-e.g. in glaucoma, iritis, sclerotitis, etc. When dropped into the eye it causes at first a smarting and burning sensation, accompanied by chemosis of the conjunctiva and swelling of the lids. These symptoms are sometimes very pronounced, and may alarm the patient greatly if he has not been forewarned of the probability of their occurrence, and told that they speedily pass off. This lymphagogue property of dionin is intimately associated with its power as an analgesic, because only after a good reaction is there much relief of the pain. This property also explains its power in promoting absorption of inflammatory deposits in the cornea. Its action in this way, very satisfactory in itself, is greatly increased if it be used along with collargol, and the best results are obtained when 5 per cent. solution of dionin is instilled in the morning, and a disc of ro per cent. collargol gelatin is placed in the conjunctival sac in the evening. This method of treatment hastens the clearing of the cornea after an attack of ulceration or of interstitial keratitis.

5. Eucain ( $\beta$ ) Hydrochloride Solution.

R Eucainæ Hydrochloridi $(\beta)$. . . gr. iv Aquæ Destillatæ . . . . . 3 ij I00.0 Solve.

\section{Eucain $(\beta)$ Lactate Solution.}
R Eucainæ $(\beta)$ Lactatis . . . . gr. iv $4^{\circ} \mathrm{O}$ Sodii Chloridi Purissimi . . . . $\quad$ gr. $\frac{1}{5} \quad 0 \cdot 2$ Aquæ Destillatæ . . . . . $3 \mathrm{ij} \quad$ го००

Solve.

Note.-Eucain ( $(B)$ is a synthetic compound allied to cocain, but less active, the solutions requiring to be double the strength of those of cocain. The solutions may be sterilised by boiling, as, unlike cocain solutions, they are not decomposed in the process. Eucain (3) must always be used, as Eucain (a) causes too much irritation to be of any service in ophthalmic work.

\section{Holocain Hydrochloride Solution.}

R Holocainæ Hydrochloridi . . . gr. Solve.

Note.-Holocain is a substitute for cocain prepared from phenacetin and paraphenetidin. A I per cent. solution is equal to a 2 per cent. solution of cocain. 
It produces anæsthesia of the eye without dilating the pupil or paralysing the accommodation.

Great care is necessary in the preparation and storing of this solution. Freshly distilled water must be slightly warmed and gradually added to the salt in a glass mortar. Both the mortar and glass-stoppered bottle in which it is to be kept must be thoroughly dealkalinised by being boiled in dilute hydrochloric acid before commencing operations. The presence of an alkali appears to throw down the base.

\section{Novocain Solution.}

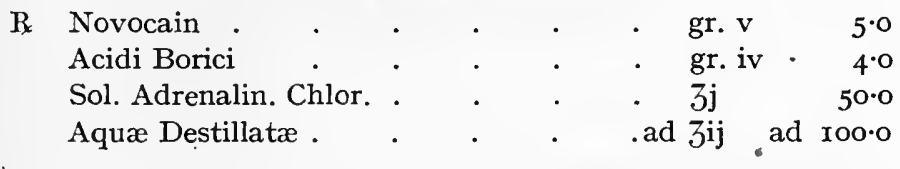

Note.-Novocain is a derivative of the new class of amino-alcohols. It is nonirritant and non-toxic, while the anæsthetic action is said to be more rapid than that of cocain. It causes neither mydriasis nor derangement of accommodation.

The following prescription is recommended for subcutaneous injection in order to induce local anæsthesia.

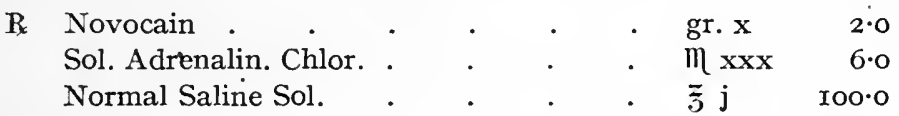

Note.-The solution is non-poisonous, and can be used very freely without fear of unpleasant results. By itself, however, it does little to allay a timid patient's nervousness and restlessness. Local anæsthesia may be perfect, but the mind of the patient is not at ease. $\mathrm{He}$ is alert and apprehensive, and in consequence has difficulty in keeping himself under proper control; hence the value of using omnopon as an adjuvant. It acts as a sedative to the brain, makes the patient feel calm and self-possessed, and thereby helps the surgeon to perform the operation easily and satisfactorily.

On the whole, omnopon acts best in elderly patients, and may be safely given in cases where the administration of a general anæsthetic might be attended by considerable anxiety and danger.

The good qualities of omnopon are greatly enhanced by combining it with scopolamin. The average dose for an adult is $\frac{2}{3}$ grain of omnopon and $\frac{1}{150}$ grain of scopolamin. It may be given by the mouth, but in most instances it is more convenient to administer it by hypodermic injection. It is supplied for that purpose in sterile ampoules, each containing I c.c. of a 2 per cent. aqueous solution of the drug.

If a patient is to have omnopon he should be prepared as he would be for a general anæsthetic. An hour and a half before the time fixed for the operation the contents of.a I c.c. sterile ampoule are injected into the muscles of the gluteal region. The patient ought to be in bed during the interval of waiting, and if the room be darkened and care be taken that he is not disturbed by noise, he will usually rest quietly or sleep until it is time to carry him to the operating table. He ought never to be allowed to walk, or to be roused more than can be avoided. If a single dose is not enough to induce sleep, an additional half dose is injected an hour after the first. This extra dose is rarely necessary; it can, however, be administered without risk, and ought always to be given if the patient be nervous 
and excitable. It is essential that the patient be thoroughly under the influence of the drug. He wakens up while he is being carried to the operation room, answers questions, and may even engage in conversation, but if the dose of omnopgn be sufficient he is quite free from excitement or from any feeling of nervous dread or apprehensiveness. If he be let alone for a few minutes after he is placed on the operating table he usually falls asleep, but he wakens at once when he is spoken to, and he is ready to assist the operator by voluntarily turning his eye in whatever direction may be necessary.

The pulse-rate may for a short time be slightly accelerated, but it very soon returns to normal, while the respirations, although slow, are regular and deep and never stertorous. The face sometimes is flushed, but there is no lividity. The patient sleeps soundly and quietly, but is easily roused. He sometimes complains of thirst, and readily takes any liquid offered to him. While awake he talks quite coherently and intelligently, but on the following morning he has no recollection of what he has said, and is often very much surprised when he is told that the operation has been performed. Sickness and vomiting occur very rarely, there is no complaint of headache or of giddiness, and on the morning after the operation the patient is usually free from any effect of the drug.

In plastic operations on the eyelids infiltration anæsthesia is employed. After the skin has been disinfected the novocain-adrenalin solution must be injected freely, and allowed to act for twenty minutes before the operation is begun. At the end of that time the skin over the area affected by the anæsthetising fluid is distinctly blanched owing to constriction, of the blood-vessels, and consequently hæmorrhage during the operation is rarely troublesome. If the local anæsthesia be carried out properly, and a sufficient dose of omnopon-scopolamin has been administered, the patient sleeps quietly, and unless he is spoken to gives no indication that he is aware of anything being done to him.

In extirpation of the lachrymal sac, if the local anæesthesia is to be satisfactory, great care must be taken to follow the technique recommended for the injection of the novocain-adrenalin solution. The skin of the side of the nose and of the eyelids should be disinfected by painting it with a 3 per cent. tincture of iodine. The needle of the hypodermic syringe, which has been carefully sterilised, is then inserted along the line of the primary incision, and as it is being withdrawn the novocain-adrenalin solution is injected slowly into the cellular tissue around the track of the needle. The lachrymal sac is filled with the anæsthetising fluid by the inferior canaliculus, and after waiting a few minutes deep injections are made, one above the dome of the sac and another below the internal tarsal ligament. The hypodermic needle must be inserted deeply until its point strikes the bone and the fluid is forced into the tissues around and behind the tear sac. Twenty minutes should elapse before the operation is begun, because all that time is necessary for the novocain-adrenalin to exercise its full effect on the nerves and blood-vessels.

For enucleation of the eyeball the majority of surgeons would prefer to have the patient under the influence of a general anæsthetic, but the operation can be performed painlessly and with entire absence of shock if omnopon-scopolamin be employed as an adjuvant to local anæsthesia. There need be no hesitation in employing this method in any case where a general anæsthetic is contra-indicated or where a patient is unwilling to take ether or chloroform. After the conjunctival sac is anæsthetised by the instillation of cocain the novocain-adrenalin solution is injected beneath the conjunctiva over the insertion of the four recti muscles. A long needle is then passed deeply into the orbit and the anæsthetising fluid injected 
into the tissues behind the eyeball. Insert the needle at each quadrant of the globe between the recti muscles and inject about 5 minims of the solution at each puncture. After waiting for twenty minutes the eyeball can be enucleated without pain and with the minimum of hæmorrhage.

\section{Stovain Solution.}

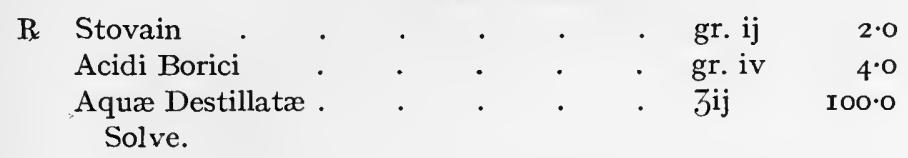

Note.-Stovain in a solution of $\mathrm{I}$ in 200 has an anæsthetic action identical with that of cocain; but it is much less toxic, and it has the advantage of being clinically a vaso-dilator. Patients do not lose their colour, and there is no fear of syncope. A valuable combination is two parts of stovain and one part of cocain.

\section{MYDRIATICS.}

Mydriatics-drugs which dilate the pupil-have, owing to the use of the ophthalmoscope, and the estimation of refraction by the shadow test, become much more generally employed than was formerly the case. One of the commonest of them is atropin, which has, for long, occupied such a high place in ocular therapeutics that the uninstructed have come to look upon it as a panacea for all the ills that the eye is heir to, and consequently the abuse of the drug has done much harm. In simple conditions, where its administration was unnecessary, much inconvenience has been caused by paralysis of the accommodation, while in the elderly its use has precipitated an attack of acute glaucoma. It ought to be a golden rule, never to be forgotten by any one who treats diseases of the eye, that atropin should in no case be instilled in a patient over forty years of age until the intraocular tension has been carefully tested and found to be normal. The caution that atropin ought never to be used in glaucoma cannot be repeated too often.

For examination purposes its disadvantages are obvious. It not only dilates the pupil but it paralyses the accommodation, and its effects remain for several days, greatly to the inconvenience of the patient, who, if he be suffering from an affection of the retina or choroid, and there has been an increase of the disease during the time the eyes are under the influence of the drug, may be easily tempted to blame the atropin used to facilitate an ophthalmoscopic examination for making his sight worse. A demand has, therefore, arisen for a mydriatic which will act quickly, and whose effects will pass off quickly, and which, while it will dilate the pupil to the maximum, will interfere as little as possible with accommodation. The dilators of the pupil are used to improve vision when it is obstructed by a central opacity of the media-e.g. nuclear cataract-to prevent the iris from becoming adherent to an ulcer or a wound near the centre of the cornea, to relieve spasm of the ciliary muscle, and to subdue pain.

\section{Atropin Sulphate Solution (Strong).}

$\begin{array}{llllllr}\text { R. Atropinæ Sulphatis } & \cdot & \cdot & \cdot & \cdot & \text { gr. ii } & 2 \cdot 0 \\ \text { Aquæ Destillatæ } & \cdot & \cdot & \cdot & \cdot & 3 \mathrm{ij} & \text { гоo } \cdot 0 \\ \text { Solve. }\end{array}$

Note.-Atropin must be avoided in glaucoma, and ought never to be used for the purpose of making an ophthalmoscopic examination in patients over forty years 
of age until it has been first ascertained that the tension of the eyeball is normal. It is the chief remedy in iritis, keratitis, and scleritis; but it must be remembered that in some few persons the drug acts as an irritant, producing redness and swelling of the eyelids, accompanied by an eczematous eruption of the skin in the neighbourhood of the eye. When this occurs the use of the drug must be stopped, and one of the other mydriatics substituted for it. Strong solutions of atropin are necessary to break down iritic adhesions; but if they require to be instilled repeatedly, care must be taken to watch for the first symptoms of general intoxication due to absorption of the drug. In old persons its use may cause acute mania.

\section{Daturin Sulphate Solution.}

R Daturinæ Sulphatis . . . . .

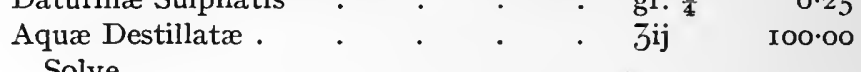

Note.-Daturin sulphate is a salt of an alkaloid obtained from stramonium leaves.

\section{Duboisin Sulphate Solution.}

R Duboisinæ Sulphatis . . . . . gr. $\frac{1}{4} 0.25$

Aquæ Destillatæ. . . . . $3 \mathrm{ij} \quad$ I00॰0

Solve.

Note.-Daturin and duboisin are useful in those cases where atropin produces irritation. It must, however, be remembered that, although duboisin rarely produces any local irritation, it requires to be used with caution, as it possesses markedly toxic properties. The following combination of mydriatics is highly recommended-

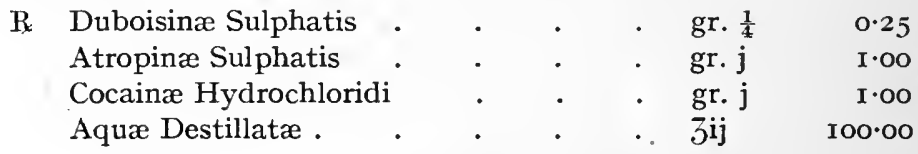
Solve.

\section{Euphthalmin Solution.}

Re Euphthalminæ . . . . . $\quad$ gr. iij $3 \cdot 0$

Aquæ Destillatæ . . . . . $3^{\mathrm{ij}}$ гоo॰o

Solve.

Note.-Euphthalmin is a mandelic acid derivative. It dilates the pupil, but does not disturb the power of accommodation. Very useful in nuclear cataract, or to dilate the pupil for the purpose of making an ophthalmoscopic examination.

\section{Homatropin Hydrobromide Solution.}

- R Homatropinæ Hydrobromidi

\section{Acidi Borici}

. $\}$ āā gr. j āā $x \cdot o$

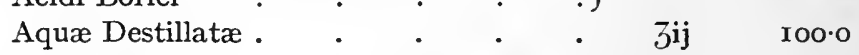

Solve.

Note.-When atropin is acted on by a mild alkali it breaks up into tropic acid and tropin. Homatropin hydrobromide is a salt of an alkaloid obtained from tropin. The white crystals are soluble in absolute alcohol, acetone, and chloroform, and in water, $I$ in 6 . Two drops of a $I$ per cent. solution produces a 
dilatation of the pupil which passes off within four hours. It dilates the pupil more quickly than atropin and the dilatation passes off sooner. It is frequently, therefore, employed to facilitate ophthalmoscopic examination; and to paralyse the ciliary muscle previous to the estimation of errors of refraction. It acts more powerfully when combined with cocain (see Formula 6). It acts best in an oily solution, and it is also more economical to use it as an oil.

\section{Homatropin Hydrobromide and Cocain Solution.}

R Homatropinæ Hydrobromidi . . gr. j $\mathrm{I} \cdot \mathrm{O}$ Cocainæ Hydrochloridi • . . . gr. ij $2 \cdot 0$ Acidi Borici . . . . . gr. ij $2 \cdot 0$ Aquæ Destillatæ . . . . . 3 ij Iоo

Solve.

7. Hyoscin or Scopolamin Hydrobromide Solution.

R Hyoscinæ Hydrobromidi vel Scopola-

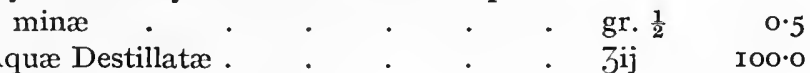
Solve.

Note.-Acts more powerfully than atropin upon old iritic adhesions; but, being very poisonous, it must be cautiously employed, and the lachrymal passages should be compressed for a few minutes after instillation. The patient ought to be kept at rest in the recumbent position for a short time after the use of the remedy. It is safer to employ this drug in the form of an ophthalmic disc.

\section{Mydriasin Solution.}
R Mydriasinæ . . . . . gr. i to $\mathrm{ij}$ I.O to $2 \cdot 0$
Aquæ Destillatæ . . . . . . 3 ij roo॰o
Solve.

Note.-Mydriasin, or atropin methyl-bromide is used instead of atropin sulphate, and is frequently combined with cocain hydrochloride (see Formula 9).

\section{Mydriasin and Cocain Hydrochloride Solution.}

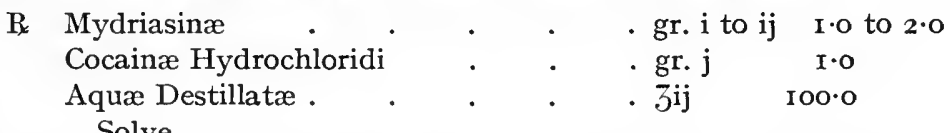

Solve.

Note - Is less expensive than homatropin and almost equally efficacious.

\section{Mydrin Solution.}

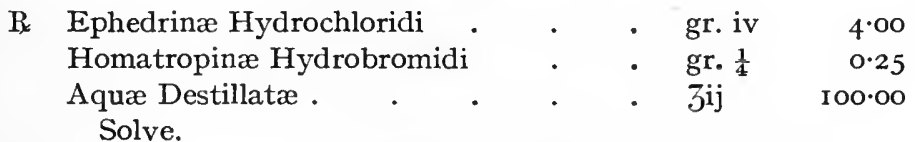

Note.-Ephedrin hydrochloride is a salt of an alkaloid obtained from Ephedra vulgaris. The solution dilates the pupil without affecting the accommodation, and its effects pass away in a few hours. It is a convenient drug to use for diagnostic purposes. 


\section{MYOTICS.}

Myotics contract the pupil and are used chiefly to diminish intraocular tension. Eserin and pilocarpin are the two most generally employed, but isophysostigmin and arecolin are also recommended. Of them all eserin is the most reliable, but when it is employed in from one-half to one per cent. solution its use is apt to be followed by supra-orbital pain, and it may cause acute follicular conjunctivitis and even inflammation of the iris.

The contractors of the pupil are used in glaucomatous states of the eye, in mydriasis, in ulceration of the cornea due to malnutrition, and in wounds and ulcers situated at the margin of the cornea. They act more rapidly than the mydriatics, but their effects pass off more speedily.

\section{Arecolin Hydrobromide Solution.}

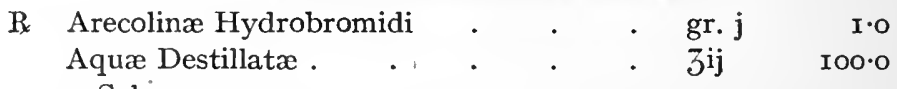
Solve.

Note.-Contracts the pupil more quickly than eserin, but its action passes off within a few hours.

\section{Eserin Sulphate Solution.}

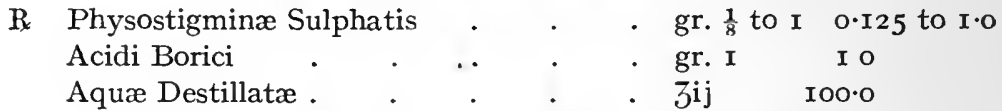

Solve.

Note.-The myotic most commonly employed to reduce intraocular tension in glaucoma; to contract the pupil and restore the power of accommodation after the use of atropin, or in paralysis due to disease, e.g. diphtheria; to prevent or reduce the prolapse of the iris after wounds of the cornea; to prevent perforation and promote the healing of marginal ulcers of the cornea. When the iris is inflamed, the use of eserin will aggravate the iritis.

As a general rule, eserin is to be preferred when a wound or an ulcer of the cornea is peripheral, and atropin when it is central.

The long-continued use of either atropin or eserin is apt to set up acute follicular conjunctivitis.

Eserin will not contract a pupil that is fully dilated by atropin.

\section{Eserin Sulphate and Cocain Solution.}

R Physostigminæ Sulphatis . . . gr. $\frac{1}{8}$

Cocainæ Hydrochloridi . . . . gr. iij

Aquæ Destillatæ . . . . . $33^{i j}$

Solve.

$100 \cdot 000$

Note.-The combination of cocain with eserin prevents the occurrence of the pain in the eye and brow so common after the instillation of the latter drug in simple solution. The dilatation of the pupil produced by cocain can readily be overcome by eserin; and the combination of eserin with cocain is often most efficacious in subduing pain in glaucomatous affections, more especially when combined with dionin. In subacute glaucoma the following combination is of great value: Eserin Salicylatis, I per cent., Pilocarpin Nitratis, 2 per cent., Dionin, ro per cent. 


\section{Iso-Physostigmin Sulphate Solution.}

R Iso-Physostigminæ Sulphatis Acidi Borici Aquæ Destillatæ .

Solve.

gr. $\frac{1}{2}$ to $\mathrm{j} \quad 0.5$ to $\mathrm{I} \cdot \mathrm{O}$

gr. iv

$3 \mathrm{ij}$

$4 \cdot 0$

IOO०O

Note.-More pure chemically but not any more efficacious clinically than eserin.

\section{Pilocarpin Nitrate Solution.}

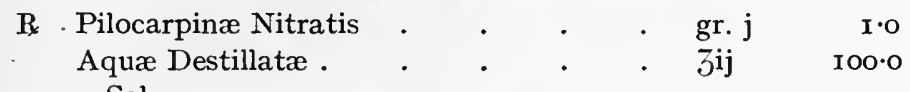

Solve.

Note.-Contracts the pupil like eserin, but its action is much weaker. It is useful in those cases where eserin causes acute pain.

\section{STIMULANTS.}

Stimulants are useful in many forms of conjunctival inflammation, but only after the acute stage has passed. They promote the absorption of the products of the inflammation, and hasten the healing of phlyctenulæ and indolent ulcers of the cornea. They ought always to be avoided when photophobia, lachrymation, and circumcorneal injection are pronounced. They are employed for the most part as ointments.

\section{Alcohol.}

R Alcohol Absoluti . . . . . 3ij

Note.-Used as a local application in herpes of the cornea.

\section{Argyrol Solution.}

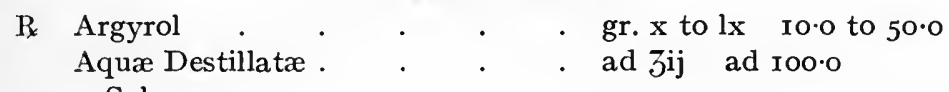

Solve.

Note.-Argyrol, fine black glistening hygroscopic scales, soluble in its own weight of water, contains the equivalent of 30 per cent. of silver combined with a proteid obtained from wheat.

In acute conjunctivitis argyrol gives good results. It does not irritate, and its value in lessening discharge and in relieving pain is very great indeed. It can be used in as great strength as 50 per cent., and may be applied either in solution or as an ointment. It is so non-irritating that it may safely be injected into the anterior chamber to control intraocular suppuration. In the treatment of purulent ophthalmia, in either the adult or the new-born child, it is one of the most valuable remedies we possess, but the best results are obtained by brushing the whole palpebral conjunctiva once thoroughly with a 2 per cent. solution of nitrate of silver at the outset of the disease, and afterwards applying a 20 per cent. solution of argyrol every few hours, the frequency of these applications being regulated solely by the amount of the discharge. In blennorrhœa of the lachrymal passages, after the nasal duct is clear of obstruction, the injection of solution of argyrol into the tear-sac checks suppuration, and thereby hastens the cure of one of the most tedious of diseases. 
Argyrol is so heavy that when a 20 per cent. solution is instilled into the eye it soon finds its way into all the folds of the conjunctiva, from which it clears out all discharge. This scavenger action of argyrol is one of its most important properties in the treatment of inflammation of the conjunctiva accompanied by profuse discharge.

\section{Collargol Solution.}

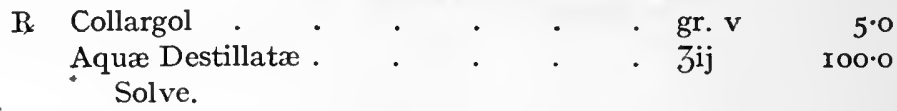

Collargol is a colloid salt of silver containing about 30 per cent. of the metal.

In recent wounds of the eyeball collargol, in the form of gelatin wafers containing ro per cent. of the drug, or in solution and as ointment, in strength varying from 5 to Io per cent. is of great value. If the injured surface be aseptic the collargol gelatine at once adheres to it, thereby sealing the wound and covering it up completely with an antiseptic plaster, under which healing goes on with great rapidity. If, however, there be sepsis the silver salt will not adhere to the wound, and consequently the presence or absence of the staining forms a trustworthy guide in prognosis. Collargol is almost always successful in its action, and it has also a wonderful power in clearing up recent opacities of the cornea, whether these be due to injury or disease; but on very rare occasions it induces so much chemosis, and causes such severe pain, that its use requires to be discontinued.

To make the gelatine wafers, rub up collargol in a mortar with melted gelatoglycerine until dissolved, taking great care to use a gentle heat, because a high temperature causes the collargol to change colour, probably as a result of partial reduction to metallic silver. The resulting mixture is spread on a warm tile, allowed to cool, and cut into discs of a suitable size.

\section{Ethylhydrocuprein Solution.}

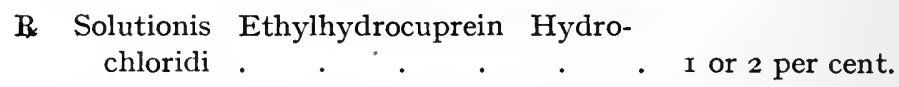

Note.-Ethylhydrocuprein hydrochloride-also known by the trade name of optochin (a derivative of quinine)-forms a white salt readily soluble in water, and is employed in I per cent. or 2 per cent. aqueous solution. It is applied for two minutes directly to the ulcerated surface by means of a swab of sterilised cotton-wool.

The application of optochin causes sharp burning pain, lasting from five to ten minutes. That irritation, however, soon lessens, and by the end of fifteen minutes there is complete anæsthesia of the cornea, which will last for fully half an hour. To begin with, these applications should be repeated two or three times dailythe good effect being maintained by instilling a few drops of $\mathrm{x}$ per cent. solution of optochin every hour during the day, while, during the night, a 1 per cent. optochin and atropin ointment may be applied as often as possible without interfering witl sleep.

The success of the treatment by ethylhydrocuprein depends upon the fact that the pneumococcus is the infecting organism, and that it is possible to kill the microbe in the substance of the cornea without injury to the cornea itself. Preliminary bacteriological examination is therefore essential, because it is very difficult to distinguish by clinical signs alone a serpiginous ulcer due to the pneumo- 
coccus from one the result of infection by other micro-organisms, and optochin has little or no effect on microbes other than the pneumococcus.

\section{Fluorescin Solution.}

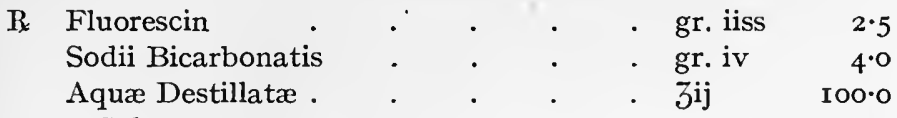

Solve.

Note.-Useful in the diagnosis of ulceration of the cornea, which it stains green wherever the epithelium is absent. Resorcin in a 10 to 20 per cent. solution stains an abrasion of the cornea red immediately after instillation.

Silver fluorescin and Zinc fluorescin are recommended by Wolff in conjunctivitis. They are prescribed in 5 per cent. ointment.

$$
\begin{aligned}
& \text { 6. Guaiacol Solution. }
\end{aligned}
$$

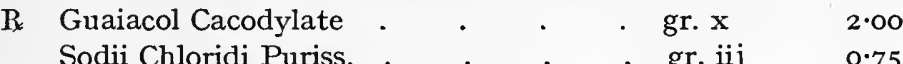

$$
\begin{aligned}
& \text { Aquæ Sterile . . . . . } \overline{3} \mathrm{j}^{\mathbf{2}} \text { I00.00 }
\end{aligned}
$$

Note.-Darier considers guaiacol to be a specific in tuberculous affections of the eye. The above solution can be used as an instillation into the conjunctival sac, or as a sub-conjunctival injection.

\section{Hetol Solution.}

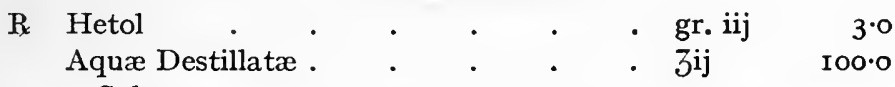
Solve.

Note.-Hetol, sodium cinnamate, is used as a sub-conjunctival injection to clear up opacities of the cornea.

\section{Ichthargan Solution.}

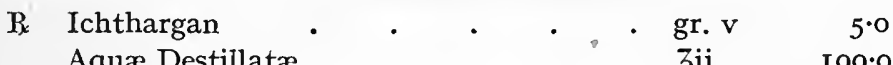

Aquæ Destillatæ . . . . . $3{ }^{\circ}$.

Solve.

Note.-Contains 20 per cent. of silver combined with ichthyol.

\section{Jequirity Solution.}

$\mathbf{R}_{k}$ Jequirity Seeds . $\quad$. . . . 3 parts

Cold Distilled Water . $\quad . \quad$. 500 parts

Macerate for 24 hours, then add Boiling

Water. . . . . . 500 parts

Filter when cold.

Note.-In obstinate cases of granular ophthalmia with pannus, paint the everted eyelids with above solution; and, after two or three applications, a purulent ophthalmia is set up which occasionally cures the granular condition and clears the cornea.

\section{Picric Acid Solution.}

$\mathrm{B}_{k}$ Solut. Acid. Picric . . . . 2 per cent.

Note.-Useful as an instillation in burns of the eye : an ointment of the same strength can be used instead of the drops. 
11. Protargol Solution.

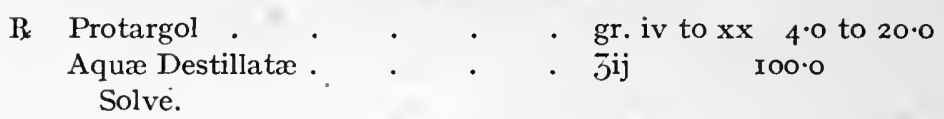

Protargol is a combination of silver with protein, containing 8.3 per cent. of silver. It is a powerful germicide, and penetrates more deeply into the tissues than nitrate of silver. It causes slight discomfort when instilled into the eye. It is very useful in ophthalmia neonatorum, blennorrhœa of the tear-sac, sloughy ulcer of the cornea, and obstinate ophthalmia tarsi.

In chronic inflammation of the conjunctiva and the edge of the eyelids, protargol, in from Io to 25 per cent. solution, brushed vigorously over the affected parts, produces quicker and better results than are to be obtained by any other method of treatment (Darier).

Protosil in 5 per cent. ointment is of great value in the treatment of ophthalmia neonatorum.

\section{Pyoktanin Solution.}

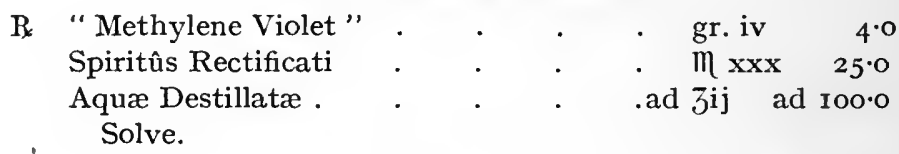

Note.-Useful in ulceration of the cornea and as an antiseptic in injuries of the eyeball. Should be freshly prepared and kept in a dark bottle, as it is decomposed by light.

\section{Silver Nitrate Solution.}

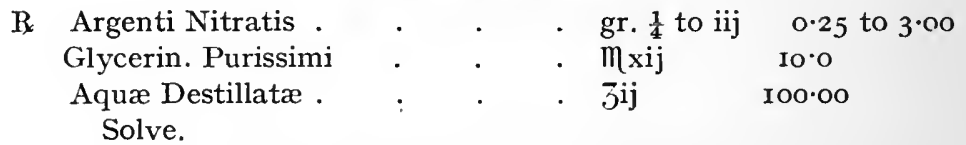

Note.-Used to paint the everted eyelids in conjunctivitis with discharge. Any excess must be washed away with a solution of table salt and water. The solution should be applied thoroughly with a brush to the palpebral conjunctiva, and the retrotarsal folds, the frequency of the applications depending upon the amount of discharge. If possible, nitrate of silver should not be used shortly before going to bed, as the pain to which it gives rise may interfere with sleep.

The addition of ${ }_{5} 5$ per cent. of pure glycerine to the nitrate of silver drops prevents them from causing severe pain, and thereby removes the chief objection to their use. There is no doubt that nitrate of silver is in the majority of cases more efficacious than any of its substitutes. The old form of nitrate has no doubt many disadvantages, and is now being, in great measure, supplanted by other salts of the same metal, which, while they are bactericidal, possess feiver undesirable qualities.

Concerning most of the drugs put on the market by manufacturing chemists for the purpose of preventing or controlling sepsis, it is necessary, however, to remember that the results obtained in a test-tube experiment are not always confirmed in actual practice.

Solutions containing nitrate of silver ought to be kept in actinic bottles. 
Nitrate of silver should not be employed continuously over a long period of time, since it produces a discoloration of the conjunctiva (argyrosis), due to a deposit of oxide in its substance.

Collosol argentum is a powerful non-irritating antiseptic used in full strength in all forms of infection of the conjunctiva; it may also be employed as a lotion diluted with three times the quantity of distilled water at blood heat.

\section{Silver Nitrate and Atropin Solution.}

$\mathrm{R}_{\mathrm{x}}$ Argenti Nitratis . . . . gr. $\frac{1}{4}$ to $\mathrm{ij} 0.25$ to $2 \cdot 00$

Liquoris Atropinæ Sulphatis B.P. . $3 \mathrm{j} \quad 50 \cdot 00$

Aquæ Destillatæ . . . . ad $3 \mathrm{ij}$ ad roo·oo

Solve.

Note--Useful to subdue pain and promote the healing of corneal ulcers accompanied by muco-purulent or purulent discharge from the conjunctiva: quickly relieves the conjunctivitis which occasionally follows extraction of cataract.

\section{Zinc Sulphate Solution.}

R Zinci Sulphatis . . . . gr. $\frac{1}{4}$ to $\mathrm{r} 0.25$ to $\mathrm{I} \cdot 00$

Sol. Adrenalin Chlor. . . . $3 \mathrm{j} \quad 50 \cdot 00$

Aquæ Destillatæ . . . . . ad $3 \mathrm{ij} \quad$ ad roo.oo

Solve.

Note.-Used in superficial injections of the conjunctiva and in follicular conjunctivitis.

\section{Zine Sulphate Solution (Strong).}

$\mathrm{R}_{\mathrm{e}}$ Zinci Sulphatis . . . . . gr. j to $\mathrm{I} \cdot \mathrm{O}$

Sol. Adrenalin Chlor. . . . . $3 \mathbf{j} \quad 50 \cdot 0$

Aquæ Destillatæ . . . . . . ad $3 \mathrm{ij} \quad$ ad roo.o

Solve.

Note.-A valuable astringent in many cases of conjunctivitis, and a specific in those associated with the Morax-Axenfeld diplo-bacillus-angular conjunctivitis. Zinc sulphate in 20 per cent. solution is used as an application to hypopyon ulcer due to Morax-Axenfeld diplo-bacillus.

\section{OLEA-OILY SOLUTIONS.}

The following advantages are claimed for oily solutions of drugs used in ophthalmic practice : (I) they can be readily rendered aseptic; (2) their instillation causes very little irritation to the eye;" (3) when the drug is employed in an oily vehicle its action is more prompt, powerful, and lasting, than in an aqueous solution.

\section{Atropin Oil.}

$\begin{array}{llllllr}\text { Be Atropinæ, Alkaloid } & \cdot & . & . & \text { gr. } \frac{1}{2} & 0.5 \\ \text { Olei Ricini Purissimi } & . & . & . & \text {. } & 3 \mathrm{ij} & 100 \cdot 0\end{array}$

Solve.

Note.-Useful in ulceration of the cornea-a valuable lubricating and sedative application in some cases of granular inflammation and xerosis of the conjunctiva.

F F 
2. Cajuput Oil (Cleland).
R Olei Cajuputi . . . . . $\quad \begin{array}{llll} & 3 i & 50 \cdot 0\end{array}$

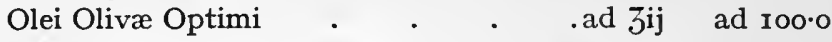 Misce.

Note.-Useful in chronic blepharitis and in hyperæmia of the eyelids due to eye-strain.

\section{Chloretone Oil.}
$\mathrm{R}$ Chloretone . . . . . . gr. viij 8.0
Olei Olivæ Optimi $\quad \cdot \quad \cdot \quad \cdot \quad \cdot \quad 3$ ij $100 \cdot 0$
Solve.

Note.-Useful as an anæsthetic and antiseptic. Valuable after a superficial burn of the cornea or conjunctiva.

\section{Cocain Oil.}

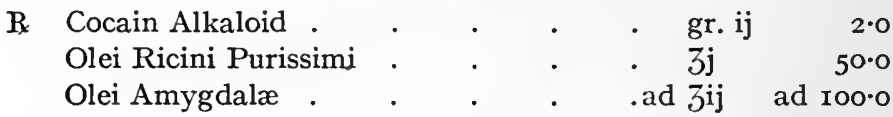

Solve.

Note.-Useful to subdue pain in burns of the eyeball and in inflammations of the cornea, the sedative action of the drug being much increased by the lubricating effects of the oil.

5. Eserin Oil.
$\begin{array}{lllllll}\text { Bk}_{k} & \text { Physostigminæ . } & \text {. } & \text {. } & \text {. } & \text { gr. } \frac{1}{8} & 0.125\end{array}$ Cocain Alkaloid . . . . . . gr. ij $2 \cdot 000$ Olei Ricini Purissimi . . . . 3j $50 \cdot 000$ Olei Amygdalæ . . . . . . ad $3 \mathrm{ij}$ ad roo.000 Solve.

Note.-This, unlike the watery solution, does not become red (rubreserine) on exposure to the air.

\section{Homatropin Oil.}

\begin{tabular}{|c|}
\hline $\begin{array}{l}\text { Homatropin Alkaloid } \\
\text { Cocain Alkaloid } \\
\text { Olei Ricini Purissimi } \\
\text {-In this form the very cos }\end{array}$ \\
\hline
\end{tabular}

Note. - In this form the very costly homatropin is used most economically and acts more quickly than an aqueous solution.

\section{Mercuric Iodide Oil (Panas).}
R Hydrargyri Biniodidi . . . . . gr. $\frac{1}{2} \quad 0.5$

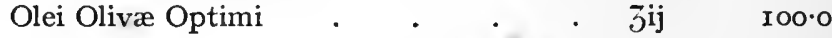 Solve.

Note.-Used as a dressing for the eye before operations to disinfect the skin of the lids and the roots of the eyelashes 


\section{THERAPEUTIC NOTES AND FORMULÆ}

\section{Mercuric Menthol Oil.}

R Unguent. Hydrargyri Nitratis diluti . 3 Menthol Purissimi . . . . gr. iv Olei Olivæ Purissimi . . . . . $\overline{3} \mathbf{j}$ IOO॰O

Note.-An admirable application to the edge of the eyelids in chronic conjunctivitis, and in blepharitis marginalis.

\section{GLYCEROLES.}

Some cases of granular ophthalmia with much congestion of the palpebral conjunctiva are benefited by the application, twice a day, of one of the following glyceroles.

\section{Glycerole of Copper.}

$\mathrm{R}$

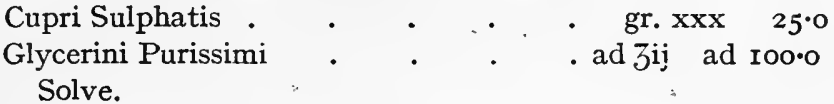

\section{Glycerole of Iodine.}

Glycerini Purissimi $\because \quad . \quad . \quad$. $\prod_{x x x} 25^{\circ}$

Vaselini Albi Purissimi . . . . ad 3 ij ad roo.o

Note.-Iodine vasogen ro per cent. is a most efficacious local application in some forms of sloughy ulcer of the cornea.

A combination of iodine with phenol is one of the best applications to a septic wound or an ulcer of the cornea.

$$
\begin{array}{lllll}
\text { R Iodi Purissimi } & \text {. } & \text {. } & \text {. } & \text {. } 20 \text { parts } \\
\text { Phenol Purissimi } & . & . & . & \text {. } 60 \text { parts } \\
\text { Glycerin Purissimi } & \text {. } & \text {. } & \text {. } & \text {. } 20 \text { parts }
\end{array}
$$

Note.-Should be applied carefully but very thoroughly by a swab of sterilised cotton-wool twisted round the end of a probe.

The following prescription is useful in chronic blepharitis marginalis-

$\mathrm{B}$. Tinct. Iodi mitis

$\begin{array}{llll}\text { Acid. Carbolici Purissimi } & . & . & . \\ \text { Chloral Hydratis } & . & . & .\end{array}$

Note.-To paint the eyelids in case of long-standing ophthalmia tarsi.

\section{Glycerole of Iodoform.}

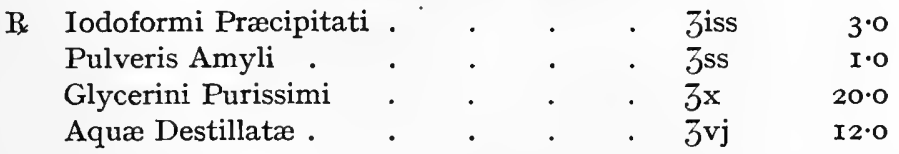

Note.-Mix the glycerine and water together and add to the powders previously mixed. Heat gradually to $240^{\circ}$ Fahr. A useful injection in suppuration of the tear passages, complicated by extensive necrosis of bone. 


\section{Glycerole of Lead Subacetate.}

B Glycerini Plumbi Subacetatis . . $m_{x v} \mathbf{1 2 . 5}$ Glycerini Purissimi . . . . ad 3 ij ad roo.o Misce.

Note.-Must never be employed if the cornea be ulcerated.

\section{Glycerole of Tannic Acid.}

R Acidi Tannici . . . . . $\quad$ gr. $\operatorname{xxx} 25^{\circ} 0$ Glycerini Purissimi . . . . $3 \mathbf{j} 55^{\circ} 0$ Syrupi Simplicis . $\quad . \quad . \quad . \quad$. ad 3 ij ad Ioo•o Solve.

Note.-A valuable astringent in chronic forms of conjunctivitis.

\section{E. UNGUENTA-OINTMENTS.}

Vaseline or lanoline forms the best basis for the ointments employed in ophthalmic practice, but, before mixing, all alkaloid salts must be dissolved in a few minims of water. Some prefer yellow vaseline to the white, which is said to be more irritating; but in practice this does not seem to be the case. Ointments are preferable to "drops" in the case of children and where there is profuse lachrymation, as watery solutions are liable to be washed out of the eye before they have time to take effect. They are conveniently dispensed in collapsible tubes, or when pure oil of theobroma is used as a base they can be cast into crayon-shaped moulds and mounted in a celluloid handle. Such pencils are of great service in the treatment of chronic forms of conjunctivitis.

\section{Aristol Ointment.}

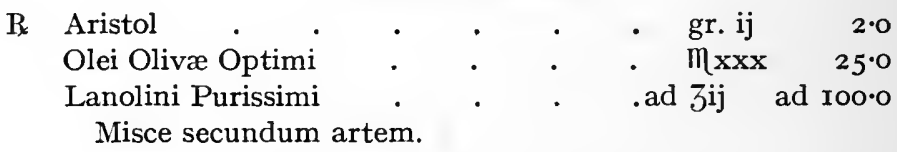

Note.-Useful in burns of the eyelids and conjunctiva, and, if pain be severe, may be combined with cocain and atropin.

\section{Argyrol Ointment.}

$\mathrm{R}_{\mathrm{x}}$ Argyrol . . . . . . . gr. viij 8.0 Vaselini Albi Purissimi . . . 3 ij $100 \cdot 0$ Misce.

Note.-A reliable application in conjunctivitis with ulceration of the cornea.

\section{Atropin and Boric Acid Ointment.}

B Atropinæ Sulphatis . . . . . gr. j I•O Pulveris Acidi Borici Subtilis . . $\quad$ gr. xv 12.5 Vaselini Albi Purissimi . . . . ad $3 \mathrm{ij}$ ad Ioo॰o Misce secundum artem. 


\section{Atropin and Quinine Sulphate Ointment.}

R Atropinæ Sulphatis

Quininæ Sulphatis . . . . gr. ij $2 \cdot 0$

Aquæ Destillatæ . . . . $m_{x x x} 25 \cdot 0$

Lanolini Purissimi . . . . gr. $x x x \quad 25 \cdot 0$

Vaselini Albi Purissimi . . . ad $3 \mathbf{i j}^{\mathrm{a}}$ ad Iо००

Misce.

Note.-Useful in phlyctenular conjunctivitis complicated by sloughy ulceration of the cornea.

\section{Atropin Sulphate Ointment.}

B Atropinæ Sulphatis . . . . gr. j I·O Vaselini Albi Purissimi $. \quad . \quad . \quad 3^{i^{i}}$ 100.0

Misce secundum artem.

Note.-As to the use of atropin, see p. 425-426.

\section{Atropin Sulphate and Cocain Ointment.}

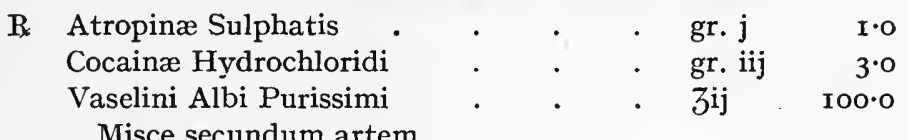

Note.-Useful in phlyctenular conjunctivitis for the relief of intolerance of light. Children suffering from photophobia due to phlyctenular keratitis should have their eyes protected by a shade, but not bandaged. The hands and face ought to be washed several times a day, and, as far as possible, the patient should be prevented from rubbing the eyes and burying the face in a pillow. Attention must also be given to keeping the nasal cavities free from discharge.

\section{Atropin Sulphate Ointment (Compound).}

R Atropinæ Sulphatis

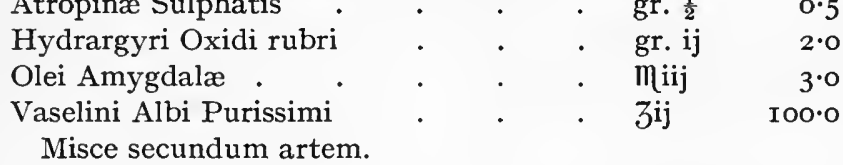

R Atropinæ Alkaloid . . . . I per cent.

Cocainæ Alkaloid . . . . 2 per cent.

Unguent. Hydrarg. oxidi flavi (Allen and Hanbury) . . . . . $\frac{1}{2}$ per cent. to 2 per cent. Misce secundum artem.

Note.-Useful in phlyctenular conjunctivitis after the acute symptoms of irritation have subsided.

\section{Boric Acid Ointment.}

B Acidi Borici Subtilis . . . . partes iij

Unguenti Paraffini Albi . . . partes xv Misce.

Note.-Useful in blepharitis and conjunctivitis and as a dressing for the eye after operation. Carefully prepared and thoroughly sterilised, this ointment causes no irritation. 


\section{Boric Acid and Cocain Ointment.}

$\mathbf{P}_{k} \quad$ Unguenti Acidi Borici (Formula 8) . $3 \mathrm{j}$ $50 \cdot 0$

Unguenti Cocainæ Hydrochloridi (Formula

I4) . . . . . 3

$3 \mathrm{j} \quad 50 \cdot 0$ Misce.

Note.-Useful in abrasions of the cornea.

\section{Cade Oil Ointment.}

\section{R Hydrargyri Oxidi Rubri Olei Cadini} Pulveris Camphoræ . Misce.

$\begin{array}{lr}\text { gr. ij } & 2 \cdot 0 \\ . \quad m \times & \text { I0.0 } \\ . \quad \text { gr. } x & \text { I0.0 } \\ . \text { ad } 3 \text { ij } & \text { ad } 100 \cdot 0\end{array}$

Note.-Useful in eczema of the eyelids.

\section{Cadmium Iodide Ointment.}

R Cadmii Iodidi . . . . . . gr. Vaselini Albi Purissimł $\quad . \quad$. $\quad 3$ j Misce.

Note.-Useful in opacities of the cornea.

\section{Calomel Ointment.}
R. Hydrargyri Subchloridi Vaselini Albi Purissimi gr. iij Misce. $100 \cdot 0$

Note--Useful to promote the absorption of opacities of the cornea. The bulk of a split pea of the ointment having been introduced into the conjunctival sac, the cornea should be gently massaged through the closed eyelids with the thumb and index finger, the patient being directed to look straight in front of him.

\section{Cassaripe Ointment.}
$\mathrm{B}_{\mathrm{e}}$ $\begin{array}{lllll}\text { Cassaripi } & . & . & \text { gr. xij } & \text { I2.0 } \\ \text { Vaselini Albi Purissimi } & . & . & \text {.ad } 3 \mathrm{jij} & \text { I00.0 }\end{array}$ Misce.

Note-Cassaripe is the inspissated juice of the cassava plant which is highly antiseptic. It is used for sloughy ulcers of the cornea, with excellent results (Risley).

\section{Cocain Hydrochloride Ointment.}

Bx Cocainæ Hydrochloridi . . . gr. ij Vaselini Albi Purissimi . $\quad 3$ ij

Misce secundum artem.

Note.-Useful application after the removal of foreign bodies from the cornea, and in superficial ulcerations and injuries. The eye ought always to be protected by a bandage for twenty-four hours, or until the epithelium has been restored. 


\section{Collargol Ointment.}

R Collargol. gr. ij to $x v$ Vaselini Albi Purissimi . . . ad 3ij $2 \cdot 0$ to $15 \cdot 0$ Misce.

Note.-Useful in blepharitis marginalis.

\section{Copper Citrate Ointment.}

B Cupri Citratis . . . . . gr. ij $2 \cdot 0$ Vaselini Albi Purissimi . . . ad $\xi_{\mathrm{ij}}$ ad Ioo.o Misce.

Note.-Useful in conjunctivitis, trachoma, etc.

\section{Creolin Ointment.}

R Acidi Cresylici - $m j$ Aquæ Destillatæ . . . . . $m x x x \quad 25 \cdot 0$ Lanolini Purissimi . . . . . ad 3ij ad roo.o Misce.

Note.-Useful occasionally in ulcerative blepharitis.

\section{Dionin Ointment.}
$\mathrm{R}$ Dionin . . . . . . gr. $\mathrm{xij} \mathrm{I} 2 \cdot 0$ Vaselini Albi Purissimi . . . .ad Zij ad Ioo.o Misce.

Note.-Useful to subdue pain in the eye, and promote the absorption of opacities of the cornea following ulceration, or interstitial inflammation.

\section{Eserin Sulphate Ointment.}

R Physostigminæ Sulphatis . . . . gr. $\frac{1}{20} \quad 0.05$ Unguenti Cocainæ Hydrochloridi (Formula
I4)
Misce.
3ij $\quad$ I00.00

Note.-Useful in ulcers situated at the margin of the cornea. Must not be employed if the iris be inflamed. For note on the use of eserin, see page 428 .

\section{Gallic Acid Ointment.}

$\mathrm{P}_{*}$ Acidi Gallici . . . . . . gr. viij $8 \cdot 0$

Olei Lavandulæ . . . . . $m_{j} \mathbf{j} I \cdot 0$

Olei Ricini Purissimi. . . . $m$ xxx $25^{\circ} 0$

Vaselini Albi Purissimi . . . ad $3 \mathbf{i j}$ ad Ioo.o

Misce.

Note.-Useful in blepharitis marginalis after loss of the eyelashes. 


\section{Ichthyol Ointment.}

$\mathrm{P}$

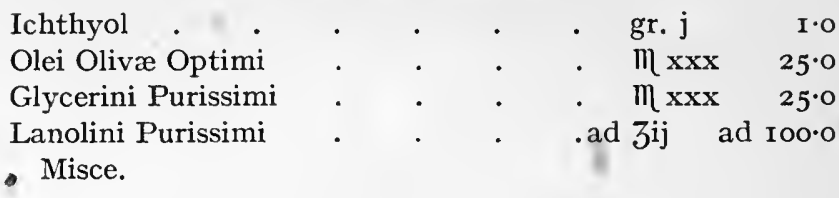

Note.-Useful in eczema of the eyelids and for conjunctivitis with fissure of the external canthus. The ichthyol may be combined with oxide of zinc.

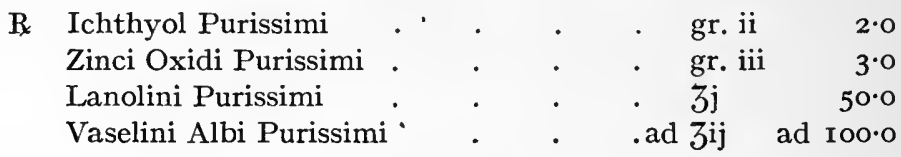

Note.-This ointment is of great service along with zinc drops in the treatment of angular conjunctivitis.

\section{Iodide of Potassium Ointment.}

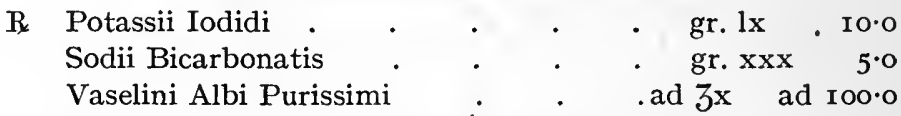

Note.-Heisrath's ointment for massage of the lids in trachoma. Recommended to be used along with alibour water.

\section{Iodoform Ointment.}

P Iodoformi Precipitati . . . .

Vaselini Albi Purissimi

Lanolini Purissimi . . . . ad 3 ij ad Ioo.o

Misce.

Note.-Useful in follicular conjunctivitis, ulcers, and burns. Iodol, which is odourless, may be substituted for iodoform. Atropin may be added to the mercurial or iodoform ointments in those cases where it is necessary to cause dilatation of the pupil; in cases of phlyctenular ulcer accompanied by distressing photophobia, or in burns of the conjunctiva. Cocain may be added to relieve pain.

\section{Iodoform, Atropin, and Cocain Ointment.}

R Iodoformi Precipitati . . . . gr. xv I2.5 Atropinæ Sulphatis . . . . . gr. $\frac{1}{2} \quad 0.5$

Cocainæ Hydrochloridi . . . gr. iij $3 \cdot 0$

Vaselini AJbi Purissimi . . . . ad 3 ij ad roo.o

Misce secundum artem.

Note.-Useful application in burns of the conjunctiva, and in ulceration of the cornea. 


\section{Iodol Ointment.}

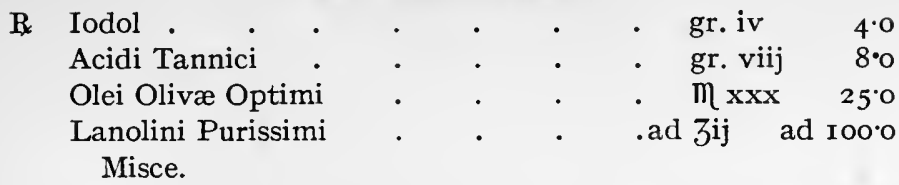

Note.-Useful as an application to the nasal mucous membrane when inflamed and ulcerated in connection with phlyctenular conjunctivitis. The crusts must first be removed by means of a nasal douche (see page 445).

26. Lead Acetate Ointment.

$\begin{array}{lllllr}\text { R. Plumbi Acetatis } & . & . & . & \text { gr. ij } & 2 \cdot 0 \\ \text { Cocainæ Hydrochloridi } & \cdot & . & . & \text { gr. ij } & 2 \cdot 0 \\ \text { Vaselini Albi Purissimi } & \cdot & \cdot & . & 3_{i j} & \text { I00 } \\ \text { Misce. }\end{array}$

Note.-Useful to relieve itching in blepharitis marginalis.

\section{Lead Ointment.}
R Emplastri Plumbi

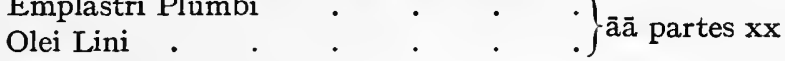
Balsami Peruviani . . . . . partem j
Misce.

Note.-This is the pommade anti-blépharatique of De Wecker. It should always be freshly prepared, and is applied to the eyes at bedtime, smeared upon discs of old linen. The application ought to be repeated every night for several weeks. Very efficacious in blepharitis hypertrophica.

\section{Menthol and Cocain Ointment.}

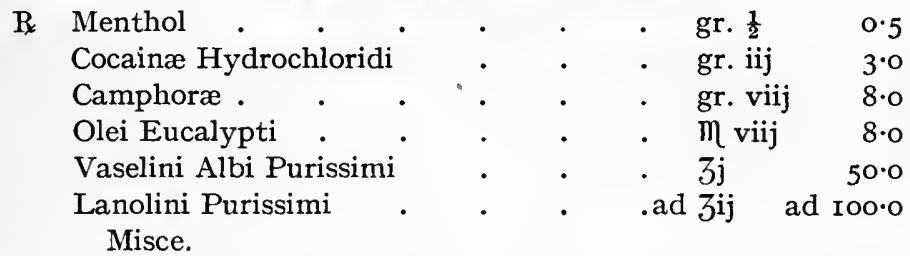

Note.-Useful to anoint the nostrils in cases of "watery eye " accompanied by swelling of the nasal mucous membrane. Cocain may be omitted from the prescription if there is a fear that the patient may form the cocain habit.

Where there is simply congestion of the erectile tissue over the inferior turbinated bone, much relief will be got from the use of the following prescription-

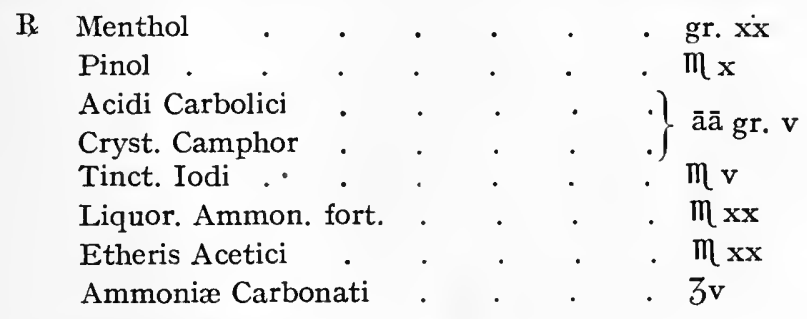

Note.-The smelling salts. 


\section{Mercuric Oleate Ointment.}

B. Unguenti Hydrargyri Oleatis

$3 \mathrm{ij}$

Note.-A good application for killing pediculi and for administering mercury by inunction.

\section{Mercuric Oleate and Morphin Ointment.}
B Morphinæ . . . . . . gr. ij $2 \cdot 0$
Unguenti Hydrargyri Oleatis . . ad $3 \mathrm{ij} \quad$ ad Ioo.o
Misce.

Note.-Useful to relieve pain round the orbit. Forms a good dressing after the epidermis has been removed by a fly blister.

\section{Mercury Ointment.}

$\mathrm{R}_{\mathrm{x}}$ Unguenti Hydrargyri . $\quad . \quad$. $\quad \bar{\jmath} \mathrm{j}$

Note.-Used as an inunction-one drachm to be rubbed into the skin below the armpit, one night on one side of the body and the next on the other. The ointment should not be washed off till morning. The patient should be clad in flannel and carefully protected from cold during the course of the treatment.

\section{Mercury and Lead Ointment (De Wecker).}

R. Hydrargyri Oxidi Rubri . . . . gr. $\mathrm{j} \quad \mathbf{I} \cdot \mathbf{O}$

Liquoris Plumbi Subacetatís diluti . $m x$ ro.0

Unguenti Paraffini Albi . . . $3 \mathrm{ij}$ Iо०॰o

Misce.

Note.-Useful in ophthalmia tarsi associated with acne and seborrhœa of the face generally.

\section{Mercury and Belladonna Ointment.}

R Extracti Belladonnze

Hydrargyri Ammoniati $\quad . \quad$. $\quad$. $\operatorname{gr.x} r \begin{array}{rr}5^{\circ} \\ \text { I0.0 }\end{array}$

Unguenti Paraffini Albi . . . . ad 3 ij ad Ioo.o

Misce.

Note.-Smeared on the brow in cases of iritis, cyclitis, or other deep-seated inflammation.

34. Mercury Vasogen.

Vasogen is an oxygenated petroleum. Mixed with mercury in the proportion of two-thirds vasogen and one-third mercury it forms a cleaner and more active preparation than ordinary mercury ointment.

\section{Mercuric Chloride Ointment (White).}

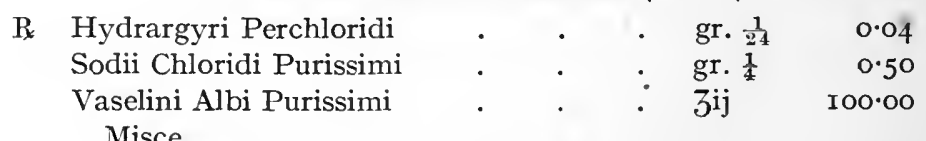

Note.-Useful as a dressing for the eyes before operation, to disinfect the skin of the lids and the roots of the eyelashes. Dissolve the mercury and the chloride of sodium in a few drops of alcohol and add to the vaseline which has previously 
been sterilised by boiling. Stir till cold, and put up in a pot, which ought to be hermetically sealed. A separate pot should be used for each patient.

(a) Ammoniated Mercury Ointment.

\section{Mercury Ointments (Various).}

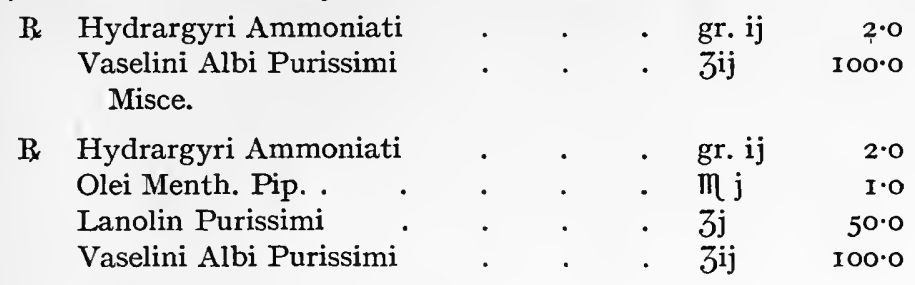

(b) Red Mercuric Oxide Ointment.

B Hydrargyri Oxidi Rubri . . . gr. ij $2 \cdot 0$

Olei Amygdalæ . . . . . M viij $8 \cdot 0$

Trite bene. Deinde adde
. . . ad $3 \mathrm{ij}$ '

Misce.

(c) Yellow Mercuric Oxide Ointment (Pagenstecher).

R Hydrargyri Oxidi Flavi . . . gr. iv $4^{\circ} \circ$ Vaselini Albi Purissimi . . . 3ij 100.0 Misce.

(d) Yellow Mercuric Oxide Ointment (Terson).

R Hydrargyri Oxidi Flavi, precipitat . gr. iss $\mathbf{1} \cdot \mathbf{5}$

Guaiacol Synthet. . . . . gr. j 0.5

Lanolin Purissimi . . . . . 3 iss $60^{\circ} 0$

Vaselini Albi Purissimi . . . $3 \mathrm{j} \quad 40^{\circ} 0$

Note.-The mercurial ointments are favourite applications in all forms of inflammation of the conjunctiva and of the edge of the eyelids. The salve containing the white precipitate is the mildest, and is useful in those cases when the others cause undue irritation. Children are more tolerant of the use of those ointments than are adults; all ointments containing mercury are more serviceable in inflammation of the bulbar conjunctiva than they are in palpebral conjunctivitis. The salve containing the yellow precipitate, commonly known as Pagenstecher's Ointment, is the strongest, and is specially recommended in pustular ophthalmia, and in old opacities of the cornea. The red oxide is the active ingredient in the popular remedy, "Singleton's Golden Eye Ointment."

\section{Orthoform Ointment.}
$\mathrm{B}$ Orthoformi . . . . . . gr. $\mathrm{xv} 15^{\circ} \mathrm{O}$ Unguenti Acidi Borici . . . . . ad 3 ij ad roo.o Misce.

Note.-Orthoform, obtained from the amido derivative of oxybenzoic acid, is a substitute for cocain, but it has little action on healthy mucous membrane. It is non-toxic and powerfully antiseptic. Useful in superficial ulceration of the cornea, as, on account of its sparing solubility, its action is maintained for several hours. 


\section{THERAPEUTIC NOTES AND FORMULE}

\section{Potassium Iodide Ointment.}

Re Potassii Iodidi . . . . . . gr. ij Vaselini Albi Purissimi . . $\quad \overline{3}^{\mathrm{ij}}$ Misce secundum artem.

Note.-This ointment, as well as those containing calomel and iodide of cadmium, dionin, etc., are used to promote absorption of opacities of the cornea after ulceration.

\section{Protargol Ointment.}

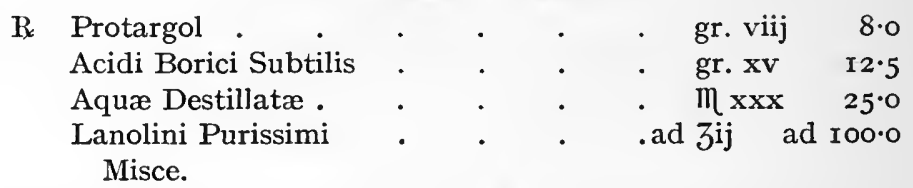

Note.-Useful in ophthalmia neonatorum, granular conjunctivitis, and serpiginous ulcer of the cornea, etc.

\section{Scarlet Red Ointment.}

\section{R Scarlet Red} (Michaelis Grübler)

Lanolin Purissimi . . . . 3iv $50 \cdot 0$

Vaselini Albi Purissimi " . . . ad $弓 j$ ad roo.o

Note.-To promote cicatrisation in ulceration of the cornea.

\section{Sulphur Ointment.}

R Sulphuris Precipitati . . . . gr. viij 8.0 Vaselini Albi Purissimi . . . ad 3 ij ad roo•o Misce.

Note.-Useful in phtheiriasis ciliorum and blepharitis marginalis.

\section{Suprarenalin Ointment.}

I Extracti Suprarenalis Siccalis . . $\quad$ gr. $\frac{1}{2} \quad 0.5$ Chloretone . . . . . . gr. vj 6.0 Vaselini Albi Purissimi . . . ad 3 ij ad roo.o Misce.

Note.-Suprarenalin is dried suprarenal. This ointment is used for the relief of red and irritable eyes. It may be combined with cocain gr. i.

\section{3. . Stavesacre Ointment}

Bc Olei Staphisagriæ . . . . $m_{l x} \mathbf{l} 2.5$ Adipis . . . . . . . . ad $\bar{j} \mathrm{j}$ ad roo.o Misce.

Note-Useful to destroy pediculi. Oil of sassafras, rubbed thoroughly into the roots of the hair, is even more efficacious. 
44. Thiosinamin Ointment.
R Thiosinamin . . . . . . . gr. xij Io.o
Vaselini Albi Purissimi . . . ad Zij ad Ioo•o
Misce.

Thiosinamin is " formed by warming oil of mustard with alcoholic solution of ammonia."

Note.-Useful to, clear up opacities of the cornea, owing to its power to dissolve scar tissue.

\section{Veratrin Ointment.}
$\mathrm{B}_{x}$ Veratrinæ . $\quad . \quad$. $\quad . . \quad$. $\quad . \quad$ gr. $\mathrm{xx} \quad 20 \cdot 0$
Adipis $\quad . \quad . \quad . \quad . \quad . \quad . a d ~ 3 i j \quad$ ad $100 \cdot 0$
Misce.

Note.-Applied to the forehead to relieve pain in iritis and more particularly in neuralgic affections of the eye.

This ointment is much stronger than that of the B.P., but it may be safely used when there is no abrasion of the skin.

\section{F. COLLUNARIA-NASAL DOUCHES.}

For nasal douches ten ounces of fluid are generally sufficient. The temperature, when they are injected into the nostrils, ought to be $95^{\circ}$ Fahrenheit, and care should be taken not to employ too much force, or to blow the nose for at least twenty minutes after the douche. In ophthalmic practice these douches are of service in phlyctenular conjunctivitis, and before operations on the eyeball, when there is much discharge from the nasal mucous membranes.

\section{Alkaline Douche (Simple).}
R Sodii Bicarbonatis

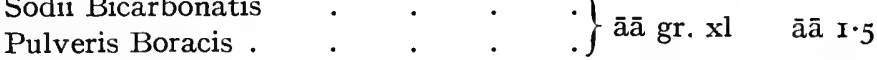

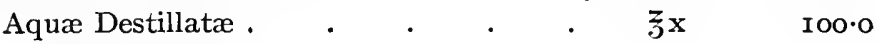
Solve et misce.

Note.-Cleansing alkaline douche.

\section{Alkaline Douche (Compound).}

R Sodii Bicarbonatis Pulveris Boracis

Sodii Chloridi Purissimi Sacchari Albi Aquæ Destillatæ

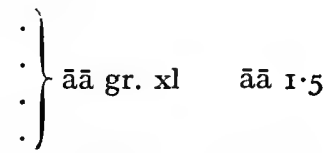
Solve et misce.

Note.-Cleansing alkaline douche. 


\section{Alkaline Antiseptic Douche.}

R Sodii Bicarbonatis

Pulveris Boracis

Glycerini Acidi Carbolici . . . $3 \mathrm{ij} \quad 3 \cdot 0$

Aquæ Destillatæ . . . . . ad $3 \mathrm{x}$ ad $100 \cdot 0$

Solve et misce.

Note.-Cleansing alkaline douche in cases where there is acrid, foul-smelling discharge from the nostrils.

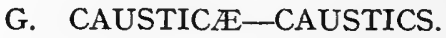

Caustics are substances which destroy the parts to which they are applied

1. Alum.

R. Aluminis Crystallini . . . . q.s.

Note.-Useful as a crayon in many forms of chronic hyperæmia of the palpebral conjunctiva. May be safely employed by the patient, or by a nurse.

\section{Chromic Acid.}

R Acidi Chromici • . . . . q.s.

Note.-Used in the treatment of morbid growths of the eyelids. The pure acid fused on the point of a probe is applied to the part to be destroyed.

\section{Copper.}

R Cupri Sulphatis . . . . . q.s.

Note.-Select a suitable crystal, and shape it into a point on fine emery paper. Mount in a quill or use in a port-crayon.

\section{Lapis Divinus (Cuprum Aluminatum).}

B Cupri Sulphatis Aluminis Potassii Nitratis .

..$\}$ partes æquales.

Fuse together in a glazed earthenware crucible, and then add camphor equal to one-thirtieth of the whole.

Note.-In using the sulphate of copper, or the lapis divinus, care should be taken to touch only the retrotarsal fold. The lapis divinus seems to give the patient more pain than the pure sulphate of copper. Employed in the treatment of granular ophthalmia and other forms of chronic conjunctivitis.

\section{Silver Nitrate.}

B. Crayon Argenti Nitratis.

Note.-May be used pure or mitigated by dilution with nitrate of potash. Nitrate of silver is to be preferred to sulphate of copper when there is much discharge from the conjunctiva. Before applying the nitrate of silver, the parts should be carefully dried, so that the action of the caustic may be limited as far as possible to the part touched. Any excess should be washed away at once with 
a solution of salt and water. Forms a convenient mode of blistering the skin of the eyelids in interstitial keratitis.

\section{Sodium Ethylate.}

\section{R Liquoris Sodii Ethylatis . . . q.s.}

Note.-Used in the treatment of nævi. It may be applied by means of a glass rod, the skin surrounding the nævus being carefully protected.

\section{Trichloracetic Acid.}

\section{R Acid Trichloraceti . . . - . q.s.}

Note.-Colourless diliquescent crystals, readily soluble in water and alcohol : a good escharotic, producing a dry adhering eschar, which is quickly thrown off with little secondary inflammation; one of the best applications after a chronic suppurating lachrymal sac has been scraped. It is applied thoroughly by means of a glass rod, and all excess is soaked up by a pad of absorbent cotton-wool.

\section{Zine Chloride Paste.}

R. Liquoris Zinci Chloridi č Opio . . そj I00.0 Farinæ Tritici . . . . . gr. cxx $25^{\circ} \mathrm{O}$

Note.-Mix smoothly in a mortar, and heat over a water-bath until of a proper consistence. The following is a formula for the chloride of zinc and opium liquor-

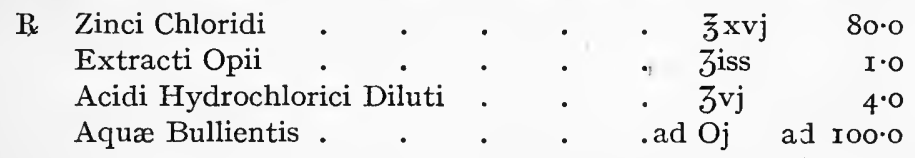

Triturate the extract of opium with twelve ounces of hot water, add the acid, and filter. In this dissolve the chloride of zinc, and make up the filtrate to twenty ounces with distilled water.

Note.-Used to destroy those portions of a malignant tumour of the orbit which have escaped excision by the knife. The paste should be spread on pieces of lint, which are to be smoothly applied to the walls of the orbit, care being taken to protect the eyelids.

\section{H. LINIMENTA-LINIMENTS.}

Liniments are not applied to the eye, but to the surrounding skin, and are commonly employed to relieve circumorbital pain.

\section{A.B.C. Liniment.}

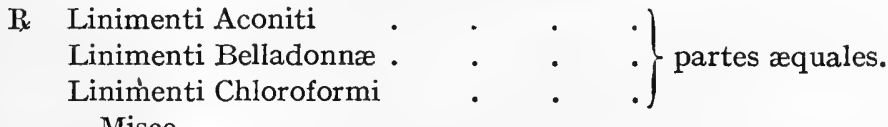

Note.-Useful to relieve circumorbital pain. 
2. Liniment of Ammonia.

$\mathrm{R}$ Liquoris Ammoniæ Fortis . • • $\quad \overline{3} \mathrm{j} \quad 25^{\circ} \mathrm{O}$ Olei Lavandulæ . $\quad . \quad$. $\quad . \quad 3 \mathrm{j} \quad 3 \cdot 0$ Spiritûs Rectificati . . . . . ad ऊiv ad roo.o Misce.

Note.-Useful to relieve pain after bites or stings of insects. A saturated solution of aloes in eau de cologne is almost a specific for bites or stings of insects, if applied at the time, but its dark colour and disagreeable smell are drawbacks to its use.

\section{Liniment of Extract of Belladonnæ.}

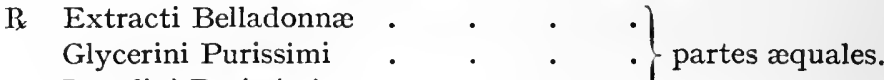

Lanolini Purissimi Misce.

Note.-When applied to the brow affords great relief in purulent ulcers of the cornea, iritis, etc. This method of treatment sometimes causes the pupil to dilate after atropin instillations have failed.

\section{Liniment of Belladonna with Chloroform.}

R Linimenti Belladonnæ . • • . $\quad \mathrm{j}_{\mathrm{ij}} 5^{\circ} \mathrm{\circ}$ Chloroformi Belladonnæ . . . . ad $\lesssim$ iv ad ıoo.o Misce.

Note.-Used to relieve circumorbital pain.

\section{Liniment of Iodine.}

R Tincturæ Iodi Fortis . . . . q.s.

Note.-Used as a counter-irritant.

\section{Liniment of Lime.}

$\mathrm{R}_{\mathrm{x}}$ Liquoris Calcis . $\quad . \quad$. $\quad . \quad$. $\quad \overline{3} \mathrm{ij} \quad 50 \cdot 0$ Olei Olivæ Optimi $\quad . \quad$. . . . ad $\bar{z}$ iv ad Ioo.o Misce.

Note.-Used as a dressing in burns and scalds of the eyelids.

7. Liniment of Menthol, Chloral, and Camphor.

R Chloral Hydratis

Menthol Purissimi $\quad . \quad \cdot \quad \cdot \quad \cdot \quad \cdot \quad$ partes æquales.

Camphor Purissimi

Note.-A valuable application painted on painful parts in neuralgia and rheumatism.

8. Liniment of Soap and Carbolic Acid (Carbosapol).

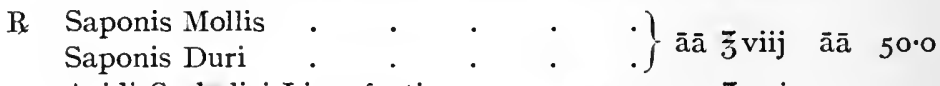

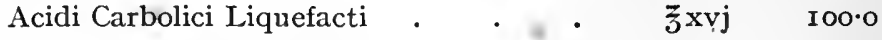

Misce secundum artem.

Note.-A valuable cleansing agent; used to disinfect the skin of the face before operations. 


\section{J. OPHTHALMIC TABLOIDS.}

All the drugs usually employed in ophthalmic practice are now to be had in the form of minute tabloids or gelatine discs readily soluble in the lachrymal secretion. In this form they are very convenient, as they can be easily carried about, and the dose administered with great accuracy. The country doctor is advised to have a selection of ophthalmic tabloids always at hand, because in an emergency it may be difficult to get the drugs he requires freshly dispensed.

\section{K. 'DUSTING POWDERS.}

Dusting powders are flicked into the eye from a dry camel-hair brush.

\section{Boric Acid.}

R Acidi Borici Subtilis . . . . q.s.

\section{Borotannic Powder.}

R Pulveris Acidi Borici Calendulati . . partes iij Pulveris Acidi Tannici . . . partem j Misce.

Note.-Useful in chronic conjunctivitis.

\section{Calendulated Boric Acid Powder.}

R Tincturæ Calendulæ Florum B.P.C. . „ „ij $50 \cdot 0$ Evaporate Sec. Art., and add Pulveris Acidi Borici Subtilliss. . . . $う \mathrm{iv}$ I00.0 Misce.

The essential oils, e.g. cajuput, neroli, fennel, or otto of roses, may be added . to the powder in the proportions of 2 minims to the $\xi i$.

Note.-Useful in all forms of conjunctivitis. A small teaspoonful of the powder to be dissolved in a tea-cupful of warm water, and used as an eye douche.

\section{Calomel.}

\section{R Hydrargyri Subchloridi . . . q.s.}

Note.-Useful in phlyctenular conjunctivitis, and in all cases of passive congestion of the conjunctiva; ought not to be used when iodides or bromides are being administered internally. It must be thoroughly dried.

\section{Iodoform.}

R Iodoformi Præcipitati . . . q.s.

Note.-Useful in sloughy corneal ulcers (the surface of which should be carefully dusted with the powder), and in massage of the conjunctiva in granular ophthalmia. Crystalline iodoform should never be used in ophthalmic practice.

G G 


\section{Iodoform and Sugar of Milk.}

R Iodoformi Præcipitati . . . . partem $\mathbf{j}$ Sacchari Lactis . . . . . partes $i j$

Note.-Useful in the fleshy condition of the conjunctiva which frequently follows purulent ophthalmia.

\section{Orthoform.}

\section{$\mathrm{R}$ Orthoformi . . . . . . q.s.}

Note.-Local anæsthetic as well as a powerful antiseptic; it acts better when diluted with boric acid. It may also be combined with dried calomel, which intensifies its antiseptic properties.

\section{SUB-CONJUNCTIVAL INJECTIONS.}

For the purpose of controlling and arresting infection some have advocated the injection of weak solutions of mercurial salts beneath the conjunctiva, on the ground that the nearer to the site of the disease the remedy is applied the more likely is it to prove effective. My own experience of this method in the treatment of suppurative keratitis, or of infective iridocyclitis, does not warrant me in speaking strongly in its favour; but in some diseases-in choroiditis, for example, accompanying high myopia, and in detachment of the retina where the usual remedies have but little effect-I have obtained very encouraging results from the use of sub-conjunctival injections. The fluid I usually employ is 8 per cent. chloride of sodium in a $I$ in 2000 solution of bicyanide of mercury, 5 to 20 minims being injected slowly beneath the conjunctiva, which, as the fluid enters, rises in a bleb. The great drawback to this method of treatment is its exceeding painfulness, but the suffering can be largely mitigated by using only chemically pure chloride of sodium, and by adding a few drops of a 2 per cent. solution of alypin or of a I per cent. solution of acoin to each dose immediately before injection. A pledget of cotton-wool soaked in ether and inserted into the nostrils gives instantaneous relief, but the pain may return later, and when it does a repetition of the, ether application is not nearly so successful.

\section{THE APPLICATION OF HEAT.}

The application of heat is of great value in all inflammations of the cornea, sclerotic, iris, ciliary body, etc. The effects vary according as the heat employed is dry or moist.

(a) Dry heat is applied to the eye by means of masses of heated cotton-wool frequently changed, by a thermophore, by a Japanese muff-warmer, or by the electric heater devised by Maddox. The action is directly stimulant, and is consequently of great service in cases where the vitality is low-e.g. in rapidly progressive ulceration of the cornea, and in the later stages of purulent cyclitis.

(b) Moist heat is usually applied by means of fomentations. It diminishes the engorgement of the blood-vessels, and so, by reducing tension, relieves pain in all acute inflammations and suppurations. Unless the case be a very severe one, moist heat should be applied not continuously, but three or four times daily for periods of from half an hour to an hour. Between the applications the eye must be protected by a pad of cotton-wool kept in position by a bandage. In very 
acute and deep-seated inflammations-e.g. subacute glaucoma-the heat must be as great as can be borne, and the application kept up unremittingly.

Boracic acid fomentations.-A double layer of sterilised boracic lint, $3 \frac{1}{2}$ in. by $3 \frac{1}{2}$ in., is laid on a sterilised towel in a basin, and boiling water, to which a solution of carbolic acid ( $I$ in 20 ) has been addéd in the proportion of about four to six drachms to the pint-just sufficient to give an odour of phenol to the fomentation-is poured over it. "The lint is then squeezed as dry as possible by wringing it in the towel, applied over the closed eyelids, and covered by a piece of jaconet or oiled silk $3 \frac{3}{4}$ in. by $3 \frac{3}{4}$ in. Over this is next placed a thick layer of absorbent cotton-wool, which is kept in position by a roller bandage applied as lightly as possible. When pain is very severe, fomentations are used every two or three hours, day and night; but as soon as the pain begins to abate, they are applied only morning, noon, and evening, and during the night and early morning if the pain be sufficiently severe to prevent the patient from sleeping.

Corrosive sublimate fomentations are used of the strength of $\mathrm{x}$ in 5000 , and are prepared and applied in a manner similar to that just described. They are used, in cases of accident and of sloughy ulcer, to allay pain and to render the eyelids and surrounding parts as clean and aseptic as possible previous to operation.

Chamomile fomentations are prepared by boiling the flowers mixed with poppy heads (both should be rubbed down into a fine flour) for a quarter of an hour in a saturated solution of boracic acid. The infusion is then strained through sterilised muslin, and a fomentation prepared and applied to the eye in a manner similar to that already described.

\section{THE APPLICATION OF COLD.}

The application of cold is of great value in subduing pain immediately after accidental injuries to, or surgical operations upon, the eye; at the outset of all acute inflammations of the conjunctiva; and in some cases of iritis; when the pain is deep-seated, throbbing, and markedly nocturnal in character. The applications are generally made three or four times daily for periods varying from a quarter of an hour to half an hour, the methods most frequently employed being as follows-

(a) An ice bag.-A small bag is made of jaconet or gutta-percha tissue, filled with chips of ice, sealed carefully, and laid over the dressing, care being taken that the patient experiences no discomfort from the weight of the application.

(b) Iced compresses.-A lump of ice is allowed to float in a solution of boracic acid, and cotton-wool sponges are dipped in this and applied directly to the closed eyelids, or the dressing is kept constantly wet by the iced water. Care must be taken that, in thus applying an iced compress, no septic material comes in contact with the eye.

(c) Leiter's tubes.-These consist of a flat spiral lead coil made to fit over the eye, and connected with two india-rubber tubes. The eye is protected by a moist compress, over which the lead coil is accurately adjusted. One of the rubber tubes is then placed in a jugful of water raised above the patient's head, when a stream of water runs through the apparatus, and is conveyed by the other rubber tube into a basin placed below the bed. These tubes may be used for hot as well as for cold applications. 


\section{BLOOD-LETTING.}

General blood-letting is very rarely employed, but the local abstraction of blood is of great service in relieving pain in all acute inflammations of the uveal tract-e.g. iritis, cyclitis, choroiditis, etc. Local blood-letting is usually carried out either by natural leeches or by Heurteloup's artificial leech. Leeches are best applied round the external canthus, or over the mastoid region. Cleanse the part thoroughly, then take the leech in a narrow tube and place it over the spot, which may require to be moistened with a drop of milk to induce the leech to suck. It should be allowed to quit its hold of its own accord. If the subsequent bleeding be excessive, it may be checked by pressure over a pad of absorbent cotton-wool dusted with powdered alum or soaked in adrenalin solution. The amount of blood which should be withdrawn will vary according to the individual case, but, generally speaking, the bleeding should be continued until the pain is relieved. The patient, for some hours after the leeching, should be kept in bed in a dark room, and, if necessary, the bleeding encouraged by the application of fomentations. In acute iritis the pupil will often yield to the influence of atropin immediately after blood-letting, though previously the drug had been inefficacious, or had even caused increased irritation.

\section{COUNTER-IRRITATION.}

Counter-irritants are useful in many eye diseases, but must always be employed with caution, especially in dealing with children or elderly persons, lest they cause severe inflammation of the skin, or a sore which may prove very difficult to heal. They may be used as liniments or ointments, as blisters or setons. The liniments and ointments are useful in relieving photophobia due to inflammation of the cornea, sclerotic, or iris; but in the treatment of deep-seated inflammatory diseases of the eye, more especially when due to syphilis, better results are obtained from the use of an open blister, a seton, or a fixation abscess. Should a blister be employed, it is usually applied to the temporal region or over the mastoid process. The ordinary cantharidis plaster may be used, but canthos or Smith's blistering fluid will generally be found more convenient. If the object of the blister be to. relieve pain, its efficacy is greatly increased by dressing the raw surface with an ointment of cocain and morphin; but if continuous counter-irritation be desired, the best dressing is d'Albespeyre's No. 2 Papier Epispastique.

Carbon dioxide snow, radium, and the Rontgen rays have been employed in the treatment of malignant affections of the eye, rodent ulcer, nævus, trachoma, Spring catarrh, etc. Brilliant results have been recorded by different observers, but these methods of treatment require expert knowledge and considerable experience, and should be carried out by those who have made a special study of the subject.

In the treatment of diphtheritic conjunctivitis antitoxic serum is indispensable, but serum therapy and the use of vaccines in the treatment of eye diseases must be acknowledged to be still in the experimental stage. Occasionally their use is followed by most gratifying and remarkable results, but too frequently they fail to maintain that brilliant promise. The use of sterilised cows' milk has been advocated, and in certain cases has seemed to convey the stimulus required to 
promote recovery, but in this case also failure is commoner than success. Autogenous vaccines undoubtedly are always to be preferred, if obtainable, but in eye diseases where the tissues are so delicate and vulnerable time can ill be spared for their preparation. Accordingly, where this line of treatment is indicated recourse should be had, in the first instance, to a stock vaccine.

\section{CONSTITUTIONAI, REMEDIES.}

On account of their special application, local remedies naturally occupy a foremost place in ocular therapeutics, but as diseases of the eye are, in most instances, related to some constitutional condition, the general treatment of the patient must never be neglected. Indeed, in syphilis, tubercle, rheumatism, or gout, constitutional remedies are as important as local applications

\section{A. MISTURÆ-MIXTURES.}

(a) Alteratives.-This group includes such remedies as arsenic, calcium, mercury, and the iodides.

\section{Ammonium and Iron Iodide Mixture.}

$\mathrm{R}$

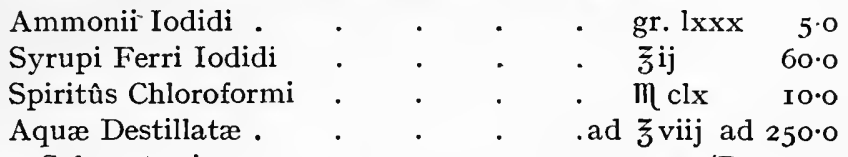

Solve et misce.

(Dose, 15 mils.)

Dose.-A tablespoonful in water three times a day after meals.

Note.-Useful in diseases of the uveal tract in old and anæmic subjects.

\section{Arsenical Mixture.}

$\mathrm{B}_{x}$ Liquoris Sodii Arsenatis . .

Glycerini Purissimi $\quad . \quad 3 j \quad 4.0$

Aquæ Destillatæ . $\quad . \quad$. $\quad . \quad$ ad 3 viij ad $250^{\circ} 0$
Misce.
(Dose, I 5 mils.)

Dose.-A tablespoonful in water three times a day after meals.

Note.-Useful in many diseases of the cornea.

\section{Calcium Chloride Mixture.}

$\mathrm{R}$

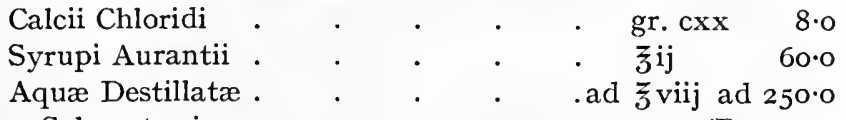

Solve et misce.

(Dose, 15 mils.)

Dose.-A tablespoonful in water three times a day after meals.

Note.-Useful in phlyctenular conjunctivitis with enlargement of the lymphatic glands. Tincture of perchloride of iron can be added to the calcium chloride in cases where there is anæmia (see Formula 4 ). 


\section{THERAPEUTIC NOTES AND FORMULÆ}

\section{4.' Calcium Chloride and Iron Mixture.}

$\mathrm{B}$

Calcii Chloridi
Tincturæ Ferri

Aquæ Chloroformi . . . . . ad $\overline{3}$ viij ad $250 \cdot 0$

Solve et misce.
(Dose, 15 mils)

Dose.-A tablespoonful in water three times a day after meals.

\section{Mercuric Chloride Mixture.}

P. Liquoris Hydrargyri Perchloridi P.B. . $\quad \bar{j} \mathrm{ij} \quad 60 \cdot 0$ Infusi Gentianæ Compositi . . . . ad $\bar{\jmath}$ viij ad 250・0
Misce.
(Dose, $x_{5}$ mils.)

Dose.-A tablespoonful in water three times a day after meals.

Note.-Useful in syphilitic diseases of the eye.

\section{Mercuric Iodide Mixture.}

R Hydrargyri Perchloridi . . . gr. j 0.06 Potassii Iodidi . . . . . gr. xlviij $3 \cdot 20$ Infusi Gentianæ Compositi . . . . ad 弓viij ad 250.00

Solve et misce secundum artem. (Dose, 15 mils.)

Dose.-A tablespoonful in water three times a day between meals.

Note.-A valuable combination in all chronic affections of the uveal tract, more especially when of syphilitic origin.

\section{Potassium Iodide Mixture.}

R Potassii Iodidi . . . . . . $3 \mathrm{ij} \quad 8.0$ Spiritûs Chloroformi . . . . . $m$ clx 10.0 Aquæ Menthæ Piperitæ . . . . ad うviij ad $250 \cdot 0$
Solve et misce.
(Dose, I 5 mils.)

Dose.-A tablespoonful in water three times a day between meals.

Note.-Useful in the late stages of syphilis.

\section{Potassium Iodide and Acetate Mixture.}

$\mathrm{P}$

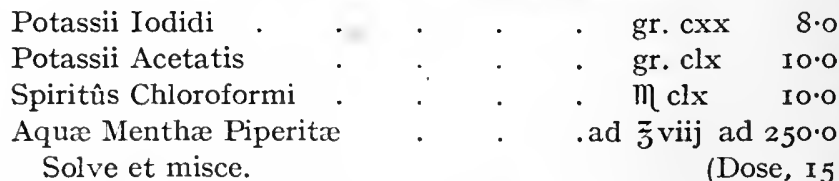

Dose.-A tablespoonful in water three times a day between meals.

Note.-In all mixtures containing iodide of potassium the addition of pepsencia seems to be helpful in promoting the assimilation of the drug and in preventing iodism. 


\section{Potassium Iodide and Sodium Bicarbonate Mixture.}

. gr. clx 10.0

. . $m$ clx $10 \cdot 0$

Aquæ Menthæ Piperitæ . . . . ad jviij ad $250 \cdot 0$

Solve et misce.

(Dose, I 5 mils.)

Dose.-A tablespoonful in water three times a day between meals.

\section{Syrup of Iodide of Iron.}

R Syrupi Ferri Iodidi . . . . q.s.

Dose.-A teaspoonful or less, according to age.

Note.-A convenient form of administering an iodide.

(b) Alterative and Sedative.

11. Potassium Iodide and Bromide Mixture.

R Potassi Iodidi

Potassii Bromidi

Spiritûs Chloroformi . . . . 3iij $12 \cdot 0$

Decocti Sarsæ Compositi . . . . ad 弓viij ad $250 \cdot 0$

Solve et misce.

(Dose, 15 mils.)

Dose.-A tablespoonful in water three times a day after meals (see Note under No. 13).

(c) Alterative and Tonic.

12. Mercuric Chloride, Quinine, and Iron Mixture.

$\mathrm{R}_{6}$ Liquoris Hydrargyri Perchloridi, B.P. . $\quad \mathrm{z}_{\mathrm{ij}} \quad 60 \cdot 0$ Quininæ Sulphatis . . . . gr. viij 0.5

Tincturæ Ferri Perchloridi . . . M lxxx $5 \cdot 0$

Glycerini Purissimi . . . . 3iij $12 \cdot 0$

Aquæ Chloroformi . . . . . ad $弓 v i i j ~ a d ~ 250 \cdot 0$

Solve et misce. (Dose, 15 mils.)

Dose.-A tablespoonful in water three times a day after meals.

Note.-Valuable combination in the late stages of interstitial keratitis, and in chronic diseases of the deep structures of the eye, especially when these are due to syphilis.

\section{Potassium Iodide with Nux Vomica Mixture.}

R Potassii Iodidi . . . . . gr. xlviij $3 \cdot 2$

Tincturæ Nucis Vomicæ . . $m \operatorname{lxxx} 5 \cdot 0$

Spiritûs Chloroformi . . . . m clx ro.0

Decocti Sarsæ Compositi . . . . ad ऊviij ad 250.0

Solve et misce.

(Dose, 15 mils.)

Dose.-A tablespoonful in water three times a day after meals.

Note.-This mixture and Formula No. I I are of use in inflammation of the deep structures of the eyeball, especially when due to syphilis. 
(d) Antiseptic.

14. Sodium Sulphocarbolate Mixture.

R Sodii Sulphocarbolatis . . . . 3iv

Tincturæ Zingiberis . . . . 3iij $12 \cdot 0$

Syrupi Zingiberis . . . . . $\quad$. $3 \mathrm{ij} \quad 60 \cdot 0$

Aquæ Menthæ Piperitæ . . . . ad گ̌ viij ad $250 \cdot 0$
Solve et misce.
(Dose, 15 mils.)

Dose.-A tablespoonful in water three or four times a day as required.

Note.-Used to relieve flatulence, which often causes great trouble after operation, in cases where it is necessary for the patient to lie on his back. In such circumstances this mixture is usually most helpful in removing the discomfort.

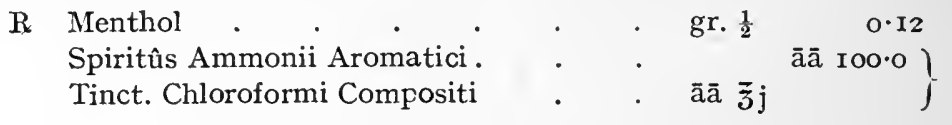

Dose.-A teaspoonful in water when required.

(Dose, 4 mils.)

Note.-Is a very valuable prescription, but the best remedy for the relief of flatulence after operations is 2 minims of oil of cajuput, in a teaspoonful of brandy.

(e) Diuretic.

15. Potassium Acetate and Iron Perchloride Mixture (Basham).

R Potassii Acetatis . . . . gr. cl Io.o

Tincturæ Ferri Perchloridi . . . 3ij $8 \cdot 0$

Liquoris Ammonii Acetatis . . . $\bar{z}$ iss $45^{\circ} \mathrm{O}$

Spiritûs Chloroformi . . . . . 3ij $8 \cdot 0$

Glycerini Purissimi . . . . 3vj $24{ }^{\circ}$

Aquæ . . . . . . . ad う ₹iij ad $250^{\circ} 0$

Solve et misce.
(Dose, $\mathrm{I}_{5}$ mils.)

Dose.-A tablespoonful in water three times a day.

Note.-Used when a chalybeate is to be given along with an eliminant.

\section{Potassium and Caffein Mixture.}

R Potassi Citratis . . . . . $3 \mathrm{v}$

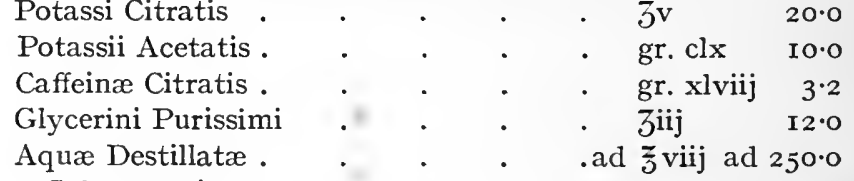

Solve et misce.
(Dose, I 5 mils.)

Dose.-A tablespoonful in water three times a day.

Note.-Promotes elimination. Useful in cases of toxic amblyopia, and in all ocular diseases accompanied by renal inadequacy.

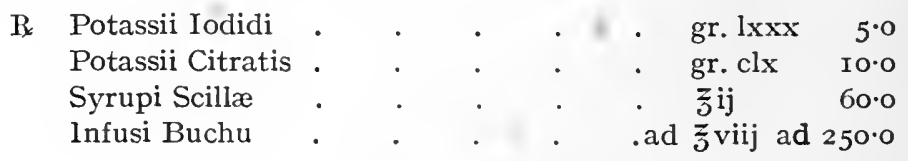


R Potassii Acetatis

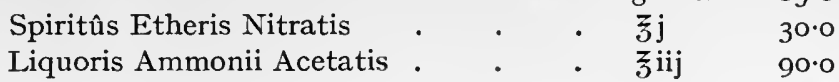

Aquæ Chloroformi . . . . . ad $う$ viij ad $250^{\circ} 0$

Note.-Probably the most reliable diuretic is theobromine in ro to 15 grain doses three times a day. It may be prescribed in cachets and given along with any of the three mixtures the prescription for which is given above.

(f) Saline.-This group includes the salts of magnesium and sodium, and their chief physiological action is to unload the portal circulation.

\section{Magnesium Sulphate Mixture.}

$\mathrm{R}$ Magnesii Sulphatis . . . . $\quad \tilde{z}$

Sodii Sulphatis . . . . . 3iv ${ }_{5} 5^{\circ}$

Acidi Sulphurici Diluti . . . 3 iij $12 \cdot 0$

Glycerini Purissimi . . . . 3iv 15.0

Aquæ Menthæ Piperitæ . . . . ad ろviij ad 250.0

Solve et misce.

(Dose, 15 mils.)

Dose.-A tablespoonful in water two or three times a day before food.

Note.-Mild aperient.

\section{Magnesium and Ferrous Sulphate Mixture.}

R Magnesii Sulphatis . . . . $j_{j} 30^{\circ} 0$

Ferri Sulphatis . . . . . gr. xlviij $3 \cdot 2$

Sodii Sulphatis . . . . . 3iv 15.0

Acidi Sulphurici Diluti . . . 3 iij $12 \cdot 0$

Glycerini Purissimi . . . . Jiv $15 \cdot 0$

Aquæ Menthæ Piperitæ . . . . ad $ろ$ viij ad $250^{\circ} \circ$

Solve et misce.

(Dose, 15 mils.)

Dose.-A tablespoonful in water two or three times a day before food.

Note.-A valuable saline and chalybeate.

19. Magnesium Sulphate and Quinine Mixture.

$\mathrm{R}_{k}$ Magnesii Sulphatis . . . . $\overline{3} \mathrm{j} \quad 30^{\circ} \mathrm{O}$

Quininæ Sulphatis . . . . gr. xvj $\mathrm{I} \bullet$

Acidi Sulphurici Diluti . . . J jiij $\quad$ I2·O

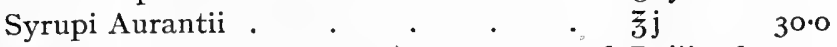

Aquæ Chloroformi . . . $\quad$ ad $\zeta$ viij ad $250^{\circ} \circ$

Solve et misce. (Dose, 15 mils.)

Dose.-A tablespoonful in water two or three times a day before food.

Note.-Tonic and aperient. 


\section{THERAPEUTIC NOTES AND FORMULA}

\section{Magnesium Sulphate, Quinine, and Iron Mixture.}

R Magnesii Sulphatis

Quininæ Sulphatis

Ferri Sulphatis

Acidi Sulphurici Diluti

Glycerini Purissimi

Aquæ Menthæ Piperitæ

Solve et misce.

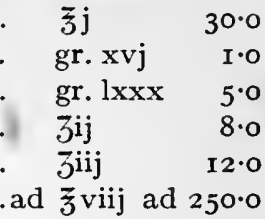

Dose.-A tablespoonful in water three times a day before food.

Note.-Useful in chronic conjunctivitis, in asthenopia associated with anæmia, and as a general tonic.

21. Magnesium Sulphate and Sodium Bicarbonate Mixture.

$\mathrm{R}_{\mathrm{k}}$ Magnesii Sulphatis . . . . $\mathrm{j}_{\mathrm{j}} 30 \cdot 0$

Sodii Bicarbonatis . . . . Jiiss I0.0

Spiritûs Chloroformi . . . . $\quad$.

Aquæ Menthæ Piperitæ . . . . ad J $\operatorname{vij}$ ad $250 \cdot 0$

Solve et misce. (Dose, 15 mils.)
(D)

Dose. - A tablespoonful in water three times a day before food.

Note:-Saline and antacid.

\section{Sodium Salicylate and Aloes Mixture.}

$\mathrm{R}_{\mathrm{k}}$ Sodii Salicylatis . . . . . $\operatorname{gr} . \operatorname{lxxx} 55^{\circ}$

Sodii Sulphatis . . . . . gr. ccclx $22 \cdot 5$

Potassii Bicarbonatis . . . . gr. clx IO॰O

Glycerini Purissimi . . . . 3iv $15 \cdot 0$

Decocti Aloes Compositi . . . . ad 弓̄viij ad $250 \cdot 0$

Solve et misce.

(Dose, 15 mils.)

Dose.-A tablespoonful in water three times a day. tract.

Note.-To relieve the portal system in congestive inflammations of the uveal

23. Sodium Salicylate and Perchloride of Iron Mixture.

iv Sodii Salicylatis . . . . . gr. lx $4^{\circ} 0$

Aquæ Destillatæ . . . . . $\quad \overline{3}^{\mathrm{ij}} 60 \cdot 0$, solve.

Potassii Bicarbonatis . . . . . gr. xc $6 \cdot 0$

Aquæ Destillatæ. . . . . . $\quad \overline{3}_{\mathrm{ij}} 60 \cdot 0$, solve.

Tincturæ Ferri Perchloridi . . . $3 \mathrm{j} \quad 4 \cdot 0$

Aquæ Chloroformi . . . . ad $\overline{3}$ viij ad $250 \cdot 0$

Dose.-A tablespoonful in water three times a day after meals. (Dose, I5 mils.)

Note.-Useful in affections of the uveal tract accompanied by pains in other parts of the body usually spoken of as rheumatic. 


\section{THERAPEUTIC NOTES AND FORMULA}

24. Sodium Salicylate and Antipyrin Mixture.

R Sodii Salicylatis .

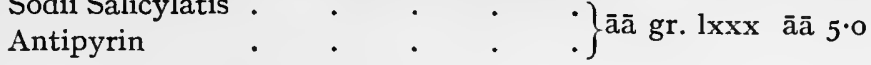

Tincturæ Chloroformi Compositi . . 3ij $8 \cdot 0$

Aquæ Menthæ Piperitæ . . ad $\zeta$ viij ad $250^{\circ} 0$

(Dose, I 5 mils.)

Dose.-A tablespoonful in water every quarter of an hour until pain is relieved. Not more than three doses to be taken.

- Note.-A valuable combination in neuralgic pain accompanying eye strain.

Giffiord advises 30 grains of Salicylate of Soda to be given five times a day dissolved in half a tumblerful of water to which two teaspoonfuls of brandy are added in cases of Sympathetic Ophthalmia. If the drug disorders the stomach, 60 grains are given per rectum two or three times a day This treatment is always combined with mercurial inunction.

\section{Sodium Sulphate and Iron Mixture.}

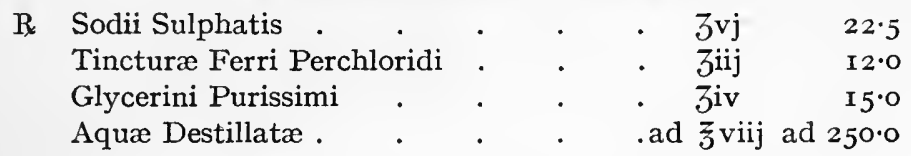

$$
\text { Solve et misce. } \quad \text { (Dose, } 15 \text { ^mils.) }
$$

Dose.-A tablespoonful in water three times a day between meals.

Note.-Saline and chalybeate.

(g) Sedative.-This group of remedies is of great service in ophthalmic practice, for overcoming nervousness, subduing pain, and promoting sleep.

26. Bromidia (Battle).

$\mathbf{R}$ Bromidix . . . . . . q.s.

Dose.-A teaspoonful as required, and repeated as directed. '

Note.-Useful as a sedative after operations.

\section{Triple Bromide Mixture.}

R Potassii Bromidi

$15^{\circ}$

Sodii Bromidi . . . . . gr. clx Io.0

Ammonii Bromidi . . . . gr. $\operatorname{lxxx} 5.0$

Spiritûs Chloroformi . . . . Jiij I $2 \cdot 0$

Aquæ Destillatæ. . . . . . ad そviij ad $250 \cdot 0$

Solve et misce. (Dose, 15 mils.)
(D)

Dose.-A tablespoonful in water as directed.

Note.-Sedative. Useful as a soothing draught previous to operations.

Sedobrol is a convenient form in which to administer bromides. 


\section{THERAPEUTIC NOTES AND FORMULE}

\section{Chloral Mixture.}

R Syrupi Chloral Hydratis

Tincturæ Chloroformi Compositi . . $m x$ 0.6

Nepenthe (Ferris) . . . . . $m_{v} 0.3$

Aquæ Destillatæ . . . . $\quad$. ad $\tilde{\jmath} \mathrm{j} \quad$ ad $30^{\circ} 0$

Solve et misce, fiat haustus. (Dose, 30 mils.)

Dose.-The draught to be taken in water at bedtime.

Note.-A reliable sleeping draught.

29. Gelsemium and Butyl-Chloral Hydrate Mixture.

$\mathrm{R}$ Tincturæ Gelsemii . . . . 3iiss 10.0

Butyl-Chloral Hydratis . . . gr. clx 10.0

Spiritûs Rectificati . . . . . そj $30 \cdot 0$

Syrupi Tolutani . . . . . . $\quad 3 i j \quad 60 \cdot 0$

Aquæ Chloroformi . . . . ad ऊviij ad $250 \cdot 0$

Solve et misce. (Dose, $\mathrm{I}_{5}$ mils.)

Dose.-A tablespoonful in water, and repeated in an hour if pain is not relieved. If a third dose be required this mixţure is not likely to prove serviceable.

Note.-Valuable combination in cases where pain radiates from the eye along the branches of the fifth cranial nerve, and more especially when the supramaxillary branches are affected.

(h) Tonic.-This group, including such important remedies as iron, quinine, a nd strychnin, should be employed in the case of anæmic and debilitated patients.

30. Compound Syrup of the Hypophosphites.

R Syrupi Hypophosphitum Compositi - q.s.

Dose.-A teaspoonful in water three times a day before food.

31. Compound Syrup of the Phosphate of Iron (Parrish).

R. Syrupi Ferri Phosphatis Compositi - q.s.

Dose.-A teaspoonful in water three times a day after meals.

Note.-When combined in equal parts, these two syrups form a valuable tonic.

\section{Quinine and Iron Citrate Mixture.}

$\mathrm{P}_{x} \quad$ Ferri et Quininæ Citratis . . . gr. clx ro॰o Aquæ Chloroformi . . . . ऊ ऊiij ad $250 \cdot 0$
Solve.
(Dose, 15 mils.)

Dose.-A tablespoonful in water three times a day before food.

Note.-General tonic. 


\section{THERAPEUTIC NOTES AND FORMULA}

33. Quinine, Iron, and Strychnin Mixture.

R Quininæ Sulphatis . . . . gr. xvj I

Tincturæ Ferri Perchloridi . . . Jiiss Io.0

Liquoris Strychninæ Hydrochloridi ' . M xlviij $3 \cdot 2$

Glycerini Purissimi . . . . $\overline{3}$ ss I $5^{\circ} \mathrm{O}$

Aquæ Destillatæ . . . . . ad ̋̋viij ad $250 \cdot 0$

Solve et misce.

(Dose, I 5 mils.)

Dose.-A tablespoonful in water three times a day before food.

Note.-General tonic.

\section{Quinine Sulphate Mixture.}

R Quininæ Sulphatis

Spiritûs Rectificati . . . . . $\quad$ ३ j $30 \cdot 0$

Syrupi Limonis . . . . . . $\quad$. 3 ij $60 \cdot 0$

Aquæ Destillatæ . . . . . . ad $ろ$ viij ad $250 \cdot 0$

Solve et misce. (Dose, 15 mils.)

Dose.-A tablespoonful in water three times a day before food.

Note.-Quinine is useful not only as a general tonic, but when administered at regular intervals in doses of from one to three grains, it appears to have a specific influence in overcoming suppuration.

The following is a convenient formula for prescribing quinine in an effervescent mixtureNo. I.

Px Quininæ Hydrochloridi

$\begin{array}{lr}\text { gr. xxx } & 2 \cdot 0 \\ \text { gr. cxx } & 8 \cdot 0 \\ \zeta^{3} \mathrm{ij} & 60 \cdot 0 \\ \text { ad } \stackrel{3}{\zeta} \mathrm{vj} \text { ad } \mathrm{r} 80 \cdot 0\end{array}$

Syrupi Limonis . . . . . $\quad$. $3 \mathrm{ij} \quad 60 \cdot 0$

Aquæ Destillatæ. . . . . ad $\overline{3} \mathrm{vj}$ ad $\mathrm{r} 8 \mathrm{o} \cdot \mathrm{o}$

No. 2.

R Potassii Bicarbonatis . . . . gr. $\mathrm{cxx} 8 \cdot 0$

Ammonii Carbonatis . . . . gr. $x x x \quad 2 \cdot 0$

Tincturæ Chloroformi Compositi . . 3ij $8 \cdot 0$

Aquæ Destillatæ . . . . . . ad $3 x i j ~ a d ~ 360 \cdot 0$

Dose.-Two tablespoonfuls of No. 2 added to one tablespoonful of No. I three times a day between meals. (Dose, 30 mils of No. 2 added to 15 mils of No. I.)

\section{Strychnin Hydrochloride and Strophanthus Mixture.}

R Liquoris Strychninæ Hydrochloridi Tincturæ Strophanthi .

. $\} \bar{a} \bar{a} \ln \operatorname{lxxx}$ āā $5 \cdot 0$ Glycerini Purissimi . . . . . $\quad$. $\bar{j} \quad 30 \cdot 0$ Aquæ Destillatæ . . . . . ad $ろ$ viij ad $250^{\circ} 0$ Misce.

(Dose, 15 mils.)

Dose.-A tablespoonful in water three times a day.

Note.-Useful in some forms of degeneration of the optic nerve, and in incipient chronic glaucoma. 


\section{Syrup of the Lactophosphate of Lime (Dusart).}

R Syrupi Calcii Lactophosphatis . . q.s.

Dose.-A teaspoonful in water three times a day after food.

Note.-Valuable tonic in many cases of phlyctenular conjunctivitis in children. after digestion improves and the tongue is clean.

\section{Syrup of Phosphate of Iron with Quinine and Strychnin.}

R. Syrupi Ferri Phosphatis cum Quininâ et

Strychninâ . • • . . q.s.

Dose.-A half to one teaspoonful in water three times a day before meals.

38. Trinitrin and Strychnin Mixture.

$\mathrm{B}_{\mathrm{k}}$ Liquoris Trinitrini . . . . $\mathrm{m}_{\mathrm{xvj}} \mathrm{I} \cdot \mathrm{O}$

Liquoris Strychninæ Hydrochloridi . Mlxxx 5.0

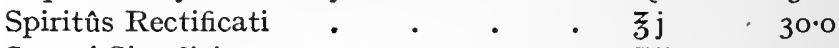

Syrupi Simplicis . . . . . $\quad z^{\mathrm{ij}} 60 \cdot 0$

Aquæ Destillatæ . . . . . . ad $\overline{3}$ viij ad $250 \cdot 0$

Misce. (Dose, 15 mils.)

Dose.-A tablespoonful in water three times a day before food.

Note.-Used in embolism and thrombosis of the retinal vessels and in atrophy of the optic nerve.

(i) Tonic and Sedative.

39. Aconite and Quinine Mixture.

R Tincturæ Aconiti (Fleming) . . . $m x v j \quad I \cdot O$

Quininæ Hydrobromidi . . . gr. xvj $\mathrm{I} \cdot 0$

Acidi Hydrobromici Diluti . . . 3vj 22.5

Aquæ Chloroformi . . . . . ad ऊ viij ad $250 \cdot 0$

Solve et misce. $\quad$ (Dose, 15 mils.)

Dose.-A tablespoonful in water three times a day between meals.

Note.-Used by the older ophthalmic surgeons to avert inflammation after injuries or operations; but its only virtue lies in its power to relieve pain.

\section{Potassium Bromide and Nux Vomica Mixture.}

R Potassii Bromidi . . . . 3iiss Io.o

Tincturæ Nucis Vomicæ . . . 3ij $8 \cdot 0$

Spiritûs Chloroformi . . . . J 3iss $10 \cdot 0$

Infusi Calumbæ . . . . . . ad 亏

Solve et misce. (Dose, 15 mils.)

Dose.-A tablespoonful in water three times a day between meals.

Note-Useful in painful conditions of the eyeball associated with chronic inflammation of its deeper structures-myopia, etc. 


\section{THERAPEUTIC NOTES AND FORMULÆ}

41. Quinine and Potassium Bromide Mixture.

R Quininæ Hydrobromidi • . . gr. xvj

Potassii Bromidi Ziiss

Acidi Hydrobromici Diluti . . . 3iv $15 \cdot 0$

Aquæ Anethi . . . . . . ad 弓viij ad 250.0

Solve et misce. $\quad$ (Dose, 15 mils.)

Dose.-A tablespoonful in water three times a day after food.

Note.-Valuable sedative, prescribed in many cases of chronic glaucoma.

\section{Valerian and Henbane Mixture.}

R Tincturæ Valerianæ Ammoniatæ .. . $\overline{\text { jis }}$

Tincturæ Hyoscyami . . . . $3 \mathrm{j} \quad 4 \cdot 0$

Tincturæ Cardamomi Compositæ . . $\quad \overline{3}$ ij $8 \cdot 0$

Aquæ Carui . . . . . . . ad ऊviij ad 250.0

Solve et misce. $\quad$ (Dose, 15 mils.)

Dose.-A tablespoonful in water three times a day between meals.

Note.-Valuable sedative in nervous and hysterical patients.

\section{B. PURGATIVES.}

In ophthalmic practice, castor oil, black draught, Gregory's powder, and calomel are the best purgatives.

Chronic constipation is one of the most important factors in the causation of many diseases of the eye.

\section{HYPODERMIC INJECTIONS.}

It is sometimes advisable, in order to influence the system rapidly, to administer remedies by means of hypodermic injections. The following are the chief drugs thus employed in ophthalmic practice-

\section{Acoin and Mercuric Cyanide Sub-conjunctival Injection.}

(a) $\mathrm{R}$ Acoin $\quad . \quad$. $\quad . \quad$. $\quad . \quad$. gr. $\mathrm{j} \quad \mathrm{I} \cdot \mathrm{O}$ Aquæ Destillatæ . . . . . $3 \mathrm{ij}$ Iоo.o Solve.

(b) $\quad \mathbf{R}_{\mathbf{x}}$ Hydrargyri Cyanidi . . . . . gr. ${ }_{80}^{\frac{1}{0}} 0.0125$ Sodii Chloridi Purissimi . . . gr. $x$ I0.0000 Aquæ Destillatæ . . . . . 3 ij I00•0000 Solve.

Note.-One part of $a$ mixed with two parts of $b$ to be injected beneath the conjunctiva in detachment of the retina, and in some forms of choroiditis of the macular region. 


\section{Antipyrine Hypodermic Injection.}

$\mathrm{P}$

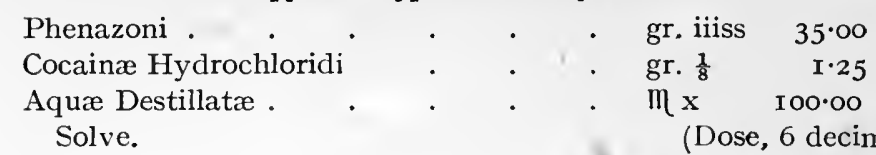

(Dose, 6 decimils.)

Note.-Relieves pain rapidly, but produces diffuse swelling around the site of puncture.

\section{Iodipin or Iodinal Hypodermic Injection.}

Either as straw-coloured oily liquid containing to per cent. iodine, or yellowishbrown viscid fluid containing 25 per cent. iodine.

Note.-A convenient method of administering iodine-3j to be injected beneath the skin between the shoulder blades, and repeated once a week.

\section{Mercuric Cyanide Hypodermic Injection.}
R Hydrargyri Bicyanidi . . . . gr. j I $\mathrm{I} O$ Aquæ Destillatæ . . . . . $3 \mathrm{ij}$ Iо०・0 Solve.

Note.-Contains one-sixteenth of a grain in seven and a half minims. A fleshy part, such as the buttock, ought to be chosen for the puncture, and the needle ought to be plunged deeply into the muscle. If the operation be performed carefully, the discomfort to the patient is slight. Is also used as a sub-conjunctival injection, see Formula I.

Mercury salicyl arsenate, generally known' as enesol, is a white powder containing 38 per cent. of mercüry, soluble in water, $I$ in 25 . Usually contains I gr. in 30 minims of a solution prescribed for intramuscular injections.

Other arsenical preparations are kharsivan, neo-kharsivan, novarsenobillon, galyl, etc. They are all reliable, and may be given intravenously or intramuscularly.

\section{Morphin Hypodermic Injection.}
R Morphinæ Hydrochloridi . . . gr. iv $4 \cdot 0$ Aquæ Chloroformi . . . . $3 \mathrm{ij}$. Iоo.0 Solve.

Note.-Five minims contain one-sixth of a grain. Sometimes it is advisable to combine from ${ }_{100}^{1}$ to ${ }_{60}^{1} \mathrm{gr}$. of atropin with each morphin injection.

\section{Pilocarpin Hypodermic Injection.}

$\mathrm{B}$

Pilocarpinæ Nitratis
Aquæ Destillatæ .
Solve.

$\begin{array}{lr}6 \cdot 0 \\ 3 \mathrm{ij} & \mathbf{1 0 0 \cdot 0}\end{array}$

Note.-Inject from two to six minims. Used to produce profuse diaphoresis in cases of separation of the retina, of choroiditis, and of retinitis. It is well to begin with a small dose once a day, and, if larger doses are indicated, to repeat the small dose night and morning, so as to avoid any danger of cardiac depression. The patient should be in bed between flannel sheets, and have the bedclothes well tucked in all round the body to keep off currents of air. A mug or a large towel should be placed at his mouth to catch the saliva. 
Guaiacol oil well rubbed into the axillæ is a convenient method of promoting diaphoresis.

\section{Saline Solution for Sub-eonjunetival Injection (Dor).}

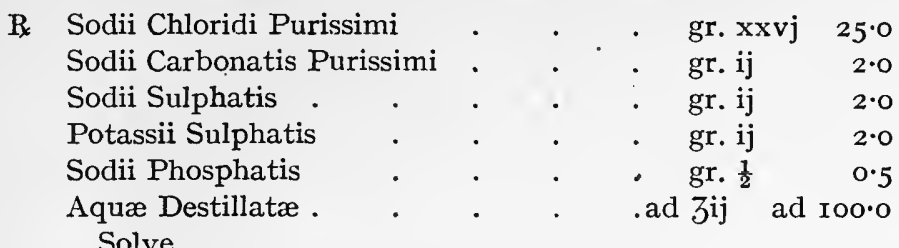

Note.-The use of this combination is always attended by very severe pain, and often followed by adhesion of the bulbar conjunctiva to the globe.

R Hydrargyri Bicyanidi . . . . $\mathrm{I}$ in 2000

Sodii Chloridi Cryst. . . . . 8 per cent.

Dionin Purissimi $\quad . \quad$. . . . I per cent.

Aquæ Sterile $\quad . \quad$. $\quad . \quad$. $3 \mathbf{j}$

Note.-Inject from 5 to 20 minims beneath the conjunctiva once a week. This is a very useful solution in the treatment of detachment of the retina.

\section{Gold and Potassium Cyanide Hypodermic Injection.}

Rx Auri et Potassii Cyanidi . . . gr. iv 20 Aquæ Destillatæ. . . . . ऊ $\mathrm{ss}$ Iо0.0

Solve.

Note.-At first inject five minims daily, and gradually increase the dose to fifteen minims. To relieve the burning pain caused by the injection, apply an ice-cold compress to the site of puncture.

Recommended by Galezowski in cases of atrophy of optic nerve, especially when associated with tabes dorsalis, but in my experience the therapeutic results are not encouraging. Bell and Croyden's hydraurium is an elegant and reliable preparation. It is a triple bromide of arsenic, mercury, and gold : $\frac{1}{32}$ grain of each is contained in to minims of the solution.

\section{Sozoiodol-Mercury Hypodermic Injection.}

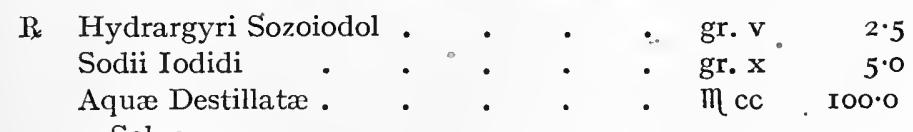

Solve.

Note.-Ten to fifteen minims for an injection. Produces very slight local reaction.

\section{Strychnin Hypodermic Injection.}

R. Liquoris Strychninæ Hydrochloridi B.P. $\quad 3 \mathrm{ij}$

Note.-Begin with two minims, and gradually increase the dose. Recommended in cases of atrophy of the optic nerve and in hysterical amblyopia.

$\mathrm{H} \mathrm{H}$ 


\section{PULVERES-POWDERS.}

\section{Aspirin Powder.}

\section{R Aspirin : . . . . gr. xv I gramme}

Note.-Aspirin, a white crystalline powder soluble in alcohol and glycerine but almost insoluble in water, is the acetic ester of salicylic acid. Given in fifteengrain doses in irido-cyclitis and other deep-seated inflammations of the eye, whether they be of rheumatic origin or not, it often acts like a charm. Should it tend to cause depression it is wise to combine it with caffein, and when the patient is sleepless its efficacy is greatly enhanced by the addition of ten grains of trional or eight grains of veronal.

2. Calomel and Compound Jalap Powder.

R Hydrargyri Subchloridi . . gr. iij to $\mathrm{x}$ Pulveris Jalapæ Compositi . . gr. xl 25 decigrams Misce.

Note.-Valuable purgative.

3. Calomel and Dover's Powder.

B Hydrargyri Subchloridi . . gr. iij Pulveris Ipecacuanhæ Compositi . gr. x Misce, fiat pulvis.

Dose.-One at bedtime.

Note.-Useful in rheumatic iritis.

\section{Phenacetin and Trional Powder.}

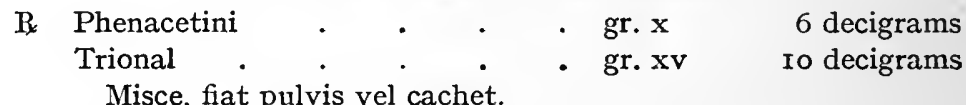

Dose.-One every eight hours.

Note.-Useful for subduing pain and promoting sleep after operations upon the eye.

\section{Quinine and Grey Powder.}

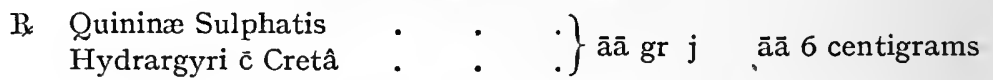
Misce, fiat pulvis.

Dose.-One three times a day.

Note.-Useful in cases where an alterative and tonic are both indicated, $e . g$. in interstitial keratitis and in sympathetic inflammation. The dose must be regulated to suit the patient.

\section{Rhubarb and Antimony Powder.}

R Pulveris Rhei Optimi . . . gr. v Antimonii Tartarati . . . gr. $\frac{1}{6}$ Misce, fiat pulvis.

30 centigrams

I centigram

Dose.-One at bedtime after a hot bath.

Note.-Useful in phlyctenular conjunctivitis with great intolerance of light. 
Must always be given in slightly nauseating doses. When the tongue is very foul, a little grey powder may be added to each dose as in the next formula.

7. Rhubarb, Antimony, and Grey Powder.

\section{$\mathrm{R}$}
Hydrargyri č Cretâ
Misce, fiat pulvis.
- gr. $\frac{1}{6}$
- gr. $\mathrm{j}$

\section{Sodium Bicarbonate Powder (Compound).}

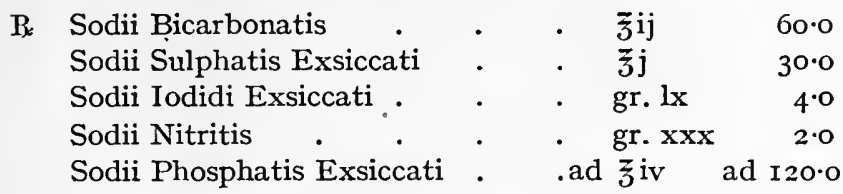

Antimonii Tartarati
- gr. $\mathbf{v}$

\section{0 centigrams \\ I centigram \\ 6 centigrams}

Dose.-A heaped teaspoonful in a large tumblerful of hot water twice a day between meals.

Note.-Useful to promote elimination in diseases of the uveal tract, toxic amblyopia, etc.

\section{Sodium Salicylate Powder.}
R

$\begin{array}{llllll}\text { Sodii Salicylatis } & \cdot & \cdot & & \text { gr. } x x & \text { I2 decigrams } \\ \text { Sodii Bromidi } & . & \cdot & \cdot & \text { gr. } x x & \text { I2 decigrams } \\ \text { Trite, fiat pulvis. } & & & & & \end{array}$

Dose.-One at bedtime.

Note.-As a diaphoretic administered in hot tea. Prescribed for relief of headache. Salicylate of soda by itself useful in choroidal and retinal inflammations, and in much larger doses in sympathetic ophthalmia (Gifford), in which disease salvarsan has also been given intravenously with encouraging results on the theory that it is of protozoal origin.

\section{Sodium Salicylate and Dover's Powder.}
R Sodii Salicylatis . . . . gr. xv . Io decigrams
Pulveris Ipecacuanhæ Compositi . gr. xij 7 decigrams
Potassii Nitratis . . . . gr. v 3 decigrams
Misce, fiat pulvis.

Dose.-Repeat as required.

Note.-Useful to relieve pain in rheumatic affections of the eye.

\section{Veronal Powder.}

R Sodium Veronal . . . . gr. vilj 5 decigrams

Note.-A trustworthy hypnotic.

Dial Ciba is a very reliable form in which to prescribe this drug. 


\section{E. PILULA-PILIS}

\section{Alterative Pill.}

R Aloes Barbadensis . . . . gr. iij Hydrargyri Subchloridi . . gr. $\frac{1}{6}$ Ferri Sulphatis Exsiccati . . gr. $\frac{1}{3}$ Pulveris Saponis Duri . • . gr. $\frac{1}{2}$ Ebor Usti . . . . . gr. $1^{1}$ Misce, fiat pilula.

Dose.-One every night at bedtime.

Note.-A mild mercurial pill, used in disease of the deeper structures of the eye, especially when due to syphilis. Commonly known as Anderson's Pill.

\section{Calomel and Opium Pill.}

B Hydrargyri Subchloridi . . gr. ij Extracti Opii . . . . . gr. j Misce, fiat pilula.

Dose.-One every night at bedtime, as directed.

Note.-In iritis continue the pills until the gums become slightly tender, and then gradually diminish the dose. Four to six pills are usually sufficient to induce the symptoms; afterwards a pill every second or third night will be required to keep the patient slightly under the influence of mercury.

\section{Digitalis and Squill Pill.}

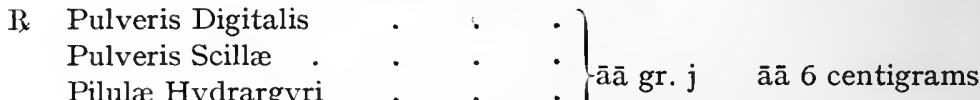
Extracti Hyoscyami

Dose.-One thrice daily.

Note.-Useful as a diuretic in some forms of retinitis associated with cardiac disease.

\section{Digitalis, Blue, and Squill Pill (Bailey).}

Rx Pulveris Digitalis . . . gr. ss

Pilulæ Hydrargyri . . . . gr. iij

Pulveris Scillæ . . . . . gr. iss

3 centigrams

Syrupi Glucosi . . . . . q.s.

Misce, fiat pilula.

Dose.-One three times a day.

Note.-Diuretic pill. Useful in some forms of toxic amblyopia, and in retinal and choroidal affections associated with cardiac disease. 
5. Donovan's Pill.

R Hydrargyri Iodidi Rubri . . gr. $\frac{7}{16}$ Arsenii Iodidi . . . . gr. $\frac{1}{20}$ Potassii Iodidi . . . . gr. j Pulveris Glycyrrhizæ, . . . gr. ss Aquæ Destillatæ . . . . q.s. 4 milligrams 3 milligrams 60 milligrams 3o milligrams q.s.

Dose.-One three times a day.

Note.-Used in chronic inflammation of the eyeball, especially when due to syphilis.

\section{Compound Gelsemium Pill.}

R Gelsemin (resinoid) • . . gr, ss

Butyl-Chloral Hydratis . . gr. j

30 milligrams

Camphoræ Monobromatæ . . gr. j .60 milligrams

Extracti Cannabis Indicæ . . gr. $\frac{1}{4} \quad x 6$ milligrams

Glycerini Tragacanthæ . . q.s.

Misce, fiat pilula.

Dose.-One every two or three hours until three doses have been taken.

Note.-To relieve pain in the head, associated with asthenopia.

\section{Iron Pill (Blaud).}

$\mathrm{R}$ Pilulæ Ferri . . . . gr. $\mathrm{v} \quad 3$ decigrams

Dose.-One three times a day.

Note.-Useful in anæmia.

\section{Iron Pill with Arsenic.}

R Pilulæ Ferri . • . - gr. v Sodii Arsenatis Anhydrosi . . gr. $\frac{1}{20} \quad 3$ milligrams Misce, fiat pilula.

Dose.-One three times a day.

Note.-Useful in anæmia.

\section{Mercury Pill.}

$\mathrm{R}_{k}$ Pilulæ Hydrargyri . . . gr. v 3 decigrams

Dose.-One three times a day, as directed.

Note.-Used in diseases of the eye due to syphilis. Should be continued until the physiological effects of the drug begin to manifest themselves.

Calomel is the most active preparation of mercury in the treatment of syphilis ; the green iodide and Hutchison's pill are very useful after a course of treatment by the arsenical preparations. 


\section{Mercury, Arsenic, and Iron Pill.}

R Hydrargyri Iodidi Rubri . . ) aagr. Acidi Arseniosi $\because \quad . \quad . \quad . \quad$ āā gr. $\overline{3}^{\frac{1}{2}} \quad \bar{a} a \bar{a} 2$ milligrams Ferri Iodidi . . . . . gr. iij 200 milligrams Pulveris Glycyrrhizæ et syrupi . q.s.

Misce, fiat pilula.

Dose.-One three times a day் after food.

Note.-Used in syphilis when a tonic effect is desired.

\section{Mercury and Quinine Pill.}

P Pilulæ Hydrargyri . . . . gr. j Quininæ Sulphatis . . . gr. j

Misce, fiat pilula.

Dose.-One three times a day.

Note.-Used as a tonic and alterative in syphilis.

\section{Phosphorus Pill.}

\section{Pe Phosphori}

\begin{tabular}{|c|c|}
\hline Phosphor1 • • • & \\
\hline Pulveris Tragacanthæ & āâ gr. j \\
\hline Pulveris Acaciæ & gr. vj \\
\hline Pulveris Glycyrrhizæ & gr. vij \\
\hline Carbonis Bisulphidi & $m i i j$. \\
\hline
\end{tabular}

$\bar{a} \bar{a} 6$ centigrams

36 centigrams 42 centigrams I 8 centimils

Confectionis Rosæ Gallicæ gr. xxxiv or q.s. 200 centigrams or q.s.

Mix the powders in a mortar; dissolve the phosphorus in the bisulphide of carbon in a test-tube; add to the mixed powders, and with the confection of roses make into a mass which is to be divided into thirty pills.

Dose.-One three times a day.

Note.-The strength of the phosphorus may be altered to suit individual cases, but it is advisable to adhere to the other quantities of the formula.

Used in degeneration of the optic nerve.

\section{Phosphorus and Nux Vomica Pill.}

Prepare as No. I2, adding-

Extracti Nucis Vomicæ to each pill.

gr. $\frac{1}{4} \quad 16$ milligrams

Dose.-One three times a day.

Note.-Used in optic atrophy.

\section{Plummer's Pill.}

R Pilulæ Hydrargyri Subchloridi Compositæ gr. v 3 decigrams Dose.-One at bedtime.

Note.-Useful in chronic iritis and in affections of the uveal tract generally. Can be borne in cases where stronger mercurials disagree. Should always be freshly prepared. 


\section{Quinine and Iron Pill.}

R Quininæ Sulphatis

Ferri Sulphatis Exsiccati

. $\}$ āā gr.j āā 6 centigrams

Extracti Aloes Socotrinæ . . gr. j 6 centigrams

Pulveris Capsici Subtilissimi $\quad$. gr. $\frac{1}{6} \quad$ I centigram

Extracti Hyoscyami . . . gr. j 6 centigrams

Syrupi Glucosi . • . . $\quad$ q.s. q.s.

Misce, fiat pilula.

Dose.-One three times a day.

Note.-Useful in weak atonic conditions, e.g. retinitis pigmentosa.

16. Compound Rhubarb Pill.

R Pilulæ Rhei Compositæ . - gr. v 3 decigrams

Dose.-One or two as required.

Note.-A mild laxative. May be combined with Pil. Hydrargyri.

R. Pilulæ Rhei Compositæ

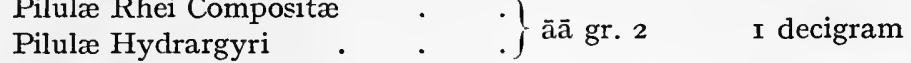

Extracti Hyoscyami . . . . gr. $\frac{1}{2} \quad 3$ centigrams

17. Thiosinamin Pills.
$\mathrm{R}$ Thiosinamin . . . . . gr. $\frac{1}{2} \quad 3$ centigrams
Extracti Gentianæ . . . . q.s.
Misce.

Note.-Prescribed along with Thiosinamin Ointment to promote the absorption of opacities of the cornea.

18. Triple Valerianate Pill.

P. Quininæ Valerianatis Ferri Valerianatis . Zinci Valerianatis. Syrupi Glucosi Misce, fiat pilula.

Dose.-One three times a day.

Note.-Useful in neurasthenia and in hysterical affections.

19. Zine Phosphide Pill.

R Zinci Phosphidi . . . . gr. ${ }_{12}^{\frac{1}{2}} 5$ milligrams

Sacchari Lactis . . . . gr. ij 120 milligrams

Extracti Centianæ . . . . gr. j 60 milligrams

Misce, fiat pilula.

Dose.-One three times a day.

Note.-Used in degeneration of the optic nerve. 


\section{GLOSSARY}

Amaurosis (ả $\mu a v \rho o ́ s=$ dark). A state of more or less absolute blindness without visible external disease of the eye.

Amblyopia ( $\dot{\alpha} \mu \beta \lambda u ́ v=$ blunt ; $\omega \psi=$ sight). Defectiveness of vision without external disease of the eye.

Ametropia $(\alpha=$ privative ; $\mu$ é $\tau \rho o \nu=$ measure $; \omega \psi=$ sight $)$. Imperfection in the refraction of the eye ; a general term including hypermetropia, myopia, and astigmatism.

Aniridia ( $\alpha \nu=$ privative ; $\bar{i} \rho t s=$ iris). A Asence of the iris.

Anisocoria ( $\alpha \nu=$ privative; $\nearrow \sigma o s=$ equal ; $\kappa \circ \eta^{\prime}=$ pupil). Inequality of the pupils.

Anisometropia ( $\alpha \nu=$ privative ; $\tau \sigma o s=$ equal ; $\mu \epsilon \tau_{\rho} \rho \nu=$ measure; $\omega \psi=$ sight). Difference in the refraction of the two eyes.

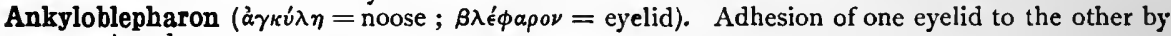
its edge.

Anophthalmos ( $\dot{a} \nu=$ privative ; $\grave{\partial} \phi \theta a \lambda \mu \dot{s}=$ eye). Absence of the eyeball.

Aphakia ( $\alpha=$ privative; $\phi \alpha \kappa o ́ s=$ lentil). Absence of the lens.

Argyria (ápqupos $=$ silver). Discoloration of the conjunctiva, etc., from prolonged use of silver preparations.

Asthenopia ( $\dot{\alpha}=$ privative; $\sigma \theta \dot{e} \nu o s=$ strength; $\omega \psi=$ sight). Weakness or speedy tiring of the eyes.

Astigmatism ( $\grave{a}=$ privative ; $\sigma \tau i \gamma \mu \alpha=$ point). A defect of the eye in which light rays in different meridians are not brought to a focus at the same point.

Blennorrhœa ( $\beta \lambda e^{\prime} \nu_{\nu n s}=$ mucus ; poí ${ }^{\circ}=$ flow). A free discharge from the conjunctival or - lachrymal sac.

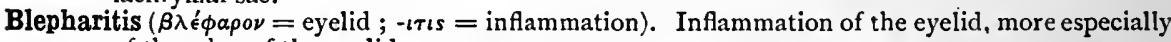
of the edge of the eyelid.

Blepharophimosis ( $\beta \lambda \lambda^{\prime} \phi \alpha \rho o \nu=$ eyelid; $\phi(\mu \omega \sigma \iota s=$ muzzling). A narrowing of the slit between the eyelids.

Buphthalmos $(\beta o v s=o x ; \dot{o} \phi \theta a \lambda \mu \hat{o} s=$ eye. Infantile glaucoma-a condition characterized by general enlargement of the eyeball.

Capsulotomy (L. = capsula, capsule ; $\tau \epsilon^{\epsilon} \mu \epsilon \epsilon \nu=$ to cut). The operation of cutting the capsule of the lens.

Chalazion ( $\left.\chi^{\prime}{ }^{\lambda} \lambda a\right\}_{i o \nu}=$ hailstone). A cyst of one of the Meibomian glands.

Chemosis ( $\chi \eta^{\prime} \mu \eta=$ cockleshell). Excessive œdema of the ocular conjunctiva, generally inflammatory in origin.

Chiasma ( $\chi^{\prime} \alpha \sigma \mu \alpha=a$ crossing). The point at which the optic nerves meet each other and the optic tracts, one half of the fibres of each optic nerve crossing to join the optic tract of the other side.

Chromatopsia $(\chi \rho \hat{\omega} \mu \alpha=$ colour ; $\omega \psi=$ sight $) . A$ disorder of vision in which objects appear abnormally coloured.

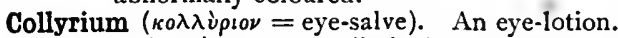

Coloboma ( $\kappa \delta \lambda \delta \beta \omega \mu \alpha=$ mutilation). A defect or fissure of the iris, choroid, or optic nerve, generally congenital or surgical.

Cyclitis ( $\kappa \dot{v} \kappa \lambda o s=$ ring ; $-\iota \tau \iota s=$ inflammation). Inflammation of the ciliary body.

Cycloplegia $(\kappa \dot{v} \kappa \lambda o s=$ ring ; $\pi \lambda \eta \gamma \eta \dot{\eta}=$ stroke). Paralysis of the ciliary muscle.

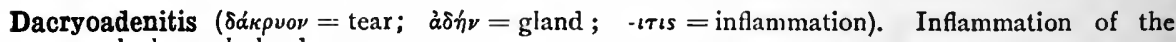
lachrymal gland.

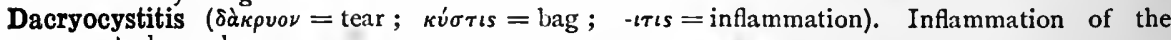
lachrymal sac.

Dacryops ( $\delta a \varkappa \kappa \rho v o \nu=$ tear $; \omega_{\psi} \psi=$ eye). Distension of one of the ducts of the lachrymal gland.

Descemetitis (Descemet, a French anatomist ; $-\imath \tau \iota s=$ inflammation). Deposition of flakes of lymph on the back of Descemet's membrane due to inflammation of the iris or ciliary body. 
Diplopia $(\delta \iota \pi \lambda d o s=$ double ; $\omega \psi=$ sight $)$. Double vision.

Distichiasis ( $\delta i s=t w i c e ; \sigma \tau i$ xos $=$ row). An affection in which there are two rows of eyelashes, some of which rub against the eyeball.

Ectasia ( $\xi^{2} \kappa=$ out $; \tau \in \dot{i} \nu \epsilon \nu=$ to stretch). A localised distension or stretching of the wall of the eyeball.

Ectropion ( $\epsilon^{2} \kappa=$ out ; $\tau \rho \in \epsilon^{\prime} \epsilon \epsilon \nu=$ to turn). Eversion of the edge of the eyelid.

Emmetropia $\left({ }^{\epsilon} \nu=\right.$ in ; $\mu \dot{\epsilon} \tau \rho o \nu=$ measure $; \omega \psi=$ sight $)$. A condition in which the refraction of the eye is normal.

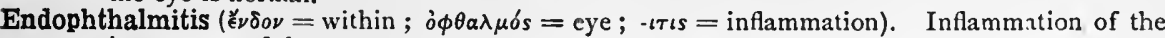
inner coats of the eye.

Enophthalmos $(\xi \nu=$ in ; $\partial \phi \theta \alpha \lambda \mu o ́ s=$ eye). A sinking backwards of the eye into the orbit.

Entropion ( $\dot{\epsilon} \nu=$ in ; $\tau \rho \epsilon \in \pi \epsilon \nu=$ to turn). Inversion of the edge of the eyelid.

Epicanthus ( $\dot{\pi} \pi l=$ upon; $\kappa \alpha \nu \theta \delta s=$ canthus). An abnormal condition in which a fold of skin, at the side of the nose, covers the inner canthus.

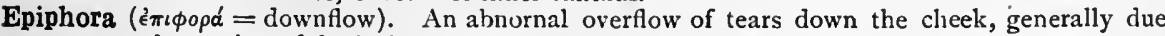
to obstruction of the lachrymal passages.

Episcleritis $\left(\dot{\epsilon} \pi i^{\prime}=\right.$ upon ; $\sigma \kappa \lambda \eta \rho \delta s=$ hard ; $-\iota \tau \iota s=$ inflammatiou $) . \quad$ Inflammation of the episcleral tissue.

Erythropsia ('́p $\rho \theta \rho \sigma ́ s=$ red $; \omega \psi=$ sight). - An affection in which objects appear abnormally red.

Esophoria ( $\ddot{\sigma} \sigma \omega=$ inward ; $\phi \dot{e} \rho \epsilon \iota=$ to bear). Latent inward squint.

Exclusion of Pupil. A condition in which the pupillary margin of the iris is adherent to the anterior capsule of the lens, so that the aqueous humour cannot pass from the posterior into the anterior chamber through the pupil.

Exophoria ( $\xi \xi \omega=$ out ; $\phi \epsilon \in \epsilon \iota=$ to bear). Latent outward squint.

Exophthalmos ( $\xi \xi=$ out ; $\dot{\phi} \phi \theta \lambda \mu \phi \delta s=$ eye). Abnormal protrusion of the eyeball.

Fovea (fovea $=\mathrm{pit}$ ). The minute depression in the centre of the macula lutea.

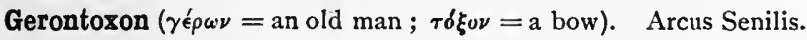

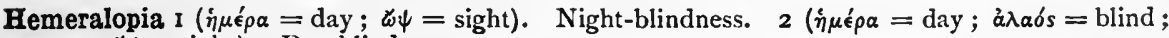
$\omega \psi=$ sight $)$. Day-blindness.

Note.-A considerable amount of confusion has arisen as to the meaning of the terms nyctalopia and hemeralopia. Ignorance of the derivation of the word nyctalopia (viz. $\nu \dot{v} \xi=$ night ; $\grave{a}^{\lambda} a \sigma_{s}=$ blind ; $\omega_{\psi}=$ sight) has led some people to believe that the correct meaning of this word was night-sight (= day-blindness), as though it were derived only from the words $\nu v \dot{\xi}$ and $\omega \psi$. In consequence, the word hemeralopia was brought into use to signify night-blindness. The correct meaning of hemeralopia is, however, day-blindness, just as the correct meaning of nyctalopia is night-blindness.

Hemianopia $(\dot{\eta} \mu \iota=$ half ; $\dot{\alpha} \nu=$ privative ; $\omega \psi=$ sight $)$. Blindness of one half of the visual field in both eyes.

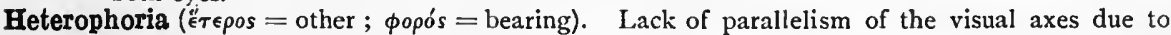
insufficiency of the orbital muscles; latent squint.

Hippus (i $i \pi \pi s=\mathrm{a}$ horse). Rapid alternate contraction and dilatation of the pupils dependent on the rhythmic activity of the nerve-centres.

Hordeolum (L. = a barley-corn). A stye.

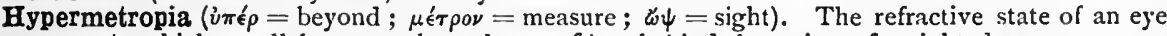
in which parallel rays are brought to a focus behind the retina; far-sightedness.

Hyperphoria ( $\dot{\varepsilon} \pi \hat{\epsilon} \rho=$ beyond; $\phi o p o ́ s=$ bearing). Defective parallelism of the visual axes such that the visual axis of one eye is directed further upwards than that of the other; a variety of latent squint.

Hyphæma ( $\dot{u} \pi \delta=$ under; $a \tau_{\mu \alpha}=$ blood). Blood in the anterior chamber of the eye.

Hypopyon $(\dot{v} \pi \dot{\delta}=$ under; $\pi \dot{v} o \nu=$ pus). Pus in the anterior chamber.

Hypotony ( $\dot{v} \pi \dot{b}=$ under ; $\tau o ́ v o s=$ tone.) Diminution in the intraocular pressure.

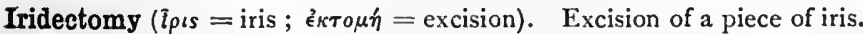

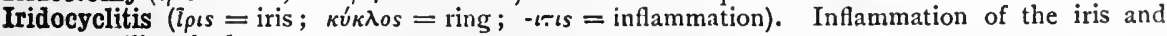
ciliary body.

Iridodialysis (īpıs $=$ iris ; $\delta \iota a^{\prime}=$ througn ; $\lambda v \epsilon \iota \nu=$ to loosen). Rupture or splitting of the iris at its root.

Iridodonesis ( $\left(\imath_{\rho i s}=\right.$ iris ; $\delta \delta^{\prime} y \eta \sigma s=$ tremor). Tremulousness of the iris.

Iridoncosis (ipls = iris; ‘ ${ }^{\prime}$ kos $=$ swelling). Bulging forward of the iris by the aqueous in the posterior chamber, due to adhesion of the pupillary margin to the anterior capsule of the lens.

Iridoplegia ( $(\bar{\rho} \iota s=$ iris $; \pi \lambda \eta \gamma \dot{\eta}=$ stroke). Paralysis of the iris. 
Iris Bombs (bombé = convex). Bulging forward of the iris by the aqueous in the posterior clamber due to adhesion of the whole of the pupillary margin to the anterior capsule of the lens.

Iritis ( $l_{\rho} \iota s=$ iris ; $-\iota \tau \iota s=$ inflammation). In!lammation of the iris.

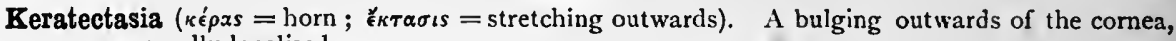
generally localised.

Keratitis punctata ( $\kappa$ épas $=$ horn ; $-\iota \tau \iota s=$ intlammation ; punctata $=$ punctate). Deposition of particles of coagulated albumen on the posterior surface of the cornea due to iridocyclitis; at one time thought to be a variety of inflammation of the cornea itself.

Keratoconus ( $\kappa \dot{\epsilon} \rho \alpha s=$ horn; $\kappa \hat{\omega} \nu o s=$ cone). A deformity of the cornea in which the latter becomes cone-shaped.

Keratoglobus ( $\kappa$ épas = horn: L., globus = globe). General enlargement of the cornea that occurs in infantile glaucoma.

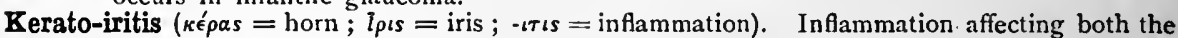
iris and the cornea.

Keratomalacia ( $\kappa$ e $\alpha s=$ horn ; $\mu a \lambda \alpha \kappa i \alpha=$ softening). A form of wasting of the cornea that occurs, as a rule, in syphilitic children.

Lagophthalmos ( $\lambda \alpha \gamma \omega \dot{s}=$ hare $; o ̋ \phi \theta a \lambda \mu \delta ́ s=e y e)$. A condition in which the eyelids cannot be properly closed-so called because a hare is supposed to sleep with its eyes open.

Lamina Cribrosa (lamina = plate; cribrum = sieve). The sieve-like part of the sclera through which the fibres of the optic nerve pass when leaving the eye.

Limbus (L. $=$. border). The corneo-scleral margin.

Lymphoma (L. = lympha; $-\omega \mu \alpha=$ tumour). A tumour made up of lymphoid tissue-found rarely in the eyelids or orbit.

Macropsia ( $\mu \alpha \varepsilon \rho \delta s=$ large $; \varpi \psi=$ ight). An anomaly of vision in which objects appear to be larger than normal.

Macula corneæ (macula $=$ a spot). Minute scars of the cornea.

Macula lutea (macula $=$ spot ; lutea $=$ yellow). The yellow-spot ; the central part of the retina.

Madarosis $(\mu \alpha \delta \alpha \rho b ́ s=$ bald). Loss of the eyelashes following disease of the edge of the eyelid.

Megalocornea ( $\mu$ '́ $\gamma a s=$ large ; cornea). A familial disease in wlich there is simple gigantism of the cornea-thought by many to be identical witl infantile glaucoma arrested at an early stage.

Meibomian Glands (Meibom, a German anatomist). Sebaceous acinous glands situated in the eyelids.

Melanoma ( $\mu \epsilon_{\lambda}^{\prime} a s=$ black ; $-\omega \mu \alpha=$ tumour). A Melanotic tumour.

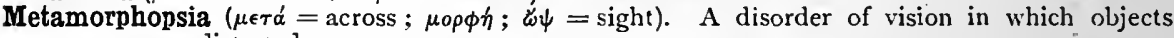
appear distorted.

Microphthalmos ( $\mu \iota \kappa \rho \delta s=$ small ; $\dot{\phi} \phi \alpha \lambda \mu \delta s=$ eye). Abnormal smallness of the eyes.

Myosis or Meiosis ( $\mu \in \epsilon^{\prime} \omega \sigma \iota s=$ contraction). Contraction of the pupil.

Mydriasis ( $\mu \nu \delta \rho i \alpha \sigma s=$ dilatation of the pupil). Dilatation of the pupil.

Mydriatic. A drug which induces mydriasis.

Myodesopsia $(\mu v \iota \omega \delta \eta s=$ fly-like; $\omega \downarrow=$ sight). An anomaly of vision in which the patient sees specks.

Myopia ( $\mu v_{\epsilon} \iota \nu=$ to shut ; $\omega \psi=$ sight). Short-sightedness ; the refractive state of an eye in which parallel rays are brought to a focus at a point in front of the retina.

Nyctalopia ( $\nu v ́ \xi=$ night ; $\measuredangle \lambda \alpha o s=$ blind ; $\psi_{\psi} \psi=$ sight). Night-blindness. N.B. - See note after Hemeralopia.

Nystagmus ( $\nu v \sigma \tau a \zeta \epsilon t \nu=$ to nod). Short jerking movements of the eye, which are rapidly repeated and always occur in the same direction.

Ophthalmia ( $\phi \phi \theta \alpha \lambda \mu \delta ́ s=$ eye). Inflammation of the conjunctiva, of the conjuncliva and cornea, or of the edge of the lids.

Ophthalmoplegia ( $\delta \phi \theta a \lambda \mu d s=$ eye ; $\pi \lambda \eta \gamma \dot{\eta}=$ stroke). Paralysis of the.extrinsic or intrinsic muscles of the eye.

Pannus ( $\mathrm{L} .=\mathrm{rag}$ ). Vascularization of the superficial layers of the cornea, generally due to trachoma.

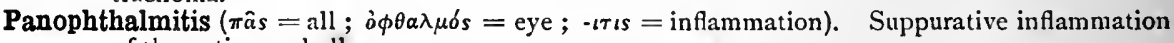
of the entire eyeball.

Papilla (L. = nipple-shaped projection). The optic disc.

Papillo-œdema (papilla; ot $\eta \mu \alpha=$ swelling). Oedema of the optic nerve, such as occurs with increased intracranial pressure ; choked disc.

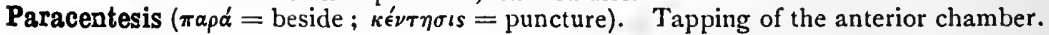


Perimeter ( $\pi \epsilon \rho^{\prime}=$ around; $\mu \epsilon ́ \tau \rho o \nu=$ measure). An instrument for measuring the extent of the visual fields.

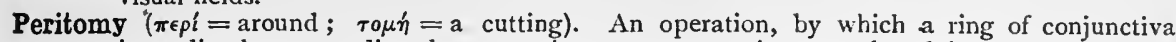
immediately surrounding the cornea is cut out; sometimes employed in the treatment of pannus.

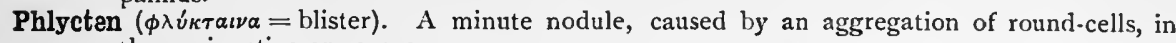
the conjunctiva or cornea.

Photophobia ( $\phi \hat{\omega} s=$ light $; \phi \delta \beta$ Bos $=$ fear $)$. Intolerance of light.

Photopsiæ $(\phi \hat{\omega} s=$ light ; $\ddot{\psi} \psi=$ sight $)$. An anomaly of vision in which flashes are seen.

Phtheiriasis (ciliorum) ( $\phi \theta \epsilon i p=$ louse). The occurrence of lice on the eyelashes.

Phthisis Bulbi ( $\phi \theta i \sigma \iota s=$ consumption). Shrinkage and wasting of the eye.

Pinguecula (pinguis = fat). A triangular yellowish patch occurring between the corneal margin and the inner (or outer) canthus, due to an increase in the number and size of the yellow elastic fibres in the conjunctiva.

Placido's Disc. A disc having concentric circles marked on it, used in examining the cornea.

Plica Semilunaris (L. = semilunar fold). The fold of the conjunctiva at the inner canthus.

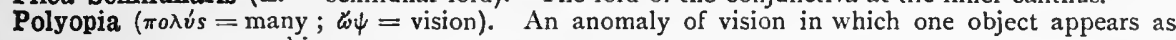
two or more objects.

Presbyopia ( $\pi \rho \epsilon \in \beta v s=$ an old $\operatorname{man} ; \omega \psi=$ sight). The condition in which, owing to advancing years, near objects cannot be seen plainly, through loss of power of accommodation, whilst objects at a distance are clearly discerned.

Pseudo-glioma $(\psi \in v \delta \dot{\eta} ;=$ false ; $\gamma \lambda \hat{\imath} \alpha=$ glue; $-\omega \mu a=$ tumour $)$. An exudate, generally of pus, lying in the vitreous, which may be mistaken for a glioma of the retina.

Pseudo-neuritis $\left(\psi \in v \delta \eta^{\prime} s=\right.$ false $; \nu \in \hat{v} \rho o \nu=$ nerve $:-\iota \tau \iota s=$ inflammation. An appearance of the optic disc, occurring in some cases of hypermetropia or hypermetropic astigmatism, which simulates optic neuritis, but which is of no pathological significance.

Pterygium ( $\pi \tau \dot{\epsilon} \rho v \xi=a$ wing). A patch of conjunctiva extending over part of the cornea, either at its inner, or (less commonly) at its outer side.

Ptosis $(\pi \tau \hat{\omega} \sigma \iota s=$ fall $)$. Drooping of the upper eyelid.

Retinitis (rete $=$ a net ; $-\iota$ ris $=$ inflammation). Inflammation of the retina.

Retinoscopy (retina ; $\sigma \kappa o \pi \epsilon \hat{\imath} \nu=$ to examine). Determination of the refractive state of the eye by observing the movements of light and shadow across the pupil when light is cast into the eye from a moving mirror; the shadow-test.

Retrotarsal Fold (retro = behind; tarsus). The portion of the conjunctival sac that lies behind the eyelids.

Schiøtz Tonometer (Schiфtz, a Norwegian oculist ; $\tau \delta \dot{\nu} o s=$ tone ; $\mu \epsilon ́ \tau \rho o \nu=$ measure). An instrument for estimating the intraocular pressure.

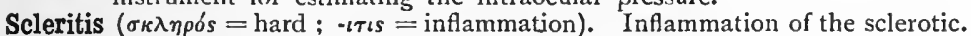

Sclerectomy ( $\sigma \kappa \lambda \eta \rho \delta s=$ hard ; $\epsilon^{2} \tau \tau \rho \mu \dot{\eta}=$ excision). An operation whereby a portion of the sclerotic is excised.

Scotoma $(\sigma \kappa \delta$ tos $=$ darkness; $-\omega \mu \alpha)$. An area of relative or absolute blindness in the visual field.

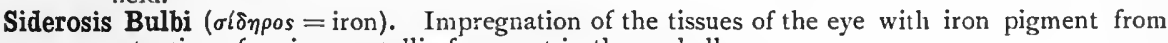
retention of an iron metallic fragment in the eyeball.

Staphyloma $(\sigma \tau i \phi v \lambda \delta s=\mathrm{a}$ bunch of grapes; $-\omega \mu \alpha=s w e l l i n g)$. A protrusion of the cornea or sclera due to weakening of the membrane at that point.

Stillicidium (stilla = a drop; cadere $=$ to fall). An abnormal flow of tears over the edge of the eyelid, generally due to obstruction of the lachrymal passages; epiphora.

Strabismus $(\sigma \tau \rho \alpha \beta \iota \sigma \mu \delta s=$ squint). Squint.

Symblepharon $(\sigma \dot{v} \nu=w i t h ; \beta \lambda \epsilon ́ \phi \alpha \rho o \nu=$ eyelid). An adhesion of the eyeball to an eyelid consequent on a burn or wound.

Synchysis Scintillans $(\sigma v \gamma \chi \in \hat{\imath} \nu=$ to pour together; scintillans = scintillating). Fluidity of the vitreous with the presence in it of cholesterin crystals which glitter when light strikes them.

Synechia ( $\sigma \nu \nu^{\prime} \chi \epsilon \iota a=$ continuity). An adhesion of the iris to the cornea (anterior), or to the lens capsule (posterior).

Tarsorrhaphy ( $\tau \alpha \rho \sigma \delta s=$ tarsus; $\beta \alpha \phi \dot{\eta}=$ suture). The operation of suturing a portion of the eyelids together.

Teichopsia $(\tau \epsilon \hat{\imath} \chi 0 s=$ wall; $\epsilon \psi=$ sight). A disorder of vision in which luminous, zigzag appearances are seen, resembling the outline of a fortification as delineated on a map; also called scintillating scotoma.

Tenonitis (Tenon, a French surgeon; - $\iota \tau \iota s=$ inflammation). Inflammation of Tenon's capsule.

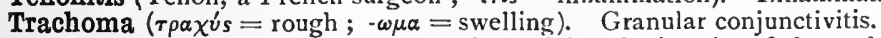

Transillumination (L.). A method of examining the interior of the eyeball by means of a strong light made to pass througl its walls. 
Trichiasis $(\theta \rho i \xi=$ hair). A condition of ingrowing eyelashes.

Tylosis (rúnos = a callous spot). Hypertrophy of the margin of the eyelid with drooping in consequence of its increased weight, following on blepharitis marginalis.

Uvea (L. = grape). The iris, ciliary body, and choroid considered together; otherwise, uveal tract.

Uveitis. Inflammation of the uveal tract.

Venæ Vorticosæ (vena $=$ vein $;$ vortex $=\mathrm{a}$ whirl). The venous trunks which carry off the blood from the choroid.

Wernicke's Hemiopic Pupillary Reaction (Wernicke, a German alienist). A reaction in certain cases of hemianopia in which light cast on one half of the retina causes no pupillary reaction, whilst light cast on the other half causes pupillo-constriction. When present, it indicates a lesion of one of the optic tracts.

Xanthelasma ( $\xi \alpha \nu \theta \delta s=$ yellow ; $\epsilon^{\prime} \lambda \alpha \sigma \mu \alpha=$ plate). A disease characterised by the formation of yellow plaques upon the skin, especially in the neighbourhood of the eyelids.

Xerophthalmos $\left(\xi \eta \rho{ }^{\prime} s=\right.$ dry ; $\dot{\phi} \phi \theta \alpha \lambda \mu \delta \delta^{\prime}=$ eye). Abnormal dryness of the conjunctiva.

Xerosis $(\xi \eta \rho o ́ s=$ dry). A disease characterised by abnormal dryness of the conjunctiva.

Zona Ophthalmica (zona $=$ a belt). Herpes zoster ophthalmicus. 


\section{INDEX}

Abrasions, corneal, 250, $25 \mathrm{I}$

traumatic, 263

Accidents, injuries due to, $260-70$

protective mechanism of the eye against, 242

Workmen's Compensation Act (I906) in relation to, 300-I2.

see also Occupational injuries.

Accommodation, $337,338,346$

amplitude of, 3I5, 316

and convergence, relation between, 346

definition of, 31 5, 346

in hypermetropia, 338

paralysis of, 347

spasm of, 320

Accommodation theory of strabismus, 337

Acid burns, 255

Acriflavine lotion, formula, 4 13

Adherent leucoma, 91

Adhesions, breaking-down of, 256

Adrenalin, acute coryza following injections of, 29

advantages and value of, 420

anæsthesia by, 205

for excessive lachrymation, 29

Age, influence on myopia, 328

Aged sight, see Presbyopia.

Albugo, corneal, $\mathrm{I}_{42}$

Albuminous fluids, in glaucoma, 197

Albuminuria, $383,391-6$

associated with cataract, 2 I 2

ocular changes in, 390-6

see also Retinitis, albuminuric.

Alcohol amblyopia, 402, 403, 408

Alibour water, $4 \mathrm{I} 6$

Alkaline douches, 445

Alkaline lotions, 4 I I

Alteratives, formulæ for, $453-5,468$

Alum, 446

Alum lotions, formulæ, 416

Alum sulphate, in follicular conjuncti-

Alumnol, 4 I9 vitis, 99

Alypin solution, 42 I

Amaurosis, 399

traumatic, $40 \mathrm{I}$

uræmic, 390

Amaurotic cat's eve, 236
Amblyopia, 399

albuminuric retinitis causing, 393

and nystagmus, 369

congenital, 399,400

ex anopsia, 400

glycosuric, 408

hysterical, $381,400,401$

strabismus and, 400

toxic, 402

causes and characteristics, 379, 402-6

- scotoma of, 404

tabetic atrophy from, 407

treatment of, 408

traumatic, 3II, 4OI, 402

Ambrine, 256

Ametropia, examination for, 347

low degrees of ill-health due to, 344

Ammonia burns, 253

Ammonium tartrate, in lime burns, 256

Amplitude of accommodation, 315, 3 I 6

Anæmia, of toxic neuritis, 169

Anæsthesia, for iridectomy, 205

Anæsthetics, administration of, 424

formulæ for, $420-33$

general, 42 I

local, 420

Anaphylaxis, 178

Anchyloblepharon, 255

Angioma, orbital, 229

Angio-neurotic œdema, $3^{8}$

Angle gamma, $33^{8}$

Angle of deviation, how measured, 339

Angular conjunctivitis, 98

Aniridia, traumatic, 266

Anisometropia, 338 eye-strain and, 346

Ankles, œdema of, œdema of eyelids associated with, 37

Anodynes, use of, 256

Antidiphtheritic serum, administration of, 177,178

in iritis, 177

in orchitis, 178

in membranous conjunctivitis, 87

Antiphlogistic treatment, of gonococcal conjunctivitis, 85 
Antiseptics, 77, 87, $4 \mathbf{1 2}$

following operation for cataract, 222

for burns, 256

formulæ for, 4I2-I 5

in cataract, 216,217

in conjunctivitis, 77

in corneal ulcer, 127

in lachrymal catarrh, 30

in ophthalmia neonatorum, 93-5

in serpiginous ulceration, 137

in trachoma, 103

nasal, 30

use of, 243

Antisyphilitic treatment, $165^{-7}$

Apparent strabismus, 338

Applanatio corneæ, I 44

Aqueous, premature escape of, during operation for cataract, 2 I9

Arcus juvenilis, $14^{8}$

Arcus senilis, $147, \mathrm{I}_{4} 8$

opacities of cornea due to, $\mathrm{I}_{42}$

Arecolin, 204, 428

Argyll Robertson pupil, 360

Argyrol, 33, 87, 95

action and characteristics of, 96, 429

administration of, 429,436

in catarrhal conjunctivitis, 8 I

in corneal ulcer, 127

in ophthalmia neonatorum, 95

in septic infection, 280

Argyrosis, of the conjunctiva, 82 .

Aristol ointment, formula, 436

Arnica, 4 I 8

Arsenical compounds, $165-7,178$

Arsenical mixtures, formulæ, 453, 454

Arsenical pills, formulæ, 469,470

Arsenobillon, in syphilitic iritis, 166

Arterial tension, detection of, 392

Arteries, ocular, 69, 385, 386

changes in, ophthalmoscopic examination for, 392

degenerative changes in, 39I

engorgement of, how diminished, I 27

in glaucoma, 196,198

injection of, $69-73$

Arterio-capillary fibrosis, $39 \mathrm{I}$

Arterio-sclerosis, 392

Arthritic foam, I68, 221

Artificial eye, $28 \mathrm{I}, 287$

Asphyxia, traumatic, 382

Aspirin, I6I, 466

Asthenopia, 329

causes of, $349-5$ I

eye-strain and, 345

muscular, characteristics and symptoms, 16,338

see also Eye-strain.

Astigmatism, cause and characteristics, 315,321

correction of, 323

definition of, $32 \mathrm{I}$
Astigmatism, diagnosis of, Io

how measured, 323

hypermetropic, 320, 322, 323

irregular, 32 I

mixed, 322

myopic, 322, 329

regular, 32 I

test for, 10, 323

Astringents, 98, 4I 5-I 7

Atropin, action and characteristics of, I 27, I 59, 425

advantages and disadvantages of, 318,425

causing glaucoma, 318

in corneal ulceration, $96,127,136$

in episcleritis, 183

in interstitial keratitis, 152

in iritis, I 59

in phlyctenular conjunctivitis, I I I

in sclerotitis, I83

in strabismus, $34 \mathrm{I}$

in toxic iritis, 169

precautions in administration of, I27

Atropin oil, formula, 433

Atropin ointments, formulæ, 436, 437

Atropin solutions, formulæ, 425,426

Bacterial infection, I30, 132

Bactericides, 77, 85, 94, 137, 243

Bacteriological examination, of secretions, 76

Bagginess of the eyelids, $3^{8}$

Bailey's pill, 468

Balls of light, 22

Bandaging, in corneal ulcer, 126

Belladonna, I37, 4I9

Binocular diplopia, 2 I

Binocular vision, 307

causes of loss of, 336

establishment of, 34I

tests of, 307

Workmen's Compensation Act in relation to, 307

Bjerrum's sign, I90

Black eye, $26 \mathrm{I}$

Blear eye, 43

Blenorrhœa, and œdema of eyelids, 40 definition of, 26,27 of the lachrymal sac, 27

Blepharitis, causes and treatment of, 44 simple, 43. stye complicating, 46

Blepharitis hypertrophica, 43, 60

Blepharitis marginalis, 26 causes and treatment of, 42, 44

Blepharitis ulcerans, causes and treatment of, 43,45

Blepharospasm, 49

of interstitial keratitis, 150

of phlyctenular conjunctivitis, Io8

treatment of, 49 
Blindness, bilateral mydriasis with, 356 choroid tumour causing, 233

hysterical, 400, 401

in children, 88

see also Ophthalmia neonatorum.

in glaucoma, 193

iritis causing, 176

malingering and, 360

monocular, traumatic causes, 262

primary g! aucoma resulting in, 202

sudden, causes of, 17

sympathetic inflammation causing, - 293

temporary, blepharospasm causing. 49

toxic iritis leading to, $I 68$

see also Vision.

Blood changes, causing paralysis of ocular muscles, 54

in sympathetic ophthalmia, 295, 296 toxic, 133

Blood clot, $264-6$

treatment of, 269

Blood letting, 160,452

Blood-pressure, high, ocular changes associated with, 393

Blood-shot eye, 2, 69

in iritis, 156

without inflammation, 73

Blood-stream, septic infection by, 294, 295,296

Blood supply, to the eye-ball, 69

Blood-vessels, congestion of, 70, 73, 127

Blows, injuries due to, 260, 264, 269

Bluestone, in trachoma, 103

Bony fractures, 26I

Boracic acid, I 35

Boracic acid fomentations, 45 I

Borax, 258, 259

Boric acid lotion, formula, $4 \mathrm{I} 3$

Boric acid ointment, formula, 437

Boric acid powder, 449

Borotannic powder, 449

Bowman's membrane, I0 5

Brain, tumour of, see Cerebral tumour.

Bright's disease, ocular changes in, 389 , 390,392

Bromides, 459, 462, 463

Brows, wrinkling of, 44

Bulbar conjunctiva, affections of, I I4-I9

Buller's shield, for purulent conjunctivitis, 84

Bullet injuries, $260,27 \mathrm{I}$

Buphthalmos, in glaucoma, $x 9 x$

Burns, varieties and treatment of, 253-9. 288

Cade oil ointment, 438

Cadmium iodide ointment, 438

Cajuput oil, 434

Calcium chloride mixtures, formulæ, 453 . 454
Calcium iodide lotion, 419

Calomel, action of, II I

in phlyctenular conjunctivitis, I I I

in syphilitic iritis, 166,167

Calomel lotion, formula, $4{ }^{1} 3$

Calomel ointment, formula, 438

Calomel pills, formulæ, 468, 469

Calomel powder, 449

Canaliculi, incision of, 30

obstruction in, 24,27 treatment, 30, 3I

probing of, technique, 3 I

Canine tooth, abscess at root of, 28

Canthus, inner, swelling at, 26

Capsulotomy, 279

Carbolic acid lotion, formula for, $4 \mathrm{I} 3$

Cassaripe, 438

Cataract, 2 I I-23

anterior polar, 92

causes, characteristics and signs of, I9, 2II, 2I2, 2I 3

complicating iritis, 180

concussion type, 268

development and progress of, 212

diabetic, $2 \mathrm{I} 2$

diagnosis of, 213

entropion following operation for, 57

examination of cases of, II, $2 \mathrm{I}_{4}$

following injuries, 267, 268

following iritis, 175

gait of the patient with, 2I 3

incipient, symptoms of, 21

Morgagnian, $2 \mathrm{I}_{4}$

myopia complicating, 2I 4,327

penetrating wounds causing, 272

prevention of, 175

pyramidal, leucoma with, 9 I

senile, I99, 212, 268

traumatic, 3 ro

treatment of, 2I 5,279

operative, $216-23$

Cataract knife, use of, 205, 206

Catarrh, spring, 99-I00

Catarrhal conjunetivitis, $76,80-82$

Cat's eye, amaurotic, 236

Caustics, 103, 446

Cauterisation, of the cornea, I4O, I 47

Cavernous sinus thrombosis, $4 \mathrm{I}, 227$

Cerebral arteries, retinal lesions and, 392

Cerebral cortex, lesion of hemianopia due to, 22

mind-blindness associated with, 20

Cerebral lesions, mydriasis accompanying, 357

causing facial paralysis, .50, $5 \mathrm{I}$

mind-blindness and, 22

optic symptoms of, 22

paralysis of ocular muscles due to, 54

Cerebral tumour, 386

diagnosis of, 387 
Cerebro-spinal disease, ocular symptoms of, 20

Cervical sympathetic nerves, irritation Chalazion, 46 of, as canse of glaucoma, I95

chancre simulating, 64

characteristics and features of, 46 hordeolum compared with, 46, 47 suppurating, 48

treatment of, 48

Chalybeate, Iso

Chamomile fomentations, 45 I

Chancre, diagnosis of treatment of, 63 , 64

in iritis, $16_{4}$

Chemical burns, treatment of, 255

Chemosis, 70, 133

conjunctival, 8 I, 92

due to burns, 254

Chemo-therapy of serpiginous ulcer, I37-9

Children, amblyopia in, 399, 400

blepharitis in, 42

blindness in, 88

catarrhal conjunctivitis in, 80

cicatricial ectropion in, 59

concomitant convergent squint in, $336-42$

corneal opacities in, I 44

corneal ulceration in, I 2 I

diphtheria of conjunctiva in, 86

ectropion in, 59

examination of, 2

eyestrain in, 349

follicular conjunctivitis in, 99

interstitial keratitis in, I 49

hypermetropic, development of myopia in, 329

myopic, spectacles for, 331, 332

œdema of eyelids in, 38

phlyctenular conjunctivitis in, see Phlyctenular conjunctivitis.

ptosis in, 53, 55

purulent conjunctivitis in, 82

retinal glioma in, 236

sympathetic ophthalmia in, 296

syphilis of eyelids in, 63

trachoma in, Ioo

tuberculosis of eyelids in, 65

see also Infants; Ophthalmia neonatorum.

Chinosol lotion, $4^{\mathrm{I}} 4$

Chloral-hydrate, 204

Chloral mixture, 460

Chloretone, 255, 434

Chlorine lotion, 4I 4

Choked dise, 386, 389

Cholesterine crystals, in the vitreous, 288

Choroid, atrophy of, in glaucoma, I9 I

hæmorrhage from, 2 I9, 266

pathological changes in, 397
Choroid, sarcoma of, 73, 232-5

sympathetic ophthalmia due to, 290

treatment of, 235

tubercle of, 237

\section{Choroiditis, 38 I}

associated with myopia, 326, 332

cataract following, 2 I I

disseminated, preceding interstitial keratitis, ${ }^{5} 5$

exudative, 237

non-suppurative, 397

ophthalmoscopic examination in, 397

prevention of, in myopia, 330

relation to myopia, 326, 332, 333

serous, and glaucoma, I95

sympathetic, 293

symptoms of, 19, 397

syphilis causing, $39^{8}$

treatment of, 398

Choroido-retinitis, characteristics, 379, $38 \mathrm{I}$

Chromic acid, 446

Cicatrices, varieties of, $\mathrm{I}_{42}$

Cicatricial ectropion, 59

treatment of, $6 \mathrm{I}$

Cicatricial entropion, 56

treatment of, 58

Cilia, see Eyelashes.

Ciliary arteries, 69, $7 \circ$

Ciliary body, involved in iritis, 174 paralysis of, 265

see also Cyclitis.

Ciliary injection, and conjunctival, differential diagnosis, 70

Ciliary muscle, activity in myopia, 329, 330

paralysis of, 54

spasm of, 320

strain on, $34^{6}$

working power of, tests of, 350

Ciliary nerves, septic infection by, 294, 296

Ciliary processes, starting-place of sympathetic ophthalmia, 296

Circulation, diplopia following disorders of, 367

toxins in, I33

Claims for compensation in eye injuries, 301, 302, 319

Cocain, advantages and disadvantages of, 420

anæsthesia by, in superficial injuries, 248

in adrenalin, in treatment of epiphora 29

in serpiginous ulceration, I35

in iritis, 159

Cocain oil, 434

Cocain ointment, 438

Cocain solutions, 421,422 
Cold, applications of, 256, 45I

Cold in the eye, 80

Cold in the head, and excessive lachry.

Collargol, 95 mation, 26

action and characteristics of, 430

in septic infections, 280

Collargol ointment, 439

Collosol argentum, 95, 433

Collyria, I 26

in purulent conjunctivitis, 86

in trachoma, Io3

Coloboma, congenital, 353

Colour-blindness, 404,405

Colour sense, in glaucoma, I93

Coloured rings, round gas or candle flame, significance of, I 89

Commotio retinæ, 264

Compensation, claims for, examination of cases of, 304

medical evidence in, 301, 302

Concussion injuries, 260, 26 4

cataract due to, 268

Congenital amblyopia, 399

Congenital nystagmus, 368

Congenital ptosis, 53,55

Congenital word blindness, 22

Conjunctiva, antiseptics for, 87

argyrosis of, 82

arteries of, 69-73

bulbar, affections of, I I4-I9

burns of, 253

chemosis of, $70,92, \mathrm{I} 33$

cleansing of, $8 \mathrm{I}$

dermoid cyst of, I 6

deep injection of, $7 \mathrm{I}$

diphtheria of, 86

discharge from, 7 I

diseased, complicating ectropion, 6I, 62

dislocations of, 270

epithelioma of, I I8, I I9

examination of, scheme of, 4

foreign bodies in, 249

healthy, transparency of, 69

hæmorrhage from, 73, 264

hypertrophy of, I0 3

in ophthalmia neonatorum, 89

infiltration of, removal of, Io3

inflammation of, see Conjunctivitis.

injuries to, $25^{2}$

lipoma of, I I 5, I I 6

local applications to, 77,78

pain in, relief of, 85

palpebral, cicatricial contraction of, IOI, IO2

sarcoma of, I I 7, I I 8

superficial injection of, 70

syphilitic sore on, 63, 64, I I7

tuberculosis of, I I6, I I7

xerosis of, see Xerosis.

see also Eyelids.
Conjunctivitis, 74

abnormal secretion in, 7 I

acute, $74-77$

and chronic, clinical distinction between, 97

discharge in, 75,86

treatment of, 77

and sclerotitis, differential diagnosis, I 5

angular, 98

antiseptics in, 77

bacteriology of, 75,76

blood-shot eve characteristic of, 69

catarrhal, 8o

characteristics and features of, 80

corneal ulceration in, $\mathrm{I} 2 \mathrm{O}$

specific organism of, 76

treatment of, $8 \mathrm{I}$

chrónic, $84,97-$ IO 4

and acute, clinical distinction between, 97

causes, characteristics and features of, 97

treatment of, 97

chronic catarrhal, 98

clinical signs of, 77

corneal ulcer complicated by, 126

diagnosis of, $7 \mathrm{I}, 74$

discharge in, nature of, $7 \mathrm{I}$

essential characteristics of, 74

follicular, 99, I00

frequency of, 74

gonococcal, see Gonorrhœal ophthalmia.

granular, see Trachoma.

in infants, 88

see also Ophthalmia neonatorum.

indications of attack of, 70

lymphatic, see Phlyctenular conjunctivitis.

membranous, 86,87

characteristics and features, 75, 86

treatment of, 87

pain of, nature of, 14

Parinaud's, Ioo

phlyctenular, see Phlyctenular conjunctivitis.

purulent, $76,82-4$

corneal ulceration in, I 20

œdema of lids differentiated from, $4^{\circ}$

opacities of cornea following, I 42 rupture of cornea in, I44

specific organism of, 76

treatment of, 84

uveal inflammation following, 92 see also Gonorrhœal ophthalmia; Ophthalmia neonatorum.

pustular, see Phlyctenular conjunctivitis.

simple, ; I 
Conjunctivitis, strumous, corneal ulceration in, 121 treatment of, 77 see also Blepharitis.

Consensual reflex, $358,360,375$

Constipation, 177

Constitutional remedies, formulæ for, 453

Contusions, $260-70$

treatment of, 269

Convergence, and accommodation, relation between, 346

excessive, in myopes, 325

insufficiency of, 323

Convergence reflex, test of, 360

Convex lenses, ro

Copper citrate ointment, 439

Copper sulphate, in corneal ulcer, 126 in trachoma, 103

Cornea, abrasions of, $130,132,250,25$ I alterations in, due to increased intraocular pressure, I9I, I93

burns of, 253

cicatrices of, I $42, \mathrm{I} 44$

cicatricial ectropion complicating, 6I congestion of vessels of, I 50

conical, see Keratoconus.

curvature of, 6

destruction of, in diphtheritic conjunctivitis, 87

diffuse cloudiness of, 19 I

dynamite injuries to, 288

examination of, 2, 5

extraction of foreign bodies through, 284

exudate in, absorption of, I 52

flattening of, $\mathrm{I} 44$

foreign bodies in, 248

galvano cauterisation of, 147

hæmorrhage into, 73

herpes of, 122,128

in glaucoma, I9I, 193

in ophthalmia neonatorum, 92

in trachoma, IoI

inflammation of, see Keratitis.

injection of, I 50

leucocytic action in, 134

malformations of, 146

massage of, 145,152

mixed infection of, I39

opacities of, characteristics and

- features of, $\mathrm{r}_{42-7}$

keratoconus following, 146

keratoglobus following, I47

peritomy for, 185

permanent, I5I

results of, I 43,144

treatment of, $145^{-7}$

paracentesis of, $16 \mathrm{I}$

penetrating wounds of, $271,272,274$ treatment of, 275

perforation of, 124, 134 complicating ulcer, 125
Cornea, perforation of, leucoma following, I 43

panophthalmitis following, I25

prevention of, 128

prevention of staphyloma, I 45

prolapse of iris following, I43

treatment following, 129

phlycten of, ro6

pneumococcal infection of, I3I, I33

protection of, I22

pyogenic infection of, 130,132

rupture of, I44

scars of, I 42

sensibility of, 6,52

serpiginous ulcer of, see Serpiginous ulcer.

size of, 6

strumous affection of, 105, 107

superficial injuries to, 250

surface of, 6

sympathetic inflammation of, 292

transparency of, 6

ulceration of, $52,120-30$

dacryocystitis causing, I3I

in gonorrhœal ophthalmia, 83

in ophthalmia neonatorum, 90 , 9I, 93

in phlyctenular conjunctivitis, I 2

in purulent conjunctivitis, 82

opacities due to, 126

primary, 130

scar resulting from, $9 \mathrm{I}$

silver nitrate for, 85

sympathetic ophthalmia due to, 290

treatment of, $96, \mathrm{II} 2, \mathrm{I2} 6, \mathrm{I} 28$

see also Serpiginous ulcer.

vascular condition of, see Pannus.

Corrosive sublimate, fomentations, $45 \mathrm{I}$

Cortical lesions, facial monoplegia due to, $5 \mathrm{I}$

Coryza, acute, following adrenalin injections, 29

Coughing, sub-conjunctival ecchymosis following, 73

Counter irritation, methods of, 452

Cranial nerve, seventh, see Facial nerve.

Creolin ointment, 439

Cross-eye, see Strabismus.

Crossed diplopia, 363

Croupous membranous conjunctivitis,

Cyclitis, complicating iritis, 157,174

diagnosis of, I5, I74

onset of, 157

pain of, 15

plastic, 286

Cycloplegies, in diagnosis, I I

in eye-strain, 347

Cystitome, use of, 2 I 8

Cystoid cicatrix, 209 
Dacryocystitis, acute, 27,28

treatment of, 33

characteristics, 28

complicating serpiginous ulcer, I35

erysipelas simulating, 28

hordeolum simulating, 46

œdema of lids simulating, 46

ulcer of cornea resulting from, I3 I

Daturin, I27, 426

Dazzling, 397

Deafness, in interstitial keratitis, $I_{52}$

Degenerative changes, sympathetic ophthalmia due to, 290

Demodex folliculorum, causing blepharitis, 44

Dermoid cyst, II 6

Dermo-lipoma, I 5

Descemetitis, I 74, I 76

Descemet's membrane, deposits on, 132 destruction of, $83, \mathbf{1 2 4}$

in ophthalmia neonatorum, 90

Deviating eye, in strabismus, 3.37

Deviation, angle of, how measured, 339

Diabetic cataract, 2 I 2

Diabetic iritis, I 70

Diabetic retinitis, 396

Diagnosis, important factors in, 2, I3 ophthalmoscope in, II, I3, 38.4, 398 principles of, I

scheme of examination in, 3

significance of pain in, I4 test types in, 9

Diaphoresis, 178

Diet, following operation for cataract, 220 improper, as cause of phlyctenular conjunctivitis, I09

in corneal ulcer, 126

in strumous ophthalmia, 126

in toxic iritis, 170

Digestive agents, I Io

Digestive disturbances, causing amblyopia, 403

in iritis, 177

in phlyctenular conjunctivitis, 108

Digitalis pills, 468

Dionin, action and characteristics, 422 in interstitial keratitis, 153

in iritis, 159

Dionin ointment, 439

Diphtheria, and cedema of eyelids, 40 loss of accommodation following, 316

Diphtheritic membranous conjunctivitis, 86

Diplopia, as sign of tabes dorsalis, 54 binocular, 21

causes of, 20, 363, 367.

characteristics and features of, 308 , 362,366

correction of, 332

crossed, 363

degeneration of nervous system and, $2 I$
Diplopia, examination of cases of, 21,364 , 365,366

homonymous, 363,365

in paralytic ptosis, 54

monocular, 21, 363

of myopes, 325

syphilis and, 21

transient and recurrent, 2I, 54

treatment of, 367

vertical, 364,365

Discharges, bacteriological examination of, 76

in ophthalmia neonatorum, 90

in phlyctenular conjunctivitis, I I 2 plastic, 86

Distorted vision, I9

Diuretics, formulæ for, $45^{6}$

Dmegon, for gonococcal infections, 86, 96

Donovan's pill, 469

Donovan's solution, 128

Double vision, see Diplopia.

Douches, nasal, 445

Dover's powder, 466,467

Dressings, 219,220

Duboisin, I27, 426

Dust, removal of, 248

Dusting powders, formule for, 4.49

Dynamic refraction, 3 I 5

Dynamite explosions, injuries due to, 288

Ear diseases, nystagmus due to, 369

Ecchymosis, 73, 265

Ectasia, of the iris, I 43

Ectropion, 59

blepharitis hypertrophica complicating, 60

causes and characteristics of, 43 , $59,6 \mathrm{I}$

chalazion with, 47

chronic conjunctivitis with, 97

cicatricial, 6I

effect on the puncta, 25

epiphora due to, 60

facial paralysis causing, 52

lagophthalmos complicated by, 50

senile, 60

spasmodic, 59

syphilitic and tubercular causes, 59

treatment of, 30, 6I, I03

Eczematous eruptions, 42, 43, Ios

Electric ophthalmia, 257

Electrotherapy, of inflammations of the sclerotic, 183,184

Emergency cases, 240

Emmetropia, correction of, 347

definition of, 9, 3I 4

estimation of, 9

eye-strain in, 347

myopia and, difference between, 330

Emotion, causing over-secretion of lachrymal ducts, 24 
Emphysema, and œdema of eyelids, 37. $26 \mathrm{I}$

Employer's Liability Act (I880), 300

\section{Entropion, 57}

causes of, 57

cicatricial, $57,5^{8}$

corneal complications of, $5^{8}$

effect on the puncta, 25

following operation for cataract, 22 I

in trachoma, IOI, IO2

senile, treatment of, $5^{8}$

spasmodic, $57,5^{8}$

Enucleation, 237, 238

anæsthesia during, 424

deformity produced by, 28 I

for removal of foreign bodies, 286 , 287

for sympathetic ophthalmia, 297

indications and contra-indications, I 46, 280, 297

Epicanthus, congenital ptosis with, 53

Epilation, by electrolysis, 59

Epiphora, 29

ectropion with, 59, 60

treatment of, $29,34,36$

Episcleritis, I 81

causes and characteristics of, I8I

gout and rheumatism in relation to, $I 81,182,183,184$

treatment of, 183

Episeleritis periodica fugax, $\mathrm{I} \delta 2$

Epistaxis, 26I

Epithelioma, 66

characteristics and features of, 66

epibulbar, I I 9

of the eyeids, 66,118

rodent ulcer compared with, 67

syphilis and tuberculosis, differential diagnosis of, 66

treatment of, 68

Erysipelas, simulating dacryocystitis, 28

Eserin, action and characteristics of, I $28,203,428$

advantages and disadvantages of, 203

anæsthesia by, 205

in corneal ulceration, 96,128

in glaucoma, 203

in serpiginous ulceration, 137

Eserin oil, formula, 434

Fserin sulphate ointment, 439

Eserin sulphate solutions, formula, $4^{28}$

Ethyl hydrochloride, action and characteristics of, 430

Ethylhydrocuprein, I 37,430 technique of treatment by, 138 uses of, I37, I39

Ethyl sulphide, burns due to, $25^{8}$

Eucain, action and characteristics, 422

Eucain solutions, formulæ, 422

Euphthalmin, 426

Evaporant lotions, formulæ, 4 I 8
Evidence, medical, difficulties in, 30I, 302,304

Examination of cases, general principles of, I, IO

ophthalmoscopic, technique of, II scheme of, 3

Exophthalmos, 40, 224

bilateral, 228

causes of, 225, 230, 235, 236

cavernous sinus thrombosis complicating, 227

characteristics and features of, 224 , 228

lagophthalmos complicating, 50

monocular, 229, 230

nasal examination in, 225

orbital abscess complicating, 226, 229

panophthalmitis complicating, 227

pulsating, 229

tarsorraphy for, 229

with signs of inflammation, 226

without signs of inflammation, 228

Explosions, amaurosis and amblyopia following, 40I

injuries due to, 260

Extraocular muscles, examination of, 8

Exudates, fibrinous, I57

of iritis, I74

Eye, action of atropin on, 127

anatomical structure of, 6,384

anterior chamber of, 6

artificial, 28I, 287

blood-shot, see Blooci-shot eye.

blood-vessels of, 69-73

cleansing of, $8 \mathrm{I}, 85$

" cold "in, 8o

destruction of, in gonorrhœal ophthalmia, 83

effects of a blow on, 260,264

examination of, I, 3, II, I3

foreign bodies in, 70,247

" hot," I82

injuries to, see Eye injuries.

local applications to, 77,78

muscles of, see Ocular muscles.

" pink," 8o

protective mechanism of, 242

rest for, 127,349

secretions from, examination of, 76

"sparking" of, 263

syphilitic affection of, I 64

Eyeball, abnormal enlargenient of, I 47

abnormal exposure of, I2 I

anatomy of, 384

blood supply to, 69

bursting of, 270

complete atrophy of, 193

concussion of, 260,264

contusions of, 268,269

destruction of, 87

distension of, 92,288 
Eyeball, enucleation of, see Enucleation, excision of anterior segment of, I 46

eyelids adherent to, 87

foreign bodies in, 282-9

forward displacement of, see Exophthalmos.

- impaired movements of, 226

inflammation of, 39, 69, $7 \mathrm{I}$

injection of, 69, 72

injuries to, see Eye injuries.

malignant tumour of, 232

movements of, see Nystagmus.

muscles of, 362

see a!so Ocular muscles.

paralysis of muscles of, 263

pustules and phlyctens on, 10 8

rupture of tissues of, 268

septic infection of interior of, 280

shrunken, 287

tension of, see Intraocular tension.

tuberculosis of, 172

vascular tunic of, 174

Eye-drops, formulæ, $420-32$

Eye injuries, antiseptics in, 243

burns and scalds, 253-9

contusions, $260-70$

due to accidents, $260-70$

dynamite, 288

first aid in, 247

foreign bodies in, 282-9

malingering in relation to, 3 Io

penetrating wounds, $271,282-9$

superficial, 246

treatment of, $240-5,269$

warfare, 258,260

Workmen's Compensation Act (I906) in relation to, 300-I2

see also Occupational injuries.

Eyelashes, crusts on, 43, 4.5

disease of, 60

falling out of, 60

loss of, 42

matting of, 43

pustules at roots of, 43

see also Trichiasis.

Eyelids, abnormal winking of, 49

adherence to eyeball, 87, 254

application of ointments to, 44

"arthritic foam" of, 168

atrophy of, 53

changes in shape of, 43

contractions of, 26, 50

cyst of, see Chalazion.

drooping of, see Ptosis.

destruction of skin of, 61

ecchymosis of, 26I

emphysema of, 26I

epithelioma of, 66,68

eversion of, see Ectropion.

examination of, 3,4

habit of partial closing of, 44
Eyelids, inability to close the, see I.agophthalmos.

inversion of, see Entropion.

itching and burning sensation of, 26

margin of, wounds implicating, 252

method of everting, 247

morbid changes in, 60

movements of, 4,47

œdema of, 37-4I

restoration of, 6 I

rodent ulcer of, 67,68

senile changes in, 60

suture of, 62

swelling of, in phlyctenular conjunctivitis, I I 2

syphilis of, 63,65

traumatic injury to, 26I

tuberculosis of, 65

ulceration of, $65,66,67$

union of, 255

Eyelids, see also Blepharitis, Chalazion, Styes, etc.

Eye protectors, 244

Eye-strain, 328, 343-52

asthenopia and, 345

consequences of, 343

headache and, $344,345,35^{\circ}$

ill-health due to, 343

in emmetropia, 347

in myopia, 346

in neurasthenics, 349

influence on myopia, 328

lithiasis and oxaluria associated with, 349

mechanism of, 345

occupational causes, 349

of children, 349

prevention of, $34^{8-52}$

psychical causes of, 352

spectacles in relation to, 348

symptoms of, 345

treatment of, $34^{8-52}$

Face formation, peculiarities of, 376

Facial nerve, alcohol injections along trunk of, 50

blepharospasm in relation to, 49

injury to, $50,5 \mathrm{I}$

Facial paralysis, causes and characteristics of, 50-52

ectropion resulting from, 52, 6I

Fasting spittle, 63

Fibrinous exudation, ${ }_{57}$

Fistula, of lachrymal sac., 34

Fixing eye, in strabismus, 337

occlusion of, $34^{\circ}$

Floating bodies, 1 76,327

Fluorescin solution, $43 \mathrm{I}$

Follicular conjunctivitis, 99

Fomentations, 127, I 59, 45 I

Food, mal-assimilation of, 107, 109 
Foreign bodies, 70, 131, 247, 2\$2

classification of, 286

degenerating processes following removal of, 287

diagnosis and symptoms of, 282-4

extraction of, 247, 249, 284-9

sympathetic ophthalmia due to, 29:

Formalin lotion, 4I 4

Formulæ, alkaline lotions, 4 I I

alteratives, 453-5

anresthetics, $420-33$

antiseptic lotions, $4^{12-15}$

astringent lotions, 4I5

caustics, 446

constitutional remedies, 4.53

diuretics, $45^{6}$

dusting powders, 449

evaporant lotions, $4 \mathrm{I} 8$

eye-drops, $420-32$

eye lotions, 4 I I

gìceroles, 435

hypodermic injections, 463

liniments, 447

mydriatics, 425-7

myotics, 428-9

nasal douches, 445

oily solutions, $433-5$

ointments, 436-45

pills, $468-7$ I

powders, 466-7

salines, $457-9$

sedatives, 459

stimulants, 429-33

tonics, $460-62$

Fowler's solution, 153

Fractures, gravity of, 264, 265 visual defects due to, 262

Fundus, anatomical structure of, 384 changes in, interpretation of, I 2

Fungus hæmatodes, 237

Fusion faculty, development of, 336, $3+1$

Gallic acid ointment, 439

Galvano-cautery, application to cornea, I 47

Galyl, in syphilitic iritis, 166

Gases, burns due to, $25^{8}$

Gasserian ganglion, extirpation of, 50

Gelsemium, 460, 469

Gerontoxon corneæ, I47

Glasses, see Spectacles.

Glaucoma, absolute, I9I

acute, atropin producing, 3 r 8

characteristics and symptoms of, 200, 201

deep injection a sign of, 72

diagnosis of, 201

iridectomy for, 207

acute unilateral, 234

age and sex incidence, I 88

albuminous fluids in, 197
Glaucoma, and iritis, differential diagnosis of, 157

anterior, 195

atrophy of optic nerve mistaken for, I99

attacks of, prevention of, 204

Bjerrum's sign in, rgo

causation of, 188, I94, 195

characteristics and features, I87-20I

chronic, 380

treatment of, 202, 207

congestive, $189,200,201$

iridectomy for, 205-7

corneal alterations in, 191, 193

diagnosis of, $187^{-201}$

following irido-cyclitis, 194

following iritis, 175

green reflex of, I99

hæmorrhagic, 197

heart in relation to, $\mathrm{I} 88$

incipient, signs of, 190

intraocular hæmorrhage in, 201

intraocular tension in, 194

loss of vision in, 193

malignant, 208

mydriasis in relation to, 356

non-congestive, 198

ocular fluids in, 194, 198

optic nerve changes in, I92

pain of, 72, 204

pathology of, 193-197

posterior, 195

predisposing causes of, $\mathbf{I} 88$

premonitory symptoms of, I9, 316

primary, characteristics, 187, 194, 196, 198

diagnosis of, $\mathrm{I} 5$

treatment of, 202

race incidence, 188

retention theory of, 194,198

retinal changes in, $192,197,198$

sclerotic alterations in, I9I

secondary, 132, 327

simple chronic, 198

subacute, 15,72

symptoms of, 72, 189, I96

treatment of, 202-2 ro

tumour of choroid causing, 234

uveal tract alterations in, I9I

Glaucomatous iritis, 157

Glioma, 235-238

Globe, see Eyeball

Glossary of terms, $472-476$

Glycerine, addition to eserin, 204

Glyceroles, formulæ, 435

Glycosuria, ocular changes due to, 396

Glycosuric iritis, I70

Glycosuric retinitis, 396

Gonorrhœal ophthalmia, 75, 82, 83

cause and characteristics, 82

destruction of the eye in, 83

metastatic, 84 
Gonorrhcal ophthalmia, prognosis in, 84 purulent conjunctivitis resembling, 75

specific vaccine for, 86 .

treatment of, 84

ulceration of cornea'in, 82

see also Ophthalmia neonatorum.

Gouty affections, amblyopia during, 407 treatment of, 184

Gouty episcleritis, I81, I82, I83, I84

Gouty iritis, $168,176,178,180$

Graefe knife, use of, 205, 206

Granular conjunctivitis, see Trachoma.

Granular ophthalmia, cicatricial entropion following, 56 ectropion with, 59

Granulations, treatment of, I03

Graves' disease, 229

see also Exophthalmos.

Green reflex, of non-congestive glaucoma, 199

Grey powder, 466,467

Guaiacol, I72, 43I

Gummata, of the eyelids, 64

Hæmatoma, of optic nerve, 262

Hæmorrhage, 264, 382

exophthalmos and, 229

gravity of, 265,266

in glaucoma, $20 \mathrm{I}$

sub-conjunctival, 73

sub-retinal, 267

treatment of, 269

Halos, coloured, I9

Hard eye, clinical significance of, I 87

Head, position of, in diagnosis, 3

Headache, cause of, 22 diagnostic significance of, I4 eye-strain and, $344,345,35^{\circ}$

Head înjuries, diplopia following, 367 danger of, 264,265

mydriasis following, 357

paralysis of ocular muscles following, 367

visual defects due to, 262

Health, influence on myopia, 328, 332

Heart, glaucoma in relation to, I 88

Heat applications, $256,45^{\circ}$ in iritis, I 59

Hemianopia, cause and characteristics of, 20,22

mind blindness with, 23

Hemianopsia, hysterical amblyopia with, 401 homonymous, $38 \mathrm{I}$

Hereditary nystagmus, 368

Hereditary optic neuritis, 380

Heredity, influence on myopia, 327

Herpes, of the cornea, 122

Heterophoria, $33^{2}, 33^{8}$

Hetol, $43 \mathbf{I}$

Hippus, $35^{8}$
Holocain, 205, 122

Homatropin, 426, 434

Homonymous diplopia, 363

Homonymous hemianopsia, $38 \mathrm{I}$

Hordeolum, see Stye.

Hot eye, I82

Hot metals, burns from, 253

Hydrotherapy, 184,185

Hyosein, 127

Hyperæmia, of ciliary vessels, 70 of conjunctival vessels, 70

Hypermetropia, 316 accommodation in, 338

correction of, $316-318$

definition of, 9, 3I 4

estimation of, 9

latent and manifest, 9, 317, 318

strabismus and, $33^{8}$

tests for, 3I7

Hypermetropic astigmatism, 320, 322, 323

Hypertrophic ptosis, 53

Hyphæma, 275

Hypodermic injections, formulæ for, 463 465

Hypophosphites, 460

Hypopyon, I25, I56

cause of, I 2 I, I34

characteristics and features of, I25 conditions predisposing to, I25

Hysteria, ocular stigmata of, $40 \mathrm{I}$ ptosis due to, 55

Hysterical amblyopia, 38r, 400

Hysterical symptoms, premonitory of organic disease, 2 I

Ice, application of, $45 \mathrm{I}$

Ichthargan, 43I

Ichthyol ointment, formula, $44^{\circ}$

Ill-health, eye-strain causing, 343 mydriasis accompanying, 357

Infants, blenorrhœa of, 27 blindness of, 93

corneal opacities of, I 44

corneal ulceration of, I2I

dacryocystitis of, 28

distension of eye-ball of, 92

leucoma of, 9I

nystagmus of, 92,368

purulent ophthalmia of, 88

staphyloma of, 9I

strabismus of, 92

syphilitic iritis of, I 65 see also Children.

Infection, see Septic infection.

Inflammatory pain, I 4, I5

Injections, hypodermic, formulæ, 463465

Insufficiency of convergence, 323

Insular sclerosis, 388

Instruments, sterilisation of, 2 I 8

Interstitial keratitis, see Keratitis. 
Intestinal antiseptics, in toxic iritis, 170 Intoxications, diplopia following, 367 Intraocular fluids, drainage by iridectomy, 209

Intraocular hæmorrhage, 264, 266

due to blows, 264-5, 269

in glaucoma, $20 \mathrm{I}$

profuse, 382

treatment of, 269

Intraocular tension, 187

choroid sarcoma and, 233

how kept normal, I94

how recorded, 8,189

Intraocular tension, high, I80, I89, I9I

following operation for cataract, 221

causes of, 194

in glaucoma, $157,187,189,190$

relief of, 185

retinal changes due to, 192

treatment of, 202, 205

Intraocular tumours, $232-237,381$

Iodides, in syphilitic iritis, 167

Iodipin, injections of, 179

Iodoform ointment, 87,440

in corneal ulcer, I27, I35

Iodoform powder, 449

Iodol ointment; $44 \mathrm{I}$

Ionic medication, 183

Iridectomy, complications of, 207, 208 drainage of intraocular fluids by, 209

for cataract, 216, 217

for glaucoma, 205-207

for toxic iritis, I 70

for sclerotic inflammations, 186

indications for, 179

technique of, 205, 206

Iridescent vision, I9I

Irido-choroiditis, $158,{ }_{7} 8$

Irido-cyclitis, chronic, treatment of, 298 complicating penetrating wounds, 273 following operation for cataract, 22 I glaucomatous symptoms in, I94 herpes of cornea and, I 22

relief of pain and insomnia of, I6I serous, 293, 297

sympathetic, 293, 294

see also Sympathetic ophthalmia.

Irido-dialysis, 265

Iridoncosis, $1_{58}, 1_{74}, I_{75}$

Iris, action of atropin on, 159

adhesion to lens capsule, I75, I76

atrophy of fibres of, I9I, I92

collapse of, 90

contusions of, 265

congestion of vessels of, I 57

ectasis of, I 43

examination of, 7

excision of, technique, 206

foreign bodies in, 285

implication in interstitial keratitis, I 5 I
Iris, implication in corneal opacities, 143 incarcerated, I 43

lack-lustre appearance of, I50, I76 nodules in, I64

paralysis of, 265

penetrating wounds of, 271, 272, 276

Iris, prolapse of, I24

avoidance of infection in, 276, 277 complicating corneal ulcer, 129

due to penetrating wounds, 272

during operation for cataract, 2 I9

following corneal perforation, I43

in phlyctenular conjunctivitis, Io 7

treatment of, $129,276-280$

sympathetic inflammation of, 293

total inversion of, 266

tubercles in, I7I, I72

Iris bombé, $158,174,175$

Iritis, I 55

acute, I56, I57

behaviour of the exudation in, 174

blood-shot eye in, 69, 72, 156

cause of, I55, I63

characteristics and features of, I63

chronic, I68

clinical types of, I63-I73

complicating serpiginous ulceration, 132

complications of, I $57,174-180$

cyclitis with, I 57

deep injection a sign of, 72

development of, 158

diabetic or glycosuric, I 70

diagnosis of, $\mathrm{I}^{7} 5,163$

digestive disturbances in, 177

diphtheritic, 177

effect on the pupil, 353

eserin contra-indicated in, 128

exclusion and occlusion of pupil in, I 57

exudation in, 157,158

following operative treatment of cataract, 22 I

frequency of, I 55

glaucomatous, I 57, I 75

gouty, I68, I 76,180

hypopyon accompanying, 125, 156

idiopathic, 155

infantile, 165

iridectomy for, I79

pain of, I5, I6o, I8o

photophobia in, 72

primary, 158

progress and spread of, 174, I75

prophylaxis of, 85

quiet, 156

reaction to, 156

recurrent, 169

rheumatic, I69, I76, I8o

sclerotitis with, I 82

secondary, 158

syphilitic, I64-I66, I76 
Iritis, toxic, $167-170$

traumatic, 155

treatment of, $159-161,176-180$

tuberculous, $171-173,176$

urinary condition in, 177

Iron pills, 469

Iron splinters in the eye, 283,284

Irritation, sympathetic, see Sympathetic irritation.

Isophysostigmin, in glaucoma, 204

Itching, diagnostic significance of, 15

Jequirity, I03, 43 I

Joint affections, 90, 108

Keratalgia, recurrent traumatic, $25 I$

Keratitis, blood-shot eye in, 69, 72 deep injection a sign of, 72

Keratitis, interstitial, I $49-$ I $_{54}$

causation of, I 49

characteristics and features of, I 49

circumscribed form of $\mathbf{I} 49$

complications of, $\mathbf{I}_{5} \mathrm{I}$

diffuse form of, I49

mercury and salvarsan in, I53

opacities in, I 50

symptoms of, 149

syphilis in relation to, 149

treatment of, $152-154$

neuro-paralytic, 52, 122

non-suppurative, opacities following, I $42, I_{43}$

parenchymatous, see Interstitial, above.

photophobia in, 72

phlyctenular, 72

sclerotinising, 182

suppurative, 246

sympathetic inflammation causing, 292

ulcerative, II 7

Keratoconus, 146

operative treatment, $I_{47}$

Keratoglobus, I 47

treatment of, 147

Keratomalacia, characteristics of, 123

Keratome, use of, 206

Kharsivan, in syphilitic iritis, I66

Kidney disease, ocular changes in, 389396 ophthalmoscopic examination in, 392

Klebs-Lœffler bacillus, 75,87

Koch-Weeks bacillus, $76,8 \mathrm{I}$

Lachrymal eatarrh, 25

treatment of, 29

Lachrymal ducts, anæsthesia of, 34

blocking of, 24

establishment of permeability of, 3I, 32

examination of, 4
Lachrymal ducts, forcible dilatation of, 33 micro-organisms in, 130, I32

obliteration of, 34

over-secretion of, $24,29,43$

suppuration of, 135,249

Lachrymal sac, adrenalin injections into, 29

antiseptic treatment of, $30,3 \mathrm{I}$

blenorrhœa of, 27

catarrh of, 30

extirpation of, anæsthesia during, 424 technique, 34

examination of, 4

fistula of, 34

incision of, 34

inflammation of, see Dacryocystitis.

permanent enlargement of, 34

pus formation in, 33,35

septic infection of, 30

syringing of, $3 \mathrm{I}, 33$

tumours near, diagnosis of, 29

Lachrymal secretion, micro-organisms in, 27

Lachrymation, excessive, causes and characteristics of, $24,25,27,29$

lotion for, 4 I 2

treatment of, 29, 36

in interstitial keratitis, 150

in phlyctenular conjunctivitis, I07

simple, 29

treatment of, 29

Lagophthalmos, 50

causes of, 50,52

ectropion complicating, 50

in exophthalmic goitre, 50

treatment of, 52

Lamina cribrosa, backward displacement of, 192, 199

Lapis divinus, 446

Latent hypermetropia, 317,318

Latent strabismus, 338

Lead acetate lotions, 4 16

Lead ointments, $44 \mathrm{I}$

Leber's disease, 380

Leeches; application of, 127 in gonococcal conjunctivitis, 85 in iritis, 160

in serpiginous ulceration, 136

Legal aspects of eye injuries, 300-3 I 2

Lenicet, 96

Lens, adhesion of iris to capsule of, 174, I 75, I 76

Lens, dislocation of, 267,327

during iridectomy, 207, 208

treatment of, 270

implication in myopia, 327

incision of capsule of, 279

opacity of, see Cataract.

penetrating wounds of, 27I, 272, 273

Lenses, convex, ro

examination with, Io

Lenticular astigmatism, 32 I 
Lenticular opacities, see Cataract.

Letters, loss of visual memory for, 22

Leucocyte deposits, I 82

Leucocytic action, I33, I34

Leucoma, I42

adherent, 9I, I43

characteristics and causes of, 90, 9I, I 42,143

following ophthalmia neonatorum, 90, 9 I

Leucosarcoma, 232

Levator palpebræ, injury to, 53

weakened action of, 54

Light, action on the pupil, 359

Light reflex, $35^{8}, 360$

loss of, 360,388

test of, 375

Liniments, 447

Lipoma, sub-conjunctival, I I 5

Lithiasis, eye-strain accompanied by, 349 corneal ulceration in, I 2 I

Liver, functional disorders of, eye-strain and, 349

Long sight, see Presbyopia, Hypermetropia.

Lotions, application of, $7^{8}$

Lupus, and syphilis, differential diagnosis, 65

Lymphaties, dilatation of, 65, I I 5

Lymphectasia, characteristics and features of, I I 5

Macropsia, I9, 379

Madarosis, see Eyelashes, loss of.

Magnesium sulphate mixtures, $457,45^{8}$

Magnet, extraction of foreign bodies by, 284,285

Malformations of the cornea, $I_{4} 6$

Malingering, $310^{\circ}$

blindness and, 360

claims for compensation in relation to, 3 Io

Mania, acute, atropin producing, 127

Manifest hypermetropia, 317, 318

Massage, of the cornea, I $45,{ }_{52}$

Meibomian gland, inflammation of, chalazion resulting from, 46

obstruction of, 47

Melano-sarcoma, 232

Membranous conjunctivitis, 86,87

Memory, loss of, see Mind blindness.

Menthol and cocain ointment, $44 \mathrm{I}$.

Mercury, in interstitial keratitis, I 53 in iritis, I6o, 178

in sympathetic ophthalmia, 298

in syphilitic iritis, I66, I67

methods of administration, I66, I67 tolerance of, I66

Mercury chloride mixtures, 454,455

Mercury iodide mixture, 454

Mercury lotions, 4I 4 , 4 I 7

Mercury oil, 434
Mercury ointments, $442-443$

Mercury oxide, action of, I I I

in absorption of corneal opacities, I 45 in phlyctenular conjunctivitis, I I I yellow, massage of cornea with, I 52

Mercury perchloride, in tuberculous iritis, I73

Mercury pills, 469,470

Mercury-vasogen, 298

Metabolism, defective, and ocular defects, $35 \mathrm{I}$

Metal splinters, in the eye, 283, 284

Metamorphopsia, 19, 379

albuminuric retinitis causing, 394

and myopia, 326, 327

cause of, 326

clinical significance of, 379

examination in, ro

Metastatic gonococeal conjunctivitis, 84

Metastatic infection from intraocula tumours, 235

Methylene blue lotion, 4 I 4

Miero-organisms, classification of, 76 in lachrymal passages, I30, I32

sympathetic ophthalmia due to, 294, 295

Micropsia, I9, 379

Migraine, ophthalmic, 22

Mind-blindness, cause and characteristics of, 20,22

clinical aspect of, case illustrating, 23 hemianopia with, 23

Mineral waters, 185

Miners' nystagmus, 369

characteristics and features of, 370

symptoms of, 37 I

treatment of, 37I

Mixtures, formulæ for, $453-463$

Monocular diplopia, 2 I, 363

Monocular polyopia, I9, 2 I cause of, 2 I 3

Monocular vision in.workmen, 308

Monoplegia facialis, $5^{\mathrm{I}}$

Morax-Axenfeld bacillus, 76, 98

Morgagnian cataract, $2 \mathrm{I}_{4}$

Morphia, in glaucoma, 204 in iritis, $16 \mathbf{I}$

Mouth wash, I 66

Mucocele, formation of, 27

Muscæ volitantes, 20

in toxic neuritis, I 68

Muscles, extraocular, examination of, 8 see also Ocular muscles.

Muscular asthenopia, characteristics and symptoms of, I6

Muscular twitchings, 49

Mustard gas, burns due to, $25^{8}$

Mydriasis, I 59, 356-358

blindness due to, 356

causes and characteristics of, 354 , 356,357

diagnosis of, 357,359 
Mydriasis, drugs producing, 127

glaucoma in relation to, $35^{6}$

ill-health accompanied by, 357

paralytic and spasmodic, 356

Mydriaties, $127,178,425$

action of, 354

harmful in primary glaucoma, 204

in cataract, $2 \times 5$

in strabismus, 340

Myopia, 324-335

astigmatism and, 329

cataract and, $2 \mathrm{I}_{2}, 2 \mathrm{I} 3,2 \mathrm{I} 4$

causes and characteristics of, 324, 327,329

definition of, 3 I4

diplopia associated with, 325

emmetropia and, difference between, 330

eye-strain and, 328, 346

implication of macula in, 326

inflammatory changes in, 326

influence of age on, 328

influence of health on, 328, 332

influence of heredity on, 327

onset of, $x 9$

prescription of spectacles for, 330, $33 \mathrm{I}$

simple, 3I8, 319

structures implicated in, 326, 327

symptoms of, 325

sympathetic irritation causing, 292

traumatic detachment of retina in, 267

treatment of, 330,332

Myopic astigmatism, 322

Myosis, 203, 355

causes and characteristics of, 354, 355

drugs producing, 128

exposure to light causing, 359

in iritis, 156

in toxic iritis, 168

spasmodic and paralytic, 355

Myoties, 128, 203, 428

in cataract, 2 I 5

in glaucoma, 203

Myxœdema, œdema of eyelids and, 38

Nasal douches, 445

Nasal duct, antiseptic treatment of, 30, $3^{x}$

blocking of, 27 treatment, 34

introduction of style into, 33

probing of, technique, 32

stricture of, 30,33

Nasal mucous membrane, affected in excessive lachrymation, 25

inflammation of, in phlyctenular conjunctivitis, $\mathrm{x}$ I 2

œdema of lids and inflammation of, $3^{8}$

treatment of, $3 x$
Nasal tabloids, 30

Nebula, corneal, I $_{42}$

permanent, I5I

Neisser, gonococcus of, 76,88

Neo-kharsivan, in sympathetic ophthalmia, 298

in syphilitic iritis, I66

Nephritis, ocular changes of, 389-96

Nerve fibres, 384,385

Nerve-storm, 350

Nerve supply, interference with, corneal ulceration and, $x 22$

Nervous diseases, nystagmus following, 369

Nervous system, degeneration of, ocular symptoms of, 2 I

irritability of, and ocular defects, $35^{\circ}$

Neuralgia, 16

Neurasthenia, 347

Neurasthenics, eye-strain of, 349

Neuritis, retrobulbar, see Retrobulbar.

Neuro-paralytic keratitis, 52

characteristics and causes of, $x 22$

Neuro-retinitis, 385

New-born children, ophthalmia of, see Ophthalmia neonatorum.

Night blindness, 18,380

Nitrate of silver, see Silver nitrate.

Nitrogen retention, ocular changes associated with, 393

Nodules of the iris, 164

Non-inflammatory pain, 16

Nose, empyema of sinus of, 225 see also Nasal.

Nostrils, dryness of, 25,27

Novocain, action and characteristics, 423

Novocain solution, 423,424

Numerals, loss of visual memory for, 22

Nutrition, disorders of, causing amblyopia, 403

in phlyctenular conjunctivitis, 107, 109

Nux vomica, $455,462,470$

Nystagmus, $368-72$

acquired, 369

amblyopia in relation to, 369

central scotoma associated with, 378

characteristics and features of, 368

clinical significance of, 368

congenital, 368

due to ear diseases, 369

due to nervous diseases, 369

examination of cases of, 370

following ophthalmia neonatorum, 92

miners', $369-71$

prognosis of, $37 \mathbf{I}$

symptoms of, $37 \mathrm{I}$

treatment of, $37 \mathrm{I}$

Objects, apparent alterations in size of, I9 
Occupational injuries, $241,244,248$

burns, 253-59

dynamite explosions, 288

eye-strain due to, 349

first aid in, 247, 248

malingering in, 3 Io

medical evidence in, $30 \mathrm{r}, 302$

nystagmus due to, 369

prevention of, 244

sepsis in, 243, 244

treatment of, 246,247

Workmen's Compensation Act (1906) in relation to, 300-12

Occupations, various, standards of vision in, 306

Ocular fluids, and increased tension, 194 in glaucoma, 194, r98

hypersecretion of, in glaucoma, 195

Ocular muscles, action and function of, 362

anatomy and physiology of, 362

paralysis of, 20, 54, 363

causes and characteristics, $2 \mathrm{r}$, $54,363-65$

Ocular nerves, affections of, 20

Oculo-motor nerve, lesion of, paralytic ptosis due to, 54 paralysis of, 354

Edema, angio-neurotic, 38 of the eyelids, $37-4 \mathrm{I}$

Oily solutions, formulæ for, $433-35$.

Ointments, formulæ for, $436-45$

Omnopon, 423, 424

Onyx, I2 I

Opacities, lenticular, see Cataract. of the cornea, see Cornea.

Ophthalmia, electric, 257

gonorrhœal, see Gonorrhœal ophthalmia.

strumous, see Phlyctenular conjunctivitis.

sympathetic, see Sympathetic ophthalmia.

Ophthalmia migratoria, see Sympathetic ophthalmia.

Ophthalmia neonatorum, 88-96

antiseptics in, 93, 94, 95

bacteriology of, 88

cataract following, 2 I I

cause and characteristics of, 88

corneal ulceration complicating, 9r, 93

definition of, 94

discharge in, 90

distension of eyeball following, 92

frequency of, 89

leucoma with pyramidal cataract following, 91

joint affections due to, 90

notification of, 94

nystagmus following, 92

palpebral fissure in, 93
Ophthalmia neonatorum, prevention of corneal complications, 92

prognosis of, 92

prophylaxis of, 93

signs and symptoms of, 89

silver nitrate instillations in, 93, 95

staphyloma anterior due to, $9 \mathrm{I}$

strabismus following, 92

syphilis in relation to, 93

treatment of, 94

Ophthalmia tarsi, see Blepharitis marginalis.

Ophthalmic artery, 69

Ophthalmic tabloids, 449

Ophthalmic veins, superior, tortuous enlargements of, 230

Ophthalmitis, sympathetic, 288

Ophthalmoplegia, causes of, 54

due to accidents, 263

syphilitic, 55

Ophthalmoplegia externa, 55

Ophthalmoplegia interna, 55,316

Ophthalmoscopic examination, direct, 319

for failing vision, 373,375

for hypermetropia, 317

for simple myopia, 319

general considerations on, 373, 374

importance of, II, I3, 399

in cases of cataract, 214

in general medicine, $384-98$

indirect, 319

methods and technique of, I I

Opium, in syphilitic iritis, $x 66,167$

Opsonic index, in phlyctenular conjunctivitis, Io8

Optic atrophy, 262, 38o, 38r, 383

causing blindness, 18

diagnosis of, $387,388,407$

mydriasis following, 356

primary, $3^{8} 7$

retrobulbar neuritis and, 407

tabes dorsalis and, 388

Optic nerve, changes in glaucoma, 192 hæmatoma of, 262

paralysis of, 263

pressure on, 388

septic infection by, 294, 295

structure of, $3^{8} 4$

tumour of, 230

visual and pupillary fibres of, $35^{8}$

see also Optic atrophy.

Optic neuritis, 385

causes of, 386,389

detection of, 385

general diseases accompanied by, 389

hereditary, 380

treatment of, 389

Optic tracts, hemianopia associated with disturbances in, 20

Optochin, see Ethylhydrocuprein.

Optologists, $34^{8}$ 
Orbicularis, paralysis of, $5^{\circ}$ spasm of, see Blepharospasm. want of tone in fibres of, 25

Orbit, dilatation of blood-vessels of, 229 examination of, 3

hæmorrhage into, and exophthalmos, 229

tumour of, 230

causing lagophthalmos, $5^{\circ}$

Orbital abscess, 226, 295

causes of, 40,226

diagnosis and symptoms of, 226

exophthalmos in relation to, 226

pain of, 226

Orbital cavity, inflammations of, 226, 228

Orbital cellulitis, 4o

Orchitis, treatment of, I 78

Organic disease, hysterical symptoms and, 21

Orthoform ointment, 443

Orthophoria, re-establishment of, 347

Otitis media, phlyctenular conjunctivitis and, ro 8

Oxaluria, eye-strain accompanied by, 349

Ox-eye, see Buphthalmos.

Pain, accompaniments of, I 5 aspirin in relief of,-I6I

diagnostic significance of, $I_{4}$

due to burns, 254

inflammatory, I4

in superficial injuries, $25^{\circ}$

neuralgic, 16

non-inflammatory, 16

of glaucoma, relief of, 204

of muscular asthenopia, 16

of iritis, I 55

relief of, I 60

of orbital abscess, 226

of retinal asthenopia, 16

of toxic iritis, 169

of various ocular conditions, 15

phenomenon of, $\mathrm{I}_{4}$

relief of, $85,127,180,252,298$

significance of, I8o

subjective nature of, 14

Palpebral fissure in ophthalmia neonatorum, 93

Palpebral syphilis, 63

Palpebral tuberculosis, 65

Pannus, 106, I50

in trachoma, ror, ro2

scrofulous, 106, 107

treatment of, 103

Panophthalmitis, $4^{\circ}$

acute, following suppuration, 25 I

displacement of eyeball in, $4^{\circ}$

ectasia of iris and, $\mathrm{I}_{43}$

following corneal perforation, 125

following corneal ulcer, 123

following orbital abscess, 227

Papilla, Stauung's, 386
Papillitis, 295

causes of, 386

Papillœdema, 385

Paracentesis of cornea, in iritis, I6I

Paraffin applications, 256

during operative measures, 277

in fitting of artificial eye, $28 \mathrm{I}$

Paralytic ectropion, 6I

Paralytic mydriasis, 356

Paralytic myosis, 355

Paralytic ptosis, 54

Paralytic squint, 336

Parenchymatous keratitis, see Keratitis, interstitial.

Parinaud's conjunctivitis, Ioo

Patient, exaggeration of symptoms by, I 3 manner of, aiding diagnosis, 2

Pediculi, causing blepharitis, 44 in phlyctenular conjunctivitis, II 2

Penetrating wounds, $27 \mathrm{I}-89$

classification of, $27 \mathrm{I}$

conditions due to, 27 1, 272

diagnosis of, 274,275

legal aspect of, 308

prognosis of, 272

sepsis complicating, 273

sympathetic ophthalmia due to, 290,294

treatment of, $273-8$ I

with retention of foreign body, 282-89

Perchloride of iron, $456,45^{8}$

Peritomy, for sclerotic inflammations, I 85,186

Permanganate of potash, 87,95

Phagocytosis, 133

Phenacetin, 466

Phenosalyl lotion, 4I 5

Phlycten, ro6

Phlyctenular conjunctivitis, $2, \mathrm{IO}^{-1} 3$

characteristics and features of, 105 . 107

corneal ulceration in, II 2, I 2 I

diet in, 109, 126

differential diagnosis of, II 7

digestive disturbances in, 107, 108, I09

drugs in, I09, I Io

entropion with, 56

inflammation of nasal mucous membrane in, II 2

mal-assimilation of food in, Io9

muco-purulent discharge in, I I 2

œedema of the lids with, 38

opacities of cornea following, I 42

opsonic index in, Io8

pediculi complicating, I I 2

photophobia of, I0 5

scrofula in relation to, 107

signs and symptoms of, I07

swelling of eyelids complicating, I I 2

treatment of, ro8-II

tuberculosis and, 107, 108 
Phlyctenular keratitis, 72

Phosphates, 460,462

Phosphorus pills, 470

Photophobia, I6

blepharitis causing, 43

conjunctival injection with, 7I

gas burns causing, 258

manner of patient in diagnosis of, 2 of iritis, 72

of keratitis, 72,150

of phlyctenular conjunctivitis, 105 pain of, 15

Phtheiriasis, 44

Phthisis bulbi, 144, 172, 293

Physiognomy, interstitial keratitis associated with peculiarities of, I 5 I

Physostigmine lotion, 4 I9

Picric acid, 256

formula, 43 I

Pigmentation, I 75, 232

Pills, formula for, $468-7$ I

Pilocarpin, action of, 203, 204

and eserin combined, 129

in corneal ulceration, 128

in glaucoma, 203

in toxic iritis, I7o

Pinguecula, characteristics and features of, II4

Pinhole test, 3 I $_{4}$

Pink eye, 8o

v. Pirquet's tuberculin reaction, $I_{72}^{2}$

Plummer's pill, 178,470

Pneunococcal ulceration, I3I, I32

antiseptic treatment, I 57

cauterisation of, $\mathrm{x}_{4} \mathrm{O}$

ethylhydrocuprein treatment of, 137 I 38

first-aid in, 135

pathology of, I33

treatment of, I36-4I

Pneumococei, antiseptics destroying, I37 effect of ethylhydrocuprein on, 137 in conjunctivitis, 76

in lachrymal secretion, 27

Pneumococcus lanceolatus, infection by, I 30

Poison gases, burns due to, $25^{8}$

Polyopia, 146

monocular, I9, 2I, 213

Pons, lesions of, facial paralysis due to, 51

Poppy-heads, and chamomile flowers, infusion of, $x 52$

Posterior synechiæ, I 56

Post-neuritic atrophy, characteristics of, 387,388

Potassium iodide mixtures, 454,455

Potassium iodide ointment, formula, 440

Potassium permanganate lotion, formula, 4 I 5

Powders, formulæ for, 466
Pregnancy, albuminuric retinitis of, 392-94

Presbyopia, $3 x_{5}$

characteristics of, 315

premonitory sign of glaucoma, 316 spectacles for, 315

Probes, use of, $30,32,58$

Proptosis, see Exophthalmos.

Protargol, 95

action and characteristics of, 430 in trachoma, ro3

use of, 33

Protargol ointment, 444

Protargol solution, 432

Protective bodies, 133

Protective mechanism of the eyes, 242

Pseudo-glioma, 237

Pseudo-pterygium, I I 5

Psychical causes of eye-strain, 352

Pterygium, II 4 I I 5

Ptosis, 53

characteristics and features of, 53

congenital, 53

treatment of, 55

hypertrophic, 53

hysterical, 55

in childhood, 55

in exophthalmos, 228

operations for, 55

paralytic, 54

recurring, 55

senile, 54

trachomatous, 102

traumatic, 53.

treatment of, 55

unilateral, 53

Puffiness of the eyelids, 37-4I

Pulsating exophthalmos, 229, 230

Puncta, congenital absence of, 25

contraction of, 29

displacement of, $24,25,43,60$

treatment of, 30

partial occlusion of, 30

Pupil, abnormalities of, 353

appearance of, diagnostic significance of, 7

action of light on, 359

Argyll Robertson type of, 360

colour and shape of, 8,353

contraction of, see Myosis.

dilatation of, see Mydriasis.

examination of, 7,353

exclusion and occlusion of, 157,158 , 175

in health and disease, 353-6I

inequality of, $357,35^{8}$

jet-black appearance of, conditions affecting, 353

mobility of, 358

normal condition of, 353

optic nerve in relation to, 358

physiology of the movements of, 354 
Pupil, position, size, and shape of, 7,354 , $35^{8}$

reaction of, 8

reflex dilatation of, 36 I

reflexes of, 354, 358, 375

optic atrophy in relation to, 388 synechia of, 8

Purgatives, I I0, I 77, 463

Purulent conjunctivitis, $76,82-84$

Pustular conjunctivitis, see Phlyctenular conjunctivitis.

Pustular elevations, 106

Pyogenic infection, see Septic infection.

Pyogenic micro-organisms, prevention of cultivation of, 222

Pyoktanin, 432

Pyramidal cataract, leucoma with, 9 I

Quicklime burns, 255

Quiet iritis, I 56

Quinine, and grey powder, 153, 466 in phlyctenular conjunctivitis, I IO

Quinine lotion, 4r 5

Quinine mixtures, $460,46 I$

Quinine sulphate, 87,461

Quinsey, I69

Radium, burns due to, 257 for sarcoma of conjunctiva, I 8

Reaction of degeneration, in facial paralysis, 52

Reading, position of the body during, 329

Recti muscles, action and function of, 362

advancement of, $34 \mathrm{I}$

paralysis of, 362-66

tenotomy of, $34 \mathrm{I}$

Recurrent iritis, I69

Reflexes, pupillary, 375

abnormalities of, 359

examination of, $358,359,375$

Refraction, atropin in, 318

dynamic, 3 I 5

errors of, blepharitis due to, 42

diagnosis of, 322

differential diagnosis of conditions due to, Io

eye-strain and, 348

ill-health due to, 343

in chronic conjunctivitis, 97

tests for, 314

treatment of, $3 r_{4}$

examination of, 8 ,

static, 315

Refractionists, 348

Relative scotoma, I90

Renal inadequacy, 177

Resorcin lotion, 4I5

Retina, arteries of, degenerative changes in, 391

embolism of, 17,382

pallor of, I93
Retina, central and peripheral parts of distinction between, 376

cerebral arteries in relation to, 392

change in relative position of cones of, 19

changes in glaucoma, 192

coagulation of blood in, 198

detachment of, $\mathrm{I} 7,38 \mathrm{I}$

foreign bodies causing, 286, 287

in myopia, 327

in sympathetic ophthalmia, 295

premonitory symptoms of, 20

traumatic, 267,269

tumours causing, 233, 234

glioma of, $235-37$

case illustrating, 238

characteristics and features of, 235

treatment of, 237

hæmorrhage from, 392

" hole" in, 266

hyperæsthesia of, 20

macula of, 376

progressive degeneration of, 18

rupture of vessels of, 266

thrombosis of veins of, 197, 198, $3^{82}$ traumatic injury to, 264

Retinal asthenopia, 16

Retinitis, diabetic, diagnosis of, 397 treatment of, 396

sympathetic, 293

Retinitis albuminurica, characteristics and features of, 390-96

diabetic retinitis and, 396

diagnosis of, 392

metamorphopsia due to, 394

prognosis in, 393

prognosis in relation to sight, 393

prognosis in regard to life, 394

treatment of, 395

Retinitis pigmentosa, $18,380,381$

Retinitis proliferans, 266

Retinoscopy, for hypermetropia, 317 for simple myopia, 318

Retrobulbar neuritis, 382, 405 acute, 382

and toxic amblyopia, 405

characteristics and features of, 405 optic atrophy and, 407

prognosis of, 406

sudden failure of vision due to, 379 treatment of, 408

Retrotarsal fold, upper, method of ex posing, 247

Rheumatic affections, treatment of, 184

Rheumatic episcleritis, I8I, I82, I84

Rheumatic facial paralysis, 52

Rheumatic iritis, $168,176,180$

Rheumatic sclerotitis, I83, I84

Rheumatism, I68

amblyopia during, 407

Rhubarb and antimony, 466, 467

Rhubarb pill, 47 I 
Rodent ulcer, case illustrating, 68 epithelioma compared with, 67 growth and spread of, 67, 68 treatment of, 68

Round shoulders, 333

\section{Saemisch's ulcer, I 3 I}

Sago-grain bodies, in trachoma, IO2

Salicylic acid and borax, in follicular conjunctivitis, 99

Saline lotions, 4 I I-I 2, 4 I9

Salines, 35I, 457-9

Salivation, 178

in antisyphilitic treatment, I66

Salvarsan, in interstitial keratitis, I 53

Sarcoma, 232

of the choroid, 232

of the conjunctiva, II 7

Scabs, on eyelashes, 43,45

Scalds, 254

Scars, corneal, $\mathrm{I}_{42}$

School children, myopia of, 333

Scleritis, blood-shot eye in, 69 characteristics, 72

Sclerotic, alterations in, I9 I cicatrisation of, 182, 209 dynamite injuries to, 288 examination of, 5

foreign bodies in, extraction of, 284 , 285

healing process of, 273

incision of, 209, 2 Io

inflammation of, I 8 I -6

see also Episcleritis; Sclerotitis, etc.

in glaucoma, I9I

leucocyte deposit in, I82

penetrating wounds of, $27 \mathrm{I}-3$

puncture of, technique, I85, 205

rupture of, 268, 269

thickened patches of 182

traumatic injury to, 268

Sclerotinising keratitis, I 82

Sclerotitis, causes and characteristics of, $\mathrm{I} 8 \mathrm{I}, \mathrm{I} 82$

definition of, I8I

iritis with, I 82

treatment of, I $83-6$

Sclerotomy, for glaucoma, 209

technique, 2 IO

Scopolamin, I27, 423

Scotoma, 233

associated with myopia, 326

causes of, $377-9$

central, 377, 379, 404

clinical significance of, 377

failing sight due to, 377,378

nature of the defects due to, 377,378

negative, 378,404

positive, 377,378

relative, $190,377,378,404$

of toxic amblyopia, 404
Scrofula, blepharitis in relation to, 44 phlyctenular conjunctivitis and, I07

Serofulous pannus, I06, I07

Second sight, I9

Secretions, examination of, 76 method of obtaining specimens of, 76

Sedatives, I28, 459, 460

Senile cataract, 2 I2

Senile ectropion, 60

Senile entropion, $5^{8}$

Senile ptosis, 54

Senile reflex, simulating cataract, 2 I 2

Sensibility, general, disorders of, ${ }_{4}$

Septic infection, 130, 132, 222, 243

complicating penetrating wounds, 273

complicating prolapse of iris, 276

diplopia following, 367

following operation for cataract, 22I, 222

pain in diagnosis of, ' 15

paths of, 294, 295

sympathetic ophthalmia due to, 293

treatment of, 280

Serpiginous ulcer of cornea, $130-4 \mathrm{I}$

bacteriology of, $130-2$

characteristics and features of, I30, I3I

dacryocystitis complicating, 135

diagnosis of, 132

opacities following, $\mathbf{I}_{42}$

pathology of, I33

prognosis of, I 33

spread of, I 33

treatment of, İ $34-4$ I

Serum therapy, 452

of iritis, 177

of orchitis, 178

of serpiginous ulceration of cornea, I 39

Shell injuries, $260,40 \mathrm{I}$

Short sight, see Myopia.

Sideroscope, use of, 284

Siderosis bulbi, 283

Sight, see Vision.

Silver nitrate, action and characteristics of, $95,412,432$

administration of, 432

as caustic, 446

for conjunctival infiltration, I03

in burns, 256

in corneal ulceration, 85,126

in gonococcal conjunctivitis, 85

in ophthalmia neonatorum, 93,95

in purulent conjunctivitis, 85

in suppurative conditions, 85

Silver nitrate solutions, 432, 433

Silver salts, 95

-Sinusitis, displacement of eye-ball in, 40

Skull fractures, gravity of, 265

mydriasis following, 357

visual defects due to, 262 
Skull, malformations of, 389

Snellen's test types, 9, 305

Sodium ethylate, 447

Sodium salicylate mixtures, $45^{8}$

Sodium salicylate powders, 467

Soporifics, in glaucoma, 204

Spa treatment, $184,185,408$

Sparking of the eye, 263

Spasmodic ectropion, 59

Spasmodic entropion, 57,58

Spasmodic mydriasis, 356

Spectacles, eye-strain in relation to, $34^{8}$ following operation for cataract, 220

frequent changing of, 189

in neurasthenic cases, 347,348

prescribing of, 312,313

for astigmatism, 323

for eye-strain, 348

for myopia, 330, 33 I

for presbyopia, 3I5, 3I6

for simple myopia, 319

Spinal cord, disease of posterior columns of, sudden loss of sight due to, I 8

optic atrophy related to disease of, 387,388

Spindle spot, 292

Spirochætes, I65

Splinters, removal of, 283,285

Spring catarrh, 99, roo

Squint, see Strabismus.

Staphylococcus, in conjunctivitis, 76

Staphyloma, anterior, characteristics and features of, $9 I$

following ophthalmia neonatorum, 9 I

following serpiginous ulcer, 132

formation of, 144

causes and characteristics of, 144

ciliary, 183

complicating interstitial keratitis, I 5 I

complicating sclerotitis, 182

complicating myopia, 327

intercalary, 183

multiple, 183

prevention of, 145

partial, I44

total, $\mathrm{I}_{44}, \mathrm{I}_{4} 6$

treatment of, 146

Static refraction, $3 \mathrm{I} 5$

Stauung's papilla, 386

Stavesacre ointment, 444

Steel splinters, in the eye, 283,284

Stillicidium, see Epiphora.

Stimulants, I 26, 429

formulæ for, 429-33

Stovain solution, 425

Strabismus, $336-42$

accommodation theory of, 337

alternating, 337

amblyopia and, 400

K K
Strabismus, apparent, 338,339

atropin in, 34I

cerebral lesion causing, 20

characteristics and causes of, 336

concomitant, characteristics, 336

treatment of, $34^{\circ}$

concomitant convergent, in children, 336-42

constant, 337

convergent, 337

deorsumvergent, 337

diplopia and, 364

divergent, 337

examination of cases of, 339

"fixing " and "deviating" eye in, 337

following ophthalmia neonatorum, 92

hypermetropia and, 338

latent, 338

occlusion of the fixing eye in, 340

paralysis of ocular muscles causing, 364

paralytic, 336

sursumvergent, 337

training of fusion sense in, 34I

treatment of, 340, 34I

Streptococcus, in conjunctivitis, 76

Strophanthus, 46 I

in glaucoma, 203

Strumous ophthalmia, see Phlyctenular conjunctivitis.

Strychnin, $46 x$

Stye, blepharitis complicating, 46

chancre simulating, 64

chalazion compared with, 46,47

characteristics and features of, 28,45 , 47

definition of, 45

differential diagnosis of, 46,47

evacuation of contents of, 47

predisposing causes of, 46

treatment of, 46

Styles, use of, 33

Sub-conjunctival ecchymosis, 73

Sub-conjunctival hæmorrhage, 73

Sub-conjunctival injections, $45^{\circ}$

Sugar, effect on lime, 255

Sulphur ointment, 444

Suppuration, $243,244,250$

acute panophthalmitis following, 25 I

causes of, 246

following operation for cataract, 22I, 222

see also Septic infection.

Suprarenalin ointment, 444

Symblepharon, cicatricial entropion with, 57

from burns, 255

prevention of, 256

treatment of, 257

Sympathetic, cervical, paralysis of, 354 . 355 
Sympathetic inflammation, see Sympathetic ophthalmia.

Sympathetic irritation, 287

characteristics and features of, $29 \mathrm{I}$

and inflammation, distinction between, 291

mechanism of, 294

Sympathetic ophthalmia, 288, 290-9, 309

blood changes in, 295, 296

cause and characteristics, 290, 292, 293, 296

claims for compensation for, 309

detachment of retina in, 295

diagnosis of, 295

drugs in, 298

enucleation for, 297

mechanism of production of, 294

onset and progress of, 29I, 296

paths of infection in, 294, 295

pathogenesis of, 293

prevention of, 297

sympathetic irritation and, difference between, 29I

symptoms of, 292,296

toxremia of, 296

treatment of, 297-9

Symptoms, exaggeration by patient, I3

hysterical, and organic disease, 2 I

importance of correct interpretation of, 13

subjective and objective, I3

Synchysis scintillans, 288

Synechiæ, anterior, complicating prolapse of iris, 276

detection of, I I

posterior, I5, I56, I58, I69, I74, 293

Syphilis, 63, I76, 304

a cause of choroiditis, 398

and lupus, differential diagnosis, 65

causing ocular paralysis, 2 I

congenital, I65, 399

as cause of interstitial keratitis, I 49

diplopia and, 2 I

ophthalmia neonatorum in relation. to, 93

ophthalmoplegia of, 55

paralysis of ocular muscles due to, 54

tuberculosis resembling, 65, I I7

Syphilitic iritis, I 64-6

Syringes, use of, 95

Tabes dorsalis, diplopia as sign of, 54 optic atrophy and, 388

Tabetic atrophy, characteristics of, 407

Tabloids, 449

Tannic acid lotion, formula, $4^{\mathrm{I}} 7$

Tannin, in trachoma, I03

Tarsal cartilage, inflammatory thickening in trachoma, 101, 102

Tarsal eyst, see Chalazion.

Tarsorraphy, for exophthalmos, 229
Tarsus, syphilitic disease of, 64

Tartar emetic, in phlyctenular conjunctivitis, 109

Tear passages, diseases of, $24-36$ see also Lachrymal ducts.

Tears, action of, on calomel, i I I oversecretion of, causes of, 24, 29, 43 treatment of, 29, 36

Tenon, capsule of, function of, 242

Tenonitis, characteristics and causes, 40, 226

diagnosis of, $4 \mathrm{I}, 226$

Tenotomy, of recti muscles, $34 \mathrm{I}$

Test types, use of, 9 visual acuity measured by, 305

Thiocol, I72

Thiosinamin ointment, 445

Thiosinamin pill, $47 \mathrm{I}$

Thrombosis, retinal, 197, 198

Tic douloureux, 50

Tobacco amblyopia, 3 II, 402, 408

Tonies, formulæ, $460-2$

Toxæmia, 402, 408, 409 in sympathetic ophthalmia, 296

Toxic amblyopia, 379,402

Toxic iritis, $167-70$

Toxins, action of, 133, 134 hypopyon due to action of, 134 in circulation, 133

Trachoma, Ioo-4

antiseptics in, 103

cause and characteristics of, Ioo, IOI caustics in, 103

corneal ulceration in, I2I

diagnosis and onset of, IOo, IOI

incurable stage of, IO4

ptosis in, 102

pannus in, 102

treatment of, 102

trichiasis in, I0I, I02

xerosis in, IOI, IO2

Transillumination, in diagnosis of exophthalmos, 226

in diagnosis of tumours of choroid, 233

method of, 200

Transparent media, changes in glaucoma, I92

Transplantations, 257

Traumatic amblyopia, 401

Traumatic asphyxia, 382

Traumatic cataract, 310

Traumatic detachment, of retina, 267, 269

Traumatic injuries, 260-70

Traumatic keratalgia, recurrent, 25I

Traumatic ptosis, 53

Trephining, for intraocular tension, I85

Trichiasis, cause of, 43

cicatricial entropion with, $57,5^{8}$

corneal ulceration in, I2 I

in trachoma, IOI, IO2

treatment of, $5^{8}$ 
Trichloracetic acid, 447

Trional, $\mathrm{x} 6 \mathrm{r}$

Tubercle bacillus, phlyctenular conjunctivitis in relation to, Io7

Tuberculin, in tuberculous iritis, 172

Tuberculin diagnosis, 65

Tuberculin reaction, 172 in phlyctenular conjunctivitis, ro7

Tuberculosis, and syphilis, differential diagnosis, 65

" candidates for," ro7

differential diagnosis of, $\mathrm{I} I 7$

interstitial keratitis in relation to, r 49

of the conjunctiva, Ix 6

of the eyelids, 65

phlyctenular conjunctivitis in relation to, 107, I0 8

Tuberculous iritis, $163, \mathrm{r} 7 \mathrm{r}-3$

Tumours, 230, 232

cerebral, 386

diagnosis of, 233

intraocular, $38 \mathrm{I}$

Turbinate, inferior, congestion of erectile tissue over, 29, $3 \mathrm{I}$

Twitching, and eye-strain, 345

Tylosis, see Blepharitis hypertrophica.

Typists, eye-strain of, 349

Tyrosine crystals, in the vitreous, 288

Ulceration, bandaging in, $\mathbf{I} 26$

cicatrix resulting from, $\mathrm{I}_{42}$

conjunctival, 83

corneal, see Cornea.

diagnosis of, 123

in ophthalmia neonatorum, 90

in phlyctenular conjunctivitis, ro6, I 12

of the eyelids, $65,66,67$

pneumococcal, $13 x, x 33,135$

treatment of, $134-4 \mathrm{r}$

primary, 130

serpiginous, see Serpiginous ulcer.

treatment of, 126

Uræmia, failing vision due to, 383

Uræmic amaurosis, 390

Urine, examination of, $35^{\mathrm{r}}$

in iritis, 163,177

in toxic amblyopia and tabetic atrophy, 407, 408, 409

in toxic iritis, 168

Urticaria, œdema of eyelids in relation to, 38

Uveal tract, alterations in glaucoma, rgr implication in iritis, 157

inflammation following purulent conjunctivitis, 92

sarcoma of, 232

Vaccine, anti-gonococcic, 96

Vaccine therapy, $45^{2}$

of iritis, 177
Vaccine therapy of serpiginous ulceration of cornea, 139

of toxic iritis, 170

Valerian, 463, 47 $\mathrm{x}$

Varicose vessels, 73

Vaso-motor disturbance, in sympathetic ophthalmia, 295

Venæ vorticosæ, I97

Veratrin ointment, 445

Veronal, in glaucoma, 204

Vertical diplopia, 364,365

Vessels of repair, 124

Vinum opii, in interstitial keratitis, I 53

Vision, acuity of, how measured, 305

how expressed, 305

in myopes, $33 \mathbf{I}$

on what dependent, 376

test for, 375

binocular, see Binocular vision.

central and peripheral, loss of, 376 , 382

comfortable, on what dependent, 346

defects of, claims for compensation for, 304

due to opacities, $\mathrm{r}_{43}$

examination of, for spectacles, $3 \times 3$

keratoconus causing, $\mathrm{r}_{4} 6, \mathrm{I}_{47}$

malingering and, 3 I I

tests for, $3{ }^{1} 4$

distorted, I9

double, see Diplopia.

examination of, methods of, 8

failing, albuminuric retinitis causing, 393

causes of, 373

choroiditis causing, 397

clinical significance of, 373-83

examination for, $373,375,377$

kidney disease causing, 389

mydriasis and, $35^{6}$

of both eyes, 379

toxic causes of, 403,404

field of, 306

central loss of, 377

contraction of, $380,38 \mathrm{I}, 400$

examination of, 376

factors influencing the extent of, 376

hand measurement of, 376

how mapped out, 306

peripheral loss of, 38I

restriction of, in glaucoma, 193

symmetrical defects in, 2 I

total loss of, 382

iridescent, $\mathrm{r} 9 \mathrm{I}$

long, see Presbyopia; Hypermetropia.

loss of, gradual, I8, 379

sudden, $17,378,379$

transient, 19

see also Blindness.

monocular, 307,308 


\section{INDEX}

Vision, peripheral, 380,407

"second," I9

short, see Myopia.

standards of, in various occupations, 306

temporary obscuration of, I 89

tests of, 9, 10, I I

Visual function, disorders of, 17-23

Visual memory, loss of, 22

Vitriol burns, 255

Vitreous, cholesterine and tyrosine crystals in, 288

escape of, during operation for cataract, 219

excess of pressure in, I9r

hæmorrhage into, causing loss of sight, I 7

in penetrating wounds, 272

opacities in, 176

post-traumatic retraction of, 267

Warfare gases, burns due to, $25^{8}$

Warfare injuries, 260 see also Penetrating wounds.

Warts, of the eyelids, 66,67

Waste products, elimination of, 395,408 imperfect elimination of, toxic amblyopia and retrobulbar neuritis due to, 403, 406

Watering-places, treatment at, I 84

Watery eye, 24

blepharitis causing, 43

causes and characteristics of; 24,26 , 29
Watery eye, lotion for, $4^{12}$

of keratitis and iritis, 72, I50

sudden onset of, 29

treatment of, 29, 35

see also Lachrymation.

White of the eye, 69

Whooping cough,sub-conjunctival ecchymosis in, 73

Windage, eye injuries due to, 260

Word-blindness, 22

Workmen, injuries to, see Occupational injuries.

Workmen's Compensation Act (I906), provisions of, 300

Wounds, see Penetrating wounds.

Writing, position of the body during, 329

$\mathrm{X}$-rays, burns due to, 257

detection of foreign bodies by, 284

Xerosis, in trachoma, IOI, IO2

Xerosis bacillus, 76

Yellow oxide of mercury, action of, I I I

in phlyctenular conjunctivitis, I II

Zigzag lines, 22

Zine chloride lotion, 4 I 5

Zine chloride paste, 447

Zine phosphide pill, $47 \mathrm{I}$

Zine sulphate lotions, $98,4^{17}$

Zine sulphate solutions, 433

Zona ophthalmica; I22, 128 


\section{OXFORD $\mathscr{H} E D I C A L$ PUBLICATIONS}

\section{TROPICAL OPHTHALMOLOGY}

By R. H. Elliot, M.D., L.R.C.P., F.R.C.S., late Superintendent Government Ophthalmological Hospital, Madras; Professor of Ophthalmology, Medical College, Madras.

Demy 8vo. Cloth. 520 pages, fully Illustrated, and with seven coloured plates.

In the Press

\section{DISEASES OF THE EYE}

A Manual of Essential Facts for Students and Practitioners

By M. Stephen Mayou, F.R.C.S., Assistant Surgeon, Central London Ophthalmic Hospital, etc.

Crown 8vo. Cloth. Third Edition. About 400 pages, well Illustrated in black and white, with five plates, four in colour, and one monochrome.

\section{THE PATHOLOGY OF THE EYE}

[In the Press

By J. Herbert Parsons, B.S., D.Sc. (Lond.), F.R.C.S. (Eng.), Assistant Surgeon, Royal London (Moorfields) Ophthalmic Hospital ; Assistant Ophthalmic Surgeon, University College, London; Ophthalmic Surgeon, Hospital for Sick Children, Great Ormond Street.

\section{In 4 volumes, with over 700 lliustrations.} Royal 8vo. Cloth.

(Awarderl the Nettleship Gold Medal of the Ophthalmological Society.) Vols. I and 4 out of print.

\section{THE FUNDUS OCULI : An Ophthalmoscopic Atlas}

Illustrating its Physiological and Pathological Conditions: By W. Adams Frost, F.R.C.S., Consulting Ophthalmic Surgeon, St. George's Hospital.

In one 4 to volume, extra cloth, gilt top, with 47 plates, containing 170 beautifully coloured figures and numerous engravings in the Text.

\section{INJURIES OF THE}

\section{EYES, NOSE, THROAT AND EARS}

By A. Maitland Ramsay, M.D. (Glas.), Major R.A.M.C. (T.), 3rd Scottish General Hospital, Glasgow ; J. Dundas Grant, M.D., F.R.C.S (Eng.), late Major R.A.M.C. (Post Office Rifles Volunteers), King George Hospital, London; Lord Knutford's Special Hospital for Officers ; H. Lawson Whale, M.D. (Camb.), Captain R.A.M.C. (T.), formerly No. I 3 General Hospital, British Expeditionary Force, Overseas; The County of London War Hospital, Epsom; and C. Ernest West, F.R.C.S.(Eng.), Captain R.A.M.C. (T.), Ist City of London General Hospital.

Pp. 160. 11 Figures.

HENRY FROWDE, HODDER AND STOUGHTON

17 Warwick Square, Newgate Street, London, E.C. 4 


\section{MANUAL OF THE PATHOLOGY OF THE EYE}

\section{A Manual for Students and Post-Graduates}

By P. E. H. Adams, M.B., F.R.C.S.

Honorary Surgeon, Oxford Eye Hospital.

Crown 8vo. Cloth, pp. 160. 45 Illustrations.

"Bears the stamp of practicality throughout. Evidently it has been written from personal experience in the teaching of ocular pathology."-Liverpool Medico-Chirurgical Journal.

\section{OPHTHALMOLOGICAL ANATOMY}

With Some Illustrative Cases.

By J. Herbert Fisher, M.B., B.Sc. (Lond.), F.R.C.S.

Royal 8vo. Cloth. Illustrated, pp. 188.

\section{TRACHOMA}

By Dr. J. Boldt.

Translated by J. Herbert Parsons, D.Sc., F.R.C.S. and Thos. Snowball, M.B.

Royal 8vo. Cloth.

\section{EPIDEMIC OPHTHALMIA}

Its Symptoms, Diagnosis and Management

By Sydney Stephenson, F.R.C.S. (Edin.).

Ophthalmic Surgeon, Evelina Hospital and N.E. Hospital for Children, London.

8vo. Cloth, pp. xvi, 278. Illustrated with a Coloured Plate and 25 Figures in the Text.

\section{MANUAL OF PRACTICAL OPHTHALMOLOGY}

By George A. Berry, M.B., F.R.C.S. (Edin.).

Consulting Ophthalmic Surgeon, Edinburgh Royal Infirmary; Surgeon, Edinburgh Eye Dispensary; Lecturer on Ophthalmology, University of Edinburgh.

Crown 8vo. Cloth, pp. $x x, 570$, with 223 Illustrations, mostly from original drawings.

"Dr. Berry has the knack of telling the practitioner exactly the kind of thing he wants to know and often looks for in vain in the average textbook."-Liverpool Medico-Chiruvgical Journal.

\section{THE ARRIS AND GALE}

\section{LECTURES ON THE NEUROLOGY OF VISION}

By J. Herbert Parsons, B.S., D.Sc. (Lond.), F.R.C.S. (Eng.).

Two Coloured Plates and 22 Illustrations. Demy 8 vo, paper.

HENRY FROWDE, HODDER AND STOUGHTON

17 Warwick Square, Newgate Street, London, E.C. 4 






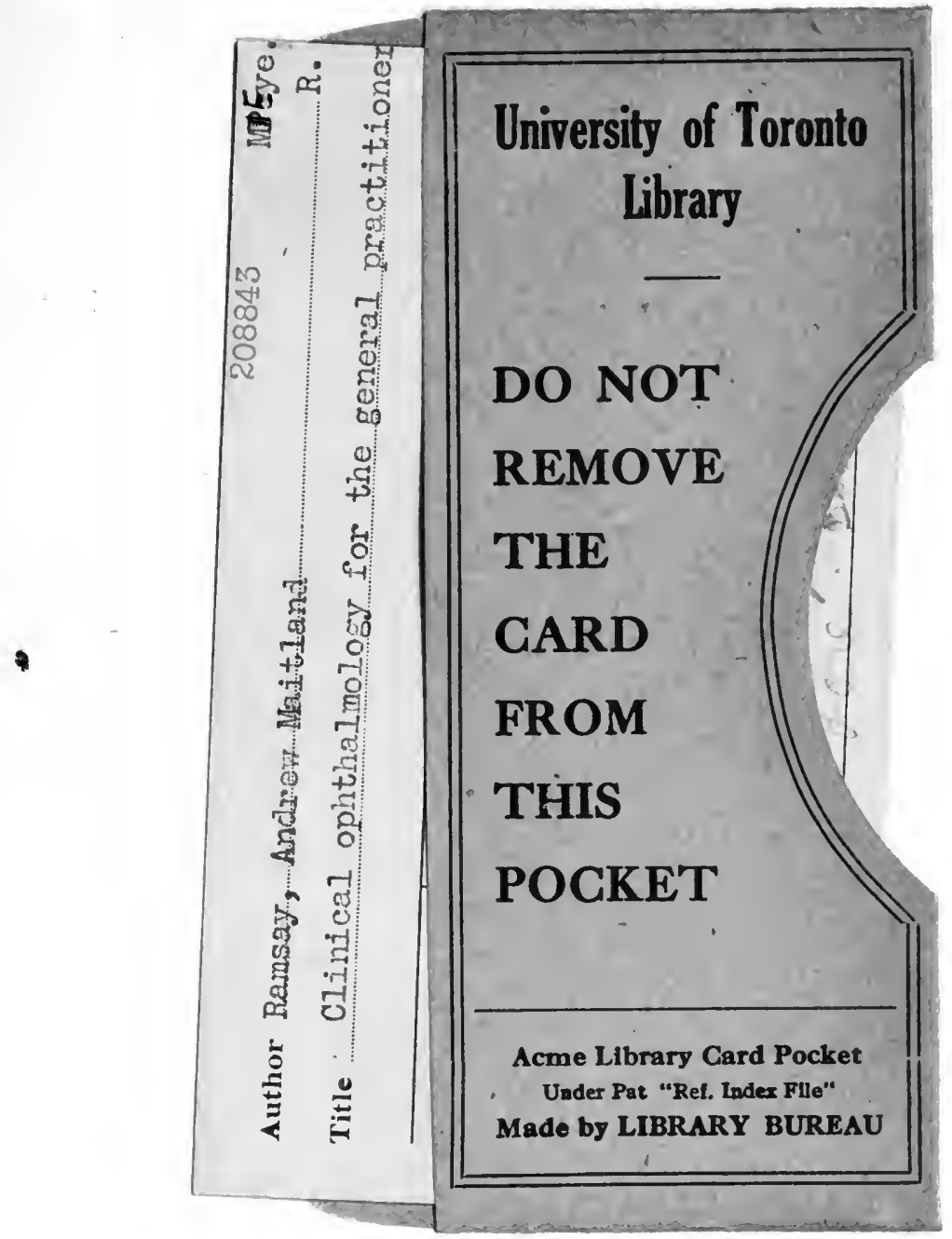


